Monatsschr Kinderheilkd 2016 · [Suppl 2]:

164:S121-S230

DOI 10.1007/s00112-016-0091-1

(c) Springer-Verlag Berlin Heidelberg 2016

CrossMark

\section{Abstracts der 42. Jahrestagung der Gesellschaft für Neonatologie und Pädiatrische Intensivmedizin (GNPI)}

\author{
2. bis 4. Juni 2016, Frankfurt a. M.
}

Wissenschaftliche Leitung:

Prof. Dr. Rolf Schlößer, Frankfurt a.M.

PD Dr. Lothar Schrod, Frankfurt-Höchst

Karin Schnabel, Frankfurt a. M.

Susanne Ressel, Frankfurt-Höchst

\title{
Freie Vorträge
}

\section{Freie Themen 01: Frühgeborene}

\section{FV01}

Senkt SMOFLipid ${ }^{\circledR}$ die Inzidenz der Cholestase bei Frühgeborenen? - Eine prospektive, randomisierte, doppelblinde Studie

A. Repa', C. Binder', M. Thanhäuser', A. Kreissl', M. Dangl', A. Berger', N. Haiden ${ }^{1}$

'Universitätsklinik für Kinder- und Jugendheilkunde, Medizinische Universität Wien, Abteilung für Neonatologie, pädiatrische Intensivmedizin und Neuropädiatrie, Wien, Österreich

Hintergrund: Frühgeborene mit extrem niedrigem Geburtsgewicht (ELBW) haben ein erhöhtes Risiko eine mit parenteraler Ernährung assoziierte Cholestase (PNAC) zu entwickeln. Bei Kindern werden Fettemulsionen die Fischöl enthalten zur Therapie der PNAC erfolgreich eingesetzt. Ob das Auftreten der PNAC durch primäre Verwendung in einer Hochrisikogruppe wie ELBW Frühgeborene verhindert werden kann ist wenig untersucht.

Fragestellung: Inwiefern reduziert eine Fischöl enthaltende Fettemulsion $\left(\mathrm{SMOFlipid}^{\circ}\right)$ im Vergleich zu einer rein auf Sojaöl basierenden Fettemulsion (Intralipid ${ }^{\star}$ ) das Auftreten von PNAC bei ELBW Frühgeborenen.

Material und Methoden: In dieser randomisierten, doppelblinden, nach Geburtsgewicht stratifizierten prospektiven Studie wurde an unserer Abteilung geborenen ELBW Frühgeborenen entweder Intralipid ${ }^{\varpi}$ (IL) oder SMOFlipid $^{\circ}$ (SMOF) für die parenterale Ernährung verabreicht. Der Hauptzielparameter war PNAC (konjugiertes Bilirubin $>1,5 \mathrm{mg} / \mathrm{dl}$ in zwei aufeinander folgenden Messungen). Zur Fallzahlberechnung wurde die PNAC Inzidenz an unserer Abteilung bei ELBW Kindern vor Studienbeginn bei Verwendung von Intralipid (25\%) herangezogen.

Ergebnisse: Insgesamt wurden 230 Kinder eingeschlossen, davon konnten 207 (IL: 101; SMOF: 106) Kinder ausgewertet werden. Die demographischen Parameter zeigten eine gleichmäßige Verteilung zwischen den beiden Gruppen. Der Hauptzielparameter PNAC zeigte keinen statistisch signifikanten Unterschied (IL: $14,9 \%$ vs. SMOF: $11,3 \% ; p=0,451$ ). Andere klinische Parameter die auch als durch die Gabe von Fischöl positiv beein- flussbar beschrieben wurden zeigten ebenfalls keinen Unterschied (therapiebedürftige ROP: IL: 9,3\% vs. SMOF: 9,7\%; $p=0,917$; Sepsis: IL 24,8 \% vs. SMOF: $23,6 \% ; p=0,844)$.

Schlussfolgerung: Die Verwendung einer Fischöl enthaltende Fettemulsion $\left(\right.$ SMOFlipid $^{\circ}$ ) hatte keinen protektiven Effekt auf das Auftreten von PNAC. Ebenso konnten wir keinen Effekt auf ROP oder Sepsis nachweisen.

\section{FV02}

\section{Körperfettanteil bei Frühgeborenen} zum Zeitpunkt der Entlassung

C. Wiechers' ${ }^{1}$ B. Luger', V. Avellina' ${ }^{2}$, S. Huber', L. Storz'ㄹ, R. Weber', M. Hallschmid ${ }^{3}$, C. Poets ${ }^{1}$

'Eberhard-Karls-Universität Universitätsklinik für Kinderheilkunde und Jugendmedizin, Neonatologie, Tübingen, Deutschland, ${ }^{2}$ Eberhard-KarlsUniversität Universitätsklinik für Kinderheilkunde und Jugendmedizin, Tübingen, Deutschland, ${ }^{3}$ Universität Tübingen, Institut für Medizinische Psychologie und Verhaltensneurobiologie, Tübingen, Deutschland

Hintergrund: Die Ernährung Frühgeborener (FG) soll Wachstum und Entwicklung wie in utero ermöglichen, da eine postnatale Wachstumsrestriktion mit schlechterer neurokognitiver Entwicklung assoziiert ist. Auch bei zufriedenstellendem d. h. percentilenparallelem Wachstum scheint jedoch die Körperzusammensetzung am errechneten Termin nicht derjenigen Reifgeborener (RG) zu entsprechen. Eine veränderte Körperzusammensetzung im frühen Kindesalter ist mit einem erhöhten Risiko für spätere Erkrankungen wie z. B. metabolischem Syndrom assoziiert. Daher ist die Entwicklung der Körperzusammensetzung bei FG zur Optimierung der Ernährung wichtig.

Fragestellung: Ziel war, den Körperfettanteil (FA) und die fettfreie Körpermasse (FFM) FG mit dem RG zu vergleichen.

Material und Methoden: In dieser Studie wurden von Juni 2014 bis August 2015 reife, gesunde Einlings-Neugeborene (RG) (Gestationsalter bei Geburt (GA): 37,0-42,0 SSW, kein Gestationsdiabetes o. ä., Alter bei Messung $<96$ h, „Referenzkohorte") und ab September 2015 FG (GA <36,0 SSW) eingeschlossen. Der FA wurde mittels Air Displacement Plethysmographie 
(PEA POD ${ }^{\circ}$ Infant Body Composition System, Cosmed, Rome, Italy) bestimmt. Die Daten sind als Median und Interquartilbereich dargestellt und wurden mittels Wilkoxon-Test ausgewertet.

Ergebnisse: In die Referenzkohorte wurden 277 RG eingeschlossen: GG $3420 \mathrm{~g}$ (3020-3670), GA 39,9 SSW (38,9-40,4), GG-SDS 0,04 (-0,61 $+0,62)$, Alter bei Messung $42 \mathrm{~h}(29-56)$, Gewichts-SDS bei Messung $-0,63$ $(-1,17--0,04)$, FA 10,8\% (7,7-13,4), FFM $2840 \mathrm{~g}(2589-3096)$.

Es wurden 30 FG untersucht: 18 FG mit einem GA von 32,0-36,0 SSW (Gruppe A): GG 1895 g (1430-2700), GA 33,9 SSW (32,0-34,7), GG-SDS $-0,85(-1,76-+0,30)$. Das postmenstruelle Alter (PMA) bei Messung betrug 36,4 SSW (34,6-36,9), die Gewichts-SDS-Differenz (Messung-Geburt) betrug $-0,31(-0,62--0,12)$, der FA $11,6 \%(10,5-13,3)$ und die FFM $1956 \mathrm{~g}$ (1828-2174). Weiterhin 12 FG mit GA <32,0 SSW (Gruppe B): GG 902 g (515-1615), GA 28,3 SSW (23,3-31,6), GG-SDS -0,70 $(-1,77--0,27)$. Das PMA bei Messung betrug 38,6 SSW $(34,7-40,4)$, die Gewichts-SDS-Differenz (Messung-Geburt) betrug +0,23 (-0,25- +0,62), der FA 18,9\% (15,5-20,9) und die FFM $2139 \mathrm{~g}(2031-2568)$. FG beider Gruppen hatten im Vergleich mit RG niedrigere GG-SDS-Werte (je $p<0,01$ ), ähnliche Gewichts-SDS-Werte bei Messung, einen höheren FA ( $p<0,01$ für Gruppe A vs. RG, $p=0,12$ für Gruppe B vs RG) und eine geringere FFM (je $p<0,01$ ).

Schlussfolgerung: Sehr unreife FG, die eine Gewichtsentwicklung in der Nähe intrauteriner Wachstumskurven aufwiesen, zeigten vor Entlassung im Vergleich zum Referenzkollektiv einen höheren FA und eine geringere FFM. Die Ursachen für die unterschiedliche Körperzusammensetzung und deren langfristige Auswirkungen sind zur Zeit noch unklar, insofern können aus diesen Daten noch keine Konsequenzen für die Ernährung FG abgeleitet werden.

\section{FV03 \\ Ergebnisse der Geburtskohortenstudie EcoCare-PIn I. Stationäre Leistungen}

K. Arnold', D. Druschke', M. Scheibe ', V. Stephan', L. Heinrich', J. Reichert ${ }^{2}$, M. Rüdiger ${ }^{2}$, J. Schmitt ${ }^{1}$

'Universitätsklinikum Carl Gustav Carus an der Technischen Universität Dresden, Zentrum für Evidenzbasierte Gesundheitsversorgung, Dresden, Deutschland, ${ }^{2}$ Universitätsklinikum Carl Gustav Carus, Klinik und Poliklinik für Kinder- und Jugendmedizin, Dresden, Deutschland

Hintergrund: Für Neugeborene mit einem Geburtsgewicht unter $1500 \mathrm{~g}$ (very low birthweight infants - VLBW) besteht ein hohes Risiko für negative psychische, physische und soziale Langzeitfolgen. Die BMBF-geförderte Geburtskohortenstudie EcoCare-PIn (Early comprehensive Care of Preterm Infants - effects on quality of life, childhood development, and healthcare utilization; Förderkennzeichen: 01GY1323) dient der versorgungsepidemiologischen Analyse von Morbiditätsmustern Frühgeborener unter Beachtung der Inanspruchnahme ausgewählter stationärer Leistungen.

Fragestellung: Wie gestalten sich Inanspruchnahme und Kosten perinataler sowie in der weiteren Kindheit auftretender Krankenhausaufenthalte für Kinder mit geringem Geburtsgewicht; lassen sich regionale Versorgungsunterschiede (Stadt-Land) beobachten?

Methoden: Analysiert wurden Routinedaten von sächsischen Kindern, die in den Jahren 2007 bis 2013 geboren wurden und im Perinatalzeitraum bzw. zusätzlich bis Ende 2013 durchgängig AOK PLUS-versichert waren; verglichen wurden Kinder mit einem Geburtsgewicht von $<1500 \mathrm{~g}$ (VLBW), 1500-2499 g (low birthweight - LBW) und $\geq 2500$ g (normal birthweight - NBW).

Ergebnisse: Zur Verfügung standen Daten von 139.383 Kindern (57\% der 2007-2013 in Sachsen geborenen Kinder). In der Perinatalzeit wurden $98 \%$ der VLBW- $(n=1235), 78 \%$ der LBW- $(n=4885)$ und $16 \%$ der NBW-Kinder $(n=17.400)$ stationär behandelt, wobei die Kosten für die initiale Behandlung im Median bei 37,667 $€(I Q R=37,683 €)$ für VLBW, bei $7389 €(I Q R=5958 €)$ für LBW- und bei $2032 €(I Q R=1219 €)$ für NBW-Kinder lagen. Eine Subgruppenanalyse mit in den Jahren 2007 oder 2008 geborenen Kindern über den Zeitraum 2009 bis $2013(n=26.667)$ ergab, dass $66 \%$ der VLBW-, 55\% der LBW- und $43 \%$ der NBW-Kinder mindestens einen Krankenhausaufenthalt hatten, wobei der Anteil dieser Kinder in allen Geburtsgewichtsgruppen im ländlichen Raum höher lag als im städtischen (VLBW 70 bzw. $63 \%$, LBW 58 bzw. 47\%, NBW 46 bzw. $35 \%)$. Hospitalisierte VLBW-Kinder hatten im betrachteten 5-Jahres-Zeitraum im Mittel 3,5 (SD 3,8) Krankenhausaufenthalte, verglichen mit 2,6 $($ SD 3,6) Aufenthalten bei LBW- und 1,9 (SD 1,9) bei NBW-Kindern. Die stationären Kosten betrugen im Median $3476 €$ (VLBW), $2556 €$ (LBW) bzw. $2229 €(\mathrm{NBW})$. In allen drei Gewichtsgruppen hatten anteilig mehr Jungen mindestens einen Krankenhausaufenthalt.

Diskussion: Die Folgen eines Geburtsgewichtes von unter $1500 \mathrm{~g}$ auf individueller Ebene und für das Gesundheitssystem sind enorm und beschränken sich nicht auf die Perinatalperiode. VLBW-Kinder weisen bis zum Schuleintrittsalter eine häufigere Inanspruchnahme stationärer Gesundheitsleistungen und erhöhte Gesundheitskosten auf. Häufigkeitsunterschiede in den post-perinatalen Hospitalisierungen von VLBW-Kindern zwischen Stadt und Land weisen auf Effizienzreserven im Gesundheitssystem hin; eine verbesserte ambulante pädiatrische Versorgung im ländlichen Raum könnte zu einer Reduktion stationärer Behandlungen bei VLBW-Kindern beitragen.

\section{FV04}

\section{Cholin und polyunsaturierte Fettsäuren in der Muttermilch} nach Frühgeburt

C. Maas', A. Franz ${ }^{2}$, A. Shunova', M. Mathes', C. Bleeker', J. Bürkle', M. Kovarova ${ }^{3}$, E. Schleicher ${ }^{3}$, C. Poets ${ }^{1}$, W. Bernhard ${ }^{1}$

'Eberhard-Karls-Universität Universitätsklinik für Kinderheilkunde und Jugendmedizin, Neonatologie, Tübingen, Deutschland, ${ }^{2}$ Eberhard-KarlsUniversität Universitätsklinik für Kinderheilkunde und Jugendmedizin, Neonatologie und CPCS, Tübingen, Deutschland, ${ }^{3}$ Medizinische Universitätsklinik - Department für Innere Medizin, Abteilung IV, Tübingen, Deutschland

Hintergrund: Cholin, Arachidonsäure (ARA) und Docosahexaensäure (DHA) sind essentiell für Gehirn- und allgemeine Entwicklung. Sie werden durch kontinuierlichen \& selektiven diaplazentaren Transport im Feten angereichert, während Linolsäure (LA) im mütterlichen Organismus retiniert wird. Bei Frühgeborenen entfällt dieser diaplazentare Transport und wird durch die nicht-selektive enterale Nährstoffresorption ersetzt. Die Versorgung ist nun von der Zusammensetzung der Muttermilch (MM) oder Formualnahrung abhängig, die hinsichtlich essentieller Nährstoffe nicht zwingend an die schnelle Wachstumsrate und das Entwicklungsstadium von Frühgeborenen adaptiert ist.

Untersuchungsziele: Bestimmung der Konzentrationen und Variabilität von cholin-, ARA- und DHA-haltigen Komponenten in MM nach Früh (FG)- und Termingeburt (TG). Design: MM-Proben $(n=354$; Tag 6-85 post partum) von 34 Müttern (PMA 30 (range: $25,4-31,0$ ) Wochen) und von Müttern nach Termingeburt $(n=9$; Tag $6-118)$. Bestimmung von Cholin und seinen Metaboliten mit Tandem-Massenspektrometrie. Bestimmung von Gesamtlipid-Fettsäuren mittels Gaschromatographie. Daten sind Mediane und 25/75-Perzentilen soweit nicht anders angegeben. Ergebnisse: Phosphocholin und Glycerophosphocholin stellten den größten Anteil der Cholinkomponenten in MM, gefolgt von freiem Cholin, Phosphatidylcholin, Sphingomyelin \& Lyso-phosphatidylcholin. Nach Termingeburt betrug das Gesamtcholin in MM 259 (211-283) $\mathrm{mg} / \mathrm{L}$, nach Frühgeburt nur 158 (131-191)mg/L $(p<0,01)$. Der jeweilige Range betrug $142-334 \mathrm{mg} / \mathrm{L}$ und $61-360 \mathrm{mg} / \mathrm{L}$. Milchfett enthielt $0,81(0,71-$ $0,93) \%$ ARA und $0,43(0,35-0,59)$ \%DHA nach Frühgeburt (Termingeburt: 0,66(0,61-0,73) \% bzw. 0,35(0,31-0,40)\%). Die Konzentrationen aller Parameter nahmen über die Zeit ab, mit einem Plateau nach 28 Tagen.

Schlussfolgerung: Nach Frühgeburt ist Gesamtcholin in der MM erniedrigt. ARA und DHA sind generell niedrig in MM. Die Konzentrationen schwanken um den Faktor 6. Diese Daten rechtfertigen weitere Studien zur kombinierten Supplementierung von MM mit Cholin (bzw. anderen Cholinderivaten), ARA and DHA zur Verbesserung des Ernährungsstatus Frühgeborener. 


\section{FV05}

Ergebnisse der Geburtskohortenstudie EcoCare-PIn III. Erste Auswertungen zu kindlicher und elterlicher Lebensqualität

M. Scheibe ', K. Arnold', D. Druschke', F. Tesch', L. Heinrich', V. Stephan', M. Rüdiger ${ }^{2}$, J. Schmitt ${ }^{1}$, J. Reichert ${ }^{2}$

'Universitätsklinikum Carl Gustav Carus an der Technischen Universität Dresden, Zentrum für Evidenzbasierte Gesundheitsversorgung, Dresden, Deutschland, ${ }^{2}$ Universitätsklinikum Carl Gustav Carus, Klinik und Poliklinik für Kinder- und Jugendmedizin, Dresden, Deutschland

Hintergrund: Für Neugeborene mit einem Geburtsgewicht unter $1500 \mathrm{~g}$ (very low birthweight infants - VLBW) besteht ein hohes Risiko für spätere negative Auswirkungen auf die elterliche wie auch kindliche Lebensqualität. Ferner wurde ein Einfluss ausgewählter Merkmale elterlichen Belastungserlebens auf die kindliche Verhaltensentwicklung beobachtet. Im Rahmen der BMBF-geförderten Geburtskohortenstudie EcoCare-PIn (Early comprehensive Care of Preterm Infants - effects on quality of life, childhood development, and healthcare utilization; Förderkennzeichen 01GY1323) können über Primärdaten einzelne Merkmale elterlicher und kindlicher Lebensqualität erfasst und hinsichtlich ihrer versorgungsepidemiologischen Bedeutung beurteilt werden.

Fragestellung: Wie gestalten sich ausgewählte Merkmale gesundheitsbezogener Lebensqualität von Kindern mit geringem Geburtsgewicht und deren Eltern?

Methoden: Für eine EcoCare-PIn-Teilstichprobe wurde die gesundheitsbezogene Lebensqualität bei Kindern (KINDL Elternversion: Ravens-Sieberer \& Bullinger, 1998) und ihren Eltern (WHOQOL BREF: Angermeyer et al., 2000) erfasst. Verglichen wurden Kinder mit einem Geburtsgewicht $<1500 \mathrm{~g}$ (VLBW), 1500-2500 g (low birth weight - LBW) und $>2500 \mathrm{~g}$ (normal birth weight - NBW).

Ergebnisse: Eine erste Analyse der Daten für 3955 Kinder (Alter 0 bis 7 Jahre) erbrachte, dass sich mit abnehmendem Geburtsgewicht auch die gesundheitsbezogene Lebensqualität der Kinder verringert. Z.B. zeigt sich insbesondere im Kita-Alter, dass VLBW- im Vergleich mit LBWund NBW-Kindern aus Sicht der Eltern in Bezug auf Peer-Kontakte eine geringere Lebensqualität haben $(p=.016 ; p=.005)$ sowie ein vermindertes psychisches Wohlbefinden aufweisen $(p=.019 ; p=.013)$; hierin unterscheiden sich LBW- und NBW-Kinder nicht. Parallel fallen Unterschiede im Selbstwerterleben auf $(p=.007 ; p<.001)$, wobei LBW- und NBW-Kinder in diesem Bereich auch differieren $(p=.021)$. Die elterliche Lebensqualität (Globalwert) ist bei VLBW- und LBW- gegenüber NBW-Kindern vermindert ( $p=.007 ; p=.003)$, scheint jedoch nicht vom Geburtsgewicht des Kindes abzuhängen (VLBW vs. LBW: n. s.). Dieses Muster zeigt sich bei allen weiteren Domänen (Psychisch, Soziale Beziehungen) mit Ausnahme der Domäne Umwelt.

Diskussion: Die Daten zeigen Einschränkungen in der Lebensqualität frühgeborener Kinder und ihrer Eltern. Bei den Kindern steht das Geburtsgewicht in einem Zusammenhang u. a. mit Teilhabeprozessen und - mehr noch - deren Einfluss auf das kindliche Selbstwerterleben. Für Eltern scheint allein die Tatsache der Frühgeburt ausschlaggebend für ihre Beurteilung ihrer Lebensqualität zu sein. Weitere Analysen werden diese Zusammenhänge näher aufklären müssen, um erforderliche Versorgungsleistungen begründen zu können.

\section{FV06}

\section{Elevated International normalised Ratio (INR) is associated with an increased risk of intraventricular haemorrhage in extremely preterm infants}

\section{S. Bates' , D. Odd ' , G. Russell', A. Heep ${ }^{2}$}

${ }^{1}$ Southmead Hospital, Neonatal Service, Bristol, United Kingdom, ${ }^{2}$ University of Bristol, Neonatal Neurology Group, Bristol, United Kingdom, ${ }^{3}$ Southmead Hospital, Department of Clinical Biochemistry, Bristol, United Kingdom

Background: The International Normalised Ratio (INR) presents a standardised method of reporting the prothrombin time (PT) and can be a surrogate marker of the vitamin $\mathrm{K}$ dependent coagulation pathways.
Objective: To study the relationship between INR measurements in the first 48 hours of life, and subsequent development of Intra Ventricular Haemorrhage (IVH) in extremely preterm infants and to evaluate the influence of early blood product administration on any association.

Study Design: Single centre retrospective, observational cohort study. All infants born at less than 28 completed weeks gestational age admitted within 48 hours of age to the tertiary NICU at Southmead hospital, Bristol, UK between March 2008 and January 2012 were eligible for this study $(n=218)$. Infants were excluded if they did not have ultrasound data recorded $(n=18)$ or had no coagulation studies performed in the first 48 hours of life $(n=91)$. Data was collected on clinical parameters of the study cohort and coagulation studies (INR, aPTT, fibrinogen). Main outcome measure was defined as the degree of IVH seen on cranial ultrasound examinations at day 7 postnatal age. (Ethical approval: North West NRES committee; 12/NW/0903).

Results: 109 infants (mean gestational age 25.2 weeks (SD 1.27)) had coagulation results available. 26/109 developed IVH. Excluded infants did not differ in major perinatal characteristics except a higher incidence of complete antenatal steroids $(p=0.005)$. An elevated INR in the first 48 hours of life was significantly related to the degree of IVH in the studied patients $(p=0.003)$. In the logistic regression model adjusting for gestation, birth weight and gender increasing INR was associated with increased risk of a severe IVH (OR $6.50(1.65-25.62), p=0.008)$. INR remained strongly associated with severe IVH in infants who did not receive blood products (OR 64.60 (1.35-3081.25)), $p=0.035$ ), but not in those who did (OR 2.93 $(0.67-12.71),(p=0.151)$ (pinteraction $=0.086)$.

Conclusion: An elevated INR in the first 48 hours of life is associated with an increased risk of severe IVH at day 7 postnatal age and this relationship may be attenuated by the early administration of blood products.

\section{FV07}

\section{Gibt es einen Anstieg der Frühgeborenen-Retinopathie} durch veränderte Sauerstoff-Leitlinien? Vorläufige Daten aus der Neonatalerhebung 2010 bis 2015

\section{R. Hentschel', J. Arand', T. Böhler', M. Mohrmann², P. Ruef', I. Bruder ${ }^{2}$}

${ }^{1}$ Institut/Klinik: Universitätsklinikum Freiburg, Zentrum für Kinder- und Jugendmedizin, Funktionsbereich Neonatologie/Päd. Intensivmedizin, Freiburg, Deutschland, ${ }^{2}$ AG Neonatologie der GeQiK Baden-Württemberg, Stuttgart, Deutschland, ${ }^{3}$ Heilbronn

Hintergrund: Der optimale Zielbereich der Sauerstoffsättigung bzw. des $\mathrm{paO}_{2}$ für Frühgeborene, ggf. abgestuft nach Gestationsalter und postnatalem Alter sowie relevanten zusätzlichen Erkrankungen, ist bis heute nicht sicher definiert worden. Mangels einer eigenen deutschen Leitlinie orientieren sich die meisten Kliniken an den Ergebnissen mehrerer randomisierter internationaler Studien aus den Jahren 2010 bis 2013 (SUPPORT, BOOST II, COT). Aus einer Metaanalyse dieser Daten in der NEOPROM-Studie ergibt sich die Empfehlung, den Zielbereich der Sauerstoffsättigung bei 90-95\% zu halten (O. D. Saugstad 2014). Die Rationale für diese Empfehlung beruht auf einer signifikanten Reduktion von Mortalität $(-30 \%)$ und NEC-Rate $(-20 \%)$, wenngleich dadurch die Rate an Frühgeborenen-Retinopathien (ROP) ansteigt (+35\%). Da die Risikoadjustierung für jedes Bundesland und jede einzelne Klinik Bezug nimmt auf die Gesamtrate in Deutschland (DE) ist ein bundesweiter Anstieg nur durch Analyse der Rohdaten erkennbar.

Fragestellung: Ist die Rate an schwerer ROP $>{ }^{\circ} 2$ in Baden-Württemberg (BW) oder in DE insgesamt signifikant angestiegen, seitdem die Daten internationaler Studien zur Optimierung der Sauerstoffgrenzen bei Frühgeborenen bekannt wurden und deren Ergebnisse mutmaßlich in den Kliniken umgesetzt wurden?

Material und Methoden: Analyse der ROP-Raten an Hand der Daten der bundesweiten Neonatalerhebung durch das Aqua-Institut (DE) und/oder der baden-württembergischen Neonatalerhebung durch die GeQiK (BW) für den Zeitraum 2010 bis 2015.

Ergebnisse: In BW beobachteten wir 2014 einen sprunghaften Anstieg der schweren ROP-Fälle um $84 \%$, während die Zahl in den Vorjahren kon- 


\begin{tabular}{|c|c|c|c|c|c|c|c|c|c|c|c|c|}
\hline Jahrgang & 2010 & & 2011 & & 2012 & & 2013 & & 2014 & & 2015 & \\
\hline Kohorte & BW & DE & BW & $\mathrm{DE}$ & BW & $\mathrm{DE}$ & BW & $\mathrm{DE}$ & BW & $\mathrm{DE}$ & BW & DE \\
\hline Fälle mit ROP $>{ }^{\circ} 2(n)$ & 53 & & 60 & & 61 & 369 & 63 & 347 & 116 & 465 & & \\
\hline $\begin{array}{l}\text { Fälle mit ROP }>^{\circ} 2 \\
\text { zwischen } 24 \text { und } 32 \text { SSW } \\
\text { (bzw. }<1500 \text { g) }(\%)\end{array}$ & & & 3,9 & 3,0 & 3,3 & 3,4 & 4,2 & 3,2 & 5,7 & 3,3 & & \\
\hline untersuchte Patienten $(n)$ & 1400 & & 1337 & & 1307 & 10830 & 1346 & 11226 & 1447 & 11758 & & \\
\hline Fgb. $>22<29$ SSW $(n)$ & 640 & & 568 & & 546 & & 503 & & 621 & & & \\
\hline $\begin{array}{l}\text { Fgb. }>22<29 \mathrm{SSW} \text { in Re- } \\
\text { lation zu allen erfassten } \\
\text { Neugeborenen (\%) }\end{array}$ & 5,3 & & 4,8 & & 5,0 & & 4,4 & & 5,6 & & & \\
\hline $\begin{array}{l}\text { Fgb. }>22<33 \mathrm{SSW} \text { in Re- } \\
\text { lation zu allen erfassten } \\
\text { Neugeborenen (\%) }\end{array}$ & 13,8 & & 13,7 & & 14,2 & & 13,7 & & 16,1 & & & \\
\hline $\begin{array}{l}\text { ROP-Befund lag bei Auf- } \\
\text { nahme bereits vor (alle } \\
\text { Stadien) (\%) }\end{array}$ & & & & & 13,9 & 11,2 & 17,5 & 10,1 & 15,4 & 11,3 & & \\
\hline
\end{tabular}

stant war. In DE fiel dieser Anstieg 2014 schwächer aus. Zugleich stieg aber auch der Anteil der Frühgeborenen mit dem höchsten Risiko in beiden Kohorten leicht an. Der Anteil der Patienten, die bereits bei Aufnahme in die Klinik einen auffälligen ROP-Befund hatten, blieb konstant.

Schlussfolgerung: Vorbehaltlich einer endgültigen statistischen Analyse unter Einbeziehung von zurzeit noch fehlenden Daten aus BW und DE für das Jahr 2015 und einer Risikoadjustierung deutet vieles auf einen signifikanten Anstieg der schweren Retinopathien in Deutschland und insbesondere in BW hin. Da gleichzeitig der Anteil sehr unreifer Frühgeborener ebenfalls anstieg ist die absolute Fallzahl jedoch mit Vorsicht zu interpretieren. Allerdings war der prozentuale Anstieg der ROP-Fälle größer als der Anstieg der Hoch-Risikogruppe. Eventuell ist auch die ROP-Erfassung in den Kliniken besser geworden. Ob diese tatsächlich ihre Sauerstoffgrenzen im untersuchten Zeitraum verändert haben, oder ob andere Ursachen zu dem Anstieg führten, kann leider derzeit noch nicht sicher festgestellt werden. Ein Anstieg der Doppelerfassung durch Weiterverlegung ist unwahrscheinlich, da der Anteil der Zweitbefunde („lag bereits vor") annähernd gleich blieb.

\section{Freie Themen 02: Erstversorgung}

\section{FV08 \\ Experimentell induzierte Wehen zur Stimulation einer milden fetalen Stressantwort vor elektivem Kaiserschnitt}

S. Wellmann 1', A. Koslowski², K. Spanaus ${ }^{3}$, R. Zimmermann ${ }^{2}$, T. Burkhardt ${ }^{2}$ ${ }^{1}$ Universitäts-Kinderspital beider Basel (UKBB), Abteilung für Neonatologie, Basel, Schweiz, ${ }^{2}$ Universitätsspital Zürich, Klinik für Geburtshilfe, Zürich, Schweiz, ${ }^{3}$ Universitätsspital Zürich, Institut für Klinische Chemie, Zürich, Schweiz

Hintergrund: Die Plasmaspiegel des hochsensitiven Stressmarkers Copeptin, ein Spaltprodukt der Arginin-Vasopressin (AVP) Synthese, sind in Nabelschnurblutproben von Neugeborenen nach Spontangeburt massiv erhöht im Vergleich zu Kaiserschnittentbindungen. Verschiedene Stressoren wie Sauerstoffmangel bewirken eine Ausschüttung von Copeptin und AVP. Es ist aber unbekannt, ob milde Wehen ohne Zeichen einer fetalen Hypoxie ausreichend sind, um eine fetale Copeptinausschüttung zu induzieren. Fragestellung: Bewirkt eine milde Wehentätigkeit per se den Anstieg fetaler Copeptinspiegel?
Material und Methoden: In diese prospektive, randomisierte und kontrollierte Studie wurden Frauen mit Einlingsschwangerschaft und geplanter elektiver Kaiserschnittentbindung nach 36 vollendeten Schwangerschaftswochen eingeschlossen, sofern weder Wehen noch ein Blasensprung zum Zeitpunkt des Studienbeginns vorlagen (Studienregistrierung ClinicalTrials.gov NCT01962701). Die Studienintervention bestand aus einer intravenösen Oxytocingabe, beginnend mit 5 IU Oxytocin/500 ml Ringerlaktatinfusion und einer Laufgeschwindigkeit von $12 \mathrm{ml} / S t u n d e$, welche alle 10 Minuten verdoppelt wurde bis 3 Wehen pro 10 Minutenintervall im Kardiotokograph aufgezeichnet wurden. Die Kontrollgruppe erhielt eine Ringerlaktatinfusion. Die Schnittentbindung folgte 1-2 Stunden anschließend. Primärer Endpunkt war der Copeptinspiegel im arteriellen Nabelschnurblut unmittelbar nach Geburt. Sekundäre Endpunkte waren laborchemische und klinische Parameter der fetalen und maternalen Gesundheit, insbesondere der arterielle Nabelschnur-pH, die Primäradaptation und das Abklingen der experimentell erzeugten Wehen nach Ende der Oxytocininfusion.

Ergebnisse: 156 Frauen wurden insgesamt in die Studie eingeschlossen, je 78 in die Interventions- und Kontrollgruppe, wovon 12 respektive 11 ausgeschlossen werden mussten aufgrund zu geringer Nabelschnurblutmengen. Die Copeptinspiegel im arteriellen Nabelschnurblut waren in der Interventionsgruppe dreimal höher als in der Kontrollgruppe mit einem Median von 22,2 (Bereich 3,2-2319) gegenüber 7,39 (2,5-344,6) pmol/L, $p<0,001$. Zwei Kinder der Interventionsgruppe (3\%) benötigten Atemhilfe bei Flüssigkeitslunge gegenüber 4 Kindern der Kontrollgruppe (6\%), $p=0,414$. In keinem Fall musste die Oxytocininfusion vorzeitig gestoppt werden, es kam weder zu schmerzhaften Wehen noch pathologischen Befunden in der Kardiotokographie. Kein Kind hatte eine perinatale Azidose und nur 36 der 66 Schwangeren der Interventionsgruppe (55\%) bemerkten die experimentell erzeugten Wehen

Schlussfolgerung: Milde Wehen vor elektiver Schnittentbindung reichen aus, um eine milde fetale Stressantwort ohne Zeichen einer fetalen Hypoxie zu erzeugen. Die exzellente Verträglichkeit der Studienintervention bildet die Basis für eine neue Studie mit der Hypothese, dass künstlich induzierte Wehen vor einer elektiven Schnittentbindung die Rate an neonatologischen Hospitalisationen reduziert. 


\section{FV09}

Der Einfluss einer pränatalen Caffeine Administration auf die kardiovaskuläre Adaptation nach der Geburt.

C. Binder ${ }^{1}$, K. Crossley ${ }^{2}$, A.te Pas ${ }^{3}$, G. Polglase ${ }^{2}$, D. Blank ${ }^{2}$, V. Zahra ${ }^{2}$, A. Moxham ${ }^{2}$, K. Rodgers', S. Hooper ${ }^{2}$

${ }^{1}$ Medizinische Universität Graz, Graz, Österreich, ${ }^{2}$ Hudson Institute of Medical Research, Clayton, Australien, ${ }^{3}$ Leiden University Medical Centre, Leiden, Niederlande

Hintergrund: Caffeine, ein Adenosin Rezeptor Antagonist, ist eines der am häufigsten benutzten Medikamente im Bereich der Neonatologie und wird zur Therapie von Frühgeborenenapnoen eingesetzt. Viele Studien untersuchten die Wirksamkeit von Caffeine in Bezug auf die Prävention von Apnoen, es gibt jedoch nur wenig Wissen über die hämodynamischen Effekte dieser Therapie, vor allem während der Adaptationsphase. Das Ziel dieser Studie war es, den Effekt einer pränatalen Caffeine Administration auf die Herzfunktion und auf den pulmonalen Blutfluss während der Adaptationsphase, im Speziellen zum Zeitpunkt des Abklemmens der Nabelschnur, von beatmeten, frühgeborenen Lämmern zu untersuchen.

Methoden: Diese prospektive Interventionsstudie wurde an frühgeborenen Lämmer $(\approx 125$ Tage Gestationsalter) durchgeführt. Kurz vor einer geplanten Kaiserschnittgeburt wurden den Lämmern, in utero, Blutdruckkatheter (A. carotis) und Blutflusssonden (A. pulmonalis und A. carotis) eingesetzt. Vor dem Abklemmen der Nabelschnur wurden sie intubiert und es wurde sowohl ihnen, als auch dem Muttertier, eine intravenöse Caffeinelösung (10 mg/kg Caffeine-Base) oder Kochsalzlösung über 15 Minuten verabreicht. Danach wurde die Nabelschnur abgeklemmt. Nach einer Beobachtungsperiode von 2 Minuten wurde eine Sustained Inflation ( $35 \mathrm{~cm} \mathrm{H}_{2} \mathrm{O}$ für 30 Sekunden) durchgeführt.

Ergebnisse: Insgesamt wurden 11 Lämmer, 6 in der Caffeine und 5 in der Saline Gruppe, untersucht. Sowohl vor bzw. nach der Infusionstherapie, als auch vor bzw. nach dem Abklemmen der Nabelschnur zeigte sich zwischen den Gruppen kein signifikanter Unterschied hinsichtlich der Herzfrequenz (HR), des mittleren arteriellen Carotisblutdrucks (meanCBP), des pulmonalarteriellen Blutflusses (PBF) und des Blutflusses in der A. carotis (CBF), siehe $-A b b$. FV09. Weiter kam es auch während der ersten 10 Herzschläge nach dem Abklemmen der Nabelschnur zu keinen signifikanten Unterschieden zwischen den Gruppen hinsichtlich dieser Parameter. Schlussfolgerung: Eine pränatale Caffeine Administration hat keinen signifikanten Effekt auf das kardiovaskuläre System kurz nach der Geburt.
FV10

Verhindert eine höhere Raumtemperatur des Erstversorgungsraums die Auskühlung von Frühgeborenen?

J. Nufer', P. Neuberger', M. Vochem ${ }^{2}$

'Olgahospital Päd. Zentrum, Neonatologie, Stuttgart, Deutschland, 2Olgahospital, Neonatologie, Stuttgart, Deutschland

Einleitung: Während der Erstversorgung sind Neugeborene der Gefahr der Auskühlung ausgesetzt; besonders für sehr kleine Frühgeborene ist ein Zusammenhang zwischen Körpertemperatur und Kurzzeitoutcome belegt. Um Auskühlung zu verhindern werden während der Erstversorgung diverse Maßnahmen (Wärmezufuhr durch Strahler und Heizmatte, sterile Tüte, warme Tücher, Mützen etc.) ergriffen. Der Einfluss der Raumtemperatur des Erstversorgungsraums (EVR) auf die Aufnahmetemperatur von Frühgeborenen wurde kaum untersucht. Durch Erhöhung der Raumtemperatur von $28^{\circ} \mathrm{C}$ auf $34^{\circ} \mathrm{C}$ im Rahmen eines Neubaus hatten wir die Möglichkeit, den Effekt der Temperaturerhöhung auf die Aufnahmetemperatur der Frühgeborenen in zwei Untersuchungszeiträumen zu vergleichen.

Methoden: Verglichen wurden Frühgeborene mit einem Geburtsgewicht (GG) < 1500 g, die im Zeitraum zwischen Juli 2014 bis Oktober 2015 per sectio caesarea im Kreißsaal der Frauenklinik Stuttgart zur Welt kamen (Temperatur im EVR $34^{\circ} \mathrm{C}$ ), mit einer Kontrollgruppe von Frühgeborenen, die zwischen Juli 2012 und Oktober 2013 in der Frauenklinik Stuttgart, Bad-Cannstatt (Temperatur im EVR $28^{\circ} \mathrm{C}$ ) geboren wurden. Ausgeschlossen wurden Extrem-Frühgeborene mit Palliativbehandlung und Kinder, die den Eltern zum Bonden gegeben wurden. In beiden Zeiträumen waren die Massnahmen zum Wärmeerhalt identisch: Übernahme des Kindes im vorgewärmtem Handtuch, Verbringen in sterile Tüte bei GG $<1000 \mathrm{~g}$, Wärmebett mit Gelmatratze und Strahler, Zudecken mit warmen Tüchern, Mütze, angewärmtes Atemgas aus F120-Respirator. Die Aufnahmetemperatur wurde durch rektale Messung bei Aufnahme auf der Intensivstation ermittelt.

Ergebnisse: In Gruppe 1 - EVR $34^{\circ} \mathrm{C}$ - erfüllten 111 Frühgeborene die Kriterien, in Gruppe 2 - EVR $28^{\circ} \mathrm{C}$ - 91 Frühgeborene. Mittleres Geburtsgewicht (1075 g vs $1091 \mathrm{~g}$ ) sowie Gestationsalter $(29+1$ SSW) war in beiden Gruppen vergleichbar. Die mittlere Aufnahmetemperatur der Gruppe 1 betrug $36,87^{\circ} \mathrm{C}$ (Standardabweichung $0,56^{\circ} \mathrm{C}$ ). 4 Kinder $(3,6 \%)$ hatten eine Aufnahmetemperatur von $<36,0^{\circ} \mathrm{C}$. 14 Kinder $(12,6 \%)$ wiesen eine Temperatur $>37,5^{\circ} \mathrm{C}$ auf. Die mittlere Aufnahmetemperatur der Gruppe 2 war $36,51^{\circ} \mathrm{C}$ (Standardabweichung $0,61{ }^{\circ} \mathrm{C}$ ). 10 Kinder $(11 \%)$ hatten eine Aufnahmetemperatur von $<36,0^{\circ} \mathrm{C}$. 1 Kind ( $\left.1 \%\right)$ hatte eine Temperatur $>37,5^{\circ} \mathrm{C}$.

Fazit: Bei einer Raumtemperatur im Erstversorgungsraum von $34^{\circ} \mathrm{C}$ verglichen mit $28^{\circ} \mathrm{C}$ war der Anteil hypothermer Frühgeborener signifikant niedriger (3,6\% vs $11 \%)$, der Anteil von FG mit Hyperthermie jedoch auch signifikant höher (12,6\% vs 1,1\%).

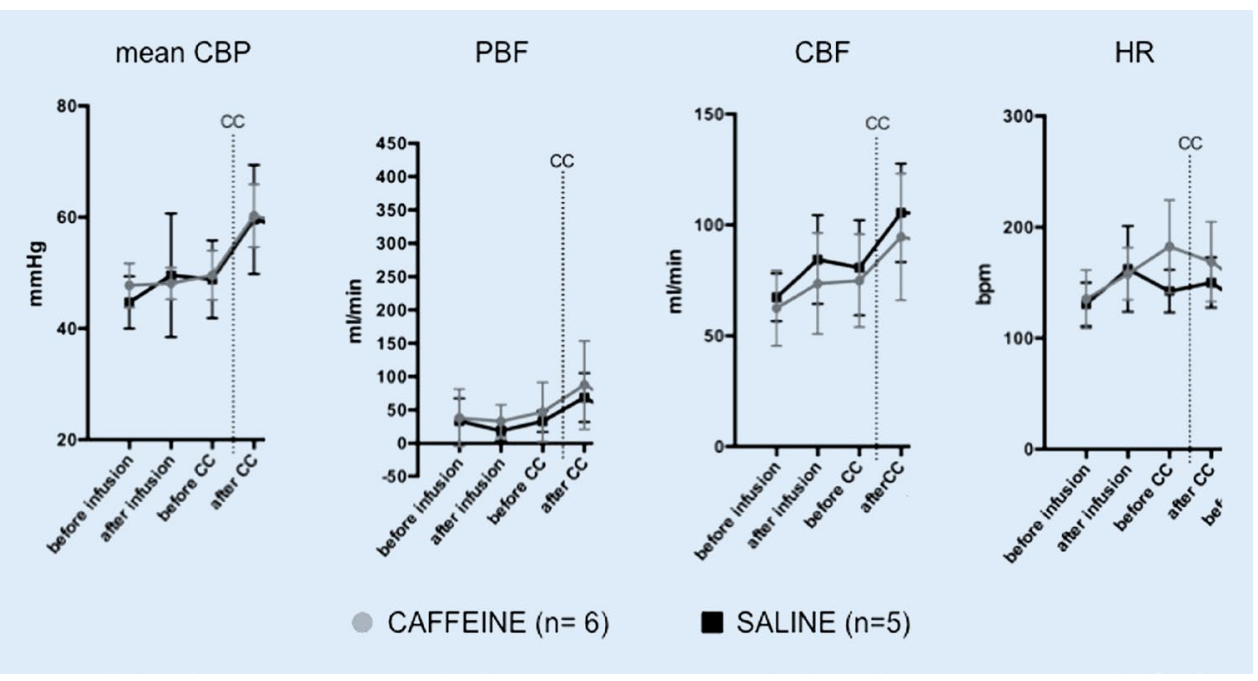

Abb. FV09: $>$ Angegebene Werte sind Mittelwerte ( \pm Standardabweichung) 


\section{FV11}

\section{Peripherer venöser Zugang bei Frühgeborenen während der Erstversorgung}

\section{N. Baik', A. Karnberger', L. Mileder', B. Schwaberger', C. Binder-Heschl', B. Urlesberger', G. Pichler ${ }^{1}$ \\ 'Universitätsklinik für Kinder- und Jugenheilkunde, Klinische Abteilung für Neonatologie, Graz, Österreich}

Hintergrund: Das Legen eines peripher venösen Zuganges für die Verabreichung von Medikamenten ist eine häufig notwendige Intervention während der Erstversorgung von Frühgeborenen unmittelbar nach der Geburt. Neben den zentralvenösen Zugängen wird hier häufig auch der peripher venöse Zugang gewählt.

Zielsetzung: Ziel dieser Studie war es, zu analysieren, wann während der Erstversorgung bei Frühgeborenen ein peripher venöser IV-Zugang geleg werden konnte, wie viele Punktionsversuche benötigt wurden und ob das Legen des peripher venösen Zuganges einen Effekt auf die Vitalparameter von Frühgeborenen hatte.

Material und Methode: Es wurde eine Analyse von Videoaufzeichnungen, die im Rahmen von prospektiv durchgeführten Beobachtungs-Studien an der Abteilung für Neonatologie/Medizinischen Universität Graz bei Frühgeborenen während der Erstversorgung gemacht wurden, durchgeführt Periphere arterielle Sauerstoffsättigung $\left(\mathrm{SpO}_{2}\right)$ und die Herzfrequenz (HF) wurden kontinuierlich mittels Pulsoxymetrie gemessen. Zerebrale Oxygenierung (cTOI) wurde mittels Nah-infrarot Spektroskopie (NIRS) gemessen. Der NIRS Sensor wurde an der Stirn rechts frontal angelegt. Neben Monitoringparameter wurden auch Zeitpunkt und Versuche von Legen eines peripheren venösen Zuganges erhoben. Herzfrequenz, $\mathrm{SpO}_{2}$ und cTOI wurden vor, nach und während Manipulation gemessen und verglichen. Ergebnisse: Insgesamt wurden 72 Frühgeborene in die Studie inkludiert Das mittlere Gestationsalter betrug 30,9 $\pm 2,7$ Wochen und das mittlere Geburtsgewicht $1517 \pm 523$ Gramm. Es wurde bei allen Frühgeborenen ein peripher venöser Zugang im Rahmen der Erstversorgung gelegt. Der durchschnittliche Zeitpunkt von peripheren venösen Zugang war 6,9 \pm 4.4 Minuten nach der Geburt. Im Median war 1 Versuch (IQR 1-2) notwendig, um einen peripheren IV-Zugang zu legen. $\mathrm{SpO}_{2}$ (vor: $82,8 \% \pm 18,1 \%$ versus nach: $87,1 \% \pm 10,5 \%$ ) und cTOI (vor: $59,6 \% \pm 17,4 \%$ versus nach $63,2 \% \pm 16,7 \%)$ stiegen vor, während und nach der Manipulation statistisch signifikant an, jedoch entsprechend der Perzentilen in den ersten 10 Minuten nach der Geburt. Die Herzfrequenz (vor: 149,6/min $\pm 26,8 / \mathrm{min}$ versus nach: $150,4 / \mathrm{min} \pm 24,5 / \mathrm{min}$ ) änderte sich nicht signifikant.

Schlussfolgerung: In dieser Beobachtungsstudie konnte gezeigt werden, dass der periphere venöse Zugang bei Frühgeborenen während der Erstversorgung rasch gelegt werden können und die Adaptation nicht zu beeinflussen scheint.

\section{FV12}

\section{Koordination von Herzdruckmassage und Beatmung bei der simulierten Reanimation von Neugeborenen. Vergleich von Beatmungsbeutel, Beatmungsgerät und T-Stück-System.}

M. Schmid', M. Hoenes' ${ }^{2}$, J. Essers' ${ }^{2}$, M. Mendler' ${ }^{2}$, B. Mayer ${ }^{3}$, H. Hummler ${ }^{2}$ ${ }^{1}$ Klinik für Neonatologie, Universitätsspital Zürich, Zürich, Schweiz, ${ }^{2}$ Klinik für Kinder- und Jugendmedizin, Universitätsklinik Ulm, Sektion für Neonatologie und pädiatrische Intensivmedizin, Ulm, Deutschland, ${ }^{3}$ Universität Ulm, Institut für Epidemiologie und Medizinische Biometrie, Ulm, Deutschland

Einleitung: Aktuelle Richtlinien zur Neugeborenen-Reanimation empfehlen beim nicht intubierten Kind die Koordination von 90 Thoraxkompressionen und 30 Beatmungshüben (Verhältnis 3:1) mit einer Frequenz von 120 Ereignissen pro Minute. Die große Bedeutung der Beatmung ist durch die meist hypoxämische Ursache von Atem- und Kreislaufstillstand beim Neugeborenen begründet. Entsprechend bedeutsam ist es, dass die Beatmungshübe effizient zur Belüftung der Lunge führen. Die Koordination von Thoraxkompression und Beatmung ist aber bei zwei Wechseln und vier Ereignissen pro zwei Sekunden nicht einfach und es besteht die Ge- fahr, dass Beatmungen durch Thoraxkompressionen gestört werden oder dass unnötige Pausen entstehen. Theoretische Vorteile von T-Stück-System und Beatmungsgerät bestehen in der definierten und effektiven Anwendung von endexspiratorischem Druck und Spitzendruck. Beim Beatmungsgerät können außerdem Frequenz und Inspirationszeit festgelegt werden und als Taktgeber für die Koordination dienen. In einer randomisierten Cross-over-Studie untersuchten wir die Koordination zwischen Beatmungshüben und Thoraxkompressionen mit allen drei Geräten bei der simulierten Reanimation von Neugeborenen.

Methoden: Teams aus je einem Arzt und einer Hebamme oder Pflegenden wurden unvorbereitet in den Kreißsaal gerufen, wo sie auf der Erstversorgungseinheit ein Neugeborenen-Modell vorfanden. Sie wurden gebeten, das Modell für jeweils 30 Sekunden zu reanimieren und danach die Rollen (Beatmung und Thoraxkompressionen) zu tauschen. Dann wurde die Sequenz mit den beiden anderen Geräten wiederholt. Die Reihenfolge der Geräte folgte dem Zufallsprinzip. Die gesamte Sequenz wurde dann wiederholt, wobei der Person in der Kopfposition (Beatmung) Fragen aus einem Quizspiel gestellt wurden. Die Software des Modells zeichnete Beatmungen und Thoraxkompressionen millisekundengenau auf. Primäre Zielgrösse war die Anzahl der ungestörten Beatmungshübe pro $30 \mathrm{Se}$ kunden, definiert als Abstand zwischen Beginn Ventilation und Beginn nachfolgender Kompression $>=0,35 \mathrm{sec}$ und Maximum der vorhergenden Kompression vor Beginn der Ventilation. Korrektes Tidalvolumen war gemäß den Eigenschaften des Modells definiert als 20-35 ml. Die Auswertung erfolgte mit einem gemischt linearen Modell zur Abbildung der Wiederholungsmessungsstruktur.

Ergebnisse: 18 Teams nahmen Teil. Die Zahl (Standardabweichung) der ungestörten Beatmungshübe pro 30 Sekunden war 12,8 $(2,8), 11,6(3,6)$ und 10,1 $(3,7)$ für Beatmungsbeutel, Beatmungsgerät und T-Stück-System $(p<0,0001)$. Die höchste Anzahl an Beatmungshüben mit korrektem Tidalvolumen wurde mit 12,0 (5,2) dem Beatmungsgerät erzielt (Beatmungsbeutel 3,8 (4,7), T-Stück-System 8,1 (3,9)). Berufserfahrung, Berufsgruppe und simultan gestellte Fragen wirkten sich nicht signifikant aus. Diskussion: Die beste Koordination war mit dem Beatmungsbeutel möglich, die schlechteste mit dem T-Stück-System. Die höchste Anzahl Atemhübe mit adäquatem Tidalvolumen gelang mit dem Beatmungsgerät.

\section{FV13}

\section{Prospektives monatliches Monitoring von Qualitätsindikatoren} der perinatalen und neonatologischen Versorgung von VLBW im Rahmen einer Perinatalkonferenz nach GBA-Beschluss

\section{Mögel', A. Koch', K. Nitzsche², M. Rüdiger ${ }^{1}$}

'Universitätsklinikum Carl Gustav Carus, Klinik und Poliklinik für Kinder- und Jugendmedizin, Abteilung Neonatologie/pädiatrische Intensivmedizin, Dresden, Deutschland, ${ }^{2}$ Universitätsklinikum Carl Gustav Carus, Klinik und Poliklinik für Frauenheilkunde und Geburtshilfe, Dresden, Deutschland

Einleitung: In der Neonatologie steht die Reduktion der neonatalen Morbidität der kleinen Frühgeborenen im Vordergrund. Der Gemeinsame Bundesausschuss (GBA) fordert im Rahmen seiner Empfehlungen zur Versorgung von Früh- und Neugeborenen die Durchführung einer interdisziplinären Fallkonferenz, bisher gibt es hierzu kein strukturiertes und evaluiertes Konzept.

Methoden: Nach Entwicklung einer strukturierten, wöchentlichen interdisziplinären Perinatalkonferenz im Jahr 2014, wurde diese 2015 in die klinische Routine implementiert und um eine monatliche Auswertung wichtiger perinatologischer Qualitätsparameter erweitert, um so eine prospektive Qualitätsanalyse der perinatalen Versorgung von Frühgeborenen $<1500 \mathrm{~g}(\mathrm{VLBW})$ zu ermöglichen. Die Qualitätsindikatoren wurden in perinatale Merkmale (Geburt per Sectio, Geburt im Frühdienst, Amnioninfektionssyndrom), in die postnatale Versorgung (Surfactantgabe, INSURE-Rate, Bondingrate, Aufnahmetemperatur) und in die neonatale Morbidität in der 1. Lebenswoche (IVH, Katecholamintherapie, neonatale Infektion, Dauersedierung, therapiepflichtiger PDA) unterteilt. Dafür wurden Zielwerte definiert und deren Erreichen im Rahmen einer monatlichen Auswertung diskutiert. 
Ergebnisse: In den Jahren 2014/2015 konnte im Rahmen von jeweils 35 strukturierten Perinatalbesprechungen für ca. $80 \%$ der VLBW die perinatologische Versorgung innerhalb der ersten Lebenswoche evaluiert werden. Bei vergleichbaren Patientenkollektiven wurden im Rahmen der beschriebenen Qualitätssicherungsmaßnahme folgende Veränderungen der perinatologischen Versorgung bzw. des neonatalen Outcomes in der 1. Lebenswoche beobachtet: die Sectiorate im Frühdienst wurde bei $50 \%$ stabilisiert (2014: $51 \%$ vs. 2015: 53\%), die Häufigkeit der Surfactant-Applikationen (2014: $82 \%$ vs. 2015: 69\%) und die Rate an Hirnblutungen (2014: $21 \%$ vs. $2015: 17 \%$ ) wurden reduziert, die Bonding-Rate im Kreissaal wurde nahezu verdoppelt (2014: $18 \%$ vs. $2015: 32 \%)$, die Kreislaufunterstützung mittels Katecholamine (2014: $13 \%$ vs. 2015: $7 \%$ ) und die Sedierung per Dauerinfusion (2014: $25 \%$ vs. 2015: 11\%) wurden deutlich reduziert. Somit ist eine Tendenz zur Verbesserung der perinatologischen Versorgung zu beobachten. Aktuell steht als wichtiger Qualitätsparameter die Aufnahmetemperatur im Fokus der Betrachtungen.

Diskussion: Durch interdisziplinären Austausch und kontinuierliches Feedback im Sinne des PDCA-Zyklus (Plan-Do-Check-Act) konnte im Rahmen der Perinatalbesprechung eine Verbesserung der Versorgungsqualität und der kurzfristigen Morbidität erreicht werden. Mittels Implementierung des Videofeedbacks der Erstversorgung ist eine Erweiterung der beschriebenen perinatalen Qualitätssicherungsmaßnahme möglich. Zusammenfassung: Eine strukturierte interdisziplinäre Perinatalbesprechung nach GBA-Beschluss kann als sinnvolle und wirksame Maßnahme zur Qualitätssicherung der perinatalen Versorgung von Frühgeborenen bewertet werden.

\section{FV14}

\section{Zerebrale regionale Gewebe-Sauerstoffsättigung als Monitoringparameter während der Surfactant-Administration}

K. Hanke', P. Paul ${ }^{2}$, C. Bösche ${ }^{3}$, C. Schrapel ${ }^{3}$, W. Göpel', E. Herting ${ }^{5}$, C. Wieg ${ }^{6}$, C. Härtel ${ }^{7}$

'Universitätsklinikum Schleswig Holstein, Campus Lübeck, Klinik für Kinderund Jugendmedizin, Lübeck, Deutschland, ${ }^{2}$ Universitätsklinikum SchleswigHolstein, Klinik für Kinder- und Jugendmedizin, Abteilung Neonatologie, Lübeck, Deutschland, ${ }^{3}$ Klinikum Aschaffenburg, Kinderklinik, Aschaffenburg, Deutschland, ${ }^{4}$ Universitätsklinikum Schleswig-Holstein, Klinik für Kinderund Jugendmedizin, Lübeck, Deutschland, ${ }^{5}$ Universitätsklinikum SchleswigHolstein Campus Lübeck, Direktor der Klinik für Kinder- und Jugendmedizin, Lübeck, Deutschland, ${ }^{6}$ Klinikum Aschaffenburg, Neonatologie und pädiatrische Intensivmedizin, Aschaffenburg, Deutschland, ${ }^{7}$ Universitätsklinikum Schleswig Holstein/Campus Lübeck, Klinik für Kinderund Jugendmedizin, Lübeck, Deutschland

Fragestellung: Surfactant-Therapie ist ein wichtiges Element in der Behandlung des schweren Atemnotsyndroms. Die Applikation von Surfactant kann zu unerwünschten Nebenwirkungen (u. a. Bradykardie, Hypoxie, Atemwegsobstruktion) führen, weshalb ein optimales Monitoring notwendig ist. Ziel unserer Studie ist die Evaluation der zerebralen regionalen Gewebe-Sauerstoffsättigung $\left(\mathrm{rStO}_{2}\right)$ als zusätzlichen Parameter der Überwachung von Frühgeborenen in den ersten 120 Lebensstunden, insbesondere während einer Surfactant-Applikation.

Material und Methoden: Wir führen eine bizentrische prospektive Beobachtungsstudie von Frühgeborenen mit einem Gestationsalter von $26+0-31+6$ Schwangerschaftswochen durch. Neben Standard-Monitoringverfahren erfolgt zusätzlich die Evaluation der $\mathrm{rStO}_{2}$ mittels Nah-Infrarot-Spektroskopie (Stirnsensor). Ergebnisse: Die aktuelle Studienkohorte umfasst $n=44$ Frühgeborene (mittleres Gestationsalter \pm SD: 29,4 $\pm 1,8$ Wochen, mittleres Geburtsgewicht \pm SD: $1296 \pm 316$ g). Insgesamt wurden $n=32$ Surfactant-Gaben bei $n=24$ Frühgeborenen $(n=24$ Frühgeborene 1 Gabe, $n=9$ Frühgeborene 2 Gaben, $n=1$ Frühgeborenes 3 Gaben) erforderlich. 19 Frühgeborene erhielten die 1. Surfactant-Gabe unter Spontanatmung. Während der Surfactant-Administration wurden Abfälle der peripheren Sauerstoffsättigung $\left(\mathrm{SpO}_{2}\right.$, Mittelwert/IQR; Ausgangswert vor Gabe: 87/82-92\%, minimaler Wert während Gabe: 69/43-95\%, $p<0,001$ ) und $\mathrm{rStO}_{2}$ (Median/IQR; Ausgangswert vor Gabe: 75/49-96\%, minima- ler Wert während Gabe: 66/40-92\%, $p=0,01$ ) beobachtet. Bei 15 Surfactant-Gaben wurden $\mathrm{SpO}_{2}$-Abfälle um $>20 \%$ des Ausgangswertes dokumentiert, bei 8 Surfactant-Administrationen wurden $\mathrm{rStO}_{2}$-Abfälle um $>20 \%$ des Ausgangswertes beobachtet. Bradykardien $<100 /$ min traten während 9 Surfactantgaben auf.

Schlussfolgerung: Nicht-invasive Monitoringverfahren wie die Bestimmung der $\mathrm{rStO}_{2}$ können einen wichtigen Beitrag zur Patientensicherheit während der Surfactant-Administration leisten. Für eine bessere Einschätzung der klinischen Relevanz ist die Etablierung von Gestations- und Lebensalter-bezogenen Referenzwerten notwendig.

\section{Freie Themen 03: Fehlbildungen}

\section{FV15}

Trio-basierte Exom-Sequenzierung bei Familien mit Ösophagusatresien

R. Zhang ', F. Marsch', H. Thiele', A. Hilger', F. Kause', E. Jenetzky', M. Ludwig', A. Müller ${ }^{5}$, J. Schumacher', H. Reutter ${ }^{6}$

'Universität Bonn, Institut für Humangenetik, Bonn, Deutschland, ${ }^{2}$ Universität Köln, Cologne Center for Genomics (CCG), Köln, Deutschland, ${ }^{3}$ Deutsches Krebsforschungszentrum (DKFZ), Klinische Epidemiologie und Alternsforschung (C070), Heidelberg, Deutschland, ${ }^{4}$ Universität Bonn, Abteilung für klinische Chemie und klinische Pharmakologie, Bonn, Deutschland, ${ }^{5}$ Zentrum für Kinderheilkunde am Universitätsklinikum

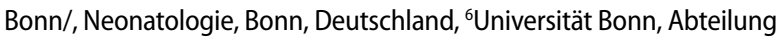
für Neonatologie und Pädiatrische Intensivmedizin und Institut für Humangenetik, Bonn, Deutschland

Hintergrund: Ösophagusatresien mit oder ohne Fistel zur Trachea (ÖA/ TÖF) treten zumeist sporadisch bei etwa 1 von 3500 Neugeborenen auf. Die unterschiedlichen Formen werden hinsichtlich dem Vorhandensein und der Position der Fistel zur Trachea anatomisch klassifiziert. Die Ursachen der ÖA/TÖF sind in den meisten Fällen unklar.

Fragestellung: Erklärt sich das sporadische Auftreten dieser früher meist letal verlaufenden Fehlbildung durch dominante de novo Mutationen. Material und Methoden: In der vorliegenden Studie führten wir bei 30 ÖA/ TÖF Patienten und ihren Eltern (Trio-basierte Exom-Sequenzierung) eine Exom-Sequenzierung durch. Dabei führten wir eine sogenannte 100bp umfassende "paired-end-Sequenzierung" auf dem Illumina HiSeq2000 Sequenzierer durch. Für die Datenanalyse nutzen wir u. a. „bwa-aln, gatk, samtools" und „DeNovoGear“. Zusammengefasst wurden die Analysen mittels VARBANK (CCG, Cologne).

Ergebnisse: Unter Anwendung von standardisierten Filterkriterien ergaben unsere vorläufigen Analysen 26 vermeintliche „de novo"Varianten bei 17 verschiedenen Patienten. Fünf dieser Varianten konnten bereits mittels Sangersequenzierung bestätigt werden. Zwei der Varianten liegen in den ÖA/TÖF assoziierten Krankheitsgenen CHD7 und TRPS1, wodurch die Ursache in beiden Fällen geklärt werden konnte ( 2 von $30 \sim 7 \%$ ). Varianten in MTA3, FANCB, und PLEC werden derzeit wissenschaftlich hinsichtlich eines ursächlichen Zusammenhangs zu ÖA/TÖF aufgearbeitet.

Schlussfolgerung: Bei einem Teil der früher meist letal verlaufenden ÖA/ TÖF liegen ursächliche dominante de novo Varianten zugrunde. Der tatsächliche Umfang ursächlicher de novo Varianten unter allen ÖA/TÖF kann nur durch die systematische Untersuchung großer Kohorten ermittelt werden. Wir gehen davon aus, dass ÖA/TÖF hier stellvertretend für andere, früher (als auch heute noch) letal verlaufende Fehlbildungen stehen. 
FV16

\section{Zungenbändchen und Stillerfolg -}

\section{Ergebnis einer prospektiven Studie}

S. Schlatter', D. Stavropoulou', W. Schupp ${ }^{2}$, J. Otten ${ }^{2}$, R. Rasenack ${ }^{3}$, S. Harnisch' ${ }^{1}$ R. Hentschel ${ }^{1}$

'Institut/Klinik: Universitätsklinikum Freiburg, Zentrum für Kinder- und Jugendmedizin, FB Neonatologie/Intensivmedizin, Freiburg, Deutschland, ${ }^{2}$ Universitätsklinik für Zahn-, Mund- und Kieferheilkunde, Abt. Mund-KieferGesichtschirurgie, Freiburg, Deutschland, ${ }^{3}$ Universitätsklinikum Freiburg, Department Frauenheilkunde Abt. Geburtshilfe, Freiburg, Deutschland

Hintergrund: Mangelnder Stillerfolg ist eines der häufigsten Probleme auf Wochenbettstationen. Dieser kann durch die Stillanamnese und verschiedene quantitative Scores erfasst werden. Die Relevanz eines verkürzten Zungenbändchens (ZB), das nach dem ATLFF-Score von A. Hazelbaker in Schweregrade eingeteilt werden kann, und die Notwendigkeit einer Frenulotomie zur Verbesserung des Stillerfolgs werden dabei oftmals thematisiert, in der wissenschaftlichen Literatur jedoch kontrovers diskutiert Fragestellung: Welche Bedeutung haben das ZB, die Stillerfahrung, das Geburtsgewicht und weitere Variablen auf den Stillerfolg? Wie werden Stillprobleme bei ZB durch die unterschiedlichen Scores abgebildet, und lässt sich der ATLFF-Score mit seiner Untereinheit ATLFF-Funktionsscore (ATLFF-FS) noch verbessern? Welchen Effekt hat die Durchführung einer Frenulotomie?

Material und Methoden: Prospektive, multidisziplinäre Beobachtungsstudie über einen Zeitraum von 10 Monaten. Klinische Inspektion der Mundhöhle des Neugeborenen und Bewertung des Zungenbändchens mit dem ATLFF-Score, Erhebung der Stillanamnese und des LATCH- und BBAT-Scores als objektive Stillskalen, sowie einer numerischen Schmerzskala und ggf. Brustinspektion, jeweils durch eine einzige Untersucherin (S.M.S). Standardintervention: Stillberatung durch eine zertifizierte Stillberaterin, bei ZB ggf. Kontrollevaluation durch eine einzige MKG-Chirurgin (W.S.) und, falls erforderlich, Frenulotomie. Telefon-Interview nach ca. 2 Wochen bei allen Fällen mit Stillproblemen und/oder ZB, dabei Wiederholung der Stillanamnese. Statistik: logistische Regressionen, Wilcoxon Rangsummentest, Mann-Whitney-U-Test, Fisher-Exakt-Test.

Ergebnisse: Erfassung von 776 Neugeborenen (NGB), davon $345 \mathrm{mit}$ Stillproblemen und 116 mit einem ZB, von denen 30 eine Frenulotomie erhielten. Signifikant häufiger schwere Stillprobleme bei NGB mit ZB $(p=.000)$, mit kürzeren Still- und vermehrten Flaschenmahlzeiten und signifikant schlechteren LATCH-, BBAT- und Schmerzscores. Nach logistischer Regression Risikofaktor von $\mathrm{OR}=2,6-2,8$ für schwere Stillprobleme bei einem ZB. Weitere Risikofaktoren: fehlende Stillerfahrung $(\mathrm{OR}=4,4-6,4)$ und Untergewicht des NGB $(\mathrm{OR}=2,9-3,7)$. Erhöhung des Risikos für schwere Stillprobleme um den Faktor von $\mathrm{OR}=3,5-3,8 \mathrm{ab}$ einem ATLFF-FS von $<11$ Punkte. Bei pathologischer Peristaltik der Zunge (einzelne Variable des ATLFF-FS) 4-fach erhöhtes Risiko für schwere Stillprobleme $(\mathrm{OR}=4,1-4,6)$. Signifikante Reduktion der Stillprobleme nach Frenulotomie im Follow-up-Interview $(p=.010)$. Bessere Erfassung von Stillproblemen durch die Variablen „keine Stillerfahrung“, Untergewicht, pathologische Peristaltik der Zunge, und Gesamtwert des ATLFF-FS < 11 Punkte im Vergleich zum Original-ATLFF-Score (AUC in der ROC-Kurve 0,84 vs. 0,66 ).

Schlussfolgerung: Nach unserer Untersuchung hat das ZB einen signifikanten Einfluss auf den Stillerfolg, ebenso aber auch fehlende Stillerfahrung und kindliches Untergewicht. Die Frenulotomie ist bei entsprechender Indikation erfolgreich. Es bleibt zu überprüfen, ob der Faktor „fehlende Stillerfahrung" durch eine noch konsequentere Stillberatung kompensiert werden kann.
FV17

Inzidenz der angeborenen Hüftgelenksydysplasie bei Frühgeborenen. Populationsbasierte Untersuchung aus der SNiP-Studie

\section{A. Lange' ${ }^{1}$, H. Bahlmann ${ }^{1}$, M. Heckmann ${ }^{2}$}

${ }^{1}$ Ernst-Moritz-Arndt Universität, Kinderklinik, Greifswald, Deutschland, ${ }^{2}$ Klinik und Poliklinik für Kinder und Jugendmedizin, Neonatologie u. Päd. Intensivmedizin, Greifswald, Deutschland

Hintergrund: Die angeborene Hüftgelenksdysplasie hängt von unterschiedlichen Faktoren ab. Evtl. wirken sie v. a. im letzten Trimester der Schwangerschaft, was zu einer geringeren Inzidenz der angeborenen Hüftgelenksdysplasie bei Frühgeborenen führen könnte.

Objektive: Inzidenz der angeborenen Hüftgelenksdysplasie bei reif geborenen Neugeborenen vs. Frühgeborenen.

Material und Methoden: Wir untersuchten 2534 termingeborene Neugeborene und 376 Frühgeborene mit Hilfe eines Hüftgelenksultraschalls im Rahmen der populationsbasierten Studie "Survey of Neonates in Pomerania (SNiP)“. Die Einteilung erfolgte nach Graf.

Ergebnisse: $42(1,66 \%)$ termingeborene Neugeborene zeigten eine angeborene Hüftgelenksdysplasie vom Typ IIc oder höher (Graf Typ II c, 0,8\%; Typ D, 0,3\% links und 0,4\% rechts; Typ III a, 0,2\% links). Bei achtzehn Kindern lag ein beidseitiger Befund vor. Es zeigte sich eine deutliche Prädisposition des weiblichen Geschlechts (32/1,182 vs. 10/1,302, $p<0,023$; $95 \%$ CI 0,012-0,022, $\chi 2$ test). Eine familiäre Prädisposition fand sich bei 169 (6,7\%) termingeborenen Neugeborenen und bei 181 (7,1\%) in der übrigen Population. Bei Frühgeborenen trat nur bei drei late preterm infants mit einem Gestationsalter zwischen 36-37SSW $(n=97)$ eine angeborene Hüftgelenksdysplasie auf. Kein Frühgeborenes unter 36SSW zeigte eine angeborene Hüftgelenksdysplasie $(n=279)$. In der durchgeführten Regressionsanalyse konnten wir einen engen Zusammenhang zwischen einer angeborenen Hüftgelenksdysplasie und dem Gestationsalter bei Geburt aufzeigen. (relative risk $=1,17 ; 95 \%$ confidence interval 0,99-1,37; $p=0,065$ ). Zusammenfassung: Unsere Studie zeigt, das Frühgeborene Kinder unter 36 SSW ein reduziertes Risiko für eine angeborene Hüftgelenksdysplasie haben.

Level of Evidence: Level 1 - prognostic study, all patients were enrolled at the same point in their disease with $\geq 80 \%$ follow-up of enrolled patients.

\section{FV18}

Anästhesie in der Neonatalperiode - Entwicklungsneurologisches Outcome von Kindern mit angeborenen Fehlbildungen und Anästhesie in der Neonatalperiode und dessen Zusammenhang mit den verabreichten Anästhetika und Analgetika

N. Doberschütz' ', A. Allendorf', R. Dewitz' ${ }^{2}$ U. Rolle ${ }^{3}$, R. Schlößer ${ }^{1}$

'Klinikum der J.W. Goethe-Univ. Zentrum der Kinderheilkunde, Neonatologie, Frankfurt, Deutschland, ${ }^{2}$ Klinikum der J.W. Goethe-Univ. Zentrum der Kinderheilkunde, Neuropädiatrie, Frankfurt am Main, Deutschland, ${ }^{3}$ Universitätsklinikum der Johann-Wolfgang-GoetheUniversität Frankfurt am Main, Klinik für Kinderchirurgie, Frankfurt, Deutschland

Hintergrund: Durch die verbesserten Therapiemöglichkeiten für Kinder mit angeborenen Fehlbildungen haben sich deren Überlebensraten innerhalb der letzten Jahre stark verbessert, weshalb sich der Fokus der aktuellen Forschung zunehmend auf die Qualität des Überlebens legt. Hierbei tritt besonders die Frage nach dem Einfluss der Anästhesie auf das neurologische Outcome in den Mittelpunkt, gerade in Anbetracht der im Tierexperiment nachgewiesenen neurotoxischen Wirkungen von Anästhetika und Analgetika.

Fragestellung: Ziel dieser Studie war es, einen möglichen Zusammenhang zwischen der verabreichten Medikation und dem entwicklungsneurologischen Outcome von Kindern mit angeborenen Fehlbildungen des Gastrointestinaltraktes zu ermitteln. 
Material und Methoden: In einer prospektiven Fall-Kontroll-Studie untersuchten wir 40 Patienten mit relevanten Fehlbildungen des Gastrointestinaltraktes im Alter von 24 Monaten mittels des Bayley Scales of Infant Development II-Assessments, wobei ein psychomotorischer (PDI) und ein kognitiver Entwicklungsindex (MDI) ermittelt wurden. Die Outcomedaten der Patienten wurden mit einer Kontrollgruppe aus 40 gesunden Kindern, welche noch nie einer Allgemeinanästhesie unterzogen werden mussten, verglichen. Zudem wurde für alle Patienten die im Rahmen der stationären Aufenthalte verabreichte Medikation ausgewertet, wobei ein besonderer Fokus auf die Anästhetika und Analgetika gelegt wurde. Diese Daten wurden mittels Spearman-Rangkorrelation mit den neurologischen Outcomeparametern korreliert.

Ergebnisse:

Es zeigte sich hinsichtlich des psychomotorischen Outcomes kein signifikanter Unterschied zwischen Patienten- und Kontrollgruppe $(p=0,244)$ In Bezug auf das kognitive Outcome bestand hingegen ein signifikanter Unterschied zwischen den Gruppen $(p=0,0217)$ mit einem mittleren MDI von 102 in der Patienten- und 110 in der Kontrollgruppe. Die Untersuchung eines möglichen Zusammenhangs des entwicklungsneurologischen Outcomes unserer Patienten mit den verabreichten Anästhetika und Analgetika ergab für keine der Substanzen eine signifikante Korrelation. Insbesondere in Hinsicht auf die neurotoxischen Wirkstoffe zeigte sich keine signifikante Korrelation der verabreichten Dosen mit dem entwicklungsneurologischen Outcome der Kinder.

Schlussfolgerung: In unserer Studie konnten wir einen signifikanten Unterschied im Bereich der kognitiven Entwicklung von Kindern mit angeborenen Fehlbildungen des Gastrointestinaltraktes und neonataler Allgemeinanästhesie im Vergleich zu gesunden Kindern feststellen. Ein signifikanter Zusammenhang mit den verabreichten Anästhetika und Analgetika in der Neonatalperiode konnte hingegen nicht festgestellt werden.

\section{FV19}

\section{Postnatale Adaptation des pulmonalen Blutflusses bei Lungenhypoplasie durch Zwerchfellhernie}

A. Flemmer', M. Thio Lluch', M. Wallace ${ }^{3}$, K. Lee ${ }^{4}$, M. Kitchen ${ }^{5}$, L. Kerr ${ }^{6}$, J. Jani', R. Carnibella ${ }^{8}$, A. tePas ${ }^{9}$, P. DeKoninck ${ }^{10}$, A. Fouras ${ }^{11}$, C. Roehr ${ }^{12}$, S. Hooper ${ }^{13}$

'LMU-München, Neonatologie der Kinderklinik am Perinatalzentrum Großhadern, München, Deutschland, ${ }^{2}$ The Royal Women's Hospital, Division of Neonatal Research, Parkville, Australien, ${ }^{3}$ The Ritchie Centre, Monash Institute of Medical Research, Melbourne, Australien, ${ }^{4}$ School of Physics, Monash University, Melbourne, Australia, Melbourne, Australien, ${ }^{5}$ School of Physics, Monash University, Melbourne, Australia, Melbourne, Australien, ${ }^{6}$ The Ritchie Centre, Monash University, MIMR-PHI Institute of Medical Research, Clayton, Australien, ${ }^{7}$ Brugman University Hospital, Obstetrics and Gynecology, Brüssel, Belgien, ${ }^{8}$ Monash University, Division of Biological Engineering, Clayton, Australien, ${ }^{\circ}$ University Medical Center, Department of Pediatrics, Division of Neonatology, ZA Leiden, Niederlande, ${ }^{10}$ The Ritchie Centre, MIMR-PHI Institute of Medical Research, Melbourne, Australien, ${ }^{11}$ Monash University, Laboratory for Dynamic Imaging, Clayton, Australien, ${ }^{12}$ Oxford University Hospitals, Women's Centre John Radcliffe Hospital, Oxford, United Kingdom, ${ }^{13}$ Department of Physiology, Monash Institute for Medical Resarch, Monash University, Melbourne, Australien

Hintergrund: Die kongenitale Zwerchfellhernie (DH) kommt bei ca. 1:4000 Neugeborenen vor. Die potsnatale Mortalitätist ist hoch, wenn die durch die Herneation induzierte Lungenhypoplasie ausgeprägt ist. Das klinische Bild nach der Geburt ist neben einer Gasaustauschstörung geprägt von einer pulmonalen Durchblutungsstörung, ohne dass es ausreichend Daten über den pulmonalen Blutfluss bei der postnatalen Transition während der initialen Lungenbelüftung gibt. In unserem experimentellen DH-Modell wurde der pulmonale Blutfluss (rel-PBF) aus dem rechten Herzen während der ersten Atemzüge im Synchrotron zeitlich und räumlich untersucht

Methode: Fetalen Kaninchen (25./31 Gestationstag) wurden an der uteroplazentaren Perfusion ex-utero eine linksseitige Zwerchfellhernie angelegt. DH-Feten $(n=6)$ sowie nicht operierte Kontrollen (Ko; $n=8)$ wurden am
Termin per Sektio entbunden. Vor dem ersten Atemzug wurden die Feten in den Strahlengang eines Synchrotron-Teilchenbeschleunigers (Spring-8, Osaka, Japan) eingebracht. Die ersten Atemzüge wurden volumenkontrolliert $\left(5 \mathrm{ml} / \mathrm{kg}\right.$ ) bei einem PEEP von $5 \mathrm{cmH}_{2} \mathrm{O}$ appliziert. Gleichzeitig wurde über einen in der Jugularvene liegenden Katheter Kontrastmittel injiziert. Die regionale Perfusion der Lungen wurde mit Hilfe der Phasenkontrast Röntgen Technik $(33,2 \mathrm{keV})$ mit $20 \mathrm{~Hz}$ bei $30 \mathrm{msec} /$ frame aufgezeichnet und hinsichtlich räumlichen und zeitlichen Verlauf ausgewertet.

Ergebnisse: Zu Beginn der Lungenbelüftung stieg der relative pulmonale Blutfluss in der linken und der rechten Pulmonalarterie (PA) bei Ko signifikant an $(2,0 \pm 0,8$ und 2,1 $\pm 1,0 \% / \mathrm{sek}$ auf $14,1 \pm 3,0$ und $11,1 \pm 2,0 \%$ /sek, jeweils PA li/re. Bei DH stieg der relative PBF mit Beginn der Lungenbelüftung noch deutlicher an (von 4,1 $\pm 1,0$ und $3,1 \pm 0,5 \% /$ sek auf $23,2 \pm 4,0$ und $17,5 \pm 2,0 \% / \mathrm{sek}$, jeweils li/re PA). Der Anstieg des rel-PBF mit Beginn der Lungenbelüftung bei Geburt unterschied sich nicht zwischen den beiden Gruppen.

Schlussfolgerung: Unsere Daten zeigen erstmals in Echtzeit in vivo, dass die postnatale Lungendurchblutung bei Lungenhypoplasie durch Zwerchfellhernie während der Transition zunächst nicht beeinträchtigt ist. Unsere Daten lassen vermuten, dass die wesentliche Komponente der klinisch beobachteten pulmonalen Hypertension bei Kindern mit angeborener Zwerchfellhernie durch ein hyperreagibles pulmonales Gefäßbett verursacht sein könnte. Inwieweit unterschiedliche Beatmungsmodalitäten nach der Geburt diese Hyperreagibilität beeinflussen ist bisher nicht bekannt.

\section{FV20}

\section{Uroaszites als prognostisch günstiges Zeichen bei} Urethralklappen? Vier Fälle mit unterschiedlichem Ausgang

\section{S. Römer', D. Müller ${ }^{2}$, K. Rothe ${ }^{3}$, C. Bührer}

${ }^{1}$ Charité Universitätsmedizin, Neonatologie, Berlin, Deutschland, ${ }^{2}$ Charité Universitätsmedizin, Kindernephrologie, Berlin, Deutschland, ${ }^{3}$ Charité Universitätsmedizin, Kinderchirurgie, Berlin, Deutschland

Einleitung: Hintere Urethralklappen stellen die häufigste obstruktive Harnröhrenerkrankung dar. In bis zu 30 \% führen sie zur terminalen Niereninsuffizienz. Wir stellen eine Fallserie von vier Patienten mit Urethralklappen und unterschiedlichen Verläufen vor.

Fallberichte: Fall 1: FG 36+ 4 SSW, Geburtsgewicht 3150 g. Pränatal Balkenblase und Harnstauung beider Nieren, Perforation der rechten Niere mit Urinom, Uroaszites. Im MCU Nachweis der Urethralklappe, VUR ${ }^{\circ} \mathrm{IV}$ rechts. Kreatinin postnatal $0,9 \mathrm{mg} / \mathrm{dl}$, nach Klappenresektion und vor Entlassung 0,4 mg/d. Fall 2: FG 36+5 SSW, Geburtsgewicht $3020 \mathrm{~g}$. Pränatal isolierter Aszites, Oligohydramnion. Fetale Aszitespunktion mit 31 SSW. Postnatal Intubation bei respiratorischer Insuffizienz durch das hochdrückende Abdomen. Aszitesdrainage $>500 \mathrm{ml}$. Beidseits NBKS-Erweiterung. Urethralklappenschlitzung. Kreatinin max. 0,6 mg/dl, vor Entlassung 0,3 mg/dl. Fall 3: RG 38+5 SSW, Geburtsgewicht 3390 g. Pränatal Megacystis und Hydronephrose beidseits. Kein Aszites. Schlitzung der Klappe, offene Pyelostomie rechts. Initial Kreatinin $1,1 \mathrm{mg} / \mathrm{dl}$, im Verlauf $4,7 \mathrm{mg} / \mathrm{dl}$, progrediente Niereninsuffizienz. Peritonealdialyse ab 11. Lebenstag. Fall 4: FG $25+5$ SSW, Geburtsgewicht 960 g, keine pränatalen Auffälligkeiten. Ab 3. Lebenstag zunehmend Aszites, Drainage bei respiratorischen Problemen. Megacystis und Kreatinin 2,5 mg/dl im Aszitespunktat. Passagere Niereninsuffizienz mit max. Kreatinin 1,7 mg/dl, vor Entlassung 0,3 mg/dl. Nach Katheterentfernung unauffällige Miktion, keine chirurgische Intervention erforderlich.

Diskussion: In unserer Fallserie haben die drei Kinder mit Uroaszites einen günstigen Verlauf genommen; der Patient ohne Aszites entwickelte eine terminale Niereninsuffizienz. Das Pop-off-Phänomen als Entlastung des Harntraktes kann daher als nephroprotektives Kriterium diskutiert werden. 
FV21

Einfluss der kongenitalen zystisch-adenomatoiden Malformation der Lunge (CCAM) auf die postnatale Lungenfunktion

P. Barikbin' ', C. Roehr' ${ }^{2}$, S. Wilitzki', K. Kalache ${ }^{3}$, P. Degenhardt', C. Bührer', G. Schmalisch ${ }^{1}$

'Klinik für Neonatologie CVK, Charité Univ.med. Berlin, Neonatologie, Berlin, Deutschland, ${ }^{2}$ Oxford University Hospitals, Women's Centre John Radcliffe Hospital, Oxford, United Kingdom, ${ }^{3}$ Charite Centrum 17 für Frauen-, Kinder- und Jugendmedizin, Klinik für Geburtsmedizin, Berlin, Deutschland, ${ }^{4}$ Universitätsmedizin Berlin Charité, Klinik und Poliklinik für Kinderchirurgie, Berlin, Deutschland

Hintergrund: Die CCAM wird zunehmend bereits in der pränatalen Ultraschalluntersuchung diagnostiziert. Das optimale therapeutische Vorgehen bei postantal asymptomatischen Läsionen wird kontrovers diskutiert Fragestellung: Untersuchung der Auswirkungen von CCAM auf die postnatale Lungenfunktion und der Frage, ob eine frühe postnatale Lungenfunktionstestung (LFT) zur Identifikation von Patienten beitragen kann, die von einer frühen Operation profitieren würden.

Methoden: LFT wurden bei 26 Säuglingen mit CCAM in einem korrigierten von 42, 4 Schwangerschaftswochen (Median, Interquartilsabstand 39,6-44,0) durchgeführt und die Ergebnisse mit 30 lungengesunden Kontrollkindern verglichen. Untersucht wurden die Parameter der Ruheatmung, die funktionelle Residualkapazität mittels Ganzkörperplethysmographie (FRCpleth), die Atemmechanik (respiratorische Compliance (Crs) und respiratorische Resistance (Rrs)) mittels Okklusionstest sowie die Blutgase.

Ergebnisse: Kinder mit CCAM zeigten ein für restriktive Ventilationsstörungen typisches Atemmuster mit erhöhter Atemfrequenz $(p=0,006)$ und geringfügig reduziertem Atemzugvolumen $(p=0,043)$. Außerdem war die Crs im Vergleich mit den Kontrollpatienten signifikant erniedrigt $(p=0,007)$. Es fanden sich keine signifikanten Unterschiede zwischen den Gruppen in Bezug auf Rrs, FRCpleth und die kapillären Blutgase. Insbesondere CCAM-Kinder, die in den ersten zwei Lebensjahren operiert wurden, wiesen in der präoperativen Lungenfunktionsuntersuchung eine ausgeprägte Reduktion der Crs auf $(p<0,001)$.

Die CCAM kann zu einer restriktiven Ventilationsstörung führen, welche mittels LFT im frühen Säuglingsalter erkannt und im Verlauf kontrolliert werden kann. Daher stellt die postnatale LFT ein zusätzliches diagnostisches Werkzeug dar, mit dem die Entscheidung für oder gegen eine chirurgische Intervention unterstützt werden kann.

\section{Freie Themen 04: Hypoxie, Ischaemie, Neuroprotektion}

\section{FV22}

Repeated Antenatal Corticosteroid Exposure Disrupts Myelination in the Auditory Nerve of Preterm Sheep

J. Rittenschober-Böhm' ', J. Rodgers', A. Jobe 3 , S. Kallapur ${ }^{3}$, D. Doherty', B. Kramer ${ }^{5}$, M. Payne 4 , Y. Miura ${ }^{4}$, A. Berger ${ }^{1}$, J. Newnham ${ }^{4}$, M. Kemp ${ }^{4}$

'Univ. Klinik für Kinder- und Jugendheilkunde, Medizinische Universität Wien, Abteilung für Neonatologie, pädiatrische Intensivmedizin und Neuropädiatrie, Wien, Österreich, ${ }^{2}$ University of Western Australia, Experimental and Regenerative Neurosciences, Perth, Australien, ${ }^{3} \mathrm{Cincinnati}$ Children's Hospital, Division of Pulmonary Biology, Ohio, USA, ${ }^{4} S$ chool of Women's and Infant's Health, The University of Western Australia, Subiaco, Perth, Australien, ${ }^{5}$ Maastricht University Medical Center, Department of Pediatrics, Maastricht, Niederlande

Background: Using antenatal corticosteroids (ACS) to improve neonatal survival is one of the greatest advances in obstetric medicine to date. However, whether ACS course should be repeated in women remaining at risk for preterm birth is controversially discussed. A rapidly evolving area of research suggests that repeated exposure to ACS is associated with reduced fetal growth, adverse effect on brain development and delayed myelination. The potential for adverse effects on fetal neurosensory development is of particular interest.

Aim: We aimed to determine the impact of repeated antenatal corticosteroid exposures on auditory nerve development in an ovine model of pregnancy.

Materials and Methods: Following ethics approval, ewes with a single 100d gestational age (GA, term 145d GA) fetus received an intramuscular (IM) injection of $150 \mathrm{mg}$ medroxyprogesterone acetate to minimize the risk of preterm labor and abortion induced by glucocorticoids in sheep followed by either: i) IM saline on GA days 104, 111, 118 (control group; $n=5$ ) ii) IM $\beta$-methasone $(0.5 \mathrm{mg} / \mathrm{kg})$ on GA days 104 and IM saline on GA days $111,118$ (single treatment group; $n=5)$; or iii) IM $\beta$-methasone $(0.5 \mathrm{mg} /$ $\mathrm{kg}$ ) on GA days 104, 111, 118 (repeated treatment group; $n=7$ );. Animals were euthanized and lambs were delivered at $125 \mathrm{~d}$ GA. Fetal brains were perfusion fixed with paraformaldehyde before embedding and sectioning. Transmission electron microscope images were digitally analysed to determine auditory nerve morphology and myelination. Comparisons of treatment effects were conducted using linear (continuous outcomes) and generalised logistic (categorical outcomes) regression analysis.

Results: Relative to control, mean myelin area was significantly increased in the repeated treatment group $(p<0.001)$, but not in the single treatment group $(p=0.624)$. Analysis of morphological assessments suggested that the increased myelin area in the repeated treatment group derived from an increased likelihood of decompacted areas $(p=0.005, \mathrm{OR}=2.14))$ and irregular myelin deposition $(p=0.001, \mathrm{OR}=5.91)$.

Discussion and Conclusions: In preterm sheep, repeated antenatal corticosteroid exposure increased auditory nerve myelin area, potentially due to disruption of myelin deposition. This pathology may result in a functional deficit, as conduction velocity in central fibres is adversely affected by myelin decompaction. Further large scale studies are needed to clarify these observations.

\section{FV23}

\section{Erythropoietin als Neuroprotektivum bei der Hyperoxie-} vermittelten neonatalen Hirnschädigung im Tiermodell

\section{Brait' ' J. Palasz', M. Serdar' ', S. Sizonenko², Y. van de Looij', J. Fandrey4,} U. Felderhoff-Müser', I. Bendix ${ }^{?}$

'Universitätsklinikum Essen, Klinik für Kinderheilkunde I/Neonatologie, Essen, Deutschland, ${ }^{2}$ Univerisitätsklinikum Genf, Klinik für Kinderheilkunde, Genf, Schweiz, ${ }^{3}$ Ecole Polytechnique Fédérale de Lausanne,

Laboratory of Functional and Metabolic Imaging, Lausanne, Schweiz, ${ }^{4}$ Universitätsklinikum Essen, Institut für Physiologie, Essen, Deutschland

Hintergrund und Fragestellung: Aufgrund der Anfälligkeit des unreifen Gehirns haben frühgeborene Kinder ein erhöhtes Risiko für die Entwicklung neurologischer und kognitiver Langzeitprobleme. Pathophysiologisch liegt dabei häufig eine Schädigung der weißen Substanz zugrunde. Erythropoietin findet in seiner rekombinanten Form (rEPO) schon seit geraumer Zeit Anwendung in der Behandlung der Früh- und Neugeborenen-Anämie. Darüber hinaus besitzt EPO neuroprotektive Eigenschaften im Modell der Hyperoxie-induzierten Hirnschädigung sowie anderen neonalen Schädigungsmodellen. Das Ziel der aktuellen Untersuchungen war daher die Evaluation der Effekte von EPO im neonatalen experimentellen Modell der Hyperoxie-induzierten Hirnschädigung auf die graue und weiße Substanz sowie die kognitive und motorische Langzeitentwicklung.

Material und Methoden: Wistar-Ratten erhielten am 6. Lebenstag eine intraperitoneale Injektion mit 20.000 I.E./kg Körpergewicht rEPO oder isotoner Kochsalzlösung und wurden anschließend einer 24-stündigen Hyperoxie bei $80 \%$ Sauerstoff ausgesetzt oder bei Raumluft gehalten. Die motor-kognitive Langzeitentwicklung wurde sowohl bei adoleszenten als auch adulten Tieren evaluiert. Es erfolgte eine Untersuchung der weißen Substanz anhand von Proteinanalysen des Myelinisierungsmarkers MBP (myelin basic protein) sowie post-mortaler diffusionsgewichte- 
ter MRT-Aufnahmen. Des Weiteren wurden RNA-Analysen neuronaler Plastizitätsmarker (Synaptophysin, Neuregulin-1) durchgeführt.

Ergebnisse: Die Analysen der motor-kognitiven Langzeitentwicklung durch EPO ergaben eine signifikante Verbesserung des Erinnerungsverhaltens. In den durchgeführten Protein- sowie MRT-Untersuchungen zeigte sich keine Protektion der weißen Substanz, die Analysen der neuronalen Plastizitätsmarker ergaben jedoch eine Hyperoxie-abhängige Verminderung, welcher durch EPO-Applikation signifikant entgegengewirkt werden konnte.

Diskussion und Schlussfolgerung: Unsere Untersuchungen zeigen erstmalig eine Verbesserung der Langzeitkognition durch EPO im Hyperoxie-vermittelten Schädigungsmodell und bestätigen so seine Bedeutung für die potentielle Neuroprotektion bei Frühgeborenen. Die Verbesserung des Erinnerungsverhaltens lässt sich dabei nicht auf einen Schutz der weißen Substanz zurückführen, scheint aber in Zusammenhang mit der Protektion neuronaler Plastizität zu stehen und sollte durch weitere Untersuchungen der zugrunde liegenden Mechanismen genauer evaluiert werden.

\section{FV24}

\section{Fentanyl induziert zerebelläre Apoptose in gesunden,} neugeborenen Schweinen

\section{H. Sabir', E. Maes ${ }^{2}$, X. Liu' ${ }^{3}$, J. Dingley ${ }^{4}$, M. Thoresen ${ }^{3}$}

${ }^{1}$ Med. Einricht. d. Universität Kinderklinik, Neonatologie und pädiatrische Intensivmedizin, Düsseldorf, Deutschland, ${ }^{2}$ University of Oslo, Department of Physiology, Institute of Basic Medical Sciences, Oslo, Norwegen, ${ }^{3}$ University of Bristol, Child Health, School of Clinical Sciences, Bristol, United Kingdom, ${ }^{4}$ Morriston Hospital, Department of Anaesthetics and Intensive Care, Swansea, United Kingdom

Hintergrund: Opioide, wie Morphin oder Fentanyl, werden routinemäßig zur Analgosedierung in der Neonatalologie eingesetzt. Soweit bekann sind sie sicher und geeignet für den Einsatz bei Neonaten. Das Kleinhirn ist in den letzten Jahren in den Fokus der Neurowissenschaften gerückt, da es eine sehr wichtige Rolle in der motorischen und kognitiven Entwicklung spielt. Das Ziel dieser Studie war es, zu untersuchen, ob eine $24 \mathrm{~h}$ Fentanyl-Dauertropfinfusion einen Einfluss auf den Zelltod im Kleinhirn bei gesunden, neugeborenen Schweinen hat.

Material und Methodik: Dreizehn neugeborene Schweine (Alter $<24 \mathrm{~h}$ ) wurden in zwei Gruppen randomisiert: (1) $24 \mathrm{~h}$ Fentanyl Dauerinfusion bei Normothermie und Spontanatmung (NT, Trektal $\left.38^{\circ} \mathrm{C}, n=6\right)$ oder (2) Kontrollgruppe bei Normothermie und Spontanatmung $(n=7)$. Nach der jeweiligen Therapie endeten die Experimente. Pathologische Zellen wurden im zerbellären Kortex untersucht und nach immunhistochemischer Färbung quantifiziert (Caspase 3).

Ergebnisse: Alle Tiere waren spontanatmend und hatten stabile Vitalparameter während des $24 \mathrm{~h}$ Untersuchungszeitraums. Die durchschnittliche Fentanyldosierung in Guppe (1) betrug $12,85 \mathrm{mcgr} / \mathrm{kg} / \mathrm{h}( \pm 8,9)$. Bei den Tieren in Gruppe (1) zeigte sich eine um ca. $50 \%$ erhöhte Zelltodrate in der inneren Körnerzellschicht der Kleinhirnrinde (3-fach erhöhte Caspase-3 Aktivität). Die Anzahl der Purkinje Zellen war nicht unterschiedlich in den beiden Therapiegruppen.

Schlußfolgerung: Mit Sorge haben wir festgestellt, dass eine $24 \mathrm{~h}$ Fentanyl-Dauertropfinfusion Neuroapoptose im Kleinhirn bei gesunden, neugeborenen Schweinen induziert.

\section{FV25}

\section{Prediction of outcome in neonates with HIE grade II: role of aEEG and cerebral oxygen saturation measured by NIRS}

K. Goeral', B. Urlesberger', V. Giordano', G. Kasprian'3 , M. Wagner', L. Schmidt ${ }^{1}$, A. Berger', K. Klebermaß-Schrehof', M. Olischar ${ }^{1}$

'Univ. Klinik für Kinder und Jugendheilkunde, Abt. für Neonatologie, Pädiatrische Intensivmedizin und Neuropädiatrie, Wien, Österreich, ${ }^{2}$ Universitätsklinik für Kinder- und Jugendheilkunde, Abt. für Neonatologie, Graz, Österreich, ${ }^{3}$ Univ. Klinik für Radiologie, Abt. für Neuroradiologie, Wien, Österreich
Background and Objective: Hypoxic-ischemic encephalopathy (HIE) is still a major cause of neonatal mortality and morbidity. In this patient population, only few data have been published on the combined use of amplitude-integrated electroencephalography (aEEG) and near-infrared spectroscopy (NIRS) for prognostication. The aim of this study was firstly to determine the changes observed in continuous aEEG and NIRS monitoring during the first 102 hours of life. Secondly, we aimed to evaluate the predictive value, the most powerful predictive combination of single aEEG and NIRS parameters and respective cut-off values for outcome in neonates with HIE grade II. Study Design: In term neonates with HIE grade II according to Sarnat, aEEG (including background pattern (BP), sleepwake cycling (SWC) and seizure activity) and NIRS (including $\mathrm{rScO}_{2}$ and cFTOE) were prospectively studied from birth up to $102 \mathrm{hrs}$ of life in relation to magnetic resonance imaging.

Material and Methods: Eighteen neonates diagnosed with HIE grade II treated with hypothermia at the Department of Paediatrics and Adolescent Medicine at the Medical University of Vienna between 2012 and 2015 were studied. The measurement period of $102 \mathrm{hrs}$ was divided into six hour epochs for futher analyses. According to MRI, two outcome groups were defined and predictive value of aEEG parameters in various combinations, $\mathrm{rScO}_{2}$ in \%, and the additional value of both methods combined was studied. Therefore, the following scores were created: ,background pattern' (BP), ,BP+seizures', , aEEG SUM' (including BP, SWC and seizure activity) and a combination of aEEG and NIRS entitled, $\left(\mathrm{BP}+\right.$ seizures ${ }^{*}{ }^{*} \mathrm{rSCO}_{2}{ }^{\prime}$. Receiver-operating curves (ROCs) were obtained and area under the curve (AUC) values were calculated for mentioned scores. ROCs were then used to detect the optimal „cut-off“" points and to calculate sensitivity, specificity, positive and negative predictive value (PPV, NPV).

Results: Infants with abnormal MRI showed a higher percentage of abnormal BP and longer time to normal trace, a higher percentage without SWC and longer time to mature SWC and a higher percentage of seizure activity. They also showed higher $\mathrm{rScO}_{2}$ and lower cFTOE values measured by NIRS. Differences in NIRS values were largest on the second day of life. At all studied time epochs, combined parameter scores were more predictive with regard to short term outcome than single parameter scores. Highest AUCs were observed during the first $60 \mathrm{hrs}$ of life for ,BP+seizures' and ,(BP+seizures) $)^{*} \mathrm{rScO}_{2}$, being between 0.91 and 1 at $42-60 \mathrm{hrs}$. Highest sensitivity, specificity, PPV and NPV was observed at 54-60 hrs for ,(BP+seizures) ${ }^{*} \mathrm{rScO}_{2}$ ' being $100 \%$ each.

Conclusions: In HIE grade II, aEEG and NIRS are important predictors of short-term outcome. Highest predictive abilities were observed during the first $60 \mathrm{hrs}$ of life. The combination of both methods further improves prognostication.

\section{FV26}

\section{Factors associated with coagulopathy and intracranial bleeding} (ICB) in cooled neonates with hypoxic ischaemic encephalopathy (HIE).

\section{N. Calder', B. Cordon', D. Odd ${ }^{3}$, A. Heep 4}

'Southmead Hospital, Neonatal Service, Bristol, United Kingdom, ${ }^{2}$ Southmead Hospital, Department of Medicine, Bristol, United Kingdom, ${ }^{3}$ University of Bristol, Bristol, United Kingdom, ${ }^{4}$ University of Bristol, Neonatal Neurology Group, Bristol, United Kingdom

Background: Therapeutic hypothermia (TH) and hypoxic ischaemic encephalopathy (HIE) have been associated with coagulopathy, which can result in intracranial bleeding (ICB).

Aims: to describe the incidence of ICB in neonates undergoing TH and to identify factors associated with this adverse outcome. We hypothesised that, in neonates undergoing TH, more severe HIE would result in a more adverse coagulation profile and therefore a higher risk of developing ICB. Methods: A single centre retrospective case study of 50 term neonates treated with TH as per the Western Neonatal Network guideline over a two-year period (2010-2011) was performed. Data were collected from electronic result databases and BadgerNet patient data management system on HIE grade (1-3), clotting (APTT, INR, PT, fibrinogen, FBC), 
blood product use, vitamin $\mathrm{K}$ administration, co-existing diagnosis, mode of delivery and demographics. Co-existing diagnoses included i) sepsis (CRP $>10 \mathrm{mg} / \mathrm{l}$, positive blood culture), ii) placental insufficiency, iii) placental abruption, iv) meconium aspiration syndrome, and v) persistent pulmonary hypertension of the newborn. Cerebral bleeding was diagnosed on MRI at day 7-10 (available in 48/50 patients). Logistic regression models were performed to identify factors independently associated with ICB.

Results: HIE grade 1 was seen in $16 \%$ of neonates, HIE grade 2 in $58 \%$ and HIE grade 3 in $26 \%$. ICB was diagnosed on MRI in 19\% (9/48). Prevalence of co-existing diagnosis was as follows: placental abruption in $16 \%$, persistent pulmonary hypertension in $18 \%$, placental insufficiency in $2 \%$ and sepsis in $66 \%$. Coagulation parameters worsened as HIE grade increased. INR: $1.6-1.5-2.5, p=0.03$; fibrinogen: $1.4-1.0 .1 .0 ; p=0.03$; platelets: $157-$ $137-92 ; p=0.01$ ); median values given.More severe HIE was associated with a higher risk of cerebral bleed (OR 7.3; $p=0.009$ ). After adjusting for potential confounders - gender and doses of coagulopathy-correcting blood products (OR 28.3; $p=0.03$ ). A history of placental abruption was associated with an increased risk of cerebral bleeding, independently of HIE severity and neonatal gender $(p=0.02)$. Other co-existing diagnoses were not associated with bleeding. After adjusting for the effect of HIE grade, placental abruption (no/yes) was associated with a higher APTT (39.1/77.7; $p=0.001)$ and INR $(1.5 / 2.1 ; p=0.01)$, median values given. Conclusions: Severe HIE is associated with more adverse coagulation parameters and therefore with higher risk of ICB. In addition, our study found that placental abruption is independently associated with coagulopathy and risk of ICB. Further work is required to confirm this relationship and explore potential mechanisms.

\section{FV27}

\section{Therapeutische Hypothermie plus inhalatives Xenon: keine Therapieoption nach schwerer Hypoxie-Ischämie im neonatalen Rattenmodell}

\section{H. Sabir', D. Osredkar' ${ }^{2}$ E. Maes², T. Wood², M. Thoresen ${ }^{2}$}

'Med. Einricht. d. Universität Kinderklinik, Neonatologie und pädiatrische Intensivmedizin, Düsseldorf, Deutschland, ${ }^{2}$ University of Oslo, Department of Physiology, Institute of Basic Clinical Sciences, Oslo, Norwegen

Hintergrund: Die therapeutische Hypothermie (TH) ist Standardtherapie zur Behandlung der perinatalen Asphyxie. Im präklinischen Rattenmodell ist die TH nach moderatem hypoxisch-ischämischem Hirnschaden (HI) neuroprotektiv, jedoch nicht nach schwerem HI. Das inhalative Anästhetikum Xenon verdoppelt im moderaten HI Tiermodell den neuroprotektiven Effekt von TH. Das Ziel dieser Studie war es zu untersuchen, ob die
Kombination aus TH und inhalativem Xenon neuroprotektiv im schweren HI Rattenmodell ist.

Material und Methodik: Einhundertzwanzig 7-Tage alte Ratten erhielten experimentell einen schweren HI (unilaterale Ligatur der A. carotis, $8 \%$ $\mathrm{O}_{2}$ für 150 min bei experimenteller Normothermie, NT-37:Trektal $37^{\circ} \mathrm{C}$ ). Anschließend erfolgte eine Randomisierung in 3 Therapiegruppen: (1) direkte NT-37 für $5 \mathrm{~h}(n=36)$; (2) direkte HT-32:Trektal $32^{\circ} \mathrm{C}$ für $5 \mathrm{~h}$ $(n=25)$ oder (3) direkte HT-32 plus $50 \%$ inhaltives Xenon für $5 \mathrm{~h}$ (HT$32+\mathrm{Xe} 50 \%)(n=24)$. Nach 7 Tagen Überleben wurden die Gehirne mittels „brain area loss (BAL)“ ausgewertet. Um den Hirnschaden weiter zu quantifizieren wurden die Neurone in der subventrikulären Zone gezählt und zwischen den Therapiegruppen verglichen. Ergebnisse: In der NT-37 Gruppe betrug der mediane (IQR) BAL 63,5\% (55,5-75,0). Weder HT32 , noch HT-32+Xe50\% waren neuroprotektiv. Die Anzahl der Neurone in der subventrikulären Zone zeigte keinen Unterschied zwischen den Therapiegruppen.

Schlußfolgerung: Weder die direkte therapeutische Hypothermie, noch die Kombination aus direkter therapeutischer Hypothermie plus $50 \%$ inhalativem Xenon sind neuroprotektiv nach schwerem hypoxisch-ischämischem Hirnschaden im neonatalen Rattenmodell. Die spiegelt höchstwahrscheinlich die klinische Situation nach schwerer Asphyxie wieder und bedarf dringend weiterer Therapieoptionen.

\section{FV28}

\section{Der Einfluss von Doxapramtherapie auf} das Entwicklungsneurologische Outcome von Frühgeborenen mit einem Gestationsalter unter $\mathbf{3 0}$ Schwangerschaftswochen

C. Czaba-Hnizdo', K. Klebermaß-Schrehof', V. Giordano', M. Olischar', E. Schwindt', L. Schmidt' ', K. Goeral' ', A. Berger', M. Weninger ${ }^{1}$

'Univ. Klinik für Kinder und Jugendheilkunde, Abt. für Neonatologie, Pädiatrische Intensivmedizin und Neuropädiatrie, Wien, Österreich

Hintergrund: Zur Behandlung von Apnoen bei Frühgeborenen (FG) wird neben Methylxanthinen auch das zentrale Atemstimulans Doxapram verwendet. Bezüglich Coffeinzitrat gibt es Studien, die einen positiven Effekt auf das entwicklungsneurologische Outcome von FG zeigen. Doxapramtherapie scheint jedoch einen negativen Einfluß sowohl auf die Hirnaktivität, als auch auf die neurologische Entwicklung zu haben, allerdings gibt es bezüglich beider Medikamente wenige Daten, die den Effekt auf die Hirnaktivität und das entwicklungsneurologische Outcome von sehr kleinen FG zeigen.

Fragestellung: dieser Studie war, ob Doxapramtherapie einen Effekt auf die Hirnaktivität und das entwicklungsneurologische Outcome von FG unter der 30. Schwangerschaftswoche (SSW) hat.

\section{Tab. FV28}

\section{Ergebnisse Outcome}

\begin{tabular}{|lllll}
\hline PDI & Signifikanz (2-sided) & $\mathbf{0 , 0 0 0}$ & & $\mathbf{2 0 - 8 4}$ \\
\hline & PDI Score & $\mathbf{8} \mathbf{8 5}$ & $n=25 / 31,3 \%$ & $n=9 / 11,25 \%$ \\
\hline & Kontrollgruppe & $n=46 / 57,5 \%$ & $n=10 / 35,7 \%$ & $n=10 / 35,7 \%$ \\
\hline MDI & Doxapramgruppe & $n=8 / 28,6 \%$ & & $\mathbf{5 5 - 6 9}$ \\
\hline & Signifikanz (2-sided) & $\mathbf{0 , 0 0 4}$ & $\mathbf{7 0 - 8 4}$ & $n=12 / 15,0 \%$ \\
\hline & MDI Score & $\mathbf{8 5 - 1 1 4}$ & $n=15 / 18,8 \%$ & $n=7 / 39,3 \%$ \\
\hline Kontrollgruppe & $n=53 / 66,25 \%$ & $n=5 / 17,9 \%$ & Sehhilfe \\
\hline & Doxapramgruppe & $n=12 / 42,9 \%$ & & $n=4 / 5,0 \%$ \\
\hline & Signifikanz (2-sided) & $\mathbf{0 , 0 8 1}$ & $\mathbf{K e i n e ~ S e h h i l f e ~}$ & $n=0 / 0 \%$ \\
\hline
\end{tabular}


Methodik: Es wurden alle 2009 und 2010 unter der 30. SSW geborenen FG mit einer Messung mit dem amplitudenintegrierten EEG (aEEG) in den ersten 2 Lebenswochen und mit verwertbaren Outcomedaten inkludiert. Patienten mit intraventrikulärer Hirnblutung Grad III und IV, mit periventrikulärer Leukomalazie Grad III und IV, sowie mit zerebralen Infektionen oder Fehlbildungen wurden exkludiert. Folgende aEEG-Parameter wurden erhoben: die Prozentsätze der Hintergrundmuster, das Auftreten und die Qualität der Schlaf-Wach-Zyklen, das Auftreten von elektrophysiologischer Krampfaktivität, sowie der Burdjalov Score. Bezüglich des entwicklungsneurologischen Outcome wurden folgende Variablen von FG mit Doxapramtherapie mit jenen von FG ohne Doxapramtherapie verglichen: Mental (MDI) und Psychomotor (PDI) Developmental Index der Bayley Scales of Infant Development II mit 3,5 Jahren, visuelle und akustische Beeinträchtigung. Die statistische Auswertung erfolgte mittels SPSS 21 mittels Mann Whithney U- Test, Chi2- Test sowie logistischer Regression.

Ergebnisse: In diesem Kollektiv konnte kein eindeutiger Einfluss von Doxapram auf die aEEG- Hintergrundaktvitität nachgewiesen werden, die Dopramgruppe zeigte jedoch signifikant weniger Schlaf-Wach-Zyklen und signifikant häufiger Krampfaktivität. Auch der Burdjalov Score war mit 5,9 versus 6,4 in der Doxapramgruppe signifikant niedriger Kein inkludiertes Kind zeigte eine Hörbehinderung. Im binären logistischen Regressionsmodell war Doxapramtherapie neben männlichem Geschlecht, chronic lung disease, Frühgeborenenretinopathie und nekrotisierender Enterokolitis signifikant, in der multiplen Regression blieben nur chronic lung disease und männliches Geschlecht als eigenständige Risikofaktoren bestehen.

Schlussfolgerung: FG unter Doxapramtherapie zeigten im Vergleich zur Kontrollgruppe ein signifikant schlechteres entwicklungsneurologisches Outcome, sowie vermehrte Sehbehinderung, auch wenn Doxapramtherapie nicht als eigenständiger Risikofaktor identifiziert werden konnte. Die Ergebnisse dieser Studie bestärken allerdings, dass Doxapram nur in Ausnahmefällen verabreicht werden soll.

\section{Freie Themen 05: Beatmung}

\section{FV29}

\section{Lasergestützte Untersuchung der Assoziation von Langzeit-CPAP mit Veränderungen von Schädelform und Mittelgesichtskonfiguration Frühgeborener}

\section{E. Biskupski', M. Rüdiger ${ }^{1}$, A. Koch' ${ }^{1}$, W. Burkhardt'² S. Ifflaender ${ }^{1}$}

'Medizinische Fakultät Carl Gustav Carus an der TU Dresden, Fachbereich Neonatologie und Pädiatrische Intensivmedizin, Dresden, Deutschland, ${ }^{2}$ HELIOS Klinikum Schwerin, Klinik für Kinder- und Jugendmedizin, Bereich Neuropädiatrie, Schwerin, Deutschland

Hintergrund: Continuous Positive Airway Pressure (CPAP) findet bei Frühgeborenen (FG) seit Jahrzehnten routinemäßig Anwendung. Die Therapie wird aufgrund ihrer Vorteile gegenüber der mechanischen Ventilation geschätzt. Insbesondere bei der oft notwendigen Langzeitanwendung von CPAP können Nebenwirkungen wie Druckschäden und Hautnekrosen auftreten. Bedingt durch die Fixierung der CPAP-Systeme sowie die relative Weichheit des knöchernen Schädels von FG sind neben lokalen Weichteilschäden auch Veränderungen der Schädelkonfiguration und besonders des Mittelgesichtes denkbar.

Fragestellung: Ist die Langzeitanwendung von CPAP mit Veränderungen der Schädelform und des Mittelgesichts Frühgeborener assoziiert?

Methoden: Im Rahmen einer retrospektiven klinischen Kohortenstudie wurden 3D-Laserscans von FG vermessen und einschließlich zugehöriger klinischer Daten analysiert. Verglichen wurden zwei Kohorten: I.) Extremely Low Birthweight FG mit CPAP Langzeitanwendung $(>200 \mathrm{~h})(n=65)$ vs. II.) FG mit Geburtsgewicht $\geq 2500 \mathrm{~g}$ ohne/mit minimaler CPAP Anwendung $(<30 \mathrm{~min})(n=72)$. Beide Gruppen wurden im postmenstruellen Alter von 35+0-38+0 SSW mittels 3D-Laserscan untersucht. Neben dem Kopfumfang (KU) wurden anthropometrische Größen zur Schädelsym- metrie (Cranial Vault Asymmetry Index - CVAI) und -proportion (Cranial Index - CI) und speziell das Mittelgesicht beschreibende Strecken (obere und untere Mittelgesichtstiefe - oMGT, uMGT; innere Interkanthaldistanz - IKD; Länge der Nase) erfasst.

Ergebnisse: FG mit CPAP-Langzeitanwendung wiesen signifikant veränderte Schädelmaße im absoluten Vergleich zu FG ohne CPAP-Therapie auf. Sie zeigten u. a. einen niedrigeren $\mathrm{KU}(31,0 \mathrm{~cm}$; IQR $30,3-32,1 \mathrm{~cm}$ vs. $33,3 \mathrm{~cm}$; IQR 32,8-34,0 cm), eine erhöhte Neigung zur Dolichozephalie (CI $72,3 \%$; IQR $68,4-76,6 \%$ vs. $81,1 \%$; IQR 78,4-83,9\%) sowie asymmetrischere Schädelformen (CVAI 4,1 \%; IQR 2,5-6,1\% vs. 1,8\%; IQR 1,12-3,5\%). Alle Mittelgesichtsmaße erwiesen sich im absoluten Vergleich als signifikant verringert in der CPAP-Gruppe. Um eine bessere Vergleichbarkeit zu erreichen, wurden diese zusätzlich in Relation zum KU betrachtet. Auch diese proportionalen Mittelgesichtsmaße waren signifikant verringert nach CPAP-Langzeitanwendung: oMGT/KU 0,206 (IQR 0,2000,213 ) vs. 0,215 (IQR 0,208-0,219), uMGT/KU 0,210 (IQR 0,204-0,217) vs. 0,218 (IQR $0,209-0,222$ ), IKD/KU 0,060 (IQR 0,056-0,066) vs. 0,064 (IQR 0,060-0,069).

Diskussion: FG zeigen nach Langzeitanwendung von CPAP Veränderungen der globalen Schädelform sowie der Mittelgesichtskonfiguration. Dies könnte auf eine Mittelgesichtsimpression sowie eine Verkürzung der Nase durch die Therapie hinweisen. Weitere Untersuchungen, etwa im Sinne einer prospektiv randomisierten Studie zum Vergleich von CPAP-Therapie und High Flow Nasal Cannula (HFNC), wären zum Nachweis einer Kausalität sowie langfristiger Konsequenzen dieser Veränderungen wünschenswert.

\section{FV30}

In-vitro Untersuchungen zum Einfluss des Mundlecks auf die Atemgaskonditionierung bei der neonatalen CPAP-Therapie

H. Fischer', T. Ullrich', C. Bührer', C. Czernik' , G. Schmalisch ${ }^{1}$

'Charité - Universitätsmedizin Berlin, Klinik für Neonatologie, Berlin, Deutschland

Hintergrund: Klinische Studien bei der CPAP-Therapie von Erwachsenen zeigen einen negativen Einfluss des Mundlecks auf die Atemgaskonditionierung. Vergleichbare Untersuchungen bei Neugeborenen fehlen.

Fragestellung: Ziel der vorliegenden in-vitro Studie war, mit einem „spontanatmenden" mechanischen Modell die oropharyngeale Gaskonditionierung bei der neonatalen CPAP-Therapie zu simulieren, und den Einfluss des Mundlecks auf Temperatur und Luftfeuchte im Oropharynx zu untersuchen.

Material und Methoden: Zur Simulation der physiologischen Atemgasbefeuchtung wurde ein individuell präparierter Befeuchter zwischen einem aktiven mechanischen Lungenmodell und einem vereinfachten Oropharynx-Modell eingefügt. Eine verschließbare Öffnung am Modell diente der Simulation des Mundlecks. Mit einem digitalen Thermo-/Hygrosensor wurden die Temperatur $(\mathrm{T})$, relative Feuchte $(\mathrm{rF})$ und absolute Feuchte $(\mathrm{aF})$ im Oropharynx gemessen. Zunächst erfolgten Messungen ohne Atemunterstützung, bei Atemfrequenzen von 30,60 und 90/min, mit und ohne Mundleck. Anschließend wurde der Einfluss des Mundlecks während einer binasalen CPAP-Therapie untersucht, wobei drei Szenarien getestet wurden: keine Atemgaskonditionierung im CPAP-Schlauchsystem, nur Erwärmung des Atemgases, Erwärmung und Befeuchtung des Atemgases.

Ergebnisse: Ohne Atemunterstützung hatte das Mundleck keinen signifikanten Effekt auf T, rF oder aF, aber T und aF stiegen bei höheren Atemfrequenzen an $(p<0,001)$. Bei einer konstanten Atemfrequenz von $60 / \mathrm{min}$ und verschlossenem Mundleck war die oropharyngeale T $32,1 \pm 0,2{ }^{\circ} \mathrm{C}$ und die aF 33,7 $\pm 0,6 \mathrm{~g} / \mathrm{m}^{3}$. Unter CPAP-Therapie hatten Mundlecks einen signifikanten Einfluss. Ohne Atemgaskonditionierung fiel die aF von $23,2 \pm 2,0 \mathrm{~g} / \mathrm{m}^{3}$ bei verschlossenem Mundleck auf $3,0 \pm 0,3 \mathrm{~g} / \mathrm{m}^{3}$ mit Mundleck $(p<0,001)$, und bei ausschließlicher Erwärmung des Atemgases, von $20,3 \pm 2,8 \mathrm{~g} / \mathrm{m}^{3}$ auf $4,3 \pm 0,3 \mathrm{~g} / \mathrm{m}^{3}(p<0,001)$. Bei Erwärmung und Befeuchtung war die aF $42,6 \pm 0,9 \mathrm{~g} / \mathrm{m}^{3}$ bei verschlossenem Mundleck und $35,7 \pm 1,9 \mathrm{~g} / \mathrm{m}^{3}$ mit Mundleck $(p<0,001)$. 
Schlussfolgerung: Im in-vitro Modell verschlechtert ein Mundleck die oropharyngeale Befeuchtung bei der neonatalen CPAP-Therapie in allen drei Testszenarien. Bei Erwärmung und Befeuchtung des Atemgases im CPAP-Schlauchsystem wird jedoch eine klinisch ausreichende Atemgaskonditionierung auch bei Vorhandensein eines Mundlecks erreicht.

\section{FV31 \\ Maschinelle Beatmung und nCPAP: Gibt es Unterschiede im Pflegearbeitseinsatz? Ergebnisse einer empirischen Studie}

\section{K. Langhammer', S. Sülz' ${ }^{2}$, M. Becker-Peth ${ }^{3}$, A. Kribs' ${ }^{1}$, B. Roth}

'Universitäts-Kinderklinik Köln, Neonatologie und Pädiatrische Intensivmedizin, Köln, Deutschland, ${ }^{2}$ Institute of Health Policy \& Management Erasmus University Rotterdam, Rotterdam, Niederlande, ${ }^{3}$ Department of Supply Chain Management \& Management Science, Köln, Deutschland

Hintergrund: Die GBA-Richtlinie zur Qualitätssicherung der Versorgung Früh-und Neugeborener, die ab dem 1.1.2017 gilt, definiert den Pflegepersonalbedarf u. a. unter Berücksichtigung des eingesetzten Verfahrens zur Atemunterstützung (maschinelle endotracheale Beatmung vs. nCPAP). Hiernach sollen Früh- und Neugeborene mit Beatmung jederzeit und Frühgeborene $<1000 \mathrm{~g}$ mit nicht-invasiver druckunterstützter Atemhilfe in den ersten drei Interventionstagen eine 1:1 Betreuung erhalten. Die Empfehlungen wurden u. a. auf Grundlage der British Association of Perinatal Medicine (BAPM) entworfen, empirische Daten aus deutschen Abteilungen existieren nicht.

Fragestellung: Ist der Pflegearbeitseinsatz insbesondere bei Frühgeborenen $<1500 \mathrm{~g}$ abhängig vom Beatmungsverfahren und Lebensalter?

Material und Methoden: Wir analysierten unsere Forschungsfrage mittels eines prospektiven Studiendesigns an einem Perinatalzentrum Level 1. Die Aufzeichnung des zeitlichen Arbeitseinsatzes der Pflegekräfte erfolgte durch Study Nurses, die die mit der Betreuung des Patienten täglich anfallenden Gesamtaktivitäten des Pflegepersonals mittels Exceltool via Tablet erfassten. Per Stichprobenverfahren wurde die Aufnahme über Früh-, Spät- und Nachtdienst durchgeführt, so dass ein repräsentativer Ausschnitt des Arbeitstages erreicht wurde. Erfasst wurden alle Pflegetätigkeiten (direkte Pflege inkl. Elternanleitung, indirekte Pflege, administrative Aufgaben und Sonstiges) zusammen mit den Beatmungstypen der Patienten.

Ergebnisse: Im fünfmonatigen Studienzeitraum wurden an 84 Erfassungstagen und 155 Stunden 39 Pflegekräfte beobachtet, die 113 Frühund Neugeborene betreuten; 57 davon mit einem Geburtsgewicht $<1500 \mathrm{~g}$ (VLBW-Patienten). Im Durchschnitt entfielen 48,2\% der täglichen Arbeitszeit auf direkte Pflegetätigkeiten an VLBW-Patienten, 10,2\% auf direkte Pflegetätigkeiten an Nicht-VLBW-Patienten (Patienten $>1500 \mathrm{~g}$ ), $15,5 \%$ auf indirekte Pflegetätigkeiten, 2,2\% auf Verwaltungstätigkeiten und $23,9 \%$ auf sonstige, patientenferne Tätigkeiten. Auf Patientenebene zeigten sich nur kleine Unterschiede in Abhängigkeit des Atemunterstützungsverfahrens und des Lebensalters (siehe - Tab. FV31). Zum Vergleich wurde zusätzlich die Patientengruppe $<1000 \mathrm{~g}$ (ELBW-Patienten) aufgeführt.

Schlussfolgerung: Unsere Ergebnisse suggerieren, dass vor allem die Form der Atemhilfe des Patienten den zeitlichen Arbeitseinsatz der Pflegekräfte beeinflusst. Die Replikation der Daten im Rahmen einer Multicenter-Studie wird angestrebt. In Richtlinien zum Pflegearbeitseinsatz sollten solche empirisch gewonnenen Ergebnisse berücksichtigt werden.

\section{FV32}

\section{Triggerschwellen von Heimbeatmungsgeräten mit Offen-,} Ein- oder Zweischlauchsystem im Lungenmodell

J. Schmidt' , C. Brickmann' ', D. Klotz', S. Schumann' ${ }^{2}$, H. Fuchs ${ }^{1}$

'Universitäts - Kinderklinik, Neonatologie und pädiatrische Intensivmedizin, Freiburg, Deutschland, ${ }^{2}$ Klinik für Anästhesiologie und Intensivmedizin, Experimentelle Anästhesiologie, Freiburg, Deutschland

Hintergrund: Säuglinge und Kleinkinder vermögen insbesondere bei größeren Leckagen oft nicht Heimbeatmungsgeräte suffizient zu triggern, was $\mathrm{zu}$ asynchroner Beatmung führt.

Fragestellung: Welche Unterschiede in den Triggerschwellen bestehen zwischen verschiedenen Heimbeatmungsgeräten und welchen Einfluss hat die Auswahl des Schlauchsystems.

Methoden: Die Trigggerschwellen von 7 verschiedenen Heimbeatmungsgeräten mit Offenschlauchsystem, Einschlauchsystem mit patientennahem Exspirationsventil oder Zweischlauchsystem wurden unter Bedingungen ohne Leckage, bei moderater Leckage (4 mm Öffnung) und bei großer Leckage $(2 \times 4 \mathrm{~mm}$ Öffnung) in einem Lungenmodell mit Flussgenerator im assistiert druckkontrollierten Modus (PIP: $16 \mathrm{~cm} \mathrm{H}_{2} \mathrm{O}$, PEEP: $4 \mathrm{~cm} \mathrm{H} \mathrm{H}_{2} \mathrm{O}$, Ti 0,4 s, Backup Atemfrequenz 15/min) bestimmt. Zunächst wurde die Triggerempfindlichkeit ermittelt, bei der keine Autotriggerung stattfand. Als Triggerschwelle wurde der Fluss festgelegt, bei dem $\geq 95 \%$ der Atemzüge (30/min) durch das Beatmungsgerät synchronisiert unterstützt wurden. Die Messungen wurden je $3 \mathrm{x}$ wiederholt. Mean (Standardabweichung) sind angeben. Die Signifikanz der Unterschiede wurde durch one way-ANOVA bzw T-Test überprüft.

Ergebnisse: Die 4 Turbinengeräte der neusten Generation (Gerät A, B, C, D) triggerten Atemzüge zuverlässig ab Inspirations-Flussraten von $(2,02$ $(0,02)$ vs $2,96(0,01)$ vs $1,67(0,01)$ vs $1,45(0,01) 1 / \mathrm{min} ; A$ vs $B$ vs $C$ vs $\mathrm{D}, p<0,01)$. Auch große Leckage erhöhte die Triggerschwelle bei diesen Beatmungsgeräten nur unwesentlich $(+9,5 \% ;+6,5 \% ;+/-0 \%,+50 \%)$. Die Triggerschwelle bei Gerät A war am niedrigsten mit Offenschlauchsystem $(2,02(0,02)$ vs $3,91(0,03) 1 / \mathrm{min}, p<0,01)$ bei Gerät $\mathrm{C}$ und $\mathrm{D}$ mit geschlossenem Schlauchsystem (gesteuertem Exspirationsventil (C: $2,11(0,02)$ vs $1,67(0,01) 1 / \mathrm{min} ; p<0,01 ; \mathrm{D}: 2,18(0,01$ vs $1,45(0,01) 1 / \mathrm{min} ; p<0,01)$. Die Triggerschwelle eines Gerätes der älteren Generation (Gerät $\mathrm{E}$ ) war erheblich höher $(8,13(0,04)$ 1/min) und damit im Bereich der Triggerschwelle eines Intensivrespirators (Evita 4). Bei zwei Heimbeatmungs-Geräten (F, $\mathrm{G})$ konnte auch bei hohen Flusswerten $(10 \mathrm{l} / \mathrm{min})$ keine Inspiration getriggert werden.

Schlussfolgerung: In unserem Lungenmodell waren die Triggerschwellen von Heimbeatmungsgeräten der neusten Generation ähnlich sensibel und übertrafen Geräte der älteren Generation und einen Intensivrespirator. Leckage wurde gut kompensiert und hatte nur wenig Einfluss auf die Triggerperformance. Es gibt gerätespezifische Eigenheiten bzgl. der Voroder Nachteile von Offen- oder Geschlossenschlauchsystemen in Bezug auf die Triggerschwelle. 2 Heimbeatmungsgeräte erlaubten im Versuchsaufbau keine Triggerung.

Tab. FV31: Durchschnittliche pflegerische Gesamtarbeitszeit unterteilt nach Lebensalter sowie $<1500 \mathrm{~g}$ und $<1000 \mathrm{~g}$

\begin{tabular}{|lllll}
\hline & Erste drei Lebenstage & \multicolumn{3}{l}{ Nach drei Lebenstagen } \\
\hline & $<1500 \mathrm{~g}$ & $<1000 \mathrm{~g}$ & $<1500 \mathrm{~g}$ & $<1000 \mathrm{~g}$ \\
\hline Maschinell beatmet & $45,3 \%$ (IQR: 36,7-52,2\%) & $45,0 \%$ (IQR: 36,7-52,2\%) & $43,8 \%$ (IQR: 19,4-67,8\%) & $43,8 \%$ (IQR: 19,4-67,8\%) \\
\hline nCPAP & $37,3 \%$ (IQR: 20-48,0\%) & $41,5 \%$ (IQR: 25,8-62,0\%) & $35,5 \%$ (IQR: 12,5-48,6\%) & $34,8 \%($ IQR: 12,2-46,7\%) \\
\hline
\end{tabular}


FV33

\section{Endotracheale vs. intravenöse Gabe eines wasserlöslichen, nano-verkapselten Vitamin A Präparats - Pilotstudie in frühgeborenen Lämmern}

H. Wahl', M. Hütten' ${ }^{2}$, D. Monz', E. Tutdibi', D. Ophelders' ${ }^{3}$, M. Nikiforou', T. Tschernig ${ }^{4}$, L. Gortner' ${ }^{1}$ D. Nohr ${ }^{5}$, H. Biesalski ${ }^{5}$, B. Kramer ${ }^{3}$

'Universitätsklinik für Kinder- und Jugendmedizin, Neonatologie, Homburg, Deutschland, ${ }^{2}$ Universitätsklinikum, RWTH Aachen, Sektion Neonatologie, Klinik für Kinder- und Jugendmedizin, Aachen, Deutschland, ${ }^{3}$ Maastricht University Medical Center, Department of Pediatrics, Maastricht, Niederlande, ${ }^{4}$ Universität des Saarlandes, Institut für Anatomie und Zellbiologie, Homburg/Saar, Deutschland, ${ }^{5}$ Universität Hohenheim, Stuttgart, Deutschland

Hintergrund: Vitamin A (VA) ist ein notwendiger Faktor für die physiologische Organentwicklung, insbesondere für die Lungenreifung. Frühgeborene mit extrem niedrigem Geburtsgewicht (ELBW) haben erniedrigte VA Plasma-Spiegel und ein erhöhtes Risiko, an einer Bronchopulmonalen Dysplasie (BPD) zu erkranken. VA Supplementation wird bei ELBW Frühgeborenen zur Prävention der BPD eingesetzt. Die regelmäßige intramuskuläre (i. m.) VA-Gabe verringert die Mortalität und die Sauerstoffpflichtigkeit im korrigierten Alter von 36 Wochen. Aufgrund einer Anzahl vor allem praktischer Vorbehalte, wie z. B. die schmerzhafte i. m. Gabe, konnte sich die VA-Gabe bisher nicht als Therapiestandard zur Vermeidung der BPD bei ELBW Neonaten durchsetzen.

Fragestellung: Diese Pilotstudie im frühgeborenen Lamm Modell diente zur Beurteilung eines alternativen VA-Präparates, einer wasserlöslichen, nano-verkapselten Retinylpalmitat (RP)-Präparation, hinsichtlich Machbarkeit, Gewebsverteilung und Einfluss auf homöostatische Gene des VA Stoffwechsels (CYP26B, LRAT, STRA6) nach intravenöser (i.v.) und endotrachealer (e.t.) Einmalgabe.

Material und Methoden: 10 frühgeborene Lämmer wurden per Kaiserschnitt entbunden, intubiert und für drei Stunden beatmet. Randomisiert erhielten sie I) einmalig 5000 IE/kg RP i. v., II) einmalig Poractant alfa plus 5000 IE/kg RP e.t. via Tubus, oder III) keine RP Gabe (Kontrolle).

Ergebnisse: Retinol (ROH) und RP waren $3 \mathrm{~h}$ nach Gabe im Serum beider Behandlungsgruppen im Vergleich zur Kontrollgruppe signifikant erhöht, wobei die Kinetik des Anstiegs unterschiedlich war. In der Lunge war die Gewebekonzentration von $\mathrm{ROH}$ und RP beider Behandlungsgruppen signifikant erhöht, in der Leber zeigte sich nur nach i.v. Gabe ein signifikanter Anstieg. Ferner beobachteten wir einen mRNA-Anstieg des im VA Katabolismus wichtigen Gens CYP26B nach i.v.-RP, während nach e.t.-RP ein Abfall der LRAT mRNA (Veresterung) zu beobachten war.

Schlussfolgerung: Sowohl die endotracheale als auch die intravenöse VA-Gabe erhöhte in unserem Modell innerhalb von $3 \mathrm{~h}$ die VA-Konzentration im Serum und im Zielorgan Lunge. Bereits in diesem kurzen Zeitraum konnten Effekte auf molekularer Ebene nachgewiesen werden. Serumkinetik und Enzyminduktion sollten hinsichtlich ihrer Langzeiteffekte auf die Lunge weiter untersucht werden.

\section{FV34}

Ergebnisse der Nachuntersuchung ehemaliger sehr kleiner beatmeter Frühgeborener nach Randomisierung in unterschiedliche $\mathrm{PCO}_{2}$-Zielbereiche (PHELBI-Studie)

U. Thome', O. Genzel-Boroviczény' ${ }^{2}$, B. Bohnhorst', M. Schmid', H. Fuchs ${ }^{5}$, O. Rohde ${ }^{6}$, S. Avenarius ${ }^{7}$, H. Topf ${ }^{8}$, A. Zimmermann ${ }^{9}$, D. Faas ${ }^{10}$, K. Timme ${ }^{11}$, B. Kleinlein ${ }^{12}$, H. Buxmann ${ }^{13}$, W. Schenk ${ }^{14}$, H. Segerer ${ }^{15}$, N. Teig ${ }^{16}$, C. Gebauer ${ }^{17}$, R. Hentschel ${ }^{18}$, M. Heckmann ${ }^{19}$, R. Schlößer ${ }^{13}$, J. Peters ${ }^{12}$, R. Rossi ${ }^{11}$, W. Rascher ${ }^{22}$, R. Böttger ${ }^{23}$, J. Seidenberg ${ }^{24}$, G. Hansen ${ }^{25}$, M. Zernickel ${ }^{26}$, G. Alzen ${ }^{27}$, J. Dreyhaupt ${ }^{28}$, R. Muche ${ }^{28}$, H. Hummler ${ }^{30}$

'Universitätsklinikum Leipzig, Klinik und Poliklinik für Kinder und Jugendliche der Universität, Abteilung Neonatologie, Leipzig, Deutschland, ${ }^{2}$ Klinikum der Universität München Innenstadt, Perinatologie, München, Deutschland, ${ }^{3}$ Kinderklinik der Med. Hochschule, Abt. Pädiatrische Pneumologie, Neonatologie und Allergologie, Hannover, Deutschland, ${ }^{4}$ Neonatologie Zürich, Zürich, Schweiz, ${ }^{5}$ Universitäts - Kinderklinik, Freiburg, Deutschland, ${ }^{6}$ Elisabeth-Kinderkrankenhaus, Kinder- und Jugendmedizin, Oldenburg, Deutschland, ${ }^{7}$ Universitätsklinik für Allgemeine Pädiatrie und Neonatologie, Magdeburg, Deutschland, ${ }^{8}$ Universitäts-Kinderklinik, Neonatolgie, Erlangen, Deutschland, ${ }^{9}$ Kinderklinik der TU Krankenhaus München Schwabing, Klinik und Poliklinik für Kinder- und Jugendmedizin, München, Deutschland, ${ }^{10}$ Zentrum für Kinderheilkunde der Justus-v.-Liebig-Universität, Allgemeinpädiatrie und Neonatologie, Giessen, Deutschland, "VivantesKlinikum Neukölln, Kinder- und Jugendmedizin, Berlin, Deutschland, ${ }^{12}$ Kinderklinik Dritter Orden, Klinik für Kinder- und Jugendmedizin, München, Deutschland, ${ }^{13}$ Klinikum der J.W.Goethe-Universität, Zentrum der Kinderheilkunde, Abteilung für Neonatologie, Frankfurt am Main, Deutschland, ${ }^{14} \mathrm{Klinikum}$ Augsburg, II. Klink für Kinder und Jugendliche, Augsburg, Deutschland, ${ }^{15}$ Klinik St. Hedwig, Krankenhaus Barmherzige Brüder Regensburg, Abteilung für Neonatologie, Regensburg, Deutschland, ${ }^{16}$ Klinik für Kinder- und Jugendmedizin St.Josefs-Hospital, Abteilung für Neonatologie, Bochum, Deutschland, ${ }^{17}$ Universitätskinderklinik, Neonatologie, Leipzig, Deutschland, ${ }^{18}$ Institut/Klinik: Universitätsklinikum Freiburg, Zentrum für Kinder- und Jugendmedizin, Neonatologie/Intensivmedizin, Freiburg, Deutschland, ${ }^{19} \mathrm{~K}$ linik und Poliklinik für Kinder und Jugendmedizin, Neonatologie u. Päd. Intensivmedizin, Greifswald, Deutschland, ${ }^{20} \mathrm{Klinikum}$ der J.W.Goethe-Universität, Zentrum der Kinderheilkunde, Abteilung für Neonatologie, Frankfurt am Main, Deutschland, ${ }^{21}$ Vivantes Klinikum Neukölln, Klinik für Kinder - und Jugendmedizin, Berlin, Deutschland, ${ }^{22}$ Kinder- und Jugendklinik, Universitätsklinikum Erlangen, Erlangen, Deutschland, ${ }^{23}$ Universitäts-Kinderklinik Magdeburg, Perinatalzentrum/Neo1, Wellen, Deutschland, ${ }^{24}$ Klinikum Oldenburg gGmbH, Klinik für Kinder- und Jugendmedizin, Oldenburg, Deutschland, ${ }^{25}$ Kinderklinik der Med. Hochschule, Abt. Pädiatrische Pneumologie, Neonatologie und Allergologie, Hannover, Deutschland, ${ }^{26}$ Klinik für Kinder- und Jugendmedizin, Universitätsklinikum Ulm, Sektion Neonatologie und Pädiatrische Intensivmedizin, Ulm, Deutschland, ${ }^{27}$ Medizinische Fakultät JLU Gießen, Kinderradiologie, Heuchelheim, Deutschland, ${ }^{28}$ Universität Ulm, Institut für Epidemiologie und Medizinische Biometrie, Ulm, Deutschland, ${ }^{29} \mathrm{Klinik}$ für Kinder- und Jugendmedizin, Universitätsklinikum Ulm, Sektion Neonatologie und Pädiatrische Intensivmedizin, Ulm, Deutschland

Hintergrund: In einer randomisierten Multizenterstudie wurde untersucht, ob permissive Hyperkapnie das pulmonale Outcome verbessert. Durch Steigerung der zerebralen Durchblutung oder mittels Beeinträchtigung der Zellfunktionen durch die respiratorische Azidose könnte permissive Hyperkapnie Auswirkungen auf das entwicklungsneurologische Outcome haben.

Tab. FV34.1

\begin{tabular}{|lllllll}
\hline & Gewicht $(\mathbf{k g})$ & Größe $(\mathbf{c m})$ & KU $(\mathbf{c m})$ & MDI & PDI & EfkE \\
\hline Hyperkapnie & $10,5(9,75-11,7)$ & $84(82-87)$ & $47(45-48)$ & $82(60-96)$ & $84(57-100)$ & $22(15-28)$ \\
\hline Kontrollgruppe & $10,5(9,6-11,55)$ & $85(82-87)$ & $47(46-48)$ & $84(58-96)$ & $84(65-96)$ & $23(16-29)$ \\
\hline$p$ & 0,99 & 0,41 & 0,58 & 0,79 & 0,73 & 0,56 \\
\hline \multicolumn{2}{ll}{ Angegeben sind Median und Interquartilbereich (25.-75. Perzentile). } \\
\end{tabular}




\begin{tabular}{|lllll|}
\hline Tab. FV34.2 & & & & \\
\hline $\boldsymbol{n}$ (\%) & $\mathrm{MDI}<85$ & $\mathrm{PDI}<85$ & $\mathrm{MDI}<70$ & $\mathrm{PDI}<70$ \\
\hline Hyperkapnie & $67(55)$ & $56(51)$ & $37(30)$ & $36(33)$ \\
\hline Kontrollgruppe & $64(50)$ & $62(53)$ & $41(32)$ & $39(33)$ \\
\hline$p$ & 0,47 & 0,81 & 0,74 & 0,96 \\
\hline Angegeben sind $n$ (\%). & & & \\
\hline
\end{tabular}

Fragestellung: Beeinflusst eine permissive Hyperkapnie die Ergebnisse der entwicklungsneurologischen Nachuntersuchung in einem korrigierten Alter von 2 Jahren?

Material und Methoden: Die entwicklungsneurologische Nachuntersuchung erfolgte anhand der Bayley Scales for Infant Development II mit Ermittlung der mentalen und psychomotorischen Entwicklungsquotienten (MDI und PDI) sowie der Gross Motor Function Classification Scale (GMFCS) und des Elternfragebogens für die kindliche Entwicklung (EfKE). Hörstörungen und Sehstörungen wurden anamnestisch erfasst. Ergebnisse: Randomisiert wurden im Rahmen der PHELBI-Studie 359 Frühgeborene. Nachuntersucht wurden 265 Probanden; 48 waren zum Untersuchungszeitpunkt verstorben und 46 Überlebende konnten nicht nachuntersucht werden. Das ergab eine Follow-up-Rate von $85 \%$ der Überlebenden. In den demografischen Daten der nachuntersuchten Kinder gab es keine signifikanten Unterschiede zwischen den beiden Gruppen (FV34.1). Auch gab es keine signifikanten Unterschiede zwischen den nachuntersuchten und nicht nachuntersuchten Überlebenden, außer einem häufigeren vorzeitigen Blasensprung bei den nicht nachuntersuchten Überlebenden ( $35 \%$ vs. $20 \%, p=0,03$ ).

Auch für die kombinierten Ergebnisse Tod oder MDI $<70$ und Tod oder PDI $<70$ ergaben sich keine signifikanten Unterschiede (Tab. FV34.2). Ebenso unterschieden sich die Ergebnisse des GMFCS nicht signifikant $(p=0,23)$ Hörbehinderungen gab es bei $8(6 \%)$ bzw. $5(4 \%, p=0,35)$ und Sehbehinderungen bei $24(19 \%)$ bzw. $26(20 \%, p=0,89)$. Multiple logistische Regressionsanalysen ergaben für MDI $<70$ die Risikofaktoren Hirnblutungen (alle Schweregrade), Hydrocortisonbehandlung und periventrikuläre Leukomalazie. Risikofaktoren für PDI $<70$ waren bronchopulmonale Dysplasie, Hirnblutungen (alle Schweregrade), und Hydrocortisonbehandlung. Keinen signifikanten Beitrag zum Risiko für einen MDI $<70$ oder PDI $<70$ leisteten Gruppenzuteilung, Geschlecht, Geburtsgewicht, Gestationsalter, Klassifizierung als SGA, Behandlung mit Natriumbicarbonat oder Dexamethason. Schlussfolgerungen: Eine Beatmungsstrategie mit permissiver Hyperkapnie mit dem untersuchten Zielbereich hatte keinen Einfluss auf das entwicklungsneurologische Outcome mit 2 Jahren. Die insgesamt niedrigen Entwicklungsquotienten sprechen für eine Negativselektion der eingeschlossenen Patienten, da nur Patienten eingeschlossen wurden, die frühzeitig einer invasiven Beatmung bedurften.

\section{FV35}

\section{NT-proBNP und rechtsventrikulärer myokardialer Performance Index zur Prädiktion einer brochopulmonalen Dysplasie bei Frühgeborenen $<32 \mathrm{SSW}$}

\section{Czernik', A. Minke', B. Metze', C. Bührer ${ }^{1}$}

${ }^{1}$ Charite Universitätsmedizin Berlin, Klinik für Neonatologie, Berlin, Deutschland

Hintergrund: Die bronchopulmonale Dysplasie (BPD) ist eine der am häufigsten auftretenden Morbiditäten bei kleinen Frühgeborenen. Vielversprechende Studienresultate weisen daraufhin, dass zirkulierende Peptide wie BNP (B-type natriuretic peptide) einen prädiktiven Wert für die Entwicklung einer BPD haben. Ebenso konnte in einigen Studien gezeigt werden, dass der myokardiale Performance Index des rechten Ventrikels (RIMP) zur Abschätzung eines erhöhten pulmonalvaskulären Widerstands geeignet ist. Inwieweit beide Parameter (BNP, gemessen als NT-proBNP im Urin, und RIMP) bereits in der ersten Lebenswoche mit der Entwicklung einer BPD korrelieren, ist bisher unklar.
Fragestellung: Ziel dieser prospektiven Studie war, die Bestimmung von NT-proBNP im Urin und des echokardiographischen Parameters RIMP am 7. Lebenstag von Frühgeborenen $<32$ SSW, und den Bezug auf die Prädiktion einer BPD zu überprüfen.

Patienten und Methodik: Bei 66 Frühgeborenen $<32$ SSW wurden im Alter von 7 Lebenstagen NT-proBNP Konzentrationen im Urin mit einem Chemilumineszenz-Immunoassay-Verfahren (Roche Diagnostics, Mannheim, Germany) gemessen und der echokardiographische Parameter RIMP bestimmt.

Ergebnisse: In der Studienpopulation entwickelten 10 Frühgeborene eine BPD (Sauerstoffsupplementierungsbedarf mit 36 Schwangerschaftswochen). Die NT-proBNP Konzentration im Urin war bei Frühgeborenen mit einer BPD im Vergleich mit der Kontrollgruppe im Durchschnitt über 10 fach erhöht (median 8873 pg/ml [IQR 5676-25743] vs. 770 pg/ml [379_ 2823], $p<0,001)$. Der echokardiographische Paramter RIMP war bei den Frühgeborenen, die später eine BPD entwickelt haben, signifikant erhöht. (median 0,31 [IQR 0,21-0,43] vs. 0,18 [0,1-0,24], $p<0,05$ ). Zwischen beiden Parametern NT-proBNP und RIMP konnte eine signifikante Korrelation nachgewiesen werden. (Rs $=0,35 ; p=0,008)$. NT-proBNP und RIMP erwiesen sich im Alter von 7 Tagen anhand der ROC-Kurve (AUC: 0,88 $(95 \%$ KI: 0,78-0,98)) bzw. 0,72 (0,52-0,92)) als gute prädiktive Marker für eine BPD.

Schlussfolgerung: Frühgeborene mit einer BPD zeigen bereits am 7. Lebenstag sowohl erhöhte NT-proBNP Konzentrationen im Urin als auch einen erhöhten RIMP. Somit erscheint die frühzeitige Bestimmung beider Parameter hilfreich in der Prädiktion einer BPD.

\section{Freie Themen 06: Psyche}

\section{FV36}

The role of maternal care behavior in the effects of maternal immune activation on depression-like behavior in the mouse

\section{Lindtner ${ }^{1}$, M. Ronovsky², S. Berger ${ }^{2}$, A. Berger ${ }^{3}$, D. Pollak ${ }^{2}$}

'Universitätsklinik für Kinder- und Jugendheilkunde, Klinische Abteilung für Neonatologie, pädiatrische Intensivmedizin und Neuropädiatrie, Medizinische Universität Wien, Wien, Österreich, ${ }^{2}$ Zentrum für Physiologie und Pharmakologie, Abt. f. Neurophysiologie und Neuropharmakologie, Medizinische Universität Wien, Wien, Österreich, ${ }^{3}$ Universitätsklinik für Kinder- und Jugendheilkunde, Klinische Abteilung für Neonatologie, pädiatrische Intensivmedizin und Neuropädiatrie, Medizinische Universität Wien, Wien, Österreich

Background: In a mouse model of gestational infection we have previously reported that maternal immune activation (MIA) is associated with depression-like behavior in the adult male and female offspring and correlated with long-lasting cellular and molecular deficits in the brain. Enduring behavioral and neural adaptations can be modulated by specific elements of the perinatal environment, most prominently by maternal care behavior (MB). These life-long influences of $\mathrm{MB}$ on offspring behavior are thought to be engrained in the brain through epigenetic mechanisms which confer long-lasting changes in gene expression without alterations in DNA sequence.

Aim: To investigate whether a paradigm of MIA related to the development of depression-like behavior is also associated with alterations in $\mathrm{MB}$ and modifications of the expressional level of genes known to be epigenetically controlled through $\mathrm{MB}$ in the offspring brain.

Material and Methods: MIA was induced by administration of Poly(I:C) at embryonic day 12.5 (E12.5) to pregnant C57BL/6N mice and maternal behavior was recorded on the postnatal days 1 to 6 . mRNA levels of glucocorticoid and mineralocorticoid receptors (GR and MR), confirmed targets of regulation by $\mathrm{MB}$ and implicated in the pathophysiology of depression, were evaluated by quantitative real-time PCR (qRT-PCR) in hippocampal tissue of adult female offspring. 
Results: MIA significantly reduced the percentage of time mothers engaged in licking-grooming behavior, the main form of physical contact experienced by the newborn pups, which is known to modulate behavioral, autonomous and endocrine functions later in life. A significant enhancement in hippocampal mRNA expression of GR and MR were observed in MIA as compared to control female offspring. Discussion: We here firstly describe the significantly dampening effect of Poly(I:C)-induced MIA on maternal licking and grooming behavior in the mouse and provide evidence that the expression of prime targets of epigenetic regulation is also altered in the adult female offspring brain. It is conceivable to speculate that MIA may, through hitherto undefined mechanisms, impact MB which in turn epigenetically regulates corticosteroid receptor expression in the offspring brain, potentially leading to or causing emotional disturbances later in life. The present study could be of significant translational value, as it may elucidate the specific relevance of physical contact in the postnatal environment as relevant determining factor for susceptibility or resilience to the impact of prenatal infection on the regulation of emotional function later in life.

\section{FV37}

Hat die Länge des präpartalen Krankenhausaufenthaltes einen Einfluss auf den mütterlichen Stress nach Geburt eines frühgeborenen Kindes (Eine 2-Zentren prospektive PilotBeobachtungsstudie)

\section{E. Pichler-Stachl', G. Pichler', N. Baik', B. Urlesberger', A. Avian², P. Urlesberger', P. Cheung ${ }^{3}$, G. Schmölzer ${ }^{3}$}

'Univ. Klinik für Kinder- und Jugendheilkunde Graz, Abt. für Neonatologie, Graz, Österreich, ${ }^{2}$ Medizinische Universität Graz, Institut für Medizinische Informatik, Statistik und Dokumentation, Graz, Österreich, ${ }^{3}$ Department of Neonatology, Royal Alexandra Hospital, Edmonton, Canada, Edmonton, Kanada

Hintergrund: Frühgeburt ist assoziiert mit erhöhtem elterlichem Stress und mit Ängsten, die in Folge die Mutter-Kind Interaktion beeinflussen können.

Fragestellung: Ziel der Studie war es, einen möglichen Einfluss der Länge eines präpartalen mütterlichen Krankenhausaufenthaltes auf den mütterlichen Stress nach der Geburt eines frühgeborenen Kindes zu untersuchen. Methoden: Eine 2-Zentren prospektiven Beobachtungsstudie wurde an der Neonatologie in Graz (Österreich) und in Edmonton (Canada) durchgeführt. Mütter von Frühgeborenen $<36$ Schwangerschaftswochen (SSW), deren Kinder an der Neonatologie stationär aufgenommen werden mussten, wurden untersucht. Mütter mit einem präpartalem mütterlichen Krankenhausaufenthalt $<12$ Stunden wurden verglichen mit Müttern mit einem präpartalem mütterlichen Krankenhausaufenthalt $\geq 12$ Stunden. Der mütterliche Stress wurde innerhalb von drei Tagen nach der Geburt mit dem PSS:NICU Fragebogen - bestehend aus drei Bereichen („Aussehen und Verhalten des Kindes“, „Elterliche Rolle/Beziehung zum Kind“ und „Staionäre Umgebung“") erhoben. Soziodemographische Daten wurden aus den Krankenakten und mit einem zusätzlichen mütterlichen Fragebogen erhoben. Ergebnisse: 20 Mütter mit einem präpartalem mütterlichen Krankenhausaufenthalt $<12$ Stunden wurden verglichen mit 20 Müttern mit einem präpartalem mütterlichen Krankenhausaufenthalt $\geq 12$ Stunden. Die Gruppen unterschieden sich nicht in den soziodemographischen Daten. Der mütterliche Stress im Bereich „Stationäre Umgebung“ war bei Müttern mit präpartalem Aufenthalt $\geq 12 \mathrm{~h}$ signifikant erhöht $(2,48 \pm 0,69)$ verglichen mit Müttern mit präpartalem Aufenthalt $<12 \mathrm{~h}(1,95 \pm 0,73)(p=0,024)$. Kein Unterschied zwischen den Gruppen gab es in den Bereichen „Aussehen und Verhalten des Kindes" $(2,73 \pm 0,80$ vs. $2,72 \pm 0,91 ; p=0,962)$ und „Elterliche Rolle/Beziehung zum Kind" $(3,31 \pm 1,08$ vs. $3,58 \pm 1,18 ; p=0,484)$. Zusammenfassung: Die vorliegende Studie zeigt, dass mütterlicher Stress nach einer Frühgeburt von der präpartalen mütterlichen Krankenaufenthaltsdauer beeinflusst wird. Interventionsprogramme sollten daher bereits in dieser Zeit beginnen, mit dem Ziel den mütterlichen Stress zu reduzieren, um somit möglicherweise eine die Mutter-Kind Interaktion positiv zu beeinflussen.

\section{FV38}

Pränatale Beratungsgespräche bei drohender Frühgeburtlichkeit an der Grenze der Lebensfähigkeit: Basis für eine vertrauensvolle Beziehung zwischen Eltern und medizinischem Personal

\section{B. Bohnhorst ${ }^{1}$, T. Sengpiel' ${ }^{2}$, C. Peter ${ }^{1}$, S. Pirr ${ }^{1}$}

'Medizinische Hochschule Hannover, Klinik für Pädiatrische Pneumologie, Allergologie und Neonatologie, Hannover, Deutschland, ${ }^{2}$ Städtisches Klinikum Wolfenbüttel, Klinik für Anästhesie, operativ Intensiv-, Notfall- und Schmerzmedizin, Wolfenbüttel, Deutschland

Hintergrund: Die Geburt eines Frühgeborenen (FG) an der Grenze der Lebensfähigkeit sowie die Phase der anschließenden stationären Behandlung stellen für die Eltern ein einschneidendes Erlebnis dar. In der Regel erlebt die Familie ein Auf und Ab der Gefühle mit emotionalem Stress an der Grenze der Belastungsfähigkeit.

Fragestellung: Welchen Einfluss hat die pränatale Beratung der Eltern auf deren späteres Erleben der stationären Behandlung ihres/ihrer FG sowie die Zeit nach der Entlassung? Gibt es generelle Unterschiede in den persönlichen Erfahrungen zwischen Eltern mit ausschließlich lebenden Kindern einerseits und Eltern mit verstorbenen Kindern andererseits?

Methoden: Ein strukturierter Fragebogen (35 Fragen) wurde an 99 Familien versendet, deren FG zwischen 1.1.2000 und 31.12.2008 in der Medizinischen Hochschule Hannover geboren wurde und ein Gestationsalter $<260 / 7$ Wochen hatte. Die statistische Auswertung erfolgte mit IBM SPSS Statistics for Windows, Version 20.0. Es wurden chi-Quadrat- und exakte Fisher-Tests durchgeführt.

Ergebnisse: Die Rücklaufquote war 73\%. Eltern mit ausschließlich lebenden Kindern beantworteten den Fragebogen signifikant häufiger als Eltern mit verstorbenen Kindern ( 78 vs. $62 \%, p=0,027)$. Unabhängig vom Outcome des Kindes fühlten sich Eltern, welche angaben, durch eine empathische pränatale Beratung gut in die pränatalen Entscheidungsprozesse einbezogen worden zu sein, signifikant häufiger auch gut in die stationäre Behandlung ihres Kindes eingebunden ( 79 vs. $43 \%, p=0,006$ ). Sie würden sich auch signifikant häufiger erneut für eine lebenserhaltende Therapie ihres extrem unreifen FG entscheiden ( 95 vs. $65 \%, p=0,007$ ). Außerdem hatten sie im Verlauf ungeachtet des kindlichen Outcome signifikant seltener Zweifel an der Richtigkeit der Behandlung ihres Kindes als Eltern, die sich pränatal nicht genügend involviert fühlten ( 18 vs. $43 \%, p=0,013$ ). Eltern mit ausschließlich lebenden Kindern entschieden sich später signifikant seltener für ein weiteres Kind als Eltern mit mindestens einem verstorbenen Kind ( 19 vs. $56 \%, p=0,004)$. Schlussfolgerung: Diese Studie hebt die Bedeutung einer umfassenden, empathischen pränatalen Beratung der Eltern hervor und zeigt, dass unabhängig vom Outcome des Kindes die Grundlage für eine vertrauensvolle Beziehung zwischen Eltern und medizinischem Personal bereits vor der Geburt gelegt wird.

\section{FV39}

\section{Charakterisierung und Vorhersage des postnatalen} Gewichtsverlaufs von Neugeborenen

S. Kasser', M. Wilbaux' ${ }^{2}$, N. Klarer', H. Rickenbacher', J. van den Anker ${ }^{2}$, O. Lapaire ${ }^{3}$, M. Pfister', S. Wellmann

'Universitäts-Kinderspital beider Basel (UKBB), Abteilung für Neonatologie, Basel, Schweiz, ${ }^{2}$ Universitäts-Kinderspital beider Basel (UKBB), Abteilung für Klinische Pädiatrische Pharmakologie, Basel, Schweiz, ${ }^{3}$ Universitätsspital Basel, Frauenklinik, Basel, Schweiz

Hintergrund: Neugeborene verlieren postnatal vorübergehend Gewicht. Dies ist Bestandteil der physiologischen Umstellungsprozesse. Ein zu hoher Gewichtsverlust ist mit schwerwiegenden Komplikationen verbunden, weshalb die frühzeitige Identifikation von Risikokindern wichtig ist. Fragestellung: Ziel dieser Arbeit war es, (1) klinische Schlüsselfaktoren zu identifizieren, welche den natürlichen Gewichtsverlauf von vollgestillten, gesunden, termingeborenen Kindern bestimmen, (2) in einem mathematischen Modell den Gewichtsverlauf zu charakterisieren und (3) eine nutzerfreundliche Applikation zu entwickeln, um den individuellen Gewichtsverlauf mit einem Minimum an Kerndaten vorhersagen zu können. 
Material und Methoden: In einer retrospektiven Studie wurden zwei komplette Geburtsjahrgänge (2009 und 2010) von termingeborenen gesunden Kindern an der Frauenklinik Universitätsspital Basel untersucht $(n=4128)$. Kombinierte schrittweise Vorwärtsselektion und Rückwärtselimination (Kovarianzanalyse) von zahlreichen Variablen wie Alter der Mutter, BMI vor Schwangerschaft, Versicherungsstatus, Gravidität, Parität, Anästhesie, Geburtsmodus, intrapartaler Blutverlust, Gestationsalter, Geburtsgewicht, Körperlänge, Geschlecht, Nabelschnur pH und Apgar. Entwicklung eines semi-mechanistischen Modells zur Charakterisierung der postnatalen Gewichtsveränderung mittels nichtlinearer gemischter Modellierung (NONMEM7.3). Externe Validierung anhand von 300 zufällig ausgewählten Kindern eines weiteren Jahrgangs (2011). Programmierung einer Online-Applikation.

Resultate: Insgesamt konnten 1335 vollgestillte, gesunde, termingeborenen Kinder in die Analyse eingeschlossen werden nach Ausschluss von Kindern mit Zusatznahrung (Anfangsmilch oder Maltodextrin, $n=2428$ ), nur einer Gewichtsmessung ( $n=24)$, Verlegung in Neonatologie $(n=267)$, Mehrlingsgeburten $(n=72)$ und/oder eine Gewichtszunahme bei der ersten Folgemessung $(n=2)$. Die mathematische Analyse ergab, dass sich die Gewichtsänderung der ersten Lebenswoche ideal als eine Funktion der Nettobilanz zwischen zeitabhängiger Rate an Gewichtsgewinn (KIN) und Gewichtsverlust (KOUT) beschreiben lässt. Knaben hatten ein höheres Geburtsgewicht als Mädchen. Das Gestationsalter hatte einen positiven Effekt auf das Geburtsgewicht und KIN. Das Alter der Mutter zeigte einen positiven Effekt auf das Geburtsgewicht wirkte sich jedoch negative auf KIN aus. KOUT war grösser nach Kaiserschnitt als nach Spontangeburt. Die externe Validierung des Modells war sehr zuverlässig (Bias $=0,011 \%$, Präzision $=0,52 \%$ ) und erlaubte eine akkurate Vorhersage der individuellen Gewichtsänderungen bis zu einer Lebenswoche anhand von nur drei initialen Gewichtsbestimmungen (Geburtsgewicht und zwei Folgegewichte, Bias $=-0,74 \%$, Präzision $=1,54 \%$ ).

Schlussfolgerungen: Wir haben das erste semi-mechanistische Modell entwickelt, dass den postnatalen Gewichtsverlauf von voll gestillten, gesunden termingeborenen Kindern in der ersten Lebenswoche charakterisiert. Die frei zugängliche Online-Applikation (http://neoweight.mashframe.com) erlaubt eine individuelle Vorhersage der Gewichtsentwicklung und hilft Risikokinder früh zu erkennen. Mit Daten von Neugeborenen anderer Zentren und unter Berücksichtigung des Effekts von Zusatznahrung und Frühgeburtlichkeit werden das Modell und seine Anwendbarkeit ausgebaut.

\section{FV40 \\ Unerfüllte psychosoziale und spirituelle Bedürfnisse von Müttern kranker Neugeborener oder Frühgeborener}

\section{U. Waßermann' ${ }^{1}$, M. Thiel ${ }^{2}$, A. Längler ${ }^{3}$, A. Büssing}

${ }^{1} G$ emeinschaftskrankenhaus Herdecke, Kinder- und Jugendpsychiatrie, Herdecke, Deutschland, ${ }^{2}$ Sana-Klinikum Remscheid, Klinik für Kinder und Jugendliche, Remscheid, Deutschland, ${ }^{3}$ Gemeinschaftskrankenhaus Herdecke, Herdecke, Deutschland, ${ }^{4}$ Professur für Lebensqualität, Spiritualität und Coping, Institut für Integrative Medizin, Herdecke, Deutschland

Hintergrund: Spiritualität gehört zu den menschlichen Grundbedürfnissen, wird aber häufig in Krankenhäusern ignoriert, da es von Angehörigen der Gesundheitsberufe als außerhalb der beruflichen Tätigkeit angesehen wird. Inzwischen gibt es zunehmend Hinweise darauf, dass auch in säkularen Gesellschaften Patienten mit chronischen Erkrankungen bestimmte spirituelle Bedürfnisse haben. Diese tragen möglicherweise zur Heilung, mindestens aber zur Krankheitsverarbeitung bei. Über die spirituellen Bedürfnisse der Mütter von Frühgeborenen oder kranken Neugeborenen ist wenig bekannt.

Ziel: Die Identifikation und Quantifizierung unerfüllter Bedürfnisse von Müttern und auf diese beziehen ihre wahrgenommene Stress und Neigungen Leben Bedenken müssen.

Methoden: Anonyme Querschnittsbefragung mit standardisierten Instrumenten (SpNQ, FACIT-Sp, BMLSS, PSS etc.) bei 124 Müttern aus zwei pädiatrischen Abteilungen.
Ergebnisse: „Religiöse Bedürfnisse“ $(0,6 \pm 0,8)$ und „Existentielle Bedürfnisse“ $(0,4 \pm 0,5)$ zeigten die niedrigsten Scores, erzielte während „Geben/ Großzügigkeit“ $(1,0 \pm 0,8)$ von mittelmäßiger und das Item „Innerer Frieden“ $(1,4 \pm 0,7)$ die höchste Relevanz zeigte. Das Item „spirituelles Wohlbefinden“ erzielte ein hohes Ergebnis vor allem die Aspekte „Bedeutung“" $(3,2 \pm 0,6)$ und „Frieden“ $(2,4 \pm 0,8)$, allerdings nicht die Komponente „Glauben“ $(1,5 \pm 1,1)$. Die Mütter spürten einen Einfluss auf „Aspekte des Alltagslebens" (57 \pm 22$)$ und fühlten sich ,unter Druck“ (53 \pm 26$)$, hatten aber nur moderate „Stress-Scores“ ( $23 \pm 6)$, und ihre Lebenszufriedenheit war dennoch sehr hoch $(82 \pm 13)$. Sie fühlten sich von ihrem ihre Partner $(5,6 \pm 0,9)$ und vom Personal auf der Intensivstation $(5,1 \pm 1,1)$ sehr unterstützt. Durch Gespräche mit dem medizinischen Personal hatten $82 \%$ von ihnen das Gefühl, dass sie sich um die Prognose ihres Kindes keine Sorgen machen müssen. In bezug auf die Prognose des Kindes (Frühgeburt - krank mit guter - krank mit unklarer/schlechte Prognose), gab es keine signifikanten Unterschiede zwischen den spirituellen Bedürfnissen, nur einen Trend für höhere religiöse Bedürfnisse $(f=2,8 ; p=.065)$. Die Komponente für „spirituelles Wohlbefinden“, „Frieden“ war jedoch höher bei Müttern von Kindern mit schlechter oder unklarer Prognose $(f=5,5$; $p=.005)$, und ihre Scores für „positive Stimmung “ waren am niedrigsten $(f=7,8 ; p=.001)$ und sie fühlte sich mehr ,unter Druck“ $(f=4,4 ; p=.014)$. Besonders das Item „Innerer Frieden“ korrelierte nur schwach mit der Wahrnehmung von Stress $(r=.25)$, Einfluss auf die Lebensqualität $(r=.25)$ und Trauer $(r=.23)$, und nur am Rande mit der Komponente „Frieden“ des spirituellen Wohlbefindens ( $r=-.19)$. Das „spirituelle Wohlbefinden“ war mäßig negativ korreliert zum Empfinden von Stress $(r=-.44)$ und zur „Lebenszufriedenheit“ $(r=.36)$. Diese Dimensionen decken offensichtlich verschiedene Aspekte des „inneren Friedens“ ab.

Schlussfolgerungen: Mütter von kranken Neugeborenen oder Frühgeborenen fühlen sich unterstützt vom Team der Intensivstation und ihrem Partner, aber empfinden dennoch Stress und eine Beeinflussung des täglichen Lebens Zuneigungen, und haben unbefriedigte Bedürfnisse nach Innerem Frieden. Auf diese spezifischen Bedürfnisse in der Klinik einzugehen kann helfen, sie in dieser schwierigen Situation zu unterstützen und eine gesunde Bindung zu ihrem Kind zu fördern.

\section{FV41}

Zusammenhang zwischen Zeitverständnis und Bedürfnisaufschub bei ehemaligen Frühgeborenen $<32$. SSW im genauen Alter von 4 Jahren

B. Hüning ${ }^{1}$, B. Assing ${ }^{1}$, E. Weishaupt ${ }^{1}$, U. Felderhoff-Müser ${ }^{1}$, N. Zmyj ${ }^{2}$

'Universitätsklinikum Essen, Klinik für Kinderheilkunde I, Neonatologie, Essen, Deutschland, ${ }^{2}$ Technische Universität Dortmund, Institut für Psychologie, Dortmund, Deutschland

Hintergrund: Die Fähigkeit auf die Befriedigung eines Bedürfnisses zu warten, gehört zu den grundlegenden Entwicklungsaufgaben im Kindesalter. Dabei spielt die inhibitorische Kontrolle, d.h. einen dominanten Handlungsimpuls zu unterdrücken, eine entscheidende Rolle. Sehr kleine Frühgeborene (FG) besitzen häufig geringere exekutive Funktionen, z. B. im Bereich der Selbstregulation oder Impulskontrolle. In dieser Studie wird die Fähigkeit zum Bedürfnisaufschub (inhibitorische Kontrolle) bei FG im Vergleich zu Reifgeborenen (RG) untersucht. Zusätzlich wurde der Zusammenhang zwischen Zeitverständnis und Bedürfnisaufschub untersucht, um weitere Aufschlüsse über die kognitiven Mechanismen der inhibitorischen Kontrolle zu erhalten. Ziel der Studie ist es, die Auswirkungen der Frühgeburtlichkeit auf die o.g. Fähigkeiten nachzuvollziehen. Material und Methoden: Es wurden 20 FG (11 männlich, 9 weiblich; Median Gestationsalter: $28+4$ SSW, Gewicht: 1005 g, korr. Alter: 4J 21 Tage) und 22 RG (14 weiblich, 8 männlich; Median Gestationsalter: $40+1$ SSW, Gewicht 3310 g, Alter: 4J23 Tage) untersucht. Die Kinder erhielten zwei Testverfahren: Beim Sanduhrentest (Bischof-Köhler, 2000) wurde das Zeitverständnis durch den Vergleich der Laufzeiten von 3 Sanduhren gemessen (z. B. „Welche Sanduhr ist am schnellsten fertig?"), hierbei wurde der Prozentsatz richtiger Antworten gewertet. Beim Bedürfnisaufschubtest (Mischel\& Ebbesen, 1970) wurde den Kindern die Wahl gelassen, eine 
Süßigkeit sofort oder eine zusätzliche Süßigkeit nach ungewisser Wartezeit zu erhalten. Die Kinder hatten darüber hinaus die Möglichkeit, den Testleiter vorzeitig durch Benutzen einer Klingel zurück zu holen, im Gegenzug erhielten sie nur eine Süßigkeit. Die maximale Wartezeit betrug 15 Minuten, das Kind befand sich in dieser Zeit allein im Testraum.

Ergebnisse: Es zeigte sich eine mediane Wartezeit der FG von 1:22 min sowie der RG von 9:19 $\min (z=2,058, p=.020$, einseitig, Mann-Whitney U Test), im Sanduhrentest erreichten die FG median 39,3\% richtige Antworten, die RG $50 \%$. Die Wartezeit korrelierte nicht mit Zeitverständnis $(r=.286, p=.066$, zweiseitig, Spearman-Test).

Schlussfolgerung: Die FG zeigen eine signifikant geringere Fähigkeit zur Impulskontrolle, gemessen im Bedürfnisaufschubtest. Ein Zusammenhang zum Zeitverständnis zeigte sich nicht. Erkenntnisse über kognitive Prozesse, die an der Impulskontrolle von Kinder beteiligt sind, eröffnen neue Perspektiven für Interventionsmöglichkeiten.

\section{FV42}

\section{Spiritualität in der Neonatologie - Ergebnisse einer systematischen Literaturrecherche}

\section{Thiel'}

'Sana-Klinikum Remscheid, Klinik für Kinder und Jugendliche, Remscheid, Deutschland

Einführung: Die Prognose von Frühgeborenen und kranken Neugeborenen hat sich in den letzten Jahrzehnten wesentlich verbessert. Neben konventionellen Methoden spielen additive Interventionen eine wichtige Rolle. Dazu zählen auch spirituelle Belange. Daten zu diesem Thema wurden bisher nicht systematisch evaluiert.

Methode: Es wurde eine systematische Literaturübersicht nach den PRISMA-Kriterien durchgeführt. Schlüsselworte waren Neonaten, Neonatologie, Neugeborenen, Frühgeburten und Eltern und Spiritualität; gesucht wurden alle Formen klinischer Studien auf Englisch und Deutsch.

Ergebnisse: Es wurden über 70 Studien gefunden. In die abschließende Analyse kamen 45 Veröffentlichungen. Studien-Typen waren Interviews (qualitative Untersuchungen), Fallberichte und Reviews. Die Themenbereiche lassen sich gliedern in 1.Eltern und ihre Bedürfnisse, 2.ethnische Besonderheiten und Unterschiede, 3. medizinisches Personal und 4. Übergangsbereich zur Palliativmedizin.

Diskussion und Fazit: Es gibt eine Vielzahl von Studien über Spiritualität in Neonatologie mit einem breiten Spektrum von Studientypen. Eine Meta-Analyse ist nicht möglich, vor allem wegen der Heterogenität der Studiendesigns, der Zahl der Patienten und der Art der verwendeten Fragebögen. Einige Studien sind vielversprechend, aber weder Kurz- noch Langzeitergebnisse lassen sich ableiten. Interessante Endpunkte wie z.B. der Einfluss auf Mortalität, Morbidität, Dauer des Krankenhausaufenthaltes oder die Entwicklung und psychosoziale Aspekte sollten in weiteren Studien herausgearbeitet werden. Empfehlungen für das medizinische Personal können im Sinne von Denkanstößen, nicht aber als wissenschaftlich fundierte Empfehlung gegeben werden.

\section{Freie Themen 07: Erstversorgung/Reanimation}

\section{FV43}

\section{Stimulation bei Frühgeborenen während der Erstversorgung}

N. Baik', M. Rock', K. Binder', B. Urlesberger', L. Mileder', B. Schwaberger', J. Stadler', G. Pichler'

'Universitätsklinik für Kinder- und Jugenheilkunde, Klinische Abteilung für Neonatologie, Graz, Österreich

Hintergrund: In den ERC-Guidelines für Neugeborenen-Erstversorgung werden das Abtrocknen und eine „kurze“ Stimulation empfohlen. Effekte einer Stimulation wurden bisher jedoch nicht untersucht.
Zielsetzung: Ziel dieser Studie war es, zu analysieren, welche Körperregion wie lange bei den Frühgeborenen während der Erstversorgung stimuliert wurden und ob die Stimulation einen Effekt auf die Vitalparameter hatte. Material und Methode: Es wurde eine Analyse von Videoaufzeichnungen, die im Rahmen von prospektiven Beobachtungs-Studien an der Abteilung für Neonatologie/Medizinischen Universität Graz bei Frühgeborenen während der Erstversorgung gemacht wurden, durchgeführt. Die Videoaufzeichnungen wurden von zwei Untersuchern analysiert hinsichtlich Dauer und Körperregion von Stimulationen. Peripher-arterielle Sauerstoffsättigung $\left(\mathrm{SpO}_{2}\right)$ und die Herzfrequenz $(\mathrm{HF})$ wurden kontinuierlich mittels Pulsoxymetrie gemessen. Um eventuelle Effekte der Stimulation auf die Vitalparameter zu untersuchen, wurden $\mathrm{SpO}_{2}$ und $\mathrm{HF}$ vor und nach der Stimulation verglichen.

Ergebnisse: Insgesamt wurden 59 Frühgeborene in dieser Studie analysiert. Das mittlere Gestationsalter betrug 33,2 $\pm 2,5$ Wochen und das mittlere Geburtsgewicht $1950 \pm 426$ g. 25 von 59 Frühgeborenen wurden im Rahmen der Erstversorgung stimuliert. Es wurden im Median 1 Stimulation pro Neugeborenes durchgeführt. Die Stimulation erfolgte bei 11 Frühgeborenen an der Brust, bei 11 an den Füßen und bei 4 an mehreren Körperregionen (Brust, Fuß). Die durchschnittliche Stimulationsdauer betrug $20,7 \pm 17,4$ Sekunden. Im Vergleich vor und nach der Stimulation konnte ein statistisch signifikanter Anstieg sowohl von Herzfrequenz (vor: 125,5/ min versus nach: $139,1 / \mathrm{min} ; p=0,045$ ) als auch von $\mathrm{SpO}_{2}$ (vor: $67,3 \%$ versus nach: $71,1 \% ; p=0,00018$ ) beobachtet werden.

Schlussfolgerung: Die vorliegende Beobachtungstudie beschreibt erstmalig Stimulationen während der Erstversorgung bei Frühgeborenen wobei die Stimulationen einen Effekt auf $\mathrm{HF}$ und $\mathrm{SpO}_{2}$ hatten.

\section{FV44}

Implementierung und Evaluation eines interprofessionellen, simulationsbasierten Ausbildungskonzepts in Lehre und Studium

\section{E. Heimberg' ', E. Lang ${ }^{2}$, J. Schmutz ${ }^{3}$, M. Kerry' ${ }^{4}$, A. Wosnik ${ }^{5}$, F. Neunhoeffer ${ }^{6}$,} M. Hofbeck ${ }^{7}$, O. Heinzel ${ }^{8}$

'Eberhard-Karls-Universität Universitätsklinik für Kinderheilkunde und Jugendmedizin, Abteilung für Kinderkardiologie, Intensivmedizin und Pulmologie, Tübingen, Deutschland, ${ }^{2}$ Eberhard-Karls-Universität Universitätsklinik für Kinderheilkunde und Jugendmedizin, Tübingen, Deutschland, ${ }^{3}$ ETH Zürich, Arbeits-u. Organisationspsychologie WEV K 504, Zürich, Schweiz, ${ }^{4}$ ETH Zürich, Arbeits-u. Organisationspsychologie, Zürich, Schweiz, ${ }^{5}$ Universitätsklinik Tübingen, Dekanat der Medizinischen Fakultät, Bereich Studium und Lehre, Tübingen, Deutschland, ${ }^{6}$ EberhardKarls-Universität Universitätsklinik für Kinderheilkunde und Jugendmedizin, Kinderkardiologie, Pulmologie und pädiatrische Intensivmedizin, Tübingen, Deutschland, ${ }^{7}$ Universitätsklinik für Kinderheilkunde und Jugendmedizin/ Eberhard-Karls-Universität/, Kinderheilkunde II (Kardiologie, Pulmologie, Intensivmedizin), Tübingen, Deutschland, ${ }^{8}$ Universitäts-Kinderklinik, Abteilung für Allgemeine Pädiatrie, Hämatologie und Onkologie, Tübingen, Deutschland

Hintergrund: In der komplexen Versorgung kritisch kranker Kinder ist die interprofessionelle Zusammenarbeit von entscheidender Bedeutung. Obwohl in Studien interprofessionelle Teamtrainings im medizinischen Sektor die Teamarbeit verbessern, konzentrieren sich bisherige Ausbildungskonzepte hauptsächlich auf die Vermittlung von Fachwissen und technischen Fähigkeiten und finden überwiegend für die beteiligten Berufsgruppen getrennt statt. Simulationsbasierte Konzepte ermöglichen die gemeinsame Zusammenarbeit in komplexen Situationen zu proben ohne Patienten zu gefährden. Im Rahmen eines Pilotprojekts wurde an der Universitätsklinik Tübingen ein interprofessionelles, simulationsbasiertes Ausbildungskonzept entwickelt und implementiert.

Fragestellung: Ziel dieser Studie ist die Evaluation des interprofessionellen Ausbildungsprojekts und die Erhebung der Einstellung der Studenten und der Auszubildenden gegenüber interprofessionellem Lernens vor und nach Einführung des Lernmoduls.

Material und Methoden: In einer prospektiven Studie nahmen 280 Medizinstudenten (9. Fachsemester) und 90 Auszubildende der Gesundheits- 
und (Kinder)-Krankenpflege (1.-3. Ausbildungsjahr) teil. Das Lernmodul umfasste insgesamt 7 Lehrstunden. Es bestand aus einem theoretischen Teil, einem Hands-on Workshop zu Basismaßnahmen der Notfallversorgung und anschließend drei simulierten Kindernotfällen. Die Simulationen wurden mit Hilfe einer High-Fidelity Simulationspuppe (SimBaby $^{\text {min }}$, Laerdal) in realem klinischen Setting und mit realem medizinischem Equipment durchgeführt. Anschließend erfolgte eine videounterstützte Nachbesprechung der medizinischen Inhalte, der Teamzusammenarbeit und der Kommunikation. Die Einstellung der Studenten und Auszubildenden wurde mittels eines validierten Scores (RIPLS; Readiness for Interprofessional Learning Scale) vor und nach dem Training erhoben. Zusätzlich erfolgte die qualitative und quantitative Evaluation des Lernmoduls nach dem Training.

Ergebnisse: 277 Fragebögen vor Einführung und 199 Fragebögen nach Einführung des Lernmoduls konnten ausgewertet werden. Die Einstellung gegenüber interprofessionellem Lernen änderte sich in allen Subskalierungen. In der Versorgung von Notfällen verbesserte sich das subjektive Sicherheitsempfinden, das Rollenverständnis und die Kommunikationskompetenz. Der eigene Aufgabenbereich und der der anderen beteiligten Berufsgruppen wurde klarer wahrgenommen.

Diskussion: Ein einmaliges siebenstündiges interprofessionelles Lernmodul war prinzipiell durchführbar und hatte einen sofortigen positiven Effekt auf die Einstellung gegenüber interprofessionellem Lernen. Das Rollenverständnis, die Aufteilung von Verantwortlichkeiten sowie die gezielte Kommunikation konnte subjektiv verbessert werden.

Schlussfolgerung: Interprofessionelles Lehre ist möglich und verbessert das Teamverständnis. Es bleibt zu zeigen, ob die Qualität der Patientenversorgung dadurch verbessert werden kann.

\section{FV45}

\section{Extrauterine plazentare Transfusion bei Frühgeborenen - eine Alternative zum delayed cord clamping?}

B. Kühne ', C. Kirchgäßner', M. Valter', G. Weißhaar' ', A. Kribs' ', A. Oberthuer' 'Universitätskinderklinik Köln, Neonatologie und Pädiatrische Intensivmedizin, Köln, Deutschland, ${ }^{2}$ Uniklinik Köln, Klinik und Poliklinik für Frauenheilkunde und Geburtshilfe, Köln, Deutschland

Hintergrund: Ein spätes Abnabeln, auch „delayed cord clamping“ (DCC) genannt, führt zu höheren kindlichen Blutvolumina und trägt zur hämodynamischen Stabilität während der postnatalen Anpassungsphase Frühgeborener bei. Es ist zudem mit einer reduzierten Inzidenz neonataler Morbiditäten, wie z. B. intraventrikulärer Blutungen (IVH), verbunden. Im klinischen Alltag besteht die Schwierigkeit, dass durch DCC verzögert mit einer bei Frühgeborenen notwendigen Atemunterstützung begonnen werden kann, da meist nicht bettseitig neben der Mutter mit einer CPAP-Therapie oder positiven Druckbeatmung (PPV) begonnen werden kann. Somit können durch DCC natürlicherweise parallel ablaufende Prozesse der Lungenbelüftung und Vorlasterhöhung durch plazentare Transfusion unphysiologisch gestört werden. Um die Vorteile des verzögerten Abnabelns zu nutzen und dabei eine CPAP-Unterstützung zu ermöglichen, wurde in unserem Level 1 Perinatalzentrum die sogenannte ,intraamniale Entwicklung" etabliert. Dabei werden Frühgeborene per Sectio entbunden und im Amnionsack zur Erstversorgungseinheit gebracht. Nach Eröffnen der Fruchtblase erfolgt durch Hochhalten der Plazenta etwa 40-50 cm über Herzhöhe eine plazentare Transfusion, während simultan mit nCPAP oder nPPV via Maske begonnen wird. Dieses Vorgehen nennen wir „extrauterine plazentare Transfusion“ (EPT). Da die Auswirkungen der EPT bislang nicht untersucht sind und theoretisch die Gefahr einer plazentaren Transfusion von deoxygeniertem $\mathrm{Hb}$ besteht, analysierten wir in dieser Studie erstmalig die Effekte des EPT-Verfahrens.

Studiendesign: Eingeschlossen in die retrospektive Untersuchung wurden Kinder mit einem Gestationsalter von 22+0-32+0 SSW, die zwischen 03/2015 und 11/2015 in unserem Zentrum geboren wurden und bei denen ein erweitertes Kreißsaalmonitoring mit Atemfunktionsmonitoring mittels der NewLifeBox Neo-RSD (Applied Biosignals) und Videoaufzeichnung erfolgte. Ausschlusskriterien waren: Versterben am ersten Lebenstag sowie schwere angeborene Fehlbildungen. 25 VLBW Neugeborene, mit einem Gestationsalter von $23+1$ bis $31+4$ SSW erfüllten die Einschlusskriterien. Bei 14 erfolgte eine EPT (EPT-Gruppe), bei den restlichen 11 erfolgte eine Standardversorgung mit variablen Zeiten bis zum Abnabeln, aber keine EPT (Standardgruppe). Die Outcome-Daten wurden bis zur Entlassung dokumentiert.

Ergebnisse: Das mittlere Geburtsgewicht der Kinder der EPT und Standard-Gruppe betrug $906 \mathrm{~g}$ (SD $365 \mathrm{~g}$ ) und $992 \mathrm{~g}$ (SD 365); das mittlere Gestationsalter war 27+3 SSW (SD 2 Tage) bzw. 27+2 SSW (SD 3). Die mittlere Zeit bis zur Abnabelung war in der EPT-Gruppe 4,6 Min (SD 1,3). Zwischen den Gruppen zeigten sich keine signifikanten Unterschiede hinsichtlich der Kriterien postnatale Hämoglobinkonzentration, Transfusionsbedürftigkeit, sowie Sauerstoffsättigung und Herzfrequenz während der ersten 10 Lebensminuten (alle $p>0,1$ ). Weiterhin gab es keine signifikanten Unterschiede in der Inzidenz von IVH, ROP, Bronchopulmonaler Dysplasie und Hyperbilirubinämie zwischen den Gruppen ( $p$ jeweils $>0,1$ ) Schlussfolgerung: In dieser retrospektiven Pilotstudie traten keine negative Effekte der EPT auf die erfassten Outcome-Parameter auf. Es erscheint daher gerechtfertigt, die Effekte des EPT-Verfahrens in einer prospektiv, randomisiert-kontrollierten Folgestudie genauer zu charakterisieren.

\section{FV46}

Neonatale Mortalität und Morbidität in fünf Provinzkrankenhäusern in der Demokratischen Volksrepublik Laos

S. Schmidt ${ }^{1}$, H. Sabir ${ }^{2}$, S. Brenner ${ }^{3}$, M. Schmid ${ }^{4}$, T. Kühn ${ }^{5}$, P. Duangdala ${ }^{6}$, S. Bounnack', T. Höhn ${ }^{8}$

${ }^{1} \mathrm{TU}$ München, München, Deutschland, ${ }^{2}$ Med. Einricht. d. Universität Kinderklinik, Neonatologie und pädiatrische Intensivmedizin, Düsseldorf, Deutschland, ${ }^{3}$ Universitätsklinikum Carl Gustav Carus Kinderklinik, Kinderklinik Kinderintensivstation, Dresden, Deutschland, ${ }^{4}$ Neonatologie Zürich, Zürich, Schweiz, ${ }^{5}$ Klinik für Kinder- und Jugenmedizin, Klinikum Neukölln, Berlin, Neonatologie, Berlin, Deutschland, ${ }^{6}$ Provinzkrankenhaus Luangnamtha, Luangnamtha, Laos, People's Democratic Republic of, ${ }^{7}$ Ministry of Health, Vientiane, Laos, People's Democratic Republic of, ${ }^{8}$ Med. Einricht. d. Universität Kinderklinik, Allgemeine Pädiatrie, Düsseldorf, Deutschland

Hintergrund: Obwohl sich die Mortalität von Kindern unterhalb eines Alters von 5 Jahren (U5MR) über die letzten Jahre verringert hat, ist der relative Prozentsatz der neonatalen Mortalitätsrate (NMR) gestiegen. Derzeit hat Laos die höchste NMR in der WHO-Region Westlicher Pazifik. Ziel: Ziel der Studie war retrospektiv Inzidenzen und Gründe für neonatale Erkrankungen und Todesfälle in fünf Provinzkrankenhäusern (Luangnamtha, Oudomxay, Xamnua, Phonsavan, Paksan) in den Jahren 2010 bis 2012 darzustellen.

Material und Methoden: Daten von Neugeborenen wurden vor Beginn eines mindestens 3-jährigen Ausbildungsprogramms für alle medizinischen Berufsgruppen, die an der Versorgung von Neugeborenen beteiligt sind, in fünf Provinzkrankenhäusern in Laos gesammelt.

Ergebnisse: In den Jahren 2010, 2011 und 2012 wurden insgesamt 1673 Neugeborene in den fünf Provinzkrankenhäusern behandelt. $48 \%$ der hospitalisierten Neugeborenen wurden wegen einer Infektion, $17 \%$ wegen Komplikationen von Frühgeburtlichkeit, $14 \%$ wegen geburtshilflicher Komplikationen und 9\% aufgrund anderer, nicht-kategorisierter Erkrankungen behandelt. Insgesamt starben 108 von 1673 behandelten Neugeborenen während des Krankenhausaufenthalts. Die durchschnittliche Mortalität in allen Krankenhäusern lag bei 6,5\%. Häufigste Todesursache waren Komplikationen auf dem Boden von Frühgeburtlichkeit, gefolgt von Infektionen (Sepsis und Pneumonie) und Asphyxie.

Schlußfolgerung: Mortalitätsraten hospitalisierter Neugeborener in Provinzkrankenhäusern in Laos bewegten sich zwischen 2,3\% in Xamnua und $10,2 \%$ in Luangnamtha. Diese Daten können als Grundlage für Ausbildungsprogramme dienen, die darauf gerichtet sind, die Neugeborenensterblichkeit in Laos zu reduzieren. 


\section{FV47 \\ Reflexionsverhalten von In-action Teams in simulierten Kindernotfällen}

\section{E. Heimberg' ${ }^{1}$, J. Schmutz', M. Hofbeck', W. Eppich ${ }^{3}$}

'Lehrstuhl für Arbeits- und Organisationspsychologie, Departement für Management, Technik und Ökonomie, ETH Zürich, Zürich, Schweiz, ${ }^{2}$ Universitätskinderklinik Tübingen, Abteilung für Kinderkardiologie, Intensivmedizin und Pulmologie" Tübingen, Deutschland, ${ }^{3}$ Northwestern University Feinberg School of Medicine, Chicago, USA

Teamreflexion beschreibt die Fähigkeit, gemeinsam Ziele, Prozesse und Strategien regelmäßig zu reflektieren. Teamreflektion ist ein wichtiger Faktor zur Unterstützung von Teamverhalten, von Lernprozessen und medizinischer Performanz. In Notfallsituationen mit hohem Zeitdruck tendieren Menschen meistens dazu, sofort Aktionen auszuführen. Teamreflexion in solchen schwierigen und oft unklaren Situationen kann verhindern, dass unnötige Aktionen ausgeführt werden, welche für den Patienten in dieser Situation keinen Nutzen haben oder sogar schädlich sind. Studien, die Teamreflektion in medizinischen Teams während komplexen Situationen, z. B. eines pädiatrischen Notfalls, untersuchen, sind rar. Die vorliegende Studie soll den Nutzen von Teamreflexivität in medizinischen Notfallteams untersuchen. In einem simulationsbasierten Notfalltraining soll reflektierendes Verhalten beobachtet werden und mit klinischer Leistung (Expertenrating) in Verbindung gebracht werden. Erste Ergebnisse sollen präsentiert werden. Die Studie fand im Rahmen eines wöchentlichen Notfalltrainings an der Universitätskinderklinik Tübingen statt. An jedem Training nahmen 7 Pflegekräfte und 4 Ärzte mit unterschiedlichem klinischen Erfahrungslevel von Allgemein- und Intensivstation teil. Jeder Teilnehmer nahm entweder aktiv oder passiv als Zuschauer an insgesamt 3 simulierten Kindernotfällen teil. Die Simulation wurde mit Hilfe eines High-Fidelity Simulators (SimBaby ${ }^{\text {Tix }}$, Laerdal) in einem realen klinischen Setting mit realem medizinischen Equipment durchgeführt. Nach jedem Szenario wurde eine videounterstützte Nachbesprechung durchgeführt. Diese beinhaltete ein Kurzreferat und ein Lehrvideo zum Thema Teamreflektion. Die aufgezeichneten Videos wurden anschliessend mit Hilfe eines neuen Beobachtungsinstruments zur Teamreflexion von unabhängigen Beobachtern codiert. Zusätzlich wurde die medizinische Leistung mittels einer validierten Evaluationscheckliste bewertet. Das Beobachtungsinstrument wurde entwickelt und validiert. Teamreflexionsverhalten und medizinische Performanz zwischen dem ersten und dem dritten Szenario wurden verglichen. Vorabergebnisse zeigen eine Verbesserung von Teamreflexionsverhalten und der medizinischen Leistung im 2. und 3. Szenario. Ein positiver Einfluss zwischen Teamreflexion und klinischer Performanz konnte gezeigt werden. Die Videocodierungen sind noch nicht abgeschlossen zum aktuellen Zeitpunkt. Die ersten Ergebnisse zeigen, dass interprofessionelle Teamtrainings und eine Intervention mittels Kurzreferat und Video zu einer Verbesserung des Reflexionsverhaltens von Teams in simulierten Notfallsituationen führen.

\section{FV48 \\ Topographisch-anatomische Untersuchung der Ausdehnung des neonatalen Herzbeutels zur Optimierung der ZVK-Lage in der Neonatologie}

\section{F. Eifinger' ', A. Vierzig' ', F. Körber², M. Scaal ${ }^{3}$}

${ }^{1}$ Univ.-Kinderklinik, Intensivstation, Köln, Deutschland, ${ }^{2}$ Uniklinik Köln, Kinderradiologie, Köln, Deutschland, ${ }^{3}$ Uniklinik Köln, Institut für Anatomie, Köln, Deutschland

Hintergrund: Die Anlage eines zentral venösen Katheters (ZVK) zur medizinischen Versorgung wird in der Neonatologie und pädiatrischen Intensivmedizin vielfach durchgeführt. Komplikationen treten bei Früh- und Neugeborenen signifikant häufiger als im Erwachsenenalter auf. Im ungünstigsten Fall führt die Perforation der Katheterspitze in den Herzbeutel zu einer vielfach letal endenden Herzbeuteltamponade. Die bestmögliche Lageposition für die ZVK-Spitze sollte deshalb optimaler Weise außerhalb des Herzbeutels sein. Als Orientierung und Abschätzung dient neben der
Echokardiographie vielfach eine Röntgen-Thorax Aufnahme. Dabei bildet der radiologisch sichtbare Herzschatten nicht immer die perikardiale Umschlagfalte ab. Überdies ist der Herzschatten zum Teil nicht eindeutig identifizierbar (Infiltrate, RDS, Atelektasen u. a.) oder die echokardiographischen Kompartimente sind nicht klar erkennbar. Untersuchungen der thorakalen Ausdehnung und Lage des neonatalen Perikardiums, insbesondere in Relation zu den knöchernen (d.h. radiologisch erkennbaren) Strukturen, fehlen unseres Wissens bislang.

Fragestellung: Unsere anatomische Untersuchung beschreibt die Ausdehnung des Perikardbeutels bei Früh- und Neugeborenen im Verhältnis zu radiologisch erkennbaren thorakalen Strukturen.

Material und Methoden: Die Studie wurde von der lokalen Ethikkommission (No. 14-288, 2015) genehmigt. An 20 neonatalen Demonstrationspräparaten (mittleres Gestationsalter $33+0$ Wochen, range: $24-42$ Wochen) erfolgte eine vorsichtige oberflächliche thorakale und nuchale Präparation. Mittels offener Punktion des Perikardbeutels wurde dieser mit Kontrastmittel gefüllt $\left(5 \mathrm{ml}\right.$ Imeron $\left.^{\star}\right)$. Im Weiteren wurde die rechte $\mathrm{V}$. jugularis interna mit einem $4 \mathrm{Fr}$. Katheter langstreckig kanüliert. Abschließend erfolgte eine Röntgendarstellung (Mobile C-arm ${ }^{\circledR}$ BV25, Philips Electronics) sowie Analyse (InterVideoWin ${ }^{\circ}$ DVR3) der Beziehung zwischen den radiologisch-erkennbaren knöchernen thorakalen Strukturen und dem kontrastieren Herzbeutel.

Ergebnisse: Die Entfernung zwischen oberer perikardialer Umschlagfalte und der ersten dorsal liegenden rechten Rippe beträgt $1,26 \mathrm{~cm} \pm 0,29$ $( \pm$ SD). Die perikardiale Umschlagfalte endet unterhalb der 3.dorsalen Rippe in allen untersuchten Präparaten. Die Entfernung zwischen Eintritt der V. cava superior in das Perikard und der ersten rechtsseitigen dorsalen Rippe beträgt $2,34 \mathrm{~cm} \pm 0,48$.

Schlussfolgerung: Das äußerste kraniale Ende der Perikardumschlagfalte in Autopsien bei Früh- und Neugeborenen liegt unterhalb der 3.Rippe auf der rechten Seite. Ein von der oberen Körperhälfte gelegter ZVK sollte den 3. Intercostalraum nicht überschreiten.

\section{FV49}

Zieltemperatur sehr kleiner Frühgeborener:

\section{Eine Umfrage zur Wärmetherapie auf 87 deutschsprachigen} neonatologischen Intensivstationen

\section{A. Perez ${ }^{1}$, F. van der Meer ${ }^{1}$, E. Vettorazzi ${ }^{2}$, D. Singer ${ }^{1}$}

'Universitätsklinikum Eppendorf, Sektion Neonatologie und Pädiatrische Intensivmedizin, Hamburg, Deutschland, ${ }^{2}$ Universitätsklinikum Eppendorf, Institut für Medizinische Biometrie und Epidemiologie, Hamburg, Deutschland

Hintergrund und Fragestellung: Die Wärmetherapie ist ein essentieller Bestandteil der Neonatologie. Sie erfolgt meist anhand von Richtwerten für die adäquate Lufttemperatur und -feuchte, welche schon in den 1970-er Jahren entwickelt wurden; für die adäquate Körpertemperatur sehr kleiner Frühgeborener gibt es hingegen bis heute keine belastbare wissenschaftliche Evidenz. Ziel unserer Untersuchung war es zu erfassen, wie Zentren der neonatologischen Maximalversorgung die anzustrebende Körperkerntemperatur sehr kleiner Frühgeborener definieren und diese Definition umsetzen; aufgrund der weitgehend fehlenden Evidenz erwarteten wir eine große Streuung zwischen den verschiedenen Zentren.

Material und Methoden: Von Oktober 2014 bis Januar 2015 wurde eine elektronische Umfrage an 149 „Level 1“ - Zentren in Deutschland, Österreich und der Schweiz versandt. Mit insgesamt 21 Fragen wurden die Temperaturziele und die tägliche Umsetzung wärmetherapeutischer Maßnahmen bei Frühgeborenen $\leq 30$ Wochen und $\leq 1500$ g erfragt. Die Frühgeborenen wurden hierzu in 3 Kategorien (30-27-24 Schwangerschaftswochen bzw. 1500-1000-500 g) eingeteilt und die Daten zur Thermoregulation für jede Kategorie separat erhoben.

Ergebnisse: Die Rücklaufrate dieser Umfrage lag bei 75\%, 87 Antworten $(58,4 \%)$ wurden in die Analyse aufgenommen. Die Zieltemperatur wird weitgehend unabhängig von Gestationsalter und Geburtsgewicht mit $37^{\circ} \mathrm{C}\left(36,99 \pm 0,19^{\circ} \mathrm{C}\right)$ angegeben. Die Definitionen für Hypo- und Hyperthermie sind ebenfalls für alle drei Kategorien von Frühgeborenen 
sehr ähnlich und unterscheiden sich nicht zwischen den Zentren. Alle Zentren bevorzugen zur Wärmetherapie den Inkubator gegenüber dem Wärmestrahler. Die Einstellung von Lufttemperatur und -feuchte des Inkubators zeigt die üblichen Unterschiede in Abhängigkeit von Gestationsalter und Körpergewicht. In den meisten Zentren wird die Temperaturkontrolle und Wärmetherapie von der am Bett tätigen Pflege(fach-)kraft ausgeführt. In $>40 \%$ der Zentren erfolgt die Steuerung der Wärmetherapie allein anhand der klinischen Einschätzung, in den übrigen Zentren anhand schriftlicher SOPs.

Diskussion und Schlussfolgerung: Hinsichtlich der Zieltemperatur sehr kleiner Frühgeborener besteht im deutschsprachigen Raum offenbar ein stillschweigender Konsens, dem zufolge - unabhängig von Gestationsalter und Geburtsgewicht $-37,0^{\circ} \mathrm{C}$ die normale und somit anzustrebende Körperkerntemperatur sei. Diese Erkenntnis überrascht, ist doch bekannt, dass die Körpertemperatur des Feten in utero $0,5-1,0{ }^{\circ} \mathrm{C}$ höher als die der Mutter liegt. Die Praxis, den Frühgeborenen bei einem so grundlegenden Vitalparameter wie der Körpertemperatur dieselben Normwerte wie reifen Neugeborenen oder älteren Kindern zuzuordnen, muss kritisch hinterfragt werden. Versorgen wir unsere jüngsten und vulnerabelsten Patienten womöglich mit einer zu niedrigen „Bebrütungstemperatur“?

\section{Freie Themen 08: Perinatale Infektionen}

FV50

Materno-fetaler Transfer von IgG-Antikörpern gegen das humane Zytomegalievirus (HCMV) und das Varizella-Zoster Virus (VZV) bei Früh- und Reifgeborenen von 24 bis 41 vollendeten Schwangerschaftswochen (SSW)

H. Buxmann', C. Lüdeke' , H. Hürter ${ }^{2}$, F. Louwen'², R. Schlößer', H. Rabenau ${ }^{3}$ 'Universitätsklinikum Frankfurt, Klinik für Kinder- und Jugendmedizin, Schwerpunkt Neonatologie, Frankfurt am Main, Deutschland,

2Universitätsklinkum Frankfurt, Klinik für Frauenheilkunde und Geburtshilfe, Funktionsbereich Geburtshilfe und Pränatalmedizin, Frankfurt am Main, Deutschland, ${ }^{3}$ Universitätsklinikum Frankfurt, Zentrum für Hygiene, Institut für Medizinische Virologie, Frankfurt am Main, Deutschland

Hintergrund: Der diaplazentare anti-HCMV und anti-VZV IgG-Antikörper(AK)-Transfer korreliert positiv mit dem Gestationsalter. Bisherige Studien untersuchten dieses Phänomen bei Früh- und Reifgeborenen von SSW 28 an aufwärts unter Zusammenfassung mehrerer SSW.

Zielsetzung: Detaillierte Analyse des materno-fetalen anti-HCMV und anti-VZV IgG-AK-Transfers bei Früh- und Reifgeborenen von 24 bis 41 vollendeten SSW.

Material und Methoden: Prospektive, analytische Kohortenstudie; Eingeschlossen werden nach Einverständnis Mütter und ihre Neugeborenen von SSW $24+0$ bis $41+6$. Die anti-HCMV und anti-VZV IgG-Konzentrationen werden im Nabelschnurblut der Neugeborenen und im mütterlichen Blut bestimmt.

Ergebnisse: Bisher wurden 297 Mütter in die Studie eingeschlossen, 282 $(94,9 \%)$ waren anti-VZV-IgG positiv und $195(65,7 \%)$ waren anti-HCMV positiv. Die anti-HCMV-IgG-Konzentrationen $[\mathrm{AE} / \mathrm{ml}]$ der bis dato

\section{Tab. FV50.1}

\begin{tabular}{|c|c|c|c|c|c|c|c|c|c|c|c|c|c|c|c|c|c|c|c|}
\hline \multirow[t]{5}{*}{ NG } & GW & 24 & 25 & 26 & 27 & 28 & 29 & 30 & 31 & 32 & 33 & 34 & 35 & 36 & 37 & 38 & 39 & 40 & 41 \\
\hline & $n=$ & 2 & 4 & 5 & 9 & 9 & 7 & 13 & 9 & 14 & 13 & 21 & 8 & 9 & 14 & 19 & 19 & 25 & 19 \\
\hline & Min. & 380 & 240 & 370 & 220 & 280 & 70 & 170 & 250 & 280 & 32 & 130 & 1300 & 200 & 67 & 39 & 470 & 490 & 640 \\
\hline & MW & 1090 & 645 & 1834 & 1320 & 1212 & 913 & 1495 & 1576 & 1151 & 833 & 1595 & 2325 & 1546 & 1703 & 1952 & 2114 & 2917 & 2681 \\
\hline & Max. & 1800 & 920 & 2600 & 2800 & 2700 & 3600 & 4200 & 3900 & 4400 & 2000 & 4700 & 3900 & 2100 & 3900 & 6700 & 4800 & 8200 & 6700 \\
\hline \multirow[t]{4}{*}{ Mütter } & $n=$ & 2 & 4 & 4 & 6 & 8 & 5 & 11 & 9 & 10 & 13 & 16 & 7 & 7 & 12 & 19 & 19 & 24 & 19 \\
\hline & Min. & 510 & 560 & 710 & 460 & 170 & 110 & 200 & 290 & 320 & 61 & 110 & 630 & 180 & 30 & 39 & 340 & 190 & 270 \\
\hline & MW & 2805 & 1305 & 1898 & 1404 & 1504 & 967 & 1518 & 1562 & 1313 & 879 & 1457 & 1954 & 1266 & 1293 & 1491 & 1467 & 2121 & 1827 \\
\hline & Max. & 5100 & 2400 & 3300 & 2600 & 4300 & 2000 & 5900 & 3900 & 4400 & 2200 & 5600 & 3700 & 2100 & 3200 & 6700 & 4100 & 5700 & 5300 \\
\hline \multicolumn{2}{|c|}{$\begin{array}{l}\text { Differenz } \\
\text { (MW) }\end{array}$} & 715 & -660 & -64 & -84 & -292 & -54 & -23 & 14 & -162 & -46 & 138 & 371 & 280 & 410 & 461 & 647 & 796 & 854 \\
\hline
\end{tabular}

Tab. FV50.2

\begin{tabular}{|c|c|c|c|c|c|c|c|c|c|c|c|c|c|c|c|c|c|c|c|}
\hline \multirow[t]{5}{*}{ NG } & GW & 24 & 25 & 26 & 27 & 28 & 29 & 30 & 31 & 32 & 33 & 34 & 35 & 36 & 37 & 38 & 39 & 40 & 41 \\
\hline & $n=$ & 2 & 3 & 6 & 13 & 10 & 14 & 12 & 10 & 18 & 19 & 38 & 13 & 20 & 28 & 29 & 34 & 31 & 28 \\
\hline & Min. & 700 & 84 & 89 & 120 & 150 & 290 & 230 & 140 & 0 & 120 & 120 & 96 & 520 & 160 & 110 & 130 & 54 & 350 \\
\hline & MW & 775 & 171 & 162 & 778 & 575 & 757 & 592 & 953 & 984 & 972 & 851 & 711 & 1389 & 1203 & 1161 & 1536 & 1436 & 1528 \\
\hline & Max. & 850 & 250 & 270 & 1600 & 2300 & 1300 & 1500 & 3300 & 3400 & 3100 & 2200 & 2000 & 3000 & 2500 & 3600 & 3800 & 5400 & 4600 \\
\hline \multirow[t]{4}{*}{ Mütter } & $n=$ & 2 & 3 & 4 & 9 & 8 & 12 & 11 & 10 & 14 & 18 & 29 & 10 & 15 & 24 & 27 & 34 & 27 & 25 \\
\hline & Min. & 980 & 150 & 89 & 130 & 290 & 210 & 240 & 110 & 190 & 170 & 87 & 55 & 310 & 130 & 72 & 89 & 77 & 130 \\
\hline & MW & 1490 & 427 & 264 & 886 & 650 & 739 & 658 & 849 & 981 & 982 & 767 & 545 & 969 & 834 & 722 & 1055 & 953 & 1013 \\
\hline & Max. & 2000 & 620 & 480 & 1500 & 1900 & 1300 & 1800 & 2900 & 3400 & 3300 & 2100 & 1400 & 2800 & 2200 & 2700 & 3800 & 5100 & 4900 \\
\hline $\begin{array}{l}\text { Differen } \\
\text { (MW) }\end{array}$ & & -715 & -256 & -102 & -108 & -75 & 18 & -66 & 104 & 3 & -10 & 84 & 166 & 420 & 369 & 439 & 481 & 483 & 515 \\
\hline
\end{tabular}


eingeschlossenen 219 Neugeborenen (NG) von 195 Müttern sind tabellarisch in Form von Minimum (Min.), Mittelwert (MW), Maximum (Max.) und feto-maternaler Differenz für jede Gestationswoche (GW) dargestellt (Tab. FV50.1).

Die anti-VZV-IgG-Konzentrationen [IE/ml] der bis dato eingeschlossenen 318 Neugeborenen (NG) von 282 Müttern sind in ebenso dargestellt: Diskussion und Schlussfolgerung: In den sehr frühen SSW 24-28 sind die anti-HCMV und anti-VZV-IgG-Konzentrationen der Frühgeborenen im Mittel niedriger als die ihrer Mütter. In den SSW 29-33 sind diese Konzentrationen in etwa vergleichbar. Ab SSW 34 übersteigen die anti-HCMV und anti-VZV-IgG-Konzentrationen der Neugeborenen die ihrer Mütter regelhaft. Innerhalb der einzelnen SSW fanden sich erhebliche interindividuelle Unterschiede der AK-Konzentrationen. Die Ursachen hierfür sind zu analysieren.

\section{FV51}

\section{Vaginale Kolonisierung mit Ureaplasma parvum in der Frühschwangerschaft ist signifikant assoziiert mit Frühgeburtlichkeit: Ergebnisse einer Multicenter-Studie}

J. Rittenschober-Böhm ', T. Waldhör², S. Schulz'3 , B. Stihsen ${ }^{4}$, B. Pimpel', K. Göral', E. Hafner ${ }^{5}$, G. Sliutz ${ }^{6}$, D. Kasper ${ }^{3}$, A. Witt', A. Berger ${ }^{1}$

${ }^{1}$ Medizinische Universität Wien/Universitätsklinik für Kinder- und Jugendheilkunde, Abteilung für Neonatologie, pädiatrische Intensivmedizin und Neuropädiatrie, Wien, Österreich, ${ }^{2}$ Center of Public Health, Abteilung fürEpidemiologie, Wien, Österreich, ${ }^{3}$ Medizinische Universtität Wien, Univ. Klinik für Kinder- und Jugendheilkunde, Wien, Österreich, ${ }^{4}$ Medizinische Universität Wien, Univ. Klinik für Gynäkologie und Geburtshilfe, Wien, Österreich, ${ }^{5} \mathrm{SMZ}$ Ost, Abteilung für Gynäkologie und Geburtshilfe, Wien, Österreich, ${ }^{6}$ Rudolfstiftung, Abteilung für Gynäkologie und Geburtshilfe, Wien, Österreich

Hintergrund: Zahlreiche Studien zeigen eine Assoziation zwischen einer intrauterinen Infektion mit Ureaplasma spp. und Frühgeburt sowie einem schlechteren Outcome für die Neugeborenen. Diese entstehen zumeist durch eine aufsteigende Infektion aus dem Vaginaltrakt. Ein eindeutiger Nachweis, dass eine vaginalen Kolonisierung - nach der leicht gescreent werden kann - zu einem erhöhten Risiko für die ungeboren Kinder führt, fehlt jedoch.

Fragestellung: Ziel der vorliegenden Studie war es, Frauen während des ersten Schwangerschaftstrimenons auf das Vorliegen einer vaginalen Ureaplasma-Kolonisierung zu testen und die Biovar-Ergebnisse mit dem Schwangerschaftsoutcome zu korrelieren, um Schwangere mit einem erhöhten Risikofür eine Frühgeburt zu identifizieren.

Material und Methode: Zwischen Mai 2009 und Dezember 2012 wurden 4330 Schwangere in diese prospektive Multicenter-Beobachtungsstudie eingeschlossen. In SSW 11-16 wurde ein Vaginalabstrich abgenommen und mittels real-time PCR auf das Vorkommen von Ureaplasma spp. getestet, anschließend wurden PCR-Ergebnis und Schwangerschaftsoutcome korreliert. Primäre Outcome-Variable war die Rate an spontanen Fehlgeburten oder Frühgeburten vor der 37. SSW. Nach Ausschluss von Mehrlingsschwangerschaften, medizinisch induzierten Frühgeburten und $\mathrm{Ab}$ treibungen waren die Daten von 3643 Frauen für die Analyse verfügbar. Ergebnisse: Bei $37 \%$ der Schwangeren (1347) wurde eine vaginale Kolonisierung mit Ureaplasma parvum nachgewiesen, bei 5,9\% (214) eine Kolonisierung mit Ureaplasma urealyticum. Eine vaginale Kolonisierung mit Ureaplasma parvum war signifikant assoziiert mit einem erhöhten Risiko für spontane Frühgeburt (OR 1,7, CI 1,3-2,2, $p<0,001$ ), im Gegensatz zu einer Kolonisierung mit Ureaplasma urealyticum $(p=0,19)$. Diese statistisch signifikante Assoziation war zutreffend für alle untersuchten Untergruppen von Frühgeburten, besonders häufig war eine vaginale Kolonisierung mit Ureaplasma parvum bei Schwangerschaften mit extremer Frühgeburt (70,6\% bei Frühgeburten in SSW 21-23, 62,5\% in SSW 24-26). Je niedriger die SSW bei Geburt, desto höher die Rate der vaginalen Ureaplasma parvum Kolonisierungen in der Frühschwangerschaft $(p=0,001)$. Das Risiko für eine Frühgeburt war besonders hoch bei Schwangeren mit einer vorhergegangenen Frühgeburt und einer Koloni- sierung mit Ureaplasma parvum. Die vorhergesagte Wahrscheinlichkeit für spontane Frühgeburt erhöhte sich von 5,5\% $(4,6-6,7)$ bei Schwangeren ohne Kolonisierung mit Ureaplasma parvum und ohne vorhergegangenen Frühgeburt auf 14,2\% (9,8-20,2) bei Schwangeren mit vaginaler Kolonisierung mit Ureaplasma parvum und vorhergegangener Frühgeburt. Diskussion: Aufgrund der hohen Rate an vaginaler Kolonisierung mit Ureaplasma parvum und der Tatsache, dass die meisten Schwangeren dennoch zum Termin gebären, müssen noch weitere Risikofaktoren identifiziert werden, um jene Schwangere herausfiltern zu können, die potentiell von einer Therapie profitieren. In einem nächsten Schritt erhoffen wir uns durch die Analyse der Serovare der Ureaplasma parvum positiven Schwangeren, diese Risikogruppe näher eingrenzen zu können.

Schlussfolgerung: Schwangere mit vaginaler Ureaplasma parvum Kolonisierung in der Frühschwangerschaft haben ein erhöhtes Risiko für Frühgeburt.

\section{FV52}

\section{Endogene alarmine S100A8/S100A9 induzieren mikrobielle Toleranz in Neugeborenen}

S. Pirr', J. Austermann', J. Burgmann', J. Friesenhagen', S. Fass' ', K. BarczykKahlert $^{2}$, T. Ortkras ${ }^{2}$, M. von Köckritz-Blickwede ${ }^{3}$, J. Roth ${ }^{2}$, T. Vogl'

D. Viemann

'Medizinische Hochschule Hannover, Pädiatrische Pneumologie, Allergologie und Neonatologie, Hannover, Deutschland, ${ }^{2}$ Universität Münster, Institut für Immunologie, Münster, Deutschland, ${ }^{3}$ Tierärztliche Hochschule Hannover, Institut für Physiologische Chemie, Hannover, Deutschland

Hintergrund: Trotz enormer Fortschritte auf dem Gebiet der Neonatologie ist die neonatale Sepsis noch immer eine der führenden Ursachen für die peripartale Mortalität. Klinisch kennzeichnend für die neonatale Sepsis ist der hyperdyname Verlauf. Eine Schlüsselrolle hierbei spielt das angeborene Immunsystem. Fatale Verläufe einer adulten Sepsis hingegen sind charakterisiert durch Hypoinflammation und Endotoxintoleranz. Endotoxintoleranz kann in Monozyten durch niedrig dosierte Stimulation des Toll-like-Rezeptor-4 (TLR4) mit Lipopolysaccharid (LPS) hervorgerufen werden. Die Alarmine S100A8 und S100A9 wurden kürzlich als endogene TLR4-Liganden identifiziert.

Fragestellung: Welche Rolle spielen Alarmine in der neonatalen Sepsis? Können sie mikrobielle Toleranz induzieren?

Material und Methoden: S100A8/S100A9 wurde im Serum gesunder NG bestimmt. In vitro wurden die Effekte von S100A8/S100A9 auf die Immunreaktion adulter und neonataler Monozyten mittels Genexpressionsund Zytokinanalysen untersucht. Neonatale S100A9 knock-out Mäuse wurden im neonatalen Endotoxinämiemodell analysiert und mit Wildtyp-Mäusen verglichen.

Ergebnisse: Überraschenderweise sind bei gesunden reifen Neugeborenen die S100A8/S100A9 Serumspiegel bei Geburt extrem erhöht und fallen in der ersten Lebenswoche auf Normwerte Erwachsener ab. Die endogenen Alarmine S100A8 und S100A9 lösen in humanen Monozyten ähnlich dem Mechanismus von LPS eine Hyporesponsivität gegenüber bakteriellen Stimuli aus. Wir konnten zeigen, dass die massive Freisetzung von S100A8/ S100A9 bei Geburt für die Induktion einer mikrobiellen Hyporesponsivität neonataler Monozyten verantwortlich ist. Im Mausmodell resultierte dieser Mechanismus in einem verbesserten Überleben der Wildtyp-Mäuse im Vergleich zu S100A9 knock-out Mäusen.

Schlussfolgerung: Wir zeigen erstmals, dass S100A8/S100A9 in Monozyten mikrobielle Toleranz vermittelt. Endogene TLR4-Stressliganden erweisen sich als kritische Regulatoren von Toleranz und Abwehr in der Neonatalzeit und stellen völlig neue immunmodulatorische Therapieansätze dar. 
FV53

Infektanfälligkeit bei Neugeborenen: Myeloide Suppressorzellen (MDSC) aus dem Nabelschnurblut induzieren Th2- und Treg-Zellen und hemmen Th1-Zellen

M. Vogelmann ${ }^{1}$, N. Köstlin' ', C. Härtel' ${ }^{2}$, B. Spring ${ }^{1}$, T. Orlikowsky³, C. Poets', J. Pagel ${ }^{4}$, C. Gille

'Eberhard-Karls-Universität Universitätsklinik für Kinderheilkunde und Jugendmedizin, Neonatologie, Tübingen, Deutschland, 2Universitätsklinikum Schleswig Holstein/Campus Lübeck, Klinik für Kinder- und Jugendmedizin, Lübeck, Deutschland, ${ }^{3}$ Universitätsklinik für Kinder- und Jugendmedizin, Sektion für Neonatologie, Aachen, Deutschland, ${ }^{4}$ Universitätsklinikum Schleswig-Holstein, Klinik für Kinderund Jugendmedizin, Abteilung Neonatologie, Lübeck, Deutschland

Hintergrund: Infektionen sind die Hauptursache neonataler Morbidität und Mortalität. Die immunologischen Mechanismen, welche die erhöhte Infektanfälligkeit von Neu- und Frühgeborenen bedingen, sind bis heute allerdings nur unvollständig verstanden. Myeloide Suppressorzellen (MDSC) sind myeloide Zellen, die hemmend auf andere Immunzellen, insbesondere auf T-Zellen wirken und in erhöhter Zahl im Nabelschnurblut gesunder Neugeborener vorkommen. Bekannte Mechanismen der inhibitorischen Wirkung von MDSC sind die Expression der Effektorenzyme Arginase1 (Arg1), induzierbare NO-Synthetase (iNOS), Indolamin-2,3-Dioxygenase (IDO) sowie die Produktion reaktiver Sauerstoffspezies (ROS). Ob und über welche Mechanismen MDSC aus dem Nabelschnurblut (CB-MDSC) die Differenzierung von T-Helfer-Zellen beeinflussen ist bisher nicht untersucht. Hypothese: CB-MDSC beeinflussen die Polarisierung von T-Helfer-Zellen und führen zu einer Induktion von regulatorischen T-Zellen (Treg).

Methoden: Mononukleäre Zellen (v. a. Myeloide Zellen ohne reife Granulozyten, T-Zellen, B-Zellen, NK-Zellen) wurden aus dem peripheren Blut von gesunden Erwachsenen (PBMC) und aus Nabelschnurblut (CBMC) isoliert. MDSC und T-Zellen wurden mittels MACS aus CBMC angereichert. Es folgte eine Co-Kultur von PBMC mit CB-MDSC über 96-120 Stunden im direkten Zellkontakt, und getrennt durch eine Membran (transwell). Als Kontrolle dienten PBMC ohne Zugabe von MDSC. Daneben erfolgte die Co-Kultur von MDSC mit reinen T-Zellen, um Effekte anderer Zellreihen auszuschließen. Anschließend wurden Th1-, Th2- und Treg-Zellen durchflusszytometrisch quantifiziert. Typische MDSC-Effektormechanismen wie Arg1, ROS, iNOS und IDO wurden durch Zugabe spezifischer Inhibitoren während der Co-Kultur blockiert.

Ergebnisse: Die Zugabe von MDSC zu PBMC führte zu einer Induktion von Th2-Zellen und Tregs und zu einer Reduktion von Th1-zellen in der Kultur. Die Induktion von Th2-Zellen und Tregs war Zellkontakt-unabhängig, während die Reduktion von Th1-Zellen Zellkontakt-abhängig erfolgte. Die Induktion von Th2-Zellen gelang nur aus PBMC in Co-Kultur mit MDSC, nicht jedoch aus reinen T-Zellen. Die Treg-Induktion und Th1-Hemmung durch CB-MDSC war hingegen aus reinen T-Zellen möglich. Eine Blockade von Arg1 und eine Blockade der ROS-Produktion konnte die Induktion von Th2-Zellen durch MDSC aufheben.

Schlussfolgerung: MDSC aus dem Nabelschnurblut können die Th-Zell-Differenzierung spezifisch verändern. Die zugrundeliegenden Mechanismen scheinen für die einzelnen Th-Zell-Subpopulationen unterschiedlich zu sein. Die Ergebnisse legen die Vermutung nahe, dass MDSC die Immunantwort des Neugeborenen entscheidend beeinflussen und zur hohen Infektanfälligkeit in der Neonatalzeit beitragen.
FV54

Mechanismen der feto-maternalen Toleranz: HLA-G führt zu einer Induktion und Aktivierung von myeloiden Suppressorzellen (MDSC)

N. Köstlin' ', A. Ostermeir' ${ }^{2}$, B. Spring' ', C. Poets' ', C. Gille

${ }^{1}$ Eberhard-Karls-Universität Universitätsklinik für Kinderheilkunde und Jugendmedizin, Neonatologie, Tübingen, Deutschland, ${ }^{2}$ Universitätskinderklinik Tübingen, Abteilung Neonalogie, Tübingen, Deutschland

Hintergrund: Myeloide Suppressorzellen (MDSC) sind myeloide Zellen mit suppressiver Wirkung auf andere Immunzellen. MDSC kommen im Nabelschnurblut gesunder Neugeborener in erhöhter Zahl vor und fallen postpartal rasch ab, was vermuten lässt, dass diese Zellen einerseits zur Aufrechterhaltung der fetomaternalen Toleranz während der Schwangerschaft und andererseits zur veränderten Immunantwort des Neonaten beitragen könnten. Bisher ist nur wenig über Faktoren bekannt, die zur Induktion von MDSC in der Fetalzeit führen. HLA-G ist ein MHC Klasse I Molekül, das hauptsächlich durch Trophoblastzellen exprimiert wird und über Bindung an immunoglobulin-like transcript (ILT) Rezeptoren immunsuppressiv auf verschiedene Zellen wirkt. Bisher ist nichts über die Bedeutung von HLA-G für die Induktion von MDSC in der Fetalzeit bekannt.

Hypothese: HLA-G führt zu einer Induktion und Aktivierung von MDSC. Methoden: Mononukleäre Zellen wurden aus dem Nabelschnurblut (CBMC) und dem peripheren Blut gesunder Erwachsener (PBMC) aufgereinigt. Die Expression von ILT2 und ILT4 auf MDSC aus CBMC wurde durchflusszytometrisch ermittelt. PBMC wurden über sechs Tage mit oder ohne Zugabe von $1 \mathrm{ng} / \mathrm{ml}$ HLA-G kultiviert. Dann wurden CD33+ myeloide Zellen mittels MACS aufgereinigt und zu frisch isolierten, CFSE-markierten und mit OKT3/IL-2 stimulierten PBMC desselben Spenders gegeben. Nach $96 \mathrm{~h}$ wurde die Proliferation von T-Zellen durchflusszytometrisch ermittelt. Die Rezeptoren ILT2 und ILT4 wurden während der 6tägigen Induktion durch spezifische Antikörper blockiert. Die Expression des Transkriptionsfaktors signal transducer and activator of transcription 3 (pSTAT3) in myeloiden Zellen nach Stimulation mit HLA-G wurde durchflusszytometrisch bestimmt.

Ergebnisse: MDSC aus CBMC exprimierten die Rezeptoren ILT2 und ILT4. Die Zugabe von HLA-G zu PBMC führte zu einer quantitativen Induktion von MDSC in PBMC ( $10 \%$ vs. $28 \%$ aller PBMC, $p<0,05$, $n=5)$. HLA-G-induzierte MDSC zeigten im Vergleich zu Kontroll-Zellen eine erhöhte suppressive Aktivität auf T-Zellen ( $74 \%$ vs. $53 \%$ Proliferations-Hemmung, $p<0,05, n=5$ ). Die Induktion von MDSC aus PBMC konnte durch Blockade des von ILT4, nicht aber durch Blockade von ILT2 unterdrückt werden. Eine Stimulation mit HLA-G bewirkte eine erhöhte Expression von phosphoryliertem STAT3 in myeloiden Zellen.

Schlussfolgerung: HLA-G führt zu einer quantitativen und funktionellen Induktion von MDSC aus PBMC über die Interaktion mit dem Rezeptor ILT4 und die Aktivierung des Transkriptionsfaktors STAT3. Die Induktion von MDSC durch HLA-G könnte ein bisher unbekannter Mechanismus für die Aufrechterhaltung der feto-maternalen Toleranz während der Schwangerschaft und die im Vergleich zum Erwachsenen veränderte Immunantwort des Neugeborenen sein. 


\section{FV55}

\section{Charakterisierung der von Staphylococcus epidermidis} induzierten Immunantwort bei Neugeborenen

\section{B. Tröger ${ }^{1}$, E. Herting ${ }^{2}$, C. Härtel ${ }^{3}$}

'Universitätsklinikum Schleswig Holstein, Kinderklinik, Lübeck, Deutschland, ${ }^{2}$ Universitätsklinikum Schleswig-Holstein Campus Lübeck, Direktor der Klinik für Kinder- und Jugendmedizin, Lübeck, Deutschland, ${ }^{3}$ Universitätsklinikum Schleswig Holstein/Campus Lübeck, Klinik für Kinderund Jugendmedizin, Lübeck, Deutschland

Hintergrund: Infektionen mit gram-positiven Erregern wie koagulase negativen Staphylokokken (CoNS) sind eine wichtige Ursache für Langzeitmorbidität und Mortalität von Frühgeborenen. Dabei ist der zu den CoNS gehörende S. epidermidis der am häufigsten in der Blutkultur nachgewiesene Erreger. Neben einer Resistenz gegen Antibiotika zählen zu den wesentlichen Virulenzfaktoren die Fähigkeit zur Biofilmformation und die Expression extrazellulärer Virulenzfaktoren. In einem Vollblutassay (Nabelschnurblut) untersuchten wir die S. epidermidis-induzierten Immunantworten mit invasiven und nichtinvasiven Klinikisolaten, sowie den Einfluss verschiedener Virulenzfaktoren (S. epidermidis-Wildtyp vs. Mutanten). Weiter wurde der immunmodulatorische Einfluss von häufig in der Neonatologie verwendeten Medikamenten studiert.

Material und Methoden: Nabelschnurblutproben wurden mit S. epidermidis-Vollkeimen im Vollblutstimulationsassay untersucht und die Expression pro- und antiinflammatorischer Zytokine unter folgenden Bedingungen gemessen:

1. Vergleich der Immunantwort zwischen Sepsis- und Kolonisationsisolaten (2 Sepsis- und 7 Kolonisationsisolate)

2. Immunantwort auf S. epidermidis-Wildtyp und Mutanten:

1. 1457 Wildtyp: stark biolfilmbildend, geringe Expression extrazellulärer Virulenzfaktoren

2. 1457M10: PIA/HA negativ, gering biolfilmbildend, Expression extrazellulärer Virulenzfaktoren

3. 1457sigB: vermindert biolfilmbildend, vermehrte Expression extrazellulärer Virulenzfaktoren

4. 1457sigBagr: stark biolfilmbildend, keine extrazellulären Virulenzfaktoren

3. Einfluss von potentiell immunmodulatorischen Medikamenten wie Indomethacin und Ibuprofen (jeweils Konzentrationen 0-10 $\mu \mathrm{g} /$ $\mathrm{ml})$

Ergebnisse:

1. Es zeigte sich eine signifikant höhere IL-6-Produktion und ein Trend zu einer höheren IL-10-Produktion nach Stimulation mit S. epidermidis-Sepsisisolaten im Vergleich zu Kolonisationsisolaten

2. Biofilmformation und Expression extrazellulärer Virulenzfaktoren hatten in unserem Modell keinen Einfluss auf die Zytokinproduktion

3. Nach Inkubation mit Indomethacin und Ibuprofen zeigte sich nach Stimulation mit S. epidermidis eine erhöhte TNF- $\alpha$-Produktion

Diskussion: Es zeigten sich Unterschiede in proinflammatorischen Antworten bei S. epidermidis-Sepsisisolaten im Vergleich zu Kolonisationsisolaten, wobei sich Biofilmproduktion und extrazelluläre Virulenzfaktoren dabei nicht als ursächlich erwiesen. Immunmodulatorische Medikamente wie Indomethacin und Ibuprofen führten zu einer marginalen Erhöhung des proinflammatorischen Zytokins TNF- $\alpha$. Ob diese Ergebnisse einen klinisch bedeutsamen Effekt haben, müssen weitere Untersuchungen mit einer höheren Zahl von S. epidermidis-Sepsis- und Kolonisationsstämmen mit unterschiedlich modifizierten Virulenzfaktoren zeigen.

Schlussfolgerung: Unsere Daten bieten eine deskriptive Basis für die Etablierung von Risikomodellen für S. epidermidis-Sepsitiden, um langfristig individuelle Risikoprofile für besonders vulnerable Früh- und Neugeborene zu erstellen.
FV56

Der Effekt einer antiinfektösen Katheterbeschichtung von Einschwemmkathetern auf Katheterkolonisierung und Sekundärsepsis bei Frühgeborenen

A. Flemmer', N. DeMaio', S. Schubert ${ }^{2}$, T. Wurster ${ }^{3}$, M. Klemme' ${ }^{1}$, H. Messner ${ }^{3}$, P. Innocenti ${ }^{4}$, S. Herber-Jonat ${ }^{1}$, A. Staffler ${ }^{3}$

'LMU-München, Neonatologie der Kinderklinik am Perinatalzentrum Großhadern, München, Deutschland, ${ }^{2}$ Max v. Pettenkofer Institut, Mikrobiologiew, München, Deutschland, ${ }^{3}$ Zentrales Lehrkrankenhaus Bozen, Neonatologie und Neugeborenen-Intensivstation, Bozen, Italien, ${ }^{4}$ Zentrales Lehrkrankenhaus Bozen, Labor für Mikrobiologie, Bozen, Italien

Hintergrund: Nosokomiale Infektionen spielen eine wesentliche Rolle für die Morbidität und Mortalität Frühgeborener. Das Risiko für Infektionen ist erhöht, wenn die Kinder invasive Devices benötigen, die als Eintrittspforte für Bakterien dienen. Seit Kurzem sind Einschwemmkatheter verfügbar, die mit einem Antibiotikum (Rifampicin) und einem Antimykotikum (Miconazol) beschichtete sind verfügbar, ohne dass bekannt ist, ob diese Katheter zu einer Verminderung von bakteriellen Kolonisierung oder der Entwicklung von klinisch manifesten Infektionen führen.

Methoden: Wir führten eine dizentrische, randomisierte, kontrollierte Beobachtungsstudie an Neugeborenen, die aus klinischer Indikation einen Einschwemmkatheter benötigten, durch. Die Studie war von den Ethikkomissionen beider Perinatalzentren (München, Bozen) begutachtet und alle Eltern stimmten der Beobachtung zu. Es wurden insgesamt 83 Katheter untersucht (43 Kontrollkatheter \& 40 Verumkatheter). Die untersuchten Katheter wurden nach einem standardisierten Protokoll nach der Entfernung bakteriologisch untersucht. Als primäres Outcome-Kriterium wurde die Kolonisierung der Katheter mit $>15 \mathrm{KBE} / \mathrm{ml}$ (Slobbe, El Barzouhi et al. 2009) in der Standard-Rollout Kultur angesehen. Als Katheter assoziierte Infektion wurde ein besiedelter Katheter mit lokaler Reaktion, z. B. Hautrötung oder Induration der Einstichstelle angesehen. Als Kathetersepsis wird eine Besiedelung des Katheters und eine klinische SIRS-Reaktion (systemic inflammatory response syndrome) des Patienten mit CRP Anstieg $>1,5 \mathrm{mg} / \mathrm{dl}$ angesehen.

Ergebnisse: Die Kinder beider Kathetergruppen unterschieden sich nicht hinsichtlich Gestationsalter, Geburtsgewicht oder Geschlechtsverteilung. Eine Kolonisierung des Katheters fand sich Kontrollkathetern bei 14,3\%, bei Verumkathetern bei 5,1\% ( $p=0,27)$. Eine Katheter-assoziierte Infektion trat bei 7,1\% der Kontrollkatheter und bei 2,6\% der Verumkatheter auf $(p=0,62)$. Eine Katheter-assoziierte Sepsis trat bei 7,1\% der Kontrollkatheter und bei keinem der Verumkatheter auf $(p=0,24)$. Bakteriell bedingte Komplikationen des Katheters (Kolonisierung, Infektion oder Sepsis) traten bei Kontrollkathetern viermal häufiger wie bei Verumkathetern auf $(p=0,05)$. Die Odds-Ratio, eine Kolonisierung des Katheters ohne Beschichtung zu erleben, lag bei 3,0 (95\% CI: 0,41-22,42), die Odds-Ratio für eine Sepsis bei 4,43 (95\% CI: 0,44-44,27).

Schlussfolgerung: In dieser Pilotstudie präsentieren wir erstmals einen Vergleich von antiinfektös beschichteten Einschwemmkathetern mit Standardkathetern für die Anwendung bei Früh- und Neugeborenen. Die Katheterbeschichtung führte zu einer Reduktion der Katheter-assoziierten bakteriologischen Komplikationen. Diese Daten können nun für eine Fallzahlkalkulation für eine größer angelegte Untersuchung verwendet werden. 


\section{Freie Themen 09: Kardiologie}

\section{FV57}

Sedierungen mit Propofol oder 4-Hydroxybutansäure für diagnostische und interventionelle Herzkatheteruntersuchungen: Eine prospektive, randomisierte Studie bei pädiatrischen Patienten unter 18 Jahren

H. Sauer', L. Grünzinger' ${ }^{2}$, J. Pfeifer', S. Gräber ${ }^{3}$, H. Abdul-Khaliq ${ }^{4}$

'Unversitätsklinik für Kinder und Jugendmedizin Gebäude 9, Klinik für Pädiatrische Kardiologie, Homburg, Deutschland, ${ }^{2}$ Klinikum Lippe, Dermatologie, Detmold, Deutschland, ${ }^{3}$ Universitätsklinikum des Saarlandes, Institut für Medizinische Biometrie, Epidemiologie und Medizinische Informatik, Homburg, Deutschland, ${ }^{4}$ Universitätsklinikum des Saarlandes, Klinik für Pädiatrische Kardiologie, Homburg, Deutschland

Hintergrund: Päd. Patienten benötigen für eine diagn. od. intervent. Herzkatheteruntersuchung (HKU) in den meisten Fällen eine tiefe Sedierung od. Allgemeinanästhesie. Im Rahmen eines hausinternen Standards führen wir diese HKUs i.d.R. in tiefer Sedierung bei erhaltener Spontanatmung durch. Über mehrere Jahre hinweg haben wir sowohl mit Propofol (P) bei HKUs als auch 4-Hydroxybutansäure $(4 \mathrm{H})$ bei anderen Sedierungen gute Erfahrungen gemacht.

Fragestellung: Zur Optimierung des standardisierten Vorgehens be HKUs initiierten wir eine prospektive, randomisierte Studie, um die bd Wirkstoffe miteinander zu vergleichen (pos. Votum d. Ethikkomm., Registr. im dt. Studienreg.).

Material und Methoden: Wir analysierten die Daten unserer Sedierungsprotokolle aller HKUs aus einem Zeitraum von 1 Jahr (4.2011-3.2012) unter Berücksichtigung der Ein- u. Ausschlusskriterien. Für den ersten Teil der Auswertung erfolgte eine Verblindung der Protokolle. Neben den prim. Endpunkten (EP), Durchführbarkeit der HKU u. Auftreten von schw. Komplikationen, waren die sek. EP: Auftreten von leichten od. mittelschw. Komplikationen inkl. Veränderungen von kardiozirk. Parametern u. Änderungen der transkutan gemessenen Sauerstoffsättigungen, Laborparameter am Ende der $\mathrm{HKU}$ (u. a. $\mathrm{pH}$, Laktat, $\mathrm{pCO}_{2}$ ) sowie Auftreten von Komplikationen in der postsedativen Phase (u. a. PONV).

Ergebnisse: In bd. Gruppen konnten insgesamt 36 Patienten eingeschleust werden. Bzgl. der Basisparameter (Alter b. HKU, Geschlecht, Gewicht, Schwere der Grunderkrankung (Differenzierung gemäß ASA-Klassif.), Dosis von Midazolam zur Prämedikation, Dauer der Sedierung) ergaben sich keine signifikanten Unterschiede zw. den bd. Gruppen. Die Medikamentendosierungen bewegten sich in bd. Gruppen innerhalb der vom Hersteller empfohlenen Rahmen. In der P-Gruppe ließen sich signifikant niedrigere RR-Werte gegen Ende der Sedierung nachweisen sowie niedrigere $\mathrm{pH}-\mathrm{u}$. höhere $\mathrm{pCO}_{2}$-Werte. In der 4H-Gruppe fiel vor allem die signifikant höhere Rate an PONV im Rahmen einer Zwischenanalyse auf. Durch die Gabe von Dimenhydrinat 5-10 min vor Ende der Sedierung konnte die PONV-Häufigkeit auf die der P-Gruppe gesenkt werden. Insgesamt war die Komplikationsrate niedrig ohne ausgeprägten Interventionsbedarf (jeweils 2 schwere Komplikationen in bd. Gruppen). Alle HKUs konnten problemlos durchgeführt werden.

Diskussion: Die Durchführbarkeit von diagn. u./od. intervent. Prozeduren sowie die Sicherheit der Patienten sind die maßgeblichen Qualitätskriterien von tiefen Sedierungen bei erhaltener Spontanatmung. Ein standardisiertes Vorgehen halten wir für unumgänglich. Mit Blick auf die prim. EP lässt sich festhalten, dass bd. Sedierungsverfahren die Durchführbarkeit einer diagn. od. intervent. HKU in vollem Umfang gewährleisten. Schw. Komplikationen traten selten auf $u$. ließen sich mit einfachen Maßnahmen beherrschen (Absaugen, Esmarchhandgriff, Einbringen eines Wendl-Tubus). Bei keinem unserer Patienten bestand die Notwendigkeit zur Maskenbeatmung, Intubation od. Gabe von kardiozirkulatorisch wirksamen Medikamenten. Bei Sedierungen mit $4 \mathrm{H}$ empfiehlt sich zur Vermeidung von PONV die prophylaktische Gabe eines Antiemetikums.

Schlussfolgerungen: Beide Verfahren eignen sich für die sichere Durchführung von tiefen Sedierungen in Spontanatmung für HKUs. Die Applikation von $4 \mathrm{H}$ scheint in Bezug auf die Atmung u. den Gasaustausch sowie die Sedierungsqualität einige Vorteile zu bieten.
FV58

Mortalitätseinschätzung mittels echokardiographischer Beurteilung des pulmonalarteriellen Flussprofils bei Kindern mit angeborener Zwerchfellhernie während ECMO-Therapie

F. Kipfmueller', K. Heindel', L. Schröder', A. Müller ${ }^{1}$

'Universitätsklinik Zentrum f. Kinderheilkunde, Neonatologie und

Pädiatrische Intensivmedizin, Bonn, Deutschland

Einleitung: Der Schweregrad der pulmonalen Hypertonie (PH) ist ein prognostisch wichtiger Parameter bei Neugeborenen mit angeborener Zwerchfellhernie (CDH). Eine genaue Einschätzung der PH ist insbesondere unter ECMO-Therapie wichtig. In der Echokardiographie kann über verschieden Messmethoden der pulmonalerterieller Druck (PAP) berechnet werden. Echokardiographisch korreliert der Quotient aus pulmonalerterieller Azzelerationszeit (TPV) und rechtsventrikulärer Ejektionsdauer (RVET) mit der Höhe des PAP. Hierbei sprechen niedrigere Werte für einen höheren PAP. Bei Neugeborenen mit CDH ist eine niedrige TPV/ RVET $(<0,29)$ in den ersten Lebensstunden mit einem deutlich höherem ECMO-Risiko assoziiert. Der Stellenwert der TPV/RVET während einer ECMO-Therapie ist bisher nicht untersucht worden.

Fragestellung: Wir untersuchten bei Neugeborenen mit $\mathrm{CDH}$, ob die TPV/RVET während einer ECMO-Therapie (nach 24 Std und nach 5 Tagen) mit dem Outcome korreliert.

Methodik: Verglichen wurden die Werte bei Patienten, die aus dem stationären Aufenthalt entlassen werden konnten (Gruppe 1), mit den Patienten, die im Krankenhaus verstarben (Gruppe 2). Es erfolgte ein Vergleich der Medianwerte mit dem Mann-Whitney-U-Test. Mittels ROC-Analyse wurde der optimale Grenzwert für TPV/RVET berechnet und die Sensitivität und Spezifität in Bezug auf die Krankenhausmortalität kalkuliert. Ergebnisse: Zwischen Januar 2013 und Januar 2016 wurden in unserer Abteilung 68 Neugeborene mit CDH behandelt. Davon benötigten 25 Patienten $(36,8 \%)$ eine ECMO-Unterstützung, von denen 21 Patienten (84\%) erfolgreich dekanüliert werden konnten. In der ECMO-Gruppe verstarben insgesamt 9 Patienten während des stat. Aufenthaltes (36,4\%). Nach Ausschluss von 5 Patienten mit einer ECMO-Dauer von $<0,310$ ) und nach 5 Tagen von $88,9 \%$ und $72,7 \%($ TPV/RVET $<0,325)$ für die kalkulierten Grenzwerte.

Schlussfolgerung: Bei Neugeborenen mit $\mathrm{CDH}$ eignet sich die TPV/RVET als Parameter zur Risikoeinschätzung während einer ECMO-Therapie. Ein ausbleibender Anstieg der TPV/RVET unter ECMO-Therapie ist bei Neugeborenen mit CDH mit einer höheren Mortalität assoziiert. Dies gilt insbesondere auch im Hinblick auf die Mortalität nach erfolgreicher ECMO-Dekanülierung.

\section{FV59}

Ein neuer Algorithmus zur Analyse von Phonokardiogrammen zur Diagnostik kongenitaler Herzfehler

R. Grgic-Mustafic', N. Baik', B. Schwaberger', L. Mileder', J. Pansy', M. Köstenberger ${ }^{2}$, B. Urlesberger ${ }^{1}$, G. Pichler ${ }^{1}$

'Univ.-Klinik für Kinder- und Jugendheilkunde, Klinische Abteilung

für Neonatologie, Graz, Österreich, ${ }^{3} U n i v$.-Klinik für Kinder- und Jugendheilkunde, Klinische Abteilung für pädiatrische Kardiologie, Graz, Österreich

Hintergrund: Die kardiale Auskultation ist eine Standard-Screeningmethode zur Identifizierung von kongenitalen Herzfehlern in Rahmen der postnatalen klinischen Routineuntersuchung. Die Sensitivität und Spezifität der Auskultation ist jedoch insbesondere bei Neugeborenen abhängig von der Herzfrequenz und der Erfahrung des Untersuchers. Automatisierte Phonokardiographie als Screeningmethode von Herzgeräuschen und kongenitalen Herzfehlern ist aufgrund der immer noch unzureichender Präzision und mangelnder klinischer Studien derzeit nicht Bestandteil der klinischen Routine.

Ziel: Ziel unserer Pilotstudie war die Bestimmung der Sensitivität und Spezifität eines neuen automatisierten Algorithmus in der Detektion von Herzgeräuschen mittels Phonokardiogrammen bei Neugeborenen. 
Methoden: Es wurde an 40 Neugeborenen eine prospektive Beobachtungs-/Pilotstudie durchgeführt. Echokardiographie, Auskultation durch Kinderfachärzte, und die Aufnahme der Phonokardiogramme wurden innerhalb von $12 \mathrm{~h}$ durchgeführt. Die Phonokardiogramme wurden mittels eines neuen automatisierten Algorithmus zur Detektion von Herzgeräuschen, entwickelt von der CSD Labs GmbH, in zwei Durchgängen analysiert. Sensitivität und Spezifität der Detektion von Herzgeräuschen bei echokardiografisch bestätigten, kongenitalen Herzfehlern wurden mittels des automatisierten Algorithmus in den Phonokardiogrammen während eines ersten Durchlaufes bzw. eines zweiten konsekutiven Durchlaufes (nach erneutem Training des Algorithmus mit erweiterter Datensatz) sowie mittels Auskultation durch Kinderärzte errechnet und verglichen. Resultate: Von 40 inkludierten Neugeborenen wurden 36 (mittleres Gestationsalter $36 \pm 3$ Wochen, Geburtsgewicht $2572 \pm 847 \mathrm{~g}$ ) analysiert. Davon hatten 23 Neugeborene (64\%) pathologische und $13(36 \%)$ unauffällige Befunde in der Echokardiografie. Sensitivität und Spezifität der Auskultation von Herzgeräuschen mit echokardiografisch bestätigten Pathologien durch Kinderfachärzte waren $17 \%$ und $100 \%$. Der neue automatisierte Algorithmus zeigte im ersten Durchgang eine im Vergleich dazu signifikant höhere Sensitivität von 70 \% und eine niedrigere Spezifität von $77 \%$. Im zweiten Durchgang zeigten sich im Vergleich zum ersten Durchgang eine deutlich höhere Sensitivität von $83 \%$ und eine Spezifität von $85 \%$.

Konklusion: Die Phonokardiographie mit dem untersuchten automatisierten Algorithmus zur Detektion von Herzgeräuschen bei echokardiografisch bestätigten Pathologien zeigte eine höhere Sensitivität, aber eine niedrigere Spezifität als die kardiale Auskultation durch Kinderfachärzte mit verbesserter Detektion nach dem der Algorithmus mit einer größeren Datensatz trainiert wurde. Eine größere Fallzahl in weiteren Studien könnte zu einer weiteren Verbesserung der Sensitivität und Spezifität der Phonokardiographie führen und somit eine Implementierung der Phonokardiographie in die klinische Routine ermöglichen.

\section{FV60 \\ Hämodynamische Assoziationen der fetalen Stunts beim Frühgeborenen}

\section{S. Baumgartner', L. Pitterle², A. Berger', G. Fischer ${ }^{3}$, U. Salzer-Muhar ${ }^{2}$}

'Medizinische Universität Wien/Universitätsklinik für Kinder- und Jugendheilkunde, Abteilung für Neonatologie, pädiatrische Intensivmedizin und Neuropädiatrie, Wien, Österreich, ${ }^{2}$ Universitätsklinik für Kinderund Jugendheilkunde, Medizinische Universität Wien, Abteilung für pädiatrische Kardiologie, Wien, Österreich, ${ }^{3}$ Institut für Medizinisches Informationsmanagement und Bildverarbeitung, Wien, Österreich

Hintergrund: Der Ductus Venosus verschließt sich beim reifen gesunden Neugeboren gegen Ende der ersten Lebenwoche. Ein persistierender Ductus Venosus (PDV) im Sinne eines portosystemischen Shunts wird sowohl bei angeborenen Herzfehlern als auch bei Lebererkrankungen, insbesonders der Leberzirrhose und bei pulmonaler und portopulmonaler Hypertension beschrieben.

Fragestellung: Ziele der Studie waren einerseits den Zeitpunkt des Verschlusses des PDV bei Frühgeborenen (FG) darzustellen sowie mögliche Zusammenhänge zwischen dem PDV und dem Bestehen eines persitierenden Ductus arteriosus (PDA) und persistierenden Foramen ovale (PFO) sowie deren Shuntrichtungen zu ermitteln.

Material und Methoden: Es handelt sich um eine retrospektive Datenanalyse im Zeitraum von Jänner 2010 bis März 2013. Erhoben wurden alle funktionellen echokardiographischen Untersuchungen von FG der SSW $23+0$ bis $36+6$ in den ersten acht Lebenswochen. Ausgeschlossen wurden funktionelle echokardiographische Befundblätter bei denen die Präsenz des Ductus Venosus nicht dokumentiert wurde und FG mit angeborenen Herzfehlern.

Ergebnisse: 249 echokardiographische Untersuchungen und standardisierte elektronische Befundblätter wurden von $162 \mathrm{FG}$ erhoben. Ein PDV wurde im Vergleich von FG der SSW $\geq 28+0(n=73)$ vs. SSW $\leq 27+6$ $(n=89)$ zu folgenden Zeitpunkten beschrieben: 1.-3. Lebenstag (LT) $87 \%$ vs. $92 \%$, LT $4-6: 58 \%$ vs. $78 \%$, LT 7-9: $33 \%$ vs. $42 \%$ und LT $16 .-18: 0 \%$ vs. $22 \%$. Es konnte ein signifikanter Zusammenhang zwischen dem Verschluss des PDV und der Präsenz eines PDA $(p=0,005)$ sowie eines PFO $(p=0,040)$ festgestellt werden. Ein bidirektionaler oder Rechts-LinksShunt waren bei diesen beiden fetalen Shunts signifikant häufiger mit einem PDV assoziiert als ein Links-Rechts Shunt ( $p$-Wert jeweils $<0,001$ ). Dem entsprechend wurde bei FG mit pulmonaler Hypertension häufiger ein PDV dokumentiert ( $90 \%$ vs. $41 \%$ ). Die Analyse der Flussmuster über dem PDA bei Links-Rechts Shunt ergab, dass Flussmuster die auf einen erhöhten pulmonalen Widerstand hinwiesen häufiger mit einem PDV assoziiert waren als jenen mit Hinweis auf einen hämodynamisch wirksamen Shunt und pulmonaler Überflutung.

Diskussion: Bei FG mit SSW $\leq 27+6$ wurde im Vergleich zu SSW $\geq 28+0$ eine Verzögerung des PDV-Verschlusses dokumentiert. „Fetale“ Blutflussmuster über PDA bzw. PFO wie ein bidirektionalem oder Rechts-Links Shunt könnten relevante Faktoren beim Verschluss des PDV darstellen, während hämodynamisch wirksame Links-Rechts Blutflussmuster über dem PDA keinen Einfluss auf den PDV-Verschluss haben. Damit dürfte der erhöhte pulmonale Druck/Widerstand eine größere Rolle im Verschluss des PDV spielen als Faktoren die in Assoziation mit einem hämodynamisch wirksamen PDA stehen. Ob und inwieweit dem PDV bei der Entstehung der pulmonalen Hypertension eine pathogenetische Bedeutung zukommt bzw. inwieweit die pulmonale Hypertension den Verschluss des PDV verzögert bedarf noch weiterer Untersuchungen.

\section{FV61}

\section{Vergleich einer Ibuprofen-Kurz- oder -Dauerinfusion zur Therapie} des offenen Ductus arteriosus Botalli bei Frühgeborenen

S. Bagci' , K. Hajiyeva' , D. Katzer' , P. Bartmann' ', A. Müller ${ }^{1}$

'Zentrum für Kinderheilkunde, Universität Bonn, Neonatologie und Pädiatrische Intensivmedizin, Bonn, Deutschland

Fragestellung: Das Ziel dieser retrospektiven Studie war es, die Erfolgsrate des Ductusverschlusses mit Ibuprofen bei Frühgeborenen (FG) $<1500 \mathrm{~g}$ mit zwei unterschiedlichen Applikationsformen (Kurzinfusion (KI) und Dauerinfusion (DI)) bzgl. der renalen (quantitative Urinausscheidung) und gastrointestinalen Nebenwirkungen (Nahrungs-intoleranz, nekrotisierende Enterokolitis (NEK) oder fokale intestinale Perforation (FIP)) zu vergleichen.

Patienten und Methodik: In die retrospektive Studie wurden 100 FG mit einem hämodynamisch relevanten offenen Ductus in den ersten drei Lebenstagen eingeschlossen. Die Patienten erhielten während des ersten Behandlungzyklus entweder Ibuprofen als KI über 30 Minuten ( $n=55$, initial $10 \mathrm{mg} / \mathrm{kg}$, dann $5 \mathrm{mg} / \mathrm{kg}$ nach $24 \mathrm{und} 48 \mathrm{~h}$ ) oder Ibuprofen als DI ( $n=45$, initial $10 \mathrm{mg} / \mathrm{kg}$ für $24 \mathrm{~h}$, dann $5 \mathrm{mg} / \mathrm{kg} / \mathrm{d}$ für $48 \mathrm{~h}$ ).

Ergebnisse: Mittleres Geburtsgewicht und mittleres Gestationsalter der FG beider Gruppen unterschieden sich nicht signifikant (KI vs. DI; 26,9 $\pm 2,2$ vs. $27,0 \pm 2,1$ und $844 \pm 292 \mathrm{~g}$ vs. $835 \pm 258 \mathrm{~g} ; p=0,760$ und $p=0,909)$. AuBer dem arteriell gemessenen Base Exzess (BE) bei Aufnahme (KI vs. DI, $-5,5$ vs. $-6,8 \mathrm{mmol} / \mathrm{l}, p=0,012)$, gab es keinen signifikanten Unterschied zwischen beiden Gruppen: Arterieller $\mathrm{pH}$ bei Aufnahme, minimaler $\mathrm{pH}$ und BE in den ersten 72 Lebensstunden (LS), Häufigkeit eines fetofetalen Transfusionssyndrom (FFTS) und einer Mehrlingsschwangerschaft, Infektion bei Aufnahme oder in den ersten $72 \mathrm{~h}$ unterschieden sich nicht signifikant $(p>0,05)$. Nach dem ersten Zyklus war die Verschlussrate des Ductus in der KI-Gruppe $80,0 \%$ gegenüber $73,3 \%$ bei Patienten mit DIGruppe $(p=0,433)$. Bei drei (5,5\%) Patienten der KI-Gruppe, aber bei keinem Patienten der DI-Gruppe, war im Verlauf eine Ligatur erforderlich $(p=0,113)$. Gestationsalter, Häufigkeit eines FFTS und einer Mehrlingsschwangerschaft, arterieller $\mathrm{pH}$ und $\mathrm{BE}$ bei Aufnahme, minimaler $\mathrm{pH}$ und BE in den ersten 72 LS, Häufigkeit einer Infektion bei Aufnahme oder in den ersten 72 LS hatten allesamt keinen Einfluss auf die Ductusverschlussrate $(p>0,05)$. Ein Geburtsgewicht unter $1000 \mathrm{~g}$ konnte als einziger Prädiktor für einen erfolglosen Duktusverschluss nach dem ersten Behandlungszyklus für das gesamte Kollektiv festgestellt werden $(r=0,200$, $p=0,048)$. Im Vergleich mit der KI-Gruppe hatten DI-Patienten statistisch signifikant mehr Magenreste am 2. (T2) und 3. Tag (T3) des Zyklus 
(12,9 vs. $18,5 \mathrm{ml} / \mathrm{kg} / \mathrm{d}$ an T2 und 16,9 vs. $25,3 \mathrm{ml} / \mathrm{kg} / \mathrm{d}$ an T3, $p=0,015$ und $p=0,013$ ), obwohl der maximale Magenrest an T1, T2 und T3 in beiden Gruppen nicht signifikant different waren $(3,1 \mathrm{vs.} 3,4 \mathrm{ml} / \mathrm{kg}$ an T1, 3,9 vs. $5,0 \mathrm{ml} / \mathrm{kg}$ an T2 und 4,7 vs. $5,8 \mathrm{ml} / \mathrm{kg}$ an T3, $p=0,305, p=0,118$ und $p=0,246)$. Die Rate einer NEK/FIP war in beiden Gruppen vergleichbar (KI vs. DI, 7,3 vs. $13,3 \%, p=0,317$ ).

Zwischen beiden Gruppen fand sich während des Zyklus kein signifikanter Unterschied bei der Urinausscheidung vor und nach der Ibuprofengabe (KI vs. DI, vor und nach der Ibuprofengabe; 4.0 vs. $4.3 \mathrm{ml} / \mathrm{kg} / \mathrm{h}$ und $3.7 \mathrm{vs.}$ $3.9 \mathrm{ml} / \mathrm{kg} / \mathrm{h}$ an T1, 5.0 vs. $4.3 \mathrm{ml} / \mathrm{kg} / \mathrm{h}$ und 4.5 vs. $4.1 \mathrm{ml} / \mathrm{kg} / \mathrm{h}$ an T2, 5.4 vs. $4.6 \mathrm{ml} / \mathrm{kg} / \mathrm{h}$ und $5.7 \mathrm{vs.} .7 \mathrm{ml} / \mathrm{kg} / \mathrm{h}$ an T3, $p>0.05$ ).

Diskussion: Sowohl in Bezug auf die Erfolgsraten als auch die Nebenwirkungen ergaben sich keine signifikanten Unterschiede zwischen beiden Applikationsformen. Die vorliegende Evidenz zeigt, dass der Einsatz einer Dauerinfusionstherapie im untersuchten Kollektiv und bezüglich der analysierten Parameter nicht zu einem klinisch besseren Ergebnis führt.

\section{FV62}

Funktionelle Echokardiografie in der Neonatologie Referenzwerte für das Velocity-Time-Integral über der Aortenklappe als potentiellem Surrogatparameter für das linksventrikuläre Schlagvolumen

\section{K. Gründler', C. Schwarz', G. Wiegand', C. Poets', A. Franz' \\ 'Eberhard-Karls-Universität, Universitätsklinik für Kindermedizin und Jugendheilkunde, Neonatologie, Tübingen, Deutschland, ${ }^{2}$ Eberhard-Karls- Universität Universitätsklinik für Kinderheilkunde und Jugendmedizin Kinderkardiologie, Tübingen, Deutschland}

Hintergrund: Für die Beurteilung der Kreislauffunktion bei Frühgeborenen (FG) stehen in der Regel nur der arterielle Blutdruck, die Urinproduktion sowie subjektive Parameter wie Hautkolorit und Rekapillarisierungszeit zur Verfügung. Der Fluss in der oberen Hohlvene ist für weniger Geübte schlecht reproduzierbar. Das Velocity-Time-Integral über der Aortenklappe (AK-VTI) könnte als leicht ermittelbarer und gut reproduzierbarer Verlaufsparameter helfen, das linksventrikuläre Schlagvolumen und damit das systemische Herzminutenvolumen bei sehr kleinen Frühgeborenen ohne relevanten Ductus arteriosus abzuschätzen.

Zielsetzung: Erstellung von Referenzwerten für das AK-VTI bei FG.

Material und Methoden: Retrospektive Analyse klinisch indizierter Echokardiografien bis zum 10. Lebenstag bei FG mit einem Gestationsalter (GA) bei Geburt von <29 SSW, die in den Jahren 2012-2014 in der neonatologischen Abteilung des Universitätsklinikums Tübingen behandelt wurden und keine schwerwiegenden angeborenen Herzfehler hatten.

Ergebnisse: Insgesamt wurden 120 Frühgeborene $<29$ SSW im Studienzeitraum geboren. Innerhalb der ersten 10 Lebenstage wurden 66 Patienten mit einem GA $26( \pm 1,57)$ SSW und einem Geburtsgewicht von $740 \mathrm{~g}$ $( \pm 220)$ echokardiografisch untersucht. 1 Patient wurde bei Tod innerhalb der ersten 10 Lebenstage ausgeschlossen. Bei 87 von 109 Echokardiografien wurde das AK-VTI bestimmt. Die Ergebnisse sind in $\bullet$ Tab. FV62, nach Alter bei Messung aufgeteilt, zusammengefasst. Dabei ging in jede Gruppe jeweils nur die erste echokardiografische Untersuchung eines Patienten im jeweiligen Intervall ein.

In dieser Kohorte erhielten 4/4 Patienten, die am 1. Lebenstag ein AKVTI $<6,0 \mathrm{~cm}$ aufwiesen eine Kreislauftherapie mit Katecholaminen, Volumen und Hydrocortison im Vergleich zu 7 Patienten mit Katecholaminen,

\begin{tabular}{|c|c|c|c|c|c|}
\hline & d1 & d2-3 & d4-5 & d6-10 & Alle \\
\hline$n$ & 24 & 27 & 18 & 18 & 87 \\
\hline median & 8,65 & 8,50 & 10,15 & 9,20 & 8,80 \\
\hline P10 & 5,56 & 5,68 & 6,91 & 6,21 & 5,76 \\
\hline P90 & 10,49 & 11,28 & 12,51 & 13,2 & 11,8 \\
\hline SD & 2,20 & 2,20 & 2,89 & 2,96 & 2,57 \\
\hline
\end{tabular}

15 mit Volumengaben und 4 mit Hydrocortison mit einem VTI $\geq 6,0 \mathrm{~cm}$ $(n=20) .2 / 4$ Patienten im Vergleich zu 6/20 Patienten entwickelten eine intraventrikuläre Hämorrhagie $\geq 2^{\circ}$ oder eine periventrikuläre Leukomalazie im Verlauf.

Schlussfolgerung: Der Referenzbereich für das AK-VTI bei FG < 29SSW liegt in den ersten Lebenstagen zwischen 6 und $-11 \mathrm{~cm}$ (entsprechend P10 und P90 der vorliegenden Werte). In dieser retrospektiven Analyse lässt sich kein Zusammenhang des AK-VTI mit Kreislaufinterventionen bzw. mit Hirnschädigung belegen. Unserer Erfahrung nach ist die Messung des AK-VTI eine einfach erlernbare, wenig zeitintensive und wenig belastende Untersuchung, die bei FG eine Einschätzung des linksventrikulären Schlagvolumens ermöglicht. Wenn nicht gleichzeitig ein hämodynamisch relevanter Ductus arteriosus vorliegt, könnte das AK-VTI zur Steuerung der Kreislauftherapie beitragen. Eine prospektive Studie ist in Vorbereitung.

\section{FV63}

Einfluss eines persistierenden Ductus arteriosus auf die periphermuskuläre Oxygenierung und Perfusion bei Neugeborenen

T. Müller', L. Mileder', N. Baik', J. Pansy', B. Schwaberger', C. Binder', B. Urlesberger', G. Pichler

'Univ.-Klinik für Kinder- und Jugendheilkunde, Medizinische Universität Graz, Klinische Abteilung für Neonatologie, Graz, Österreich

Hintergrund: Mittels Nahinfrarotspektroskopie (NIRS) können Konzentrationsänderungen von oxygeniertem und desoxygeniertem Hämoglobin in Geweben kontinuierlich und nichtinvasiv gemessen werden. Aus diesen Messwerten lassen sich der Tissue Oxygenation Index (TOI) und die Fractional Oxygen Extraction (FOE) errechnen. Durch venöse Okklusion können zusätzlich Sauerstoffangebot $\left(\mathrm{DO}_{2}\right)$, Sauerstoffverbrauch $\left(\mathrm{VO}_{2}\right)$ und venöse Sauerstoffsättigung $\left(\mathrm{SvO}_{2}\right)$ bestimmt werden.

Fragestellung: Ziel der Studie war es herauszufinden, ob ein persistierender Ductus arteriosus (PDA) einen Einfluss auf die peripher-muskuläre Oxygenierung und Perfusion bei Neugeborenen hat.

Material und Methoden: Die Daten wurden im Rahmen von prospektiven Beobachtungsstudien an Früh- und Reifgeborenen zwischen dem ersten und dritten Lebenstag erhoben. Ausschlusskriterien waren kongenitale kardio-pulmonale Malformationen. Für die venöse Okklusion wurde mittels einer Blutdruckmanschette an Oberarm oder Oberschenkel der venöse Abfluss mit einem Druck zwischen diastolischem und geschätztem venösen Blutdruck des Neugeborenen $(\sim 20 \mathrm{mmHg})$ für 20 Sekunden unterbunden. Die NIRS-Elektroden (NIRO 200-NX, Hamamatsu, Japan) wurden postduktal am linken Unterarm oder am Unterschenkel angebracht, wobei die Messungen bis zum Erreichen zumindest eines validen Ergebnisses wiederholt wurden.

Innerhalb von sechs Stunden vor oder nach der peripheren NIRS-Messung wurde eine Echokardiographie durchgeführt, im Rahmen derer der PDA-Durchmesser $[\mathrm{mm} / \mathrm{kg}]$ standardisiert bestimmt wurde. Die Daten wurden in Abhängigkeit von der Normalverteilung mittels Korrelationsanalyse nach Pearson oder Spearman bei einem Signifikanzniveau von $p \leq 0,05$ untersucht.

Ergebnisse: Es wurden 40 Neugeborene (28 Frühgeborene, 12 Reifgeborene) mit einem mittleren Gestationsalter von 35,7 $\pm 3,3$ Schwangerschaftswochen eingeschlossen. Es fanden sich signifikante Unterschiede bei Herzfrequenz und arterieller Sauerstoffsättigung zwischen jenen Neugeborenen mit bzw. ohne PDA. Die Korrelationsanalysen ergaben signifikante negative Korrelationen zwischen PDA-Durchmesser und arterieller Sauerstoffsättigung, $\mathrm{SVO}_{2}$ und pTOI, wohingegen der PDA-Durchmesser positiv mit Herzfrequenz und FOE korrelierte.

Diskussion: Unsere Daten zeigen einen signifikanten Zusammenhang zwischen dem PDA-Durchmesser und der peripheren Oxygenierung bzw. Perfusion bei Früh- und Reifgeborenen. 


\section{Freie Themen 11: Neugeborene drogenabhängiger Mütter}

\section{FV64}

Langzeitoutcome von Neugeborenen mit Neonatalem Abstinenzsyndrom (NAS) unter besonderer Berücksichtigung von sozialmedizinischen und entwicklungsneurologischen Aspekten

N. Kopf', U. Maurer-Fellbaum ', E. Pichler-Stachl', B. Urlesberger', W. Raith ${ }^{1}$

'Universitätsklinik für Kinder-und Jugendheilkunde, Medizinische

Universität Graz, Klinische Abteilung für Neonatologie, Graz, Österreich

Hintergrund: Maternaler Opioidkonsum während der Schwangerschaft geht häufig mit einer postnatalen Entzugssymptomatik (NAS) des Neugeborenen einher. Die Langzeitprognose dieser Kinder, hinsichtlich ihrer weiteren Entwicklung, und der sozialmedizinischen Umstände, in denen die weitere Entwicklung erfolgt, wurde bisher kaum erforscht. In einigen Studien konnte eine Tendenz für deutliche kognitive und entwicklungsneurologische Einschränkungen der Kinder gezeigt werden.

Fragestellung: Unsere Studie untersucht das Auftreten von Entwicklungsdefiziten bei Kindern nach intrauteriner Opioidexposition und NAS. Der mögliche Einfluss von sozialmedizinischen Aspekten wie der Fremdunterbringung von Kindern substanzabhängiger Frauen soll analysiert werden. Material und Methoden: Die vorliegende Studie beinhaltet eine retrospektive Analyse von Daten der Jahre 2000-2012 der Klinischen Abteilung für Neonatologie in Graz. Es wurden Kinder mit Opioidexposition während der Schwangerschaft und diagnostiziertem NAS eingeschlossen. Die Entwicklungsdiagnostik wurde durch Anwendung von Bayley Scales of Infant Development (BSID-II) und Denver Entwicklungsskalen durchgeführt. Ergebnisse: Es wurden 63 Kinder in die Studie eingeschlossen. 69,8\% $(n=44)$ der Kinder wurden zu vereinbarten entwicklungsneurologischen Untersuchungen vorgestellt. Prinzipiell wurden nur Kinder mit Auffälligkeiten wiedereinbestellt. Von den 23 im zweiten Lebensjahr vorgestellten Kindern zeigten 43,3 \% Auffälligkeiten. Im 3. Lebensjahr $75 \%$ von 12 wiedervorgestellten Kindern, wobei ein Kind eine schwere Entwicklungsverzögerung aufwies. Im 4 . Lebensjahr waren es $71,4 \%$ von 7 vorgestellten Kindern, und im 5.Lebensjahr $100 \%$ der kontrollierten Kinder $(n=4)$. Es fielen 18,3\% ( $n=9)$ der Kinder mit ADHS, Hyperkinetischem Syndrom, Wahrnehmungs-, Verhaltens-, oder Bindungsstörungen auf. In 50,8 \% der Fälle kam es zu einem Wechsel in der Obsorge, mit erstem Betreuungswechsel bei 81,3\% im 1. Lebensjahr. Es zeigt sich, in Abhängigkeit der Unterbringung, bei leiblichen Eltern beziehungsweise Fremdunterbringung, kein Unterschied in der Vorstellung der Kinder zur vereinbarten Entwicklungsuntersuchung im 1. Lebensjahr $(p=0,595)$. Entwicklungsauffällige Kinder unterscheiden sich bezüglich der Aufenthaltsdauer $(p=0,761)$ und der Dauer des Entzuges $(p=0,822)$ nicht signifikant von in der Entwicklung unauffälligen Kindern. Bezüglich der Art der Opioidexposition während der Schwangerschaft zeigten sich Kinder mit Substitol-Exposition seltener in ihrer Entwicklung auffällig $(p=0,005)$, als Kinder die Methadon oder Buprenorphin ausgesetzt waren. Als relevante Nebendiagnosen fanden wir in 4,8\% $(n=3)$ ein Battered Child Syndrom, und in 4,8\% $(n=3)$ eine notfallmässige Spitalsaufnahme bei Intoxikation.

Schlussfolgerung: Kinder mit Opioidexposition in der Schwangerschaft zeigen eine große Variabilität in ihrer weiteren Entwicklung: sowohl unauffällige Entwicklungsverläufe, als auch Verläufe mit Defiziten in Sprachentwicklung, Feinmotorik, Grobmotorik oder im Sozialkontakt werden gesehen. Auch bei einer bis zum Alter von 2 Jahren unauffällig verlaufenen Entwicklung können sich bis zum Alter von 5 Jahren neue Auffälligkeiten zeigen. Diese Ergebnisse implizieren die Wichtigkeit von Entwicklungskontrollen über das 2. Lebensjahr hinaus, und eine interdisziplinäre Vernetzung im medizinischen sowie sozialen Bereich in der Weiterbetreuung der Familien.

\section{FV65}

Zunahme der Therapiedauer beim neonatalen Abstinenzsyndrom von 2000 bis 2011

S. Mücke', M. Nagel', J. Siedentopf ${ }^{2}$, C. Bührer ${ }^{1}$, D. Hüseman ${ }^{3}$

${ }^{1}$ Charité Universitätsmedizin Berlin, Klinik für Neonatologie, Berlin, Deutschland, ${ }^{2}$ Charité Universitätsmedizin Berlin, Klinik für Geburtsmedizin, Berlin, Deutschland, ${ }^{3}$ Werner-Forßmann Krankenhaus Barnim, Klinik für Kinder- und Jugendmedizin, Eberswalde, Deutschland

Hintergrund und Fragestellung: Intrauterin opiatexponierte Neugeborene können postnatal ein neonatales Abstinenzsyndrom (NAS) entwickeln. Fast alle opiatabhängigen Schwangeren erhalten eine Substitutionstherapie mit Methadon, Polamidon oder Buprenorphin. Wir stellten uns die Frage, wie sich die durchschnittliche Dauer der medikamentös behandelten NAS-Symptomatik und die Hospitalisationsdauer in Abhängigkeit von Art und Dosis des Substitutionsmedikament über einen Zeitraum von 12 Jahren verändert haben.

Material und Methoden: Im Zeitraum von Januar 2000 bis Dezember 2011 wurden aus den archivierten Patientenakten von 378 opiatexponierten Neugeborenen Gestationsalter, Geburtsgewicht, Aufenthalts- und medikamentöse Therapiedauer mit Morphin oder Phenobarbital erhoben. Von den insgesamt 352 substituierten Müttern wurde die Wahl des Opiatsubstituts (Methadon, Polamidon oder Buprenorphin) erfasst, bei 297 Müttern war die genaue Substitutionsdosis bei Entbindung bekannt. Die Daten wurden in drei Zeitabschnitte $\left(t_{1}=2000-2004, n_{1}=147 ; t_{2}=2005-\right.$ 2007, $\left.\mathrm{n}_{2}=101 ; \mathrm{t}_{3}=2008-2011, \mathrm{n}_{3}=130\right)$ zusammengefasst und mit Hilfe von Microsoft Excel 2007 (Kruskal-Wallis-Test) und Statpoint Inc. Statgraphics Centurion1 Version 16.0 (MANOVA) miteinander verglichen. Ergebnisse: Im untersuchten Zeitraum blieben das mediane Geburtsgewicht $\left(\mathrm{t}_{1}: 2760 \mathrm{~g}, \mathrm{t}_{2}: 2900 \mathrm{~g}, \mathrm{t}_{3}: 2890 \mathrm{~g}, p=0,43\right)$ und die Frühgeburtlichkeitsrate (21-22\%) stabil. Auch der Anteil der Kinder, deren Entzug medikamentös unterstützt werden musste, schwankte nicht signifikant $\left(t_{1}: 65 \%\right.$, $\left.\mathrm{t}_{2}: 69 \%, \mathrm{t}_{3}: 62 \%\right)$. Währenddessen nahm die mediane Dauer der medikamentösen Entzugstherapie in den Vergleichszeiträumen von $t_{1}: 5$ Tagen über $t_{2}: 13$ Tage auf $t_{3}: 18$ Tage zu $(p<0,01)$. Analog verlängerte sich auch die mediane Aufenthaltsdauer von $t_{1}: 11$ Tage auf $t_{3}: 19$ Tage $(p<0,01)$. Zeitgleich war eine Zunahme der maternalen Methadon- $\left(t_{1}: 13,5 \mathrm{mg} / \mathrm{d}\right.$, $\left.\mathrm{t}_{3}: 25 \mathrm{mg} / \mathrm{d}, p<0,01\right)$ und Polamidon-Dosierung $\left(\mathrm{t}_{1}=22,5 \mathrm{mg}, \mathrm{t}_{3}=30 \mathrm{mg}\right.$, $p=0,02)$ zu beobachten, während die mediane Buprenorphin-Tagesdosis über den untersuchten Zeitraum (ca. 4-5,5 mg/d) sich nicht veränderte. Die multivariate Varianzanalyse (MANOVA) zeigte einen signifikanten Einfluss der maternalen Methadon- bzw. Polamidondosis auf Therapie$(p=0,04$ und $p<0,01)$ und Aufenthaltsdauer $(p=0,03$ und $p<0,01)$ unabhängig von anderen bekannten Einflussfaktoren wie dem neonatalen Entzugsmedikament.

Diskussion: Die Wahl des Substitutionsmedikaments wird kontrovers diskutiert, wobei die Gefahr eines Drogenbeigebrauchs und die plazentare Pharmakokinetik als relevante Aspekte zu nennen sind. Höhere Dosen erleichtern möglicherweise die Führung der Schwangeren, sind aber mit einer längeren kindlichen Entzugssymptomatik assoziiert. Die Krankenhausaufenthaltsdauer wird aber auch durch die Komplexität des sozialen Entlassungsmanagements und die Verfügbarkeit von Familienhilfen sowie Mutter-Kind-Einrichtungen bestimmt.

\section{FV66}

Neonatales Abstinenzsyndrom (NAS) in Graz. Eine retrospektive Analyse der Jahre 2000-2012: Prä-, peri-, und postpartale Daten von Neugeborenen, Müttern und Vätern

N. Kopf', U. Maurer-Fellbaum' ', J. Pansy' ', B. Urlesberger', W. Raith ${ }^{1}$

'Universitätsklinik für Kinder-und Jugendheilkunde, Medizinische Universität Graz, Klinische Abteilung für Neonatologie, Graz, Österreich

Hintergrund: Der Opioidkonsum wird als größter Anteil des problematischen Drogenkonsums in Österreich beschrieben. Maternaler Drogenkonsum während der Schwangerschaft geht mit einer postnatalen Entzugssymptomatik des Neugeborenen einher, wobei die Neugeborenen in 
der Regel eine medikamentöse Therapie benötigen. Ein Bedarf an einem standardisierten Programm zur Betreuung substanzabhängiger Frauen, Mütter und Neugeborener prä-, peri-, und postpartal soll evaluiert werden.

Fragestellung: Ziel dieser Studie war die Erfassung des NAS am Standort Graz, sowie die Erhebung der prä-, peri-, und postpartalen Situation von Neugeborenen, Müttern und Vätern.

Material und Methoden: Die vorliegende Studie beinhaltet eine retrospektive Analyse der Daten (2000-2012) der Klinischen Abteilung für Neonatologie der Universitätsklinik für Kinder-und Jugendheilkunde in Graz, Österreich. Es wurden alle Neugeborenen mit einer Opioidexposition in der Schwangerschaft und folgendem NAS in die Studie eingeschlossen. Ergebnisse: Die untersuchte Studienpopulation von 63 Kindern setzte sich zu 52,4\% aus Mädchen $(n=33)$, und zu 47,6\% $(n=30)$ aus Knaben zusammen. Das mittlere Geburtsgewicht der Neugeborenen betrug $2812,13 \mathrm{~g}$ (SD 506,66 g), die Kinder waren 48,02 cm gross (SD 2,64), und der Kopfumfang betrug $33,266 \mathrm{~cm}$ (SD 1,85). Bei einem mittleren Gestationsalter von $38,68 \mathrm{SSW}$ mit einem Nabelschnur $\mathrm{pH}$ von 7,28. In unserer Population zeigte sich eine Frühgeburten - Rate von $14,3 \%(n=9)$ Das Geburtsgewicht Neugeborener $(p=0,036)$ als auch das Gestationsalter $(p=0,020)$ zeigte sich bei Neugeborenen von Müttern mit Beikonsum signifikant geringer. 98,4\% der Neugeborenen wiesen ein behandlungsbedürftiges NAS auf. Die mediane Dauer des Entzuges betrug 32 (4-251) Tage, die mediane Dauer des stationären Aufenthaltes der Neugeborenen betrug 42 (11-116) Tage. Die Buprenorphin-Gruppe unterschied sich signifikant in der Aufenthaltsdauer ( $p=0,019 ; 27$ versus 53 Tage), und der Entzugsdauer ( $p=0,030 ; 19$ versus 42 Tage) von der Gruppe mit retardierten Morphinen. Die Mütter sind bei Geburt des Kindes im Median 26 Jahre alt. Sie haben im Durchschnitt 1,47 lebende Kinder, bei einer Anzahl von 1,97 Schwangerschaften pro Frau, geboren. Jede 4. Frau hatte einen Abort, jede 5. Frau eine Interruptio. 82,7\% der Mütter waren zum Zeitpunkt der Geburt Hepatitis-C positiv. 78,7\% der Frauen konsumierten in der Schwangerschaft Opioide im Rahmen einer Erhaltungstherapie. Diese erfolgte in 70,5\% mit retardiertem Morphin, in 13,1\% der Population mit Methadon, und bei 8,2\% mit Buprenorphin. 19,6\% der Frauen konsumierten Heroin oder Opioide in illegaler Form. In der vorliegenden Population von Müttern wurde bei 63,5\% ein Beikonsum dokumentiert. 35,3\% der Väter wiesen keinerlei Drogenkonsum und keinen Kontakt zum Drogenmilieu auf, 23,5\% waren zum Zeitpunkt der Geburt des Kindes selbst substanzabhängig, 35,3\% waren zum Zeitpunkt der Geburt in Erhaltungstherapie

Schlussfolgerung: Schwangerschaften in dieser Population stellen sowohl klinisch als auch sozialmedizinisch, in der Behandlung der werdenden Mütter und der Neugeborenen, eine große Herausforderung dar. Die Ergebnisse dieser Studie zeigen die Notwendigkeit einer adäquaten prä-, peri-, und postpartalen Betreuung der Mütter, Kinder und auch die Einbeziehung der Väter durch ein multidisziplinäres Team.

\section{FV67}

Beurteilung der Neurologie von Neugeborenen mit Neonatalem Abstinenz Syndrom anhand der General Movements nach Prechtl

J. Pansy', J. Stöger', A. Scheuchenegger', B. Urlesberger', E. Ziehenberger', W. Raith ${ }^{1}$

'Universitätsklinik für Kinder- und Jugendheilkunde, Klinische Abteilung für Neonatologie, Medizinische Universität Graz, Graz, Österreich

Hintergrund: Maternaler Drogenkonsum während der Schwangerschaft ist häufig mit einer postnatalen Entzugssymptomatik des Neugeborenen (neonatales Abstinenzsyndrom, NAS) verbunden. Zusätzlich zeigen Studien der letzten Jahre eine Tendenz für kognitive und entwicklungsneurologische Einschränkungen von Neugeborenen nach intrauteriner Opioidexposition und NAS. Die Beurteilung der Spontanbewegungen - „General Movements (GMs)“ - nach Prechtl ist eine verlässliche Methode, um spätere neurologische Defizite bereits sehr früh zu erkennen. GMs um den Geburtstermin können normal, normal non-optimal, Poor Repertoire (PR), Cramped Synchronised oder Chaotic sein. Zusätzlich ist eine de- taillierte Analyse mit dem General Movement Optimality Score (GMOS, max. 42 Punkte) möglich. Um das 3. Lebensmonat (LM) werden die GMs als „Fidgety Movements“ (FMs) bezeichnet: Normal FMs, Abnormal FMs or Absent FMs und eine detaillierte Analyse ist mit dem Motor Optimality Score (MOS, max. 28 Punkte) möglich.

Fragestellung: Ziel der Studie war es, die Qualität der GMs von Neugeborenen nach intrauteriner Opioidexposition und mit NAS zur Erfassung des frühen neurologischen Outcomes global und detailliert zu beurteilen (DRKS-ID: DRKS00008306).

Methode: In dieser prospektiven klinischen Studie wurden alle Reifgeborene mit NAS von Juli 2015 bis Jänner 2016 eingeschlossen und ihre GMs in der 2. LW noch während des NAS global und detailliert (GMOS) analysiert. Im 3. LM erfolgte ebenfalls eine globale und detaillierte Analyse (MOS) ihrer GMs.

Ergebnisse: 14 Reifgeborene nach intrauteriner Opioidexposition und NAS (medianes Gestationsalter 39/2 (37/2-41/6), 7 weiblich) konnten eingeschlossen werden. In der 2. LW konnten 14 und im 3. LM 11 der eingeschlossenen Neugeborenen analysiert werden (3 Neugeborene lost to follow up). In der 2. LW zeigten 12 Neugeborene (86\%) PR GMs und 2 (14\%) normale, aber non-optimal GMs mit reduzierter und monotoner Sequenz $(n=11 / 78 \%)$, abnormer Amplitude $(n=11 / 78 \%)$, abnormer Geschwindigkeit ( $n=11 / 78 \%)$, limitiertem Ausnützen des Raumes $(n=12 / 86 \%)$, wenigen oder fehlenden Rotationen (proximal $n=11 / 78 \%$, distal 12/86\%), limitierten Fluktuationen von Beginn und Ende des Bewegungsablaufes $(11 / 78 \%)$ und einen Tremor $(12 / 86 \%)$. Der mediane GMOS betrug 25 (17-39,5). Im 3. LM hatte ein Kind (9\%) fehlende FMs (Absent FMs) und zeigte monotone, steife und langsame Bewegungen, eine abnorme Körperhaltung, ein reduziertes Repertoire co-existenter Bewegungen: MOS 11. Von 10 Kindern (91\%) mit normalen FMs bewegten sich 3 (27\%) Kinder sehr ruckartig und ein Kind (9\%) sehr monoton bei guter Qualität und normalem Repertoire co-existenter Bewegungen und normaler Körperhaltung: $\operatorname{MOS} 26(n=3 / 27 \%)$ und $27(n=1 / 9 \%)$. Sechs Kinder (55\%) bewegten sich völlig unauffällig: MOS 28. Der mediane MOS lag bei 28 (11-28).

Schlussfolgerung: Fast alle Neugeborene von Müttern mit Drogenkonsum zeigten während des NAS abnorme GMs (PR) und $45 \%$ zeigten Auffälligkeiten mit 3 Monaten. Für die entwicklungsneurologische Prognose der PR GMs ist eine Analyse der GMs mit 3 Monaten notwendig. Mit Hilfe wiederholter globaler und detaillierter GMs Analysen können jene Kinder mit anhaltend auffälligem Bewegungsmuster und einem zu erwartenden höheren Risiko für spätere neurologische Defizite frühzeitig identifiziert werden.

\section{FV68}

Neonataler Score zur Erfassung von Entzugs-Symptomen bei Neugeborenen nach mütterlichem Methamphetamin-Abusus

M. Schreiber, E. Robel-Tillig

Klinikum St. Georg gGmbH, Klinik für Neonatologie, Leipzig, Deutschland

Vor allem in Sachsen und Thüringen ist in den letzten Jahren ein sprunghafter Anstieg des Methamphetaminabusus auch bei Schwangeren zu verzeichnen. Charakteristisch ist das sehr hohe Abhängigkeitspotential insbesondere bei jungen Frauen. Eine steigende Zahl von Neugeborenen, die bereits intrauterin den negativen Effekten der synthetischen Droge ausgesetzt sind, ist die Folge. Aus der Literatur ist bekannt, dass die entstehenden Probleme für die Kinder gravierend sind.

Die üblichen Scores zur Erfassung von neonatalen Entzugssymptomen sind für die Beurteilung der Folgen der pränatalen Methamphetaminexposition nicht anwendbar. Ziel ist es daher, geeignete klinische Parameter zu finden, die Aussagen zum Risikoprofil der Neugeborenen zulassen und einen prädiktiven Wert besitzen. Der dargestellte Score wurde anhand der Daten der Literatur entwickelt und beinhaltet klinische, neurologische, dopplersonographische und echokardiographische Parameter. Seit 2014 erfassen wir alle in der Klinik geborenen Kinder und untersuchen sie mithilfe des Scores während der ersten 7 Lebenstage. Damit wird erstmals eine kontinuierliche und objektivierbare Erfassung der Symptome mög- 
lich. Die Ergebnisse werden mit den ambulanten Nachuntersuchungen während der ersten 3 Lebensjahre verglichen, um den prädiktiven Wert beurteilen zu können.

Der Score soll damit eine Möglichkeit vergleichbarer Daten schaffen und frühzeitig Hinweise auf körperliche, neurologische und psychische Folgeschäden aufzeigen.

\section{Freie Themen 10: Nachsorge}

\section{FV69 \\ Bei Neugeborenen und Säuglingen mit hereditärer Epidermolysis bullosa ist eine strukturierte, multiprofessionelle Betreuung unverzichtbar}

\section{A. Asgeirsson' ${ }^{1}$ F. Guthmann ${ }^{2}$, B. Ludwikowski ${ }^{3}$, H. Ott ${ }^{4}$ \\ ${ }^{1}$ Kinderkrankenhaus auf der Bult, Neonatologie, Hannover, Deutschland, ${ }^{2}$ Perinatalzentrum Hannover, Auf der Bult- Kinder- und Jugendkrankenhaus, Neonatologie, Hannover, Deutschland, ${ }^{3}$ Kinderkrankenhaus auf der Bult, Kinderchirurgie/Kinderurologie, Hannover, Deutschland, ${ }^{4}$ Kinderkrankenhaus auf der Bult, Fachbereich Pädiatrische Dermatologie und Allergologie, Hannover, Deutschland}

Hintergrund: Als molekulargenetisch und klinisch heterogene Gruppe kongenitaler, blasenbildender Erkrankungen wird die Epidermolysis bullosa hereditaria (EB) je nach Spaltbildungsebene in vier Haupttypen unterschieden. Diese gehen in unterschiedlicher Ausprägung und Häufigkeit mit zusätzlichen, auch extrakutanen Symptomen einher, die bereits im Neugeborenen- und Säuglingsalter beginnen können.

Fragestellung: Ziel dieser Untersuchung war es, bei Neugeborenen und Säuglingen mit EB sowohl kutane als auch extrakutane Komplikationen und den hieraus resultierenden Bedarf an Nachsorgemaßnahmen zu charakterisieren

Material und Methoden: In einer retrospektiven Analyse identifizierten wir alle Neugeborenen und Säuglinge mit EB, die während des 20-monatigen Studienzeitraums (07/2014 bis 02/2016) in unserem Epidermolysis bullosa-Zentrum betreut wurden. Durch Analyse elektronischer Krankenakten sowie der klinischen Fotodokumentation wurden neben demographischen Daten, dem EB-Subtyp und kutanen Symptomen zusätzlich mukosale und extrakutane Beschwerden erfasst. Außerdem wurden die eingesetzten Verbandsmaterialien sowie die analgetische Medikation dokumentiert.

Ergebnisse: Im Untersuchungszeitraum wurden von uns 10 Neugeborene und Säuglinge ( $n=5$ weiblich) mit EB behandelt, deren mittleres Alter bei Erstvorstellung 117 Tage betrug (Range 1-330 Tage). Bei vier Patienten lagen nicht-schwere EB-Subtypen vor: EB simplex (EBS, $n=2)$, autosomal-dominante EB dystrophica (DDEB, $n=2$ ). Fünf Kinder wiesen EB-Subtypen mit obligat letalem oder potenziell schwerwiegendem Verlauf auf: schwere, generalisierte EB junctionalis vom Typ Herlitz (JEBGS; $n=2$ ), schwere, generalisierte, autosomal-rezessive EB dystrophica (RDEB-GS, $n=2)$, JEB non-Herlitz $(n=1)$. Bei einer Patientin war eine sichere Zuordnung des EB-Subtyps nicht möglich. Alle Patienten wiesen eine krankheitstypische Blasenbildung auf, die sich in der Mehrzahl akral betont zeigte, jedoch bei Neugeborenen mit JEB-GS $(n=2)$, RDEBGS $(n=1)$ und EBS $(n=1)$ generalisiert auftrat. Das Wundmanagement erfolgte bei allen Kindern mit einer primären Wundauflage, Polyurethan-Schaumverbänden, elastischen Schlauchverbänden und ggf. topischen Antiseptika. Aufgrund enoraler und/oder perioraler Läsionen litten drei Patienten mit schwerer Verlaufsform unter einer Trinkschwäche. Bei vier Kindern wurde in der Neugeborenen- und frühen Säuglingsperiode eine Analgesie mit oraler Glukose-Lösung, einem nicht-steroidalen Antiphlogistikum und Morphin erforderlich. Zwei Patientinnen mit JEB-GS benötigten eine intensive, palliativmedizinische Betreuung und verstarben bei schwerer Gedeihstörung noch im frühen Säuglingsalter.

Schlussfolgerung: Unabhängig vom Erkrankungs-Subtyp treten bei Neugeborenen und Säuglingen mit EB potenziell schwerwiegende Komplikationen auf. Eine multiprofessionelle Betreuung durch ärztliches, pflegeri- sches und psychologisch-pädagogisches Fachpersonal ist daher in dieser Patientengruppe unverzichtbar.

\section{FV70}

\section{Aachener Nachsorge-Modell: Frühzeitige Anbindung, langfristige Begleitung}

S. Trepels-Kottek', A. Schoberer' ${ }^{2}$ K. Hoberg², M. Schoberer' ${ }^{1}$ M. Häusler², T. Orlikowsky ${ }^{1}$

'Uniklink RWTH Aachen, Kinderklinik Sektion Neonatologie und Kinderintensivmedizin, Aachen, Deutschland, ${ }^{2}$ Universitätsklinikum Aachen, SPZ der Klinik für Kinder- und Jugendmedizin, Aachen, Deutschland

Hintergrund: Für Level 1 Neonatologien ist die Nachuntersuchung mit entwicklungsneurologischer Testung für Kinder unter 32 Schwangerschaftswochen $/<1500 \mathrm{~g}$ Geburtsgewicht mit 24 Monaten verpflichtend.

Ziel: Unser Ziel war es, diesen Frühgeborenen und ihren Familien zusätzlich zum Besuch beim Kinderarzt auch im Zeitraum zuvor ein strukturiertes Nachsorgeangebot zu geben, welches vor der Entlassung individuell mit einem Unterstützungsplan erarbeitet und durch unser Case Management koordiniert wird. Physiotherapeutische Übungsbehandlungen, Angebote durch den „Bunten Kreis“, „Frühe Hilfen“, SPFH oder in speziellen Fällen dem Jugendamt werden initiiert. Seit 2010 finden Vorstellungen der Familien im korrigierten Alter von 3 und 12 Monaten in der SPZ-Nachsorgeambulanz („Neoambulanz"), der Neonatologie angeschlossen, statt. Methodik: Neben psychomotorischer Entwicklung (MÜFED), körperlicher Untersuchung, Blutbild und Sonographie erfolgt eine ausführliche Ernährungs- und Impfanamnese.

Ergebnisse: 2015 waren zu den Nachsorgeterminen 87 Kinder mit 3 Monaten eingeladen, davon kamen alle, zu den Nachsorgeterminen nach 12 Monaten noch $91 \%$ erschienen. Mit 3 Monaten kommen bei ca. 75\% der Kinder beide Elternteile zum Termin, mit 12 Monaten in etwa 50\%. Bei $90 \%$ wurden zusätzliche Hilfsmaßnahmen verordnet, die nur in Einzelfällen nicht angenommen wurden. In $60 \%$ wird die bis dahin oft noch nicht begonnene Beikosternährung ausführlich besprochen. In $30 \%$ gab es Ernährungskonzepte, die nichtaltersentsprechend waren (Fruchtzwergfütterung mit 3 Monaten, oder überwiegend flaschenbasierte Ernährung mit 12 Monaten). Bei dem 3-Monats-Termin sehen wir in $50 \%$ leichte und in $8 \%$ schwere Entwicklungsverzögerungen, denen mit Elternanleitung und Intensivierung der Therapien entgegengewirkt wird. Im Kollektiv der 12 Monatskinder sehen wir nur noch in ca. 30\% leichte und in 5\% schwere Verzögerungen. Der Impfstatus war trotz der konsequent in der Klinik begonnen Impfungen mit 14-16 Monaten in $90 \%$ nicht den STIKO Empfehlungen entsprechend. Dies wird aktuell mit dem Impfstatus von Reifgeborenen verglichen. Häufige Auffälligkeiten sind erweiterte äußere Liquorräume bis zu $1 \mathrm{~cm}$ bei $23 \%$ der ehemaligen Frühgeborenen, die aber keinen signifikanten Einfluss auf die Entwicklung hatten. Es fanden sich bei ca. $10 \%$ milde Nephrokalzinosen, die aber nicht behandlungsbedürftig waren. In 3 Fällen benötigten Eltern, meist die Mütter, weitere psychologische Unterstützung, wobei die Kontaktanbahnung zu Therapeuten unterstützt wird.

Fazit: Durch intensive Anbindung der Frühgeborenen über die Entlassung hinaus können Probleme frühzeitig erkannt werden. Die Eltern schätzen die Nachsorge durch das ihnen bereits vertraute Team, so dass auch die Termine mit 2 und 5 Jahren sehr häufig wahrgenommen werden.

\section{FV71}

Zur Praktikabilität der Klassifikation der General Movements nach Prechtl in der neonatologischen Nachsorge

P. Hinner', S. Ifflaender' ${ }^{1}$, B. Eger' ${ }^{1}$, B. Seipolt' ${ }^{1}$, J. Reichert ${ }^{1}$, M. Rüdiger ${ }^{1}$ 'Universitätsklinikum Carl Gustav Carus Kinderklinik, Neonatologie/ Pädiatrische Intensivmedizin, Dresden, Deutschland

Hintergrund: Die Beobachtung der Langzeitentwicklung in der neonatologischen Nachsorge dient auch dem rechtzeitigen Erkennen von Entwicklungsproblemen Frühgeborener und kranker Neugeborener. Der 
prädiktive Wert der eingesetzten Untersuchungsinstrumente erlaubt es, nachfolgende - und ggf. ressourcenintensivere - Diagnostik und Interventionsmaßnahmen an die individuellen Bedarfe der Kinder anzupassen. So kann mit der Untersuchung der General Movements nach Prechtl innerhalb der ersten Lebensmonate das Entwicklungsrisiko für eine Zerebralparese mit hoher Sensitivität (98\%; $95 \%$-KI 74-100\%) als auch Spezifität (91\%; 95\%-KI 83-93\%) eingeschätzt werden [1]. Aktuel wird die Praktikabilität der Methode als Verlaufsuntersuchung im Rahmen der neonatologischen Nachsorge am Universitätsklinikum Dresden geprüft.

Fragestellung: Ist die Diagnostik der General Movements nach Prechtl ein praktikables Screening-Verfahren für die neonatologische Nachsorge Früh- und kranker Neugeborener?

Material und Methode: Die Diagnostik wird bei Frühgeborenen mit einem Geburtsgewicht $<1500 \mathrm{~g}$ und/oder einem Gestationsalter $<32$ SSW sowie kranken Neugeborenen nach perinataler Asphyxie im Zusammenhang mit einem ph-Wert $<7,0$ und Hypothermie-Behandlung angewandt. Dabei werden die spontanmotorischen Bewegungen im späten Frühgeborenenalter $(\mathrm{PMA}=34+0 \mathrm{SSW}$ bis $36+6 \mathrm{SSW})$, im Reifealter $(\mathrm{PMA}=37+0$ SSW bis $42+0$ SSW) und mit korrigiert 3 Lebensmonaten videographiert. Die erstellten Videoaufnahmen der kindlichen Bewegungsmuster werden entsprechend der Klassifikation der General Movements nach Prechtl beurteilt.

Ergebnisse: In einem ersten Erhebungszeitraum von Juli bis Dezember 2015 wurde das Verfahren zunächst im ambulanten Setting eingesetzt; es wurden die General Movements für 73 Kinder im Alter von korrigiert 3 Monaten erfasst. Es ergaben sich bei $12 \%$ der Kinder auffällige Bewegungsmuster im Sinne sporadischer Fidgety Movements. Für den zweiten Erhebungszeitraum Januar bis Mai 2016 werden die spontanmotorischen Bewegungsmuster zu drei Erhebungszeitpunkten für Frühgeborene und kranke Neugeborene untersucht und in ihrer Entwicklung analysiert. Besonderes Interesse gilt dabei der Stabilität normaler und abnormaler Bewegungsmuster über die Zeit. Desweiteren werden zeitlicher, technischer und personaler Aufwand für die Umsetzung des Erhebungsverfahrens erhoben.

Diskussion: Im Vordergrund der aktuellen Studie steht die Praktikabilität des Verfahrens im Rahmen der stationären Behandlung und Nachsorge. In Abhängigkeit von den Ergebnissen ist die Formulierung eines Erhebungsstandards geplant, welcher weiteren Anwendern zugänglich gemacht werden kann. In einem weiteren Schritt sollen die Ergebnisse der Genera Movements-Untersuchung mit dem neuromotorischen Entwicklungsoutcome der Kinder in Bezug gesetzt werden.

\section{Literatur}

1. Bosanquet et al. Dev Med Child Neurol 2013;55:418-426

\section{FV72}

Gesundheitsbezogene Lebensqualität sehr kleiner Frühgeborener vom Jugend- bis zum Erwachsenenalter

\section{N. Baumann', P. Bartmann'2, D. Wolke ${ }^{3}$}

'University of Warwick, Department of Psychology, Coventry, United Kingdom, ${ }^{2}$ Universitätsklinikum Bonn, Neonatologie, Bonn, Deutschland, ${ }^{3}$ University of Warwick, Department of Psychology and Division of Mental Health and Wellbeing, Warwick Medical School, Coventry, United Kingdom

Hintergrund: Diese Studie befasst sich mit Veränderungen der gesundheitsbezogenen Lebensqualität bei Frühgeborenen $(<32$ Gestationswochen und/oder $<1500 \mathrm{~g}$ Geburtsgewicht) vom Jugend- bis zum Erwachsenenalter.

Fragestellung: Wird gesundheitsbezogene Lebensqualität von den Patienten und ihren Eltern ähnlich wahrgenommen und gibt es einen Zusammenhang zwischen gesundheitsbezogener Lebensqualität und ökonomischem sowie sozialem Status?

Material und Methoden: In einer prospektiven populationsbasierten Langzeitstudie (Bayerische Entwicklungsstudie, Beginn 1985) wurden $260 \mathrm{sehr}$ kleine Frühgeborene und 229 Termingeborene als Kontrollen von Geburt bis zum Erwachsenenalter verfolgt. Eine Beurteilung der gesundheitsbezo- genen Lebensqualität im Alter von 13 und 26 Jahren erfolgte mit Hilfe von Selbst- und Elternberichten mit dem Health Utility Index Mark 3 (HUI3). Ökonomische und soziale Parameter im Alter von 26 Jahren wurden mit Interviews sowie standardisierten Fragebögen evaluiert.

Ergebnisse: Zu beiden Erhebungszeitpunkten wurde, von den Teilnehmer selbst und deren Eltern, ein niedrigerer HUI3 Wert für die Frühgeborenen im Vergleich zu den Kontrollen berichtet. Mit Ausnahme der Selbstberichte von den Teilnehmern im Jugendalter $(p=0,13)$ waren alle anderen vergleichenden Unterschiede signifikant $(p<0,05)$. Im Gegensatz zu den Frühgeborenen selbst, berichteten ihre Eltern von einer Verschlechterung der gesundheitsbezogenen Lebensqualität ihrer Kinder (Veränderung des HUI3 Mittelwerts: 0,88 zu 0,86, $p=0,03$ ). Sie stellten insbesondere negative Veränderungen bezüglich emotionaler Parameter und Schmerzen bei den Frühgeborenen im Laufe der Zeit fest. Aus der Sicht der Teilnehmer als auch deren Eltern konnte ein negativer Zusammenhang zwischen gesundheitsbezogener Lebensqualität und ökonomischen und sozialen Parametern, wie der Bezug von Arbeitslosengeld II, Arbeitslosigkeit, sowie geringere romantische und Freundschaftsbeziehungen, nachgewiesen werden.

Schlussfolgerung: Im Vergleich zu Kontrollen wird gesundheitsbezogene Lebensqualität von Frühgeborenen als auch von deren Eltern niedriger als im Vergleich zu Reifgeborenen wahrgenommen. Es besteht ein Zusammenhang zwischen niedriger gesundheitsbezogener Lebensqualität und ökonomischen und sozialen Problemen im Erwachsenenalter. Eine Verbesserung der gesundheitsbezogenen Lebensqualität bis zum Erwachsenalter konnte für sehr kleine Frühgeborene in dieser populationsbasierten bayerischen Längsschnittstudie nicht nachgewiesen werden.

\section{FV73}

\section{Langzeitoutcome nach niedriggradiger intraventrikulärer Blutung: Vorsicht bei der Vorhersage des Outcomes!}

\section{S. Pfahl', K. Lohmeier' ${ }^{2}$ R. Richter-Werkle², T. Höhn ${ }^{3}$, H. Sabir}

'Med. Einricht. d. Universität Kinderklinik, Pädiatrische Intensivstation/ Neonatologie, Düsseldorf, Deutschland, ${ }^{2}$ Med. Einricht. d. Universität Kinderklinik, Düsseldorf, Deutschland, ${ }^{3}$ Med. Einricht. d. Universität Kinderklinik, Allgemeine Pädiatrie, Düsseldorf, Deutschland, ${ }^{4}$ Med. Einricht. d. Universität Kinderklinik, Neonatologie und pädiatrische Intensivmedizin, Düsseldorf, Deutschland

Hintergrund: Die intraventrikuläre Blutung (IVH) ist eine der gefürchtetsten neurologischen Komplikationen bei Frühgeborenen. Das Überleben ohne neurologische Beeinträchtigung wird hierbei mit der Gradeinteilung der IVH nach Papile in Korrelation gebracht. Danach gelten die IVH 1. und 2. Grades als geringer Risikofaktor für eine neurologische Beeinträchtigung mit 24 Monaten. Es gibt jedoch zunehmend Zweifel daran. Das Ziel dieser Analyse war zu untersuchen, welches Langzeitoutcome Frühgeborenen mit IVH 1. oder 2. Grades haben und welche Risikofaktoren sich für eine IVH detektieren lassen.

Methode: Dies ist eine Retrospektivanalyse aller Frühgeborener, die zwischen 2005-2013 bei uns behandelt wurden und bei denen eine IVH 1 . oder 2.Grades (ein- oder beidseitig) detektiert wurde. Alle Kinder wurden im Alter von 20-24 Monaten kognitiv (MDI) und motorisch (PDI) nachuntersucht (Bayley II). Eine Regressionsanalyse wurde durchgeführt mit IVH Grad, MDI und PDI als abhängige Variablen.

Ergebnisse: Im Untersuchungszeitraum wurden die Daten von Kindern zwischen der $23+5$ und $32+3$ (Median 26+4) Schwangerschaftswoche erhoben. Insgesamt wurden 52 Kinder ausgewertet, die eine IVH 1. $(n=33)$ oder 2. Grades hatten. Der Median MDI war 96 und PDI 94. In der Regressionsanalyse zeigten sich der IVH Grad $(p=0,030)$, das Gestationsalter $(p=0,010)$ und die nicht antenatal verabreichten Steroide $(p=0,036)$ als signifikante unabhängige Risikofaktoren für ein schlechteres motorisches Outcome. Insgesamt hatten 17 Kinder (32\%) einen MDI $<1$ Standardabweichung (SD, MDI <85). Von diesen hatten 9 eine IVH 1 . Grades. 16 Kinder $(30 \%)$ hatten einen PDI $<1 \mathrm{SD}(\mathrm{PDI}<85)$. Von diesen hatten 6 eine IVH 1.Grades. Ein MDI/PDI <2SD $(<70)$ fand sich nur bei 3 Kindern. In der Regressionsanalyse zeigten sich bei diesem kleinen sub-Kollektiv 
keine zusätzlich signifikanten Risikofaktoren. Eine IVH 1. Grades war in unserem Kollektiv nicht immer mit einem guten Outcome verbunden. Schlussfolgerung: Bei der Interpretation und der Outcomeprognose nach IVH 1. oder 2. Grades ist Vorsicht geboten, da sie nicht immer mit einem guten Outcome verbunden sind.

\section{FV74}

\section{Ergebnisse der Geburtskohortenstudie EcoCare-PIn II. Ambulante Leistungen}

D. Druschke', M. Scheibe', K. Arnold' ', L. Heinrich', V. Stephan', J. Reichert ${ }^{2}$, J. Schmitt', M. Rüdiger ${ }^{2}$

'Universitätsklinikum Carl Gustav Carus an der Technischen Universität Dresden, Zentrum für Evidenzbasierte Gesundheitsversorgung, Dresden, Deutschland, ${ }^{2}$ Universitätsklinikum Carl Gustav Carus, Klinik und Poliklinik für Kinder- und Jugendmedizin, Dresden, Deutschland

Hintergrund: Neugeborene mit einem Geburtsgewicht unter 1500 g haben ein erhöhtes Morbiditätsrisiko insbesondere bis zum Schuleintrittsalter allerdings fehlen bisher epidemiologische Versorgungsdaten unter Beachtung ambulanter Leistungen. Die BMBF-geförderte Geburtskohortenstudie EcoCare-PIn (Early comprehensive Care of Preterm Infants - effects on quality of life, childhood development, and healthcare utilization; Förderkennzeichen: 01GY1323) gestattet die Analyse von Morbiditäts- und Inanspruchnahmemustern Frühgeborener in Sachsen mit Hilfe von Routinedaten einer Krankenkasse.

Fragestellung: Unterscheidet sich die Inanspruchnahme ambulanter Versorgungsleistungen in Abhängigkeit vom Geburtsgewicht und lassen sich regionale Unterschiede zwischen Stadt und Land finden?

Methoden: Analysiert wurden Routinedaten (ambulante Leistungen) von durchgängig versicherten Kindern, die in den Jahren 2007 bis 2008 in Sachsen geboren wurden und 2009 bis 2013 beobachtet werden konnten Verglichen wurden Kinder mit einem Geburtsgewicht $<1500 \mathrm{~g}$ (very low birthweight infants - VLBW), 1500 bis unter $2500 \mathrm{~g}$ (low birthweight LBW) und $>=2500 \mathrm{~g}$ (normal birthweight - NBW).

Ergebnisse: Analysiert wurden die Daten von 264 VLBW-, 1396 LBWund 25.007 NBW-Kindern. Das entspricht 39\% der im Analysezeitraum in Sachsen geborener Kinder. In dem untersuchten Zeitraum zeigten VLBW-Kinder die höchste Inanspruchnahme ambulanter Leistungen; der Median für die Anzahl der Abrechnungsscheine lag bei 39 (IQR 20) für VLBW-, bei 31 (IQR 18) für LBW- und bei 29 (IQR 16) für NBW-Kindern. Die ambulante Versorgung von VLBW Kindern mit geringer, durchschnittlicher bzw. häufiger Inanspruchnahme ambulanter Leistungen erfolgt im Median zu 88 \%, 79\% bzw. 63 \% durch Pädiater. Bei VLBW-Kindern mit häufiger Inanspruchnahme ambulanter Versorgung werden im ländlichen Raum mehr Erwachsenenhausärzte und -fachärzte zu Rate gezogen als im städtischen Raum mit gleichzeitig geringeren durch Pädiater erbrachten Versorgungsanteilen (61 vs. $70 \%$ ).

Diskussion: Eine zu frühe Geburt geht nicht nur mit einer erhöhten Notwendigkeit einer stationären Behandlung einher; vielmehr findet sich auch ein erhöhter Bedarf an ambulanter Behandlung. Dabei gilt, dass je größer das biologische Risiko des zu behandelnden Kindes - dargestellt über das Geburtsgewicht - ist, desto öfter werden ambulante Versorgungsleistungen in Anspruch genommen. Unterschiede in Abhängigkeit von der Versorgungsregion (Stadt vs. Land) bedürfen einer weiterführenden Analyse zur Prüfung der bedarfsgerechten Versorgungsqualität und zur Identifizierung von relevanten Effizienzreserven.

\section{FV75}

Aachener Nachsorge-Modell: Kognitive Entwicklung früher Frühgeborener bis zum fünften Lebensjahr im Längsschnitt

K. Hoberg' ', A. Schoberer', S. Trepels-Kottek², M. Häusler', T. Orlikowsky ${ }^{3}$ 'Universitätsklinikum Aachen, Klinik für Kinder- und Jugendmedizin, Sektion Neuropädiatrie und Sozialpädiatrie, Aachen, Deutschland, ${ }^{2}$ Universitätsklinikum Aachen, Klinik für Kinder- und Jugendmedizin, Sektion Neonatologie und Kinderintensivmedizin, Aachen, Deutschland, ${ }^{3}$ Universitätsklinikum Aachen, Klinik für Kinder- und Jugendmedizin, Sektion für Neonatologie, Aachen, Deutschland

1. Hintergrund und Fragestellung: Definierte Verzögerungen Frühgeborener in verschiedenen Entwicklungsdimensionen sind vielfältig untersucht und liegen im kognitiven Bereich eine Standardabweichung unter der Referenzpopulation. Ziel der vorliegenden Arbeit ist die Analyse und Vorhersage von kognitiven Outcomefaktoren längsschnittlich über mehrere Untersuchungszeitpunkte.

2. Methode: Wir untersuchten longitudinal die kognitive Entwicklung von $n=92$ ehemaligen Frühgeborenen (GG 1136 g; 29 SSW) der Geburtsjahrgänge 2007-2009 zu vier Zeitpunkten: $t_{1}$ : korrigiert 3-6 Monate, $t_{2}$ : korrigiert 12 Monate: Münchner funktionelle Entwicklungsdiagnostik; $t_{3}$ : korrigiert 24 Monate: Bayley II; $\mathrm{t}_{4}$ : 5-6 Jahre, vor Einschulung: K-ABC.

3. Ergebnisse: Frühgeborene wiesen $\mathrm{zu} \mathrm{t}_{1}, \mathrm{t}_{2}$ und $\mathrm{t}_{4}$ durchschnittliche kognitive Werte auf $\left(\mathrm{IQt}_{1}=100, \mathrm{IQt}_{2}=102, \mathrm{IQt}_{4}=96\right)$. $\mathrm{Zu} \mathrm{t}_{3}$ erreichten sie hingegen unterdurchschnittliche Werte $\left(\mathrm{IQt}_{3}=82\right)$. Damit zeigten sich auch statistisch bedeutsame Unterschiede im Messwiederholungsdesign und im paarweisen Mittelwertvergleich ( $p$ jeweils $<0,01$ ). Im Alter von zwei und fünf Jahren schnitten extrem Frühgeborene (GG $<1500$ g oder SSW <28) signifikant schlechter ab $(p<0,05)$. Der Zusammenhang mit dem IQ zu $\mathrm{t}_{4}$ stieg von $\mathrm{rt}_{1}=.1$ über $\mathrm{rt}_{2}=.5 \mathrm{zu} \mathrm{rt}_{3}=.6$. Regressionsanalytisch konnte die kognitive Entwicklung $\mathrm{zu} \mathrm{t}_{4}$ durch den kognitiven Outcome $\mathrm{zu} \mathrm{t}_{3}$ mittels Bayley II und das Geburtsgewicht vorhergesagt werden. Diese erklärten zusammen $41 \%$ der Varianz. Bei Einteilung in kognitive Leistungsgruppen blieben nur $50 \%$ der Frühgeborenen stabil in ihren Quartilen. Allerdings erreichten die Kinder, die $\mathrm{zu} \mathrm{t}_{3}$ im obersten Quartil lagen, auch zu $\mathrm{t}_{4}$ einen gut durchschnittlichen IQ, diese Vorhersagegüte sank kontinuierlich bis auf eine Differenz von 25 IQ-Punkten im ersten Quartil.

4. Schlussfolgerung und Diskussion: Im Longitudinalvergleich lag die kognitive Entwicklung der Frühgeborenen mit 24 Monaten im unterdurchschnittlichen und im Alter von fünf Jahren im knapp durchschnittlichen Bereich (vgl. Referenzpopulation). Eine Erklärung könnte der sogenannte Flynneffekt liefern, was der ausstehende Vergleich mit der gleichaltrigen Kontrollstichprobe klären kann. Möglicherweise nähern sich die Kinder aber auch bereits mit fünf Jahren ihrem genetischen kognitiven Potential an, so hatten diejenigen, die keine Therapien erhielten, bedeutsam höhere IQ-Werte. Die vorschulische kognitive Entwicklung wurde am besten durch die kognitive Entwicklung im Alter von zwei Jahren und das Gewicht bei Geburt vorhergesagt. Die höhere Risikobelastung durch andere perinatale und psychosoziale Parameter sowie auch protektive Maßnahmen blieb ohne Einfluss. Die Prognose war schwieriger, je schlechter die Kinder initial waren; die Chance aufzuholen war zwar größer, aber sie blieben unter dem Durchschnitt. Die Ergebnisse können zur Vertiefung der Diskussion um den spezifischen diagnostischen und beraterischen Nutzen einzelner Entwicklungsvariablen im Rahmen der Nachsorgekonzepte dienen. 


\section{Poster}

\section{Postersitzung 02: Atmung/Beatmung}

\section{P001 \\ Das neue synthetische Surfactant CHF5633 supprimiert die LPS- und Ureaplasmen-induzierte TNF- $\alpha$ und IL-1 $\beta$ Antwort neonataler und adulter Monozyten}

\section{K. Glaser ', M. Fehrholz' , S. Kunzmann', C. Speer \\ 'Universitäts-Kinderklinik Würzburg, Würzburg, Deutschland}

Hintergrund: Synthetische Surfactant-Präparate der neuen Generation stellen eine vielversprechende Alternative zu natürlichen Surfactant-Präparationen für die Therapie des Atemnotsyndroms Frühgeborener dar. CHF5633 ist ein synthetisches Surfactant-Präparat, das synthetische Analoga der Surfactant-Proteine B und C sowie zwei synthetische Phospholipide enthält. Seine biophysikalische Wirksamkeit konnte in vitro und in vivo bestätigt, eine Klinische Phase I-Studie erfolgreich abgeschlossen werden. Während für zahlreiche Surfactant-Präparate immunmodulatorische und antiinflammatorische Eigenschaften beschrieben wurden, liegen für CHF5633 noch keine Daten vor. Das Atemnotsyndrom ist neben Surfactantmangel mit Alveolarkollaps und pulmonaler Minderbelüftung durch eine Inflammationsreaktion unterschiedlicher Ausprägung charakterisiert. Relevante infektiöse Auslöser dieser frühen pulmonalen Inflammation sind sowohl Gram-negative Erreger als auch Ureaplasmen.

Fragestellung: Ziel der vorliegenden Studie war es, mögliche pro- und anti-inflammatorische Effekte von CHF5633 auf native und LPS- bzw. Ureaplasmen-stimulierte neonatale und adulte Monozyten zu untersuchen.

Material und Methoden: Positiv selektionierte neonatale und adulte CD14+ Monozyten, nativ, LPS- und Ureaplasma parvum-stimuliert, wurden gegenüber CHF5633 (100 $\mu \mathrm{g} / \mathrm{ml})$ exponiert. Die Viabilität der Zellen wurde durchflusszytometrisch analysiert, die Expression von TNF- $\alpha$, IL$1 \beta$, IL- 8 und IL-10 sowie TLR2 and TLR4 auf mRNA- und Proteinebene mittels quantitativer Real-time PCR and Durchflusszytometrie bestimmt. Ergebnisse: CHF5633 induzierte weder in nativen noch in LPS- oder U. parvum-stimulierten neonatalen und adulten Monozyten eine Apoptose. Sowohl in neonatalen als auch in adulten CD14+-Zellen verstärkte CHF5633 weder die LPS- noch die Ureaplasmen-induzierte pro-inflammatorische Zytokinantwort. Exposition von adulten CD14+ Zellen gegenüber CHF5633 reduzierte signifikant die LPS-und U. parvum-induzierte TNF- $a$ mRNA-Expression und die Ureaplasmen-induzierte TNF- $a$ und IL-1b Proteinsynthese. In neonatalen Monozyten führte die Inkubation mit CHF5633 zu einer signifikanten Suppression der LPS-induzierten TNF- $\alpha$ Proteinsynthese und zu ähnlichen Effekten auf die Ureaplasmen-induzierte TNF- $\alpha$ und IL- $1 \beta$ Protein-Expression.

Schlussfolgerung: CHF5633 zeigte keine ungewollten pro-apoptotischen und pro-inflammatorischen Effekte sowohl in nativen als auch in LPS- und U. parvum-stimulierten neonatalen and adulten Monozyten. Sowohl in neonatalen als auch in adulten Monozyten konnte eine signifikante Suppression der LPS-induzierten TNF-a und der Ureaplasmen-induzierten TNF- $\alpha$ und IL- $1 \beta$ Zytokinantwort beobachtet werden. Diese Daten sprechen für eine Stimulus-unabhängige, anti-inflammatorische Wirkung von CHF5633.

\section{P002}

\section{Legionella pneumophila Pneumonie eines 7 Wochen alten Säuglings}

F. Menzel', L. Schulze', A. Wroblewski', B. Schmidt

'St. Joseph-Krankenhaus Berlin Tempelhof, Neonatologie und pädiatrische Intensivmedizin, Berlin, Deutschland

Einleitung. Trotz niedriger Fallzahlen von Legionellosen in der Pädiatrie mit einer Inzidenz von $<0,3 / 100.000$ bei unter 15 jährigen Kindern in Deutschland im Jahr 2014 [1] bzw. 39 weltweit publizierten Legionärskrankheiten im Alter <2 Jahre bis 2006 [2] sind diese atypischen Erreger differentialdiagnostisch bei Infektionen vor allem im Neugeborenen- und Säuglingsalter in Betracht zu ziehen. In der jüngeren Literatur finden sich fünf Fälle neonataler Legionellosen assoziiert mit Wassergeburten [3-7] davon zwei Fälle seit 2014 [3, 7]. In den Fallbeschreibungen finden sich vergleichbare Organmanifestationen mit ähnlich schweren Verläufen, teils letalem Ausgang [3, 7].

Fallbericht. Wir berichten den Fall eines sieben Wochen alten Säuglings mit seit 3 Tagen bestehendem Luftwegsinfekt. Geboren wurde das Kind nach 39+4 SSW per Wassergeburt in einem Berliner Geburtshaus und zeigte bis zur 7. Lebenswoche einen unauffälligen postnatalen Verlauf. Bei Aufnahme präsentierte sich der Säugling mit febrilen Temperaturen bei ansonsten stabilem Allgemeinzustand und Vitalparametern. Nach Durchführung der Sepsisdiagnostik begannen wir bei massiv erhöhten Entzündungsparametern und bisher unklarem Fokus eine kalkulierte intravenöse antibiotische Therapie mit Ampicillin/Sulbactam und Gentamicin. Bei zunehmender klinischer und paraklinischer Verschlechterung sowie unauffälligen Liquor- u. Blutkulturbefunden erfolgte ein Wechsel des Antibiotikaregimes am 7. stationären Tag auf Vancomycin und Meropenem. Im Röntgenbild sowie sonographisch zeigte sich eine flaue Zeichnungsvermehrung im rechten Unterlappen, welche differentialdiagnostisch an eine infizierte CPAM (congenital pulmonary airway malformation) denken ließ. Zur weiteren Abklärung erfolgte eine Computertomographie des Thorax, welche eine einschmelzende Unterlappenpneumonie rechts mit bilateral disseminierten septischen Streuherden zeigte. Trotz der fulminanten paraklinischen Befunde war eine maschinelle Beatmung nie notwendig, lediglich HHFNC (humidified high flow nasal cannula) wurde für einige Tage benötigt. Nach Eingang des hoch positiven Befundes für Legionella-pneumophila SG-1 Antigens im Urin am 9. Tag erfolgte die leitliniengerechte Umstellung auf eine Monotherapie mit Clarithromycin. Hierunter trat eine rasche klinische Besserung des Allgemeinzustandes ein, welche mit dem Rückgang der Infektparameter korrelierte. Nach Entlassung am 17. Tag kam es vier Tage später zu einer erneuten stationären Aufnahme aufgrund erneuter Leukozytose, welche bei einer ambulanten Kontrolle auffiel. Auch hierbei sahen wir eine Diskrepanz zwischen der abermals stark entzündlich veränderten Paraklinik und dem stabilen Allgemeinzustand des Säuglings. Aufgrund Exazerbation und sonographisch-radiologisch bestätigter zunehmender Abszedierung rechts pulmonal erweiterten wir die mit Clarithromycin bis dato fortgeführte Monotherapie um die Gabe von Ceftazidim und Piperacillin/Tazobactam. Hierunter kam es schließlich zu einem Rückgang der Entzündungswerte mit Besserung der Symptomatik. Der Nachweis einer Serokonversion steht derzeit noch aus, ebenso die bildgebenden Follow-Up Untersuchungen des pulmonalen Lokalbefundes. Bei der Kindsmutter wurde keine Legionelleninfektion festgestellt. Eine Meldung über den positiven Legionellenbefund an das zuständige Gesundheitsamt ist erfolgt; die mikrobiologische Umfeldanalyse steht bis dato noch aus.

\section{P003}

Posteriore Chordotomie bei beidseitiger Stimmbandparese eine Alternative zur Tracheototomie für einen bessere Balance zwischen gutem Atemweg und Stimmqualität?

\section{K. Ruf', S. Hackenberg' ${ }^{2}$, S. Kunzmann'}

'Universitätsklinikum Würzburg, Universitäts-Kinderklinik Würzburg, Würzburg, Deutschland, ${ }^{2}$ Universitätsklinikum Würzburg, Klinik und Poliklinik für Hals-, Nasen- und Ohrenkrankheiten, plastische und ästhetische Operationen, Würzburg, Deutschland

Hintergrund: Starke Atemnot mit inspiratorischem Stridor als lebensbedrohliche Situation im Kreissaal ist stets eine Herausforderung für den Neonatologen. Neben einer Laryngomalazie, Larynxspalten, subglottischen Hämangiomen sowie aberrierenden Gefäßen stellt die angeborene Stimmbandparese eine weitere wichtige Differentialdiagnose dar.

Falldarstellung: Ein reifes Neugeborenes $(40+1$ SSW) fiel direkt postnatal mit ausgeprägtem inspiratorischem Stridor und Dyspnoe auf. Bei zuneh- 
mender Hyperkapnie und Hypoxämie erfolgte eine nasotracheale Intubation im Alter von 30 Minuten. Das Neugeborene war unter milden Beatmungsparametern kardiorespiratorisch stabil, eine flexible Laryngoskope nach Extubation zeigte eine beidseitige Stimmbandparese, aber keine weitere Fehlbildungen. Unter Spontanatmung weiterhin deutliche Dyspnoe, so dass der Patient rezidivierend intubiert sowie über eine nasogastrale Sonde ernährt werden musste. Letztlich erfolgte im Alter von 11 Wochen eine laserchirurgische posteriore Chordotomie als Therapieversuch. Direkt postoperativ bestand die Notwendigkeit einer CPAP-Atemhilfe, 3 Wochen postoperativ war der Patient jedoch in Ruhe vollkommen beschwerdefrei, lediglich bei Aufregung war ein inspiratorischer Stridor hörbar, bei jedoch stets stabiler Sauerstoffsättigung. Der Patient konnte im Alter von 14 Wochen ohne Atemhilfe sowie ohne Magensonde entlassen werden. In der Nachbeobachtung des jetzt 8 Monate alten Patienten bislang problemloser Verlauf mit regelrechter Gewichtszunahme; erste Atemwegsinfektionen wurden problemlos ohne die Notwendigkeit eines Klinikaufenthaltes überstanden, auch die Phonation ist, soweit bei einem 8 Monate alten Säugling beurteilbar, unauffällig.

Diskussion: Die posteriore Chordotomie als therapeutische Option einer Stimmbandparese im Neugeborenenalter ist bislang kaum in der Literatur berichtet. Neben einer iatrogenen Stimmbandparese nach operativen Eingriffen stellt die kongenitale Stimmbandparese die zweithäufigste Ursache dar. Auch wenn die Stimmbandparese eigentlich eine gute Prognose mit Tendenz zur spontanen Besserung hat, so ist die initiale Atemnot doch lebensbedrohlich. Somit erfolgt bei vielen Kindern die Anlage eines Tracheostomas, was außer einem deutlich invasiverem Eingriff auch postoperativ deutlich mehr, auch technischen, Aufwand für Personal und Eltern bedeutet. Die posteriore Chordotomie ist ein schonenderes Verfahren, das neben einer deutlichen Verbesserung des Atemwegs auch stimmschonend ist, und somit eine mögliche Alternative zur Tracheostoma-Anlage darstellt. Bislang sind keine Langzeitdaten für die Nachbeobachtung von Patienten mit Stimmbandparese, die mittels posteriorer Chordomtomie behandeltwurden, verfügbar; es finden sich lediglich wenige Einzelfallberichte. Es wird interessant sein, zu sehen, wie sich diese Patienten entwickeln, scheint doch dieser weniger invasive und meist einmalige Eingriff Vorteile gegenüber einer Trachotomie im Säuglingsalter darzustellen.

\section{P004 \\ Inhalativ verabreichtes Furosemid bei Neugeborenen mit Transitorischer Tachypnoe}

P. Nitsch-Felsecker', A. Kribs' ', A. Vierzig' ', B. Hero'2, B. Roth

'Universitäts-Kinderklinik, Neonatologie und Pädiatrische Intensivmedizin, Köln, Deutschland, ${ }^{2}$ Univ.-Kinderklinik, Klinisches Studienzentrum Pädiatrie, Köln, Deutschland

Einleitung: Die Transitorische Tachypnoe (TT) ist eine postnatale respiratorische Anpassungsstörung, die bevorzugt nach Sectio wenige Wochen vor errechnetem Geburtstermin auftritt. Ursache ist eine gestörte und verzögerte Resorption der fetalen Alveolarflüssigkeit. Die Standardtherapie der TT besteht in einer symptomatischen Behandlung mittels Atemunterstützung mit PEEP (nCPAP, ggfl. Beatmung) und Sauerstoffzufuhr. Eine etablierte medikamentöse Therapie gibt es zurzeit nicht. Es liegen Hinweise dafür vor, dass der kurzfristige Einsatz von inhalativ verabreichtem Furosemid die Lungenfunktion bei Neugeborenen günstig beeinflussen kann. Es sollte in einer Pilotstudie der Frage nachgegangen werden, ob inhalativ verabreichtes Furosemid bei Neugeborenen den klinischen Verlauf einer TT beeinflussen kann.

Material/Methoden: In einer prospektiven, doppelblinden, randomisierten und monozentrischen Pilotstudie sollten Machbarkeit, Effektivität und Sicherheit von inhalativ verabreichtem Furosemid bei Neugeborenen mit TT untersucht werden (NCT 01407848, EudraCT-Nr: 2011-003473-29). Vom April 2013 bis Juli 2015 wurden insgesamt 20 Neu- und Frühgeborene in einem Gestationsalter von 35+0-39+0 SSW am 1. Lebenstag (7 weibliche, 13 männliche Patienten) mit klinischen Zeichen einer TT und nicht-invasiver CPAP-Atemhilfe von $>6 \mathrm{~h}$ in die Studie eingeschlossen. Einverständis beider Eltern lag vor. Das mittlere Gestationsalter be- trug 36,4 Schwangerschaftswochen, das mittlere Geburtsgewicht 2943 g, 13 Kinder wurden per Sectio, 7 spontan geboren. Verabreicht wurde inhalativ alle $6 \mathrm{~h}$ entweder $1 \mathrm{ml} / \mathrm{kg}$ Furosemid-Lsg. mit $1 \mathrm{mg} / \mathrm{ml}$ Wirkstoff (10 Pat.) oder $1 \mathrm{ml} / \mathrm{kg} \mathrm{NaCl}$ 0,9\% als Placebo (10 Pat.) Beobachtet wurden Intensität und Dauer der TT anhand des Silvermann Scores (Hauptzielkriterium), Länge des CPAP- und Sauerstoffbedarfs sowie das Auftreten von Nebenwirkungen. Das Studienende war mit Beendigung des CPAP-Bedarfs definiert (Silvermann Score $<3$, kein zusätzl. 02 Bedarf, BGA im Normbereich). Ein positives Votum der zuständigen Ethik-Kommission lag vor.

Ergebnisse: Die demographischen Daten zeigen keine statistisch signifikanten Unterschiede zwischen den beiden Gruppen. Im Durchschnitt bedurften die Patienten der Furosemid-Gruppe 18,6 h, die der Placebo-Gruppe 25,3 h CPAP-Unterstützung. Zwar fanden sich in den Zielkriterien keine statistisch signifikanten Unterschiede, jedoch zeigten die Patienten, der Verumgruppe, im Trend einen kürzeren und weniger schweren Verlauf der TT als die Patienten der Placebogruppe (objektiviert anhand der Berechnung der area under curve des Silbermann-Scores). Unerwünschte Nebenwirkungen wurden in der Studie nicht beobachtet. Schlussfolgerung: Inhalativ verabreichtes Furosemid scheint Intensität und Dauer der TT in der Studien-Population reduziert zu haben. Es konnten keine Nebenwirkungen beobachtet werden. Aufgrund der geringen Fallzahl ist der Effekt als statistisch nicht signifikant zu bewerten. Eine Empfehlung zur Anwendung von inhalativ verabreichtem Furosemid bei Patienten mit TT kann derzeit nicht ausgesprochen werden. Nach erfolgter Nutzen-Risiko-Bewertung wäre eine größere klinische Studie erforderlich.

\section{P005}

\section{Retrospective audit on the use of high flow therapy in neonates in a tertiary care centre}

\section{G. Rajendran' ', C. Roehr' ${ }^{1}$ K. Ives' , Z. Molnar ${ }^{1}$}

'Oxford University Hospitals, Women's Centre John Radcliffe Hospital, Oxford, United Kingdom

Introduction and background: Over recent years high flow therapy (HFT) has gained popularity in the UK and almost $80 \%$ of the neonatal units use this modality of respiratory support. Interestingly, half of the units do not have a written guideline or policy for the use of HFT. High flow nasal cannula therapy (HFT) has been used for 11 years at the Oxford Neonatal Unit as a primary respiratory support after birth and following extubation. Since the last update of our guideline we have noted practice variations among junior doctors regarding the selection of starting flow rate and the rate of weaning from HFT. Our objective was to audit our current practice against the guideline.

Methods: All the neonates started on HFT in the month of September 2014 $(n=33)$ were included in the audit. Hourly data were collected from the nursing records retrospectively. Patients were categorized into 3 groups according to the weight and $\mathrm{FiO}_{2}$ requirement in accordance with the guideline. Group 1: weight $<1.5 \mathrm{~kg}$ and $\mathrm{FiO}_{2}<30 \%$, Group 2: weight $\geq 1.5 \mathrm{~kg}$ and $\mathrm{FiO}_{2}<30 \%$ and Group 3: $\mathrm{FiO}_{2} \geq 30 \%$. The HFT requirement of the neonates in various groups were plotted against time in a graph and compared with the current recommendation.

\section{Current Guideline:}

Operational flow rates range of HFT from $2-8 \mathrm{~L} / \mathrm{min}$

Start at rate of $4-6 \mathrm{~L} / \mathrm{min}$

Attempt to reduce by $1.0 \mathrm{~L} / \mathrm{min} 24$ hourly if $\mathrm{FiO}_{2} 0.21-0.3$ in neonates $>1.5 \mathrm{~kg}$

Attempt to reduce by $0.5 \mathrm{~L} / \mathrm{min} 24$ hourly if $\mathrm{FiO}_{2} 0.21-0.3$ in neonates $<1.5 \mathrm{~kg}$

Wean flow rate slowly by $0.5 \mathrm{~L} / \mathrm{min}$ every other day if $\mathrm{FiO}_{2}>0.3$

Attempt to stop if in air and requiring flow $\leq 2.5 \mathrm{~L} / \mathrm{min}$ or less

Attempt to switch to low flow if in oxygen and requiring $2.0 \mathrm{~L} / \mathrm{min}$

During the weaning phase, we routinely assess respiratory stability (presence of dyspnoea, work of breathing, blood gas examination) and weaning was only recommended in clinically stable patients. 
Results: Adherence to the guideline with regard to initial flow velocity, rate of weaning and flow velocity at the time of discontinuation of HFT was $73 \%, 43 \%$ and $31 \%$, respectively. The duration of HFT in Group 1 was similar to the projected weaning schedule of our guideline recommendation ( 7.4 days versus 8 days, $p=0.4$ ). In contrast to this finding, the duration of HFT in Group 2 was significantly shorter than the projected weaning schedule ( 1.3 days versus 4 days, $(p<0.001))$ without rebound. There were 12 neonates with transient tachypnoea of newborn in Group 2. Patients in Group 3 were weaned according to our guideline in all cases.

Conclusions: Adherence of junior doctors to our written policy needs improvement. This practice variation on the other hand revealed, that faster rate weaning from $\mathrm{HFT}$ in a subgroup of infants $\left(\mathrm{FiO}_{2}\right.$ requirements $<30 \%$ and weight $>1.5 \mathrm{~kg}$ ) is feasible. More data are needed to establish recommendations about the practical aspects of weaning form HFT.

\section{P006}

Early biomarkers and magnetic resonance imaging for diagnosing Bronchopulmonary Dysplasia

K. Förster', S. Sass' ${ }^{2}$, O. Dietrich ${ }^{3}$, P. Andreas ${ }^{3}$, L. Nährlich ${ }^{4}$, P. Oak ${ }^{5}$, A. Schulze, A. Flemmer ${ }^{7}$, S. Herber-Jonat ${ }^{8}$, H. Ehrhardt ${ }^{9}$, C. Hübener ${ }^{10}$, O. Eickelberg ${ }^{5}$, F. Theis' ${ }^{2}$ B. Ertl-Wagner ${ }^{11}$, A. Hilgendorff' ${ }^{2}$

'Klinikum der Universität München, Marchioninistraße 15, 81377 München, Neonatologie der Kinderklinik am Perinatalzentrum Großhadern, München, Deutschland, ${ }^{2}$ Helmholtz Zentrum München, Institute of Computational Biology, Neuherberg, Deutschland, ${ }^{3}$ Klinikum der Universität München, Institut für Klinische Radiologie, München, Deutschland, ${ }^{4}$ Universitätskinderklinik Gießen, Kinderpneumologie, Gießen, Deutschland, ${ }^{5}$ Comprehensive Pneumology Center, München, Deutschland, ${ }^{6}$ Dr. von Haunersches Kinderspital, Neonatologie der Kinderklinik am Perinatalzentrum Großhadern, München, Deutschland, ${ }^{7}$ LMU-München, Neonatologie der Kinderklinik am Perinatalzentrum Großhadern, München, Deutschland, ${ }^{8}$ Dr. von Haunersches Kinderspital, Neonatologie am Perinatalzentrum Großhadern, München, Deutschland, ${ }^{9}$ Dept. of General Pediatrics and Neonatology, Justus-Liebig-University, Giessen, Germany, Giessen, Deutschland, ${ }^{10} \mathrm{Klinik}$ und Poliklinik für Frauenheilkunde und Geburtshilfe, München, Deutschland, "Klinikum der Universität München Klinikum Großhadern, Institut für Klinische Radiologie, München, Deutschland, ${ }^{12}$ Perinatalzentrum Grosshadern, Neonatologische Intensivstation, München, Deutschland

Rationale: Neonatal chronic lung disease, i. e. BPD, determines long-term pulmonary and neurologic development. As to date, the diagnosis is solely stated with respect to its clinical course referring to oxygen dependency on day 28 post partum or at 36 weeks postmenstrual age (PMA). Early markers are urgently needed for timely diagnosis and personalized treatment. Objectives: The prospective study determined structural and functional changes in the preterm lung at the time of diagnosis and identified early disease markers by proteome screening in plasma in the first week of life. Method: 40 preterm infants $(27.7 \pm 2.09$ wks, $984 \pm 332 \mathrm{~g})$ were included for advanced MR imaging (3-Tesla) and complemented by Infant Lung function testing (ILFT) in spontaneously breathing infants. Samples from plasma, tracheal aspirate and urine were processed for proteomic screening. Key findings were confirmed in an independent study cohort $(n=21$ infants). Statistical analysis used penalized and Poisson regression analysis for protein analysis confounder effects were subtracted by lasso regression. Long-term follow-up with 2 and 5 years is intended.

Results: Statistical analysis confirmed a high correlation of MRI and lung function variables and identified a pattern characterizing changes in the lungs of preterm infants by T2- and T1-weighed image analysis and lung volume measurements as well as ILFT. Statistical modelling using the outcome variables ,days of oxygen' or ,days of $\mathrm{MV}^{\prime}$ instead of BPD diagnosis confirmed the analysis indicated above. Functional enrichment analysis showed overrepresentation of the GO categories ,immune function', ,extracellular matrix', ,cellular proliferation/migration', ,organ development and ,angiogenesis' in infants with BPD. One protein was identified as a potential biomarker.
Conclusions: We identified a structural pattern characterizing BPD by advanced MRI confirmed by ILFT. The findings reflect the characteristic picture outlined by previous studies of human tissue samples, i.e. the presence of interstitial and emphysematous changes. The identified protein indicated BPD development in the first week of life enabling personalized treatment strategies. Further investigation will be the establishment of this biomarker in a clinical study in a number of tertiary perinatal centers.

\section{P007}

\section{Interim Report: NeoVitaA Study Trial}

S. Meyer, Study Group NeoVitaA

Universitätsklinikum des Saarlandes, Klinik für Allgemeine Pädiatrie und Neonatologie, Homburg, Deutschland

Background: Prematurity and the associated risk for bronchopulmonary dysplasia (BPD) remain a significant threat to extremely low birth weight (ELBW) infants. Vitamin A has been considered a therapeutic alternative in reducing the rate of BPD and mortality. To investigate whether early postnatal, additional high-dose oral vitamin A supplementation for 28 days is more efficient in reducing BPD or death in ELBW infants than placebo treatment.

Objectives: To provide data on the current status of participating centers, number of included patients, and ongoing obstacles in realizing this pharmaceutical study in ELBW infants.

Methods: This is an interim report of the multicenter, double-blind RCT comparing postnatal high-dose oral vitamin A supplementation (5,000 IU vitamin $\mathrm{A} / \mathrm{kg} /$ day vs. placebo) for 28 days in ELBW neonates requiring mechanical ventilation, noninvasive ventilatory support or supplemental oxygen at $24 \mathrm{~h}$ of age. The primary end point is the proportion of children who died before 36 weeks gestational age or developed moderate or severe BPD. $\mathrm{BPD}$ is defined as the need for supplemental oxygen to maintain $\mathrm{SaO}_{2}$ of $\geq 92 \%$ at rest at 36 weeks postmenstrual age (PMA). Clinical secondary end points include the following: BPD (including mild form), IVH, PVL, ROP, NEC, total number of days of mechanical ventilation and oxygen supplementation, and safety and tolerability of high-dose vitamin A supplementation. Logistic and organizational support is provided by the IZKS, Mainz. Results and conclusions: After trial start in March 2015, a total of 20 large neonatal centers in Germany are actively recruiting patients. As of January 2016, a total of 62 ELBW infants have been enrolled into the NeoVita A trial. Major problems that have been encountered during the initial phase are mostly related to organizational and medico-legal aspects. Tight financial budgets have been proven a relevant obstacle in recruiting a larger of number of participating centers.

\section{P008}

Hemmung der RSV- und Steroid-induzierten CTGF-Expression in Lungenepithelzellen durch Koffein: Protektive Wirkung von Koffein für airway-remodeling Prozesse?

M. Fehrholz', C. Krempl'², K. Glaser', S. Seidenspinner', T. Kreuzahler², C. Speer', S. Kunzmann

'Universitäts-Kinderklinik Würzburg, Würzburg, Deutschland, ${ }^{2}$ Insitut für Virologie und Immunbiologie, Universität-Würzburg, Würzburg, Deutschland

Hintergrund: Das Respiratorische Synzytialvirus (RSV) ist der bedeutendste virale Erreger von schwerwiegenden Infektionen der unteren Atemwege in den ersten beiden Lebensjahren, insbesondere bei Frühgeborenen mit Bronchopulmonaler Dysplasie (BPD). Zusätzlich fungiert RSV als Trigger für eine erhöhte pulmonale Morbidität im späteren Kindesalter (wheezing, Asthma bronchiale). Die hierfür verantwortlichen Pathomechanismen sind dabei noch weitestgehend unbekannt.

Ziel: In dieser Studie soll untersucht werden, inwieweit eine Infektion mit RSV die Regulation von connective tissue growth factor (CTGF), einem Schlüssel-Molekül von remodeling-Prozessen, beeinflusst. Insbesondere soll dabei auch der mögliche Einfluss von Steroiden und Koffein näher charakterisiert werden. 
Material und Methoden: Typ II Lungenepithelzellen (H441) wurden mit RSV infiziert und die CTGF mRNA-Expression mittels real-time PCR bei Anwesenheit von verschiedenen Steroiden sowie Koffein quantifiziert. Ergebnisse: Eine Infektion von H441 Zellen mit RSV führte zu einem signifikanten Anstieg der CTGF mRNA Expression (10,4 \pm 1,8-fach, $p=0,0051$ ), während die Expression von TGF- $\beta 1-3$ nicht beeinflusst wurde. Eine zusätzliche Behandlung mit Dexamethason (DXM) führte hierbei überraschend zu einer zusätzlich verstärkten Induktion von CTGF durch RSV $(25,7 \pm 3,9$-fach, $p<0,0001)$. Koffein war in der Lage beide Effekt von RSV und DXM auf die CTGF-Expression zu antagonisieren $(p$ $<0,0001)$. Als möglicher molekularer Mechanismus dieser Koffein-induzierten Hemmung der CTGF-Expression fand sich eine erhöhte Expression von TNF- $\alpha(53,9 \pm 14,5$-fach, $p<0,0001)$, welcher als Inhibitor der CTGF-Expression beschrieben ist. Neben DXM induzierten auch andere Steroide wie Betamethason, Prednisolon, Budenosid und Hydrocortison die CTGF-mRNA Expression in Lungenepithelzellen, welche erneut durch Koffein antagonisiert werden konnte.

Schlussfolgerung: Diese in vitro Studie beschreibt eine Hochregulation der CTGF-Expression in Lungenepithelzellen zum einen durch eine Infektion mit RSV, zum anderen durch Steroide. Zusätzlich zeigte sich, dass Koffein den negativen Effekt von RSV und Steroiden auf die Expression von CTGF aufhebt. Hieraus könnte sich ein neuer therapeutischer Ansatz von Koffein bei RSV-Infektionen bzw. als Co-Medikation einer Steroid-Gabe bei Kindern ergeben. Zur Behandlung einer RSV-Infektion, sowie vieler anderer Lungenerkrankungen, werden in der Praxis oftmals Steroide aufgrund ihrer anti-inflammtorischen Wirkung verabreicht, ohne einen potentiell negativen Effekt auf die gleichzeitig ablaufenden remodeling-Vorgänge zu berücksichtigen.

\section{P009}

\section{Kardiorespiratorische Parameter bei reifen Neugeborenen: Perzentilen für Herz- \& Atemfrequenz, Sauerstoffsättigung, Apnoen und Hypoxämien}

\section{B. Bohnhorst ${ }^{1}$, K. Seidel ${ }^{2}$, C. Böhne ${ }^{1}$, C. Peter ${ }^{1}$, S. Pirr ${ }^{1}$}

${ }^{1}$ Kinderklinik der Med. Hochschule, Pädiatrische Pneumologie, Allergologie und Neonatologie, Hannover, Deutschland, ${ }^{2}$ St.-Bernward-Krankenhaus, Medizinische Klinik 3, Hildesheim, Deutschland

Hintergrund: Das Monitoring kardiorespiratorischer Parameter ist in der Neonatologie Standard. Dabei ist die Apnoe-Bradykardie-Hypoxämie-Symptomatik (ABHS) des Frühgeborenen (FG) ein bekanntes Phänomen. Inwieweit auch reife, gesunde Neugeborene (NG) derartige Ereignisse zeigen und in welchem Ausmaß diese physiologisch sind, ist derzeit nur wenig untersucht.

Fragestellung: Normwerterhebung für kardiorespiratorische Parameter bei reifen, gesunden NG mit Erstellung von Perzentilen für Herz- und Atemfrequenz (HF, AF), Sauerstoffsättigung $\left(\mathrm{SpO}_{2}\right)$, Apnoen (Ap, $\geq 4$ Sek.) und Hypoxämien (Hy, 81-85\% und < $80 \%$ ).

Methoden: In einer einarmigen, prospektiven Beobachtungsstudie wurden bei 225 NG (GA im Median 39 3/7, Bereich 37 0/7-41 0/7 SSW, GG im Median $3440 \mathrm{~g}$, Bereich 2520-4340 g) im Alter von 2-4 Tagen einma- lig kontinuierlich über 6 Stunden Plethysmografiekurve, $\mathrm{SpO}_{2}$, thorakale Atembewegungen und EKG aufgezeichnet (GETEMED ${ }^{\circ}$-Monitor). Der Schlaf währenddessen erfolgte in strikter Rückenlage. Falls im Aufzeichnungszeitraum eine Nahrungsaufnahme stattfandt, wurden Zeit und Dauer protokolliert. Im Alter von 3 Monaten wurde per Telefoninterview der Gesundheitszustand der NG abgefragt. Die statistische Auswertung erfolgte mit IBM SPSS Statistics for Windows, Version 15.0.

Ergebnisse: $10 \mathrm{NG}$ wurden nachträglich ausgeschlossen (6 wegen fehlender telefonischer Erreichbarkeit, 4 wegen Pathologien (VSD, Aortenstenose, Cystinurie, OSAS), so dass die Daten von 215 NG in die Auswertung einbezogen werden konnten. Bei $15 \mathrm{NG}$ (7\%) wurden Bradykardien (HF-Abfall auf $<2 / 3$ der Basalfrequenz) beobachtet. Maximale Bradykardiedauer bzw. minimale HF betrugen im Median 6 Sek. bzw. 73/min (Bereich 2-36 Sek. bzw. 59-78/min). Bei 54\% der NG traten $\mathrm{Hy}<85 \%$, bei $28 \% \mathrm{Hy}<80 \%$ und bei $98 \% \mathrm{Ap} \geq 4$ Sek. auf.

Folgende Normwerte in Form von Perzentilen wurden erhoben:

Schlussfolgerung: Während Bradykardien bei reifen NG selten sind, kommen $\mathrm{SpO}_{2}$-Abfälle und Apnoen regelmäßig vor. Die vorliegenden Daten ermöglichen eine evidenzbasierte Bewertung kardiorespiratorischer Parameter des reifen NG und stellen darüber hinaus eine Bewertungshilfe dar, um die Relevanz der ABHS eines zur Entlassung anstehenden FG einzuschätzen.

\section{P010}

Effects of automated closed loop control of inspired oxygen concentration on fluctuations of arterial and different regional organ tissue oxygen saturations in preterm infants

\section{Gajdos' ${ }^{1}$ M. Waitz' ${ }^{2}$, M. Mendler ${ }^{3}$, W. Braun ${ }^{4}$, H. Hummler ${ }^{5}$}

'Universitäts-Kinderklinik, Neonatologie und Pädiatrische Intensivmedizin, Ulm, Deutschland, ${ }^{2}$ Universitätsklinik für Kinder- und Jugendmedizin, Sektion Neonatologie und Pädiatrische Intensivmedizin, Ulm, Deutschland, ${ }^{3}$ Klinik für Kinder- und Jugendmedizin, Universitätsklinikum Ulm, Sektion Neonatologie und pädiatrische Intensivmedizin, Ulm, Deutschland, ${ }^{4}$ Fritz Stephan GmbH, Gackenbach, Deutschland, ${ }^{5}$ Klinik für Kinder- und Jugendmedizin, Universitätsklinikum Ulm, Sektion Neonatologie und Pädiatrische Intensivmedizin, Ulm, Deutschland

Objective: To assess the efficacy of a newly developed system for automated adjustment of the fraction of inspired oxygen $\left(\mathrm{FiO}_{2}\right)$ on variation of arterial and regional tissue oxygen saturation in preterm infants with spontaneous fluctuations in arterial oxygen saturation.

Methods: 12 VLBWI, (gestational age [median; interquartile range]: [25; 23-26 weeks]; birthweight [mean \pm SD]: [ $667 \pm 134 \mathrm{~g}$ ]; postnatal age: [31.5 \pm 14 days]) were studied. Infants were exposed to automated $\mathrm{FiO}_{2}$ adjustment (auto) or to manual adjustment (manual) by the clinical nursing staff during two consecutive $24 \mathrm{~h}$ periods in random sequence using a Sophie ${ }^{\oplus}$ infant ventilator (Fritz Stephan GmbH, Gackenbach, Germany). Results: There was a significant increase in time within the intended oxygen target range ( $88 \%-96 \%$ ) during the automated $\mathrm{FiO}_{2}$-control as compared with the manual period $(77.8 \% \pm 7.1 \%$ vs. $68.5 \% \pm 7.7 \%$ [mean \pm SD], $p<0.001)$. Infants spent significantly less time below the $\mathrm{SpO}_{2}$-threshold

\begin{tabular}{llllllll} 
Tab. P009 & HF/min & AF/min & $\begin{array}{l}\mathrm{SpO}_{2} \\
\text { in } \%\end{array}$ & $\begin{array}{l}\text { Anzahl Ap } \\
\geq 4 \text { Sek./h }\end{array}$ & $\begin{array}{l}\text { Ap-Dauer } \\
\text { in Sek. }\end{array}$ & Tiefste Hy in \% & Längste Hy<80 \% in Sek. \\
\hline 3. & 87 & 32 & 94,3 & 0 & 5 & 49,3 & 1 \\
\hline 10. & 95 & 35 & 96,1 & 0,2 & 6 & 68,3 & 2 \\
\hline 25. & 101 & 39 & 97,1 & 0,9 & 8 & 75,4 & 3 \\
\hline 50. & 112 & 44 & 98,1 & 2,3 & 10 & 79,7 & 10 \\
\hline 75. & 119 & 48 & 99,2 & 4,8 & 13 & 82,2 & 20 \\
\hline 90. & 126 & 54 & 100 & 8,1 & 14 & 83,3 & 69 \\
\hline 97. & 133 & 57 & 100 & 16,4 & 16 & 84,2 & 308 \\
\hline
\end{tabular}


during the automated period $(18.1 \% \pm 6.4 \%$ vs. $25.6 \% \pm 7.6 \%$; $p<0.01)$. There was a dramatic reduction in events with a $\mathrm{SpO}_{2}<88 \%$ with $>3$ mins duration $(2(0-10)$ vs. $10(0-37)$ events; $p<0.001)$. The percentage of time the infants spent above the intended arterial oxygen range $(4.1 \% \pm 3.8 \%$ vs. $5.9 \% \pm 3.6 \%$, median inspired oxygen concentration and mean $\mathrm{SpO}_{2}$ over time did not differ significantly between the two periods. The total time below a pre-specified cerebral tissue oxygen saturation threshold was not significantly different $(1.9 \pm 5.9 \%$ vs. $2.5 \% \pm 2.8 \%)$. Furthermore, time below a pre-specified regional hepatic $(36.3 \pm 3.3 \%$ vs. $32.4 \pm 4.9 \%)$ and regional renal oxygen saturation threshold $(24.6 \pm 4.7 \%$ vs. $28.5 \pm 4.1 \%)$ did not differ.

Conclusions: Closed-loop inspired oxygen concentration using the Sophie $e^{\circledast}$ infant ventilator significantly increased time of arterial $\mathrm{SpO}_{2}$ within the intended range in VLBWI when compared to the routine adjustment by staff members over a short period of time. No significant difference in time was detected within a pre-specified regional cerebral, hepatic, or renal tissue oxygen saturation range. Long-term effects of this closed loop system of $\mathrm{FiO}_{2}$-control should be evaluated in large randomized controlled trials in VLBWI.

\section{P011}

\section{Einfluss von Mundleck und Flowrate auf die adäquate Befeuchtung des Atemgases in der High-Flow-Therapie: eine in-vitro-Studie}

\section{T. Ullrich', C. Czernik', C. Bührer', G. Schmalisch' ', H. Fischer' \\ ${ }^{1}$ Charité Universitätsmedizin Berlin, Klinik für Neonatologie, Berlin, Deutschland}

Hintergrund: Die adäquate Anfeuchtung und Erwärmung des Atemgases ist von essentieller Bedeutung bei der nicht-invasiven Atemunterstützung von Neugeborenen. Bei Erwachsenen hat sich gezeigt, dass Mundlecks die Atemgaskonditionierung bei der nasalen CPAP-Therapie erschweren können. Für die High-Flow-Therapie, insbesondere des Neugeborenen, liegen bisher keine Daten vor.

Fragestellung: Die vorliegende in-vitro Studie hatte zum Ziel, mit einem neuen neonatalen Lungenmodell den Einfluss des Mundlecks und des eingestellten Flows auf die Anfeuchtung und Erwärmung des Atemgases im Oropharynx bei der High-Flow Therapie zu untersuchen.

Material und Methoden: Einem Oropharynxmodell (modifizierter Gummiball) wurde angefeuchtete und erhitzte Luft durch ein aktives mechanisches Lungenmodell zugeführt. Lungenmodell und Anfeuchter wurden in einem Inkubator $\left(37^{\circ} \mathrm{C}\right)$ platziert, das Oropharynxmodell außerhalb. Zur Simulation des Mundlecks hatte der Gummiball eine frontale Öffnung, die mit einem Silikonstopfen verschlossen werden konnte. Das Anlegen der High-Flow-Brille (Optiflow Junior ${ }^{\circ}$, Fisher \& Paykel, NZ) erfolgte über zwei weitere Bohrungen, die die Nasenlöcher des Neonaten simulierten. Zur Atemgaskonditionierung im High-Flow-System diente ein kommerzieller Oberflächenverdunster. Die Messungen von Temperatur (T), relativer $(\mathrm{rF})$ und absoluter Feuchte $(\mathrm{aF})$ wurden mit einem digitalen Thermohygrometer im Oropharynxmodell durchgeführt. Es wurden Flows von 4, 6 und $8 \mathrm{~L} \cdot \mathrm{min}^{-1}$ getestet.

Ergebnisse: Das Atemgas im Oropharynxmodell war in nahezu allen Messungen vollständig gesättigt mit einer $\mathrm{rF}>99 \%$. Unter Spontanatmung, ohne High-Flow-Brille (Flow $\left.=0 \mathrm{~L} \cdot \mathrm{min}^{-1}\right)$, gab es im in-vitro Modell keinen signifikanten Effekt des Mundlecks auf die Atemgaskonditionierung im Oropharynx. Mit High-Flow-Brille zeigte sich ein signifikanter Anstieg der oropharyngealen $\mathrm{T}(r=0,969 p<0,001)$ und der $\mathrm{aF}(r=0,968 p<0,001)$ bis zu einem Flow von $6 \mathrm{~L} \cdot \mathrm{min}^{-1}$, der unabhängig vom Mundleck war. Beim höchsten eingestellten Flow von $8 \mathrm{~L} \cdot \mathrm{min}^{-1}$ verursachte allerdings das Mundleck eine signifikante Reduktion der erreichten T $(p=0,001)$ und aF $(p=0,001)$, und es kam zu keinem weiteren Anstieg von T und aF zwischen Flowraten von 6 und $8 \mathrm{~L} \cdot \mathrm{min}^{-1}$.

Schlussfolgerung: Bei der High-Flow-Therapie haben Mundlecks erst bei hohen Flowraten einen negativen Einfluss auf die Atemgaskonditionierung. Unter Berücksichtigung aktueller Leitlinien, wie der aktuellen
ISO-Empfehlung zur non-invasiven Atemunterstützung, lagen Temperatur und Feuchte aber stets im klinisch akzeptablen Bereich.

\section{P012}

\section{Survey On The Use And Weaning Of Non-Invasive Ventilation} Among UK Tertiary Neonatal Units

\section{S. Zivanovic ${ }^{1}$, I. Bressetti ${ }^{1}$, C. Roehr ${ }^{1}$}

'Oxford University Hospitals, Women's Centre John Radcliffe Hospital, Oxford, United Kingdom

Background: High flow (HF) therapy has been increasingly used over last decade in the treatment of neonatal respiratory distress $1-5$. To date, there are no data available supporting the choice of high flow as primary respiratory support in extremely preterm babies, although encouraging data have been published regarding its use to treat late preterm infants $10-12$. There is no evidence available on the weaning of high flow 13.

Methods: We submitted an online questionnaire to the tertiary NICUs in England, Scotland, Wales and Northern Ireland. The list of centers was extrapolated from the RCPCH (Royal College of Paediatrics and Child Health) and BAPM (British Association of Perinatal Medicine) database. The questionnaire was proposed in the form of 16 multiple choice questions regarding the current practice of non-invasive ventilation term and preterm neonates. The online survey and reminders were sent over 2 months period (November 2015 to January 2016).

Results: Out of the 57 units asked to take party in the survey, a total of 40 returned the questionnaire $(70 \%)$. The number of units using HF is 22 (55\%), while 14 (35\%) use CPAP. Non-invasive ventilation is started in the delivery suite in 26 units ( $66 \%$ ), however only $3 \%$ of them use HF for transport to NICU. Minimally invasive surfactant therapy is administered in 7 units (18\%). $60 \%$ of the units have a guideline for the use and weaning of HF. There is a high variability in the starting flow rate for high HF on preterm babies less than 28 weeks of gestational age. Most common starting flow rate for both term and preterm babies is $6 \mathrm{~L} / \mathrm{min}$. HF is not commonly used for weaning CPAP. There is a large variation in the rate of weaning of the high flow, though $63 \%$ of the units are used to decrease it by $1 \mathrm{~L} / \mathrm{min}$ every 24 hours. $63 \%$ of the units would consider to use HF in preterm babies less than 27 weeks of gestational age, and $45 \%$ would start it in 25-26 weeks of gestational age upon delivery.

Conclusion: There is an emerging trend towards use of High Flow as a first line treatment for RDS in extreme preterm babies above 25 weeks of GA. Great proportion of units rely on the local guideline in the use of high flow, despite the lack of available evidence. The survey shows a high degree of variation in the use of non-invasive ventilation among tertiary units in UK. There is an urgent need for RCT studies on the use and weaning of high flow.

\section{References}

1. Hochwald $\mathrm{O}$, Osiovich $\mathrm{H}$. The use of high flow nasal cannulae in neonatal intensive care units: Is clinical practice consistent with the evidence? J Neonatal Perinatal Med. 2010;3(3):187-191

2. Holme N., Harrison C. Should we be using high flow therapy on the neonatal unit? Infant 2012; 8(6): 172- 176

3. Hough JL, Shearman AD, Jardine LA, Davies MW. Humidified high flow nasal cannulae: Current practice in Australasian nurseries, a survey. J Paediatr Child Health. 2011;48(2):106-13. 11.

4. Ojha S, Gridley E, Dorling J. Use of heated humidified high-flow nasal cannula oxygen in neonates: a UK wide survey. Acta Paediatr. 2013;102:249-53.

5. Nath P, Ponnusamy V, Willis K, Bissett L, Clarke P. Current practices of high and low flow oxygen therapy and humidification of UK neonatal units. Pediatric Int 2010;52:893-4

6. Shetty S, Greenough A. Review finds insufficient evidence to support the routine use of heated, humidified highflow nasal cannula use in neonates. Acta Paediatr. 2014;103(9):898-903 


\section{Postersitzung 03: Leben auf der NICU}

\section{P013 \\ Neu Gleiten wir in die Dienste - ein Pilotprojekt der Neonatologie am Universitätsspital Zürich}

\author{
A. Arndt ${ }^{1}$, B. Merz ${ }^{1}$ \\ 'UniversitätsSpital Zürich/FKl, Neonatologie, Affoltern am Albis, Schweiz
}

Hintergrund: Eine Umfrage zur Mitarbeiterzufriedenheit im Januar 2015 machte deutlich, dass attraktive Arbeitszeiten und die Dienstplangestaltung im Pflegeberuf ein wichtiges Führungsthema sind. Pflegefachpersonen sind heutzutage als hochqualifizierte Fachexperten angestellt. Die Bedeutung einer ausgeglichenen Work-Life-Balance hat in den vergangenen Jahren deutlich zugenommen. Die Vereinbarkeit von Familie und Beruf, das persönliche Lebensmodell, sowie das soziale Leben mit Freunden sind für die Arbeitnehmenden von enormer Bedeutung. Um diesen Ansprüchen besser gerecht zu werden und die Mitarbeiterbindung zu stärken suchte das Management nach einem neuen Arbeitszeitmodell.

Fragestellung: Ist eine Flexibilisierung der Arbeitszeit in einem 24 Stunden Betrieb möglich? Erhöhen flexiblere Arbeitszeiten die MA Zufriedenheit? Methode: Es erfolgte eine Auseinandersetzung mit der vorhandenen Literatur zu den Themen Arbeitszeit, Arbeitszeitmodelle, Flexibilisierung der Arbeitszeit und den gesetzlichen Vorgaben. (Hellert (2013), Kelm (2012), Seco (16. Juni 2015)). Zudem wurde die Literatur zum Change Management berücksichtigt (Blixt \& James (Januar/Februar 2004), Mohr, Woehe \& Diebold. (1998), Kotter (1997)). Wegen der hohen Brisanz des Themas, wurde eine bottom-up (Betroffene zu Beteiligten machen) Implementierung empfohlen, um bestmögliche Voraussetzungen für das Gelingen des Projektes zu schaffen. Nach der Gründung einer Arbeitsgruppe wurde eine SWOT-Analyse erstellt und ein nicht validierter Fragebogen zur Zufriedenheit der MA zum aktuellen Arbeitszeitmodel, sowie ihre Haltung zu möglichen Änderungen erarbeitet. Die Darstellung und Auswertung der Antworten erfolgte mittels Reporting Tool. Die Auswertung des Fragebogens ergab, dass der Spätdienst der unbeliebteste Dienst ist, gefolgt von der Wochenendarbeit. Die Nachtdienste wurden als weniger belastend empfunden. Die Bereitschaft zu 12 Stundendiensten über das Wochenende, zugunsten weniger Wochenendarbeit, war kaum vorhanden. Mit den Ergebnissen des Fragebogens wurde ein Arbeitszeitmodell entwickelt. Das Arbeitszeitgesetz und der Personalleitfaden des UniversitätsSpitals Zürich wurden darin berücksichtigt. Der wenig beliebte Spätdienst wurde um eine Stunde gekürzt, der Nachtdienst um eine Stunde verlängert. Im Früh- und Spätdienst besteht die Möglichkeit einer Gleitzeit von 45 Minuten. Die Übergaberapporte wurden gestrichen, bzw. neu organisiert.

Ergebnisse: Das neue Arbeitszeitmodell wird im April 2016 evaluiert, die Resultate liegen im Sommer 2016 vor. Eine Zwischenevaluation der Gleitzeit im Frühdienst zeigte erste positive Ergebnisse.

Schlussfolgerungen: Obwohl noch leichte Widerstände zu spüren sind, ist das Pilotprojekt gut gestartet. Eine Begleitung im Change Prozess ist nötig. Das Management ist in Zeiten des Fachkräftemangels gut beraten, den gesellschaftlichen und sozialen Entwicklungen im Arbeitsalltag Rechnung zu tragen und den neuen Erwartungen der Mitarbeitenden mit modernen, attraktiven Arbeitszeitmodellen zu begegnen.

\section{P014 \\ Die Reduktion des Wohlbefindens von Frühgeborenen während einer routinemäßigen Echokardiographie}

\section{K. Kaar' ' J. Brandner', B. Minnich'², J. Hilberath' ', C. Weisser' ', M. Wald ${ }^{1}$ \\ 'Salzburger Universitätsklinikum, Division für Neonatologie, Salzburg, Österreich, ${ }^{2}$ Universität Salzburg, Abteilung für zoologische Strukturforschung, Salzburg, Österreich}

Hintergrund und Fragestellungen: Während ihrem Aufenthalt auf einer Intensivstation sind Frühgeborene einer Vielzahl an potentiellen Stressfaktoren, verursacht durch medizinische und pflegerische Maßnahmen, ausgesetzt. Es ist bekannt, dass dieser Stress zu Entwicklungsrückstän- den führen kann. Die Echokardiographie ist ein wichtiges Diagnosemittel, aber bekannt dafür das Wohlbefinden zu beeinträchtigen. Ziel dieser Studie war es zu untersuchen, ob Frühgeborene durch eine routinemäßige Echokardiographie gestresst werden und ob das Stresslevel von der Dauer der Echokardiographie abhängt. Außerdem wurde die Aussagekraft des NIPETM Monitors (NIPE - Newborn Infant Parasympathetic Evaluation) zur Beurteilung von akuten Einschränkungen im Wohlbefinden in der täglichen Praxis untersucht. Es handelt sich dabei um eine neue technische Möglichkeit, welche das Erkennen von potentiell unangenehmen, stressigen oder schmerzhaften Situationen bei Frühgeborenen erleichtern soll. Die Methode basiert auf der Beurteilung der Herzfrequenzvariabilität. Methoden: 16 Frühgeborene, die vor der 32. Schwangerschaftswoche geboren wurden oder weniger als $1500 \mathrm{Gramm}$ wogen, wurden in die Studie eingeschlossen. Die routinemäßige Echokardiographie wurde in den ersten Lebenstagen durchgeführt und die Dauer der Untersuchung aufgezeichnet. Die physiologischen Parameter (Herzfrequenz, transkutane Sauerstoffsättigung, NIPEi Index) wurden gesammelt und für die statistische Auswertung in drei Phasen eingeteilt (vor, während und nach der Echokardiographie).

Ergebnisse: Die Herzfrequenz stieg um 3,11\% und die transkutane Sauerstoffsättigung fiel um 0,27\% während der Untersuchung im Vergleich zu davor. Beides spricht für eine akute Einschränkung im Wohlbefinden während der Untersuchung. Im Vergleich dazu stieg der NIPEi Index um $7,73 \%$, was für ein höheres Wohlbefinden sprechen würde. Es konnte kein signifikanter Zusammenhang zwischen der Veränderung der Vitalparameter zwischen den drei Phasen und der Dauer der Echokardiographie ermittelt werden.

Schlussfolgerung: In der vorliegenden Studie erwies sich die Echokardiographie als eine potentielle Stressquelle für Frühgeborene. Die Veränderungen zweier bewährter Stressindikatoren, Herzfrequenz und Sauerstoffsättigung, wiesen auf diese Einschränkung des Wohlbefindens hin. Das Ausmaß des Unbehagens war unabhängig von der Dauer der Echokardiographie. Ferner erwies sich der NIPETM Monitor in dieser Studie als nicht geeignet für die Beurteilung von akuten Einschränkungen im Wohlbefinden von Frühgeborenen. Der Einsatz des NIPETM Monitors zur Beurteilung von längerfristigen Trendlinien des Wohlbefindens wurde in dieser Studie nicht untersucht.

\section{P015}

\section{Effect of the introduction of the Microsystems care model} on parent stress and satisfaction

D. Morra', G. Fusch', A. Bakry', D. Pogorzelski', S. el Helou', S. Dutta', C. Fusch' 'McMaster University, Department of Pediatrics, Division of Neonatology, Hamilton, Ontario, Kanada

Introduction: In a Level III NICU, not all infants are equally sick. Some centers have implemented varying care models to meet the demands different infants require. No critical evaluation of the impact of these models has been conducted. McMaster Children's Hospital NICU previously followed the standard model, with infants of varying acuity diversely distributed. In 2014, the ,microsystems' care model was implemented, with infants designated to specified areas of the NICU according to their acuity level. A prospective examination of each care model was conducted to determine strengths and challenges in four domains (patient, health care personnel, process of care and administrative-related).

Objective: To assess stress and satisfaction levels before and after the implementation of microsystems and assess correlations between stress and satisfaction levels with infant characteristics.

Methods: Participants were recruited pre- and post-microsystems. Two surveys were administered to parents, the Parental Stressor Scale (PSS) and the Neonatal Instrument of Parent Satisfaction with Care (NIPS). One investigator extracted data from patient records: birth weight, gestational age, length of stay, acuity level (WANNNT score), and overall pod acuity. Overall PSS and NIPS scores were calculated for each patient and a Wilcoxon rank-sum test (alpha $=0.05)$ was used to examine the differences in scores before and after microsystems implementation. 
Results: 59 patients and parents were recruited (29 pre- and 30 post-microsystems). No statistically significant difference was found between study groups for stress and satisfaction scores, $(p=0.203$ and $p=0.117$, respectively). Birth weight and length of stay differed significantly between the two groups $(p<0.001$ and $p=0.009$, respectively). Gestational age, WANNNT score and overall pod acuity were similar.

Conclusion: The microsystems care model did not have a significant impact on stress and satisfaction among parents in the NICU (patient domain). This may indicate that the microsystems care model does not impact parents' perceptions of the care their infants are receiving. Additionally, it did not increase stress where parents were exposed to more intensive care. We have previously found that the microsystems care model has resulted in improved outcomes in other domains (noise, round length and workload distribution) and therefore may be a more effective care model. Further domains are still under evaluation.

\section{P016}

\section{Muttermilch aus dem Internet: Mikrobielle Kontamination und Nährstoffgehalt}

\section{Klotz' ${ }^{1}$, R. Hentschel', C. Möllmann}

'Universitäts - Kinderklinik, Abteilung für Neonatologie und Pädiatrische Intensivmedizin, Freiburg, Deutschland

Hintergrund: Neben der informellen Muttermilchspende im privaten Umfeld hat sich der Online-Handel als eine Bezugsquelle von Frauenmilch etabliert. Auch in Deutschland ist Frauenmilch im Internet kommerziel frei erhältlich. Angaben zu Gewinnung, Handling, Lagerung und Versand der Milch sowie zum Gesundheitszustand und individuellem Risikoprofil der Spenderin sind freiwillig, eine behördliche Aufsicht findet nicht statt. Daten zur hygienischen und nutritiven Qualität dieser Milch liegen nicht vor.

Fragestellung: Die vorliegende Untersuchung evaluiert erstmals die mikrobielle Kontamination und den Nährstoffgehalt von über das Internet aus verschiedenen deutschen Regionen bezogener Frauenmilch.

Material \& Methoden: Postalischer Bezug von Muttermilch aus Deutschland über eine online-Frauenmilchbörse im Zeitraum September 2015 bis Januar 2016. Messung der Probentemperatur bei Ankunft. Evaluation von Preis, Versanddauer und Verpackungsqualität. Kulturelle Bestimmung der mikrobiellen Kontamination und Analyse der Makronährstoffe mittels Nahinfrarotbestimmung.

Ergebnisse: 45 Anbieterinnen wurden angeschrieben. $31 \%$ antworteten nicht, von den verbleibenden Müttern boten $61 \%$ entgegen ersten Angaben doch keinen Postversand an. 12 gefriergelagerte Proben Frauenmilch wurden geliefert, medianer Preis 3,90€/100 ml (2,50-5€), Verpackungsund Portokosten 4,99-48,50€/Lieferung. Alter der Milch bei Bezug 0-6 Wochen (Median 3 Wochen). Die Temperatur bei Ankunft betrug -0,1 bis $+8{ }^{\circ} \mathrm{C}$, die Dauer des Postweges durchgehend $<24$ h. 2 Proben waren anhand Verpackung und Beschriftung als zeitweise in einer Klinik gelagert erkennbar. Hoher interindividueller Gehalt an Kohlenhydraten (7,4-5,1 mg/dl), Fett (2,5-7,2 g/dl) und Protein (1,4-0,6 g/dl) bei 11 Proben. Eine Probe mit extrem niedrigem Gehalt an Makronährstoffen (Trockenmasse 7,4 g/dl, Norm 12-13). Die aus der Klinik stammenden Proben wiesen eine Gesamtkeimzahl von max. 10E $3 / \mathrm{ml}$ auf (CNS, S. lugdunensis/ epidermidis). Alle übrigen Proben waren mit Enterococcus faecalis, Pseudomonaden oder anderen gramnegativen Bakterien mit einer Keimzahl von bis zu $10 \mathrm{E}^{5} / \mathrm{ml}$ kontaminiert.

Diskussion: Der Erwerb von Muttermilch über den Onlinehandel ist zeitund kostenintensiv. Hohe Portokosten und schneller Versand garantieren keine intakte Kühlkette. Der Nährstoffgehalt und die ausgeprägte in terindividuelle Variabilität der Nährstoffzusammensetzung entsprechen den für reife Frauenmilch publizierten Werten. Ein erhöhter Proteingehalt, der auf eine Beimengung von z. B. Kuhmilch hinweisen würde, fand sich nicht. Die analysierte Milch war relativ frisch, lange Gefrierlagerung kann jedoch den Nährstoffgehalt reduzieren. Eine extrem niedrige Trockenmasse weist auf einen erhöhten Wasseranteil in einer Probe hin und kann auf ein Zufügen von Fremdwasser hinweisen, auch wenn sich dies nicht abschließend beweisen lässt. Streckung der Milch mit Wasser oder Fremdmilchen kann ohne aufwändige Laboranalysen durch den Käufer nicht erkannt werden. Nach lebensmittelhygienischen Maßstäben wäre keine der Proben verzehrfähig. Für Spendermilch wird im Allgemeinen die Pasteurisierung empfohlen. Toxikologische und virologische Untersuchung wurden nicht durchgeführt.

Schlussfolgerung: Nach dieser explorativen Untersuchung entspricht über das Internet bezogene Frauenmilch nicht den hygienischen Anforderungen an eine Säuglingsnahrung und stellt ein potentielles Gesundheitsrisiko dar.

\section{P017}

Der informelle Online-Frauenmilch-Handel in Deutschland eine Bestandsaufnahme

\section{Klotz' ${ }^{1}$ C. Möllmann', R. Hentschel'}

'Universitäts - Kinderklinik, Abteilung für Neonatologie und Pädiatrische Intensivmedizin, Freiburg, Deutschland

Hintergrund: Abseits der institutionellen Einrichtungen der klinischen Frauenmilchbanken existiert in Deutschland ein gewerblicher Markt für Muttermilch. Aufgrund der fehlenden rechtlichen Zuordnung von Frauenmilch als Medikament, menschliches Sekret oder Lebensmittel findet der Handel mit Frauenmilch in einer rechtlichen Grauzone ohne staatlichen Kontrollaufsicht statt. Die Inbetriebnahme der ersten deutschen Online-Muttermilch-Börse Anfang 2014 führte zu einer kontroversen öffentlichen Diskussion. Pädiatrische Fachgesellschaften und Berufsverbände, Hebammenverbände und die Nationale Stillkommission warnten einhellig vor einer Verfütterung fremder Frauenmilch und insbesondere vor einem Bezug von Frauenmilch über das Internet.

Fragestellung: Über die Charakteristika und das Ausmaß des informellen Muttermilchmarktes in Deutschland liegen bisher keine Daten vor. Mit dieser Untersuchung sollen erste nationale Daten zu diesem neuen Phänomen vorgelegt werden.

Methode: An einem Stichtag im April 2015 wurde im deutschsprachigen Internet eine Recherche nach allen Angeboten von Muttermilch durchgeführt. Die Angebote wurden u. a. nach demographischen Angaben, Informationen zum Gesundheitsstatus, Hinweisen zu Medikamenteneinnahme und Ernährungsgewohnheiten der Anbieterinnen, Angaben zum Handling, zu Lagerung und Versand sowie Preis und Alter der angebotenen Muttermilch ausgewertet.

Ergebnisse: Identifiziert wurden 2 Onlineplattformen mit insgesamt 66 Angeboten (D 64, CH 2), der Preis lag im Mittel bei 3,10 €/100 ml (0-5€) ohne Porto. Angeboten wurde frische oder gefriergelagerte Frauenmilch im Rohzustand, das mediane Alter der angebotenen Frauenmilch betrug 5 Monate (2-12) Monate. Postalischer Versand wurde in 59 Fällen angeboten. Anbieterinnen waren 0-40 Wochen (median 26 Wochen) postpartum, 7 Frauen waren Mütter frühgeborener Kinder, zwei von diesen spendeten ebenfalls an eine institutionelle Frauenmilchbank. 85\% der Anbieterinnen machten Angaben zu Gesundheitsstatus und Lebensstil, $83 \%$ zu Genuss-/Rauschmittelabusus, $67 \%$ zu Ernährungsgewohnheiten. Angaben zum Handling und zur Lagerung der Milch wurde in $41 \%$ der Fälle gemacht. Falls Motive für den Milchverkauf angegeben wurden, waren diese fast ausschließlich altruistischer Art.

Diskussion: Angaben zur Gewinnung und Lagerung der Milch sowie zum Gesundheitszustand und individuellem Risikoprofil der Spenderin sind freiwillige Angaben. Handling, Lagerung und Versand sind keinen Mindeststandards unterworfen. Mikrobiologische Untersuchungen und Versandmodalitäten liegen in Eigenverantwortung des Anbieters bzw. des Käufers, eine behördliche Aufsicht findet nicht statt. Auf diesem Wege erworbene, kostenintensive Form der Ernährung stellt potentiell ein unkalkulierbares Gesundheitsrisiko dar.

Schlussfolgerung: Muttermilch ist kommerziell frei erhältlich. Momentan scheint es sich um ein Nischenphänomen zu handeln. Es gibt jedoch bisher keine Daten über den tatsächlichen Bedarf an Muttermilch im ambulanten Setting oder Zahlen zum Umsatz mit gehandelter Muttermilch. 
P018

\section{Betätigungsbalance bei Eltern von frühgeborenen Kindern}

M. Dür ${ }^{1}$, M. Brückner², B. Mattner ${ }^{3}$, K. Kugler ${ }^{4}$, A. Berger ${ }^{5}$

'IMC Fachhochschule Krems, Department Health Sciences, Medizinische Universität Wien, Universitätsklinik für Kinder- und Jugendheilkunde, Abteilung für Neonatologie, pädiatrische Intensivmedizin und Neuropädiatrie, Wien, Krems, Österreich, ${ }^{2}$ Medizinische Universität Wien, Univ. Klinik f. Kinder- und Jugendheilkunde, Abteilung für Neonatologie, pädiatrische Intensivmedizin und Neuropädiatrie, Wien, Österreich, ${ }^{3}$ Wilhelminenspital, Physikalisches Institut, Kinder - und Jugendheilkunde mit Neonatologie u. Psychosomatik, Wien, Österreich, ${ }^{4}$ Spielstudio Kindertherapie, Wien, Österreich, ${ }^{5}$ Uinversitätsklinik für Kinder- und Jugendheilkunde, MUW, Klinische Abteilung für Neonatologie, Pädiatrische Intensivmedizin und Neuropädiatrie, Wien, Österreich

Hintergrund: Betätigungsbalance ist eine als gut und befriedigend erlebte Mischung von verschiedenen Tätigkeiten. Als wesentliches Konstrukt in der Ergotherapie, beruht ein Großteil der Definitionen von Betätigungsbalance auf der Perspektive von ErgotherapeutInnen bzw. GesundheitswissenschafterInnen. Bestehende Instrumente, die zur Messung von Betätigungsbalance verwendet oder sogar entwickelt wurden, unterscheiden sich sowohl inhaltlich als auch methodisch. Obwohl es weitgehend bekannt ist, dass sich der Alltag und die Tätigkeiten von Eltern durch die Geburt eines Kindes verändern, gibt es bisher kaum Untersuchungen zur Betätigungsbalance bei Eltern. Eine spezielle Veränderung der Tätigkeiten erleben vor allem Eltern von frühgeborenen Kindern, die zusätzlich zu ihren Ängsten hinsichtlich des Überlebens ihrer Kinder häufig ein unerwartet großes Maß an gesundheitserhaltenden und pflegerischen Tätigkeiten übernehmen.

Fragestellung: Was sind wichtige Komponenten der Betätigungsbalance aus der Sicht von Eltern frühgeborener Kinder?

TeilnehmerInnen und Methoden: Es wurden Eltern von frühgeborenen Kindern mit sehr niedrigem Geburtsgewicht (< vollendete 37. SSW, $\leq 1500 \mathrm{~g}$ ) rekrutiert und im Rahmen von Fokusgruppen einmalig interviewt. Die Interviews fanden maximal 6 Monate nach der Geburt statt. Die Interviews wurden mit einem elektronischen Tonträger aufgenommen, wörtlich transkribiert und anschließend mit einer qualitativen Forschungsmethode, der Bedeutungskondensierung nach Kvale (1996), analysiert.

Ergebnisse: In Bezug auf die Betätigungsbalance konnten insgesamt 22 Komponenten identifiziert werden, die für die 36 interviewten Eltern (jeweils 18 Mütter und Väter) von frühgeborenen Kindern wichtig sind. Diese 22 Komponenten ergeben die folgenden drei Dimensionen: das Mischverhältnis verschiedener Tätigkeiten, Tätigkeiten im Kontext veränderter Lebensumstände und der sozio-kulturelle Kontext von Tätigkeiten. Die Dimension Mischverhältnis verschiedener Tätigkeiten umfasst sieben Komponenten wie zum Beispiel die Verschiedenartigkeit von Tätigkeitsbereichen, die Verschiedenartigkeit der Eigenschaften und Wirkungsweisen von Tätigkeiten sowie eine Verschiedenartigkeit von Orten bzw. Räumen an denen man tätig ist.

Diskussion: Die identifizierten Komponenten der Betätigungsbalance sind für Eltern von frühgeborenen Kindern wichtig. Die Verschiedenartigkeit von Orten bzw. Räumen an denen man tätig ist wurde bisher noch nicht als Komponente der Betätigungsbalance diskutiert. Die drei identifizierten Dimensionen bieten einen weiteren Nachweis für die Mehrdimensionalität des Konstrukts Betätigungsbalance. Künftig sollten die identifizierten Komponenten der Betätigungsbalance im klinischen Alltag und bei der Messung von Betätigungsbalance bei Eltern von frühgeborenen Kindern erfasst werden.

Schlussfolgerung: Die identifizierten Komponenten der Betätigungsbalance könnten künftig im klinischen Alltag im Umgang mit den Eltern von frühgeborenen Kindern berücksichtigt werden. Zusätzlich sollten diese als auch die Mehrdimensionalität des Konstrukts bei der Messung von Betätigungsbalance mit einbezogen werden.
P019

Verstehen, Bewältigen, Sinn stiften - Ein nicht-direktiver Ansatz zur Förderung der Elternrolle in der Neonatologie

P. Hinner ${ }^{1}$, M. Sommerfeld ${ }^{1}$, M. Rüdiger ${ }^{1}$, J. Reichert ${ }^{1}$

'Universitätsklinikum Carl Gustav Carus Kinderklinik, Neonatologie/

Pädiatrische Intensivmedizin, Dresden, Deutschland

Hintergrund: Die familienzentrierte Versorgung und Behandlung von frühgeborenen Kindern bildet zunehmend einen Schwerpunkt in den Therapiekonzepten der Perinatalzentren; dabei werden angemessene Inhalte und die praktische Umsetzung dieses Ansatzes anhaltend diskutiert. Thomson und Kollegen haben das Konstrukt des Kohärenzgefühls nach Antonovsky in die Debatte eingebracht, wonach die Verstehbarkeit, Bewältigbarkeit und Sinnhaftigkeit im Umgang mit der Frühgeburt den Grundgedanken der familienzentrierten Versorgung darstellen sollte (1, 2). Dass Eltern dem eigenen Handeln wieder einen Sinn geben können, wird als wesentliche Motivation betrachtet, mit dem eigenen Kind zu interagieren und so eine angemessene Eltern-Kind-Beziehung zu gestalten. Ein Gespräch mit den Eltern, das videogestützt das Versorgen des Kindes durch ein Elternteil thematisiert und der nicht-direktiven Gesprächsführung folgt, kann der Stärkung des elterlichen Kohärenzerlebens dienen.

Fragestellung: Welchen Beitrag leisten nicht-direktive, videogestützte Gespräche mit den Eltern zur Stärkung ihres Kohärenzerlebens und zur Auseinandersetzung mit ihrer Elternrolle?

Material und Methode: In die Datenanalyse werden Eltern, deren Kinder mit einem Geburtsgewicht unter 1500 g geboren wurden, einbezogen. Ausgewählte Videoszenen vom Baden des Kindes durch seine, darin unterwiesenen Eltern bilden den Ausgangspunkt des Gesprächs. Es wird ein Evaluationsbogen mit quantitativen und qualitativen Fragen an die Eltern ausgegeben. Ergänzend finden narrative Interviews mit den Eltern zum wahrgenommen Nutzen der Intervention statt.

Ergebnisse: Seit 2012 wurden pro Jahr durchschnittlich 67 videogestützte Gespräche durchgeführt. Bei einer Rücklaufquote von $67 \%$ ergab die Auswertung von 153 Feedbackbögen, dass Eltern eine hohe Akzeptanz für das Angebot aufbringen. Besondere Wertschätzung erfahren dabei die Möglichkeiten, das eigene sowie kindliche Verhalten zu beobachten und in seiner gegenseitigen Wechselwirkung reflektieren zu können. Die qualitative Inhaltsanalyse der narrativen Interviews $(n=5)$ wird nach Abschluss der Auswertungen weitere Aussagen zum Zusammenhang der Intervention mit dem Kohärenzerleben der Eltern erlauben.

Diskussion: Das videogestützte Gespräch stellt eine Maßnahme dar, um die Selbstreflektion von Eltern nach einer Frühgeburt zu fördern. Im Sinne der Selbstaktualisierung wird es ermöglicht, internale Inhalte der Elternrolle zu verbalisieren und insbesondere kind- und interaktionsbezogene Gefühle zu äußern. So wird die Verstehbarkeit, Bewältigbarkeit und Sinnhaftigkeit der Interaktion mit dem eigenen Kind erlebbar gemacht. Die Rolle des Beraters und die Art der Gesprächsführung stehen dabei beispielhaft für eine - nicht-direktive - familienzentrierte Versorgung, welche die Förderung des elterlichen Kohärenzgefühls in den Vordergrund stellt.

\section{Literatur}

1. Thomson G et al. BMC Pediatr 2013;13:84

2. Antonovsky. San Francisco, Jossey-Bass Publishers, 1979

\section{P020}

\section{Neue Medien für Eltern früh und krank neugeborener Kinder}

\section{J. Bergander', J. Hennig', J. Reichert', M. Rüdiger ${ }^{2}$}

'Universitätsklinikum Carl Gustav Carus Kinderklinik, Neonatologie/ Pädiatrische Intensivmedizin, Dresden, Deutschland, ${ }^{2}$ Univ. Klinikum Carl Gustav Carus, Klinik und Poliklinik für Kinder- und Jugendmedizin, Neonatologie, Pädiatrische Intensivmedizin, Dresden, Deutschland

Hintergrund: Eine zu frühe Geburt oder die Geburt eines kranken Kindes führt bei vielen Eltern zu einer starken Belastung. Die Trennung vom Kind zieht eine Beeinträchtigung in der Ausübung der Elternrolle nach sich. Dabei hat gerade der elterliche Umgang mit dem Kind eine große Bedeutung bei der Bewältigung der zu frühen Geburt oder der Auseinandersetzung 
mit gesundheitlichen Einschränkungen. In diesem Zusammenhang wurde im November 2012 auf der Kinderintensivstation des Universitätsklinikums Dresden ein Tagebuch für Eltern eingeführt. Das Tagebuch-Schreiben kann nach der „Facilitated Sensemaking Theory“ (Davidson, 2010) Eltern unterstützen, ihren Erfahrungen einen Sinn zu geben und sich mit ihrer neuen Rolle zu identifizieren. Ein digitales Tagebuch könnte dem Bedarf an Tagebüchern entsprechen und der zunehmenden „Digitalisierung “ von Informationssammlung und -austausch gerecht werden. In diesem Zusammenhang war von Interesse, in welchem Umfang Eltern Frühgeborener „Neue Medien“ für sich nutzen. Fragestellung: Wie gestalten Eltern Frühgeborener ihren Erfahrungsaustausch in einer Frühchen-Elterngruppe auf einer Social-Media-Plattform?

Methodik: Durchführung einer Online-Befragung einer Frühchen-Elterngruppe $^{\star}$ auf einer Social-Media-Plattform; erfasst wurden neben ausgewählten Angaben zu Eltern und Kindern vor allem das Nutzungsverhalten (Dauer und Häufigkeit, Gründe, Kontaktgestaltung).

Ergebnisse: Die Literaturanalyse zeigt in den vergangenen 5 Jahren eine stetige Zunahme der Nutzung von stationärem sowie mobilem Internet; mehr als $40 \%$ der 30-49-jährigen nutzen täglich Apps. Davon sind auch die Eltern früh und krank neugeborener Kinder nicht ausgenommen. Die 47 Befragten der Online-Elterngruppe, die derzeit ca. 190 aktive und passive Mitglieder umfasst, sind zum größten Teil zwischen 26 und 40 Jahren alt. Fast $50 \%$ der Befragten der Gruppe besuchen diese mehrmals täglich online, um mit anderen Eltern in Kontakt zu bleiben, die Entwicklung der Familien zu verfolgen und miteinander in Erfahrungsaustausch zu treten. Diskussion: Es zeigt sich, dass die Aktualität von digitalen Medien in den vergangenen Jahren deutlich zugenommen hat und sich die Suche nach Informationen und der Austausch mit anderen auch zukünftig noch mehr in den virtuellen Bereich hinein verlagern werden. Auch die Befragung einer Online-Gruppe von Eltern Frühgeborener macht deutlich, dass hier der digitale Austausch einen erheblichen Stellenwert hat. Die künftige psychosoziale Begleitung von Eltern mit einem früh oder auch krank neugeborener Kind muss sich dieser „digitalisierten Herausforderung“ stellen, mit der einerseits zwar ein niederschwelliger Zugang zu Eltern möglich wird, andererseits aber auch die Verantwortung für Form und Inhalt des digitalisierten Informations- und Erfahrungsangebots wächst.

${ }^{*}$ Die Online-Gruppe wurde durch eine Kinderkrankenschwester initiiert und stellt heute eine wichtige Austauchplattform für beteiligte Eltern dar.

\section{P021}

\section{Aufbau einer Frauenmilchbank am Perinatalzentrum der LMU-München, Großhadern}

V. Lieftüchter', S. Herber-Jonat' ', M. Kujawa' , M. Klemme', K. Förster', A. Schulze', A. Flemmer

${ }^{1}$ Klinikum der Universität München, Marchioninistraße 15, 81377 München, Neonatologie der Kinderklinik am Perinatalzentrum Großhadern, München, Deutschland

Hintergrund: In Deutschland liegt die Rate an operationsbedürftiger nekrotisierender Enterokolitis oder fokaler intestinaler Perforation als Komplikation extremer Frühgeburtlichkeit ( $<32$ SSW oder $<1500$ g GG) im Mittel bei 5-6\%. Eine exklusive Frauenmilchernährung scheint das Risiko schwerwiegender gastrointestinaler Komplikationen in dieser Patientengruppe zu senken. Aus diesem Grund wird empfohlen, auch extrem unreife Frühgeborene nach der Geburt bis zum Erhalt von Milch der eigenen Mutter mit pasteurisierter Spender-Frauenmilch zu ernähren. Pasteurisierung senkt zwar das Risiko einer durch Milch übertragenen bakteriellen oder viralen Infektion, es werden aber auch wichtige Bestandteile der Frauenmilch dadurch in ihrer Konzentration vermindert oder zerstört. Im Jahr 2012 wurde eine Frauenmilchbank (FMB) an unserem Perinatalzentrum etabliert. Ziel war es, durch die Auswahl der Spenderinnen, Milchgewinnung und Umgang mit der gespendeten Milch einen großen Anteil der Spendermilch unpasteurisiert zu verfüttern. Wir berichten über die Erfahrungen der ersten vier Jahre nach Einführung der FMB an unserem Zentrum. Als Spenderinnen wurden 38 Mütter rekrutiert, die vor der Spende wie bei einer Blutspende untersucht wurden. Für eine Frauen- milchspende kamen nur gesunde Mütter infrage (HIV, HBV, HCV, CMV, Lues und Toxoplasmose negativ), die über eine ausreichende Milchmenge $(500 \mathrm{ml} / \mathrm{d})$ verfügen. Von jeder gewonnen Portion wurde vor dem Schockfrosten eine Probe gewonnen und bakteriologisch untersucht. Die Milch wurde nur verwendet, wenn sie festgelegten Kriterien für Kolonisierung genügte.

Ergebnisse: Bisher wurden 90L Milch gesammelt, wovon 72,5 L verfüttert wurden, 68 L (94\%) davon unpasteurisiert. Seit Einführung der FMB 2012 wurden am PNZ 194 Kinder mit einem Geburtsgewicht unter $1250 \mathrm{~g}$ geboren. Von diesen Kindern erhielten 164 Kinder (85\%) Spendermilch. In zehn Jahren vor Einführung der FMB lag an unserem Zentrum die NEC-Rate (Bell-Stadium >2) im Mittel bei 3,2\% aller Kinder unter $1500 \mathrm{~g}$. Nach Einführung der FMB und Verfütterung von überwiegend unpasteurisierter Frauenmilch wurde vom ersten Tag an eine NEC beobachtet.

Schlussfolgerung: Der aufwändige und kostspielige Aufbau einer FMB an einem Perinatalzentrum ermöglicht es, unter Einhaltung strenger Hygienerichtlinien, auch extreme Frühgeborene exklusiv mit roher Frauenmilch zu versorgen. Dadurch scheint die Häufigkeit einer nekrotisierenden Enterokolitis vermindert werden zu können

\section{P022}

Monitoring des Stillerfolgs auf täglicher Basis in der Neonatologie der Uniklinik Köln

\section{T. Keller', R. Klein' ', A. Kribs'}

'Universitätskinderklinik Köln, Neonatologie und Pädiatrische Intensivmedizin, Köln, Deutschland

Hintergrund: Insbesondere Frühgeborene (FG) und kranke Neugeborene (NG) profitieren von Muttermilchernährung und Stillen. Erfolgreiches Stillen ist jedoch gerade für diese Gruppe von Neugeborenen erfahrungsgemäß besonders schwierig zu erreichen. Daten zum tatsächlichen Stillerfolg dieser Kinder auf neonatalen Intensiv- und Normalstationen fehlen bisher ebenso wie ein Instrument zur Bestimmung des Stillerfolges für diesen Bereich.

Fragestellung: Wie ist der Stillerfolg in der Neonatologie der Uniklinik Köln?

Patienten und Methoden: Dokumentation des Stillerfolges für NG, die zwischen dem 1.3.-31.7.2015 geboren wurden und stationär waren in der Neonatologie der Uni Köln (Säuglingsstation, Frühgeborenenstation, neonatologische Intensivstation und pädiatrische Intensivstation) mithilfe eines eigens dafür entworfenen Scores (Scorewerte 6: Vollgestillt, 5: teilgestillt \& voll Muttermilch (MM), 4: teilgestillt \& teil MM, 3: nicht gestillt \& voll MM, 2: nicht gestillt \& teil MM, 1: keine MM, 0: Karenz). Tägliche Dokumentation mit Visualisierung des Trends. Dokumentation von Gründen bei Abfallen des Scorewertes. Zusätzlich zu Basisdaten zum Mutterkindpaar, Zeitpunkt des ersten Hautkontaktes, des ersten Anlegens, Elternbefragung zu Ausbildung, Ethnizität, Familienstand und Stillerfahrung. Ergebnisse: Von 243 möglichen Fällen lagen in 179 (74\%) vollständige Bögen vor. Bei Entlassung waren $73 \%$ der Kinder gestillt, davon $38 \%$ ausschließlich. Muttermilch erhielten $85 \%$ der Kinder, davon $51 \%$ ausschließlich. Primär abgestillt worden waren 11 Frauen $(4 \times$ Wunsch der Mutter, $1 \times$ psychische Erkrankung, $1 \times$ Adoption, $4 \times$ k. A.). Für die übrigen Frauen war der Stillerfolg bei Entlassung bei $77 \%$ gestillt, davon $38 \%$

\section{Tab. P022 Nach Gestationsalter}

\begin{tabular}{|llllll}
\hline GA (SSW) & $n=$ & gestillt (\%) & voll (\%) & MM (\%) & $\begin{array}{l}\text { nur MM } \\
(\%)\end{array}$ \\
\hline$>=37+0$ & 76 & $65(86)$ & $27(42)$ & $72(95)$ & $43(60)$ \\
\hline $34+0-36+6$ & 32 & $22(69)$ & $8(36)$ & $31(97)$ & $13(42)$ \\
\hline $32+0-33+6$ & 24 & $21(88)$ & $9(43)$ & $22(92)$ & $16(73)$ \\
\hline $28+0-31+6$ & 16 & $11(69)$ & $5(45)$ & $12(75)$ & $9(75)$ \\
\hline$<=28+0$ & 22 & $12(55)$ & $1(8)$ & $16(73)$ & $12(75)$ \\
\hline
\end{tabular}


ausschließlich gestillt. MM erhielten bei Entlassung 90\% der Kinder, davon $61 \%$ ausschließlich.

Diskussion: Die Rate der bei Entlassung mit Muttermilch ernährten Kinder war mit $85 \%$ vergleichbar mit der Stillrate gesunder Neugeborener in Deutschland zwischen 81,5\% (2005 KiGGS) und 86\% (2002 SuSe). Die Stillrate selbst lag mit $73 \%$ in der Gesamtgruppe niedriger, wobei mehr als die Hälfte der Kinder frühgeboren waren. Während die Stillrate der Reifgeborenen mit $86 \%$ der zu erwartenden Rate für gesunde NG entspricht, ist sie für Frühgeborene deutlich niedriger mit $70 \%$. Hier besteht offensichtlich Unterstützungsbedarf. Überraschend zeigten die späten Frühgeborenen eine schlechtere Stillrate bei Entlassung als die etwas früheren Frühgeborenen. Denkbar ist hier ein Effekt durch die konkurrierenden Ziele "frühzeitige Entlassung “ und „volles Stillen“. Der entworfene Score erlaubte die Beschreibung des Stillerfolges differenzierter als allein die Stillrate. Die Visualisierung des Stillerfolges erleichterte das Erkennen von Problemen sowie die Zielformulierung bei der täglichen Visite.

Schlussfolgerung: Es besteht Unterstützungsbedarf für die Verbesserung der Stillraten Frühgeborener bei Entlassung. Offensichtlich besteht auch für die späten Frühgeborenen ein Bedarf für eine Verbesserung des Stillerfolges.

\section{Postersitzung 04: Infektion/Immunologie}

\section{P023 \\ Rifampicin-Miconazol-inkorporierte perkutane zentrale Venenkatheter in der Neonatologie: Ein Pilotprojekt}

\section{A. Daun' ', H. Buxmann' , B. Wittekindt'², R. Schlößer ${ }^{1}$}

${ }^{1}$ Klinikum der J.W. Goethe Universität, Klinik für Kinder- und Jugendmedizin, Neonatologie, Frankfurt am Main, Deutschland, ${ }^{2}$ Klinikum der J.W.GoetheUniv. Zentrum der Kinderheilkunde, Neonatologie, Frankfurt, Deutschland

Hintergrund: Rifampicin-Miconazol-inkorporierte zentrale Venenkatheter führten bei Studien an Erwachsenen Patienten im Vergleich zu zentralen Venenkatheter ohne antimikrobiell wirksame Agenzien zu einer signifikanten Reduktion der klinischen Infektionsrate, der Bakteriämieund der Kolonisationsrate. Für neonatologische Patienten existiert ein Rifampicin-Miconazol-inkorporierter perkutaner zentraler Venenkatheter (RMipZVK). Daten über gewünschte oder unerwünschte Effekte bei diesem Patientenkollektiv sind rar.

Zielsetzung: Primärer Endpunkt des Pilotprojektes war die Erfassung der Rate an ZVK-assoziierten Septitiden bei Verwendung von RMipZVK im Vergleich zu perkutanen zentralen Venenkathetern (pZVK) ohne antimikrobielle Agenzien bei Neugeborenen. Sekundäre Endpunkte waren: Bakteriämien und ZVK-Kolonisationen im Rahmen von ZVK-assoziierten Septitiden.

\begin{tabular}{|c|c|c|}
\hline Parameter & RMipZVK-Gruppe & pZVK-Gruppe \\
\hline Kathetertage & 117 & 114 \\
\hline ZVK-assoziierte Sepsis & 1 & 4 \\
\hline Keimnachweis an ZVK-Spitze & Kein & $\begin{array}{l}\text { 1x Staph. epi- } \\
\text { derm. } \\
\text { 1x Staph. war- } \\
\text { neri* }\end{array}$ \\
\hline $\begin{array}{l}\text { Keimnachweis in Blutkultur } \\
\text { (BK) }\end{array}$ & Kein & $\begin{array}{l}\text { 2x Staph. capitis } \\
1 \times \text { Staph. epi- } \\
\text { derm. }\end{array}$ \\
\hline $\begin{array}{l}\text { Reife der Patienten (SSW; } \\
\text { Range) }\end{array}$ & $27+4-41+3$ & $25+1-41+1$ \\
\hline Geburtsgewicht (g; Range) & $940-4400$ & $620-4400$ \\
\hline
\end{tabular}

Material und Methoden: Im Rahmen einer Fall-Kontroll-Analyse wurden für neonatologische Patienten mit RMipZVK und solche mit pZVK ohne antimikrobielle Agenzien folgende Parameter erhoben: ZVK-assoziierte Sepsis nach Neo-KISS Kriterien, ZVK-Tage, Bakteriämien und Keimnachweis an der ZVK-Spitze. Alle Fälle wurden hinsichtlich des Geschlechtes und der Grunderkrankung (Frühgeburtlichkeit, Fehlbildung, Asphyxie) gematched.

Ergebnisse: 24 Neonaten wurden in die Analyse eingeschlossen: 13 erhielten einen RMipZVK und 11 einen pZVK ohne antimikrobielle Agenzien. Bei zwei Neonaten wurden beide Arten von ZVKs gelegt, sie waren daher ihr eigener Match-Partner. Die Grunderkrankungen waren beiden Gruppen: $8 x$ Frühgeburtlichkeit, $3 \times$ Fehlbildung und $2 \times$ Asphyxie. Wesentliche Ergebnisse tabellarisch: siehe $\bullet$ Tab. P023.

Diskussion: In dieser Pilotstudie zeigte sich eine Tendenz zu weniger ZVK-assoziierten Septitiden bei der Verwendung von RMIpZVK im Vergleich zu pZVK ohne antimikrobielle Agenzien. Kritisch anzumerken sind die kleine Fallzahl dieser Pilotstudie und die fehlende Randomisierung. Weiterhin wurden keine möglichen unerwünschten Wirkungen wie z. B. Toxizität und Blutspiegel der in die RMipZVK inkorporierten antimikrobiellen Agenzien, Veränderungen der Keimbesiedlung der Neonaten und Veränderungen von Keimresistenzen untersucht.

Schlussfolgerung: Rifampicin und Miconazol inkorporierte ZVKs könnten bei neonatologischen Patienten das Potential haben, die Rate an ZVK-assoziierten Septitiden zu reduzieren. Prospektive randomisierte Studien bei Neugeborenen sind dringend notwendig, um diesen möglichen Effekt zu prüfen und denkbare unerwünschte Wirkungen dieser Katheter zu erfassen.

\section{P024}

\section{Pathogen-spezifische Clusterhäufigkeit nosokomialer} Septikämien bei Frühgeborenen unter $1500 \mathrm{~g}$ Geburtsgewicht

F. Reichert', B. Piening ${ }^{2}$, C. Geffers², P. Gastmeier ${ }^{2}$, F. Schwab², C. Bührer ${ }^{1}$ ${ }^{1} K$ linik für Neonatologie, Charité Universitätsmedizin Berlin, Berlin, Deutschland, 'Institut für Hygiene und Umweltmedizin, Charité Universitätsmedizin Berlin, Berlin, Deutschland

Hintergrund: Nosokomiale Infektionen treten oft gruppiert auf, schlimmstenfalls als Ausbrüche. Für die Abschätzung der Wahrscheinlichkeit eines solchen gruppierten Auftretens in Abhängigkeit vom jeweiligen Keim fehlten bisher solide Daten.

Fragestellung: Quantifizierung der pathogen-spezifischen Wahrscheinlichkeit einer Sepsis bei Frühgeborenen unter $1500 \mathrm{~g}$ Geburtsgewicht auf neonatologischen Intensivstationen im Gefolge eines Index-Falls.

Material und Methoden: Basierend auf Daten von in NeoKISS erfassten Frühgeborenen unter $1500 \mathrm{~g}$ Geburtsgewicht ( $n=44$,818, Jahrgänge 20002011) wurde das relative Risiko einer Sepsis mit einem bestimmten Keim nach Auftreten einer ersten Sepsis mit dem gleichen Keim auf der Station berechnet, entweder bei Überlappung der Aufenthalte oder innerhalb der ersten 30 Tage nach Auftreten der ersten Sepsis.

Ergebnisse: Das relative Risiko lag für die meisten Pathogene in einem ähnlichen Bereich (Enterokokken: 4,3 [95\% Konfidenzeintervall 2,7-6,9]; Enterobacter: 7,9 [5,4-11,4]; E. coli: 7,9 [5,1-12,1]; Candida albicans: 8,7 [5,0-15,4]; Staphylococcus aureus: 9,5 [7,6-12,1]; Klebsiellen: 13,1 [9,019,1]: Demgegenüber lag das relative Risiko bei Serratien (77,5 [41,1$146,1]$ und Pseudomonas aeruginosa $(64,5$ [25,7-162,1]) sehr viel höher. Die Sepsis-Raten bezogen auf 100 exponierte Kinder betrugen zwischen 2,21 (Enterokokken) und 8,15 (Serratien). Adjustierungen für Patientenmerkmale oder eine Auswertung bezogen auf positive Blutkulturen innerhalb von 30 Tagen ergaben ein ähnliches Muster.

Schlussfolgerung: Nosokomiale Infektionen mit Serratien oder Pseudomonas aeruginosa auf Neugeborenen-Intensivstationen sind mit einem stark erhöhten Risiko für weitere Infektionen mit diesen Keimen bei anderen Kindern auf der gleichen Station vergesellschaftet. Dies begründet bereits bei der ersten Identifizierung einer der beiden Keime auf einer Neugeborenen-Intensivstation verschärfte Hygienemaßnahmen. 
P025

\section{Abnahmehäufigkeit von Blutkulturen in einer neonatologischen Abteilung}

\section{J. Christoph', F. Guthmann'}

'Kinderkrankenhaus auf der Bult, Neonatologie, Hannover, Deutschland

Hintergrund: Septische Infektionen sind mit zunehmender Unreife Neugeborener häufiger und schwerwiegender, insbesondere im Vergleich mit Erwachsenen. Sepsisraten werden zu externen Qualitätssicherungszwecken u. a. in NEO-KISS erfasst. Ein positiver Zusammenhang zwischen Sepsis-Häufigkeit und Blutkulturfrequenz wurde berichtet1, hierzu gibt es wenige neonatologische Daten. Bei Frühgeborenen mit einem Geburtsgewicht $\geq 2000$ g erfolgte bei $15,2 \%$ eine Blutkulturdiagnostik ${ }^{2}$. Im internationalen Vergleich werden in Deutschland nur wenige Blutkulturen abgenommen ${ }^{3}$.

Fragestellung: Generierung von Kennzahlen der Blutkulturfrequenz bei Frühgeborenen mit einem Geburtsgewicht $<1500 \mathrm{~g}$ (VLBW).

Material und Methoden: Abfrage aller Blutkulturen (aerob und anaerob, peripher oder aus venösen oder arteriellen Kathetern) Frühgeborener mit einem Geburtsgewicht $<1500 \mathrm{~g}$ aus unserer Labor-EDV in den Jahren 2012 bis Juni 2015 (wegen danach einstweilen unvollständiger Behandlungsverläufe). Die ersten 3 Lebenstage (Alter 0 bis 2 Tage) wurden von der Diagnostik nosokomialer Infektionen abgegrenzt (die Geburtsstunde ist nicht in unserer Labor-EDV erfasst). Zur Vereinfachung der orientierenden Auswertung für den Zeitraum unter NEO-KISS-Surveillance wurden Blutkulturen ab Tag 80 einem Gewicht über 1800 g zugeordnet. Angesichts niedriger Sepsisraten ( $<3$ Sepsen/1000 Patiententage) in der NEO-KISS-Surveillance-Spätphase versus bis zu 20/1000 Patiententage in den ersten Wochen sehr kleiner Frühgeborener (Schwab4: Einfluß des Lebenstages auf Surveillancedaten; Leistner ${ }^{5}$ ) erschien dies vertretbar. Ergebnisse: In 2012, 2013 \& 2015 hatten 68-72\% der VLBW eine Blutkultur in den ersten 3 Lebenstagen (Alter $0-2$ Tage), in 2014 weniger. Von Tag 3-79 bekam gemittelt jedes Frühgeborene eine Kulturdiagnostik (0,921,40 pro Pat.), entsprechend 29-43 pro 1000 geschätzten (s. o.) NEO-KISSTagen (bis 1800 g, Verlegung oder Entlassung, außerhalb der ersten 3 Lebenstage) bzw. 33 bis 38 pro 1000 Tage im gesamten Verlauf von Aufnahme bis zur Entlassung. 13\% der Blutkulturdiagnostik erfolgte im Mittel der 3112 Jahre nach dem 80. Lebenstag.

Diskussion: Aufnahmediagnostik und Surveillance nosokomialer Frühgeborenen-Infektionen erfordern unterschiedliche Kennzahlen. Unsere Blutkulturfrequenz pro 1000 Patiententage liegt unter dem Median für 2006 von ITS-KISS (60/1000 Tage) ${ }^{1}$ und deutlich unter der Empfehlung von 100 Blutkultursets pro 1000 Erwachsenen-Patiententage ${ }^{6}$ (bei einer primären Sepsisrate von im Mittel 0,9 und im Median von 0,46/1000 Tage in 2006) ${ }^{1}$. Aus Budgetgründen und zur Schonung der kleinen Frühgeborenen mag auf Blutkulturentnahmen verzichtet werden.

Damit entsteht die Gefahr einer Unterdiagnostik septischer Infektionen und, vor allem angesichts zunehmender Antibiotikaresistenzen, einer inadäquaten Therapie.

\section{Literatur}

'Gastmeier 2011, ${ }^{2}$ Escobar 2000, ${ }^{3}$ Meyer 2015, ${ }^{4}$ Schwab 2010, ${ }^{5}$ Leistner 2013, ${ }^{6}$ Karch 2015

\section{P026 \\ Frühmarker nosokomialer Infektionen bei Neu- und Frühgeborenen}

\section{Kuntz' , P. Franck', R. Hentschel', H. Fuchs ${ }^{1}$}

'Universitätsklinikum Freiburg, Zentrum für Kinder- und Jugendmedizin, Klinik für Allgemeine Kinder- und Jugendmedizin, Funktionsbereich Neonatologie und päd. Intensivmedizin, Freiburg, Deutschland

Hintergrund: Nosokomiale Infektionen tragen erheblich zu Morbidität und Mortalität bei Früh- und kranken Neugeborenen bei. Die frühzeitige Erkennung und Behandlung einer Sepsis ist jedoch für das Outcome wesentlich. Das C-reaktive Protein (CRP) wird als inflammatorischer Marker für die Erkennung von Infektionen weitverbreitet eingesetzt. Nachteil ist ein - im Vergleich zu Procalcitonin (PCT) oder Interleukin (IL)-6 - verzögerter Anstieg, sodass eine Infektion in der Frühphase übersehen werden kann. In unserer Institution werden IL-6 und PCT nach dem 1. Lebenstag nicht zur Diagnose oder zum Ausschluss einer Infektion bestimmt. Auch die I/T-Ratio ist nur zu Routine-Arbeitszeiten verfügbar.

Fragestellung: Wie häufig hätte die zusätzliche Bestimmung von IL-6 und/ oder PCT parallel zum CRP eine frühere Erkennung und Behandlung nosokomialer Blutstrominfektionen erlauben können?

Material und Methoden: Wir analysierten retrospektiv alle Blutkultur-positiven septischen Episoden aus der Neonatalerhebung der Jahre 20122014 in unserer Institution. Als Beginn der Episode wurde der Beginn (oder die Umstellung) einer antibiotischen Behandlung definiert. Gesondert analysiert wurden jene Fälle, in denen max. $48 \mathrm{~h}$ vor Therapiebeginn der Sepsis ein weiteres, negatives CRP dokumentiert war.

Ergebnisse: Im untersuchten Zeitraum konnten 43 Blutkultur-positive septische Episoden bei 35 Patienten erfasst werden. Das mediane Gestationsalter der Patienten lag bei 28+3/7 Wochen, das mediane Geburtsgewicht bei $1050 \mathrm{~g}$. Am häufigsten wurden KNS isoliert (28 Episoden). 39 Episoden waren mit dem Vorhandensein eines zentralen Venenkatheters (ZVK) assoziiert. Es konnten 9 Episoden (entspr. $21 \%$ ) identifiziert werden, bei denen innerhalb von $48 \mathrm{~h}$ vor Diagnosestellung und Therapiebeginn mindestens ein CRP $<10 \mathrm{mg} / \mathrm{l}$ dokumentiert war. In 7 Fällen handelte es sich um ZVK-assoziierte KNS-Septitiden, in einem Fall um eine Infektion mit gleichzeitigem Nachweis von Staphylococcus epidermidis und Escherichia coli, sowie in einem Fall um eine septische Meningitis mit Nachweis von Escherichia coli (bei Meningomyelocele). In 4 Fällen wurde die Therapie aus dem klinischen Verdacht heraus begonnen, obwohl das CRP weiterhin $<10 \mathrm{mg} / \mathrm{l}$ war und erst später anstieg.

Diskussion: In der überwiegenden Zahl der Fälle (34 entspr. 79\%) zeigte sich in unserer Kohorte bereits zum Zeitpunkt des ersten klinischen Verdachts das CRP erhöht, sodass die Hinzunahme weiterer Biomarker keinen Nutzen erbracht hätte. Bei 9 Episoden (21\%) war in den 48 h vor Diagnose der Infektion aufgrund eines klinischen Verdachts bereits ein CRP bestimmt worden - da dieses negativ ausfiel, auf eine Behandlung jedoch zunächst verzichtet worden. Es ist denkbar, dass zumindest in einem Teil dieser Fälle die parallele Bestimmung von IL-6 und/oder PCT zu einer früheren Diagnose und Behandlung der Infektion geführt hätte. Zur Prüfung dieser Hypothese ist eine prospektive Studie in Planung.

\section{P027}

Ein intensiviertes Kolonisationsscreening- nach den Empfehlungen der Kommission für Krankenhaushygiene und Infektionsprävention (KRINKO)- identifizierte ein Cluster von Serratia marcescens in der Neonatologie

K. Dawczynski', H. Proquitté', J. Rödel', B. Edel' ${ }^{2}$, Y. Pfeifer ${ }^{3}$, H. Dobermann ${ }^{4}$ H. Hoyer ${ }^{5}$, M. Pletz

'Klinik für Kinder-und Jugendmedizin der FSU Jena, Neonatologie und Pädiatrische Intensivmedizin, Jena, Deutschland, ${ }^{2}$ Insititut für Medizinische Mikrobiologie, Jena, Deutschland, ${ }^{3}$ Robert-Koch-Institut, Wernigerrode, Deutschland, ${ }^{2}$ Zentrum für Infektionsmedizin und Krankenhaushygiene, Jena, Deutschland, Insitut für Medizinische Statistik, Informatik und Dokumentation, Jena, Deutschland

Die Kolonisation mit pathogenen oder multiresistenten Erregern stellt ein erhöhtes Infektionsrisiko für Früh-u. Neugeborene auf einer Neugeborenen Intensivstation (NICU) dar. Ende 2013 hat die Kommission für die Krankenhaushygiene- und Infektionskrankheitsprävention (KRINKO) die Empfehlungen für das wöchentliche Kolonisationsscreening im Bereich der Neonatologie von sehr kleinen Frühgeborenen (VLBW, Gewicht $<1500$ g) auf alle Patienten der NICU erweitert. Nach dem Einführen dieser Empfehlung wurde ein zuvor unbemerktes Cluster von Serratia marcescens (S. marcescens) Besiedlungen entdeckt. Eine Fall-Kontroll-Studie wurde durchgeführt, um Risikofaktoren für S. marcescens Besiedlung zu identifizieren. Über einem Zeitraum von 6 Monaten wurden 19 von 159 Säuglingen mit S. marcescens Besiedlungen entdeckt. Signifikante Unter- 
schiede zwischen kolonisierten und nichtkolonisierten Patienten zeigten sich im Geburtsgewicht (MW: 1593 vs. 2428 g; $p<0.01$ ), Gestationsalter (31.6 vs. 34.9 Wochen; $p<0.01$ ) sowie in der Länge des stationären Aufenthaltes (Median: 35 vs. 7 d; $p<0.01$ ). Zwölf von 19 kolonisierten Patienten waren non-VLBW. Frühgeborene mit einem Geburtsgewicht von mehr als $1500 \mathrm{~g}$ (non-VLBW) wurden bedeutend früher mit S. marcescens kolonisiert als VLBW Patienten (Median: 17 vs. 28 Tage; $p<0.01$ ). Unter der Adjustierung der Länge des stationären Aufenthaltes (LOS) zeigten nonVLBW Patienten ein höheres Risiko für eine S. marcescens Besiedlung als VLBW Patienten (OR 8.6; CI 1.66-44.5; $p=0.01$ ). Die Puls-Feld-Gelelektrophorese (PFGE) offenbarte einen mono- sowie polyklonalen-Prozess. Das größte monoklonale Cluster umfasste 8 Patienten. Ausgedehnte Umgebungsuntersuchungen konnten keinen Fokus für S. marcescens ermitteln. Einen Monat nach dem Einführen eines Maßnahmenbündels konnte keine weitere Häufung von S. marcescens Besiedlungen nachgewiesen werden.

\section{P028}

\section{Reduced TNFa Response in Preterm Neonates is Associated with Impaired Non-Classical Monocyte Function}

\section{Wisgrill', E. Herndl', K. Sadeghi' ', A. Spittler ${ }^{2}$, A. Berger' ${ }^{1}$, E. Förster-Waldl'}

${ }^{1}$ Medical University of Vienna, Department of Pediatrics \& Adolescent

Medicine - Division Neonatology, Vienna, Österreich, ${ }^{2}$ Medical University of Vienna, Department of Surgery, Wien, Österreich

Background: Premature infants are highly susceptible to severe bacteria infections. The impaired infection control related to the functional immaturity of the neonatal innate immune system is an important cause of infections. Different monocyte subpopulations have been described and functionally characterized. Data from preterm infants, however, are scarce. Methods: We analyzed constitutive monocyte TLR2, TLR4, CD163 and HLA-DR expression in preterm cord blood. We further investigated activation of the signaling proteins ERK1/2 and NFkB in monocyte subpopulations after ex vivo stimulation with the bacterial TLR agonists LPS and LTA. The functional outcome of the stimulation was determined by the intracellular production of TNFa. Furthermore, the phagocytic activity was measured via flow cytometry.

Results: TLR4 and HLA-DR showed a gestational age dependent increase. However, activation of ERK1/2 and NFkB was impaired in neonatal monocyte subpopulations after stimulation with TLR agonists. Accordingly, intracellular TNFa was diminished in preterm monocytes, especially in non-classical monocytes. Premature monocytes showed high phagocytic activity with significantly lower acidification of the phagosome.

Discussion: The reduced functional response of non-classical monocytes of preterm neonates appears to be part of the diminished early immune response to bacterial cell wall components and is likely to contribute to their susceptibility to bacterial infections.

\section{P029}

\section{Auswirkungen von exogenem pulmonalem Surfactant auf umbilikale MSCs}

S. Koss' ${ }^{1}$, M. Möbius' ${ }^{1}$ D. Freund ${ }^{2}$, M. Rüdiger ${ }^{3}$

${ }^{1}$ Universitätsklinikum Carl Gustav Carus und DFG Research Center and Cluster of Excellence for Regenerative Therapies (CRTD), Dresden, Klinik und Poliklinik für Kinder- und Jugendmedizin, Neonatologie, Pädiatrische Intensivmedizin, Dresden, Deutschland, ${ }^{2}$ DFG Research Center and Cluster of Excellence for Regenerative Therapies (CRTD), Dresden, Deutschland, ${ }^{3}$ Universitätsklinikum Carl Gustav Carus und DFG Research Center and Cluster of Excellence for Regenerative Therapies (CRTD), Dresden, Klinik und Poliklinik für Kinder- und Jugendmedizin, Neonatologie, Pädiatrische Intensivmedizin, Dresden, Deutschland

Hintergrund: Die Bronchopulmonale Dysplasie (BPD) ist eine schwerwiegende Lungenerkrankung von Frühgeborenen, die mit Störungen der Lungenentwicklung einhergeht. Tierexperimentelle und erste klinische Studi- en weisen auf einen therapeutischen Nutzen mesenchymaler Stamm- oder Stromazellen (MSCs) bei der Prävention bzw. Therapie der BPD hin. Dabei scheinen parakrine Effekte der Zellen eine maßgebliche Rolle zu spielen. Eine intra-tracheale Applikation der MSCs gemeinsam mit Surfactant könnte von klinischem Interesse sein, ob jedoch Surfactant die Zellen beeinträchtigt, ist nicht bekannt.

Material und Methoden: Nabelschnurgewebe reifgeborener Kinder wurde enzymatisch verdaut. Die daraus isolierten MSCs (UC-MSCs) wurden gemäß der Empfehlungen der International Society for Cellular Therapy (ISCT) charakterisiert und bis zu einer Konfluenz von etwa $80 \%$ in 5\% $\mathrm{O}_{2}$ und $5 \% \mathrm{CO}_{2}$ kultiviert. Die Zellen wurden für vier Stunden in porcinem Surfactant oder einer physiologischen NaCl-Lösung inkubiert. Die Zellen wurden dabei in Suspension gehalten. Deren Viabilität, Expressions- und Sekretionsprofil wurde mittels qPCR sowie membranbasierter Arrays verglichen.

Ergebnisse: Die Inkubation der UC-MSCs in Surfactant verringerte deren Viabilität, wobei die metabolische Aktivität der lebenden Zellen im Vergleich zur NaCl-Inkubation unverändert blieb. Die Sekretionsprofile zeigten große Unterschiede in der Konzentration der einzelnen Faktoren. Die pro-inflammatorischen Zytokine IL-23, MIF, sICAMP sowie der Serinprotease-Inhibitor Serpin E1 wurden in größeren Mengen detektiert. Interessanterweise reduzierte sich die IL-6/IL-8-Sekretion der mit Surfactant inkubierten MSCs. Ebenfalls konnte eine reduzierte Menge an der Angiogenese beteiligter Faktoren, insbesondere VEGF und PDGF-AA, nachgewiesen werden. Letztlich fand sich eine verminderte Expression des lungenprotektiven Stanniocalcin ${ }^{-1}$ der mit Surfactant inkubierten MSCs. Schlussfolgerung: Aufgrund unserer Daten muss von einer Beeinträchtigung exogener UC-MSCs durch Surfactant ausgegangen werden. Inwieweit sich dies auf die therapeutische Effizienz der MSC bei der Prävention einer BPD auswirkt, muss noch untersucht werden.

\section{P030}

\section{Reduktion der therapeutischen Kapazität umbilikaler} mesenchymaler Stromazellen durch Präeklampsie

\section{Rodriguez Cabrera' ', S. Koss' ', M. Möbius' ', K. Nitzsche², M. Rüdiger',} I. Deinert ${ }^{3}$

'Universitätsklinikum Carl Gustav Carus Kinderklinik und DFG Research Center and Cluster of Excellence for Regenerative Therapies Dresden (CRTD), Technische Universität Dresden, Neonatologie und Pädiatrische Intensivmedizin, Dresden, Deutschland, ${ }^{2}$ Klinik und Poliklinik für Frauenheilkunde und Geburtshilfe, Universitätsklinikum Carl Gustav Carus Dresden, Dresden, Deutschland, ${ }^{3}$ Universitätsklinikum Carl Gustav Carus Kinderklinik und DFG Research Center and Cluster of Excellence for Regenerative Therapies Dresden (CRTD), Technische Universität Dresden, Neonatologie und Pädiatrische Intensivmedizin, Dresden, Deutschland

Hintergrund: Die Präeklampsie ist nicht nur mit einer erhöhten maternalen sondern auch neonatalen Morbidität assoziiert; allerdings ist der $\mathrm{Pa}$ thomechanismus der kindlichen Schädigung bisher noch unzureichend verstanden. Für hämatopoetische Progenitorzellen aus dem Nabelschnurblut und zirkulierende endotheliale Progenitorzellen wurde gezeigt, dass die mütterliche Präeklampsie mit einer Reduktion der Zellzahl und der Colony-forming Efficiency assoziiert ist. Da mesenchymaler Stroma-/ Stammzellen (MSCs) aus der Wharton'schen Sulze der Nabelschnur ein großes therapeutisches Potential besitzen, wurde in der Studie untersucht, ob diese therapeutische Kapazität durch eine mütterliche Präeklampsie beeinflusst wird.

Material und Methoden: Nabelschnüre von drei reifen $(37+1,38+1,39+4$ SSW $)$ und drei frühgeborenen $(25+0,28+2,34+0$ SSW $)$ Neonaten präeklamptischer Mütter und sechs Neonaten $(25+1,28+3,34+3,38+6$, $39+0,40+0$ SSW) von gesunden Müttern wurden gesammelt. Durch mechanische Dissoziation und enzymatischen Verdau des Nabelschnurgewebes wurden die Zellen innerhalb von 24 Stunden nach Geburt isoliert. Plastikadhärenz, Differenzierungskulturen und typische Oberflächenmarkerexpression der isolierten Zellen definierten diese als MSCs. Weitere, spezifische, im Idealfall therapeutisch relevante Aktivitäten der MSCs 
wurden mittels CFU, MTT, CyQuant Proliferationsassay, Cell stress array und Cytokine Array untersucht. Die Studie wurde durch die Ethikkommission genehmigt.

Ergebnisse: Die überwiegende Anzahl (>99\%) der isolierten Zellen exprimierten die MSC-Oberflächenmarker CD73, CD90 und CD105 bei gleichzeitiger Abwesenheit $(<1 \%)$ von CD11b, CD19, CD34, CD45 und HLA-DR. Weiterhin zeigte sich eine Differenzierung entlang osteogener, adipogener und chondrogener Linien. Es zeigte sich hierbei kein Unterschied zwischen den Versuchsgruppen. Die Oberflächenmarkerexpression, Differenzierung und Sekretion verschiedener Zytokine (GROa, I-309, IL-6, IL-8, IL-13, Il-16, MIF, Serpin E1) und Stress-assoziierte Proteine wurde durch die mütterliche Erkrankung nicht signifikant beeinflusst. Isolate von Kindern präeklamptischer Müttern wiesen dagegen eine signifikant niedrigere Colony-forming Efficiency, sowie eine geringere Proliferationsrate und metabolische Aktivität auf.

Diskussion: Die Präeklampsie scheint sowohl Proliferationsrate als auch metabolische Aktivität und Colony-forming Efficiency von neonatalen MSCs zu reduzieren. Unklar bleibt, ob dieser Effekt ursächlich für die erhöhte neonatale Morbidität ist und welche Konsequenzen bezüglich eines therapeutischen Einsatzes dieser MSCs resultieren.

Die Studie wurde durch einen Grant der European Foundation for the Care of the Newborn Infant (EFCNI) gefördert.

\section{P032}

Bilirubinanstieg bei Frühgeborene $<\mathbf{1 5 0 0} \mathrm{g}$ bis zur Phototherapie

S. Hahn', C. Bührer', G. Schmalisch', M. Berns

${ }^{1}$ Charité Charité Universitätsmedizin Berlin, Berlin, Deutschland

Hintergrund: In den ersten Lebenstagen kommt es bei allen Neugeborenen zu einem Anstieg des unkonjugiertem Bilirubins. In hohen Konzentrationen wirkt Bilirubin neurotoxisch und kann zu irreversiblen Schäden des zentralen Nervensystems führen. Ab welchen Werten bei Frühgeborenen mit einer Schädigung zu rechnen, ist unklar, die Interventionsgrenzen sind willkürlich niedriger gewählt als bei reifen Neugeborenen. Ein Normalverlauf der Bilirubinkonzentration bei Frühgeborenen $<1500 \mathrm{~g}$ und Einflussfaktoren sind nie systematisch untersucht worden.

Methodik: In einer retrospektiven Studie wurde der Verlauf des Bilirubins bis zum Beginn der Phototherapie bei 342 Frühgeborenen $<1500 \mathrm{~g}$ Geburtsgewicht (Median 995, Interquartilbereich 820-1280 g) mit einem Gestationsalter von 28,1 (26,3-30,1) Wochen erfasst. Die Messungen erfolgten im Rahmen der regulären Blutentnahmen mit dem ABL 850. Spearman Rank Korrelation und Regressionsanalysen wurden verwendet, um die Einflussgrößen auf das initiale Bilirubin und bzw. den Bilirubinanstieg und die Dauer der Phototherapie zu untersuchen.

Ergebnisse: Die erste Messung erfolgte median (IQR)) im Alter von 2,4 $(1,3-4,7) \mathrm{h}$, der initiale Bilirubinwert war $1,8(1,4-2,5) \mathrm{mg} / \mathrm{dl}$ und stieg danach um $0,15(0,11-0,19) \mathrm{mg} / \mathrm{dl} / \mathrm{h}$ an. Mit der Phototherapie wurde nach $30,8(23,0-41,6) \mathrm{h}$ begonnen, sie dauerte $91,5(38,8-144,0) \mathrm{h}$. Das initiale Bilirubin zeigte nur eine schwache positive Korrelation mit dem Gestationsalter $(\mathrm{Rs}=0,172 ; p=0,002)$ und hatte keinen Einfluss auf die Dauer der Phototherapie, es war um so höher, je später die erste Messung durchgeführt wurde $(r=0,514 p<0,001)$. Der Bilirubinanstieg war unabhängig von Geburtsgewicht und Gestationsalter. Die Dauer der Phototherapie korrelierte invers mit dem Gestationsalter $(\mathrm{Rs}=-0,315 ; p<0,001)$ und positiv mit dem Bilirubinanstieg. (Rs=0,174; $p=0,002$ ).

Schlussfolgerungen: Bei Frühgeborenen $<1500 \mathrm{~g}$ hat wegen des relative schnellen Bilirubinanstiegs der Abnahmezeitpunkt einen großen Einfluss auf den Messwert. Im Gegensatz zum initialen Bilirubin ist ein schneller Bilirubinanstieg oft mit einer verlängerten Phototherapie assoziiert.
Tab. P033

\begin{tabular}{|lll}
\hline & Fold & $p$-value \\
\hline miR-16 & 3.5 & 0.0163 \\
\hline miR-22 & 5.9 & 0.0016 \\
\hline miR-146a & 2.9 & 0.0075 \\
\hline
\end{tabular}

\section{P033}

Anstieg von microRNA-16, microRNA-22 und microRNA146a im Nabelschnurblut von Neugeborenen mit niedrigem Geburtsgewicht

M. Wagner', D. Monz' , E. Maurer', E. Tutdibi' , P. Leidinger' ${ }^{2}$ E. Meese², L. Gortner'

${ }^{1}$ Klinik für Allgemeine Pädiatrie und Neonatologie, Universität des Saarlandes, Homburg, Deutschland, ${ }^{2}$ Institut für Humangenetik, Universität des Saarlandes, Homburg, Deutschland

Hintergrund und Fragestellung: Während der Fetalperiode können materne und fetale Faktoren das Wachstum und die Entwicklung des Fetus und auch das Risiko für spätere Erkrankungen beeinflussen. MicroRNAs sind über die Regulation von Zellproliferation, Zelldifferenzierung und Apoptose an Entwicklungsprozessen beteiligt. Während einige microRNAs gewebsspezifisch sind, findet sich die Mehrzahl auch im Blutkreislauf. Ihre Plasmaspiegel sind u. a. bei Tumoren und während der Schwangerschaft verändert. Es wurden z. B. microRNA-16, microRNA-21 und microRNA-146a in der Plazenta nach Kontakt mit Zigarettenrauch vermindert gemessen. Bei SGA-Neugeborenen ist die Expression von microRNA-16 und microRNA-21 in der Plazenta reduziert. Ziel der Studie war es, die Konzentrationen der microRNA-16, microRNA-21, microRNA-22 und microRNA-146a im Nabelschnurblut bei fetalen Wachstumsstörungen zu untersuchen.

Material und Methoden: Einschlusskriterien: Reife gesunde Neugeborene 37-42 SSW nach unauffälligem Schwangerschaftsverlauf. Subgruppen: appropriate for gestational age (AGA; hier: $>$ P25- $<$ P90) und small for gestational age (SGA; hier < P25) Neugeborene. Perzentilen nach Voigt (1). AGA-Gruppe $n=20$, in der SGA-Gruppe $n=19$ Neugeborene. Aus venösem Nabelschnurblut wurde microRNA isoliert und per quantitativer real-time PCR analysiert.

Ergebnisse: Von den vier untersuchten microRNAs zeigten sich microRNA-16, microRNA-22 sowie microRNA-146a im Nabelschnurblut von Neugeborenen mit fetalen Wachstumsstörungen signifikant erhöht verglichen mit dem Nabelschnurblut adäquat gewachsener Neugeborener (siehe Tabelle P033).

Schlussfolgerung: Intrauterine Wachstumsmuster zeigen einen Zusammenhang mit microRNAs im Nabelschnurblut. Dies könnte das fetale Wachstum und die weitere postnatale Entwicklung des Kindes beeinflussen.

\section{Literatur}

1. Voigt et al., Geburtsh Frauenheilk, 2006; 66

\section{P034}

Development of the endothelial glycocalyx in preterm and term neonates

\section{Nußbaum', L. Rajwich', S. Artmann', O. Genzel-Boroviczény'}

${ }^{1}$ Klinikum der Universität München, Campus Innenstadt, Neonatologie des Dr. von Hauner Kinderspital, München, Deutschland

Background: The perfused boundary region (PBR), the luminal part of the endothelial glycocalyx, is a validated parameter to estimate the glycocalyx thickness and hence its physiologic state of function. Recent research showed that the glycocalyx exists as soon as blood flow is present. Here, we investigated how the PBR evolves within the first weeks of life comparing preterm and term neonates as well as possible influences by clinical or laboratory parameters. 
Methods: The microvasculature of the ear conch was visualized with Sidestream Dark Field imaging. Automated measurements of the PBR were obtained in vessels ranging from 5-25 $\mu \mathrm{m}$ using the GlycoCheck software. The software calculates the PBR based on dynamic diameter changes of the RBC column, which are limited by the intact glycocalyx. Thus, when the glycocalyx is damaged or shed, the PBR increases. 87 term neonates and 36 preterm neonates were included in the study. The cohort of preterm infants was divided in two subgroups i. e. neonates born $<30$ weeks gestational age (WGA) $(n=19)$ and neonates born $\geq 30$ WGA $(n=17)$. The first measurement was performed between $36-48$ hours of life. In the preterm neonates, weekly follow up measurements were performed if possible.

Results: We obtained an average of 3.4 measurements per infant (range 1-10) over an average period of 4.4 weeks (range 1-13). Interestingly, we observed significant differences in the PBR between preterm and term neonates with lowest values in the group of infants born $<30$ WGA and highest values in mature neonates. Accordingly, the group of extreme premature neonates showed a significant time- and age-dependent increase in the PBR values. Remarkably, when comparing neonates born $<30 \mathrm{WGA}$ when reaching a corrected age of $>30$ WGA (ex-utero development) to neonates with $>30$ WGA at birth (in-utero development), we did not observe any difference in the PBR values. No correlation was found with clinical or laboratory parameters that changed significantly over time i. e. MAD, oxygen saturation, hematocrit, bilirubin, base excess and bicarbonate levels. None of the children had laboratory evidence of severe infection/sepsis. Conclusion: Our study demonstrates that the endothelial glycocalyx can be detected in extreme premature neonates and shows and inverse correlation with gestational age, i. e. the most immature neonates present with the largest glycocalyx dimension. Age-dependent PBR changes seem to be influenced by intrinsic rather than extrinsic factors, suggesting an ontogenetic regulation. Possibly, that larger glycocalyx dimensions in preterm neonates reflect its' physiologic importance for fetal vascular development, as has been recently demonstrated in quail embryos.

\section{Postersitzung 05: Kardiologie}

\section{P035 \\ DRESS-Syndrom-Schwere Komplikation einer Endokarditistherapie - ein Fallbericht \\ K. Fritsche ${ }^{1}$, T. Mitschke' ${ }^{1}$, J. Pohler ${ }^{1}$, T. Kriebel' \\ 'Westpfalz-Klinikum GmbH, Kinderklinik, Kaiserslautern, Deutschland}

Das DRESS-Syndrom (Drug Reaktion with eosinophila and systemic Symptoms) ist eine seltene, aber potentiell lebensbedrohliche Arzneimittelreaktion, die im Gegensatz zum Steven-Johnson-Syndrom oder der toxisch-epidermalen Nekrolyse neben der Haut weitere Organsysteme befällt. Wir berichten über eine 11 jährige Patientin, die nach Korrekturoperation einer Pulmonalatresie mit Ventrikelseptumdefekt, MAPCAS und Di George-Syndrom, mit einer Endokarditis ohne Erregernachweis stationär mit Ampicillin/Sulbactam, Tobramycin und Vancomycin 5 Wochen behandelt wurde. 3 Wochen nach Therapiebeginn kam es zu einem generalisierten makulopapulösem Exanthem mit erneutem CRP-Anstieg, Fieber sowie einer ausgeprägten Eosinophilie. Nach einer 2-tägigen Antibiotikapause zur Erregergewinnung Auftreten einer interstitiellen Pneumonie, Erbrechen, Übelkeit sowie Zunahme des Exanthems, sodass mit Meropenem und Linezolid weiterbehandelt wurde. Darunter Zunahme des Exanthems, des Fiebers und rasche klinische Verschlechterung bei gleichzeitig rückläufigen Entzündungsparametern. Die oben beschriebene Klinik lenkte den Verdacht auf das DRESS-Syndrom. 4 Tage nach Absetzen aller Medikamente außer Paracetamol und Dimenhydrinat waren Fieber und Exanthem rückläufig. Aufgrund der schwere der Erkrankung erfolgte eine systemische Steroidbehandlung über 2 Monate mit anhaltender Entfieberung und rascher Verbesserung des klinischen Zustandes. Schlussfolgerung: Das DRESS-Syndrom ist ein seltenes und lebensbedrohliches Krankheitsbild welches aufgrund seiner Heterogenität und der un- terschiedlich langen Latenzzeit oft spät erkannt wird. Somit ist es wichtig diese Medikamentennebenwirkung bei unklaren und schweren Krankheitsverläufen differenzialdiagnostisch in Erwägung zu ziehen und das auslösende Agens rasch zu identifizieren und abzusetzen.

\section{P036}

Stentimplantation via Hybrideingriff bei Aortenisthmusstenose und Aortenbogenhypoplasie bei einem Zwillingsfrühgeborenen von 27;2 Schwangerschaftswochen

\section{N. Bittkowski', H. Küster', T. Paul' ', H. Schneider', W. Ruschewski}

${ }^{1}$ Klinik für Päd. Kardiologie und Intensivmedizin mit Neonatologie und Päd. Pneumologie, Universitätsmedizin Göttingen, Göttingen, Deutschland, ${ }^{2}$ Klinik für Thorax-, Herz- und Gefäßchirurgie Universitätsmedizin Göttingen, Schwerpunktprofessur Kinderherzchirurgie, Göttingen, Deutschland

Fallbeschreibung: Weibliches Zwillingsfrühgeborenes von 27;2 Schwangerschaftswochen. Geburt durch Sektio bei unstillbaren Wehen. Intubation und Gabe von Surfactant in der Erstversorgung bei insuffizienter Atmung. Apgar 5/7/8, pH 7,27, BE -4 mmol/l. Geburtsgewicht 635 g (8. P.), Körperlänge $32 \mathrm{~cm}$ (15. P.), Kopfumfang 22,5 cm (6. P). Auf Station hoher Beatmungs- und Sauerstoffbedarf sowie Notwendigkeit von NO. In der Echokardiographie in den ersten Lebensstunden Darstellung einer ausgeprägten Aortenisthmusstenose und Aortenbogenhypoplasie, Durchmesser der Aortenklappe und der Aorta ascendens $5 \mathrm{~mm}$, Aortenisthmus $2 \mathrm{~mm}$. Bei Ductus-abhängiger Systemperfusion erfolgte eine Apostadildauerinfusion. Am 56. Lebenstag fand bei einem Körpergewicht von $1300 \mathrm{~g}$ im Hybridverfahren die Stentimplantation in die Aortenisthmusstenose nach Freilegung der A. carotis communis li. und Einführen einer $4 \mathrm{~F}$ Schleuse. Zunächst erfolgte die Implantation zweier selbstexpandierender Stents vom Typ sinus-Repo-DS mit einem Durchmesser von 5 bzw. $6 \mathrm{~mm}$ und einer Länge von $9 \mathrm{~mm}$. Bei der anschließenden Angiographie zeigte sich die Stenose noch nicht vollständig behoben, so dass ein Palmaz-Stent von $5 \mathrm{~mm}$ Durchmesser und $11 \mathrm{~mm}$ Länge implantiert wurde. Die Apostadildauerinfusion konnte beendet werden. Die Punktionsstelle der A. carotis wurde chirurgisch versorgt. Postinterventionell war die Patientin kardiorespiratorisch stabil. Es erfolgte eine Therapie mit Heparin über 72 Stunden, überlappend wurde mit ASS p.o. begonnen. Bei erhöhten Blutdruckwerten behandelten wir mit Propranolol. Der persistierende Ductus arteriosus wurde mit Indometacin über 3 Tage erfolgreich verschlossen. Die Entlassung ins häusliche Umfeld war am 97. Lebenstag mit einem Körpergewicht 3725 g (<3.P.), Kopfumfang 34 cm (<3.P.), Körperlänge $51 \mathrm{~cm}(<3 . P$.$) möglich. Die Patientin zeigte bis auf eine muskuläre$ Hypertonie keine neurologischen Auffälligkeiten.

Diskussion: In unserem Fall konnte via Hybridverfahren per Stentimplantation die Aortenisthmusstenose unter Schonung der Leistengefäße bei einem Körpergewicht von 1300 g erfolgreich behandelt werden.

\section{P037}

Einfluss physiologischer Shunts auf die Herzindexmessung mittels elektrischer Velocimetrie im Vergleich zu transthorakaler Echokardiographie bei Neugeborenen

M. Blohm ' , J. Hartwich ', D. Obrecht ${ }^{1}$, D. Singer ${ }^{1}$

'Universitätsklinik Hamburg-Eppendorf, Kinderklinik, Sektion Neonatologie/ Päd. Intensivmedizin, Hamburg, Deutschland

Hintergrund: Die „Impedanzkardiographie“ bzw. „Elektrische Velocimetrie“ (EV) ist eine Methode der Herzzeitvolumenmessung, bei der transkutan mittels EKG-Elektroden eine pulssynchrone Widerstandsänderung gegenüber einem niederamperigen hochfrequenten Wechselstrom über dem Thorax abgeleitet und zur Errechnung des Herzzeitvolumens (HZV) verwendet wird. Bei Neugeborenen hat die EV theoretisch die Vorteile der Nicht-Invasivität und einer kontinuierlichen Anwendbarkeit, die dem Nachteil einer geringen Präzision der Methode mit hoher Streuung der einzelnen Messwerte gegenübersteht. Bei der Validierung neuer 
HZV-Messmethoden gilt es auch, Faktoren, die die Messmethode beeinflussen, zu identifizieren.

Fragestellung: Es wurde der Einfluss von vorhandenen physiologischen Shunts (PFO und/oder PDA) auf die Genauigkeit der Bestimmung des Herzindex (CI) mittels EV (CIEV) im Vergleich zum CI mittels TTE (CITTE) untersucht.

Material und Methoden: Bei 99 herzgesunden spontan atmenden Neonaten (Gewicht im Median $3300 \mathrm{~g}$ ) wurden insgesamt 291 simultane Messungen von CIEV und CITTE (jeweils mittels Aortenklappenflussmessungen über drei konsekutive Herzzyklen) durchgeführt. Bei allen Neugeborenen wurde eine Echokardiographie zum Ausschluss weiterer Shunts (außer PFO und/oder PDA) durchgeführt. Es wurde die Abweichung der Methoden EV und TTE in der Bland-Altman Analyse bestimmt. Weiterhin wurde die relative (prozentuale) Abweichung von CIEV versus CITTE in Abhängigkeit des Vorhandenseins von PFO und/oder PDA errechnet [(CITTE-CIEV)/(CITTE+CIEV)/2)].

Ergebnisse: Für die gesamte Kohorte war wurde ein CIEV von $3,32 \mathrm{l} /\left(\mathrm{min} \times \mathrm{m}^{2}\right)($ Standardabweichung SD 0,64) und ein CITTE von $2,97 \mathrm{l} /\left(\mathrm{min} \times \mathrm{m}^{2}\right)(\mathrm{SD} 0,83)$ bestimmt, die Bland-Altman Analyse zeigte eine Abweichung von $-0,35 \mathrm{l} /\left(\mathrm{min} \times \mathrm{m}^{2}\right)(95 \%$ Konfidenzinterva$\left.11-1,19 / 0,501 /\left(\min \times \mathrm{m}^{2}\right)\right)$, entsprechend einem prozentualen Bias von $-11,0 \%$ und einem mittleren prozentualen Fehler von 52,7\%. Die relative Abweichung zwischen CIEV und CITTE war ohne Shunts -2,9\% (SD $33,5 \%, n=91$ ), bei isoliertem PFO -9,6\% (SD 25,2\%,n=125), bei isoliertem PDA -14,0 (SD 31,1\%, $n=12$ ), bei kombiniert vorhandenem PFO und PDA - 18,5\% (SD 22,6\%, $n=63$ ). Der Unterschied der relativen Abweichung zwischen CIEV und CITTE im Vergleich der Neugeborenen mit und ohne Shunts war statistisch signifikant $(p=0,006)$.

Diskussion: Dieser Methodenvergleich zwischen EV und TTE zeigte, dass die Methoden formal nicht äquivalent waren. Die beste mittlere Übereinstimmung der Messergebnisse mit einer Abweichung von nur -2,9\% bestand in der Gruppe ohne Shunts. Die Genauigkeit der Messungen wurde durch das Vorhandensein von PDA und/oder PFO signifikant beeinflusst, mit einer Zunahme der relativen Differenz CIEV gegen CITTE. Hierbei überschätzte die EV im Vergleich zur TTE das CI.

Schlussfolgerung: Physiologische Shunts (PDA und/oder PFO) bei Neugeborenen beeinflussen die Messgenauigkeit und sind eine Ursache für eine Überschätzung des CI bei Messung mittels EV.

\section{P038}

\section{Nicht-infektiöse Endokarditis und akutes Nierenversagen nach Intoxikation mit Metamizol und hohen Dosen eines Östrogenpräparates}

\section{J. Pohler', T. Kriebel'}

'Westpfalz-Klinikum GmbH, Kinderklinik, Kaiserslautern, Deutschland

Einleitung: Patienten mit erhöhten Antiphospholipidantikörpern haben ein deutlich erhöhtes Thromboserisiko. Vegetationen an der Herzklappe stellen die häufigste kardiale Manifestation dar. Ebenso sind Nephropathien als Komplikation dieser Autoimmunerkrankung beschrieben.

Fallvorstellung: Eine 17-jährige Patientin stellte sich in unserer Klinik mit Bauchschmerzen und Erbrechen vor. Vorausgegangen war eine Woche vor Aufnahme eine Intoxikation von 12,5 g Metamizol und 3 Blister eines Kontrazeptivums (Daylette). Im Serum war eine Kreatininerhöhung von $4,0 \mathrm{mg} / \mathrm{dl}$ auffällig. Echokardiographisch zeigten sich bedeutsame Vegetationen an der Mitralklappe mit einem Durchmesser bis $14 \mathrm{~mm}$, es bestand kein Fieber und die Blutkulturen blieben steril. Laborchemisch waren die Antiphospholipd-Antikörper mit einem Beta-2-Glykoprotein von 5933 U/l und einem Anti-Cardiolipin-IgG von 1335 U/l stark erhöht. Retrospektiv bestand seit mindestens einem Jahr eine Thrombozytopenie. Dies ließ auf eine nicht-infektiöse Ursache der Endokarditis mit thrombotischen Klappenvegetationen im Rahmen eines Antiphospholipidsyndroms schließen. Unterstützt wurde die Diagnose durch den Nachweis einer Vaskulopathie der Niere mit thrombotischen Mikroangiopathien unterschiedlichen Alters in der Nierenbiopsie. Unter einer antikoagulativen Therapie mit Marcumar bildeten sich die Klappenvegetationen lang- sam zurück. Die Retentionswerte waren im Verlauf rückläufig, die Kreatininclearance blieb vermindert. Schlussfolgerung: In unserem Fall scheint die Intoxikation von hohen Dosen eines Ostrogenpräparates bei Vorliegen eines Antiphophoplipidsyndroms das akute thrombotische Geschehen an der Mitralklappe ausgelöst zu haben. Ebenso hat die Dosis von 12,5 g Metamizol, die bei Nierengesunden ohne Folgen bleiben kann, bei dieser Patientin mit bereits vorgeschädigter Niere zu einem akuten Nierenversagen geführt.

\section{P039}

Continuous hemodialysis therapy for a very low birth weight (VLBW) premature with acute renal failure and congenital heart disease

N. Prieto Betancourt' ', U. Herberg' ', R. Knies' ', S. Müller' ${ }^{2}$, E. Muehler' ${ }^{3}$, B. Hoppe ${ }^{4}$, J. Breuer ${ }^{7}$

'Universitätsklinik Zentrum f. Kinderheilkunde, Kinderkardiologie, Bonn, Deutschland, ${ }^{2}$ Universitätsklinikum Bonn, Klinik und Poliklinik für Anästhesiologie und Intensivmedizin, Bonn, Deutschland, ${ }^{3}$ Uniklinikum RWTH Aachen, Kinderkardiologie, Aachen, Deutschland, ${ }^{4}$ Universitätsklinik Zentrum f. Kinderheilkunde, Pädiatrische Nephrologie, Bonn, Deutschland

Case description - The premature girl was born at 32 weeks of gestation with a birth weight of $1495 \mathrm{~g}$. Thereafter, Tetralogy of Fallot (TOF) with severe infundibular and valvular pulmonic stenosis as well as hypoplastic pulmonary arteries was diagnosed. Due to progressive hypoxic spells, at the age of 31 days an aortopulmonary shunt with a diameter of $3,5 \mathrm{~mm}$ was placed between the ascending aorta and the main pulmonary artery. Postoperatively, the girl developed a pronounced capillary leak syndrome with acute renal failure and peritoneal dialysis was started at the age of 32 days. Due to an intestinal perforation on day 43, a bowel resection with ileostomy was performed. Peritoneal dialysis was continued, nevertheless the fluid retention increased and the mechanical ventilation worsend. Therefore, the girl was transferred to the Pediatric Cardiology Intensive Care Unit at the University Hospital of Bonn for hemodialysis. The primary technical challenge for initiating continous hemofiltration in this VLBW premature with an actual weight fo $1800 \mathrm{~g}$ was the size of the catheter for continous veno-venous hemofiltration and the capacity of the hemofiltration set. We successfully placed the smallest available short term dialysis catheter with a diameter of 6-French (Joline, Hechingen) into the right jugular vein. Hemofiltration was initiated with the Plasauto Sigma-System (DIAMED, Cologne). Following the priming of the hemofiltration set (filling volume of the set: $47 \mathrm{ml}$ ) with heparin and sodium chloride, the set was filled with a mixture of red cell concentrate $(75 \%)$, fresh frozen plas$\mathrm{ma}(25 \%)$ and sodium bicarbonate $(2 \mathrm{ml})$. On hemofiltration, the set was run with the blood pump of $7-10 \mathrm{ml} / \mathrm{min}$, an ultrafiltration rate between $10-30 \mathrm{ml} / \mathrm{h}$ and a replacement fluid rate of $100 \mathrm{ml} / \mathrm{h}$. Despite effective anticoagulation with heparine and a prothrombin time over 60 seconds and anti-factor Xa over $0.4 \mathrm{IU} / \mathrm{ml}$ the major complication was clotting of the hemofiltration sets venous line connected to the patients venous vascular access. The hemofiltration set had to be changed 12 times, in average every 39.15 hours. As a result of the clotting, a new catheter for continous veno-venous hemofiltration needed to be placed in the left vena subclavia after 12 days. With successful dialysis, the uremia and the lung function improved quickly. In total, hemofiltration was needed over 29 days. Renal function recovered completely. A clipping of the aortopulmonary shunt to reduce pulmonary blood flow was performed after stabilizing of the patient 8 days after admission. The patient was discharged home after 88 days and complete correction of the TOF was successfully performed at the age of $101 / 2$ months without any further renal function problem. Conclusions Continuous hemodialysis therapy is a successful and safe possibility even in very low birth weight prematures with acute renal failure. 
P040

\section{LCHAD, DD MTP Mangel bei einem Frühgeborenen -} ein Fallbeispiel

\section{K. Riebe ${ }^{1}$, H. Schneider ${ }^{1}$, P. Freisinger ${ }^{1}$ \\ 'Kreiskrankenhaus, Kinderklinik, Reutlingen, Deutschland}

LCHAD Mangel oder MTP Mangel sind als Ursache einer neonatalen Kardiomyopathie und Laktatazidose bekannt. Wir berichten über ein Frühgeborenes der $36+3 / 7$ SSW mit massiver Herzinsuffizienz sowie Laktatazidose, dass bereits vor Erhalt des Ergebnisses des Neugeborenenscreening (NS) klinisch auffällig wurde. Fallbeispiel: FG der 36+3/7 SSW wdh. erhöhte Laktatwerte bei zunächst unauffälliger Klinik. Stoffwechselscreening am 3. LT abgenommen. Am 5. LT angestrengte Atmung, echokardiografisch ausgeprägte ventrikuläre Funktionsstörung. Am 5. LT Erhalt des NS: dort V.a. LCHAD- Defekt. DD MTP Mangel. Verlegung am 6. LT in Stoffwechselzentrum. Diagnostik: -Laktatazidose (max. Laktat bei $16 \mathrm{mmol} / \mathrm{l}$ ) -Echkardiografie: Herzinsuffizienz mit linksventrikulärer Dilatation. -NS: massive Erhöhung von C18:1OH und C18-OH. Verlauf: Nach Erhalt der Stoffwechselergebnisse sofortiger Stopp der Zufuhr mit Muttermilch und Beginn enterale Ernährung mit fettfreier Nahrung (Basic F). Übernahme am 6. LT in Stoffwechselzentrum. Therapie: Stoffwechseloptimierung: Weiterführung der Infusionstherapie mit Glucose (Zielbereich BZ 60-80 mg/dl). Beginn Ernährung mit Monogene und Lipistart (MCT reiche Nahrung, reduziert an langkettigen Fettsäuren) im Verlauf Kalorienanreicherung mit Maltodextrin. Bei metabolischer Azidose bedarfsgerechte Pufferung mit Nabic über 2 Tage als DTI. Milrinon 4 Tage (d) als DTI, Propanolol 5 d i.v. als DTI, danach p. o. 3 d nach Übernahme Beginn mit Captopril mit langsamer Besserung der Herzinsuffizienz mit Entwicklung einer hypertrophen Kardiomyopathie. Entlassung am 39. LT in gutem AZ nach Hause voll enteral ernährt. Bedarfsgerecht an die Stoffwechselwerte angepasst. Befunde bei Entlassung: Vitales FG, guter AZ, enteral ernährt. Neurologisch unauffällig. Echo: hypertrophe nicht obstruktive Kardiomyopathie, gute Funktion. Stoffwechsel: $\mathrm{C} 16-\mathrm{OH}<0,52$ ( $\mathrm{NB}<0,16 \mu \mathrm{mol} / \mathrm{l}), \mathrm{C} 18: 1 \mathrm{OH}$ 0,88 (NB $<0,11 \mu \mathrm{mol} / \mathrm{l}), \mathrm{C} 18-\mathrm{OH}$ 0,35 (NB $<0,19 \mu \mathrm{mol} / \mathrm{l}$ ). Molekulargenetik (HADHA- und HADHB-Gen): noch ausstehend. Vgl. mit publizierten Fallberichten Spiekerkoetter U., et al. (2009): 75 Patienten mit langkettigen Fettsäurestoffwechseldefekten aus 18 Stoffwechselzentren (D, CH, Ö) wurden auf einem Workshop im Management und Outcome verglichen. Davon waren 14 Patienten mit LCHAD Defekt. Alle Patienten wurden mit MCT Fetten supplementiert. 11 von 14 benötigten zusätzliche Kohlenhydrate. 2 von 14 benötigten eine dauerhafte Ernährung über nasogastrale Sonde während der Nacht. 6 Patienten waren an MTP- Mangel erkrankt. Davon waren 5 mit MCT Fett supplementiert. Alle 6 erhielten zusätzlich Kohlenhydratanreicherung der Nahrung. (1) Schlussfolgerung: Das klinische Bild einer neonatalen Laktatazidose mit Veränderungen der Herzfunktion sollte auch vor Erhalt des Stoffwechselscreenings an eine Fettsäureoxidationsstörung denken lassen. Das Outcome der Kinder ist stark abhängig von dem genetischen Defekt, der frühzeitigen Therapie und der kardialen Symptome. Lebenslimitierend ist die Kardiomyopathie mit hohem Risiko von ventrikulären tachykarden Rhythmusstörungen. Lebenslange diätetische Behandlung mit Verzicht auf langkettige Fettsäuren, Vermeiden kataboler Zustände ist nötig. Eine erhöhte Neigung zu kardialen Arrhythmien und einer Rhabdomyolyse lebenslang ist zu beachten. Ausführliche Schulung der Eltern notwendig. (1) U. Spiekerkötter. J Inherit Metab Dis (2010) 33:527-532, (2) U.Spiekerkötter. J Inherit Metab Dis (2009) 32:488-497

\section{P041}

Späte Korrektur einer d-Transposition der großen Arterien bei einem sudanesischen Kind

B. Wannenmacher', N. Bigl', L. Yacoub², P. Lwabi ${ }^{3}$, M. Kostelka4, I. Dähnert', M. Weidenbach

${ }^{1}$ Herzzentrum Leipzig GmbH, Klinik für Kinderkardiologie, Leipzig, Deutschland, ${ }^{2}$ Chain of hope, London, United Kingdom, ${ }^{3}$ Mulago Hospital Complex, Uganda Heart Institute, Kampala, Uganda, ${ }^{4}$ Herzzentrum Leipzig $\mathrm{GmbH}$, Klinik für Herzchirurgie, Leipzig, Deutschland

Die Transposition der großen Arterien (dTGA) ist ein kritischer angeborener Herzfehler, der gewöhnlicherweise innerhalb der ersten Lebenstage mittels der „Arteriellen Switch Operation“ korrigiert wird. Dieser frühe Operationszeitpunkt ist wichtig, um einem „Remodelling" des linken Ventrikels entgegenzuwirken, wenn der Widerstand im pulmonalen Gefäßbett sinkt. Sollte die "Arterielle Switch Operation“ in den ersten Lebenstagen nicht möglich sein, kann mittels eines zweischrittigen Herangehens mit „Pulmonalarterienbanding“ und systempulmonalem Shunt zunächst Zeit gewonnen und eine definitive Korrektur zu einem späteren Zeitpunkt vorgenommen werden. Wir berichten über ein Mädchen mit dTGA und Monokoronarostium aus dem Südsudan, das in unserer Abteilung behandelt worden ist. Nach initialer Ballonatrioseptostomie am 7. Lebenstag im Mulago Hospital in Kampala, Uganda, wurde uns das Kind über das Netzwerk „Chain of hope“, London, vorgestellt, zur dringlichen Korrektur indiziert und eine sofortige Anreise empfohlen. Der nachfolgende Verlauf war sehr erschwert aufgrund der bürgerkriegsähnlichen Zustände im Südsudan und daher großen Verzögerung bei Pass- und Visaformalitäten. Das Mädchen und seine Familie waren über mehrere Wochen nicht auffindbar und es war völlig unklar, wie es dem Mädchen ging und ob es überhaupt noch lebte. Schließlich konnte das Mädchen in der 10. Lebenswoche nach Deutschland reisen und einer weiteren Behandlung zugeführt werden. Bei Aufnahme lag die pulsoxymetrische Sättigung bei 55\%, das Kind war dystroph und zeigte moderate Zeichen der Herzinsuffizienz. Der erste Schritt der zweizeitigen operativen Korrektur konnte am 74. Lebenstag durchgeführt werden mit einem schwierigen postoperativen Verlauf aufgrund einer akuten Linksherzinsuffizienz. Nach Erholung von dem ersten Eingriff konnte am 117. Lebenstag eine „Arterielle Switch Operation“ durchgeführt werden. Der postoperative Verlauf war hiernach unkompliziert. Am 148. Lebenstag konnte das Kind entlassen und mit seiner Familie zurück in die Heimat reisen. Zusammenfassend berichten wir über einen sehr schwierigen und seltenen Fall eines Kindes mit dTGA aus einem Bürgerkrieg-gebeutelten Drittweltland in Zentralafrika, das in unserer Abteilung in Zusammenspiel mit dem Kinderkardiologen vor Ort und dem Netzwerk Chain of hope behandelt werden konnte.

\section{P042}

Sildenafil bei therapieresistenter Tachycardie eines extrem unreifen Frühgeborenen

R. Neubauer', U. Fleischer', E. Robel-Tillig'

'Städtisches Klinikum St. Georg Kinderklinik, Klinik für Neonatologie, Leipzig, Deutschland

Problem: Sildenafil wird zur Therapie der pulmonalen Hypertension des Neugeborenen klinisch als off-label-Medikament angewendet. Die Studienlage ist bisher unzureichend und Daten für die evidente Anwendung sind erforderlich.

Kasuistik: Bei einem hypotrophen Frühgeborenes von 25+6 SSW und $480 \mathrm{~g}$ GG wurde in der 6 . LW eine Tachycardie mit HF von 180-200 bpm beobachtet.

- Infektionsparameter negativ

- $\mathrm{SaO}_{2} 90-92 \%$

- Keine peripher-zentrale Temperaturdifferenz

- Zerebrale Sonographie unauffällig

Echokardiographie

- Kein PDA

- HZV links $220 \mathrm{ml} / \mathrm{kg} / \mathrm{min}$ 
- HZV rechts $140 \mathrm{ml} / \mathrm{kg} / \mathrm{min}$

- TI $34 \mathrm{mmHg}$

- Typische Pulmonaliskurve mit kurzer Akzeleration und "Schulter“ als Zeichen der pulmonalen Hypertension

Therapie

- Sildenafil per os $4 \times 0,5 \mathrm{mg} / \mathrm{kg}$

Verlauf

- Normalisierung der Herzfrequenz nach 2 Behandlungstagen

- Beendigung der Therapie nach 7 Tagen

- Keine erneute Tachykardie

Echokardiographie nach 3 Tagen

- Deutliche Normalisierung des Flussmusters

- TI $20 \mathrm{mmHg}$

- HZV rechts $230 \mathrm{ml} / \mathrm{kg} / \mathrm{min}$

- HZV links $270 \mathrm{ml} / \mathrm{kg} / \mathrm{min}$

Schlussfolgerung: Bei klinischen und echokardiographischen Zeichen einer pulmonalen Hypertension kann eine kurzfristige Anwendung von Sildenafil unter echokardiographischer Kontrolle erfolgreich sein.

\section{P043 \\ Ibuprofen- und Paracetamol-induzierte pulmonale Hypertonie bei einem ELBW-Frühgeborenen.}

\section{A. Kirchner1, L. Garten'2, D. Szekessy'3, C. Bührer ${ }^{4}$}

${ }^{1}$ Charite Kliniken $\mathrm{f}$. Kinderheilkunde und Kinderchirugie, Neonatologie, Berlin, Deutschland, ${ }^{2}$ Charite Kliniken f. Kinderheilkunde und Kinderchirugie, Klinik für Neonatologie, CVK/CBF, Berlin, Deutschland, ${ }^{3}$ Klinikum Ernst v. Bergmann, Kinderklinik, Potsdam, Deutschland, ${ }^{4}$ Charité Universitätsmedizin Berlin, Klinik für Neonatologie, Berlin, Deutschland

Ibuprofen ist für die pharmakologische Therapie des Persistierenden Ductus arteriosus (PDA) zugelassen. Es gibt jedoch auch Berichte über die erfolgreiche pharmakologische Therapie des PDA mit Paracetamol. Wir berichten über ein ELBW-Frühgeborenes (Gestationsalter 25 + 0 Schwangerschaftwochen, Geburtsgewicht $560 \mathrm{~g}$ ) mit initial unkompliziertem Verlauf (Surfactant-Gabe und Extubation am 5. Lebenstag), das ab dem 3. Lebenstag wegen eines PDA intravenös mit Ibuprofen behandelt wurde. Die Ibuprofen-Therapie wurde bei unverändertem PDA verlängert und am 5 . Tag der Therapie entwickelte der Patient eine lebensbedrohliche suprasystemische pulmonale Hypertonie. Es erfolgte daher die Reintubation und HFO-Beatmung. Außerdem wurde eine Therapie mit inhalativem NO, eine hochdosierte Katecholamin-Therapie, eine tiefe Sedierung und Dauer-Relaxierung notwendig. Die endgültige Stabilisierung gelang jedoch erst unter zusätzlicher Iloprost-Dauerinfusion. Hierunter bildete sich die pulmonale Hypertonie im Verlauf von 10 Tagen allmählich zurück. Am 36. Lebenstag wurde bei weiterhin großem PDA mit zunehmender kardialer Belastung, pulmonaler Überflutung, fehlender Nahrungsverträglichkeit, diastolisch negativen Flüssen im Truncus coeliacus und der Arteria cerebri anterior, sowie nach einem gescheiterten Extubationsversuch ein pharmakologischer Therapie-Versuch mit Paracetamol intravenös begonnen. Hierunter entwickelte der Patient nach der 4. Dosis erneut eine suprasystemische pulmonale Hypertonie. Die Paracetamol-Therapie wurde daher umgehend abgebrochen und die pulmonale Hypertonie bildete sich innerhalb der nächsten 24 Stunden zurück. Schließlich erfolgte eine chirurgische Ligatur des PDA. Dieser Fall zeigt, dass nicht nur die chirurgische, sondern auch die pharmakologische Therapie des PDA sowohl mit Ibuprofen als auch mit Paracetamol bei sehr unreifen Frühgebornen schwerwiegende Nebenwirkungen haben kann.

\section{P044}

Hohe Propofoldosierungen und mangelnde

Behandlungserfahrung sind Risikofaktoren für Komplikationen bei der Analgosedierung von Kindern

S. Apostolidou' ', K. Röher ${ }^{2}$, G. Schön ${ }^{3}$, H. Bartz', C. Zöllner ${ }^{2}$, A. von der Wense', D. Singer

${ }^{1}$ Altonaer Kinderkrankenhaus, Neonatologie und pädiatrische Intensivmedizin, Hamburg, Deutschland, ${ }^{2}$ Universitätsklinikum Hamburg-Eppendorf, Klinik für Anästhesiologie, Hamburg, Deutschland, ${ }^{3}$ Universitätsklinikum Hamburg-Eppendorf, Institut für Medizinische Biometrie und Epidemiologie, Hamburg, Deutschland, ${ }^{4}$ Universitätsklinikum Hamburg-Eppendorf, Qualitätsmanagement und klinisches Prozessmanagement, Hamburg, Deutschland, ${ }^{5}$ Universitätsklinikum Hamburg-Eppendorf, Sektion Neonatologie und Pädiatrische Intensivmedizin, Hamburg, Deutschland

Hintergrund und Fragestellung: Daten des Pediatric Sedation Research Consortium aus den USA zeigen für Analgosedierungen von Kindern außerhalb des OP niedrige Komplikationsraten unabhängig vom durchführenden Arzt. Am Universitätsklinikum Hamburg-Eppendorf wurde im August 2014 ein spezialisiertes interdisziplinäres Kinderanalgosedierungsteam (KAST) aus Fachärzten der Anästhesiologie sowie der Neonatologie und pädiatrischen Intensivmedizin eingerichtet, welches alle Analgosedierungen für diagnostische Maßnahmen und kleine Eingriffe bei Kindern durchführt. Ziel dieser Untersuchung war es, Prävalenz und Prädiktoren von Komplikationen bei Analgosedierungen durch unser interdisziplinäres Team zu erfassen.

Material und Methoden: Es erfolgte für den Zeitraum August 2014 bis August 2015 eine retrospektive Auswertung der Sedierungsprotokolle. Mittels multifaktorieller Varianzanalyse wurden Risikofaktoren für das Auftreten von Komplikationen ermittelt. Dabei wurden als unabhängige Variablen Alter, Geschlecht, ASA-Klassifikation, Art und Dosierung des Hypnotikums, Verwendung eines Analgetikums sowie Erfahrung des Arztes untersucht.

Ergebnisse und Diskussion: Innerhalb eines Jahres wurde bei 784 Kindern eine Analgosedierung durchgeführt (8,5\% Säuglinge, 43,9\% Kleinkinder, $47,6 \%$ Kinder $>6$ Jahre). Der Anteil von Patienten mit einer ASA-Klassifikation von 3 oder 4 lag bei $53 \%$. Es wurden Propofol (79\%) oder Midazolam (16\%) sowie Esketamin (48\%) oder Remifentanil (8\%) eingesetzt. Insgesamt traten bei 6,5\% der Sedierungen unerwünschte Ereignisse auf, am häufigsten Apnoen (1,7\%), Atemwegsobstruktionen (1,0\%) und Probleme mit dem intravenösen Zugang (1,0\%). Es gab keine schwerwiegenden Komplikationen wie Herz-Kreislaufstillstand oder Aspiration. In der Varianzanalyse erhöhte jede Steigerung der Propofoldosierung um $1 \mathrm{mg} /$ $\mathrm{kg}$ das Risiko für Komplikationen (OR 1,34; 95 \% CI 1,18-1,55). Eine signifikante Verringerung des Komplikationsrisikos war bei Patienten $>1$ Jahr (OR 0,12; $95 \%$ CI 0,04-0,29) und mit jedem Quartal mehr an Behandlungserfahrung des KAST (OR 0,61; $95 \%$ CI 0,44-0,83) festzustellen. Demgegenüber ergab der Schweregrad der Grunderkrankung, gemessen an der ASA-Klassifikation, keinen signifikanten Unterschied. Die beobachteten Komplikatonsraten liegen in der Größenordnung vergleichbarer Literaturangaben, wobei eine höhere Propofoldosierung, mangelnde Erfahrung in der Durchführung von Analgosedierungen bei Kindern sowie ein Patientenalter $<1$ Jahr unabhängige Risikofaktoren zu sein scheinen. Schlussfolgerung: Die Ergebnisse belegen, dass eine entsprechende Expertise im Bereich der Analgosedierung komplex kranker Kinder einen wesentlichen Faktor für die Patientensicherheit darstellt. 


\section{P045}

\section{Central venous access device-associated thrombosis with aortic embolism in a preterm infant}

\author{
M. Biermayr', B. Brunner', K. Maurer', R. Trawöger', U. Kiechl-Kohlendorfer', \\ V. Neubauer ${ }^{1}$ \\ ${ }^{1}$ Medizinische Universität Innsbruck, Department für Kinder- und \\ Jugendheilkunde, Pädiatrie II (Neonatologie), Innsbruck, Österreich, \\ ${ }^{2}$ Medizinische Universität Innsbruck, Department für Radiologie, Innsbruck, \\ Österreich
}

Background: Thrombosis in neonates is commonly a central venous access device (CVAD) associated complication. Furthermore, a patent foramen ovale (PFO) is frequently seen in preterm infants. Even though a coincidence of both is not unusual, detaching of the thrombus and subsequent pulmonary embolism or organisation of an aortic embolism has not been described. Treatment recommendations of CVAD-associated thrombosis in neonates do not consider frequently seen complications of preterm infants e.g. intraventricular haemorrhage. This is the first case of a very preterm infant with pre-existing intraventricular haemorrhage with parenchymal haemorrhagic infarction, who developed a CVAD-associated thrombosis and thromboembolic complications.

Case report: The authors report on a very preterm girl, born at a gestational age of 30 weeks, with a pre-existing intraventricular haemorrhage and a CVAD-associated thrombus that, after removal of the CVAD, led to assumed pulmonary embolism, clinically presenting as lung haemorrhage, and to an extended aortic embolism with consequent cerebral stroke. The girl was treated with unfractionated heparin (UFH) for about 50 days. During the further in-hospital stay the girl developed a mild bronchopulmonary dysplasia but no further pulmonary complications. Follow-up until the corrected age of three months revealed clinical signs of cerebral palsy.

Discussion: There are various treatment recommendations for thrombosis in infants. However, there are no clear recommendations in very preterm infants with a high risk of cerebral bleeding respectively a pre-existing intraventricular haemorrhage. Treatment with low molecular weight heparin is difficult because of the necessity of daily subcutaneous administration. Thrombolysis or thrombectomy were also considered as not feasible due to haemorrhagic complications and the small dimensions of the vascular system. We decided to treat the patient with UFH until the affected vessels were recanalised. Even though preterm infants are often diagnosed with a PFO which constitutes the risk for paradoxical embolism, such complications do not occur frequently due to the physiological heart pressure proportion. In conclusion, if possible, patients with a confirmed thrombosis should be anticoagulated before removing a CVAD. However, in our patient it was rational to remove the CVAD without prior anticoagulation due to the pre-existing intraventricular haemorrhage. Even though pulmonary or paradoxical embolism are rare complications, it is important to monitor vital parameters and cerebral perfusion after removing a CVAD with confirmed associated thrombosis. It remains a case-by-case decision how to treat CVAD-associated thrombosis and consequent embolism depending on the patient's medical history.

\section{P046 \\ Tidal volume delivery and endotracheal tube leak during CPR in intubated newborn piglets with hypoxic cardiac arrest exposed to different modes of ventilatory support}

\section{Mendler' ' C. Weber', M. Ahmad Hassan' ', B. Mayer'², H. Hummler'}

${ }^{1}$ Klinik für Kinder- und Jugendmedizin, Universitätsklinikum Ulm, Sektion Neonatologie und pädiatrische Intensivmedizin, Ulm, Deutschland, ${ }^{2}$ Institut für Epidemiologie und Medizinische Biometrie, Ulm, Deutschland

Background: There is very little data available on the interference of inflations, chest compressions (CC) and delivery of tidal volumes (Vt) in newborn infants undergoing resuscitation in the presence of endotracheal tube (ET) leaks, using different techniques of respiratory support.
Objectives: To determine the effects of different respiratory support strategies along with $\mathrm{CC}$ on changes in Vt and ET leaks in hypoxic newborn piglets with cardiac arrest.

Methods: Thirty-three newborn piglets, intubated with weight adapted uncuffed ET tubes were exposed to hypoxia until asystole occurred, randomized into three groups and resuscitated according to ILCOR 2010 guidelines. T-Piece (TPR) group: Peak inspiratory pressure (PIP)/Positive end-expiratory pressure (PEEP) $25 / 5 \mathrm{cmH}_{2} \mathrm{O}$, rate $30 / \mathrm{min}$, inflations interposed between CC (3:1 ratio). Self-inflating bag (SIB) group: PIP 25 $\mathrm{cmH}_{2} \mathrm{O}$ without $\mathrm{PEEP}$, rate $30 / \mathrm{min}$, inflations interposed between CC $(3: 1$ ratio). Ventilator group: $\mathrm{PIP} / \mathrm{PEEP}$ of $25 / 5 \mathrm{cmH}_{2} \mathrm{O}$, rate $30 / \mathrm{min}$. CC were applied with a rate of $120 / \mathrm{min}$ without synchrony to inflations.

Results: During CC we observed a significant increase of leak (average absolute increase $11.4 \%$ ) as compared to respiratory support only without CC $(p=0.0001)$. Expiratory tidal volume was larger in the SIB group as compared to the two other modes which both applied PEEP. However, Vt caused by CC only were larger in both other groups as compared to the SIB group.

Conclusions: There is interference between lung inflations and CC affecting leak and delivery of $\mathrm{Vt}$, which may be influenced by the mode and the device of respiratory support. Leak is larger in the presence of PEEP. However, CC cause additional Vt which are larger in the presence of PEEP.

\section{Postersitzung 06: Frühgeborene}

\section{P047}

\section{Raus aus dem Inku (RADI) - ein prospektive Studie zu} Auswirkungen des Verlegens vom Inkubator in ein Wärmebett bei Frühgeborenen mit einem Geburtsgewicht unter $1500 \mathrm{~g}$ (VLBW)

\section{S. Elias' ${ }^{1}$ A. Stein'2, U. Felderhoff-Müser ${ }^{3}$}

'Klinik für Kinder und Jugendmedizin der Universität, Kinderklinik I, Essen, Deutschland, ${ }^{2}$ Klinik für Kinder und Jugendmedizin der Universität, Klinik für Kinderheilkunde I, Essen, Deutschland, ${ }^{3}$ Universitätsklinikum Essen, Direktorin der Klinik für Kinderheilkunde I, Zentrum für Kinder- und Jugendmedizin, Essen, Deutschland

Hintergrund: VLBW-Frühgeborene werden aufgrund der negativen Auswirkungen einer Hypothermie direkt postnatal im Inkubator versorgt. Es liegen keine Daten vor, welches das günstigste Gewicht ist, bei dem man die Kinder aus dem Inkubator in ein Wärmebett legen kann.

Methode: Prospektive Studie von 42 VLBW-Frühgeborenen im Zeitraum von November 2011 bis September 2014 (Geburtsgewicht $1008 \pm 272 \mathrm{~g}$, range 530-1440 g, Gestationsalter 28,6 \pm SD $38+6$, range $23+6$ SSW $32+2$ SSW). Verlegung vom Inkubator in ein Wärmebett (initiale Temperatureinstellung $37^{\circ} \mathrm{C}$ ) bei einem aktuellen Gewicht zwischen $1490 \mathrm{~g}$ und $1650 \mathrm{~g}$ (mean $1558 \mathrm{~g} \pm$ SD 45,56). In der Studiengruppe waren 8 Kinder mit noch vorhandener Atemunterstützung (davon 2 mit $\mathrm{O}_{2}$-Bedarf). Ausschlusskriterien waren invasive Beatmung oder $\mathrm{O}_{2}$-Bedarf $>25 \%$. Für $72 \mathrm{~h}$ nach der Umbettung wurden Vitalzeichen und die Körpertemperatur über zwei Temperatursonden (Sternum, Axilla) und rektal erfasst, sowie das Trinkverhalten vor und nach der Umbettung und die Gewichtsentwicklung analysiert. Die Gesamtliegedauer, der Zeitpunkt bis zur voll oralen Ernährung ohne Magensonde und der Zeitpunkt, ab dem die Kinder keinen zusätzlichen Wärmebedarf mehr benötigten, wurden mit einer historischen Gruppe von 42 VLBW-Frühgeborenen aus den Jahren 2011/2012 verglichen. Diese Kinder wurden bei einem höheren mittleren Gewicht (mean $1715 \pm$ SD $125 \mathrm{~g} ; p=<0,0001$ ) in ein Wärmebett umgebettet. Die Gruppen unterschieden sich nicht signifikant bezüglich Geburtsgewicht $(p=0,143)$ und Gestationsalter $(p=0,458)$.

Ergebnisse: In der Versuchsgruppe musste kein Kind aufgrund einer Hypothermie $\left(<36,5^{\circ} \mathrm{C}\right.$ rektal) zurück in den Inkubator gelegt werden. Lediglich bei einem Kind wurde die initial eingestellte Temperatur des Wärmebettes im Verlauf auf $38^{\circ} \mathrm{C}$ erhöht. Hypoglykämien nach Umbettung traten nicht auf. Ein signifikanter Einfluss auf die Häufigkeit von Apnoen 
und Bradykardien wurde nicht beobachtet. Das Trinkverhalten (sondierte - getrunkene Menge) verschiebt sich zu Gunsten der getrunkenen Menge ( $126 \mathrm{ml} / \mathrm{kg}$ sondiert, $34 \mathrm{ml} / \mathrm{kg}$ getrunken vor Umbettung vs. $113 \mathrm{ml} / \mathrm{kg}$ sondiert, $54 \mathrm{ml} / \mathrm{kg}$ getrunken nach Umbettung; prget $=0,052$, psond $=0,08)$.

Im Vergleich zur historischen Gruppe unterscheiden sich die Gesamtliegedauer (mean $73 \mathrm{~d}$ vs. $74 \mathrm{~d} p=0,757$ ) sowie das korrigierte Alter bei Entlassung (38,3 SSW vs. 38,7 SSW, $p=0,76)$ nicht signifikant. Es gibt einen nicht signifikanten Trend zu einem höheren Entlassungsgewicht in der Versuchsgruppe ( $2771 \mathrm{~g}$ vs $2541 \mathrm{~g} ; p=0,219$ ).

Schlussfolgerung: Ein Verlegen von Frühgeboren mit einem Geburtsgewicht von unter $1500 \mathrm{~g}$ vom Inkubator in ein Wärmebett bei einem aktuellen Gewicht von $1500 \mathrm{~g}$ bis $1600 \mathrm{~g}$ ist sicher und hat keine signifikanten Auswirkungen auf Vitalparameter, Trinkverhalten, Gewichtsentwicklung oder Gesamtliegedauer.

\section{P048}

Risikofaktoren, Behandlung und Verlauf pulmonaler Hypertonie bei Frühgeborenen $<\mathbf{1 0 0 0} \mathbf{g}$

\section{K. Mehler', F. Udin ten Cate' ${ }^{2}$, A. Kribs ${ }^{3}$, T. Keller', U. Bangen' ${ }^{2}$, A. Oberthuer}

${ }^{1}$ Unikinderklinik Köln, Neonatologie, Köln, Deutschland, ${ }^{2}$ Univ.-Kinderklinik, Köln, Deutschland, ${ }^{3}$ Universitätskinderklinik Köln, Neonatologie und Pädiatrische Intensivmedizin, Köln, Deutschland, ${ }^{4}$ Univ.-Kinderklinik, Neonatologie und Pädiatrische Intensivmedizin, Köln, Deutschland, ${ }^{5}$ University of Cologne Children's Hospital, Department of Neonatology, Cologne, Deutschland

Einleitung: Die neuen Form der bronchopulmonalen Dysplasie ist durch eine gestörte pulmonale Gefäßentwicklung und Alveolarisierung charakterisiert. Die pulmonale Hypertonie (PH) ist häufig mit einer BPD assoziiert und hat eine hohe Mortalität. Daher führten wir im Jahre 2010 ein echokardiographisches Screening zum pulmonalen Hypertonus bei Frühgeborenen $<1000 \mathrm{~g}$ (ELBW) ein.

Methoden: Von 2010-2015 wurden 360 Frühgeborene $<1000 \mathrm{~g}$ in der Uniklinik Köln behandelt. Bei 188 erfolgte sowohl ein echokardiographisches Screening auf einen pulmonalen Hypertonus und die Kinder wurden nach Entlassung in unserer kinderkardiologischen Ambulanz betreut. Die Screening Echokardiographie wurde vor Entlassung oder zum Zeitpunkt einer BPD Diagnose durchgeführt.

Ergebnisse: $34(18 \%)$ der 188 Frühgeborenen $<1000$ g entwickelten eine $\mathrm{PH}$, bei 20 von diesen wurde die $\mathrm{PH}$ vor Entlassung diagnostiziert. Risikofaktoren für die Entwicklung einer $\mathrm{PH}$ waren niedriges Geburtsgewicht (Odd ratio (OR) 3 bei SGA $<$ P3), das Vorliegen einer moderaten (OR 4) oder schweren BPD (OR 13) und ein vorzeitiger Blasensprung $>7$ Tage (OR 5). Allerdings hatten $1 / 3$ der Kinder mit PH keine oder nur eine milde BPD. 14 Frühgeborene entwickelten die $\mathrm{PH}$ nach Entlassung, die Hälfte dieser Frühgeborenen zeigte zuvor vermehrte Hypoxien im häuslichen pulsoxymetrischen Monitoring. Alle Kinder mit Diagnose einer PH während des stationären Aufenthalts und Frühgeborene mit PH nach Entlassung und Symptomen wurden mit Sildenfil (mediane Behandlungszeit 13 bzw. 16 Monate) behandelt.

Conclusio: Ein echokardiographisches Screening trägt zur Identifizierung von Frühgeborenen mit PH bei. Aufgrund der mit einer PH verbundenen hohen Mortailtät und Morbidität, die durch eine medikamentöse Behandlung gesenkt werden, kann ein Screening aller ELBW Frühgeborenern sinvoll sein, auch wenn keine BPD besteht.

\section{P049}

Vortäuschung eines 11-Hydroxylasemangels im Neugeborenenscreening durch unspezifische Erhöhung verschiedener adrenaler Steroidhormonmetabolite

J. Franzel', S. Kummer' ${ }^{2}$, W. Röschinger ${ }^{3}$, T. Höhn ${ }^{4}$, H. Sabir ${ }^{5}$

${ }^{1}$ Med. Einricht. d. Universität Kinderklinik, Klinik für Allgemeine Pädiatrie, Neonatologie und Kinderkardiologie, Düsseldorf, Deutschland, ${ }^{2}$ Med. Einricht. d. Universität Kinderklinik, Düsseldorf, Deutschland, ${ }^{3}$ Labor Becker, Olgemöller \& Kollegen, München, Deutschland, ${ }^{4}$ Med. Einricht. d. Universität Kinderklinik, Allgemeine Pädiatrie, Düsseldorf, Deutschland, ${ }^{5}$ Med. Einricht. d. Universität Kinderklinik, Neonatologie und pädiatrische Intensivmedizin, Düsseldorf, Deutschland

Einleitung: Aufgrund verzögerter Ausreifung adrenaler Enzymsysteme sowie persistierender adrenaler Fetalzone sind bei Früh- und Neugeborenen häufig unspezifische Erhöhungen verschiedener adrenaler Steroidhormonvorläufer zu finden.

Fallbericht: Hier berichten wir über ein weibliches Frühgeborenes von 33 SSW, die im Neugeborenenscreening im Alter von 55 Stunden durch ein erhöhtes 17-OH-Progesteron auffiel $(176 \mathrm{nmol} / \mathrm{l}$, Norm <88). Daraufhin wurde noch vom Screeninglabor eine detaillierte Steroidprofilanalytik durchgeführt, die ein charakteristisches Muster entsprechend eines 11-Hydroxylasemangels als seltene Variante eines adrenogenitalen Syndroms ergab (massiv erhöhtes 11-Desoxycortisol von $962 \mathrm{nmol} / \mathrm{l}$, Norm < 17; Androstendion $129 \mathrm{nmol} / \mathrm{l}$, Norm <17,8). Dabei keinerlei Virilisierung oder sonstige klinische Symptome im Sinne eines Glucocorticoidmangels, keine klinischen Zeichen der übermäßigen Mineralokortidoidwirkung infolge Desoxycorticosteronerhöhung. Nach Abnahme der Konfirmationsdiagnostik am 9. Lebenstag wurde eine Therapie mit Hydrocortison eingeleitet. Unerwarteterweise zeigte die Konfirmationsdiagnostik vor Therapieeinleitung wie auch alle in der Folge bestimmten Steroidprofile Normalbefunde. Eine Sequenzierung der 11-Hydroxylase ergab keine Sequenzabweichung. Anhaltspunkte für einen transienten/medikamentösen 11-Hydroxylaseblock ergaben sich nicht (keine Etomidatexposition). Ein Identitätsabgleich mit der Zweitscreeningkarte der Patientin konnte eine Proben-/Patientenverwechslung des Erstscreenings mit >99\% Sicherheit ausschließen. Somit wurde die Therapie beendet. Ein ACTH-Stimulationstest 8 Wochen nach Therapiebeendigung ergab einen Normalbefund. Schlussfolgerungen: Adrenale Ausreifungsprozesse können überraschend ausgeprägte unspezifische Erhöhungen verschiedener Vorläufermetabolite hervorrufen, die einen klassischen 11-Hydroxylasemangel vollständig imitieren können.

\section{P050}

Evaluation von zwei kommerziell erhältlichen ELISA Kits zur Bestimmung der Melatoninkonzentration im Fruchtwasser im Laufe der Schwangerschaft

S. Bagci', Ö. Altuntas', D. Katzer', C. Berg' ${ }^{2}$, A. Willruth ${ }^{2}$, H. Reutter', P. Bartmann' ${ }^{1}$, A. Müller' , B. Zur ${ }^{3}$

'Zentrum für Kinderheilkunde der Universität Bonn, Abteilung Neonatologie und Pädiatrische Intensivmedizin, Bonn, Deutschland, ${ }^{2}$ Universitätsklinikum Bonn, Geburtshilfe und Perinatalmedizin, Bonn, Deutschland, ${ }^{3}$ Instituts für Klinische Chemie und Klinische Pharmakologie, Universität Bonn, Bonn, Deutschland

Hintergrund: Das Ziel der Studie war es, den Nutzen eines auf Extraktion basierenden gegenüber einem nicht auf Extraktion basierenden Melatonin Enzyme Linked Immunoassay (ELISA) Kit zur Bestimmung von Melatonin im Fruchtwasser aus der frühen und späten Schwangerschaft zu testen. Methodik: Schwangerschaften bis zur 28 Woche wurden als frühe Schwangerschaften, nach der 28 Woche bis zur Entbindung als späte Schwangerschaften definiert. Insgesamt wurden neun Fruchtwasserproben in der frühen und 18 in der späten Schwangerschaft gesammelt. Zwei kommerziell erhältliche Melatonin ELISA Kits (Melatonin ELISA mit einer methanol-basierten Extraktion (ELISA1), und Direct Saliva Melatonin ELISA ohne Extraktionsschritt (ELISA2), beide von IBL-International, Deutschland) wurden zur Bestim- 
mung der Melatoninkonzentration (MTc) im Fruchtwasser verwendet. Der prozentuale Unterschied (\%DMTc) zwischen der MTc mittels ELISA1 und ELISA2 wurde mit folgender Gleichung berechnet: $\% \mathrm{DMTc}=(\mathrm{MTc}$ durch ELISA2- MTc durch ELISA1)/MTc durch ELISA1 × 100 .

Ergebnisse: Der Mittelwert der MTc mittels ELISA2 war signifikant niedriger als jener mittels ELISA1 (13,6 $\pm 8,8$ vs. $100,8 \pm 158,5 ; p<0,001)$. Die mittlere \%DMTc war $-51,5 \pm 39,1 \%$ (range 19,5 to $-96,1 \%$ ). Es konnte eine signifikante negative Korrelation zwischen \%DMTc und dem Schwangerschaftsalter gezeigt werden (Pearson Korrelationskoeffizient, $r=-0,814$, $p<0,001)$. Die Subgruppenanalyse zeigte für die frühe Schwangerschaft keinen signifikanten Unterschied zwischen der MTc mittels ELISA2 und der MTc mittels ELISA1 (11,2 $\pm 7,4$ vs. $12,2 \pm 7,7 ; p=0,463)$. Allerdings konnte für die späte Schwangerschaft gezeigt werden, dass die MTc signifikant niedriger war wenn sie ohne Extraktion (ELISA2) bestimmt wurde (14,8 $\pm 9,3$ vs. $145,1 \pm 179,3 ; p<0,001)$. Die Übereinstimmung der beiden Bestimmungen in der späten Schwangerschaft war gering $\left(r^{2}=0,271, p=0,022\right)$, ganz im Gegensatz zu der guten Korrelation in der frühen Schwangerschaft $\left(\mathrm{r}^{2}=0,929\right.$, $p<0,001)$. Zwischen früher und später Schwangerschaft konnten keine Unterschiede in der Albuminkonzentration $(1,8 \pm 0,8 \mathrm{~g} / \mathrm{L}$ vs. $1,9 \pm 1,3 \mathrm{~g} / \mathrm{L}$; $p=0,857)$, der Gesamteiweißkonzentration $(2,7 \pm 1,3 \mathrm{~g} / \mathrm{L}$ vs. $3,1 \pm 2,2 \mathrm{~g} / \mathrm{L}$; $p=0,625)$ oder dem Album-Gesamteiweiß-Verhältnis (67 $\pm 13 \%$ vs. $61 \pm 11 \%$; $p=0,217)$ gezeigt werden. Es zeigte sich keine Korrelation zwischen der mittleren \%DMTc $(r=-0,160, p=0,425)$ und dem Albuminkonzentration oder der Gesamteiweißkonzentration $(r=-0,156, p=0,436)$.

Zusammenfassung: Wir konnten zeigen, dass ein Melatonin ELISA Kit ohne Extraktion nicht geeignet ist um die MTc in Fruchtwasser aus der späten Schwangerschaft zu bestimmen. Ein Grund hierfür könnte sein, dass die Affinität von Melatonin zu bindenden Proteinen im Laufe der Schwangerschaft zunimmt. Somit ist eine Methanol-basierte Extraktion für Fruchtwasserproben aus der späten Schwangerschaft notwendig um die MTc verlässlich bestimmen zu können.

\section{P051 \\ Erhöhte Deleted in Malignant Brain Tumors 1 (DMBT1)- Konzentration im Fruchtwasser bei Risikoschwangerschaften?}

H. Müller' , D. Katzer', C. Berg ${ }^{3}$, A. Willruth' ${ }^{3}$, U. Felderhoff-Müser' ${ }^{1}$, A. Müller², S. Bagci2

'Universitäts-Kinderklinik Essen, Klinik für Kinderheilkunde I, Essen, Deutschland, ${ }^{2}$ Zentrum für Kinderheilkunde, Universität Bonn, Neonatologie und Pädiatrische Intensivmedizin, Bonn, Deutschland, ${ }^{3}$ Universitätsklinikum Bonn, Geburtshilfe und Perinatalmedizin, Bonn, Deutschland

Hintergrund: Das Fruchtwasser (FW) des humanen Feten enthält viele Wachstumsfaktoren und Proteine mit Funktionen bei der Immunabwehr. Es ist bekannt, dass ein Fetus ab der 16. Gestationswoche das FW schlucken kann. Tierversuche zeigten, dass das FW den frühen Aufbau und die Entwicklung der Funktion der epithelialen Barriere beim Fetus beeinflußt. DMBT1 ist eine Glykoprotein und hat Funktionen bei der angeborenen Immunabwehr, der epithelialen Differenzierung und bei der Angiogenese und wurde in der Muttermilch nachgewiesen. Es wurde bisher noch nicht untersucht, ob und in welchen Konzentrationen DMBT1 im Fruchtwasser vorkommt und ob die DMBT1-Konzentration mit klinischen Faktoren der Mutter oder des Fetens korreliert.

Patienten und Methoden: Von 59 Schwangeren (Median (Interquartilsabstand, IQR) Alter: 34 (30-38) Jahre; Bereich: 20-47 Jahre) der Uni-Frauenklinik Bonn wurden Fruchtwasserproben entnommen (Median (IQR) Gestationsalter (GA) bei Entnahme: 38,0 (32,7-38,9) SSW, Bereich: 14,3-40,1 SSW). Die Konzentration von DMBT1 wurde mittels Elisa (Abbexa, Cambridge) und die Konzentration von Superoxid-Dismutase (SOD) als Marker für fetalen Stress ebenso mittels Elisa (IBL-International, Hamburg) bestimmt. Die Schwangerschaft wurde als eine Risikoschwangerschaft deklariert, wenn eine der folgenden Diagnosen vorlag: HELLP-Syndrom, IUGR mit pathologischem Doppler, Zwerchfell-Hernie, vorzeitiger Blasensprung ohne oder mit Amnioninfektionssyndrom, feto-fetales Transfusionssyndrom, insulinpflichtiger Gestationsdiabetes und Cholestase. Die Ergebnisse wurden als Median mit IQR angegeben.
Ergebnisse: DMBT1 konnte im humanen FW in einer Konzentration (Median (IQR)) von 19,2 (11,0-38,7) ng/ml (Bereich: 3,6-111,9 ng/ml) nachgewiesen werden. Die DMBT1-Konzentration (jeweils Median (IQR)) bei den Proben vor der 37. SSW ( $n=19$; GA: 24,6 (17,1-32,7) SSW) betrug 12,7 $(7,5-44,7) \mathrm{ng} / \mathrm{ml}$, während sie bei den Proben nach der 37. SSW $(n=40$; GA: 38,6 (38,0-39,1) SSW) 19,7 (13,3-35,1) ng/ml war $(p=0,163)$. Es zeigte sich keine Korrelation zwischen der DMBT1-Konzentration und dem Gestationsalter $(p=0,939)$ und dem mütterlichen Alter $(p=0,289)$. Risikoschwangerschaften $(n=24)$ zeigten im Vergleich zu Schwangerschaften ohne Risiko ( $n=35)$ signifikant höhere Konzentrationen im FW (Median (IQR)) an DMBT1 und SOD: 28,4 (15,3-46,9) ng/ml vs. 15,2 $(9,0-25,1)$ $\mathrm{ng} / \mathrm{ml}$ und $88,2 \mathrm{ng} / \mathrm{ml}(63,5-104,8)$ vs. $70,2 \mathrm{ng} / \mathrm{ml}(48,0-85,7) ; p=0,008$ und $p=0,038$. Es zeigte sich keine Korrelation zwischen der DMBT1- und SOD-Konzentration $(p=0,395)$.

Schlussfolgerung: DMBT1 ist im Fruchtwasser des human Feten nachweisbar und ist im Falle einer Risikoschwangerschaft in signifikant höherer Konzentration vorzufinden. Die physiologische Bedeutung von DMBT1 im Fruchtwasser für die Entwicklung des Feten ist Gegenstand weiterer Studien, wobei insbesondere der fetale Gastrointestinaltrakt von Interesse ist.

\section{P052}

Wirkung von hochdosiertem rekombinantem Erythropoietin auf den Eisen-Stoffwechsel bei sehr unreifen Frühgeborenen

C. Dame' , J. Martitz' , J. Langer' ', B. Koller ${ }^{3}$, H. Sallmon' ', J. Fauchère ${ }^{3}$, H. Bucher ${ }^{3}$, L. Schomburg ${ }^{2}$, P. Kling ${ }^{4}$

${ }^{1}$ Charité - Universitätsmedizin Berlin, Neonatologie, Berlin, Deutschland, ${ }^{2}$ Charité - Universitätsmedizin Berlin, Institut für Experimentelle Endokrinologie, Berlin, Deutschland, ${ }^{3}$ UniversitätsSpital Zürich, Neonatologie, Zürich, Schweiz, ${ }^{4}$ University of Wisconsin, Neonatology, Madison/Wi, USA

Einleitung: Hepcidin ist der primäre Regulator des Eisenstoffwechsels. Zwischen der Hepcidin-Konzentration im Plasma (HepcP) und Urin (HepcU) besteht auch bei Frühgeborenen eine enge Korrelation.

Ziel der Studie war es, vor Beginn einer Eisensubstitution durch longitudinale Messungen von Hepcidin und Eisen im Urin den Effekt von hoch-dosiertem, repetitiv verabreichtem rekombinantem humanem Erythropoietin (rhEpo) auf den Eisenstoffwechsel zu charakterisieren.

Patienten und Methode: VLBW Frühgeborene (GA $<32$ Wochen) des Swiss Erythropoietin Neuroprotection Randomized Controlled Trial (NCT00413946). In den Zeitfenstern 3-6 h, 12-18 h, und 36-42 h nach der Geburt wurden entweder jeweils $3000 \mathrm{iU}$ rhEpo $(n=52)$ oder $\mathrm{NaCl} 0,9 \%$ als Plazebo $(n=31)$ als Kurzinfusion i.v. verabreicht. Im Nabelschnurblut und in seriellen Urin-Proben, die $4 \mathrm{~h}$ und $8 \mathrm{~h}$ nach Gabe der Studienmedikation asserviert werden konnten, wurde mittels ELISA die Konzentration von Epo (R\&D Systems) und Hepcidin (DRG Diagnostics) sowie mittels Total-Reflection X-ray Fluorescence Spectroscopy die Konzentration von eisen (Fe) bestimmt. Die Ergebnisse sind als Mittelwert \pm SEM dargestellt.

Ergebnisse: Zwischen der rhEpo- und der Kontrollgruppe fand kein signifikanter Unterschied in der Epo- $(40 \pm 9,2$ vs. $27 \pm 3,9 \mathrm{mU} / \mathrm{mL})$ oder der Hepcidin-Konzentration ( $33 \pm 6,7$ vs. $24 \pm 9,5 \mathrm{ng} / \mathrm{mL})$ des Nabelschnurbluts. Während $4 \mathrm{~h}$ nach Gabe der Studienmedikation kein Unterschied in der HepcU-Konzentration zwischen rhEpo- und Kontrolle-Gruppe bestand, war die HepcU-Konzentration 8-16 h nach rhEpo signifikant erhöht. (205 vs. $92 \mathrm{ng} / \mathrm{mL} ; p=0,05) .4 \mathrm{~h}$ und $8 \mathrm{~h}$ nach Placebo-Applikation bestand die direkte Korrelation zwischen der Konzentration von HepcU und HepcP im Nabelschnurblut fort $\left(R^{2}=0,51\right.$ bzw. $R^{2}=0,52$, je $\left.p<0,0001\right)$. In der multivariaten Analyse fand sich $8 \mathrm{~h}$ nach Placebo-Applikation eine direkte Korrelation zwischen der Urin-Konzentration von Fe und Hepci$\operatorname{din}\left(R^{2}=0,15, p<0,02\right)$, nicht jedoch zwischen HepcU oder Fe und Epo im Urin. $8 \mathrm{~h}$ nach rEpo-Therapie bestand keine Korrelation mehr zwischen der Hepcidin-Konzentration im Nabelschnurblut und im Urin, wohingegen die Fe-Konzentration im Urin direkt mit der rhEpo-Konzentration im Urin korrelierte $\left(R^{2}=0,31, p<0,006\right)$. 
Schlussfolgerungen: Während die Hepcidin-Konzentration im Urin 4 Stunden nach hoch-dosierter rhEpo Behandlung nicht von den Kontrollen verschieden war, wurde im zeitlichen Verlauf die Modifikation der Hepcidin- und Eisen-Ausscheidung infolge der rhEpo-Therapie evident. Dabei zeigte sich eine direkte Korrelation zwischen der Epo- und Eisen-Ausscheidung im Urin. Da Eisen per se neuroprotektive Eigenschaften hat, sollte den kurzfristigen Veränderungen im Eisenstoffwechsel unter hoch-dosierter rhEpo-Therapie Aufmerksamkeit geschenkt werden, zumal extrem unreife Frühgeborene noch keine adäquate Menge Eisen gespeichert haben.

\section{P053}

\section{Anti-Vascular Endothelial Growth Factor-Therapie (Bevacizumab)} bei Retinopathia praematurorum

F. Pulzer', P. Meier', C. Koch', M. Knüpfer', B. Ackermann', A. Bigl', U. Thome' 'Universitätsklinikum Leipzig, Klinik und Poliklinik für Kinder und Jugendliche der Universität, Abteilung Neonatologie, Leipzig, Deutschland, ${ }^{2}$ Klinik und Poliklinik für Augenheilkunde, Leipzig, Deutschland

Hintergrund: Die Inzidenz der behandlungsbedürftigen Frühgeborenenretinopathie (ROP) beträgt in Deutschland circa 5\%. Die anti-VEGF-Therapie (VEGF, Vascular Endothelial Growth Factor) kommt aktuell verstärkt zum Einsatz. Wir berichten über unsere Screening- und Therapiemodalitäten sowie deren Ergebnisse.

Methoden: Während einer 4-jährigen Beobachtungszeit wurden $2869 \mathrm{~Pa}-$ tienten auf unserer neonatologischen Intensivstation betreut, wovon 586 Frühgeborene (FG) eine augenärztliche Screening-Untersuchung gemäß der aktuellen Leitlinie erhielten. Im Falle einer therapiebedürftigen ROP kam an unserer Klinik neben der etablierten Lasertherapie in den letzten Jahren verstärkt die medikamentöse Therapie mit 0,625 mg Bevacizumab pro Applikation zum Einsatz. Wir führten eine Analyse aller behandelten Kinder durch.

Ergebnisse: Seit Januar 2012 wurden 501 Patienten mit einem Gestationsalter (GA) von $<32$ Schwangerschaftswochen (SSW), sowie 85 FG mit supplementärer Sauerstofftherapie länger als drei Tage ophthalmologisch untersucht. Eine therapiebedürftige ROP wurde in 23 Fällen $(3,9 \%$ der gescreenten Population, GA $23+3$ SSW bis $30+4$ SSW) festgestellt, zwei Erkrankungen waren extern entstanden. 14 Patienten (2,4\%; 28 Augen) erhielten primär eine intraokuläre Gabe von Bevacizumab, in einem Fal erfolgten einseitig zwei Gaben. Zwei Kinder benötigten zusätzlich eine Lasertherapie beidseits, ein weiteres eine einseitige Kryotherapie. 9 FG (1,5\%; 18 Augen) wurden primär mittels Laserkoagulation behandelt. Die Applikation von Bevacizumab erfolgte fast ausschließlich unter assistierter Spontanatmung auf unserer Station mit Lokalanästhesie und Analgosedierung. Lokale oder messbare systemische Nebenwirkungen wurden bisher nicht beobachtet. Das primäre Outcome der mit Bevacizumab therapierten FG zeigte bei 7 Patienten eine vollständig anliegende Netzhaut, teils mit Residuen der Laser- bzw. Kryotherapie, in einem weiteren Fall eine einseitige partielle Netzhautablösung. Bei drei Kindern ist die komplette Vaskularisierung noch nicht abgeschlossen, zwei werden extern weiterbetreut und ein Patient zeigt eine beidseitige Optikusatrophie.

Schlussfolgerungen: Neben der etablierten Lasertherapie kommt bei einer behandlungsbedürftigen Frühgeborenenretinopathie zunehmend die anti-VEGF-Therapie zum Einsatz. Es fehlen jedoch noch Daten zu den verschiedenen Präparaten sowie zur Dosierung. Nach den Erfahrungen an unserem Zentrum wird diese Therapie gut toleriert. Langzeitergebnisse liegen aktuell noch nicht vor.

\section{P054}

Infrared analyzers for breast milk analysis: Fat levels can influence the accuraxy of protein measurements

G. Fusch', C. Kwan' ${ }^{1}$ N. Rochow', C. Fusch'

${ }^{1}$ McMaster University, Division of Neonatology, Department of Pediatrics, Hamilton, Ontario, Kanada

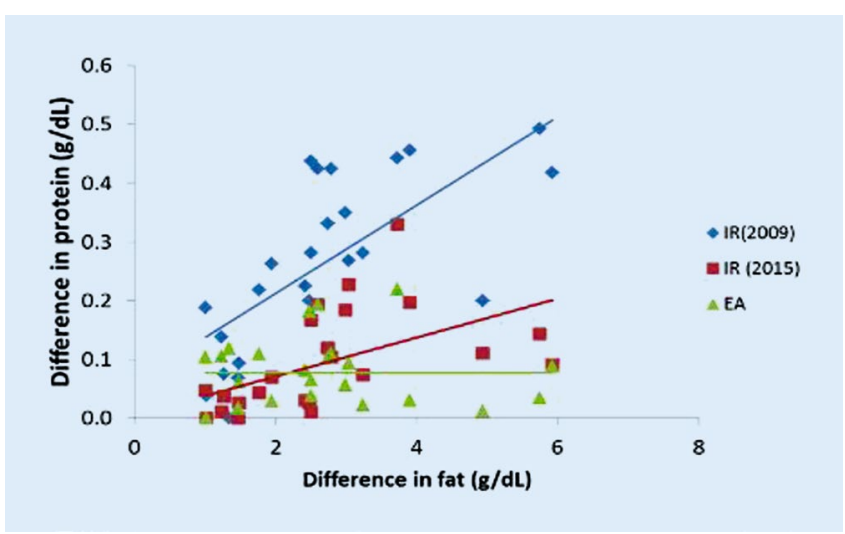

Fig. P054 $\triangle$

Background/Aim: Currently, there is a growing interest in lacto-engineering in the NICU, using infrared milk analyzers (MA) to rapidly measure the macronutrient content in breast milk before processing and feeding it to preterm infants. However, there is an overlap in the spectra information of different macronutrients, so there is a potential that they impact the robustness of the measurement. In this study, we investigate whether the measurement of protein is dependent on the levels of fat present while using a MA. Methods: Breast milk samples $(n=25)$ were measured for fat and protein content before and after being completely defatted by centrifugation, using chemical reference methods and near-infrared MA (Unity SpectraStar) with two different calibration curves (released 2009 and 2015) provided by the manufacturer.

Results: While the protein content remained unchanged, as measured by elemental analysis, measurements by MA show a difference in protein measurements dependent on fat content (• Fig. P054). This difference is less pronounced when measured using the more recent calibration curve. Conclusions: Milk analyzer measurement of protein can be dependent on the measurement of fat. This dependency varies according to the manufacturer's calibration of the device. Milk analyzers and the used calibration algorithms have to be thoroughly validated to ensure proper measurement of the macronutrients. The validation should include accuracy, precision and robustness according to the GLCP guidelines. Users of milk analyzers must be cautious of their devices' measurements, especially if they are changing the matrix of breast milk using more advanced lacto-engineering, such defatting milk for infants with chilothorax.

\section{P055}

\section{IVH in VLBW preterm babies - a role for recombinant} activated $\mathbf{F}$ VII?

M. Knüpfer', B. Ackermann' ', F. Pulzer', C. Gebauer', A. Bläser', U. Thome², N. Wolf ${ }^{2}$

'Universitätskinderklinik, Neonatologie, Leipzig, Deutschland, ${ }^{2}$ Universitätsklinikum Leipzig, Klinik und Poliklinik für Kinder und Jugendliche der Universität, Abteilung Neonatologie, Leipzig, Deutschland

Background: Intraventricular hemorrhage (IVH) remains a dangerous and frequent complication in very low birth weight infants. Activated factor VII (aFVII) activates the coagulation cascade and is a potential tool to stop bleeding, including limit the extension of IVH. This retrospective observational study compared data of infants with a growing IVH treated with fresh frozen plasma (FFP) alone or with a combination of FFP+aFVII. Methods/Intervention: All infants received cranial ultrsonography at least twice daily. When an IVH was detected, treatment with FFP $(5-20 \mathrm{ml} / \mathrm{kg}$ every 4-6 hours) was commenced and the parents were informed. If the parents endorsed aFVII treatment and IVH showed progress, aFVII (40$60 \mu \mathrm{g} / \mathrm{kg}$ body weight $4-6$ times) was given. Otherwise, infants were treated with FFP only. In a retrospective analysis, we compared the course of IVH between the control group (FFP only) and the aFVIIl group (FFP+aFVII). 
Results: 35 patients were included in the analysis (17 control group, 18 aFVIIl group). Demographic data was not different between groups. The progress of IVH was significantly smaller in the aFVII group $(p<0.01)$. During the hospital stay 4 of the infants in the control group died compared to 2 in the aFVIIl group. A posthemorrhagic hydrocephalus developed in 6 control infants and $3 \mathrm{aFVII}$ infants. All other outcome parameters (BPD, ROP, NEC) and the follow-up-results after two years were not different.

Conclusion: These data show that in case of a growing IVH, aFVII may be useful to limit the extent. A prospective randomized trial would be warranted.

\section{P056}

\section{Cerebral abscesses due to neonatal Citrobacter koseri infection}

E. Mair', H. Hummler' ${ }^{1}$ H. Haberl ${ }^{2}$

'Klinik für Kinder- und Jugendmedizin, Universitätsklinikum Ulm, Sektion Neonatologie und Pädiatrische Intensivmedizin, Ulm, Deutschland, ${ }^{2}$ Klinik für Neurochirurgie, Universitätsklinikum Ulm, Sektion Pädiatrische Neurochirurgie, Ulm, Deutschland

Introduction: Citrobacter koseri is a rare cause of neonatal meningitis and may lead to brain abscess, which is associated with a high mortality and serious neurologic damage in newborn infants.

Retrospective chart review - case report: A 35-week-old premature female baby (born via vaginal delivery 27 hours after ruptured membranes, Apgar 9/10/10, birth weight $2130 \mathrm{~g}$ ) developed fever at day of life 12 and elevated C-reactive protein $(33.5 \mathrm{mg} / \mathrm{l})$ and was admitted to our children's hospital Antibiotic therapy with piperacillin and gentamicin was started for pre-

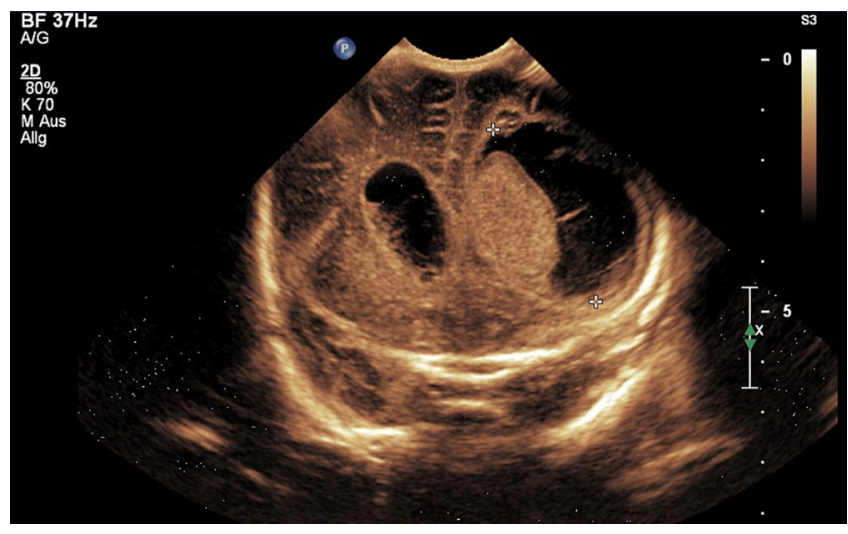

Fig. P056.1 $\Delta$ Brain ultrasound after re-admission to hospital: Circumscribed abscess in the left frontal brain (diameter approximately $4 \mathrm{~cm}$ )

Fig. P056.2

$\triangle \mathrm{MRI}$ scan after re-admission to hospital: Several bifrontal abscesses sumed bacterial sepsis. However, the blood culture was negative and $\mathrm{CrP}$ returned to normal after 6 days, when antibiotics were discontinued and the infant was discharged home. Two weeks after discharge a right-sided clonic seizure occurred and the infant was re-admitted to our hospital (CrP $31.7 \mathrm{mg} / \mathrm{l}$, WBC count normal). Antibiotic therapy with vancomycin, cefotaxime, ampicillin and phenobarbital were started. No further seizures were observed. Brain ultrasound detected a clearly circumscribed abscess in the left frontal brain. The MRI findings suggested several cerebral abscesses in the frontal region as well as a ventriculitis. We performed an endoscopic drainage of the left frontal abscess and placed an external ventricular drain. Citrobacter koseri was isolated from the intraoperative specimen. The girl received 6 weeks antibiotic treatment with meropenem and fosfomycin. Blood culture was sterile.

A following MRI scan showed several cystic residuals in the area of the former abscesses. The infant developed progressively increasing hydrocephaly requiring implantation of a rickham-reservoir and an endoscopic ventriculocisternostomy pending implantation of a ventriculo-peritoneal shunt, if necessary. At discharge, the neurological examination was normal for age.

Conclusion: This case shows the possible effects of a cerebral infection caused by Citrobacter koseri, with its tendency to affect the meninges and the brain in young infants. The propensity for C. koseri to damage the central nervous system is poorly understood. Experimental studies suggest that a specific outer membrane protein may be a neurovirulence factor. $(1,2)$

Discussion: The clinical course of this disease can be devastating because the prevalence of brain involvement is high despite early and adequate therapy with antibiotics. Our case points on the predilection for frontal injury. Clinical monitoring, sonography and MRI are important investigations to detect and treat complications of meningitis due to Citrobacter koseri: The most frequent sequelae are epilepsy and mental retardation. Because of the high risk of long-term neurologic impairment close follow-up is mandatory in these patients. $(3,4)$

\section{References}

1. The role of Citrobacter in clinical disease of children: Review, T. Doran, Clinical Infectious Diseases, 1999

2. Citrobacter brain abscesses in neonates: early surgical intervention and review of the literature, S. Chowdhry, A. Cohen, Childs Nerv Syst, 2012

3. Vertically acquired neonatal Citrobacter brain abscess - case report and review of the literature, D. Agrawal, A. Mahapatra, J Clin Neurosci, 2005

4. Citrobacter diversus brain abscess and meningitis in neonates, D. Graham, J. Band, J Am Med Assoc, 1981

\section{P057}

Die enterale Zufuhr von Formulanahrung bei frühgeborenen Schweinen induziert eine subklinische Inflammation im Darmepithel, die mit einer modifizierten Chromatinstruktur einhergeht

R. van den Bruck', R. Verena ${ }^{2}$, P. Jiang ${ }^{3}$, R. Shen ${ }^{3}$, P. Sangild ${ }^{3}$, K. Hensel', S. Wirth ${ }^{2}$, J. Postberg ${ }^{2}$, A. Jenke ${ }^{2}$

'Klinik für Kinder und Jugendmedizin der Universität, Kinderklinik I, Essen, Deutschland, ${ }^{2}$ Helios Klinikum Wuppertal, Zentrum für Kinderund Jugendmedizin, Wuppertal, Deutschland, ${ }^{3}$ Department of Human Nutrition, Faculty of Life Sciences, University of Copenhagen, Frederiksberg, Copenhagen, Dänemark

Zielsetzung: Eine überschießende Immunantwort im prämaturen Darmtrakt stellt eine zentrale Rolle in der Pathogenese der Nekrotisierenden Enterokolitis (NEK) dar. Neben einer modifizierten Expression von Proinflammatorischen- und Pattern-Recognition-Genen (PRR), scheint die enterale Nahrungszufuhr hierbei eine wesentliche Rolle zu spielen[1]. Der genaue Pathomechanismus ist jedoch noch unklar. Ziel dieser Arbeit ist es daher zu untersuchen, inwiefern die Zufuhr enteraler Nahrung in Form von Formula oder Kolostrum die intestinale Genregulation und Chromatinstruktur verschiedener PRR (z. B. IL8, TLR2, TLR4, REG3A) im Darm frühgeborener Schweine beeinflusst. 
Methoden: 15 frühgeborene Schweine wurden in drei Subgruppen stratifiziert. 5 Tiere wurden total parenteral (TPN) ernährt. Die verbleibenden 10 Schweine erhielten darüber hinaus minimale Mengen an enteraler Nahrung (MEN; 2, 4, 6, 8 mL/kgKG/3 h am 1.-4. Lebenstag), und zwar entweder bovines Kolostrum $(n=5)$ oder Frühgeborenen-Formulanahrung $(n=5)$. Vor Beginn jeglicher klinischer Symptomatik im Sinne einer NEK wurden am 5. Lebenstag Darmproben entnommen. Genexpressionsanalysen wurden mittels qRT-PCR durchgeführt mit Fokus auf PRR-, und Proinflammatorische-Genen. Die Chromatinkonformität im Bereich der untersuchten Gene charakterisierten wir mittels Endonuklease-Sensitvitäts-Assay. In funktionellen Studien wurden bei CaCo-2 Zellen unter Verwendung des Histon-Deacetylase-Inhibitors Trichostatin A (TSA) artifiziell eine offene Chromatinstruktur erzeugt und die inflammatorische Antwort auf einen bakteriellen Stimulus (Lipopolysaccharid, LPS) im Vergleich zu den nativen CaCo-2 Zellen analysiert.

Ergebnisse: Die Zufuhr enteraler Nahrung resultierte in einer signifikant gesteigerten Expression Proinflammatorischer-Gene, z. B. von IL8 und TLR4. Dieser Effekt war besonders ausgeprägt bei mit Formulanahrung ernährten Tieren. Colstrum führte darüber hinaus zu einer Induktion der Expression des anti-inflammatorischen Zytokins Interleukin-10. Passend zur gesteigerten Expression fanden sich an den meisten Genoloci (vor allem IL8 und TLR4) im Endonuklease-Sensitivität-Assay signifikant häufiger offene Chromatinstrukturen, während sich allerdings nur geringe Unterschiede in der CpG-Methylierung fanden. Die mögliche Relevanz dieser epigenetischen Signaturen zeigte sich im in vitro Zellkultur Modell. Hier führte eine durch TSA Behandlung induzierte offene Chromatinstruktur zu einer signifikant höheren IL8 Expression nach LPS Exposition im Vergleich zu unbehandelten Zellen.

Schlussfolgerung: Enterale Ernährung induziert Veränderungen in der Expression von Genen, die in der Inflammationsregulation und angeborener Immunantwort des frühgeborenen Darms involviert sind. Dieser liegt eine möglicherweise durch exogene Faktoren bedingte modifizierte Chromatinstruktur zu Grunde. Hierbei spielt die Art der enteralen Nahrung mit einem, im Vergleich zu Colostrum deutlicheren proinflammatorischen Effekt von Formulanahrung eine wesentliche Rolle. Dies prädisponiert möglicherweise zur Entstehung einer NEK beim Frühgeborenen. Weitere Studien werden benötigt, um die enterale Ernährung dieser Patientengruppe in Hinblick auf die Pathogenese NEK zu optimieren.

\section{References}

1. Morgan, J., L. Young, and W. McGuire, Delayed introduction of progressive enteral feeds to prevent necrotising enterocolitis in very low birth weight infants. Cochrane Database Syst Rev. 12.

\section{P058}

\section{High survival of infants born at 22 and 23 weeks following active} pre- and postnatal care

K. Mehler', A. Oberthuer', T. Keller ', I. Becker', M. Valter ${ }^{5}$, B. Roth ${ }^{6}$, A. Kribs 'Unikinderklinik Köln, Neonatologie, Köln, Deutschland, ${ }^{2}$ University of Cologne Children's Hospital, Department of Neonatology, Cologne, Deutschland, ${ }^{3}$ Univ.-Kinderklinik, Neonatologie und Pädiatrische Intensivmedizin, Köln, Deutschland, ${ }^{4}$ University of Cologne, Institute of Medical Statistics, Informatics and Epidemiology, Cologne, Deutschland, ${ }^{5}$ Unifrauenklinik Köln, Geburtshilfe, Köln, Deutschland, ${ }^{6}$ UniversitätsKinderklinik Köln, Neonatologie und pädiatrische Intensivmedizin, Köln, Deutschland, ${ }^{7}$ Universitätskinderklinik Köln, Neonatologie und Pädiatrische Intensivmedizin, Köln, Deutschland

Background: Rates of survival for infants born at the border of viability are still low and vary considerably between neonatal intensive care units. Objective: To determine whether higher survival rates and better short term outcome of these infants may be achieved using an active pre- and postnatal care approach. Design: We conducted a retrospective analysis of infants born between 22 0/7 and 23 6/7 weeks of gestation at the University hospital of Cologne between 2010 and 2014. Outcome parameters were survival until discharge and survival without major complications, defined as high grade intraventricular hemorrhage, surgery for abdominal complications, bronchopulmonary dysplasia or retinopathy of prematurity. Setting: Level III neonatal intensive care unit in Germany

Participants: One hundred and six liveborn infants, 45 born at 22 weeks and 61 born at 23 weeks of gestation. Exposure: Active pre- and postnatal treatment Main outcome: Survival without neonatal or short term severe complications

Results: Thirty eight percent of infants born at 22 weeks and $4.9 \%$ born at 23 weeks received palliative care. Active care was offered to 86 infants (22 weeks, $n=28$ and 23 weeks, $n=58)$. Of these, $67 \%$ (61\% born at 22 weeks and $71 \%$ born at 23 weeks) survived until discharge. Survival without severe complications was $26 \%$ ( $21 \%$ for infants born at 22 weeks and $28 \%$ of infants born at 23 weeks). Survival was predicted by the Apgar Score after 5 minutes (odds ratio (OR) 0.62, CI [0.46-0.84]) and birthweight (OR 0.001 , CI [0.00-0.40].

Conclusion: One in four infants born at the border of viability and offered active care survived without severe complications. This finding should be considered for individualized parental approach and decision making. Active follow up information is required to determine childhood outcome.

\section{Postersitzung 07: Frühgeborene II}

\section{P059}

Lactoferrin attenuates the proinflammatory response of neonatal macrophages

I. Wessely', E. Herndl', K. Sadeghi', A. Spittler', E. Förster-Waldl', A. Berger', L. Wisgrill'

'Medical University of Vienna, Department of Pediatrics \& Adolescent Medicine - Division Neonatology, Vienna, Österreich, ${ }^{2}$ Medical University of Vienna, Department of Surgery \& Core Facility Flow Cytometry, Vienna, Österreich

Introduction: Lactoferrin, a mammalian milk glycoprotein, has various effects on the host innate immune defense. In the gut, neonatal intestinal macrophages progressively acquire a gestational age- dependent non-inflammatory profile. Thus, the higher proinflammatory activity of neonatal intestinal macrophages of premature neonates might contribute to inflammatory mucosal injury. Therefore, the aim of the study was to investigate the immunomodulatory effect of human lactoferrin on LPS, LTA and classical activated (IFN-y/LPS) monocyte-derived macrophages of term neonates and healthy adults. In the next step, monocyte-to-macrophage differentiation and polarization will be investigated by whole transcriptome analysis.

Methods: Cord blood and peripheral blood monocytes were differentiated in the presence or absence of lactoferrin and then stimulated with the TLR2 agonist LTA, the TLR4 agonist LPS or IFN-y. The surface expression of TLR2/TLR4, intracellular TNFa production and cytokine secretion were evaluated by flow cytometry. Macrophage polarization will be evaluated using Human Gene ST array.

Results: Lactoferrin decreased TLR-dependent pathway activation of macrophages attenuating in a dose-dependent manner the TNFa and IL-6 secretion. Furthermore, macrophages displayed reduced phagocytic activity. Taken together, lactoferrin seems to inhibit M1 polarization driving macrophages towards a M2-driven stage. These observations will be evaluated by whole transcriptome analysis. Conclusion: In summary, human lactoferrin attenuates the proinflammatory response upon TLR2/TLR4 activation of neonatal macrophages. These data show that lactoferrin mediates anti-inflammatory effects by down-regulation of TLR2 and TLR4 expression on neonatal macrophages. We thus conclude that these anti-inflammatory properties might be a potential mechanism contributing to the preventive effects of lactoferrin in the premature gut. 
P060

\section{Magenperforation bei konnataler Listeriose bei einem Frühgeborenen der 29. SSW}

\author{
K. Riebe ${ }^{1}$, H. Schneider ${ }^{2}$, P. Freisinger ${ }^{3}$ \\ ${ }^{1}$ Kreiskrankenhaus, Kinderklinik, Reutlingen, Deutschland, ${ }^{2}$ Klinikum \\ am Steinenberg Reutlingen, Klinik für Kinder- und Jugendmedizin, \\ Reutlingen, Deutschland, ${ }^{3}$ Kreiskliniken Reutlingen, Klinik für Kinder- und \\ Jugendmedizin, Reutlingen, Deutschland
}

Hintergrund: Die konnatale Listeriose führt in der Regel zu ZNS-Infektionen oder Septikämien, deren Morbidität und Mortalität besonders bei Frühgeborenen hoch ist. Spontane Magenperforationen sind bei Frühgeborenen bekannt jedoch bisher nicht bei konnataler Listeriose in der Literatur beschrieben. Wir berichten über ein Frühgeborenes (FG) der 28+4/7 SSW mit spontaner mehrfacher und mehrzeitiger Magenperforation bei konnataler Listeriose.

Fallbeispiel: Anamnese: Männliches FG der 28 + 4/7 SSW, Geburtsgewicht $1230 \mathrm{~g}$ (50. P.) Sek. Sectio bei vorzeitiger Wehentätigkeit und Infektionszeichen der Mutter. Kind initial hypoton, Herzfrequenz > 100/min, unzureichende Spontanatmung. Dreimalig Blähmanöver und manuelle Beatmung mit Perivent. $\mathrm{Ab}$ 15. Lebensminute nur CPAP mit max. $\mathrm{FiO}_{2} 30 \%$ nötig. Temp. bei Geburt $38,3^{\circ} \mathrm{C}$, direkt Absetzen von Mekonium. Wegen Infektionszeichen der Mutter (Fieber, erhöhtes CrP mit 83 mg/l) Gabe von Ampicillin bei der Erstversorgung. 14 Tage vor Geburt hatte die Mutter bereits Fieber, der Verdacht eines viralen Infekts war gestellt worden.Kalkulierte antibiotische Therapie mit Ampicillin, Cefotaxim und Gentamycin. Bereits ab dem 1. Lebenstag(LT) massiv ausladendes Abdomen. Bei zunehmenden Magenresten und weiter ausladendem Abdomen trotz CPAP-Pausen am 3. LT Röntgen des Abdomens nach Kontrastmittelgabe über Magensonde.Dringender V.a. auf Magenperforation und Verlegung in Kinderchirurgie. Am 3. LT Nachweis von Listeria monocytogenes in den initialen Abstrichen und Magensaft bei negativer Blutkultur.

Therapie und Verlauf: Operation am 3. LT. Darstellung und Übernähung von zwei Magenperforationen. Am 4. postop. Tag erneute spontane Magenperforation. Nachweis einer Nekrose der Magenschleimhaut und Magenteilresektion. Histologisch normale Wandstruktur des Magens. Am 5. postop. Tag erneute Magenperforation- dieses Mal konservative Therapie ausreichend. Erfolgreiche Extubation am 12. postop. Tag; danach keine Atemhilfe oder $\mathrm{O}_{2}$ Bedarf mehr. Antibiotische Therapie erreger- und resistenzgerecht mit Ampicillin und Tobramycin über 21 Tage. Vom 27. LT an langsamer Nahrungsaufbau bis zum 37. LT oral und über Magensonde Als Magenschutz Ranitidin bis zum 45. LT. Am 53. LT Entlassung in gutem AZ und Körpergewicht auf der 50. Perzentile.

Schlussfolgerung: Vergleichbare Fallberichte finden sich bislang in der Literatur nicht. Spontane Magenperforationen bei Neonaten sind sehr selten $(1,2)$. Bei unserem Patienten ist ursächlich ist eine Kombination von Listerieninfektion und Frühgeburtlichkeit zu diskutieren.

\section{Literatur}

1. Korhonen P et al., Acta Paediatrica 2007, 96: 600-601

2. Jawad AJ et al., Pediatric. Surg. Int. 2002, 18: 396-399

\section{P061 \\ Auswertungsscores zum amplituden-integrierten EEG und Korrelation mit dem PIPP-R-Score bei Früh- und Neugeborenen}

\section{Stavropoulou', D. Römer' ${ }^{1}$, R. Hentschel ${ }^{1}$}

'Institut/Klinik: Universitätsklinikum Freiburg, Zentrum für Kinder- und Jugendmedizin, Neonatologie/Intensivmedizin, Freiburg, Deutschland

Hintergund: Das aEEG gewinnt zunehmend an Bedeutung als neurologische Überwachungsmethode für Früh- und Neugeborene. Eine schmerzfreie Behandlung dieser Patienten ist ebenfalls Ziel jeder Behandlung. Die Schmerzreaktion eines Früh- und Neugeborenen wird üblicherweise anhand von Schmerzscores klinisch erfasst und objektiviert. Ob der zentral verarbeitete Schmerzreiz sich im aEEG widerspiegelt ist nicht ausreichend untersucht.
Fragestellung: Wie korrelieren der aEEG-Score nach Hellström-Westas und der nach Burdjalov miteinander, und gibt es eine Korrelation mit dem PIPP-R-Schmerzscore?

Methode: Früh- und Neugeborene, die im Jahr 2015 in unserer Frauenklinik geboren wurden und in der Neonatologie behandelt wurden, erhielten nach Einverständnis der Eltern eine aEEG-Ableitung für mindestens drei Stunden. Zusätzlich wurde das Wohlbefinden des Kindes mittels des PIPPR-Scores von uns direkt nach Anlage der Elektroden erfasst. 17 Neugeborene mit einem Gestationsalter von 28 bis 42 SSW wurden eingeschlossen. Ausgeschlossen wurden Kinder mit Erkrankungen und Interventionen die nach bisherigem Kenntnisstand das aEEG beeinflussen. Zum Zeitpunkt der Ableitung des aEEG waren die Neugeborenen durchschnittlich 4 Tage alt. Zur Auswertung benutzten wir das SPSS Programm in der Version 22. Hiermit wurde der Rangkorrelationskoeffizient nach Spearman bestimmt, sowie die weitere deskriptive Analyse erarbeitet.

Ergebnis: Bei den Probanden bestimmten wir einen Burdjalov-Score zwischen 6 als Minimum und 13 als Maximum (Median 10). Die PIPP-RWerte verteilten sich zwischen 3 Punkten und 12 Punkten (Median 6). Die Auswertung nach Hellström-Westas ergab, dass alle Patienten eine kontinuierliche Hintergrundaktivität hatten, wobei der Schlaf-Wach-Rhythmus bei $12 \%$ bereits entwickelt war, bei $82 \%$ imminent und bei $6 \%$ unausgereift war. Krampfanfälle hatte während des Beobachtungszeitraumes keiner der Patienten. Bei einem Spearman-Rangkorrelationseffizienten von $-0,406$ war der kausale Zusammenhang zwischen dem PIPP-R und dem Burdjalov-Score nur mäßig signifikant $(p=0,106)$. Ebenfalls konnte keine starke Korrelation zwischen der Auswertung nach Hellström-Westas und dem PIPP-R-Score gefunden werden. Betrachtete man von den 3 Komponenten des Scores nach Hellström-Westas (Schlaf-Wach-Rhythmus, Hintergrundmuster und Krampfereignisse) den Parameter „SchlafWach-Rhythmus" so ergab sich eine Spearman-Rangkorrelation von 0,014 $(p=0,958)$ mit dem PIPP-R. Da alle Neonaten eine kontinuierliche Hintergrundaktiviät zeigten, und kein Kind Krampfananfälle erlitt, ist eine Berechnung der Korrelation dieser beiden Parameter mit dem PIPP-R nicht möglich.

Schlussfolgerung: Sowohl der aEEG-Score nach Hellström-Westas als auch der nach Burdjalov erlaubten eine übersichtliche und korrekte Klassifikation des aEEG. Vorbehaltlich der geringen Patientenzahl in unsere Studie glauben wir, dass eine Schmerzerfassung durch den PIPP-R bei Neonaten nicht durch ein aEEG ersetzt werden kann. Obwohl Schmerzen im Gehirn nachweislich mittels EEG dargestellt werden können, scheint es mit den derzeitigen aEEG-Scores keine Möglichkeit zu geben, auf Schmerzen bei Früh- und Neugeborenen zu schließen.

\section{P062}

\section{Sozialmedizinische Nachsorge als Grundlage} gesetzlicher Partizipation

\section{B. Kleemann}

${ }^{1}$ Klinikum Hanau GmbH, Sozialberatung, Hanau, Deutschland

Heutzutage kann zu einem sehr großen Teil frühgeborenen Kindern, oder Kindern mit schweren Erkrankungen oder Behinderungen weitergeholfen werden. Geschätzt können ca. 80 \% der Kinder das Erwachsenenalter erreichen, da deren Chancen auf Heilung in den letzten Jahren deutlich gesteigert werden konnte. Die Erkenntnis, dass nicht nur die medizinische Versorgung dazu beiträgt, sondern vielmehr auch die häusliche Situation und das ganze Familiensystem betrachtet werden muss, stellte eine deutlich differenziertere Art der Begleitung dar, die erst in den letzten Jahren zunehmend Beachtung findet. Die psychische und medizinisch/pflegerische Belastung, die Unkenntnis von Netzwerken, die Einschränkungen, die durch die Nichtinanspruchnahme weiterer therapeutischer Maßnahmen und die problematische Situation von Traumatisierung und Ausgrenzung der Familien findet besondere Beachtung. Die Aufspaltung des Gesundheits- und Sozialwesens in unterschiedliche Sektoren und die kategorische Trennung von stationären, ambulanten oder rehabilitativen Maßnahmen stellen eine weitere Herausforderung dar. Häufig resultiert daraus die bereits erwähnte Überforderung, die besonders zur erneuten Aufnahme der Kinder in sta- 
tionären Maßnahmen (Drehtüreffekt) führen kann. Psychosozialer Dienst, Kinderonkologische Nachsorge, Frühförderung, Sozialpädagogische Zentren und Häusliche Kinderkrankenpflege können diese Form der Nachsorge, als alleinstehende Professionen, ebenfalls nicht leisten. Durch ein interdisziplinäres Team sollen die Familien unterstützt werden um Ressourcen zu erkennen, Hilfen zu erhalten und sie damit zu echter Partizipation zu befähigen. Im ersten Teil werden die gesetzlichen Grundlagen, wie die Grundrechte bis hin zum KJHG in Bezug auf Partizipation, vorgestellt. Die Bedeutung von Partizipation im gesetzlichen und wirtschaftlichen Sinne sollen hier betrachtet werden. Im zweiten Teil wird die Arbeit des Bundesverbandes Bunter Kreis e. V. vorgestellt werden. Dieser arbeitet auf Grundlage des $₫ 43$ SGB V und erweitert diesen um die eigene Anspruchshaltung, in der weiteren Versorgung der betroffenen Familien. Ziel ist es, nicht nur die medizinische Gesundheit der Kinder herzustellen, sondern vielmehr echte Teilhabe in der Gesellschaft zu ermöglichen. Abschließend soll eine Diskussion, über die Nachhaltigkeit der Begleitung nach $\$ 43$ (2) SGB V und die Möglichkeiten von echter Partizipation, geführt werden.

\section{P063}

\section{Schwere neonatale Enterovirusinfektion mit Leberversagen und disseminierter intravasaler Gerinnung}

A. Brolund', F. Sander', S. Apostolidou'², H. Wiedemann', P. Gudowius', A. von der Wense

${ }^{1}$ Altonaer Kinderkrankenhaus, Neonatologie und pädiatrische Intensivmedizin, Hamburg, Deutschland, ${ }^{2}$ Neonatologische Intensivstation, Universitätskinderklinik, Mainz, Deutschland

Hintergrund: Humane Enteroviren sind unbekapselte RNA-Viren aus der Familie der Picornaviridae. Gemäß einer neuen Taxonomie werden Enteroviren molekulargenetisch in die fünf Gruppen humane Enteroviren A, B C, D und Polioviren einteilt. Enteroviren sind häufige Pathogene in der Allgemeinbevölkerung, im Kindesalter sind Säuglinge mit ca. einem Drittel aller Fälle die am stärksten betroffene Gruppe. Das klinische Spektrum der Nicht-Polioviren reicht von unspezifischer febriler Infektion, respiratorischem Infekt und Gastroenteritis bis hin zu schwerer Erkrankung mit Organbeteiligung wie Myokarditis, Hepatitis und Enzephalitis. In der Neonatalperiode können sie ein sepsisähnliches Krankheitsbild auslösen. Fallbeschreibung: Wir berichten über ein Frühgeborenes der $36+0$. Schwangerschaftswoche welches nach Geburtseinleitung bei Erhöhung des $\mathrm{CrP}$ und unklaren gastrointestinalen Beschwerden der Mutter mit einem Geburtsgewicht von $2630 \mathrm{~g}$ vaginal entbunden und am 4. Lebenstag (LT) nach Hause entlassen wurde. Aufgrund von Trinkschwäche und Hypothermie erfolgte die Wiedervorstellung am 5. LT. Bei Verdacht auf eine bakterielle Infektion wurde eine antibiotische Therapie mit Ampicillin und Tobramycin begonnen. Wenige Stunden nach Aufnahme war eine CPAP-Atemunterstützung notwendig. Das Kind entwickelte ein fulminantes Leberversagen mit massiver Transaminasenerhöhung, Hypalbuminämie, beidseitigen Pleuraergüssen, Aszites sowie eine disseminierte intravasale Gerinnung (DIC). In der erweiterten Diagnostik konnte mittels PCR Enterovirus RNA im Liquor und Stuhl detektiert werden. Die Sequenzierung ergab den Nachweis von Echovirus Typ 7. In der Umgebungsdiagnostik ließ sich der Echovirus 7-Stamm auch im Stuhl der Mutter nachweisen. Die massive Leberfunktionsstörung in Kombination mit der DIC erforderte mehrfache Gaben von Albumin, EKs, TKs und FFP. Die Pleuraergüsse wurden drainiert und es wurden einmalig Immunglobuline verabreicht. Unter diesen Maßnahmen verbesserte sich die klinische Situation zunehmend. Laborchemisch kam es zu einem zögerlichen Abfall der erhöhten Leberwerte. Das Kind konnte am 22. LT nach Hause entlassen werden.

Diskussion: Der Fall zeigt den Verlauf eines fulminanten Leberversagens mit begleitender DIC im Rahmen einer schweren Echovirus 7-Infektion und unterstreicht die Bedeutung einer Enterovirusinfektion als Differentialdiagnose bei Sepsis und Leberversagen in der Neonatalperiode. Die Übertragung erfolgte vermutlich postnatal von der Mutter auf das Kind. Der therapeutische Einsatz von IVIG ist in zahlreichen Fallberichten als Option beschrieben, erfordert jedoch weitere Studien.

\section{P064}

Adrenogenitales Syndrom mit nicht-funktionellen Mutationen in beiden Allelen bei einem klinisch unbeeinträchtigten Kind in Laos

T. Höhn' , Z. Lukacs², W. Huckenbeck ${ }^{3}$, T. Torresani' , O. Blankenstein ${ }^{5}$, S. Bounnack ${ }^{6}$

${ }^{1}$ Med. Einricht. d. Universität Kinderklinik, Allgemeine Pädiatrie, Düsseldorf, Deutschland, ${ }^{2}$ Univ.-Kinderklinik Eppendorf, Hamburg, Deutschland, ${ }^{3}$ Instituts für Forensische Anthropologie, Rommerskirchen, Deutschland, ${ }^{4}$ Kinderspital Zürich, Zürich, Schweiz, ${ }^{5}$ Charité Universitätsmedizin, Pädiatrische Endokrinologie, Berlin, Deutschland, ${ }^{6}$ Ministry of Health, Vientiane, Laos, People's Democratic Republic of

Hintergrund: Ergebnisse von Neugeborenenstoffwechsel-Screeningprogrammen zielen bei der Detektion des Adrenogenitalen Syndroms (AGS) auf erhöhte Werte von 17-Hydroxy-progesteron (17-OHP) ohne hierbei die Präsenz eines Salzverlusts nachweisen zu können.

Ziel: Die Vorhersage eines Salzverlusts bei Neugeborenen mit AGS.

Material und Methoden: Die erste Probe eines vermuteten AGS in einem neu etablierten Neugeborenenstoffwechsel-Screening in Laos wurde auf bekannte Mutationen mit assoziiertem Salzverlust untersucht.

Ergebnisse: Die molekulargenetische Diagnostik zeigte in beiden Allelen Mutationen, die mit einem Funktionsverlust des Enzyms assoziiert sind. Entgegen der Erwartung war das Kind ohne jegliche Substitutionstherapie klinisch komplett unbeeinträchtigt.

Schlußfolgerung: Obwohl molekulargenetische Methoden theoretisch den Funktionsverlust beim AGS vorhersagen können, ist das von uns beschriebene Kind aktuell im Alter von sechs Jahren klinisch völlig unauffällig. Wir spekulieren, dass beim AGS die verbleibende Restaktivität des Enzyms ausreichend hoch sein kann um trotz eines theoretischen Funktionsverlusts nicht zu einer klinisch Symptomatik zu führen.

\section{P065}

Der Einfluss von Infektion auf die Spiegel antimikrobieller Peptide im Blut frühgeborener Kinder

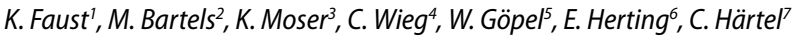

${ }^{1}$ Universitätsklinikum Schleswig Holstein Campus Lübeck, Klinik für Kinder- und Jugendmedizin, Lübeck, Deutschland, ${ }^{2}$ Universitätsklinikum Schleswig Holstein, Kinderklinik, Lübeck, Deutschland, ${ }^{3}$ Klinikum Aschaffenburg, Neonatologie und päd. Intensivmedizin, Aschaffenburg, Deutschland, ${ }^{4}$ Klinikum Aschaffenburg, Neonatologie un pädiatrische Intensivmedizin, Aschaffenburg, Deutschland, ${ }^{5}$ Universitätsklinikum Schleswig-Holstein, Klinik für Kinder- und Jugendmedizin, Lübeck, Deutschland, 'Universitätsklinikum Schleswig-Holstein Campus Lübeck, Direktor der Klinik für Kinder- und Jugendmedizin, Lübeck, Deutschland, ${ }^{7}$ Universitätsklinikum Schleswig Holstein/Campus Lübeck, Klinik für Kinderund Jugendmedizin, Lübeck, Deutschland

Hintergrund: Antimikrobielle Polypeptide (APP) sind Bestandteil des angeborenen Immunsystems. Die Rolle von APPs für die Immunantwort Frühgeborener ist bislang unzureichend untersucht.

Fragestellung: Ziel dieser bizentrischen Studie war es, die Expression von APPs in Vollblutproben von Frühgeborenen mit unterschiedlichen Gründen der Frühgeburtlichkeit zu charakterisieren und insbesondere eine Assoziation mit Infektion zu prüfen.

Material und Methoden: 250 frühgeborene Kinder mit einem Gestationsalter zwischen 23 und 35 SSW sowie 175 dazugehörige Mütter wurden für diese Studie rekrutiert. APPs (Lactoferrin, humanes neutrophiles Peptid (HNP) 1-3 und Calprotectin) wurden mittels ELISA in peripherem Blut von Mutter und Kind sowie im Nabelschnurblut bestimmt und mit klinischen Parametern assoziiert.

Ergebnisse: Bei Frühgeborenen, die aufgrund eines Amnioninfektionssyndroms (AIS) geboren wurden, zeigten sich am ersten Lebenstag signifikant erhöhte Spiegel von Lactoferrin, HNP 1-3 sowie Calprotectin im Vergleich zu Frühgeborenen ohne AIS (Lactoferrin in ng/ml: Median 91,6 mit AIS/49,5 ohne AIS, Standardfehler 24,9/22,1, $p<0,001$, HNP 1-3 in ng/ml: 5173/3120, 3444/1425, $p=0,021$, Calprotectin in ng/ml: 57,4/37,9, 
$14,3 / 9,3, p=0,004)$. Diese Assoziation war am 3. Lebenstag bereits nicht mehr nachzuweisen. Frühgeborene, die eine Early-Onset-Sepsis (EOS) entwickelten, zeigte sich am 3. Lebenstag eine Tendenz zu erhöhten Spiegeln von Calprotectin (in ng/ml, 204,7 mit EOS/116,7 ohne EOS 43,6/10,5, $p=0,044$ ), jedoch erniedrigten Spiegeln von Lactoferrin (in $\mathrm{ng} / \mathrm{ml}, 64,4 \mathrm{mit}$ $\mathrm{EOS} / 39,7$ ohne EOS $13,4 / 15,5, p=0,054)$. Es zeigte sich nur eine schwache Korrelation der Spiegel der gemessenen antimikrobiellen Peptide mit dem Gestationsalter oder mit den AMP-Spiegeln der Mutter.

Diskussion/Schlussfolgerung: Die Höhe der Plasmaspiegel von APP wird von verschiedenen Faktoren beeinflusst. Im Rahmen eines AIS scheinen die Spiegel beim Kind erhöht zu sein. Eine Infektion des Kindes geht mit veränderten Spiegeln einher, ohne dass aus den vorliegenden Daten geschlossen werden kann ob die Infektion Folge oder Ursache der veränderten Plasmaspiegel ist. Vor der Supplementation von antimikrobiellen Peptiden sollten daher weitere Untersuchungen durchgeführt werden.

\section{P066}

\section{Sapovirus Nachweis bei einem Frühgeborenen mit nekrotisierender Enterokolitis}

\section{S. Oberweis' ${ }^{1}$ T. Dresbach ${ }^{1}$, A. Müller', S. Bagci ${ }^{1}$}

'Zentrum für Kinderheilkunde der Universität Bonn, Abteilung Neonatologie und Pädiatrische Intensivmedizin, Bonn, Deutschland

Hintergrund: Die nekrotisierende Enterokolitis (NEK) ist eine der häufigsten Ursachen für ein akutes Abdomen auf einer neonatologischen Intensivstation und betrifft zumeist Frühgeborene mit sehr niedrigem Geburtsgewicht. Obwohl die genaue Ätiologie weiterhin nicht vollständig geklärt ist, scheinen Infektionserreger eine wichtige Rolle zu spielen. Neben bakteriellen Erregern wird allerdings auch eine Assoziation zwischen NEK und viralen Erreger wie Rotavirus, Astrovirus und Noravirus vermutet. Die Sapoviren bilden zusammen mit den Noroviren die Familie der humanen Caliciviridae. Infektionen mit Sapoviren lösen im Vergleich zu Noroviren eine mildere Symptomatik aus. Wir berichten über einen Fall mit NEK bei einer Sapovirus (SaV) Infektion.

Fallvorstellung: Der Patient wurde bei Uterusruptur der Mutter per Notsectio mit einem Gestationsalter von $28+5$ SSW mit einem Geburtsgewicht von $1150 \mathrm{~g}$ (50. Perz) und einem APGAR von 6/8/9 geboren. Der orale Nahrungsaufbau gelang initial sehr gut. Am 6. Lebenstag wurde eine antibiotische Therapie mit Tazobactam und Vancomycin bei einem reduzierten Allgemeinzustand, vermehrten Apnoen und IL-6 Anstieg auf $146 \mathrm{pg} / \mathrm{ml}$ (Norm < $100 \mathrm{pg} / \mathrm{ml}$ ) begonnen. Am 7. Lebenstag entwickelte das Frühgeborene blutige Stühle, sowie ein druckschmerzhaftes und abwehrgespanntes Abdomen. Die CRP-Konzentration war auf $15.3 \mathrm{mg} / \mathrm{L}$ und die Leukozytenzahl auf 13310 G/L angestiegen. Radiologisch zeigte sich eine Pneumatosis Intestinalis im Sinne einer nekrotisierenden Enterokolitis. In Abwesenheit eines abdominellen Kompartmentsyndroms wurde sich zunächst für eine konservative Therapie mit Nahrungskarenz, Magenablaufsonde und Antibiotika entschieden. Am Folgetag wurde das Kind bei deutlich vermehrten Apnoen intubationspflichtig, auf eine chirurgische Intervention konnte jedoch weiterhin verzichtet werden. Am 9. LT kam es zu einer deutlichen klinischen und radiologischen Verbesserung. Im Stuhl des Patienten konnte $\mathrm{SaV}$ nachgewiesen werden.

Schlussfolgerung: Diese Fallvorstellung zeigt, dass SaV auch mit der Entwicklung einer NEC bei Frühgeborenen assoziiert sein kann. Demnach würden wir empfehlen, dass die virologischen Untersuchungen unter Einschluss von SaV neben den bakteriologischen Untersuchungen des Stuhls bei Kindern mit NEC durchgeführt werden sollten.

\section{P067}

Korreliert die Dauer eines frühen vorzeitigen Blasensprungs (preterm premature rupture of membranes - PPROM) mit dem Auftreten eines Amnioninfektionssyndroms bei Frühgeborenen?

H. Müller' , A. Stähling' ', T. Storbeck', N. Bruns' ', A. Köninger'², U. FelderhoffMüser ${ }^{1}$

'Universitätsklinikum Essen, Klinik für Kinderheilkunde I, Essen,

Deutschland, ${ }^{2}$ Universitätsklinikum Essen, Klinik für Frauenheilkunde und Geburtshilfe, Essen, Deutschland

Hintergrund: Ein früher vorzeitiger Blasensprung (PPROM) kann mit einem primären oder sekundären Amnioninfektionssyndrom (AIS) assoziiert sein. Eine wesentliche Strategie in der Geburtshilfe ist es, durch eine adäquate maternale antibiotische Therapie und - wenn nötig - durch eine vorzeitige Entbindung ein AIS möglichst zu vermeiden, da dies mit einer erhöhten Morbidität und Mortalität bei Frühgeborenen einhergehen kann. Fragestellung: Es soll untersucht werden, ob die Prolongation der Schwangerschaft bei einem PPROM mit einem AIS und seinen Symptomen (CRP $>1 \mathrm{mg} / \mathrm{dl}$ bei der Mutter, early-onset-Sepsis beim Kind, histologischer Entzündungsnachweis in der Plazentahistologie) assoziiert ist.

Patienten und Methoden: Wir haben 52 Frühgeborene, deren Mütter am Universitäts-Klinikum Essen mit einem PPROM in Betreuung waren, in die Untersuchung eingeschlossen. Bei diesen Kindern wurde erfasst, wer Zeichen eines Amnioninfektionssyndroms aufwies.

Ergebnisse: Die 52 Frühgeborenen hatten zum Zeitpunkt der Entbindung ein Gestationsalter von 29 $\pm 0,4$ (23-36) SSW (Mittelwert \pm SEM (Bereich) und ein mittleres Geburtsgewicht von $1311 \pm 77$ (480-2940) g (Mittelwert \pm SEM (Bereich). Die Blasensprungdauer betrug im Median 382 Stunden (15,9 Tage), wobei ein Bereich von 19 Stunden bis 2700 Stunden (113 Tage) auszumachen war. Der Blasensprung war jeweils zwischen der 14. und 35. SSW aufgetreten. Bei 30/52 (58\%) Müttern war zum Zeitpunkt der Geburt ein CRP von mindestens $1 \mathrm{mg} / \mathrm{dl}$ zu beobachten. Das mittlere CRP aller Mütter lag bei 2,0 $\pm 0,3 \mathrm{mg} / \mathrm{dl}$ (Mittelwert \pm SEM). 17 von 52 Frühgeborenen ( $33 \%$ ) hatten Zeichen eines AIS. Bei den 35 Kindern ohne AIS-Symptome (67\%) betrug die Blasensprungdauer im Median 236 Stunden (9,8 Tage; Bereich: 30-2700 Stunden) und bei den 17 Kindern mit AIS-Nachweis 168 Stunden (7 Tage; Bereich: 19-1944 Stunden), was aber keinen statistischen Unterschied darstellte (U-Test nach Mann, Whitney und Wilcoxon).

Schlussfolgerung: Bei adäquater Betreuung und Therapie einer Schwangeren mit einem vorzeitigen Blasensprung über mehrere Wochen ergeben sich keine Hinweise darauf, dass die Prolongation der Schwangerschaft mit einem erhöhten Auftreten eines AIS assoziiert ist.

\section{P068}

Salmonella Enteritidis assoziierte Sepsis bei einem Frühgeborenen mit 34 + 0 Schwangerschaftswochen nach Spontangeburt

\section{Farhang', E. Panou', S. Bagci', H. Reutter', A. Müller ${ }^{1}$}

'Universitätskinderklinik, Neonatologie, Bonn, Deutschland

Kasuistik: Nach spontaner Geburt mit 34+0 Schwangerschaftswochen fiel das weibliche Frühgeborene am 2. Lebenstag mit erhöhten Temperaturen bis maximal $38,0^{\circ} \mathrm{C}$ auf. Bei positiven Entzündungszeichen wurde eine antibiotische Therapie mit Ampicillin/Sulbactam begonnen. In der Blutkultur ergab sich der Nachweis von Salmonella Enteritidis. Im Liquor zeigte sich kein Bakterienwachstum. Die antibiotische Therapie mit Ampicillin/ Sulbactam wurde für insgesamt 10 Tage durchgeführt, im Stuhl des Kindes waren bis zuletzt Salmonellen nachweisbar. Bei zügigem Nahrungsaufbau und konstanter Gewichtszunahme konnte das Kind mit korrigiert 37+0 SSW in gutem klinischen Allgemeinzustand nach Hause entlassen werden. Eine gezielte Anamnese der Mutter im Nachhinein ergab eine mütterliche Diarrhöe unter Geburt.

Diskussion: Perinatal übertragene nicht-typhoidale Salmonelleninfektionen bei Neu- oder Frühgeborenen nach Spontangeburt sind selten. Salmonellenenteritiden bei älteren Säuglingen sind hingegen häufig. Diese kön- 
nen febril oder afebril verlaufen. Tritt eine Bakteriämie auf, ist diese meist transient, kann aber auch zu einer Sepsis oder zu einer Meningitis führen. Schlussfolgerung: Kommt es in der Schwangerschaft zu einer Salmonellose der werdenden Mutter, sollte das Neugeborene postnatal intensivklinisch und paraklinisch überwacht werden. Die Übertragung des Krankheitserregers unter der Geburt ist wahrscheinlich. Das Robert-Koch-Institut empfiehlt im Falle einer Salmonellose der werdenden Mutter eine Prophylaxe mit einem wirksamen Antibiotikum, um eine Erkrankung des Neugeborenen zu verhindern.

\section{P069}

Schwerste Bronchopulmonale Dysplasie bei einem ELBWFrühgeborenen der 25.SSW - Unreife oder andere Ursachen?

M. Hohmann ${ }^{1}$, M. Wermuth ${ }^{2}$, C. Peter ${ }^{3}$, F. Brasch ${ }^{4}$, H. Körner ${ }^{5}$

${ }^{1}$ Klinikum Links der Weser, Bremen, Neonatologie, Bremen, Deutschland, ${ }^{2}$ Klinikum Links der Weser, Bremen, Bremen, Deutschland, ${ }^{3}$ Medizinische Hochschule Hannover, Abteilung für Pädiatrische Pneumologie, Allergologie und Neonatologie, Hannover, Deutschland, ${ }^{4}$ Klinikum Bielefeld Mitte, Institut für Pathologie, Bielefeld, Deutschland, ${ }^{5}$ Klinikum Links der Weser, Abteilung für Neonatologie, Bremen, Deutschland

Hintergrund: Die Bronchopulmonale Dysplasie (BPD) ist eine bekannte Komplikation bei ELBW-Frühgeborenen und tritt mit einer Häufigkeit von $15-30 \%$ bei FG unter der 28.SSW und/oder einem Geburtsgewicht unter $1000 \mathrm{~g}$ auf. Die Pathogenese ist multifaktoriell und hauptsächlich durch die anatomische und biochemische Unreife, sowie die postnatalen Belastungsfaktoren, wie z. B. Baro- und Volutrauma, Sauerstoffzufuhr und Infektionen zu erklären. Die alveolokapilläre Dysplasie ist eine seltene, aber gut beschriebene schwere Lungenerkrankung des reifen Neugeborenen.. Sie beschreibt eine genetisch bedingte Fehlbildung des pulmonalen Gefäßsystems mit einer Reduktion der Kapillaren sowie einer Mediahypertrophie der Arteriolen. Daraus resultieren eine verminderte Gas-Austauschfläche und eine insuffiziente alveoläre Perfusion. Dies verursacht eine therapierefraktäre pulmonal-arterielle Hypertonie (PHT) mit schwerer pulmonaler Insuffizienz und Hypoxämie. Die Prognose ist infaust. Kasuistik: Wir berichten über den Krankheitsverlauf eines ELBW-FG der $24+3$ SSW mit einem Geburtsgewicht von $460 \mathrm{~g}$ mit schwerer Bronchopulmonaler Dysplasie und alveolokapillärer Dysplasie. Der klinische Verlauf war vor allem durch ein schweres Atemnotsyndrom sowie eine frühe und schwere BPD mit therapierefraktärer PHTgekennzeichnet. Eine Atemunterstützung (Beatmung, CPAP, Highflow) war zu jedem Zeitpunkt notwendig. Im Rahmen der differentialdiagnostischen Überlegungen stellte sich, nach Ausschluss einiger anderer Ursachen, auch aufgrund der therapierefraktären PHT, der Verdacht auf eine alveolokapilläre Dysplasie, die im Rahmen einer Lungenbiopsie histopathologisch bestätigt wurde. Aufgrund der infausten Prognose wurde die Therapie in Absprache mit den Eltern beendet.

Diskussion: Die Darstellung des ungewöhnlich schweren Krankheitsverlaufes zeigt die Notwendigkeit differentialdiagnostischer Überlegungen bei früher, schwerer BPD und therapierefraktärer PHT auch bei ELBW-FG. Schlussfolgerung: Bei außergewöhnlich langwieriger und progredienter Ateminsuffizienz sollte auch bei ELBW-FG neben der extremen Unreife an weitere Ursachen der pulmonalen Insuffizienz wie die alveolokapilläre Dysplasie gedacht werden. Die erweiterte Diagnostik sollte neben molekulargenetischen Untersuchungen auch eine Lungenbiospsie zur histopathologischen Abklärung beinhalten. Alveolokapilläre Dysplasie- BPDPHT-Frühgeborene

\section{P070}

\section{Risikofaktoren für eine fokale intestinale Perforation} bei Frühgeborenen $<\mathbf{3 0}$ SSW

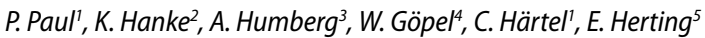
${ }^{1}$ Universitätsklinikum Schleswig-Holstein, Klinik für Kinder- und Jugendmedizin, Abteilung Neonatologie, Lübeck, Deutschland, 2Universitätsklinikum Schleswig Holstein, Campus Lübeck, Klinik für Kinder-und Jugendmedizin, Lübeck, Deutschland, ${ }^{3}$ Universitätsklinikum Schleswig Holstein, Kinderklinik, Lübeck, Deutschland, ${ }^{4}$ Universitätsklinikum Schleswig-Holstein, Klinik für Kinder- und Jugendmedizin, Lübeck, Deutschland, ${ }^{5}$ Universitätsklinikum Schleswig-Holstein Campus Lübeck, Direktor der Klinik für Kinder- und Jugendmedizin, Lübeck, Deutschland

Hintergrund: Neben dem Gestationsalter gilt die Behandlung eines persistierenden Ductus arteriosus (PDA) mit nicht-steroidalen Entzündungshemmern (Ibuprofen, Indomethacin) als möglicher Risikofaktor für eine fokale intestinale Perforation (FIP). In Zentren des Deutschen Frühgeborenennetzwerks (GNN) werden zunehmend extrem unreife Frühgeborene primär nicht-invasiv beatmet. Ob dieses Management einschließlich Surfactant-Therapie unter Spontanatmung (Less invasive Surfactant administration, LISA) ebenfalls das Risiko für eine FIP beeinflusst, ist bislang nicht hinreichend untersucht worden.

Material und Methoden: In unserer Analyse wurden $n=5753$ Frühgeborene mit einem Geburtsgewicht $<1500 \mathrm{~g}$ und einem Gestationsalter $<30$ Schwangerschaftswochen (SSW) einbezogen. In einer multivariablen logistischen Regressionsanalyse wurden folgende Einflussfaktoren auf das primäre Outcome „operationspflichtige FIP“ berücksichtigt: invasive Beatmung an Lebenstag 1, LISA-Prozedur, Gestationsalter, Geburtsgewicht, Mehrlingsstatus, Geschlecht, antenatale Lungenreife, Sauerstoffbedarf der ersten 12 Lebensstunden, sowie Medikation mit Hydrocortison, Indomethacin, Ibuprofen.

Ergebnisse: 168/5753 (2,9\%) der Frühgeborenen entwickelten eine FIP. Ibuprofen wurde häufiger eingesetzt als Indomethacin $(n=1150$ nur Ibuprofen, $n=528$ nur Indomethacin, $n=357$ Ibuprofen/Indomethacin-Kombination). Als protektive Faktoren für eine FIP zeigten sich höheres Gestationsalter (pro Woche; OR=0,67, $95 \% \mathrm{KI}: 0,59-0,77, p<0,001$ ) und weibliches Geschlecht (OR 0,60, $95 \% \mathrm{KI}$ : 0,43-0,83). Als unabhängige Risikofaktoren für eine FIP erwies sich der Einsatz von Indomethacin (OR 2,36, $95 \%$ KI: 1,48-3,76, $p<0,001$ ) jedoch nicht von Ibuprofen (OR $1,42,95 \%$ KI: $0,93-2,18, p=0,1)$. Frühgeborene, die LISA erhielten, hatten ebenfalls ein erhöhtes Risiko für eine FIP (OR 1,82, 95\% KI: 1,19$2,8, p=0,006$ ). Eine primäre Beatmung am 1 , Lebenstag ist jedoch nicht mit einem reduzierten FIP-Risiko assoziiert (OR 1,24, 95\% KI: 0,8-1,92, $p=0,34)$.

Schlussfolgerung: Die deskriptiven Daten erlauben die Hypothese, dass bei Frühgeborenen $<30$ SSW, die mittels NIV-Beatmung und LISA-Technik behandelt wurden, ein erhöhtes Risiko für eine FIP besteht. Im Risikoprofil dieser Kinder ist neben dem reduzierten Blutfluss in den mesenterialen Organen (Behandlungsnotwendigkeit eines PDA, Indomethacin als unabhängiger Risikofaktor für FIP) die mechanische Dehnung des Magen-Darm-Traktes unter NIV-Beatmung möglicherweise als ursächlich anzusehen. Weitere Studien müssen zeigen, ob und wann eine invasive Beatmung bei den eigentlich primär für NIV-Behandlung in Frage kommenden Kindern von Vorteil wäre. 


\section{Postersitzung 08: Fehlbildungen}

\section{P071}

\section{Kongenitale Zwerchfellhernie und de novo Duplikation von Chromosom 11p15.1p15.5}

\section{G. Dworschak' ${ }^{1}$ H. Reutter' ${ }^{1}$ A. Müller ${ }^{1}$ \\ 'Universitätsklinik Zentrum f. Kinderheilkunde, Neonatologie, Bonn, Deutschland}

Hintergrund: Kongenitale Zwerchfellhernien (CDH) treten mit einer Häufigkeit von 1:2000 bis 1:4000 auf und sind mit einer schweren Lungenhypoplasie und einer pulmonalen Hypertension assoziiert. Bei etwa $40 \%$ der Neugeborenen treten zudem weitere Anomalien wie zum Beispiel Herzfehler oder Fehlbildungen des Urogenitaltraktes auf. Die meisten Fälle treten sporadisch auf, familiäre Häufungen sind selten. In 10 bis $20 \%$ der pränatal diagnostizierten Fälle können chromosomale Aberrationen identifiziert werden, darunter sind Trisomien der Chromosome 13, 18 und 21 am häufigsten.

Kasuistik: Wir berichten über ein weibliches Frühgeborenes mit $34+4$ SSW mit pränatal bekannter Zwerchfellhernie (liver up, o/e LHR 29\%), das nach maximaler Therapie inklusive ECMO-Therapie aufgrund der angeborenen Lungenhypoplasie mit 2 Monaten verstarb. Aufgrund fazialer Auffälligkeiten (mongoloide Lidachse, Epikanthus) führten wir postnatal eine genetische Diagnostik durch, die in der Chromosomenanalyse eine Duplikation 46,XX,dup11p15.1p15.5 ergab. Eine Untersuchung der Eltern und eine zusätzliche Untersuchung kindlicher DNA mittels Array-CGH zeigte, dass die Duplikation de novo entstanden und $19 \mathrm{Mb}$ groß war. Interessanterweise umfasst die Duplikation auch die kritische Region des Beckwith-Wiedemann Syndroms.

Diskussion: In der Literatur finden sich zwei weitere Fälle mit kongenitaler Zwerchfellhernie und überlappender Duplikationen der Region 11p15.
Die Identifizierung einer de novo Duplikation von $19 \mathrm{Mb}$ in einem sporadischen Fall von kongenitaler Zwerchfellhernie legt eine seltene Assoziation nahe.

\section{P072}

\section{Von der Diagnose zur Dialyse: Outcome von Neugeborenen mit renalem Oligohydramnion}

K. Mehler', C. Taylan², I. Gottschalk', B. Hoppe 4 , A. Kribs', A. Vierzig', L. Weber², S. Habbig ${ }^{2}$

'Universitätskinderklinik Köln, Neonatologie und Pädiatrische Intensivmedizin, Köln, Deutschland, ${ }^{2}$ Univ.-Kinderklinik, Kindernephrologie, Köln, Deutschland, ${ }^{3}$ Univ.-Frauenklinik, Pränatalmedizin, Köln, Deutschland, ${ }^{4}$ Universitäts-Kinderklinik, Bonn, Deutschland

Hintergrund: Aufgrund der Fortschritte im neonatologischen und nephrologischen Management von Kindern mit renalem Oligohydramnion (ROH) konnte die Prognose dieser Kinder maßgeblich verbessert werden. Eine standardisierte, interdisziplinäre pränatale Beratung wird allerdings nur bei einem Teil der werdenden Eltern durchgeführt. Ziel dieser Studie war die Auswertung aller Schwangerschaften mit ROH bezüglich der elterlichen Entscheidung über die Fortführung der Schwangerschaft, sowie des neonatologischen und nephrologischen Verlaufs der geborenen Kinder. Methoden: Während des Beobachtungszeitraums von 08/2007 bis 08/2015 wurden insgesamt 103 Feten mit ROH und/oder schwerwiegender renaler Fehlbildung zur pränatalen Beratung vorgestellt.

Ergebnisse: Nach pränataler Beratung entschieden sich $38 \%$ der Eltern für einen Abbruch der Schwangerschaft. 54\% wünschten eine Fortführung der Schwangerschaft, $8 \%$ der Feten verstarben intrauterin (IUFT). Von den 53 lebend geborenen Kindern wurden $22 \%$ palliativ und $78 \%$ aktiv behandelt. Die Entscheidung zum Abbruch oder palliativer Behandlung war sowohl signifikant mit dem Vorliegen extra-renaler Symptome wie auch mit der renalen Grunderkrankung (19\% bei OU versus $73 \%$ bei

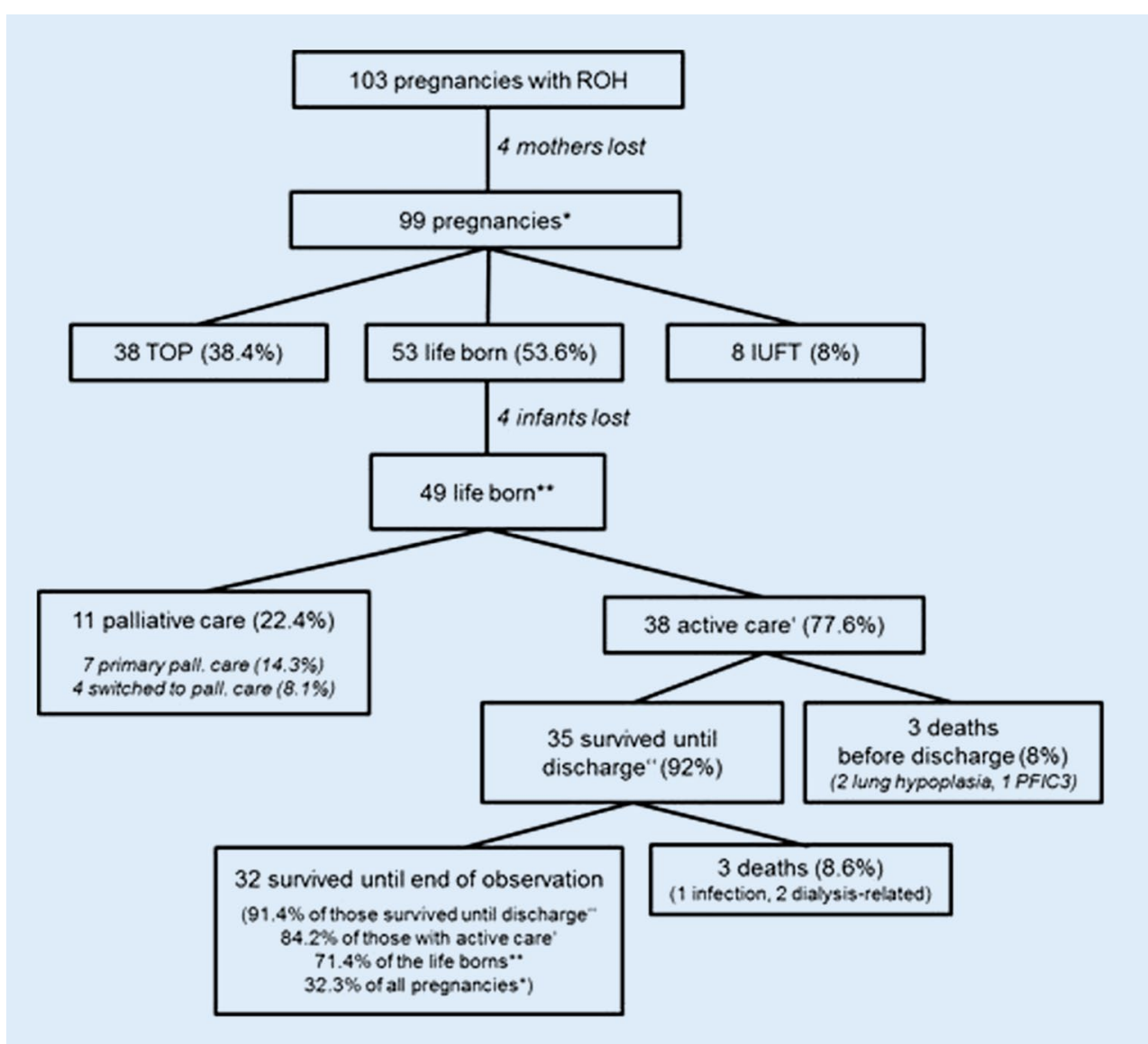


Nierenagenesie/Dysplasie) assoziiert. Pränatale Verdachtsdiagnosen waren obstruktive Uropathien (OU) in $42 \%$ der, bilaterale Nierenagenesie oder Dysplasie in 36\% und Autosomal Rezessive Polyzystische Nierenerkrankung (ARPKD) in $12 \%$ der Fälle. Komplexe syndromale Erkrankungen lagen bei $11 \%$ der Kinder vor. Die Diagnosestellung des ROH erfolgte in der medianen 22. SSW, wobei das ROH bei Kindern mit ARPKD signifikant später als bei den anderen Diagnosen auftrat. Insgesamt wurden 53 Schwangerschaften bis zur Geburt ausgetragen. Neben den 7 palliativ versorgten Kindern verstarben weitere 7 Kinder vor Entlassung, zwei davon an respiratorischen Komplikationen. Drei weitere Kinder verstarben nach Entlassung an Dialyse-assoziierten Komplikationen $(n=2)$ und pulmonaler Infektion $(n=1)$. Eine Nierenersatztherapie war bei 10 Kindern $(22 \%)$ bereits am 7. Lebenstag notwendig, bei 11 Kindern (21\%) im Alter von 4 Wochen und bei 8 Kindern (19\%) im Alter von 6 Monaten. Bei 5 der insgesamt 15 Kindern (33\%) mit Nierenersatztherapie innerhalb der ersten 4 Wochen konnte die Dialyse (temporär) beendet werden.

Schlussfolgerungen: Die vorliegenden Daten ermöglichen die Analyse dieses Patientenkollektivs vom Zeitpunkt der pränatalen Diagnosestellung an über die postnatale Phase bis ins Kleinkindesalter. Unsere Studie kann daher dazu beitragen, die pränatale Beratung zu verbessern und den Eltern zu ermöglichen, eine informierte Entscheidung für sich und ihr Kind zu treffen sowie ein interdisziplinäres Therapiekonzept zu erarbeiten.

\section{P073}

\section{Fehlbildungskonstellation aus parasitärem Thorakopagus, Omphalozele, Double-outlet-right-ventricle und doppeltem Aortenbogen}

N. Gratzki', M. Beckmann'2, R. Carbon ${ }^{3}$, R. Cesnjevar', S. Dittrich ${ }^{5}$, F. Faschingbauer ${ }^{2}$, M. Glöckler ${ }^{5}$, S. Kehl' ${ }^{2}$, V. Melichar' ${ }^{1}$, O. Rompel' ${ }^{6}$, H. Topf ${ }^{\prime}$

${ }^{1}$ Kinder- und Jugendklinik, Universitätsklinikum Erlangen, Erlangen, Deutschland, ${ }^{2}$ Frauenklinik, Universitätsklinikum Erlangen, Erlangen, Deutschland, ${ }^{3}$ Kinderchirurgische Abteilung, Universitätsklinikum Erlangen, Erlangen, Deutschland, ${ }^{4}$ Kinderherzchirurgie, Universitätsklinikum Erlangen, Erlangen, Deutschland, ${ }^{5}$ Kinderkardiologische Abteilung, Universitätsklinikum Erlangen, Erlangen, Deutschland, ${ }^{6}$ Radiologisches Institut, Universitätsklinikum Erlangen, Pädiatrische Radiologie, Erlangen, Deutschland

Hintergrund: Conjoined Twins (Siamesische Zwillinge) sind eine seltene Erkrankung, welche weltweit mit einer Inzidenz zwischen 1:50.000100.000 auftritt. Der Fallbericht beschreibt den postpartalen Verlauf eines „Zwillingsfrühgeborenen“ mit pränatal bekanntem parasitären Thorakopagus.

Fallbericht: Wir berichten über das 3. Kind einer 35-jährigen II.-Gravida, II.-Para, Z.n. IVF, „Zwillingsgeburt“ in der $35+6$ SSW bei bereits pränatal bekannter Fehlbildungskonstellation aus Omphalozele, kardialem Vitium und parasitärem Drilling. Geburt per Sectio, Apgar-Werte 7/9 (intubiert)/9 (intubiert), arterieller Nabelschnur-pH 7,29, Geburtsgewicht 1890 g, Körperlänge $42 \mathrm{~cm}$ und Kopfumfang $31,0 \mathrm{~cm}$. Postpartal Intubation bei klinischer Bestätigung der Omphalozele, echokardiografische Darstellung eines Double-outlet-right-ventricle vom Fallot-Typ bei persistierendem Ductus arteriosus (PDA). Medikamentöses Offenhalten des PDA mit Alprostadil zur Sicherung einer ausreichenden Lungenperfusion. Am ersten Lebenstag erfolgte eine CT-Bildgebung mit Kontrastmittel von Thorax/ Abdomen zur Darstellung der anatomischen Verhältnisse (insbesondere der Gefäßversorgung) vom Autosit zum parasitären Thorakopagus sowie der kardialen Verhältnisse zur operativen Planung. Es zeigte sich ergänzend zur echokardiografischen Diagnostik ein doppelter Aortenbogen mit konsekutiver Einengung der Trachea. Die Gefäßversorgung des Thorakopagus erfolgte aus einem singulären Gefäß aus der Aorta ascendens, welches sich dann vom Thorakopagus bis in die Omphalozele darstellen ließ. Am vierten Lebenstag erfolgte die chirurgische Trennung des parasitären Thorakopagus vom Autosit mit Omphalozelenverschluss, welche komplikationslos gelang. Abgesehen von einer Wundheilungsstörung postoperativ regelrechter Verlauf. Bei rezidivierenden Beatmungsproblemen mit Obstruktionen erfolgte am zwölften Lebenstag eine Bronchoskopie, wel- che, passend zum CT-Befund, im unteren Trachealbereich eine pulsierende Einengung zeigte. Am 14. Lebenstag erfolgte der kardiochirurgische Eingriff mit Durchtrennung des linksseitigen, vorderen Aortenbogens über eine posterolaterale Thorakotomie. Aufgrund der filigranen Gefäßsituation wurde auf die Anlage eines Aorto-pulmonalen Shunts verzichtet und der Alprostadil-manipulierten PDA belassen. Am 63. Lebenstag erfolgte eine palliative transanuläre rechtsventikukäre Ausflusstrakterweiterung mittels bovinem Perikardpatch mit Durchtrennung und Verschluss des PDA. Vorhof- und Ventrikelseptumdefekt wurden belassen, da eine Korrektur zu diesem Zeitpunkt als zu riskant angesehen wurde. Echokardiografisch zeigte sich vor Entlassung bei guter Pumpfunktion ein VSD mit links-rechts-Shunt, ein moderat enger Ausflusstrakt sowie ein guter Fluss in beiden Pulmonalarterien. Am 82. Lebenstag konnte die Patientin in gutem Allgemeinzustand nach Hause entlassen werden.

Diskussion/Schlussfolgerung: Siamesische Zwillinge sind eine seltene Entität, zum dargestellten Fall eines parasitären Thorakopagus mit Omphalozele und Herzvitium gibt es nur einzelne Fallberichte. Die Versorgung solcher Patienten ist aufgrund der prä- und postnatalen bildgebenden Verfahren im multidisziplinären Team möglich, so dass den Eltern heute in vielen Fällen kurative Therapieoptionen angeboten werden können. 3D-Rekonstruktionen aus CT-Bildgebungen können dabei die Planung der operativen Vorgehensweise unterstützen.

\section{P074}

\section{Hydrocephalus bei Achondroplasie, Genese und neurochirurg. Interventionsmöglichkeiten an Hand eines Fallbericht}

\section{Haftel', F. Müller', S. Asgari ${ }^{3}$}

'Oberschwabenklinik, Krankenhaus St. Elisabeth, Ravensburg, PädiatrieNeonatologie, Ravensburg, Deutschland, ${ }^{2}$ Kliniken St. Elisabeth Klinik f. Kinder.- u. Jugendmedizin, Neuburg, Deutschland, ${ }^{3}$ Klinikum Ingolstadt, Neurochirurgie, Ingolstadt, Deutschland

Einleitung: Die Achondroplaia ist die häufigste Form eine Knochendysplasie. Die Ausbildung eines Hydrocephalus wird bei den Patienten zwar sehr häufig beobachtet, die Notwendigkeit eine neurochirurgische Intervention ist aber selten indiziert. Nur bei entsprechender Symptomatik wie zum Beispiel Apnöen, Ataxie oder Kompression des Stammhirns ist so eine Intervention notwendig. Das Risiko der Ausbildung eines Hydrocephalus besteht prinzipiell lebenslang wird jedoch vor allem in den ersten zwei

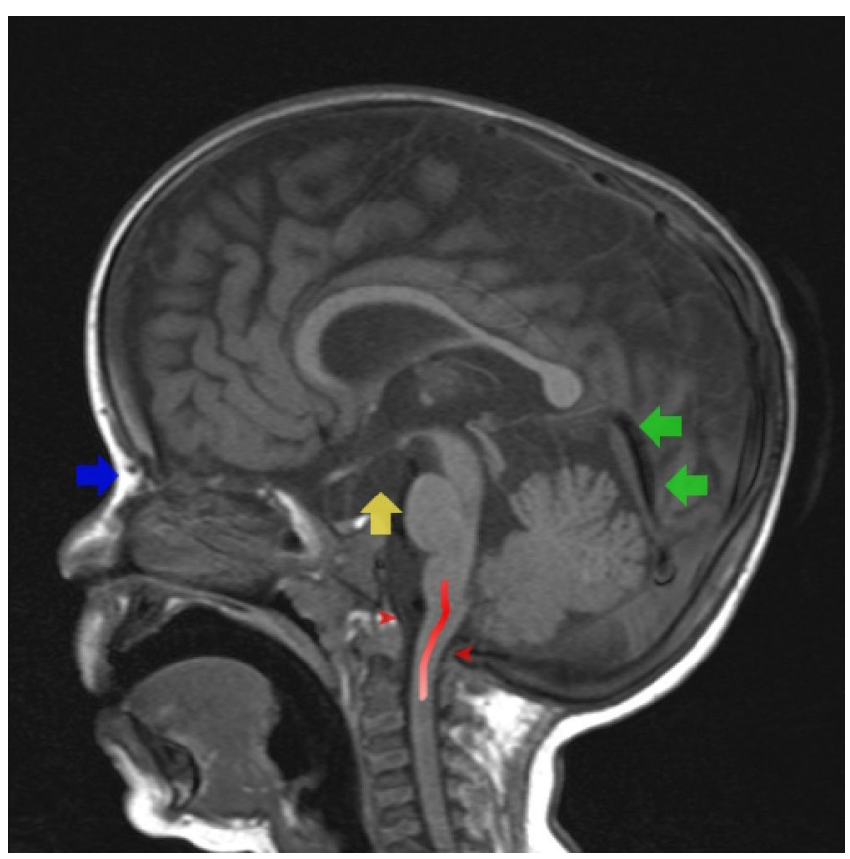

Abb. P074 
Lebensjahren beobachtet. Bezüglich der Genese existieren verschiedene Theorien: 1. Exzessives Wachstum der Schädelkalotte in Sinne eines Kompensationsversuches bei kleiner Schädelbasis. 2. Obstruktionen der Cisterna magna und der Foramina Luschkae und Magendie aufgrund Distortionen des Hirnstammes bei häufig zu engem Foramen magnum. 3. Stase des venösen Rückstroms und erhöhen des intrakraniellen venösen Druck. Fallbericht: Nach unauffälligen SS Verlauf, wurde auf Grund Makrozephalie, Ventrikulomegalie und pathologischer Hüfte-Sonographie der Verdacht auf Achondroplasie gestellt. Es wurden zwei de-novo-Mutationen im FGFR3-Gen nachgewiesen werden. Diese Mutationen sind mit der Symptomatik einer Hypochondroplasie, Achondroplasie Verlaufsformen verknüpft. Im Verlauf wurden vermehrte Apnöe-Ereignisse beobachtet und die MRT Untersuchung zeigte eine ausgeprägte Enge des Formanen magnum ohne venöse Stase (• Abb. P074). Nach subokzipitaler Dekompression zeigte sich zunächst eine leichte Besserung der klinische Symptomen, bei jedoch ausgeprägtem triventrikulärem Hydrocephalus erfolgte eine endoskopische Ventrikulostomie am Boden des 3. Ventrikels. Die erfolgte bildgebende Verfahren zeigte eine deutliche radiologische Verbesserung, und der kleiner Patient konnte ohne klinische Apnoen nach Hause entlassen.

Zusammenfassung: Hydrocephalus bei Achondroplasia ist ein komplexes Krankheitsbild, die eine sorgfältige Evaluation benötigt, um die genau Ursache zu diagnostizieren und behandeln. Die übliche Ventrikuloperitonealer Shunt Behandlung des Hydrocephalus ist bei viele diese Fälle nicht nötig.

\section{Literatur}

1. Achondroplasia, AH Menenez, J Neurosurg Pediatrics 4: 295-6, 2009

2. The role of endoscopic third ventriculostomy in the treatment of triventricular hydrocephalus seen in children aiwth achondroplasia, V Etus and S Ceylan J Neurosurg Pediatrics 3: 260-5, 2005

\section{P075}

Das Arthrogrypose-Nierenfunktionsstörung-Cholestase-(ARC)Syndrom: Eine seltene Diagnose des Säuglingsalters mit erstmals beschriebener homozygoter Mutation des VIPAS39-Gens, c.107dupA

\section{S. Christina' , J. Bialas' ${ }^{1}$, H. Müller', E. Korsch ${ }^{2}$, S. Fracassi ${ }^{3}$, S. Kathemann ${ }^{3}$,} B. Kremens ${ }^{4}$, R. Ehren ${ }^{5}$, H. Bolz ${ }^{6}$, P. Hoyer ${ }^{3}$, U. Felderhoff-Müser ${ }^{7}$ 'Universitätsklinikum Essen, Klinik für Kinderheilkunde I, Neonatologie, Essen, Deutschland, ${ }^{2}$ Kliniken Köln GmbH, Kinderklinik Amsterdamer Straße, Köln, Deutschland, ${ }^{3}$ Universitätsklinikum Essen, Klinik für Kinderheilkunde II, Nephrologie, Gastroenterologie und Endokrinologie, Essen, Deutschland, ${ }^{4}$ Universitätsklinikum Essen, Klinik für Kinderheilkunde III, Pädiatrische Hämatologie, Essen, Deutschland, ${ }^{5}$ Klinik für Kinder- und Jugendmedizin, Kindernephrologie, Universität zu Köln, Köln, Deutschland, ${ }^{6}$ Bioscientia, Zentrum für Humangenetik und Institut für Humangenetik, Universitätsklinikum Köln, Humangenetik, Mainz; Köln, Deutschland

Hintergrund: Das ARC-Syndrom ist eine sehr seltene ( $<50$ Fälle) angeborene Erkrankung, bestehend aus der Kombination von Arthrogryposis, beeinträchtigter Nierentubulusfunktion und Cholestase. Zusätzlich werden fakultativ Ichthyose, schwere Gedeihstörung, Thrombozytenauffälligkeiten, Diabetes insipidus renalis, Diarrhoe, Infektanfälligkeit, Fieberschübe, Hirnfehlbildungen, faziale Dysmorphien, Anämie und Schallempfindungsschwerhörigkeit beschrieben. Patienten mit De-Toni-Debré-Fanconi-Syndrom zeigen eine renale Resorptionsstörung des proximalen Tubulus. Die Erkrankung wird autosomal-rezessiv vererbt. Häufigste Ursache sind Mutationen (ca. 74\%) im VPS33B-Gen, gefolgt von Mutationen des VIPAR-Gens. Innerhalb einer Familie können Mutationen und Klinik jedoch variieren.

Kasuistik: Männliches Neugeborenes von $41+1$ SSW mit 3450 g Geburtsgewicht, mit typischer Trias einer Cholestase (dir. Bili. $8 \mathrm{mg} / \mathrm{dl}$, erhöhte Transaminasen), angeborenen Kontrakturen (v.a. Kniegelenke), eines Fanconi-Syndroms und einem Diabetes insipidus renalis. Das Kind entwickelte eine ausgeprägte Ichthyose an der Oberschenkelinnenseite und am Hinterkopf. Des Weiteren kam es zu einem Gewichtsverlust von 12,5\% in den ersten 12 LT trotz hochkalorischer Nahrung $(<3$. Perz.) und rezidi- vierenden Fieberschüben. Es wurde eine Schallempfindungsstörung bds., eine ausgeprägte Balken-, Falx-, und Kleinhirnunterwurmhypoplasie, erweiterte Seitenventrikel, eine Muskelhypotonie und granulierte Thrombozyten diagnostiziert (Funktionstestung ausstehend). Die Medikation besteht aus Ursofalk, Vitaminsubstitution (ADEK) und Captopril. Darunter stabilisierte sich das Fanconi-Syndrom. Die Cholestase und auch die Gedeihstörung persistierten trotz PEG-Anlage.

Bei unserem Patienten konnte erstmals eine homozygote Mutation des VIPAS39-Gens c.107dupA nachgewiesen werden (veranlasst durch Dr. E. Korsch). Die Eltern sind türkischer Abstammung und konsanguin, es gibt eine gesunde Schwester. Eine weitere Schwester verstarb mit 7 Monaten an einem ähnlichen Krankheitsbild. Die Mutationsanalyse des VPS33BGens bei ihr, der gesunden Schwester und den Eltern war unauffällig. Eine Analyse auf die VIPAS39-Mutation c.107dupA bei den gesunden Familienmitgliedern steht noch aus.

Diskussion: Eine kurative Therapie des ARC-Syndroms ist derzeit nicht bekannt. Die Patienten versterben innerhalb der ersten Lebensjahre im Rahmen einer metabolischen Entgleisung, Infektion oder Blutung. In einzelnen Fällen wurde eine Lebertransplantation beschrieben. Eine mögliche Ursache für die schwere Arthrogryposis könnte die Hirnfehlbildung mit mangelnder intrauteriner Kindsbewegung darstellen. Eine humangenetische Analyse und Beratung der Patienten ist anzustreben.

\section{P076}

\section{ECMO-Therapie bei einem Neugeborenen mit LUTO und Lungenhypoplasie}

E. Gatzweiler', B. Hoppe², M. Feldkötter², H. Reutter', A. Müller ${ }^{1}$

'Universitätsklinik, Zentrum für Kinderheilkunde, Abteilung für Neonatologie und Pädiatrische Intensivmedizin, Bonn, Deutschland, 2Universitätsklinik, Zentrum für Kinderheilkunde, Pädiatrische Nephrologie, Bonn, Deutschland

Einleitung: Das Überleben von Kindern mit kongenitaler Niereninsuffizienz wird initial maßgeblich vom Ausmaß der sekundären Lungenhypoplasie beeinflusst. Durch frühzeitige pränatale Diagnosestellung mit konsekutiver Fruchtwasserauffüllung und moderne intensivmedizinische Maßnahmen konnte die Prognose in den vergangenen Jahren verbessert werden. Trotz dieser Maßnahmen ist die Sterblichkeit aufgrund der pulmonalen Hypoplasie nach wie vor hoch. Die extrakorporale Membranoxygenierung (ECMO) ist ein etabliertes Verfahren zur Oxygenierung bei Säuglingen mit neonatalem Lungenversagen z. B. bei kongenitalen Zwerchfellhernien. Für die Anwendung bei Neugeborenen mit „lower urinary tract obstruction“ (LUTO) und sekundärer Niereninsuffizienz gibt es nur Einzelfallberichte

Fallbeschreibung: Wir berichten über ein hypotrophes männliches Frühgeborenes von $36+2$ SSW mit partieller LUTO, sekundärer Nierendysplasie und sekundärer Lungenhypoplasie. Intrauterin erfolgte eine Fruchtwasserauffüllung in der 21. SSW. Postnatal erfolgte nach intensiver Primärversorgung bei respiratorischer Insuffizienz die Intubation und Anlage von Pleuradrainagen beidseits bei Pneumo- und Hydrothorax. Unter iNO-Therapie, HFO-Beatmung sowie wiederholten Surfactant-Gaben lagen die Oxygenierungsindices (OI) ab der fünften Lebensstunde $>40$, sodass die Entscheidung zur ECMO-Therapie erfolgte. Diese wurde über insgesamt neun Tage durchgeführt. Hierunter konnten die Beatmungsparameter reduziert und der Patient nach sechs Wochen extubiert werden. Aufgrund der Niereinsuffizienz mit Anurie wurde ab dem zweiten Lebenstag eine Dialyse mittels Hämofiltration begonnen und später durch eine Peritonealdialyse fortgesetzt. Das Kind wurde nach vier Monaten stationärem Aufenthalt mit High-Flow-Atemunterstützung und Peritonealdialyse nach Hause unterlassen.

Schlussfolgerung: Der hier beschriebene Fall zeigt, dass die ECMO-Therapie das Überleben von Neugeborenen mit kongenitaler Niereninsuffizienz und sekundärer Lungenhypoplasie z. B. in Folge einer LUTO ermöglicht. 
P077

\section{Pränatales Leberversagen und Hydrops fetalis} bei einem Frühgeborenen Case-Report einer GALD

\section{S. Luibl', O. Stangl', C. Wieg'}

${ }^{1}$ Klinikum Aschaffenburg, Neonatologie und pädiatrische Intensivmedizin, Aschaffenburg, Deutschland

Einleitung: Die Ursachen für das Auftreten eines Hydrops fetalis sind sehr vielfältig und seit Einführung der Rhesusprophylaxe bis zu $90 \%$ als „nicht-immunologisch“ einzuordnen. Eine sehr seltene Ursache ist eine gestational alloimmune liver disease“ (GALD), welche durch ein pränatales Leberversagen zur Ausbildung eines Hydrops fetalis führen kann.

Case Report: Eutrophes weibliches Frühgeborenes nach $30+3$ SSW. Zunächst unkomplizierter, gut überwachter Schwangerschaftsverlauf, nach 29 SSW sonographisch rapides Auftreten einer Cardiomegalie mit verminderter Kontraktilität und eines Aszites. Bei pathologischen Dopplern der A. cerebri media eilige Sectio caesarea. Klinisch bestand ebenso wie sonographisch das Bild eines Hydrops fetalis. Primär bereits laborchemisch Zeichen einer Lebersynthesestörung mit ausgeprägter Koagulopathie und Hypalbuminämie. Außerdem bestand eine direkte Hyperbilirubinämie inklusive einer erhöhten Transferrinsättigung, allerdings ohne Transaminasenerhöhung. Nach Ausschluss infektiologischer Ursachen wies diese Befundkonstellation auf eine GALD hin. Hierbei handelt es sich um eine bereits pränatal auftretende Hepatopathie aufgrund einer Alloimmunisierung mit Bildung von mütterlichen Antikörpern (IgG) gegen ein Oberflächenantigen der kindlichen Hepatozyten und folgender Aktivierung der fetalen Komplementkaskade mit Ausbildung des Membran attackierenden Komplementkomplexes C5b-9 und nachfolgender Leberzellschädigung. Die bis vor kurzem noch als „neonatale Hämochromatose" geführte Erkrankung hatte unter einer symptomatischen Therapie mit Antioxidantien und Chelatbildnern ohne Lebertransplantation eine sehr schlechte Prognose mit einer Überlebensrate von nur 10-17\%. Seit genauerer Entschlüsselung der Pathogenese zeigt sich durch eine Therapie mit einer frühen Austauschtranfusion und Hochdosis-Immunglobulintherapie eine vielversprechende Entwicklung mit einer Steigerung der Überlebensraten auf bis zu $75 \%$.

Aufgrund der initialen Anämie bei Hydrops fetalis führten wir bei unserem Patienten sofort nach Geburt eine Austauschtransfusion durch. Wegen der oben aufgeführten Befundkonstellation folgte im Verlauf zweimalig eine Therapie mit Immunglobulinen. Hierunter kam es zu einer kompletten Remission mit Normalisierung der Leberfunktion. Eine Lebertransplantation konnte verhindert werden. Auch langfristig konnten keine Anzeichen einer Hepatopahtie mehr nachgewiesen werden.

Konklusion: Bei Anzeichen eines neonatales Leberversagens sollte die Differentialdiagnose einer GALD mit beachtet werden. Eine Diagnosesicherung kann z. B. durch Nachweis einer zellulären Eisenbeladung in einer Lippenbiopsie erfolgen .Mit einer frühzeitige Therapie inklusive Austauschtransfusion und Immunglobulinen kann eine Lebertransplantation vermieden und die Mortalität deutlich reduziert werden.

\section{P078}

\section{Akutes Abdomen beim Frühgeborenen - häufige Diagnose, seltene Ursache. Ein Case Report.}

\section{Silwedel', E. Frieauff', T. Meyer', S. Rauthe', S. Kunzmann}

'Universitäts-Kinderklinik Würzburg, Neonatologie, Würzburg, Deutschland, ${ }^{2}$ Abteilung für Kinderchirurgie, Würzburg, Deutschland, ${ }^{3}$ Pathologisches Institut, Würzburg, Deutschland

Hintergrund: Das akute Abdomen ist ein häufiges Krankheitsbild beim Frühgeborenen und von erheblicher klinischer Relevanz. Typische Ursachen sind die fokale intestinale Perforation sowie die nekrotisierende Enterocolitis. Jedoch sind auch seltenere Gründe in die Differentialdiagnose einzubeziehen.

Case Report: Spontanpartus nach vorzeitigem Blasensprung und problemlose postnatale Adaptation eines männlichen Frühgeborenen von 34+0 Schwangerschaftswochen (SSW), Apgar 6/8/9, Nabelarterien-pH 7,36, BE
$-0,4 \mathrm{mmol} / \mathrm{l}$, Geburtsgewicht 2250 g. Nach bis dahin völlig unkompliziertem Verlauf setzte am 3. Lebenstag plötzlich anhaltendes Schreien ein. In der klinischen Untersuchung fiel eine perakut aufgetretene, massive abdominelle Distension auf, das Abdomen war druckschmerzhaft, es fand sich eine vermehrte Venenzeichnung. Die Vitalparameter waren stabil, zudem kein Fieber, keine auffälligen Magenreste. In der unmittelbar durchgeführten Blutentnahme stellte sich eine ausgeglichene Blutgasanalyse dar, ein unauffälliges Blutbild, ein negatives CRP, eine Hyperglykämie von $168 \mathrm{mg} /$ dl, das Laktat lag venös bei 3,3 mmol/l. Die Sonographie erbrachte aufgrund massiver Luftüberlagerung keine wegweisenden Befunde, röntgenologisch Darstellung freier abdomineller Luft. Nach unmittelbarer Anlage einer Magenablaufsonde und Beginn einer intravenösen antibiotischen Therapie wurde das Frühgeborene intubiert und im kinderchirurgischen OP laparotomiert. Intraoperativ stellte sich ein spontan perforiertes Meckel-Divertikel als Ursache des akuten Abdomens dar, makroskopisch fanden sich keine Zeichen einer Peritonitis. Nach Resektion des divertikeltragenden Ileumabschnittes und primärer Passagerekonstruktion unkomplizerter weiterer Verlauf, problemloser Kostaufbau und Entlassung des Frühgeborenen im korrigierten Alter von 37+6 SSW.

Diskussion: Das Meckel-Divertikel als Residuum des Ductus omphaloentericus ist häufig und zumeist asymptomatisch. Spontanperforationen treten insbesondere bei Frühgeborenen sehr selten auf, typischere Symptome sind eine Blutung, Darmobstruktion oder Divertikulitis. Unabhängig von der Ursache ist eine Darmperforation immer eine Notfallsituation mit erheblicher Relevanz für Morbidität und Mortalität von Frühgeborenen und kann auch bei fehlenden Risikofaktoren für eine solche auftreten. Wenn bei dem klinischen Bild eines akuten Abdomen die Sonographie nicht aussagekräftig ist, ist die Röntgendiagnostik unerlässlich. In diesem Fall ermöglichte die rasche Diagnosestellung die Therapie noch vor Auftreten von Allgemeinsymptomen und bedingte somit den positiven weiteren Verlauf.

Dieser Fall wird ebenfalls als Case Report in der Zeitschrift Monatsschrift Kinderheilkunde vorgestellt (Monatsschrift Kinderheilkunde 2016, 164;79, DOI 10.1007/s00112-015-0017-3)

\section{P079}

Diskordante, monozygote Zwillingsfrühgeborene mit seltenem, schwerem Fehlbildungssyndrom einer fetalen Megazystis bei Kloakenfehlbildung nach künstlicher Befruchtung

\section{Schneider', I. Schmeh' ${ }^{1}$ A. Fruth ${ }^{2}$, C. Whybra-Trümpler', E. Mildenberger ${ }^{1}$}

'Universitätsmedizin Mainz - Zentrum für Kinder- und Jugendmedizin, Neonatologie, Mainz, Deutschland, ${ }^{2}$ Universitätsmedizin Mainz, Klinik und Poliklinik für Geburtshilfe und Frauenkrankheiten, Mainz, Deutschland

Hintergrund: Die Inzidenz von (monozygoten) Zwillingsschwangerschaften steigt stetig an. Eine mögliche Ursache ist die zunehmende Anwendung reproduktionsmedizinischer Maßnahmen. Monozygote Zwillinge galten in der Vergangenheit als ,identische Klone“. Seit einigen Jahren wird jedoch mehr über Diskordanz monozygoter Zwillinge, zum Beispiel für angeborene Fehlbildungen, bekannt. Wir berichten über monozygote Zwillingsfrühgeborene mit diskordanter fetaler Megazystis bei Kloakenfehlbildung nach künstlicher Befruchtung - eine bisher in der Literatur nicht beschriebene (Fehlbildungs-)Konstellation.

Fallbericht: Einzige anamnestische Besonderheit war die Konzeption mittels intrazytoplasmatischer Spermieninjektion. Nach Transfer von zwei Embryonen entstand ein monochoriales, diamniales Zwillingspaar. Im ersten Trimenon wurde eine diskordante, fetale Megazystis mit konsekutivem Oligohydramnion und Lungenhypoplasie diagnostiziert. Der Karyotyp beider Kinder war unauffällig. Nach Geburt aufgrund vorzeitiger muttermundswirksamer Wehentätigkeit bei $25+3$ SSW wurde das betroffene Kind im Einvernehmen mit den Eltern palliativ behandelt und verstarb rasch. Post mortem wurde eine Kloakenfehlbildung festgestellt. Das gesunde Geschwisterkind überlebte.

Diskussion: Die fetale Megazystis ist eine sehr seltene Fehlbildung. Typische Ursache beim männlichen Geschlecht ist eine obstruktive Uropathie wie die posteriore Urethralklappe, bei den wesentlich seltener betroffe- 
nen weiblichen Feten ein komplexes urogenitales Fehlbildungssyndrom, wie in unserem Fall die Kloakenfehlbildung. Die Entstehung der Kloakenfehlbildung geht auf eine Störung der Gastrulation oder auf eine ungleiche Verteilung der inneren Zellmasse zurück und steht damit in unmittelbarem zeitlichem Zusammenhang mit der Zwillingsentstehung. Wir fanden zehn Fallberichte/-serien über mono- oder dizygote Zwillinge mit Diskordanz entweder nur für eine Megazystis oder nur für eine Kloakenfehlbildung. Unter den sechs Zwillingspaaren mit Megazystis waren zwei monozygot, jedoch männlichen oder unbekannten Geschlechts. Bei zwei dizygoten Zwillingspaaren wurde künstliche Befruchtung erwähnt. Über eine Kloakenfehlbildung wurde in keinem Fall berichtet. Unter den sieben Zwillingspaaren mit Kloakenfehlbildung waren sechs monozygot, hierunter vier weiblichen Geschlechts. Eine assoziierte Megazystis lag nicht vor. Reproduktionsmedizinische Maßnahmen wurden nicht beschrieben. Schlussfolgerung: Diskordanz monozygoter Zwillinge ist derzeit noch unzureichend erklärt. Diskordanz für strukturelle Fehlbildungen hängt nach bisherigem Verständnis eng mit den Vorgängen der Zwillingsentstehung selbst zusammen. Bei künstlicher Befruchtung werden eine erhöhte Rate von (monozygoten) Zwillingsschwangerschaften sowie ein vermehrtes Auftreten chromosomaler Aberrationen und kongenitaler Fehlbildungen diskutiert.

Im Hinblick auf Konzeptionsmechanismus, Zygotie, Geschlecht und Fehlbildungskonstellation ist unser Fall einzigartig.

\section{P080 \\ Neugeborenes mit kombinierter Tracheal- und Ösophagusatresie - ist ein fatales Outcome unvermeidbar?}

J. Hess', T. Rechenauer', K. Daifalla', F. Faschingbauer ${ }^{3}$, M. Beckmann ${ }^{3}$, W. Rascher', S. Söder ${ }^{2}$, T. Völkl ${ }^{1}$

${ }^{1}$ Kinder- und Jugendklinik, Universitätsklinikum Erlangen, Neonatologie und pädiatrische Intensivmedizin, Erlangen, Deutschland, ${ }^{2}$ Institut für Pathologie, Universitätsklinikum Erlangen, Erlangen, Deutschland, ${ }^{3}$ Frauenklinik, Universitätsklinikum Erlangen, Erlangen, Deutschland

Hintergrund: Eine konnatale Trachealagenesie ist extrem selten (1:50.000). Männliche Neugeborene sind etwa doppelt so häufig betroffen; begleitende Fehlbildungen, z.B im Rahmen eines VACTERL, sind beschrieben. Häufig besteht eine tracheo-ösophageale Fistel, über die eine postnatale Ventilation mittels Maskenbeatmung oder ösophagealer Intubation möglich ist. Eine vollständige Atresie ohne Fistel mit congenital high airway obstruction syndroms (CHAOS) als Maximalvariante liegt nur in 10-15\% vor.

Fall: Wir berichten von einem weiblichen Neugeborenen der 36+5 SSW; 32-jährige Mutter, II. Gravida, Frühabort in der 1. Schwangerschaft, Konzeption mittels intrazytoplasmatischer Spermieninjektion (ICSI); Gestationsdiabetes und -hypertonus. Pränatal bei nur flau abgrenzbarer fetaler Magenblase und massivem Polyhydramnion V.a. eine Ö́sophagusatresie. Das fetale Lungenparenchym zeigte eine normale Echogenität, bei unauffälliger kardio-thorakaler Ratio, so dass pränatal keine Anzeichen eines CHAOS darstellbar waren. Im weiteren Verlauf erfolgten bei zunehmendem Polyhydramnion mit Zervixverkürzung zwei komplikationslose Entlastungspunktionen (insgesamt ca. 41 drainiert). Nach vorzeitigem Blasensprung und RDS-Prophylaxe (vor der ersten Entlastungspunktion) wurde eine spontane Geburt angestrebt; bei V. a. partielle Plazentalösung Indikation zur Not-Sectio. Unmittelbar nach Entbindung wurde bei ausbleibendem Schreien des Kindes und bradykarder Herzfrequenz (<60/Min.) eine Neugeborenenreanimation mit Maskenbeatmung und Herzdruckmassage initiiert. Nach Intubation bei gut darstellbaren Stimmlippen (Cormack I) war über den ca. $1 \mathrm{~cm}$ tief platzierbaren Tubus weder expiratorisches $\mathrm{CO}_{2}$ (Easycap $\left.{ }^{\star}\right)$ messbar noch ein Atemgeräusch über der Lunge auskultierbar, sodass zunächst die Maskenbeatmung (auch hier kein exspCO $\mathrm{C}_{2}$ ) fortgeführt wurde. Weitere Intubationsversuche waren sowohl konventionell als auch mittels Videolaryngoskop und Endoskopie ebenfalls frustran, da sich ein federnder Widerstand bei ca. 1-1,5 cm subglottisch spürbar, aber nicht überwindbar darstellte. Die zentralvenöse BGA (NVK) zeigte eine entsprechend schwere Azidose ( $\mathrm{pH} 6,4 ; \mathrm{pCO}_{2} 223 \mathrm{mmHg}$; Laktat
$22 \mathrm{mmol} / \mathrm{l}$ ), welche sich nicht besserte, so dass nach einer Stunde die Reanimation beendet wurde. Aufgrund des klinischen Bildes einer schweren Atemwegsobstruktion wurde eine Obduktion angestrebt. Hier zeigte sich eine negative Schwimmprobe der Lungen (Lunge war demnach nicht belüftet) sowie eine komplette, kurzstreckige Trachealatresie (Typ F nach Faro) kombiniert mit einer Ösophagusatresie. Eine tracheo-ösophageale Fistel war distal der Atresien nachweisbar, die Lungen entsprechend ausgebildet. Es bestanden keine begleitenden Fehlbildungen.

Schlussfolgerung: Eine kombinierte Tracheal- und Ösophagusatresie mit distaler Fistel ist pränatal anhand der klassischen Kriterien eines CHAOS nicht nachweisbar. Im Rahmen des perinatalen Managements wäre eine EXIT-Prozedur als mögliches Szenario der neonatalen Erstversorgung sinnvoll, dessen pränatale Indikationsstellung bei der vorliegenden Konstellation erscheint derzeit jedoch nicht möglich.

\section{P081}

\section{Eine seltener Fall: Milztorsion und funktionelle Asplenie bei einem Neugeborenen mit Prune-Belly-Syndrom:}

\section{Möllmann' ', J. Strube ${ }^{2}$, H. Fuchs ${ }^{3}$, R. Hentschel ${ }^{3}$, M. Stenzel ${ }^{2}$}

'Universitätsklinikum, Zentrum für Kinder- und Jugendmedizin, Neonatologie/Pädiatrische Intensivmedizin/Päd. Sonographie, Freiburg, Deutschland, ${ }^{2}$ Universitätsklinikum Radiologische Klinik, Sektion Pädiatrische Radiologie, Freiburg, Deutschland, ${ }^{3}$ Universitätsklinikum, Zentrum für Kinder- und Jugendmedizin, Neonatologie/Pädiatrische Intensivmedizin, Freiburg, Deutschland

Das Prune-Belly Syndrom ist ein seltenes Krankheitsbild (3,8/100.000 Lebendgeburten). Es ist charakterisiert durch eine kongenitale Trias aus: Hypoplasie der Bauchmuskulatur, Harntrakt-Malformationen und Kryptorchismus. Wir berichten den Fall eines dystrophen Neugeborenen (vermutete Schwangerschaftswoche von 37Wochen; GG 1750 g, APGAR 4/7/9) mit dilatiertem, schlaffen Abdomen, kurzem breiten Thorax, einem Kryptorchismus sowie einer IV $^{\circ} \mathrm{NBKS}$-Dilatation mit massiv geweiteten Ureteren beidseits in der abdominellen Sonographie. In den ersten Lebensmonaten kam es zu mehreren fieberhaften Harnwegsinfekten sowie einer Urosepsis trotz externer Ausleitung der gestauten Ureteren. Während dieser schweren Sepsis fiel bei der Abdomensonographie auf, dass sich im Gegensatz zu den Voruntersuchungen in der Milzloge (in der VAL links), ein scharf begrenzter, echoreicher, zentral echofreier „Tumor" ohne Perfusion darstellte. Auch in der daran anschließend durchgeführten Kontrastmittelsonographie konnte keine Perfusion innerhalb dieser Struktur dargestellt werden, so dass wir von einem Z.n. stattgehabter Milztorsion mit anschließender Autolyse des Organes ausgehen mußten. In den folgenden Ultraschallkontrollen wurde das Milzresiduum zunehmend inhomogener und kleiner. Durch den Nachweis von Howel Jolley Körperchen im Blutbild wurde die funktionelle Asplenie bewiesen. Das Kind erhielt daraufhin Penicillin als antibiotische Prophylaxe und die Infekthäufigkeit nahm erheblich ab.

Zusammenfassung: Das Prune-Belly-Syndrom ist außer den drei pathognomonischen Auffälligkeiten noch mit anderen Fehlbildungen, wie z. B. gastrointestinale Defekten oder intestinalen Malrotationen (28\%) assoziiert. Die Ätiologie dieses Krankheitsbildes ist unklar (Theorien: intrauterine Obstruktion der Urethra, mesodermaler Entwicklungsdefekt). Eine Milztorsion kommt selten bei Patienten mit Prune-Belly-Syndrom vor, in der Literatur sind 5 Fälle beschrieben. Von diesen waren drei Patienten Kleinkinder, ein Patient war 9 Jahre und der älteste war 18 Jahre alt. Bei 3 der 5 Patienten musste eine Splenektomie durchgeführt werden. Möglicherweise könnten die sehr weichen Bauchdecken eine Milztorsion begünstigen. Obwohl beim Prune-Belly-Syndrom meist die Fehlbildung des Harntraktes und deren Therapie im Vordergrund steht, sollte bei den sonographischen Untersuchungen auch die Milz lokalisiert und bei akuter abdomineller Symptomatik eine Milztorsion ausgeschlossen werden. 


\section{Abstracts}

\begin{tabular}{|c|c|c|}
\hline Publikation & Klinik/Diagnostik & Therapie \\
\hline Heydenrych, Toit 1978 & $\begin{array}{l}\text { 1-jähriges Mädchen } \\
\text { Sichtbare, weiche Masse im mittleren Abdomen } \\
\text { Abdomen Röntgen: Distendiertes proximales Kolon } \\
\text { Kolonkontrasteinlauf: extrinsisch Druck auf die linke Flexur }\end{array}$ & $\begin{array}{l}\text { Laparotomie: Gangränös veränderte Milz nach } \\
\text { Torsion, Abszess, Splenektomie }\end{array}$ \\
\hline Teramonto et al 1981 & $\begin{array}{l}12 \text { Monate alter Junge } \\
\text { Weiche Masse tastbar } \\
\text { Kolonkontrasteinlauf: Verlagerung des Kolons nach medial } \\
\text { Sonographie: gemischt solide, flüssige Masse }\end{array}$ & $\begin{array}{l}\text { Explorative Laparotomie: Milzinfarkt bei Torsion, } \\
\text { Splenektomie }\end{array}$ \\
\hline Aliabadi et al 1987 & $\begin{array}{l}\text { 9-jähriger Junge mit Bauchschmerzen und Übelkeit } \\
\text { Klinisch weiche, nicht pulsierende Masse unterhalb des } \\
\text { Bauchnabels } \\
\text { Abdomen Röntgen: Dickdarmverschluss }\end{array}$ & $\begin{array}{l}\text { Explorative Laparotomie: Milztorsion mit Milzinfarkt, } \\
\text { Splenektomie }\end{array}$ \\
\hline Villanueva et al 1990 & $\begin{array}{l}41 / 2 \text { Jahre alte Junge, Urogenit. OP, dabei zufällig frei bewegl. } \\
\text { Masse > Milz getastet }\end{array}$ & Splenopexie \\
\hline Tran et al 2012 & $\begin{array}{l}\text { 18-jähriger männlicher Patient } \\
\text { CT-Diagnostik }\end{array}$ & $\begin{array}{l}\text { intraoperative Aufhebung der Torsion mit Erhaltung } \\
\text { der Milz }\end{array}$ \\
\hline
\end{tabular}

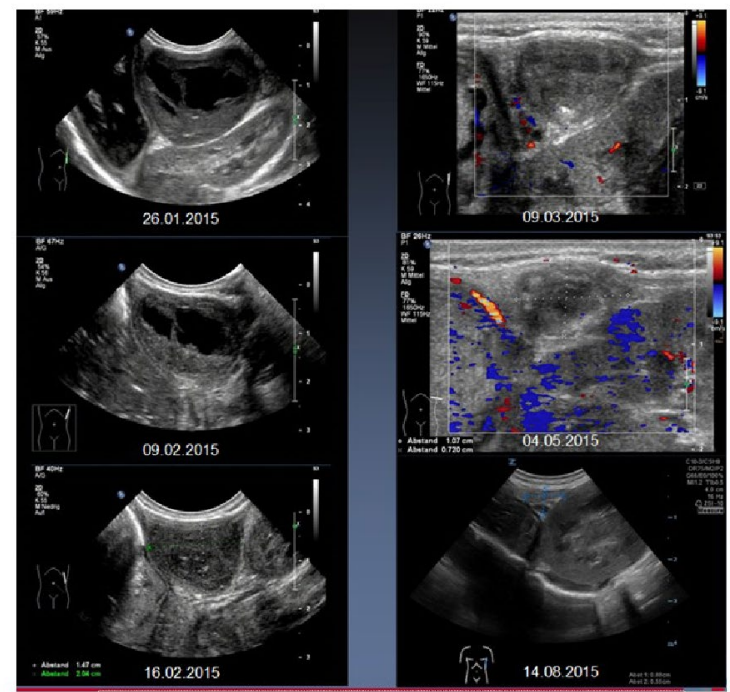

Abb. P081.1 $\triangle$ Verschiedene Zeitpunkte der Sonographien

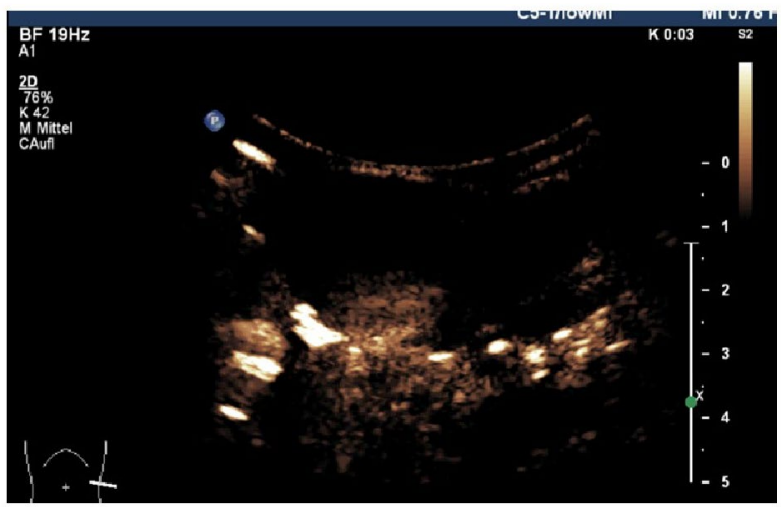

Abb. P081.2 $\triangle$ Kontrastmittelsonographie

\section{P082}

Multiple große Chorangiome als Auslöser einer schweren neonatalen respiratorischen Anpassungsstörung

H. Schneider', H. Fuchs ${ }^{2}$

'Universitäts - Kinderklinik, Neonatologie und Pädiatrische Intensivmedizin, Klinik I, Freiburg, Deutschland, ${ }^{2}$ Universitäts - Kinderklinik, Freiburg, Deutschland

Einleitung: Die respiratorische Anpassungsstörung beim reifen Neugeborenen hat verschiedene Differentialdiagnosen. Zu den häufigsten gehören die transitorische Tachypnoe, die neonatale Pneumonie, das Mekoniumaspirationssyndrom, der Pneumothorax sowie die kongenitale Zwerchfellhernie. Im Folgenden beschreiben wir die Kasuistik eines reifen Neugeborenen mit respiratorischer Globalinsuffizienz ausgelöst durch eine ausgeprägten Chorangiomatose.

Kasuistik: Ein reifes weibliches Neugeborenes wurde in einer auswärtigen Klinik in der 38. Schwangerschaftswoche per eiliger Sectio, bei pathologischem CTG, geboren. Nach Erstversorgung durch das geburtshilfliche Team mit initialem Blähmanöver adaptierte sich die Patientin zunächst gut. Anamnestisch bestand ein Polyhydramnion am Ende der Schwangerschaft. Das Fruchtwasser war klar, die Plazenta imponierte hydropisch. Maternale Infekthinweise gab es perinatal nicht. In der 1. Lebensstunde entwickelte sich eine zunehmende Oxygenierungsstörung. Nach Hinzurufen des neonatologischen Teams konnte unter CPAP-Atemunterstützung eine ausreichende Oxygenierung etabliert werden. Die weitere Versorgung erfolgte auf der pädiatrischen Intensivstation. Hier wurde die Patientin bei Verdacht auf ein Mekoniumaspirationssyndrom intubiert und für 3 Tage beatmet. Radiologisch bestand eine ausgedehnte pulmonale Minderbelüftung und Kardiomegalie. Laborchemisch imponierte eine Anämie und klinisch ein Hydrops fetalis. Intensivmedizinisch gestaltete sich der weitere Verlauf unkompliziert. Auffällig war jedoch eine prolongierte muskuläre Hypotonie und echokardiografisch eine hypertrophe Kardiomyopathie. Eine sekundäre Ursache der vorliegenden Myokardhypertrophie konnte ausgeschlossen werden. Bei Entlassung am 14. Lebenstag war die Patientin neurologisch unauffällig, auch der Echokardiografiebefund zeigte sich normalisiert. Die endgültige Diagnose konnte, kurz vor Entlassung, mit Eintreffen des histopathologischen Plazentabefundes gestellt werden. Hier zeigten sich multiple, bis $6,5 \mathrm{~cm}$ große, Chorangiome.

Diskussion: Multiple Chorangiome (größer $4 \mathrm{~cm}$ ) führen beim Fetus und Neugeborenen zu einer Herzinsuffizienz infolge high-output-failure durch arteriovenöses Shunting. Typische Komplikationen dieser hämodynamischen Veränderungen sind fetale Kardiomegalie, Anämie, Hydrops fetalis und Polyhydramnion. Diese Komplikationen erklären die im vorliegenden Fall beschriebene postnatale respiratorische Globalinsuffizienz. 
Schlussfolgerung: Eine Chorangiomatose geht aufgrund der resultierenden hämodynamischen Veränderungen mit einer perinatalen Mortalität von $30-40 \%$ einher. Eine pränatale Ultraschalldiagnostik kann die frühzeitige Diagnose ermöglichen. Therapieoptionen (z. B. intrauterine Laserablation der versorgenden Gefäße) stehen zur Verfügung und scheinen sicher sowie effektiv zu sein.

\section{Postersitzung 09: Varia}

\section{P083 \\ Isolierter kongenitaler Chylothorax: Abwägung von Therapieoptionen}

\author{
M. Schoberer', S. Trepels-Kottek', K. Heimann', J. Vásquez-Jienez², \\ F. Mottaghy ${ }^{3}$, T. Orlikowsky \\ 'Uniklinik RWTH Aachen, Kinderklinik Sektion Neonatologie und \\ Kinderintensivmedizin, Aachen, Deutschland, ${ }^{2}$ Uniklinik RWTH Aachen, \\ Kinderherzchirurgie, Aachen, Deutschland, ${ }^{3}$ Uniklinik RWTH Aachen, \\ Nuklearmedizin, Aachen, Deutschland
}

Einleitung: Der isolierte kongenitale Chylothorax ist ein seltenes Krankheitsbild, zu dem wenig Kasiustiken existieren. Bei fehlendem Ansprechen auf Therapie weist er eine Letalität von bis zu $50 \%$ auf. Klar strukturierte, evidenzbasierte Therapieempfehlungen existieren nicht. Als Eckpfeiler der Therapie gelten Drainage, Nahrungskarenz, parenterale Ernährung, MCTKost, Octreotid (Somatostatinanalogon), Versuch der operativen Sanierung. Kasuistik: Geburt des Patienten in der 38. SSW. Ein Pleuraerguss rechts wurde am 4. Lebenstag bei zunehmender Tachypnoe und fehlenden Infektionszeichen nachgewiesen, welcher sich nach Punktion von chylösem Sekret als kongenitaler Chylothorax darstellte. Keine assoziierten Fehlbildungen. Im weiteren Verlauf rezidivierende Ergussbildung, die eine Drainagenanlage mit intermittierender/kontinuierlicher Ableitungen erforderlich machte. Nach Nahrungskarenz, kompletter parenteraler Ernährung. Vergeblicher Versuch eines oralen Nahrungsaufbaus mit MCT-Kost über 4 Wochen. Octreotid-Terapie am 30. Lebenstag mit maximaler Dosis von $10 \mu \mathrm{g} / \mathrm{kg} / \mathrm{h}$ bis zum 60. Lebenstag. Bei signifikanter Lympho- und Granulozytopenie PCP-Prophylaxe (Cotrimoxazol ${ }^{\circ}$ ), Immunglobulinsubstitution und Umkehrisolierung. Die nuklearmedizinische Darstellung zur Unterscheidung zwischen diffusen oder lokal begrenzten Anomalien des Ductus thoracicus gelang nicht. Im Alter von 3 Monaten operative Intervention, die auf Höhe des BWK 5/6 mittels präoperativ oral applizierter angefärbter Sahne (30\% Fettgehalt) ein isoliertes Loch im Ductus thoracicus zeigte, welches mittels Ligatur sowie Fibrinkleber verschlossen wurde. Postoperativ rascher und unkomplizierter Kostaufbau. Im Alter von 3 Jahre gut gediehenes Kleinkind ohne pulmonale Einschränkung oder Thoraxdeformität mit unauffälliger Entwicklung.

Schlussfolgerung: Über Dauer, Dosierung und Erfolgsaussichten des isolierten kongenitalen Chylothorax existiert vor allem Expertenwissen. Als seltene Erkrankung gemeldet, sollte eine Therapieleitlinie zur optimalen Behandlung dieses Krankheitsbildes initiiert werden.

\section{P084}

\section{Case Report Frühgeborenes mit V.a. Aicardi-Goutières Syndrom}

M. Schünemann ${ }^{1}$, A. Scheuern' ', S. Ströbele' ', H. Gaspar ${ }^{2}$, W. Lindner', H. Hummler ${ }^{1}$

${ }^{1} K$ linik für Kinder- und Jugendmedizin, Universitätsklinikum Ulm, Sektion Neonatologie und Pädiatrische Intensivmedizin, Ulm, Deutschland, ${ }^{2}$ Universitätsklinikum Ulm, Humangenetik, Ulm, Deutschland

Hintergrund: Intrazerebrale Verkalkungen werden bei konnatalen Infektionen und seltenen Erkrankungen beobachtet. Differenzialdiagnostisch muss an das Aicardi-Goutières Syndrom (AGS) gedacht werden.

Fallbericht: Wir berichten über ein weibliches Frühgeborenes von $35+4 / 7$ SSW. Pränatal fielen sonographisch intrazerebrale periventrikuläre Verkalkungen auf. Diese bestätigten sich im postnatal durchgeführten MRT, daneben zeigten sich eine Pachygyrie und Hypomyelinisierung. Eine infektiöse und metabolische Genese wurde ausgeschlossen. Wir fanden auffällige Werte für Thrombozyten $89 \mathrm{G} / \mathrm{l}(>150)$, AST $160 \mathrm{U} / 1(<90)$, ALT $69 \mathrm{U} / \mathrm{ml}(<60)$, GGT $468 \mathrm{U} / \mathrm{l}(<140)$, dir. Bilirubin $94 \mu \mathrm{mol} / \mathrm{l}(<3,4)$, Na $152 \mathrm{mmol} / \mathrm{l}$ (135-145), Chlorid $114 \mathrm{mmol} / \mathrm{l}$ (95-105), passager PTH $128 \mathrm{pg} / \mathrm{ml}(15-57)$ und TSH 16,88 mIU/l (0,7-11). Klinisch bestand eine muskuläre Hypotonie, Hyperexzitabilität, gesteigerte MER bei genereller Bewegungsarmut, im Verlauf eine zunehmende Hepatomegalie -bislang unklarer Genese-, sowie im Alter von 6 Wochen Auftreten krustiger Hautläsionen an beiden Knien. Im EEG zeigten sich Allgemeinveränderungen, des weiteren temporal bds. vereinzelt sharp-waves. In Zusammenschau der Befunde stellten wir die Verdachtsdiagnose eines AGS. Zur Verifizierung erfolgte weiterführende Diagnostik. Im Liquor fanden sich erhöhte Werte für Protein $705 \mathrm{mg} / \mathrm{l}(<500)$, Interferon- $\alpha$ (IFN- $\alpha) 75 \mathrm{UI} / \mathrm{ml}(<2)$ und Neopterin $2061 \mathrm{nmol} / \mathrm{l}$ (6-59), IFN- $\alpha$ im Serum $50 \mathrm{UI} / \mathrm{ml}(<2), \mathrm{Be}-$ funde passend zum Vorliegen eines AGS $(1,3)$. Die Bestätigung durch Mutationsanalyse der Kandidatengene steht noch aus.

Diskussion: Beim Aicardi-Goutières Syndrom handelt es sich um eine seltene autosomal-rezessiv vererbte, genetisch heterogene Enzephalopathie. Neuroradiologisch zeigen sich typischerweise zerebrale Kalzifikationen, Veränderungen der weißen Substanz und Hirnatrophie, diese ähneln einer intrauterin erworbenen Infektion (Pseudo-TORCH) (2). Am zweithäufigsten betroffen ist die Haut mit „frostbeulenartigen,Effloreszenzen, ähnlich wie beim systemischen Lupus erythematodes. Im Liquor ist eine chronische Lymphozytose, eine Erhöhung von IFN- $\alpha$ und Neopterin als Zeichen autoimmuner Inflammationsreaktion nachweisbar (1,2). Die Enzephalopathie manifestiert sich früh, meist im Säuglingsalter, zeigt einen vorwiegend in den ersten zwei Jahren progredienten Verlauf (2), die Prognose ist mit schwerer psychomotorischer Retardierung ungünstig (1).

\section{Literatur}

1. Aicardi-Goutières syndrome: special type early-onset encephalopathy, J. Aicardi, E J Ped Neurol, 2002

2. Aicardi-Goutières Syndrome: Neuroradiologic Findings and Follow-Up. A J NR Pediatrics 2009. C. Uggettia et al.

3. F. Rozenberg, Pôle Biologie Pathologie Pharmacie, Service de Virologie Hôpital Cochin, Paris

\section{P085}

Bullöse kongenitale ichthyosiforme Erythrodermie ein Fallbeispiel

A. Nusche ${ }^{1}$, H. Schneider', L. Bruckner-Tuderman ${ }^{1}$, P. Freisinger ${ }^{1}$

'Klinik für Kinder und Jugendmedizin, Klinikum am Steinenberg, Reutlingen, Deutschland

Hintergrund: Die bullöse kongenitale ichthyosiforme Erythrodermie (OMIM 113800) ist eine seltene, meist autosomal-dominant vererbte Verhornungsstörung der Haut, der Mutationen in Keratinen Nr. 1 oder Nr. 10 zugrunde liegen. Letztere sind Bestandteile von Tonofilamenten, die wichtig für die Stabilität und Zusammenhalt der Keratinozyten sind. Durch den Defekt verlieren die Zellen den Zusammenhalt bei mechanischer Belastung und es kommt zur Blasenbildung und Schuppung. Im Laufe des 1. Lebensjahres nimmt die Blasenbildung ab, es kommt zu massiven, lamellären Hyperkeratosen mit einem im Verlauf ehr warzenartiges Bild. (1)(2)(3). Fallbeispiel:

Anamnese: FG der 35+3/7 SSW, spontan geboren, APGAR 9/10/10. Eine gesunde Schwester. Schwangerschaftsanamnese bis auf Gestationsdiabetes unauffällig.

Untersuchungsbefund: Großflächige Ablösung der Epidermis wie bei Verbrühung Grad 2a an Stamm und Extremitäten. An Händen und Füßen deutlich verdickte, weiße Haut. Diagnostik:Entzündgungszeichen negativ, niedrig normaler Zinkspeiegel. Hautstanze: Blasenbildung hoch intraepidermal im oberen Stratum spinosum und granulosum, indirekte Immunfluoreszenzfärbung mit 20 AK positiv für Plakophilin, Pankeratin, Keratin 1,10, 5, 14, Plektin, BPAG, alpha 1 und 6 Integrin, Desmoplakin, beta 4 Integrin, Kollagen IV, VII und XVIIBP 180, Laminin 332, à Beurteilung: Befund und Klinik: Bullöse Ichthyose. 
Genetik: Kein Nachweis einer pathogenen Mutation in KRT 1 und 10. Therapie: Zunächst hohe Flüssigkeitszufuhr wie bei Verbrennung, bei Befall von über $50 \%$ der Haut. Infektionsprophylaxe mit Cotrimoxazol (TMP-Anteil $2 \mathrm{mg} / \mathrm{kgKG} \mathrm{1x/d} \mathrm{p.o.)} \mathrm{und} \mathrm{Fluconazol} \mathrm{(4} \mathrm{mg/kgKG} \mathrm{1x/d}$ p.o.)

Minimal Handling, initial tägliche sterile Verbandswechsel, im Verlauf 2dann 3- täglich. Applikation von Octenidin, Panthenol und Urgotül. Verlauf: Ab der 4. LW langsame Besserung mit z. T. abheilenden der großflächigen Blasen, jedoch weiterhin sehr verletzlicher Haut. Bei mechanischer Beanspruchung weiterhin Bildung neuer Blasen. Ernährung mit Pregomin AS bei schmerzhaftem Abdomen und gehäuft auftretender Nahrungsmittelallergien bei Ichthyosen.

Vergleich mit publizierten Fallberichten: Das klinische Bild der Patienten mit bullöser kongenitaler ichthyosiformen Erythrodermie variiert stark. 2 Hauptgruppen werden unterschieden: 1. Mit Beteiligung von Handinnenflächen und Fußsohlen, meist Mutation in Keratin 1-Gen. 2. Ohne Beteiligung der Handinnenflächen und Fußsohlen meist Defekt im Keratin 10. (1)(2)(3) Trotz klinisch und morphologisch typischer Befunde konnte bei unserem Patient bisher keine Mutation nachgewiesen werden.

Schlussfolgerung: Die Pflege ähnlich wie bei Schwerverbrannten, mit Vermeiden einer Infektion ist sehr wichtig. Im Verlauf ist eine adäquate Hautpflege zur Infektionsprophylaxe und Vermeidung von üblem Geruch anzustreben. Eine Anbindung der Betroffenen an eine Selbsthilfegruppe und die Anbindung an ein Ichtyosezentrum wird empfohlen.

\section{Literatur}

1. Uezato Het al. J Dermat 2005; 32(10):801-8

2. Kucharekova $M$ et al. Int J Dermat 2007 Suppl 3:36-8

3. Giovanna et al J Invest Dermat 1994 102(3):390-4

\section{P086}

\section{Kongenitales Glottissegel - eine Fallvorstellung}

M. Petrasch', N. Geier', R. Petrasch' ${ }^{2}$, I. Fleischer ${ }^{2}$, P. Ruef', B. Lippert' ${ }^{2}$, T. Nicolai ${ }^{3}$ 'SLK Kliniken Heilbronn GmbH, Kinderklinik, Heilbronn, Deutschland, ${ }^{2}$ SLK Kliniken Heilbronn GmbH, Klinik für Hals-Nasen-Ohren-Heilkunde, Kopf- und Halschirurgie, Heilbronn, Deutschland, ${ }^{3}$ Klinikum der Universität München, Kinderklinik und Kinderpoliklinik im Dr. von Haunerschen Kinderspital, München, Deutschland

Anamnese: Reifes, männl. Neugeborenes der 38. SSW wird im Alter von wenigen Stunden aufgrund einer schwachen, krächzenden Stimme (,ausbleibendes Schreien") sowie erhöhten Entzündungswerten in unsere Kinderklinik aufgenommen.

Aufnahmestatus: Klinisch auffällige faziale Dysmorphie mit asymmetrischem „Schreigesicht“ bei V.a. Hypoplasie des M. Depressor anguli oris und Mikrozephalie. Dezenter inspiratorischer Stridor. Aphone Stimme. Pulmo bds. auskultatorisch frei. Cor regelmäßig ohne path. Herzgeräusch. Normales männl. Genitale. Reflexstatus und Tonus regelrecht. Weitere Untersuchung unauffällig.

Diagnostik und Verlauf: Im Routinelabor zeigte sich das CRP mit 1,3 mg/dl erhöht bei gleichzeitig bestehender, nicht behandlungsbedürftiger Thrombozytopenie. Aufgrund des initialen Verdachtes einer Infektion wurde das Neugeborene über 5 Tage mit Aminopenicillin sowie Gentamicin i.v. behandelt. Hierunter waren die Entzündungswerte rückläufig und zuletzt normwertig. Stoffwechselscreening, Schädelsonographie sowie ein Röntgen-Thorax lieferten Normalbefunde. Bei persistierender Aphonie erfolgte eine Bronchoskopie unter Spontanatmung bei sediertem Kind. Diese zeigte eine Segelbildung (Diaphragma) in der vorderen Kommissur zwischen den Stimmlippen („laryngeales Web“) mit einem Restlumen von ca. $20 \%$. Es erfolgte die Verlegung in die Kinderklinik und Kinderpoliklinik im Dr. von Haunerschen Kinderspital München. Es fand sich im Rahmen der Fehlbildungsdiagnostik in der Echokardiographie ein ASD Typ II. In einer molekulargenetischen Abklärung bestätigte sich schließlich der Verdacht eines Di-George-Syndroms. Eine erste Bestimmung der T- und B-Zellen, der Immunglobuline sowie des Calcium-/Phosphathaushaltes fiel unauffällig aus.
Therapie: Im Alter von 3 Wochen erfolgte die elektive Anlage einer Trachealkanüle zur Sicherung der Atemwege bei minimalem Restlumen. Eine Rekonstruktion der laryngealen Fehlbildung ist mit dem Erreichen des 3. Lebensjahres geplant.

Schlussfolgerung: Eine laryngeale Segelbildung beruht auf einer mangelhaften Rekanalisierung des Glottisspalts während der fetalen Entwicklung. Diese Hemmungsfehlbildung in Kombination mit einer auffälligen Fazies sowie kardialen Fehlbildungen kann im Rahmen eines Di-George-Syndroms auftreten. Eine molekulargenetische und immunologische Diagnostik sowie Kontrolle der Phosphat- und Calciumwerte sollte durchgeführt werden.

\section{P087}

\section{„Speikind“ mit Pneumonie: Wenn die Ursache „tiefer" liegt}

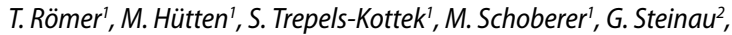
S. Schrading ${ }^{3}$, T. Orlikowsky

'Uniklinik RWTH Aachen, Kinder- und Jugendmedizin, Sektion Neonatologie, Aachen, Deutschland, ${ }^{2}$ Uniklinik RWTH Aachen, Allgemein-, Viszeral- und Transplantationschirurgie, Aachen, Deutschland, ${ }^{3}$ Uniklinik RWTH Aachen, Diagnostische und interventionelle Radiologie, Aachen, Deutschland

Fallbericht: Nach unauffälliger Schwangerschaft und Geburt wurde ein reifes (37+4 Schwangerschaftswochen), eutrophes männliches Neugeborenes am 5. Lebenstag wegen schwerer Tachydyspnoe mit Hypoxämie und respiratorischer Azidose aufgenommen. Postpartal war etwas vermehrtes Spucken bei gutem Trinkinteresse aufgefallen. Radiologisch bestanden typische Zeichen einer beidseitigen Bronchopneumonie, laborchemisch erhöhte Infektparameter (CRP 15 mg/l; IL-6789 ng/l). Eine rasche respiratorische Befundbesserung wurde unter Atemhilfe mittels CPAP sowie kalkulierter antibiotischer Behandlung erreicht. Parallel dazu entwickelte sich aber eine zunehmende Hyperemesis mit hypochlorämischer, metabolischer Alkalose bei erhaltener Stuhlpassage. Sonographisch zeigte sich eine Einschnürung des Duodenums mit massiver prästenotischer Dilatation. Radiologisch fand sich ein Double-Bubble-Phänomen. Intraoperativ bestätigte sich der Befund eines Pancreas anulare mit hochgradiger Duodenalstenose. Es erfolgte eine primäre Duodenoduodenostomie mittels Diamantanastomose. Der postoperative Verlauf gestaltete sich unkompliziert, die Entlassung erfolgte am 13. postoperativen Tag.

Schlussfolgerung: Die Sequenz der Pneumonie nach mehreren Tagen vermehrtem (aber nicht unphysiologisch starkem) Spucken lässt einen klinischen Zusammenhang mit dem Pancreas anulare im Sinne rezidivierender Aspirationen vermuten. In unserem Fall war die respiratorische Symptomatik führend und hat zur kinderärztlichen und dann stationären Vorstellung geführt. Bei dieser Konstellation sollte eine Passagestörung des oberen Gastrointestinaltraktes frühzeitig in die differenzialdiagnostischen Überlegungen einbezogen werden.

\section{P088}

\section{Mehr als nur die Haut: Adams-Oliver-Syndrom}

S. Pogodda ${ }^{1}$, W. Beyer ${ }^{2}$, E. Gilberg ${ }^{3}$, M. Rath ${ }^{3}$, S. Pietruschka ${ }^{4}$, S. Armbrust ${ }^{5}$ ${ }^{1}$ Klinikum Neubrandenburg, Kinderklinik, Neubrandenburg, Deutschland, 2Dietrich-Bonhoeffer-Klinikum, Klinik für Kinderchirurgie und Kinderurologie, Neubrandenburg, Deutschland, ${ }^{3}$ Universitätsmedizin Greifswald MVZ, Ambulantes Zentrum, Bereich Humangenetik, Greifswald, Deutschland, ${ }^{4}$ Dietrich-Bonhoeffer-Klinikum, Klinik für Radiologie und Neuroradiologie, Neubrandenburg, Deutschland, ${ }^{5}$ Dietrich-Bonhoeffer-Klinikum, Klinik für Kinder- und Jugendmedizin, Neubrandenburg, Deutschland

Das Adams-Oliver-Syndrom (AOS) ist charakterisiert als Kombination von angeborenen Kopfhautdefekten bis hin zu Ossifikationsstörungen des Schädeldachs mit terminalen transversalen Defekten der Gliedmaßen. In Einzelfällen sind auch Herzfehler, Augenveränderungen und ZNS Auffälligkeiten beschrieben. Nierenfehlbildungen sind nicht bekannt.Wir berichten über ein reifes hypotrophes Neugeborenes mit AOS und unilateraler Nierenagenesie. 
Fallbeschreibung: Reifes hypotrophes weibliches Neugeborenes mit $10 \times 8 \mathrm{~cm}$ großem nekrotischen Areal der Haut im Bereich des Vertex, Brachydaktylie der Hände und Syndaktylie Dig. ped. II/III bds. Die weitergehende Diagnostik erbrachte eine Nierenagenesie rechts, ASD II und einen großen knöchernen Defekt im Vertexbereich. Die Chromosomenanalyse inkl. einer für Lokus 13q14 spezifischen DNA-Sonde erbrachte einen normalen weiblichen Karyotyp ohne intra- oder interchromosomale Strukturveränderungen. Die Analyse des ARHGAP31-Gens (AOS 1) ergab keine Auffälligkeiten, eine Exon-Sequenzierung über NGS wurde initiiert. Im Verlauf sukzessives Abheilen und Re-Epithelialisierung des nekrotischen Areals unter Lokaltherapie.

Diskussion: Nach Erstbeschreibung 1945 wird die Inzidenz des AOS mit 1:225.000 Neugeborene angegeben. Dabei werden verschiedene Phänotypen beschrieben (AOS 1-6) mit hoher genetischer Heterogenität wobei die Vererbung zumeist autosomal-dominant erfolgt. Pathophysiologisch wird postuliert, daß ein gestörter Gefäßaufbau in Hinsicht auf die vaskulären Myozyten und Perizyten zum nachfolgenden Phänotyp führt. Die Therapie ist symptomatisch und orientiert sich an der Notwendigkeit z. B. Infektionen zu vermeiden durch plastische Deckung eines Defekts, etc. In unserem Fall war eine längerfristige Lokaltherapie unter stationären $\mathrm{Be}$ dingungen erfolgt, wodurch letztendlich eine operative Therapie vermieden werden konnte. Die Prognose ist abhängig von der Ausprägung der Symptome und bei der Variabilität des Syndroms recht verschieden. Die Entwicklung verläuft im Prinzip normal, Epilepsie oder Behinderung tritt in Abhängigkeit einer cerebralen Beteiligung auf. Conclusion: Eine renale Beteiligung ist bei bisher über 500 beschriebenen Fällen nur einmalig als Doppelniere beschrieben. Alle bekannten AOS-Genorte korrelieren nicht mit Nierenagenesien. Insoweit beschreiben wir erstmalig eine einseitige Nierenagenesie als Symptom eines AOS.

\section{P089}

\section{Frühgeborenes mit V.a. Incontinentia pigmenti}

E. Schulte-Kemna', F. Schulte-Kemna', P. Ruef', N. Geier', H. Berger², W. Weyers ${ }^{3}$

${ }^{1}$ Klinikum Heilbronn GmbH, Kinderklinik, Heilbronn, Deutschland, ${ }^{2}$ SLK Kliniken Heilbronn, Klinik für Dermatologie, Allergologie und Phlebologie, Heilbronn, Deutschland, ${ }^{3}$ Zentrum für Dermatopathologie Freiburg, Freiburg, Deutschland

Anamnese: Weibliches Zwillingsfrühgeborenes der 31+2 SSW mit einem Geburtsgewicht von $700 \mathrm{~g}$ (small for gestational age), Sectio aufgrund eines pathologischen Dopplers und diskordantem Wachstums, Apgar 4/8/9, Familienanamnese bezüglich okulokutanem Albinismus positiv. Aufnahmestatus: Weibliches, hypotrophes Frühgeborenes, Atemhilfe mittels CPAP über Rachen Tubus, ansonsten unauffälliger Organstatus, hellrotes papulöses Exanthem disseminiert am gesamten Integument mit Betonung der Extremitäten.

Diagnostik, Therapie und Verlauf: Aufnahme auf die neonatologische Intensivstation bei Frühgeburtlichkeit. Es erfolgte eine prophylaktische antibiotische Therapie mit Ampicillin und Cefotaxim aufgrund des niedrigen Geburtsgewichtes. Initial wurde der Hautbefund als toxisches Neugeborenenexanthem gewertet. Im frühen postnatalen Verlauf fielen schneeweiße Haare und rötliche Iriden auf, sodass der V.a. einen okulokutanen Albinismus gestellt wurde. Der konsiliarisch hinzugezogene Augenarzt bestätigte den Verdacht, es bestanden keine weiteren Auffälligkeiten im ophtalmologischen Befund. Aufgrund einer Persistenz der Hautveränderungen mit zunehmendem papulovesikulösem Charakter sowie der Ausbildung von honiggelben Krusten erfolgte eine topische antiseptische Therapie und eine antibiotischen Therapie mit Flucloxacillin, jedoch ohne wesentliche Befundbesserung. Laborchemisch fiel eine Eosinophilie auf, das Vorliegen eines Chediak-Higashi-Syndroms konnte ausgeschlossen werden. Es erfolgte die Mitbeurteilung durch die Kollegen der Dermatologie. Hierauf wurde die Entnahme einer Probebiopsie zur histopathologischen Begutachtung empfohlen. Im histologischen Befund zeigten sich intraepithelial kleinste Komplexe von eosinophilen Granulozyten, sowie eine Infiltration von Eosinophilen im oberen Corium. Die Dermatohistopathologen äußerten den Verdacht auf eine Incontinentia pigmenti. In der Schädelsonographie fiel eine kleine Plexuszyte auf. Weitere Auffälligkeiten ergaben sich nicht.

Diskussion: Bei der Incontinentia pigmenti (Bloch-Sulzberger Syndrom) handelt es sich um eine $\mathrm{x}$-chromosomal dominante neuroektodermale Erkrankung, welche sich meist schon beim Neugeborenen manifestiert. Es liegt eine Mutation des NF-kappa-B essential modulator Gens (Nemo Gen bei Xq28) vor. Der Krankheitsverlauf ist stadienartig und präsentiert sich initial häufig mit streifig angeordneten Bläschen, Papeln und Plaques, welche entlang der Blaschko Linien angeordnet sind. Im Verlauf treten verrukös-streifige Hyperkeratosen auf, welche unter Hyperpigmentierung abheilen. Das Endstadium, welches nach Jahren erreicht wird, ist durch streifenförmige Hypopigmentierungen und Haarlosigkeit gekennzeichnet. Als assoziierte Begleitbefunde sind Zahn-, Augen- und ZNS-Anomalien beschrieben. Eine kausale Therapie existiert nicht. In Zusammenschau der Befunde mit passendem klinischen Erscheinungsbild, dem histologischen Befund und der Eosinophilie im Blutbild erscheint die Diagnose sehr wahrscheinlich. Die molekulargenetische Sicherung der Diagnose ist aktuell noch ausstehend.

\section{P090}

\section{Trachealatresie, Va VACTERL-Assoziation,: Case Report}

P. Panagiotou', P. Kollertz' ${ }^{2}$, K. Schoner ${ }^{3}$, M. Barthel', E. Hamelmann',

\section{T. Bösing'}

'Evangelisches Krankenhaus Bielefeld, Klinik für Kinder- und Jugendmedizin, Kinderzentrum Bethel, Bielefeld, Deutschland, ${ }^{2}$ Evangelisches Krankenhaus Bielefeld,Klinik für Gynäkologie und Geburtshilfe, Bielefeld, Deutschland, ${ }^{33}$ Institut für Fetalpathologie, Universitätsklinikum Gießen und Marburg, Marburg, Deutschland, ${ }^{4}$ Evangelisches Kranknehaus Bielefeld, Klinik für Kinderchirurgie, Kinderzentrum Bethel, Bielefeld, Deutschland

Hintergrund: Die Trachealatresie ist eine sehr seltene angeborene Fehlbildung mit einer sehr hohen Letalität. Meist tritt sie in Kombination mit anderen Fehlbildungen häufiger in Form der sogenannten VACTERL Assoziation auf. Es ist deshalb insbesondere bei dem Va eine Ösophagusatresie auch an die Möglichkeit des Vorliegens einer trachealen Atresie zu denken. Fragestellung/Material/Methode: Wir sahen ein männliches Frühgeborenes (SSW $30+3$ ), GG: $1560 \mathrm{~g}$, Geburt per Sectio bei auffälligem CTG und VZB. Präpartal schon bek. Polyhydramnion, Va Ösophagusatresie und kleinen muskulären VSD, ansonsten freie Familienanamnese und unkomplizierter Schwangerschaftsverlauf. Das Kind kam tief zyanotisch, mit Schnappatmung und bradykard zur Erstversorgung. Unter intensiver druckkontrollierter Maskenventilation zunächst Stabilisierung der HF, aber keine hinreichende Eigenatmung. mehrere Intubationsversuche scheitern, es war kein Vorschieben des Tubus über den gut einstellbaren Larynxeingang in den subglottischen Raum möglich. Eine ausreichende Oxygenierung gelang über eine intensive und dichte BMV. Eine hinreichende Ventilation hingegen ließ sich nicht erreichen, so dass der V. a. eine tracheale Atresie bzw hochgradige Stenose gestellt wurde Deshalb erfolgte die notfallmäßige offene Tracheotomie durch die Kinderchirurgen. Auch über den dabei vorgeschobenen Tubus ließ sich das Kind zwar vorübergehend oygenieren, aber nicht hinreichend ventilieren, so dass die $\mathrm{pCO}_{2}$ Werte über 100mmHG anstiegen und das Kind mit einer raschen Regredienz seiner Vitalzeichen verstarb. In der postmortalen Untersuchung sahen wir radiologisch mehrere Wirbelsäulen-, Gliederanomalien, eine „weiße Lunge",luftgefüllter Magenblase. Endoskopisch zeigte sich eine hochgradige subglottische Trachealstenose bzw Atresie. Der über die offene Tracheotomie nach tracheal eingeführte Tubus erreichte über eine tracheösophageale Fistel den distalen Ösophagus und lag mit seiner Spitze unmittelbar vor der offenkundig verschlossenen Kardia im Sinne einer atypischen tiefen Ösophagusatresie. Die histopathologischen Befunde aus der Obduktion stehen noch aus. Die Autoren haben eigene Erfahrungen mit vier weiteren trachealen Atresien in 30 Jahren Neonatologie, die alle auch mit einer Ösophagusatresie kombiniert waren und postmortal aufgearbeitet worden sind. Nur einem Neugeborenen konnte vorrübergehend durch eine notfallmäßige Punktion der Trachea geholfen werden. 
Diskussion: Anders als die möglichen Begleitfehlbildungen ist die Trachealatresie oder auch die hochgradige Stenosierung mit dem präpartalen Ultraschall nicht sicher zu diagnostizieren. Es ist deshalb bei dem Verdacht einer Ösophagusatresie immer auch an das mögliche Vorliegen einer trachealen Atresie zu denken, da beide ihre gemeinsame embryonale Entstehung aus dem entodermalen Darmrohr haben, Da die Assoziation der trachealen Atresie mit einer Ösophagusatresie so überzufällig häufig ist, sollte die Vogt Klassifikation um diesen Typ V erweitert werden. Diese Entbindungen sollten deshalb von einem erfahrenen neonatologischen Versorgungsteam betreut werden. Auch die in einem Perinatalzentrum geübte interdisziplinäre Zusammenarbeit mit der Kinderchirurgie ist von großem Wert, da eine Notfalltracheotomie die einzig mögliche Lebensrettung für diese Kinder sein kann und die notfallmäßige Punktion der kleinen noch sehr weichen Trachea des neu- oder gar frühgeborenen Kindes sehr schwierig ist

\section{P091 \\ Atypischer teratoid/rabdoider Tumor als seltene Ursache eines Hydrocephalus internus bei einem Neugeborenen}

D. Giannikopoulou', H. Schwalm', C. Papastergiou', M. Friepörtner', K. Kerl', J. Sandkötter ${ }^{2}$, H. Omran'², G. Rellensmann ${ }^{2}$, A. Brentrup ${ }^{2}$, G. Selzer ${ }^{1}$

'EVK Hamm, Neonatologie und Kinderintensivstation, Hamm, Deutschland, ${ }^{2}$ Universitätsklinikum Münster, Klinik und Poliklinik für Kinderheilkunde und Jugendmedizin, Onkologie, Hämatologie, Neonatologie, pädiatrische Intensivmedizin, Münster, Deutschland

Einleitung:Hirntumore sind im Kindesalter nach den Leukämien die zweitgrößte Gruppe maligner Erkrankungen. Atypische teratoide Rabdoidtumoren (ATRT) sind die häufigsten Hirntumore bei Kindern unter einem Jahr. Wir berichten über ein männliches Neugeborenes mit einem pränatal beginnenden rasch progredienten Hydrocephalus aufgrund eines infratentoriellen Hirntumors.

Kasuistik: Drittes Kind einer 32 jährigen Mutter, ein gesundes Geschwisterkind und ein Kind in utero verstorben. Geburt nach $38+0$ SSW durch Notsectio caesarea bei pathologischem CTG. Diätetisch eingestellter Gestationsdiabetes, unauffällige pränatale Diagnostik. In der unmittelbar vor der Geburt durchgeführten Sonographie zeigte sich erstmals ein ausgeprägter Hydrocephalus, Vorkontrolle in der 36. SSW. Die postnatale Adaptation erfolgte erschwert bei insuffizienter Spontanatmung, zunächst Maskenbeatmung, dann CPAP. Apgar: 6/9/9, NS pH 7,31, Gewicht: 2750 g (10. Perzentile), Körperlänge $50 \mathrm{~cm}$ (25. Perzentile), Kopfumfang $36,5 \mathrm{~cm}$ (90.Perzentile).

Verlauf: Stationäre Aufnahme auf unsere neonatale Intensivstation. Respiratorisch stabil unter CPAP, neurologisch hypoton mit einer bestehenden Fazialis Parese rechtsseitig und den Verdacht auf eine Schluck- und Koordinationsstörung. Sonographisch ergab sich ein Hydrocephalus internus occlusus und ein inhomogen strukturiertes Kleinhirn mit zystischen Anteilen, Hirnstamm kaum abgrenzbar. Ein MRT mit Kontrastmittel bestätigte das Vorliegen eines Hydrocephalus bei Liquorzirkulationsstörung durch einen Kleinhirntumor mit Infiltration des Hirnstamms und $\mathrm{Ab}$ tropfmetastasen supratentoriell (Anlage MRT Bilder). Verlegung in das kinderonkologische Zentrum der Uni Münster, dort Entlastung des Hydrocephalus durch die Anlage eines VP Shuntes. Aufgrund des klinischen Verlaufes wurde die Diagnose eines ATRTs in Betracht gezogen, welche durch den homozygoten Verlust von SMARCB1 (ca 98\%) oder SMARCA4 (ca 2\%), zwei Untereinheiten des Chromatinremodellierungskomplexes SWIS/SNF, verursacht wird. Molekulargenetische Untersuchungen (MLPA und Sequenzierung des SMARCB1-Gens) zeigten einen heterozygoten SMARCB1 Verlust in der Keimbahn, welcher den Rückschluss zulässt, dass ein Rhabdoidtumor-Prädispositionssyndrom vorliegt. Prognostisch stellte diese Diagnose bei unserem Patient einen sehr ungünstigen Befund dar, der aufgrund der Lokalisation nicht operabel und bei Kindern unter einem Jahr wegen des neurokognitiven Outcomes nach kranialer Bestrahlung nicht mittels Strahlentherapie behandelbar ist. Eine Chemotherapie wäre grundsätzlich möglich (trotzdem hätte der Patient eine sehr schlechte Prognose unter $10 \%$ ), wurde aber wegen der vermut- lich schwerwiegenden bleibenden neurologischen Einschränkungen in Absprache mit den Eltern nicht durchgeführt. Am 10. Lebenstag ist der Patient an zentralen Atemversagen verstorben.

Schlussfolgerung: Rhabdoidtumore sind maligne Erkrankungen, die mit einem Altersgipfel zwischen der Geburt und dem 3. Lebensjahr auftreten und trotz multimodaler, intensiver Therapieansätze (Operation, Strahlentherapie, Chemotherapie) eine Gesamtüberleben von $40-50 \%$ zeigen. Aufgrund des einfachen Genoms dieser Erkrankung (ausschließlich SMARCB1 und SMARCA4 Mutationen) war es in dem hier vorgestellten Patienten möglich, die Diagnose durch molekulare Untersuchungen der Keimbahn zu stellen und bei diesem schwer kranken Patienten eine invasive Diagnostik zu vermeiden.

\section{P092}

\section{Neonatale Purpura fulminans}

B. Bubl', K. Hagemann Gysling ${ }^{2}$, M. Ragazzi ${ }^{3}$, M. Nelle ${ }^{3}$, F. Dallèves ${ }^{4}$, S. Bigi ${ }^{5}$

${ }^{1}$ Medizinische Kinderklinik/Inselspital, Bern, Schweiz, ${ }^{2}$ Universitätsklinik für Kinderheilkunde/Inselspital Bern, Hämatologie, Bern, Schweiz, ${ }^{3}$ Universitätsklinik für Kinderheilkunde/Inselspital Bern, Neonatologie, Bern, Schweiz, ${ }^{4}$ Universitätsklinik für Kinderheilkunde/Inselspital Bern, Humangenetik, Bern, Schweiz, ${ }^{5}$ Universitätsklinik für Kinderheilkunde/ Inselspital Bern, Neuropädiatrie, Bern, Schweiz

In diesem Fall stellen wir einen reifgeborenen Jungen vor mit neonataler Purpura fulminans. Pränatal wurde in der 28. SSW ein bilateraler Hydrozephalus entdeckt. Das intrauterine MRI bestätigte diesen Befund und zeigte eine Grad IV-Blutung beidseits mit Erweiterung der Seitenventrikel und des 3. Ventrikels. Die Mutter hatte während der Schwangerschaft keine Medikamente genommen und keine Infektionen gehabt. Aufgrund der bekannten Hirnblutung wurde in der 39+0 SSW eine elektive primäre Sectio durchgeführt. Postnatal war die Anpassung unproblematisch. Wenige Stunden nach Geburt entwickelte das Neugeborene eine Purpura fulminans am Gesäss und Oberschenkel beidseits, sowie am Unterschenkel links. Die Thrombozytenzahl nach Geburt war physiologisch. Jedoch war die Gerinnung auffällig (Quick 34\% und einer pTT 54.5s). In der erweiterten Gerinnungsdiagnostik ergab sich ein Protein-C-Mangel. Anschliessend an die notfallmässigen Plasma-Gaben (FFP) postnatal, wurde die intravenöse Protein-C-Substitution begonnen. Hierunter nahm die Purpura nicht mehr zu und heilte ab. Die Gaben von Protein-C (PC) wurden durch Bergund Talspiegeln gesteuert und bei $70 \mathrm{U} / \mathrm{kgKG} / 6 \mathrm{~h}$ zeigte sich eine gutes klinisches Ansprechen. Bei zu niedrigen Spiegeln ( $<30 \%$ apTT-Methode) kam es bei dem Kind zu klinischen Verschlechterungen wie Schwellung des Beins und Verschlechterung der Beatmungssituation. Nach erneutem Erreichen von angestrebten Spiegeln unter korrigierten PC-Dosen bildete sich diese Klinik schnell wieder zurück. Am wahrscheinlichsten kamen diese Verschlechterungen durch Mikrothromben zustande. Passend dazu war passager das D-Dimer in dieser Phase erhöht. Die genetische Untersuchung zeigte einen kombiniert-heterozygoten Protein-C-Mangel. In der augenärztlichen Untersuchung zeigte sich eine bilaterale PC-bedingte Glaskörper-und Retinablutung. Schädelsonographisch bestätigte sich der posthämorrhagische Hydrozephalus internus nach Hirnblutung (IVH), welcher sich im Verlauf nicht verschlechterte und nicht interventionsbedürftig wurde. Neurologisch war der Junge postnatal durch Hypertonie und Unruhe auffällig. Das Mehrkanal-EEG und das amplitudenintegrierte EEG waren unauffällig. Nachdem die anspruchsvolle Umstellung der intravenösen PC-Gaben auf subkutan erfolgreich umgesetzt werden konnte, konnte das Kind zwei Monate postnatal nach Hause entlassen werden. Zusammengefasst hatte das Neugeborene, mit der typischen Trias von postthrombotischer Hirnblutung, Purpura fulminans und retinale Blutungen, einen kombiniert-heterozygoten Protein-C-Mangel. Unter zunächst intravenöser und später subkutaner Substitution konnte die Hyperkoagulabilität beendet werden und die Purpura abheilen. Die retinalen Blutungen jedoch führten zur Blindheit. Der neurologische Verlauf ist aufgrund der zerebralen Blutungen noch ungewiss. 
P093

Management und Komplikationen von Nabelvenenkathetern auf der Neugeborenenintensivstation - Eine retrospektive Analyse über 15 Jahre

\section{S. Poralla', S. Bagci', C. Schreiner' ${ }^{1}$ A. Müller ${ }^{1}$ \\ 'Universitätsklinik Bonn Zentrum für Kinderheilkunde, Neonatologie, Bonn, Deutschland}

Hintergrund: NVK gehören seit Jahrzehnten zu den etablierten Gefäßzugangsmöglichkeiten in der Neonatologie. Als optimale Position wird in der Regel die Lage der Katheterspitze in der VCI am Übergang in den rechten Vorhof beurteilt. Wichtige Komplikation stellen Katheter-assoziierte Infektionen und Thrombosen dar. Daneben können Leberzellschäden, Perikarderguss/Perikardtamponaden oder Gefäßperforationen in Folge von NVKs auftreten.

\section{Fragestellung:}

1. Welche Patienten erhielten einen NVK?

2. In wie vielen Fällen konnte eine korrekte Lage des NVK bei der Insertion erzielt werden?

3. Welche Komplikationen traten im untersuchten Patientenkollektiv auf?

Material und Methoden: In einer retrospektiven Analyse wurden alle zwischen dem 1.1.1998 und dem 31.12.2012 geborenen Patienten der Abteilung für Neonatologie der Universitätskinderklinik Bonn untersucht, die während ihres Aufenthaltes in der neonatologischen Abteilung einen NVK erhalten hatten oder aus zuweisenden Kliniken mit liegendem NVK übernommen worden waren.

Ergebnisse: Die untersuchte Kohorte umfasste 936 Patienten.

Nach initialer Katheranlage konnte bei $71 \%$ der Gesamtkohorte eine zentrale Position der NVK-Katheterspitze dokumentiert werden. Im untersuchten Kollektiv wurden bei 27,5\% $(n=257)$ der Patienten klinische Septi-tiden, bei 43,5\% ( $n=407)$ laborchemische Septitiden diagnostiziert $12,8 \%(n=120)$ entwickelten eine Cholestase, bei $2 \%(n=19)$ fand sich ein NVK-assozi-ierter Thrombus bzw. eine Embolie, jeweils 0,2\% $(n=2)$ erlitten eine Hb-relevante Blutung oder eine Perforation durch den NVK. Im weiteren wurde der Einfluss der Katheterliegedauer auf die oben genannten Komplikationen untersucht. Dabei zeigte sich kein signifikanter Zusammenhang zwischen NVK-Liegedauer und einer NVK-assoziierten Blutung oder einer NVK-assoziierten Perforation. Für die Entwicklung einer Sepsis (sowohl nach klinischen Kriterien als auch nach laborchemischen), einer Cholestase, eines Thrombus oder einer Embolie konnte jedoch ein signifikanter Einfluss der NVK-Liegedauer nachgewiesen werden. Demnach war die Liegedauer des NVK bis zum Auftreten einer Sepsis, Cholestase, Thrombus oder Embolie signifikant länger als bei $\mathrm{Pa}$ tienten ohne eine dieser Komplikationen.

Diskussion: In der hier durchgeführten Analyse erhielten 936 Patienten einen NVK, wobei mit 36,2\% $(n=339)$ der größte Anteil der Patienten FG mit einem Geburtsgewicht $<1000 \mathrm{~g}$ waren. Die Katheterfehllage kann mit z. T. lebensbedrohlichen Komplikationen wie Gefäßruptur oder Perikardtamponade assoziiert sein. Eine zeitnahe Lagekontrolle und eine ggf. nötige Lagekorrektur sind unabdingbar. Bei $71 \%$ der Patienten konnte initial eine zentrale Katheterlage erzielt werden, jedoch nur bei 38,9\% eine

\begin{tabular}{|c|c|c|c|c|}
\hline & $\begin{array}{l}\text { Reif- } \\
\text { geborene } \\
(n=191)\end{array}$ & $\begin{array}{l}\text { FG }>1500 \mathrm{~g} \\
(n=269)\end{array}$ & $\begin{array}{l}\text { FG 1000- } \\
1499 \mathrm{~g} \\
(n=137)\end{array}$ & $\begin{array}{l}\text { FG }<1000 \mathrm{~g} \\
(n=339)\end{array}$ \\
\hline $\begin{array}{l}\text { Gestationsal- } \\
\text { ter [SSW] }\end{array}$ & $\begin{array}{l}38 \\
(37-42) \\
\end{array}$ & $\begin{array}{l}34 \\
(27-37) \\
\end{array}$ & $\begin{array}{l}29 \\
(26-36) \\
\end{array}$ & $\begin{array}{l}26 \\
(22-32) \\
\end{array}$ \\
\hline $\begin{array}{l}\text { Geburtsge- } \\
\text { wicht [g] }\end{array}$ & $\begin{array}{l}3150 \\
(1710-5300)\end{array}$ & $\begin{array}{l}2170 \\
(1500-4670)\end{array}$ & $\begin{array}{l}1210 \\
(1000-1495)\end{array}$ & $\begin{array}{l}694 \\
(257-995) \\
\end{array}$ \\
\hline NVK-Tage & $\begin{array}{l}3 \\
(1-15)\end{array}$ & $\begin{array}{l}4 \\
(1-27)\end{array}$ & $\begin{array}{l}6 \\
(1-17)\end{array}$ & $\begin{array}{l}6 \\
(1-25)\end{array}$ \\
\hline
\end{tabular}

korrekte zentrale Lage. Es zeigte sich ein signifikanter Zusammenhang zwischen längerer Katheterliegedauer und den potentiell Katheterassoziierten Komplikationen Sepsis, Cholestase, Thrombus oder Embolie. Es zeigte sich kein signifikanter Zusammenhang zwischen Katheterlage und dem Auftreten von Komplikationen.

\section{P094}

Schwere Form eines Adams-Oliver-Syndroms mit letalem Ausgang

I. Pilz-Klement ${ }^{1}$, E. Harps' ${ }^{1}$, S. Horsch ${ }^{1}$

'Helios Berlin Buch, Neonatologie, Berlin, Deutschland

Hintergrund: Das Adams-Oliver-Syndrom (AOS) ist ein seltenes Krankheitsbild mit angeborenen Haut- und Knochendefekten, Gliedmaßenfehlbildungen und Cutis marmorata teleangiectatica. Die wenigen beschriebenen Fälle zeigen eine variable Ausprägung und Prognose. Wir berichten von einem besonders schweren Fall mit letalem Ausgang. Falldarstellung: Bei dem Neugeborenen wurde ab der 30.Schwangerschaftswoche (SSW) eine schwere intrauterine Wachstumsretardierung diagnostiziert. In der fetalen Sonographie und MRT zeigte sich ein Mikrozephalus mit Verdacht auf Corpus callosum Dysgenesie und Zehenfehlbildungen. In 38+1 SSW wurde die Indikation zur Sectio gestellt. Postnatal zeigte sich der unerwartete Befund einer frontomedianen Encephalozele links, eines cephalen Knochendefekts rechts, einer ausgeprägte Cutis marmorata teleangiectatica mit ektatischen Venenkonvoluten am gesamten Integument, eine Aplasia cutis über einzelnen Hautpartien und fehlende/dysplastische Zehen sowie eine Panzytopenie und eine Hypothyreose. Im cMRT zeigte sich außer der Enzephalozele links ein strukturell normales Gehirn. Am 2. Lebenstag (LT) wurde die Enzephalozele neurochirurgisch versorgt und die intraoperativ festgestellte Kranznahtsynostose eröffnet. Jedoch wurden bei den schwierigen Hautverhältnissen und rezidivierenden Duradefekten mit Liquorleckage und Zelenrezidiv 5 weitere neurochirurgische Operationen notwendig, die zu keiner suffizienten Deckung der Defekte führten. Aus den ektatischen Venen kam es zweimal zu massiven Blutungen, woraufhin diese teils legiert und sklerosiert wurden. Im Alter von 6 Wochen zeigte sich erstmals ein hypokontraktiles Myokard bei mäßiger pulmonaler Hypertonie, die im Verlauf rasch zunahm. Ab dem 53.LT wurde bei schwerer pulmonaler Hypertension eine Therapie mit inhalativem NO begonnen, die nur kurzzeitige Besserung brachte. Aufgrund der infausten Prognose wurde im Konsens mit den Eltern von einer weiteren Eskalation der Intensivtherapie abgesehen und das Mädchen verstarb am 61. LT. Als Ursache der massiven pulmonalen Hypertonie und des kardialen Versagens sind am ehesten massive/multiple Lungenembolien anzusehen.

Diskussion: Die klinischen Merkmale des AOS mit Aplasia cutis, Cutis marmorata teleangiectatica, Ossifikationsstörung des Schädels, Syn- und Brachydaktylie der Finger/Zehen, führten in unserem Fall zur Diagnose. Vergleichend zu den in der Literatur beschriebenen Fällen handelte es sich in unserem Fall um eine besonders schwere Form des AOS. Der Verlauf wurde durch die schwierige operative Versorgung der Enzephalozelen, massive Blutungen aus den Hautvenen und rezidivierende Lungenembolien aus den ektatischen z.T. thrombosierten Venen mit daraus resultierender pulmonaler Hypertonie, kompliziert. Das in der Literatur in vielen Fällen beschriebene relativ günstige Outcome rechtfertigt den kurativen Therapieansatz, jedoch führte dieser trotz multidisziplinärer Versorgung unserer Patientin nicht zum Erfolg. 


\section{Postersitzung 10: Perinatale Infektionen}

\section{P095 \\ Nachuntersuchung von Kindern $>2$ Jahren mit einer angeborenen Infektion durch das humane Zytomegalievirus (HCMV)}

\author{
H. Buxmann', B. Schiza', M. Enders², R. Schlößer ${ }^{7}$ \\ 'Universitätsklinikum Frankfurt, Klinik für Kinder- und Jugendmedizin, \\ Schwerpunkt Neonatologie, Frankfurt am Main, Deutschland, '²abor Enders, \\ Stuttgart, Deutschland
}

Hintergrund: Das humane Zytomegalievirus (HCMV) ist die häufigste Ursache von angeborenen Infektionen. Eine antivirale Therapie mit Ganciclovir oder Valganciclovir erhalten vorwiegend symptomatisch infizierte Neugeborene mit ZNS-Beteiligung. Beide Medikamente sind für Neugeborene nicht zugelassen und mögliche unerwünschte Wirkungen im Langzeitverlauf sind in den Fachinformationen enthalten.

Zielsetzung: Ziel der Studie ist die im Verlauf nach angeborener HCMV-Infektion neu auftretenden Symptome herauszuarbeiten und nach Möglichkeit der Infektion oder der antiviralen Therapie zuzuordnen.

Material und Methoden: Retrospektive Pilotstudie von Kindern $>2$ Jahren mit angeborener HCMV-Infektion, bei denen nach Einverständnis der Eltern die anamnestischen und medizinischen Daten der Kinder, namentlich Arztberichte, Laborbefunde, Befunde apparativer Untersuchungen, Einträge ins Vorsorgeuntersuchungsheft und Impfpass systematisch analysiert werden.

Ergebnisse: Bisher wurden 54 Kinder (2-15 Jahre; Median 5 J.) mit konnataler HCMV-Infektion in die Studie eingeschlossen. Hiervon hatten 32 Kinder Symptome in der Neonatalphase und 22 waren asymptomatisch. Von den 32 symptomatischen Kindern wurden 22 Kinder mit Ganciclovir und/oder Valganciclovir behandelt, 10 erhielten keine antivirale Therapie Im Alter von > 2 Jahren (2-15 Jahre; Median 5 J.) hatten 20 Kindern neu aufgetretene Symptome: je 13 motorische und/oder kognitive Entwicklungs-verzögerungen, 5 Sprachstörungen, 4 Epilepsien, 2 Tetraspatiken, 2 Hörstörungen, 1 Mikrozephalie und 1 insulinpflichtiger Diabetes mellitus. Ein schwer mehrfachbeeinträchtigtes Kind verstarb mit 7 Jahren. 12 Kinder mit in der neonatalen Phase beschriebenen Symptomen zeigten im Alter von 2 Jahren keine Auffälligkeiten mehr. Von den 22 neonatal asymptomatischen Kindern (2-15 Jahre; Median 6 J.) wurde eines aufgrund einer hohen Viruslast im Blut und Urin mit Ganciclovir und Valganciclovir therapiert. Im Alter von > 2 Jahren waren 19 Kinder weiterhin asymptomatisch. Bei 3 initial asymptomatischen, nicht therapierten Kindern wurden im Verlauf Symptome detektiert: 3 Hörstörungen, 1 motorische und kognitive Entwicklungsverzögerung.

Diskussion: In der untersuchten Kohorte fand sich eine heterogene Indikationsstellung zur antiviralen Therapie der angeborenen HCMV-Infektion. Die jenseits der Neugeborenen-Periode neu dokumentierten Symptome sind zahlreich, zeigen eine Dominanz von Entwicklungsstörungen und sind als mögliche Folge einer HCMV-Infektion beschrieben. Bei keinem der Kinder wurden bisher Symptome detektiert, die primär einer unerwünschten Langzeitwirkung der antiviralen Therapie zuzuordnen wären. Kritisch anzumerken ist der retrospektive Ansatz der Studie und die für die Fragestellung relativ geringe Fallzahl.

Schlussfolgerung: Unsere retrospektive Studie zeigt inhomogene Indikationsstellungen der antiviralen Therapie bei Kindern mit angeborener HCMV-Infektionen, eine Dominanz von Entwicklungsstörungen als Gesundheitsproblem im klinischen Verlauf der Kinder und bisher keine Hinweise auf unerwünschte Langzeitwirkungen der antiviralen Therapie. Eine prospektive Untersuchung dieser wichtigen Zielparameter z. B. im Rahmen eines Registers für angeborene HCMV-Infektionen erscheint dringend angezeigt.

\section{P096 \\ Verminderte Bildung von Neutrophil extracellular traps bei Neugeborenen}

P. Lipp' ', J. Ruhnau' ${ }^{2}$, A. Lange' ', A. Vogelgesang ${ }^{2}$, A. Dressel' ${ }^{2}$, M. Heckmann ${ }^{1}$ 'Klinik und Poliklinik für Kinder und Jugendmedizin, Abtl. Neonatologie u. Päd. Intensivmedizin, Greifswald, Deutschland, ${ }^{2}$ Klinik und Poliklinik für Neurologie, Abtl. Neuroimmunologie, Greifswald, Deutschland

Hintergrund: Neonatale Infektionen sind mit einer erhöhten Mortalität und teilweise mit schweren neurologischen Langzeitschäden assoziiert. Die Häufigkeit und Schwere korrelieren negativ mit dem Gestationsalter, da die Immunabwehr insbesondere bei Frühgeborenen durch eine reduzierte Funktion der neutrophilen Granulozyten beeinträchtigt ist. Als Teil der neutrophilen Abwehr ist die Bildung von „Neutrophil extracellular traps" (NETs) als Strukturen aus Chromatin und antibakteriellen Proteinen zum Binden und Abtöten von Pathogenen bekannt, jedoch bei Neugeborenen bisher nicht näher untersucht.

Fragestellung: Ziel dieser prospektiven Beobachtungsstudie war der Vergleich der NET-Bildung zwischen Neugeborenen und gesunden Erwachsenen. Der Einfluss von Gestationsalter, Geburtsgewicht und -modus, Geschlecht und konnataler Infektionen auf die NET-Bildung wurde innerhalb der Gruppe der Neugeborenen untersucht.

Material und Methoden: Aus Nabelschnurblut von 9 unreifen $(\leq 37$ Wochen) und 59 reifen Neugeborenen ( $>37$ Wochen) und venösem Blut einer gesunden, adulten Kontrollgruppe $(n=18)$ wurden die neutrophilen Granulozyten mittels Dichtegradienten-Zentrifugation isoliert und die Bildung von NETs durch drei verschiedene Stimulanzien (PMA, fMLP, LPS) induziert. Die NETs wurden immunhistochemisch dargestellt und hinsichtlich Anzahl (Verhältnis zu Gesamt-Neutrophilen in \%) und Größe analysiert. Für die Studie lagen die Zustimmung der Ethikkommission und die Einwilligung der Eltern vor.

Ergebnisse: Reife, gesunde Neugeborene wiesen im Vergleich zur adulten Kontrollgruppe eine eingeschränkte NET-Bildung nach Stimulation mit PMA auf (Angaben als MW (SD), t-Test): Anzahl neonatal 14,8\% $(3,12)$ vs. adult $25,63 \%(3,97) ; p<0,0001$ und Fläche neonatal $588,46 \mu \mathrm{m}^{2}$ $(101,32)$ vs. adult $651,85 \mu \mathrm{m}^{2}(51,78) ; p=0,0166$. Dies wurde auch nach Stimulation mit fMLP und LPS beobachtet. Die NET-Bildung war bei Frühgeborenen $(35 \pm 1,9$ SSW) im Vergleich zu Reifgeborenen $(39 \pm 2,5)$ vermindert (unreif $11,98 \%(2,17)$ vs. reif $15,25 \%(3,01) ; p<0,0001)$. In der Gruppe der reifen Neugeborenen zeigten das Geschlecht, der Geburtsmodus und das Vorliegen einer Infektion keinen signifikanten Effekt auf die NET-Bildung (univariate Analyse).

Schlussfolgerung: Die verminderte NET-Bildung bei reifen Neugeborenen im Vergleich zu Erwachsenen beschreibt einen neuen Aspekt der verminderten neonatalen Immunabwehr. Schon mäßige Unreife scheint die NET-Bildung weiter zu reduzieren. Weitere Untersuchungen der NET-Bildung bei sehr unreif geborenen Kindern mit ihrem deutlich erhöhten Risiko für perinatale Infektionen sind daher dringend erforderlich.

\section{P097}

Enterovirusinfektion eines Frühgeborenen über die Muttermilch

J. Franzel', H. Sabir', O. Adams², E. Mayatepek', T. Hoehn'

${ }^{\prime} M e d$. Einricht. d. Universität Kinderklinik, Neonatologie und pädiatrische Intensivmedizin, Düsseldorf, Deutschland, ${ }^{2}$ Med. Einricht. d. Universität, Institut für Virologie, Düsseldorf, Deutschland, ${ }^{3}$ Med. Einricht. d. Universität Kinderklinik, Klinik für Allgemeine Pädiatrie, Neonatologie und Kinderkardiologie, Düsseldorf, Deutschland

Einleitung: Enteroviren sind weltweit verbreitet und können unterschiedlichste Krankheitsbilder verursachen. In der Neonatalperiode spielen sie eine wichtige Rolle, da Enterovirusinfektionen in dieser Zeit besonders schwer verlaufen können. Die Übertragung erfolgt vertikal oder fäkal-oral. Muttermilch als Infektionsquelle wurde bisher nur in einem Einzelfall beschrieben.

Fallbericht: Wir berichten den Fall eines FG von $32+0$ SSW, welches per primärer Sectio bei schwerer Enterovirusinfektion der Mutter entbunden 
wurde. Die Schwangerschaft verlief zunächst unauffällig. Nach $31+5$ SSW erfolgte die stationäre Aufnahme bei Verdacht auf Meningitis der Mutter. Bei rasch verschlechternder Klinik mit respiratorischer Insuffizienz wurde die Indikation zur Sectio gestellt.

Sowohl im Liquor der Mutter als auch im Analabstrich wurde Echovirus des Serotyps 30 nachgewiesen.

Klinischer Verlauf: Postnatal präsentierte sich das Kind mäßig deprimiert. Apgar 5/6/7. Es erfolgte die Anlage einer CPAP Atemhilfe, worunter sich der Patient kardiorespiratorisch stabilisierte. Mit dem Nahrungsaufbau wurde bereits am 1. Lebenstag (LT) begonnen. Am 4. LT entwickelte das Kind klinische Zeichen einer Infektion mit vermehrten Apnoe-Bradykardien, Zentralisation und Nahrungsunverträglichkeit. Laborchemisch zeigte sich ein CRP Anstieg auf 1,5 mg/dl und eine Thrombopenie von 18.000/ $\mu \mathrm{l}$, weshalb eine antibiotische Therapie mit Cefotaxim und Gentamicin begonnen wurde. Der Patient stabilisierte sich langsam im Verlauf und wies nach 4 Tagen weder klinische noch laborchemische Hinweise einer Infektion auf. Die abgenommene Blutkultur blieb steril. In der PCR vom Rachen- und Analabstrich waren ab dem 5. LT Echoviren des Typs 30 nachweisbar. Daraufhin wurde auch die Muttermilch untersucht, in der sich am 6. LT eine max. Kopienzahl von 8 Mill./ml feststellen ließ. Zu diesem Zeitpunkt waren im matenalen Rachenabstrich bereits keine Kopien, im maternalen Analabstrich lediglich moderate Kopienzahlen nachweisbar. Fazit: Bisher war nur eine vertikal plazentare und noskomiale Übertragung von Enteroviren bekannt. Muttermilch als mögliche Infektionsquelle ist in einem einzigen Fall beschrieben worden. Auf Grund der extrem hohen, nachgewiesenen Kopienzahlen scheint Muttermilch eine wichtige potentielle Infektionsquelle zu sein.

\section{P098 \\ Konnatale kutane Candidiasis (3C-syndrome) - ein seltenes aber klinisch relevantes Krankheitsbild bei Frühgeborenen}

A. Werlein', K. Härtel', C. Knöppel', M. Zemlin', K. Schoner' ${ }^{2}$, M. Kerwat ${ }^{3}$, S. Köhler', R. Maier ${ }^{7}$

'Universitätsklinikum Marburg, Klinik für Kinder- und Jugendmedizin, Marburg, Deutschland, ${ }^{2}$ Philipps Universität Marburg, Institut für Pathologie, Marburg, Deutschland, ${ }^{3}$ nstitut für Mikrobiologie und Krankenhaushygiene, Marburg, Deutschland, ${ }^{4}$ Klinik für Frauenheilkunde, Marburg, Deutschland

Hintergrund: Candida-Infektionen können bei Neugeborenen vertikal oder horizontal übertragen werden. Während die horizontale, d.h. nosokomiale Übertragung auf neonatologischen Intensivstationen zunehmend an Bedeutung gewinnt, ist die vertikale Übertragung selten. Eine Sonderform stellt die konnatale kutane Candidiasis (englisch: congenital cutaneous candidiasis $=3 \mathrm{C}$-syndrome) dar. In der Literatur sind bisher weniger als 100 Fälle beschrieben. Besonders gefährdet sind Frühgeborene, wobei eine Candida-Chorioamnionitis dann wahrscheinlich Auslöser der Frühgeburt ist. Als Risikofaktoren gelten ein vorzeitiger Blasensprung sowie Fremdmaterial in den Geburtswegen. Fallbericht: Schwangerschaft: Unauffällig, kein vorzeitiger Blasensprung. Im präpartalen Vaginalabstrich kein Candida-Nachweis. Geburt: Sectio caesarea mit 31+0 SSW bei Verdacht auf Amnioninfektionssyndrom mit klinischen (Fieber) und laborchemischen (CRP 53,6 mg/l, Leukozyten 10,000/ $\mathrm{ll}$ ) Entzündungszeichen bei der Mutter sowie Tachykardie beim Feten. Geburtsgewicht 1850 g. Nabelarterien-pH 7,35, Apgar-Score 7/7/9 Punkte. Intubation und Surfactant-Gabe bei insuffizienter Eigenatmung. Hautbefund bei Geburt: Generalisiertes makulo-papulöses Exanthem und weißliche miliare Pusteln auf der Nabelschnur. Laborbefunde: Leukozyten 39,200/ $\mu$ l, CRP 20,3 mg/l, Interleukin-8 114 ng/l. Mikrobiologie: Candida albicans auf der Haut und im Trachealaspirat beim Kind sowie im Uterus-Abstrich bei der Mutter. Blutkultur beim Kind steril. Plazentabefund: Eitrig-nekrotisierende Candida-Chorioamnnionitis und Candida-Funikulitis mit Nachweis von Candida-Myzelien. Behandlung: Fluconazol i.v. für 7 Tage. Verlauf: Schuppiges Abheilen der Hautläsionen innerhalb von 1 Woche. Extubation im Alter von 2 Tagen, nCPAP-Atemunterstützung für weitere 5 Tage. Weiterer Verlauf unkompliziert.
Diskussion: Der hier dargestellte Fall ist typisch für die sehr seltene konnatale kutane Candidiasis. Insbesondere bei Frühgeborenen und nach vorzeitigem Blasensprung sollte bei makulo-papulösen Hauteffloreszenzen, die schon bei Geburt bestehen und Hand- und Fußsohlen sowie die Nabelschnur einbeziehen, dieses Krankheitsbild differentialdiagnostisch in Betracht gezogen und gezielt nach Candida gesucht werden. Da systemische Ausbreitung und letale Verläufe beschrieben sind, wird eine intravenöse antimykotische Behandlung empfohlen.

\section{P099}

Messung von Aktivität und Expression des NLRP3-, NLRC4 und AIM2-Inflammasoms in einem optimierten Vollblut Stimulations-Assay

\section{S. Winkler' ', L. Grinstein ${ }^{2}$, H. Luksch ${ }^{2}$, A. Rösen-Wolff ${ }^{2}$}

'Universitätsklinikum Carl Gustav Carus Kinderklinik, Dresden, Deutschland, 2Universitätsklinikum Carl Gustav Carus Kinderklinik, Dresden, Deutschland

Hintergrund: Caspase-1 ist ein zentrales pro-inflammatorisches Enzym, das durch die Prozessierung von Interleukin (IL)-1ß und IL-18 Inflammation vermittelt. Caspase-1 wird durch „Inflammasom“ genannte Multiprotein-Komplexe aktiviert und spielt eine wichtige Rolle im Rahmen der angeborenen Immunabwehr. Weiterhin ist die Aktivierung des Inflammasom/Caspase-1 Signalwegs eng mit der Pathogenese der bronchopulmonalen Dysplasie sowie der hypoxisch-ischämischen Enzephalopathie verknüpft. Die Expression und Aktivierbarkeit der verschiedenen Inflammasome ist bei Frühgeborenen und reifen Neonaten bislang nicht untersucht.

Fragestellung: Etablierung eines Vollblut Stimulations-Assays, der die Messung der spezifischen Inflammasom-Aktivierung sowie die Expressionsanalyse NLRP3-, AIM2- und NLRC4-Inflammasom-relevanter Gene aus minimalen Blutvolumina ermöglicht.

Material und Methoden: Stimulation von Vollblut mit LPS/ATP, S. typhimurium oder LPS/polydA:dT (Aktivierung von NLRP3, NLRC4 oder AIM2). Messung von IL-1ß im Vollblut-Überstand als Surrogatparameter der Caspase-1 Aktivierung. Die Inflammasom-Spezifität wurde durch die Verwendung des NLRP3 Inhibitors MCC950 gewährleistet. Die Genexpressionsanalyse Inflammasom-relevanter Gene wurde aus den Leukozyten der jeweiligen Proben realisiert.

Ergebnisse: Die Verwendung des optimierten Vollblut Stimulations-Assays ermöglicht die Messung von IL-1ß sowie der Genexpression von IL1B, NLRP3, NLRC4, AIM2, ASC und CASP1 nach spezifischer Aktivierung des NLRP3-, NLRC4- oder AIM2-Inflammasoms. Das erforderliche Probenvolumen beträgt dabei $140 \mu \mathrm{l}$ pro Probe. Bereits die Voraktivierung der Zellen mit LPS induzierte im Vergleich zur Kontrolle eine NLRP3-abhängige IL-1ß Sekretion, welche durch zusätzliche Stimulation mit ATP weiter gesteigert werden konnte. Die Sekretion von IL-1ß nach Stimulation mit S. typhimurium war abhängig von den Inflammasomen NLRP3 und NLRC4. In der Frühphase der Stimulation wurden die Zellen durch S. typhimurium voraktiviert und sekretierten IL- 1 B durch das konstitutiv aktive NLRP3 Inflammasom. Im weiteren Verlauf war die IL-1ß Sekretion nahezu vollständig abhängig vom NLRC4-Inflammasom. Die Transfektion von polydA:dT mit Hilfe von Lipofectamine 2000 führte zu einer Aktivierung des AIM2-Inflammasoms. Im Rahmen von WesternBlot-Untersuchungen der Caspase-1 Aktivierung zeigte sich, dass die Messung von IL- $1 ß$ aus dem Überstand einen korrekten Surrogatparameter für die Caspase-1 Aktivierung darstellt.

Diskussion: Der etablierte Vollblut Stimulations-Assay ermöglicht die spezifische Untersuchung der Aktivierung von Caspase-1 durch die Inflammasome NLRP3, NLRC4 und AIM2. Aufgrund des geringen Probenvolumens eignet sich der Assay zur Charakterisierung der Inflammasom-Aktivität bei Neugeborenen. Der Assay lässt sich dabei aus Nabelschnurblut realisieren. 
P100

\section{Postnataler B-Zellmangel bei einem hypotrophen Frühgeborenen nach maternaler Chemotherapie}

\author{
S. Poralla' , T. Dresbach ${ }^{1}$, A. Müller ${ }^{1}$ \\ 'Zentrum für Kinderheilkunde am Universitätsklinikum Bonn/, \\ Neonatologie, Bonn, Deutschland
}

Hintergrund: R-CHOP (Rituximab, Cyclophosphamid, Hydroxydaunorubicin, Vincristin, Prednison) ist eine etablierte Chemotherapie bei der Behandlung maligner Lymphome. Über die Auswirkungen einer solchen Therapie während der Schwangerschaft ist bisher nur wenig bekannt, insbesondere über die Auswirkungen für das Neugeborene. Fallbericht: Hypotrophes weibliches Frühgeborenes (Geburtsgewicht 2100 g, 5.P), Sectio mit 36 + 2 SSW bei maternalem follikulärem Non-Hodgkin-Lymphom der rechten Mamma und Z.n. 4 Zyklen R-CHOP ab der 23.SSW. IUGR Verlauf: Nach unauffälliger postnataler Anpassung war die Patientin während des 11 Tage dauernden Aufenthaltes allzeit kardiorespiratorisch stabil. Der orale Nahrungsaufbau gelang problemlos, das neurologische Verhalten war altersentsprechend bei wiederholt unauffälligem Ultraschall des Gehirns. In der postnatalen Kontrolle des Immunstatus zeigte sich eine ausgeprägte Suppression der B-Zellen und der IgM-Antikörper. Bei im Verlauf der zweiten Lebenswoche rückläufigen IgG-Titern und weiterhin deutlich supprimierter B-Zellen-Zahl erfolgte an Lebenstag 11 die erste intravenöse Immunglobulin-Gabe. Es erfolgten wöchentliche Kontrollen des Immunstatus und 4 Wochen nach der ersten Immunglobulin-Gabe bei wieder abgefallenem IgG-Spiegel und unverändert supprimiertem B-Zell-Status die zweite intravenöse Immunglobulin-Gabe. Im weiteren Verlauf waren keine erneuten Immunglobulin-Gaben notwendig. Im Alter von 4 Monaten zeigten sich erstmals normalisierte B-Zell-Zahlen, im Alter von 7 Monaten schließlich auch ein adäquater IgM-Spiegel. Zur Beurteilung der kognitiven und motorischen Entwicklung wurde die Patientin im Alter von korrigiert 6 Monaten erstmals mittels Bayley III untersucht mit altersentsprechendem Ergebnis.

Zusammenfassung: Das in der Therapie des maternalen Non-Hodgkin-Lymphoms eingesetzte Rituximab führte beim Neugeborenen zu einer über 4 Monate andauernden Suppression der B-Zellen-Produktion mit anschließender vollständiger Erholung. Bisher zeigte sich in der Beurteilung der psychomotorischen Entwicklung des Kindes kein Anhalt für eine Entwicklungsverzögerung.

\section{P101 \\ Infektion mit Picornaviren in der Neonatologie - eine bisher unterschätzte Gefahr?}

\section{Richter', C. Morfeld ${ }^{2}$, F. Guthmann ${ }^{1}$, J. Richter ${ }^{1}$}

'Perinatalzentrum Hannover, Auf der Bult-Kinder- und Jugendkrankenhaus, Neonatologie, Hannover, Deutschland, ${ }^{2}$ Perinatalzentrum Hannover, Diakovere Henriettenstift, Geburtshilfe, Hannover, Deutschland

Hintergrund: Infektionen mit Picornaviren sind als Verursacher von Erkältungssymptomen und gastrointestinalen Beschwerden bei Kindern und Erwachsene verbreitet. In einzelnen Fallbeschreibungen wurde ein Sepsis-ähnliches Krankheitsbild bei Früh- und Neugeborenen beschrieben. In den vergangenen Monaten haben wir drei Fälle tödlich verlaufender Infektionen mit Picornaviren bei späten Frühgeborenen (32. bis 36. SSW) beobachtet. Bei zwei der Frühgeborenen ist eine diaplazentare Übertragung wahrscheinlich.

Fallbeschreibungen: Fall 1: 33jährige II-Grav., II-Para, HBsAg neg, Aufnahme mit $33+0$ SSW bei septischem Krankheitsbild mit Fieber, CRP-Erhöhung und rechtsseitigen abdominellen Beschwerden. Appendektomie mit $33+1$ SSW, intraoperativ keine akute Appendizitis, histologisch leicht chronische, nicht floride Entzündung. Am selben Tag primäre Sectio bei schlechtem AZ der Mutter und path. CTG. Männliches Frühgeborenes, Gewicht 2250 g, APGAR 5/7/8, Na-pH 7,33, bakt. Abstriche steril, einmalig Surfactant unter erhaltener Spontanatmung bei Atemnotsyndrom, empirische i.v. antibiotische Therapie über drei Tage. Am siebten Lebenstag Bild einer fulminanten Sepsis mit Laktat-Azidose, CRP-Erhöhung, Anämie, Thrombope- nie und disseminiert intravasalen Gerinnung (DIC). Klinisch Ileussymptomatik mit Aszites und Hepatomegalie. Im Stuhl Nachweis von Picornaviren (PCR). Unter antibiotischer Therapie bei Verdacht einer nekrotisierenden Enterocolitis sowie Gaben von Blutprodukten zunächst Stabilisierung. Am 10. Lebenstag erneut krisenhafte Verschlechterung mit Leberparenchymnekrosen und Nebennierenblutung linksseitig, renalem Nierenversagen, Hämolyse (Hb 3,4 g/dl), Thrombopenie sowie einer globalen Koagulopathie. Differentialdiagnostisch kamen eine neonatale Hämochromatose (hohes Ferritin) sowie eine lymphophagozytäre Histiozytose (hoher Spiegel von löslichem CD-25) in Betracht. Austauschtransfusion sowie mehrfache Gaben von Immunglobulinen erbrachten jedoch keine Befundverbesserung. Nach Abwägung einer nicht vertretbaren Therapieoption von Hämodialyse und Lebertransplantation Entschluss zur palliativen Therapie und Versterben am 15. Lebenstag. Postmortem Virussequenzierung mit Nachweis von Echovirus 11. Fall 2: Zwillings-Frühgeborenes der 36. SSW, am 3. Lebenstag fulminante nekrotisierende Enterocolitis, Hämolyse und DIC. Versterben am 4. Lebenstag. Nachweis von Picornavirus-RNA im Stuhl. Mutter hatte gastroenteritische Beschwerden. Fall 3: Ehem. Frühgeborenes der 32. SSW, nach 6 Wochen Bronchitis, Nachweis von Picornaviren im Rachensekret. Nach einwöchigem Infektionszeitraum Versterben im Rahmen einer akuten Myokarditis, Hämolyse und DIC.

Diskussion und Schlussfolgerung: Drei nachgewiesene Fälle einer letal verlaufenden Picornavirusinfektion innerhalb von 6 Monaten legen den Verdacht einer hohen Dunkelziffer von viralen Infektionen bei septischen Verläufen in der Neonatalperiode nahe.

Die letalen Symptome bezogen sich jeweils auf das Versagen eines Organsystems (1. Leber, 2. Darm, 3. Herz) kombiniert mit Hämolyse und DIC. Einer frühen Diagnosesicherung könnten gezielt sonographische und laborchemische Kontrollen folgen. Eine sofortige Isolation auf einer NICU bzw. geburtshilflichen Station sollte erfolgen, da Ausbrüche (Griechenland, USA) beschrieben worden sind, und das Virus sehr resistent gegen Desinfektionsmittel ist und eine lange Überlebensdauer sowie Inkubationszeit zeigt.

\section{P102}

\section{Late-presenting HIV-Infizierte in der Schwangerschaft -} führen diese zu einer vermehrten Mutter-Kind-Transmission?

K. Singer', I. Alba-Alejandre², G. Notheis ${ }^{3}$, O. Genzel-Boroviczény'

'Dr. von Haunersches Kinderspital, Ludwig-Maximilians-Universität München, Neonatologie Campus Innenstadt, München, Deutschland, ${ }^{2}$ Ludwig-Maximilians-Universität München, Gynäkologie und Geburtshilfe Campus Innenstadt, München, Deutschland, ${ }^{3}$ Dr. von Haunersches Kinderspital, Ludwig-Maximilians-Universität München, Immunologie, München, Deutschland

Hintergrund: Aufgrund des frühen Screenings und der frühzeitigen Behandlung von HIV-Infektionen in der Schwangerschaft sind Mutter-Kind-Transmissionen in Deutschland selten. Im Jahr 2014 wurden 25 Patienten vertikal infiziert, von denen $16 \mathrm{im}$ Ausland geboren wurden. Die steigende Anzahl an Flüchtlingen erschwert die Schwangerschaftsvorsorge zunehmend. Es stellen sich vermehrt nicht-behandelte HIV-Infizierte nach 28 Schwangerschaftswochen, sogenannte „late-presenting“, vor.

Methoden/Ergebnisse: Von 2010 bis 2012 wurde bei nur einer HIV-infizierten Schwangeren, bei der die Entbindung in unserem Krankenhaus stattfand, eine antiretrovirale Therapie nach 28 Schwangerschaftswochen begonnen. Von 2013 bis 2015 wurden allein in unserem Krankenhaus 10 Kinder von late-presenting HIV-infizierten Müttern geboren (13\% aller HIV-infizierten Schwangeren). Neun der 10 Mütter waren Flüchtlinge aus Subsahara-Afrika. In einem Fall trat eine Mutter-Kind-Transmission auf. Diese Mutter stellte sich zwei Wochen nach Einreise erstmals mit 35 Schwangerschaftswochen vor. Es wurden eine HIV-Infektion mit einer Viruslast von 58.607 Kopien/ml und eine latente Syphilis unbekannter Dauer diagnostiziert. Die Schwangere wurde mit Raltegravir, Tenofovir und Emtricitabin oral und Benzathin-Benzylpenicillin intramuskulär therapiert. Zum Zeitpunkt der Geburt war die Viruslast der Mutter supprimiert. Das Neugeborene zeigte eine HIV-Infektion durch intrauterine Transmission 
mit einer maximalen Viruslast von $150.000 \mathrm{Kopien} / \mathrm{ml}$. Es erfolgte eine Therapie mit Abacavir, Lamivudin, Zidovudin und Nevirapin oral. Eine konnatale Syphilisinfektion wurde nicht nachgewiesen.

Diskussion: Mit zunehmender Anzahl an Flüchtlingen betreuen wir vermehrt late-presenting HIV-infizierte Schwangere. Das Risiko einer Mutter-Kind-Transmission steigt in den letzten Wochen der Schwangerschaft sowie bei bestimmten Koinfektionen wie beispielsweise Syphilis. Eine möglichst frühe Schwangerschaftsvorsorge ist notwendig, um eine Übertragung zu verhindern.

\section{P103}

\section{Konnatale Zytomegalievirusinfektion - ein Fallbericht}

N. Alvarez Tapia', C. Leistner', S. Kelzon', C. Fremerey', M. Ehlen ${ }^{2}$

${ }^{1}$ Asklepios Klinik, Neonatologie \& Pädiatrische Intensivmedizin, Sankt Augustin, Deutschland, ${ }^{2}$ Marienhaus, Klinikum Bendorf-NeuwiedWaldbreitbach St. Elizabeth, Kinderheilkunde, Neuwied, Deutschland

Einleitung: Eine Infektion mit Zytomegalieviren (CMV) stellt die häufigste konnatale Infektionsursache dar und betrifft 0,2-2\% aller Neugeborenen. Die Transmissionsrate steigt mit der Schwangerschaftsdauer, der symptomatische Ausprägungsgrad sinkt jedoch mit zunehmendem Gestationsalter. Klinisch treten intrazerebrale Verkalkungen, Mikrozephalie, Chorioretinitis, intrauterine Wachstumsretardierung, Hepatosplenomegalie mit Transaminasenerhöhung und Thrombozytopenie, Ikterus, Pneumonie, sowie Spätschäden wie neurosensorische Hörstörungen und globale Entwicklungsretardierung auf. Wir berichten über ein Frühgeborenes von $34+5$ Schwangerschaftswochen mit dem Vollbild einer konnatalen CMV-Infektion. Der eindrucksvolle Hautbefund und die sonographischen Veränderungen werden in Bildern dargestellt.

Kasuistik: Unmittelbar postnatal zeigten sich bei unserem Patienten generalisierte Petechien, eine ausgeprägte Panzytopenie, eine beidseitige intraventrikuläre Blutung ersten Grades, eine Vaskulitis der thalamostriatalen Gefäße sowie eine Cholangitis mit Ikterus praecox und Cholestase. Aufgrund der schweren Thrombozytopenie (6/nl) und Anämie (Hb 9,2 g/dl, Hk 29\%) erfolgten eine unverzügliche Thrombozyten- und Erythrozytentransfusion und der Beginn einer antibiotischen Therapie bei differentialdiagnostischem Sepsisverdacht. Nach Nachweis der konnatalen CMV-Infektion mittels Polymerasekettenreaktion (PCR) (3840 Kopien/ml) und Immunglobulinbestimmung wurde nach Aufklärung der Eltern am 7. Lebenstag eine orale Therapie mit Valganciclovir unter engmaschiger Kontrolle von Viruslast und Medikamentenspiegel begonnen. Aufgrund einer am ehesten als Medikamentennebenwirkung auftretenden schweren Neutropenie erfolgte eine wiederholte Gabe von Granulozyten-Kolonie-stimulierendem Faktor (G-CSF). Das Kind konnte am 77. Lebenstag unter laufender Therapie nach Hause entlassen werden, nach sechs Monaten wurde die Therapie geplant beendet. Aktuell entwickeln sich Seh- und Hörvermögen unauffällig, jedoch bestehen eine Rumpfhypotonie sowie eine Hypertonie mit Koordinationsstörung der rechten oberen Extremität. Krampfanfälle sind bis dato nicht aufgetreten. Diskussion: Therapieindikationen einer konnatalen CMV-Infektion sind die symptomatische Infektion und eine zerebrale Mitbeteiligung. Therapieziel ist eine Verbesserung der neurologischen Prognose. Eine thalamostriatale Vaskulopathie gilt hierbei als Hochrisikomarker für einen späteren Hörverlust. Die Entscheidung zur primären oralen Therapie mit der Pro-Drug Valganciclovir an Stelle des intravenös zu verabreichenden Nukleosidanalogons Ganciclovir erfolgte aufgrund der gleichwertigen Bioverfügbarkeit, des wegfallenden Risikos einer Katheterinfektion und eines beschriebenen reduzierten Neutropenierisikos. Die sechsmonatige Therapie wurde gegenüber einer sechswöchigen Behandlung aufgrund der Ergebnisse einer aktuellen randomisiert-kontrollierten Studie bevorzugt, welche signifikant verbesserte Langzeitergebnisse hinsichtlich des Hörvermögens sowie eine günstigere neurologische Entwicklung in der sechsmonatigen Therapiegruppe zeigte [1]. Das Risiko einer schweren Neutropenie als Nebenwirkung des Virostatikums ist unter Überwachung beherrschbar und spricht daher nicht grundsätzlich gegen eine längere Therapiedauer.

\section{Literatur}

1. Kimberlin, Valganciclovir for Sympt. Congenital CMV Disease, N engl J Med 2015

\section{P104}

\section{Congenitale cutane Candidiasis beim Frühgeborenen}

\section{Papastergiou' ', H. Schwalm' ', M. Friepörtner', G. Selzer ${ }^{2}$}

'Evangelisches Krankenhaus Hamm, Klinik für Neonatologie und pädiatrische Intensivmedizin, Hamm, Deutschland, ${ }^{2}$ Evangelisches Krankenhaus Hamm gGmbh, Chefarzt Klinik für Neonatologie und pädiatrische Intensivmedizin, Hamm, Deutschland

Die kongenitale Candidiasis ist eine seltene Erkrankung, die mit einer hohen Morbidität und Mortilität bei Frühgenborenen unter 1000 Gramm einhergeht. Bei Reifgeborenen ist die Prognose deutlich besser, oft nur auf die kutanen Läsionen beschränkt. Es wurden bis zum Jahr 1986 nur 81 Fälle berichtet, bis 2015 wurden 95 Fälle publiziert; davon 8 Fälle von Frühgeborenen unter $1000 \mathrm{~g}$. Trotz häufiger mütterlicher Vaginalbesiedlung mit Candida albicans bis zu $30 \%$ kommt es selten zu kindlichen Infektionen. Die klinische Manifestationen haben ein weites Spektrum von lokalen Läsionen-Infiltrationen der Haut bis zu fulminanten septischen systemischen Infektionen mit Multiorganversagen. Therapie der Wahl bei candida albicans ist Amphotericin B. Kombinationen mit Flucytosine können bei ZNS Befall erwogen werden. Wir präsentieren den Fall eines Frühgeborenes von 26 1/7 Schwangerschaftswochen (Geburtsgewicht 960 Gramm). Bereits innerhalb der ersten Lebensstunden zeigte sich eine infiltrierte verdickte Haut, so dass bei Verdacht auf cutane Candidiasis eine Therapie mit liposomalen Amphotericin $B$ begonnen wurde. In dem kurzen Verlauf entwickelten sich trotz frühzeitiger und nachgewiesener suffizienter Behandlung ausgeprägte Hautläsionen sowie eine Pneumonie.Radiologisch und sonografisch zeigte sich eine Multiorgan- Beteiligung im Sinne einer Candida albicans Sepsis mit pneumonischen Infiltraten, erhöhter Nierenechcogenität und erhöhter Echogenität des Hirnparenchyms mit Verdickung des Plexus, sowie Candida albicans Nachweis in Blut,Trachealsekret, Haut und Rektalabstrichen und im Urin. Laborchemisch zeigte sich das Bild einer Sepsis mit Anämie, Thrombopenie,Laktatazidose und disseminierter intravasaler Koagulopathie. Multiorganversagen und Tod folgten am 5. Lebestag. Risiko Faktoren bei der Mutter war ein liegendes intrauterin Pessar, im vaginal Abstrich waren vereinzelt Candida albicans und physiologische Keimflora nachgewiesen. Die Plazenta Histologie zeigte eine eitrige Amnionitis und Omphalovaskulitis. Es werden die Fotodokumente der Haut und der Sonografien im Verlauf präsentiert.

\section{P105}

Retrospektive Analyse der Differentialdiagnose einer konnatalen Infektion mit dem humanen Zytomegalievirus (HCMV) bei klinisch symptomatischen Säuglingen

\section{N. Lüsebrink', M. Kieslich', R. Schlößer ${ }^{2}$, H. Buxmann²}

'Klinikum der J.W. Goethe Universität, Klinik für Kinder- und Jugendmedizin, Neurologie, Neurometabolik, und Prävention, Frankfurt am Main, Deutschland, ${ }^{2}$ Klinikum der J.W. Goethe Universität, Klinik für Kinder- und Jugendmedizin, Neonatologie, Frankfurt am Main, Deutschland

Einleitung: Hörstörungen, Entwicklungsverzögerung oder Epilepsien sind mögliche Langzeitfolgen konnataler HCMV(cHCMV)-Infektionen. Bei rechtzeitiger Diagnose stehen Therapien zur Verfügung. Der HCMV-DNA Nachweis in einer Neugeborenen-Screeningkarte (NGS-Karte) erlaubt die retrospektive Diagnose einer cHCMV-Infektion bei Säuglingen älter als drei Wochen. Ein HCMV-Nachweis jenseits von drei Lebenswochen kann Folge einer postnatal erworben HCMV(pHCMV)-Infektion sein, die sich essentiell von cHCMV-Infektionen unterscheidet.

Fragestellungen: Wie häufig wurde die Differentialdiagnose einer cHCMV-Infektion bei Säuglingen älter als drei Lebenswochen mit klinischer Symptomatik abgeklärt?

Patienten und Methoden: Retrospektive Analyse der Krankenakten aus 8 Jahren von allgemeinpädiatrischen Patienten im Alter von drei Wochen bis 12 Monaten, die mögliche Symptome einer cHCMV-Infektion aufwiesen. Die Patienten wurden mittels Stichwortsuche (mind. eines von 20 typischen Symptomen, wie z. B. Entwicklungsverzögerung, Mikrozephalie, Hörstörung, etc.) in der elektronischen Datenbank unserer Klinik detektiert und ihre Krankenakten zur Datenerhebung gesichtet. 
Ergebnisse: Es wurden 83 Säuglinge in die Analyse eingeschlossen. Eine cHCMV-Infektion wurde in 61/83, 73\% der Fälle in Betracht gezogen und virologische Diagnostik initiiert. Bei 28/61, 46 \% der Fälle war die HCMV-Serologie (IgG und IgM) negativ und somit eine cHCMV-Infektion unwahrscheinlich. In 5/61, $8 \%$ der Fälle wurde eine partielle HCMV-Diagnostik (z. B. HCMV-PCR aus Liquor) durchgeführt, die keine Hinweise auf eine cHCMV-Infektion erbrachte, diese aber auch nicht ausschloss. Bei weiteren 28/61, 46\% der Kinder war die HCMV-Serologie positiv (mind. IgG) und somit sowohl vereinbar mit einer $\mathrm{CHCMV}$-, wie auch einer $\mathrm{pH}$ CMV-Infektion. In 22/28, 79\% dieser Fälle wurde die NGS-Karte der Kinder mittels HCMV-PCR untersucht. Bei 13/22, $59 \%$ der Kinder war diese negativ. In 6/22, $27 \%$ der Fälle war die HCMV-PCR positiv und bestätigte eine cHCMV-Infektion. Drei Kinder erhielten eine antivirale Therapie. Bei 3/22, 14\% der Fälle war die NGS-Karte bereits vernichtet. Bei 22 Säuglingen der Gesamtkohorte (22/83, 27\%) wurde eine cHCMV-Infektion nicht abgeklärt.

Diskussion: Bei den meisten Säuglingen $>3$ Wochen mit Symptomen passend zu einer cHCMV-Infektion wurde diese als Ursache in Betracht gezogen. Eine gezielte Diagnostik aber nur bei 50/61, 82\% dieser Fälle konsequent verfolgt. Bei stringenter Diagnostik konnte für 41/50, $82 \%$ dieser Kinder eine cHCMV-Infektion als Ursache der Symptome weitgehend ausgeschlossen werden, bei 3/50, 6\% der Kinder konnte die Frage nach einer cHCMV-Infektion wegen bereits vernichteter NGS-Karten nicht geklärt werden. Bei 6/50, 12\% der Kinder wurde eine cHCMV-Infektion als Ursache der Symptome nachgewiesen, drei Kinder wurden antiviral therapiert. Die NGS-Karte ist oft die einzige Chance bei Säuglingen $>3$ Wochen eine cHCMV-Infektion noch diagnostizieren zu können. Schlussfolgerung: Bei Säuglingen mit Symptomen passend zu einer cHCMV-Infektion wird häufig an diese Virusinfektion gedacht. Die relevante Frage nach Vorliegen einer cHCMV- oder pHCMV-Infektion wird zum Teil nicht stringent verfolgt oder bleibt infolge vernichteter NGS-Karten offen.

\section{P106 \\ Akutes neonatales Leberversagen als Folge einer fulminanten Enterovirusinfektion - eine Fallbeschreibung}

H. Schneider ${ }^{1}$, H. Fuchs ${ }^{2}$

${ }^{1}$ Universitäts - Kinderklinik, Neonatologie und Pädiatrische Intensivmedizin, Klinik I, Freiburg, Deutschland, ${ }^{2}$ Universitäts - Kinderklinik, Freiburg, Deutschland

Einleitung: Das akute Leberversagen ist eine seltene aber schwerwiegende Erkrankung von Säuglingen, die ohne Lebertransplantation mit einer Mortalität von bis zu 70 \% einhergeht. Neben der neonatalen Hämochromatose und metababolischen Erkrankungen gehören Infektionen mit hepatotropen Viren zu den häufigsten Ursachen des akuten Leberversagens im Neugeborenenalter. Neonaten sind besonders anfällig für Enterovirusinfektionen, die häufig als schwerwiegende Sepsis, mit Kreislaufinsuffizienz, Koagulopathie, Ikterus und Multiorganversagen, verlaufen.

Kasuistik: Wir beschreiben den Fall eines Jungen der in der 36+3/7. SSW mit einem Geburtsgewicht von 2555 g (P. 20) als 4. Kind einer Viertgravida, per primärer Re-Re-Sectio, geboren wurde. Die postnatale Adaptation war unauffällig. Mutter und Kind konnten am 6. Lebenstag aus der Geburtsklinik entlassen werden. Einen Tag später wurde der Patient aufgrund einer Allgemeinzustandsverschlechterung mit Lethargie, Hypothermie, Trinkverweigerung und blutiger Diarrhoe in der pädiatrischen Notaufnahme vorstellig. Laborchemisch führend war eine ausgeprägte

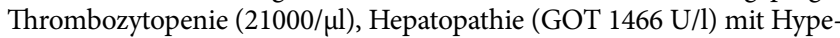
rammonämie $(209 \mu \mathrm{mol} / \mathrm{l})$ und Koagulopathie (Quick $27 \%$; PTT $85 \mathrm{~s}$ ) ohne relevante Cholestase. Desweiteren war eine deutliche Ferritinerhöhung (30240 ng/ml) bei nur unwesentlich erhöhtem CRP (23 mg/l) sowie eine Laktatazidose auffällig. Eine Tyrosinämie und Paracetamol-Intoxikation wurden ausgeschlossen. Das Vorliegen einer Hämophagozytischen Lymphohistiozytose war bei normwertigen Triglyceriden sowie fehlender Splenomegalie eher unwahrscheinlich. Bei Verdacht auf eine neonatale Hämochromatose erfolgte die 2-malige Gabe von $1 \mathrm{~g} / \mathrm{kg}$ Immunglobulin intravenös. Von einer Blutausstauschtransfusion wurde bei Nachweis von
Echovirus 11-RNA, im Serum und Stuhl des Säuglings, Abstand genommen. Die weitere Therapie war rein supportiv - es erfolgten wiederholte Transfusionen von Erythrozyten- und Thrombozytenkonzentraten sowie FFP und Fibrinogen. Vitamin K wurde intravenös substituiert. Außerdem erfolgte eine empirische antibiotische Therapie sowie passager parenterale Ernährung.

Hinweise auf eine begleitende Myokarditis gab es weder klinisch noch echokardiografisch. Außer einer Nebennierenblutung traten keine schwerwiegenden Komplikationen der Koagulopathie auf. Die Nierenfunktion war im Rahmen der Sepsis nicht beeinträchtigt. Unter der symptomatischen Therapie stabilisierte sich der Säugling ab dem 9. Krankheitstag zunehmend. Bei Entlassung in der 4. Lebenswoche bestand lediglich noch eine direkte Hyperbilirubinämie ( $4 \mathrm{mg} / \mathrm{dl})$. In den ambulanten Verlaufskontrollen wurden 2,5 Monate nach akuter Erkrankung sonografisch in der Leber multiple punktförmige Echogenitätserhöhungen nachgewiesen. Die Leberfunktionsparameter (inklusive Cholestaseparameter) waren unauffällig.

Diskussion: Enterovirusinfektionen (z. B. mit Echovirus 11) sind wichtige Differentialdiagnosen des neonatalen Leberversagens und können insbesondere bei jungen Säuglingen zu schweren systemischen Infektionen mit hoher Mortalität führen. Zu den Hauptkomplikationen dieser Erkrankung zählen schwere Hepatopathie mit Enzephalopathie und Koagulopathie, Myokarditis, sekundäre bakterielle Infektionen und akutes Nierenversagen. Die Therapie ist rein supportiv. Es gibt Hinweise darauf, dass hohe Dosen von intravenösen Immunglobulinen die Prognose verbessern können.

\section{Postersitzung 11: Varia II}

\section{P107}

Laserakupunktur an Neugeborenen mit Neonatalem

Abstinenzsyndrom (NAS) - retrospektive Datenanalyse erfasster Finnegan-Scores und Vergleich der Ergebnisse zwischen akupunktierten und nicht-akupunktierten Neugeborenen

\section{Boeckelmann', W. Raith', T. Freidl', A. Avian', B. Urlesberger'}

'Universitätsklinik für Kinder- und Jugenheilkunde, Klinische Abteilung für Neonatologie, Graz, Österreich, ${ }^{2}$ Medizinische Universität Graz, Medizinische Informatik und Statistik, Graz, Österreich

Hintergrund: Die Anzahl von Neugeborenen (NG) mit NAS ist weltweit steigend. Ergebnisse der wenigen Studien bzw. Fallberichte über die Wirksamkeit von Akupunktur bei NG mit NAS sind kontrovers. Schwarz et al. konnte keine positiven Effekte von Ohrakupressur bei NG mit NAS aufzeigen. Filippelli et al. zeigte durch retrospektive Datenanalyse einen beruhigenden Effekt auf NG und eine bessere Nahrungsaufnahme durch Anwendung nicht-invasiver Akupunktur. Auch Raith et al. dokumentiert in Fallberichten positive Effekte bezüglich Schlaf und Kalorienaufnahme der NG, welche Laserakupunktur als unterstützende Therapie erhielten. Die Pilotstudie von Raith et al. (2015) zeigt Tendenzen auf, dass NG mit NAS von Laserakupunktur, als zusätzliche Therapie zur pharmakologischen Behandlung, profitieren. Weltweit wird der Finnegan-Score zumeist zur Einschätzung der Notwendigkeit, zur Dosierung und zur Bewertung des Ansprechens einer Substitutionstherapie beim Säugling verwendet. Der Finnegan-Score selbst besteht aus Symptomgruppen (neurologische, gastrointestinale, respiratorische und vegetative Symptome), für die Einzelpunkte vergeben werden.

Fragestellung: Das Ziel dieser Arbeit war es herauszufinden, ob eine kombinierte Laserakupunktur an Ohr und Körper einzelne Symptome des NAS modifiziert und sich in den Finnegan-Scores der Symptomgruppen wiederspiegelt.

Material: Es handelt sich um eine retrospektive Datenanalyse aller NG mit Neonatalem Abstinenzsyndrom (NAS), die an einer prospektiven, randomisierten, kontrollierten, observer-geblindeten, single-center Studie teilgenommen haben (Raith et al 2015). Beide Gruppen erhielten 
eine standardisierte pharmakologische Basistherapie (orale Morphinlösung $0,04 \%$ und Phenobarbital für 10 Tage), zusätzlich erhielten die NG der Interventionsgruppe (IG) eine Laserakupunktur (LABpen MED 10 $(675 \mathrm{~nm} / 10 \mathrm{~mW}))$ an Ohr- und Körperakupunkturpunkten. Eingeschlossen wurden NG mit NAS ausgelöst durch maternale Substitutionstherapie mittels Opioide an der Klinischen Abteilung für Neonatologie der Universitätsklinik für Kinder- und Jugendheilkunde Graz. Bei jedem NG (IG und KG) wurde der Finnegan-Score dreimal täglich während der Dauer der medikamentösen Therapie erhoben.

Methoden: Die Finnegan-Scores (über 2500 Einzelscores) der eingeschlossenen NG wurden entsprechend ihrer Symptomgruppen analysiert. Die Daten wurden deskriptiv miteinander verglichen.

Ergebnisse: Zwischen IG und KG gab es keinen signifikanten Unterschied bezüglich des höchst gemessenen Finnegan-Scores (15 in der IG vs. 16 in der KG). Dieser maximale Wert wurde in beiden Gruppen am Tag 4 gemessen. Die deskriptive Datenanalyse wies auf mögliche positive Effekte in der IG hinsichtlich des Verlaufs der Einzelsymptome Tremor und Cutis marmorata hin. Der Median der Finnegan-Scores aller Symptome in der IG lagen meist innerhalb des Interquartilsbereich der KG und umgekehrt. Schlussfolgerung: Obwohl sich eine statistisch signifikante Reduzierung der Dauer der Morphintherapie sowie in der Länge des stationären Aufenthaltes zeigte, konnte kein Unterschied im Verlauf einzelner Symptome bzw. Symptomgruppen zwischen IG und KG gefunden werden.

\section{P108}

\section{Kinder drogenabhängiger Mütter - Daten aus dem Ruhrgebiet}

J. Bialas', M. Gesser', B. Hüning', U. Schara², T. Brüning ${ }^{3}$, A. Backendorf, D. Michna ${ }^{4}$, C. Roll ${ }^{3}$, U. Felderhoff-Müser ${ }^{1}$

'Universitätskinderklinik Essen, Kinderklinik I, Neonatologie, Essen, Deutschland, ${ }^{2}$ Universitätskinderklinik, Kinderklinik I Neuropädiatrie, Essen, Deutschland, ${ }^{3}$ Vestische Kinder- und Jugendklinik Datteln, Universität Witten/Herdecke, Datteln, Deutschland, ${ }^{4}$ Elisabeth Krankenhaus Essen; Klinik für Neu - und Frühgeborene, Essen, Deutschland

Hintergrund: Illegaler Drogenkonsum ist ein weltweites Problem. Es wird für Deutschland eine Zahl von 30-40.000 Kindern opiatabhängiger Eltern angenommen. Der Opiatabusus in der Schwangerschaft betraf 2011 in den USA 1,1\% aller schwangeren Frauen. Verschiedene Studien zeigen bei exponierten Kindern im Vorschulalter Defizite im Bereich der Aufmerksamkeit und Sprachentwicklung. Hinzu kommen körperliche und seelische Belastungen im familiären Umfeld während der Kindheit und ein hohes Risiko selbst Drogen zu konsumieren. 51 \% der betroffenen Mütter und $18 \%$ der betroffenen Väter leben mit Ihren Kindern zusammen. Für Deutschland gibt es keine verlässlichen populationsbasierten Daten zu diesem Thema.

Fragestellung: Ziel dieser Studie war eine Erfassung von Kindern mit intrauteriner Drogenexposition und die möglichst genaue Charakterisierung dieses Kollektives im Ruhrgebiet.

Material/Methoden: Retrospektive Analyse der Fälle intrauteriner Drogenexposition (2000-2014) in drei Perinatalzentren. Erfassung medizinischer, sozioökonomischer und psychosozialer Daten aus den Akten des primären stationären Aufenthaltes sowie aus der Nachsorge in Sozialpädiatrischen Zentren (SPZ) oder Spezialambulanzen. Es handelt sich um eine deskriptive Datenanalyse. Ergebnisse: Insgesamt wurden 297 Kinder erfasst, von denen 204 eine Entzugssymptomatik zeigten. 79 Kinder waren jünger als 37 SSW (26,6\%). In Substitutionsprogrammen hatten 47,8\% der Mütter Methadon erhalten, 11,2\% Polamidon und 9,6\% Buprenorphin. $52,7 \%$ hatten trotz Substitution einen Beikonsum. In der Polamidongruppe war die Beikonsumrate am höchsten, gefolgt von der Methadongruppe. Die mittlere stationäre Aufenthaltsdauer der Neugeborenen betrug 30 Tage. Bei Methadonsubstitution mit Beikonsum zeigte sich eine im Mittel 18 Tage längere Entzugsdauer im Vergleich zu Methadonexposition allein $(p=0,007)$. Während des stationären Aufenthaltes stieg die soziale Vernetzung und Anbindung an das Jugendamt von 21,5\% (Kontakte prästationär) auf 89,9\% (Kontakte vor Entlassung des Kindes). 74,1\% der Kinder wurden in die Herkunftsfamilie entlassen. Die aus zwei Kliniken erfasste
Nachsorgequote im SPZ und Spezialambulanzen lag im 1. Lebensjahr bei $27,3 \%$, im 2. bei $12,9 \%$, im 5 . bei $5,4 \%$ und nach dem 5 . Lebensjahr nur noch bei 3,3\%. Diskussion: Die Suchterkrankung und die schwierigen sozialen Verhältnisse erschweren die Betreuung intrauterin drogenexponierter Kinder durch ihre Eltern. Durch eine zunehmende gute Vernetzung und Verbesserung der Kommunikation zwischen den involvierten Institutionen muss die langfristige Betreuung dieser Risikokinder verbessert werden. Prospektive Studien sind gefordert, um die Situation in Deutschland $\mathrm{zu}$ analysieren und die Erarbeitung nachhaltiger Konzepte zur Unterstützung und Förderung der betroffenen Familien zu ermöglichen.

\section{P109}

Steigender Konsum von Crystal in Sachsen und dessen Risiken für Schwangere und Neugeborene - Erfahrungen eines Perinatalzentrums Level I: Versorgung der Kinder

J. Dinger' , N. Näther' , H. Menz' ', N. Zöllner' ' D. Konstantelos' , M. Rüdiger ${ }^{1}$ 'Universitätsklinikum Carl Gustav Carus an der TU Dresden, Kinderklinik, Neonatologie und Pädiatrische Intensivmedizin, Dresden, Deutschland

Einleitung: Seit Jahren bereitet den Geburtshelfern und Neonatologen in Sachsen eine stetige Zunahme von Neugeborenen, deren Mütter während der Schwangerschaft illegale Drogen konsumieren, große Sorgen. War vor 10 Jahren unser Augenmerk noch auf die Opiate gerichtet, zeichnet sich seit dieser Zeit eine beunruhigende Veränderung ab. Besorgniserregend ist die Zunahme von Neugeborenen in Sachsen, deren Mütter während der Schwangerschaft die Droge Crystal konsumieren. Allein während der letzten 7 Jahre ist auf der Grundlage der Daten der Sächsischen Neonatalerhebung ein Anstieg von 0,6 auf 7 pro 1000 Neugeborene zu verzeichnen. Fragestellung: Wie ist der Gesundheitszustand Neugeborener, die intrauterin der Droge Crystal Meth ausgesetzt waren, zu beurteilen?

Material: Die Krankenunterlagen von 129 Müttern und Neugeborenen, die im Zeitraum von Januar 2007 bis Dezember 2015 im Universitäts-Kinderund Frauenzentrum stationär wegen eines Crystal Meth-Konsums aufgenommen waren, wurden ausgewertet.

Ergebnisse: Die Analyse lässt erste Aussagen zu den unmittelbaren Folgen und Auswirkungen einer intrauterinen Crystal Meth-Exposition für das Neugeborene zu. Die Frühgeborenenrate ist im Vergleich zur der des Landes Sachsen deutlich erhöht (4-fach), ebenso die Rate an untergewichtigen Neugeborenen und Neugeborenen mit zu kleinem Kopfumfang (3-fach). Ein definiertes Abstinenzsyndrom, vergleichbar mit dem des Opiatentzuges, zeichnet sich gegenwärtig anhand der Symptome der Neugeborenen noch nicht ab. Die beobachteten Symptome sind äußerst heterogen und unspezifisch. Ursachen hierfür könnten Häufigkeit und Ausmaß des Crystal Meth-Konsums sowie der beobachtete, erhebliche Beikonsum der Schwangeren sein. Bei sonographischen Untersuchungen waren deutlich häufiger pathologische Befunde an lebenswichtigen Organen wie Hirn (26\%), Herz (12\%) und Nieren (9\%) zu beobachten.

Diskussion: Zunächst ist es zwingend erforderlich, Neugeborene nach Konsum der Droge Crystal Meth in der Schwangerschaft weiter - möglicherweise sogar im Rahmen eines „Crystal-Registers“ - zu erfassen und einer differenzierten Diagnostik zu unterziehen. Primäres Ziel ist dabei, die Drogen-induzierten gesundheitlichen Schädigungen rechtzeitig zu erkennen, zu systematisieren und optimale Behandlungsansätze zu definieren. Vor dem Hintergrund dieser Ergebnisse können sodann Geburtshelfer und Neonatologen für das Erkennen von Crystal Meth-induzierten Schädigungen des Kindes besser sensibilisiert werden. Ferner sollten Suchtpräventionsmaßnahmen nach dem Ansatz „Hilfestellung statt Stigmatisierung" etabliert werden. Letztlich bedarf es weiterer, Zentren-übergreifender Untersuchungen, um Behandlungsnormative abzuleiten und daraus eine Leitlinie für ein multiprofessionelles Betreuungsmodell zu erstellen. 


\section{P110}

\section{Steigender Konsum von Crystal in Sachsen und dessen Risiken für Schwangere und Neugeborene - Erfahrungen eines Perinatalzentrums Level I: Versorgung der Kindesmütter}

J. Reichert', J. Dinger ${ }^{1}$, K. Nitzsche ${ }^{2}$, H. Urban' ${ }^{2}$, U. Zimmermann ${ }^{3}$, J. Schmitt ${ }^{4}$, M. Rüdiger ${ }^{T}$

'Universitätsklinikum Carl Gustav Carus an der TU Dresden, Kinderklinik, Neonatologie/Pädiatrische Intensivmedizin, Dresden, Deutschland, ${ }^{2}$ Universitätsklinikum Carl Gustav Carus an der TU Dresden, Frauenklinik, Dresden, Deutschland, ${ }^{3}$ Universitätsklinikum Carl Gustav Carus an der TU Dresden, Klinik für Psychiatrie und Psychotherapie, Dresden, Deutschland, ${ }^{4}$ Universitätsklinikum Carl Gustav Carus an der TU Dresden, Zentrum für Evidenzbasierte Gesundheitsversorgung, Dresden, Deutschland

Hintergrund: Die Anzahl der bekannten Erstkonsumenten von Crysta Meth hat sich in Sachsen von 2004 bis 2013 von 655 auf 1254 nahezu verdoppelt. Für die Versorgungssysteme dürfte von Bedeutung sein, dass etwa $60 \%$ der Patienten im Alter von 20-30 Jahren und etwa ein Drittel weiblichen Geschlechts sind. Damit scheinen gerade junge Menschen in der Phase der Familienbildung einer besonderen Gefährdung zu unterliegen. Eine Unterstützung mit dem Ziel, die Auswirkungen des Drogenmissbrauches zu minimieren, sollte daher rechtzeitig, spätestens während der Schwangerschaft, besser noch davor beginnen.

Fragestellung: Wie erfolgt die optimale Begleitung von Frauen mit Crysta Meth-Konsum in der Schwangerschaft, deren neugeborenes Kind stationär in der Kinderklinik aufgenommen wird? Methoden: In die Analyse zur Beschreibung der Klientel wurden alle Mütter mit Crystal Meth-Konsum während der Schwangerschaft (Alter, berufliche und familiäre Situation, Vorsorgeuntersuchungen, Konsumverhalten, Therapiemotivation) eingeschlossen, deren Kinder von 2007 bis 2015 in der Kinderklinik stationär aufgenommen wurden. Eine zeitgleich zur Behandlung des Kindes erfolgende Entzugsbehandlung der Mutter wird aktuell erprobt und hinsichtlich Therapieverlauf qualitativ analysiert.

Ergebnisse: Von 129 Frauen mit Crystal Meth-Konsum in der Schwangerschaft waren etwa zwei Drittel zum Zeitpunkt der Entbindung zwischen 20 und 30 Jahren alt; hinsichtlich beruflicher und familiärer Situation fanden sich keine Auffälligkeiten. Nur 37\% der Frauen im Vergleich zu $90 \%$ für Sachsen nahmen die erste Vorsorgeuntersuchung bis zur vollendeten 12 . Schwangerschaftswoche wahr. Bei jeder fünften war bei ihrer Entbindung ein Drogenkonsum vorab bekannt. Mit einem Drogenscreening erklärten sich etwa $80 \%$ der Frauen noch im Kreißsaal einverstanden; es fand sich ein erheblicher Beikonsum (Alkohol, Cannabis, Nikotin, Psychopharmaka u. a.). Dem stand - in der Phase der Adaptation an die Mutterrolle - eine hohe Motivation für die eigene Entzugsbehandlung gegenüber. Eine erste qualitative Datenanalyse für fünf, zeitgleich tagesklinisch bis stationär in der Universitätsklinik für Psychiatrie behandelte Kindesmütter zeigte für alle positive Behandlungsverläufe, die eine unmittelbare Überleitung in kommunale Versorgungsangebote ermöglichten (z. B. Mutter-Kind-Einrichtungen).

Diskussion: Ein optimaler Versorgungspfad zur bedarfsgerechten Betreuung von Schwangeren bzw. Mutter und Kind nach Crystal Meth-Exposition sollte vorsehen, dass beide Betroffenen zeitgleich behandelt werden Sein Vorteil liegt vor allem darin, dass er den Kontakt zwischen Mutter und Kind nicht nur nicht unterbricht, sondern fordert und fördert und für die Mutter möglicherweise sogar zur „Sinnstiftung“ dieser komplexen Behandlung beiträgt.

\section{P111}

\section{Zusammenhang des General Movement Optimality Scores mit klinischen Parametern des Neonatalen Abstinenzsyndroms}

J. Stöger', A. Avian', E. Ziehenberger', B. Urlesberger', W. Raith', J. Pansy', A. Scheuchenegger

'Univ.-Klinik für Kinder- und Jugendheilkunde, Medizinische Universität Graz, Klinische Abteilung für Neonatologie, Graz, Österreich, ${ }^{2}$ Medizinische Universität Graz, Medizinische Informatik und Statistik, Graz, Österreich
Hintergrund: Das Neonatale Abstinenzsyndrom (NAS) wird bei Neugeborenen beobachtet, deren Mütter während der Schwangerschaft Drogen konsumierten, oder sich in einem Substitutionsprogramm befanden. Zur Evaluierung der postnatalen Entzugssymptomatik wird der Finnegan-Score verwendet, der klinische Symptome wie z.B. Muskeltonus, Reflexe, Schlafeigenschaften, Körpertemperatur, Schwitz- und Schreiverhalten, Niesen und Stuhlkonsistenz berücksichtigt. Die Behandlung des NAS basiert auf einem multimodalen Therapiekonzept mit pharmakologischen und nicht pharmakologischen Therapieansätzen. Als pharmakologische Basistherapie erhalten Neugeborene mit NAS an der Klinischen Abteilung für Neonatologie der Universitätsklinik für Kinder- und Jugendheilkunde Graz in den ersten Lebenstagen eine orale Phenobarbitaltherapie und zusätzlich eine $0,04 \%$ orale Morphintherapie. Die Beurteilung der „General Movements (GMs) " anhand von Videoanalysen nach Prechtl ist eine verlässliche Methode zur Beurteilung des frühen neurologischen Outcomes. Die GMs Analyse ist sowohl global als auch detailliert mit Hilfe des General Movement Optimality Scores (GMOS) möglich.

Fragestellung: Gibt es einen Zusammenhang zwischen dem GMOS als Bestandteil der GMs Analyse nach Prechtl mit klinischen Parametern des NAS (Finnegan-Score, Dosierung und Dauer der Phenobarbital- und Morphintherapie).

Methode: Im Zuge einer Beobachtungsstudie beurteilten wir die postnatalen GMs am Untersuchungstag (UT, Tag 8-12) anhand von Videoanalysen nach Prechtl von 14 Neugeborenen (7 weiblich) mit NAS global und detailliert mit dem GMOS. Zusätzlich wurden klinische Parameter wie Finnegan Score und Dosierung der Phenobarbital- und Morphintherapie am UT und die Dauer der medikamentösen Therapie erhoben.

Ergebnisse: 12 der Neugeborenen (86\%) zeigten am UT abnormale GMs (Poor Repertoire) und zwei Neugeborene zeigten normal- non optimal GMs. Der mediane GMOS lag bei 25 von 42 möglichen Punkten (IQR 22-32,125). Wir fanden keinen Zusammenhang zwischen dem GMOS und der Dosierung der Phenobarbital- und Morphintherapie am UT oder der Dauer der medikamentösen Therapie. Es wurde ein Trend gefunden, dass Neugeborene mit einem höheren GMOS einen niedrigeren Finnegan Score haben (Spearman's $r=-0,498, p=0,07$ ).

Schlussfolgerung: Ein höherer Wert des GMOS scheint mit einem niedrigeren Finnegan-Score und somit einer geringeren Entzugssymptomatik zu korrelieren. Der GMOS scheint keinen Zusammenhang mit Dauer und Höhe der Phenobarbital- und Morphintherapie zu haben.

\section{P112}

\section{Erfolgreiche Therapie eines akuten Nierenversagens} bei einem Frühgeborenen nach „,akzidenteller“ pränataler Candesartan-Exposition

\section{H.v. Osten ${ }^{1}$, K. Linnemann ${ }^{2}$, M. Heckmann ${ }^{3}$}

'Ernst-Moritz-Arndt Universität, Kinderklinik, Greifswald, Deutschland, ${ }^{2}$ Ernst-Moritz-Arndt Universität, Neonatologie und Pädiatrische Intensivmedizin, Greifswald, Deutschland, ${ }^{3}$ Klinik und Poliklinik für Kinder und Jugendmedizin, Neonatologie u. Päd. Intensivmedizin, Greifswald, Deutschland

Hintergrund: Das typische Schädigungsmuster des Feten nach pränataler ACE-Hemmer-Exposition umfasst: Oligohydramnion, teilweise mit Lungenhypoplasie und Kontrakturen, postnatal akutes Nierenversagen und arterielle Hypotension. Die Mortalität in der Neonatalperiode ist mit bis zu $18 \%$ hoch. Häufig bleibt eine chronische Niereninsuffizienz zurück. Erheblich seltener wurde bisher das ähnliche Muster bei pränataler Exposition mit Angiotensin-II-Rezeptor-Blockern beschrieben. Schwere der Symptomatik und Gesamtprognose korrelieren dabei mit dem Zeitpunkt der Exposition. Insbesondere bei Einnahme bis zum 2. oder 3. Trimenon findet sich eine deutlich schwerere neonatale Symptomatik mit einer Mortalität bis $40 \%$. Die Wahrscheinlichkeit einer vollständigen Erholung der Nierenfunktion ist ca. 50\%. Daher sind Angiotensin-II-Rezeptor-Blocker in der Schwangerschaft kontraindiziert.

Fallbericht: Wir berichten über eine akzidentelle Medikation mit Candesartan bei nicht festgestellter Schwangerschaft. Die wegen einer chroni- 
schen Glomerulonephritis dialysepflichtige Frau wurde zusätzlich zu ihrer Dauermedikation mit Xipamid, Colestilan und Bicanorm seit 14 Tagen mit Bisoprolol und Candesartan bei zunehmender Blutdruckentgleisung behandelt. Die Schwangerschaft (Schätzgewicht 1500 g, Oligohydramnion) wird zufällig bei geplanter Anlage eines Peritonealdialysekatheters entdeckt. Noch am selben Tag erfolgt die Spontangeburt bei vorzeitiger Wehentätigkeit: weibliches Frühgeborenes von klinisch etwa 33 SSW Reifealter, GG 1490 g (11. Perz.), Apgar 9/9/10, Na-pH 7,28.

Verlauf: Klinisch findet sich ein Atemnotsyndom (2 Tage CPAP mit FiO von max. 0,21), eine arterielle Hypotension mit mittleren Blutdrücken (Manschette) bis minimal $19 \mathrm{mmHg}$, die einer Therapie mit Dopamin und Noradrenalin (Dosisbereich 4,5-9 bzw. 0,2-1 $\mu \mathrm{g} / \mathrm{kg} / \mathrm{min}$ ) über 6 Tage bedurfte. Die Echokardiographie war unauffällig. Es bestand eine Anurie in den ersten 12 Lebensstunden. Das initiale Kreatinin betrug $416 \mu \mathrm{mol} / \mathrm{l}$, maximal $468 \mu \mathrm{mol} / \mathrm{l}$ am 2. Lebenstag. Nach einmaliger Verabreichung von 1,5 mg Furosemid i.v. beginnende Urin-Ausscheidung über die nächsten 12 Stunden. Ab dem 2. LT. 72stündige polyurische Phase mit bis zu $60 \mathrm{ml} /$ $\mathrm{kg} / \mathrm{h}$. Sonographisch waren die Nieren normal groß mit echoreicher Binnenstruktur. Die Urinausscheidung und Retentionsparameter normalisierten sich bis zum 7.LT. Im Follow-Up mit 9 Monaten waren die Retentionsparameter und der Blutdruck normal (Kreatinin $21 \mu \mathrm{mol} / \mathrm{l}$, Cystatin C 1,08 mg/l), eine Proteinurie lag nicht vor und sonographisch fand sich noch eine minimal angehobene Parenchymechogenität.

Schlussfolgerungen: Selbst eine kurze Exposition des Feten mit Angiotensin-II-Rezeptor-Blockern wie Candesartan kann beim Neugeborenen ein akutes Nierenversagen auslösen, dass eine intensivmedizinische Therapie erfordert. Dieser Fall bestätigt erneut, dass Angiotensin-II-Rezeptor-Blocker in der Schwangerschaft kontraindiziert sind.

\section{P113 \\ Erfolgreiche Lysetherapie bei zentraler Pulmonalembolie eines ELBW-Frühgeborenen nach 24 1/7 SSW Fallbericht}

J. Brandner', E. Hattinger-Jürgenssen' ${ }^{1}$ J. Brandner ${ }^{1}$, M. Wald ${ }^{1}$, W. Sperl ${ }^{1}$ 'Universitätsklinik für Kinderheilkunde Salzburg, Division für Neonatologie, Salzburg, Österreich

Einleitung: Eine zentrale Pulmonalembolie (PE) bei einem frühgeborenen Kind stellt ein extrem seltenes, zumindest sehr selten diagnostiziertes Krankheitsbild dar. In der Literatur sind wenige Fälle bei reifen Neugeborenen oder Late Preterms beschrieben, die chirurgisch mittels Embolektomie behandelt wurden. Eine Lysetherapie bei lebensbedrohlicher Thrombembolie wird auch bei Neugeborenen beschrieben. Thrombolytica sind allerdings bei Kindern unter der 32. SSW grundsätzlich kontraindiziert. Unseren Recherchen nach ist dies der erste dokumentierte Fall einer erfolgreich behandelnden zentralen PE bei einem ELBW-Frühgeborenen (FG).

Fallbericht: Bei einem ELBW-Mädchen (24 1/7 SSW, GG 400 g) mit bisher weitgehend stabilem Verlauf kommt es am 22. LT zu einer akuten Reanimationssituation mit deutlichem Sättigungsabfall $(<10 \%)$ und Bradykardie $(<40 / \mathrm{min})$ an der invasiven Beatmung. Nur unter Reanimationsdosen Suprarenin ist eine jeweils zeitlich begrenzte Stabilisierung möglich. Echokardiographisch zeigt sich eine massive Rechtsherz(RH)Belastung. Die Behandlung einer initial vermuteten pulmonalhypertensiven $(\mathrm{PH})$ Krise bringt keine Besserung. Nur durch unphysiologisch sehr hohe RR-Werte unter Suprarenin/Dopamin ist eine akzeptable Oxygenierung zu erreichen. In den Echo- Kontrollen fallen für eine $\mathrm{PH}$ untypische Flußmuster der Pulmonalarterien (MPA, LPA, RPA) auf. Im Verlauf gelingt die Darstellung eines großen Embolus an der Pulmonalisbifurkation, welcher die RPA und LPA fast komplett verschließt. Die LPA wird vermutlich nur über den Ductus arteriosus Botalli (DAB) versorgt. Unter Maximierung der Therapie mit extrem hohen Katecholamindosen und Prostingabe zur weiteren DAB-Öffnung kann eine Besserung des pulmonalen Durchflusses erreicht werden, eine zufriedenstellende Oxygenierung wird aber nicht erzielt. Es besteht eine anhaltend akut lebensbedrohliche Situation. Eine Behandlung der PE durch Embolektomie oder herzkathetergestützte Verkleinerung ist technisch bei dem $600 \mathrm{~g}$ schweren FG nicht möglich. Es wird die quoad vitam-Entscheidung zur Lyse-Therapie getroffen. Unter Gabe von $0,5 \mathrm{mg} / \mathrm{kg} / \mathrm{h}$ rtPA für $6 \mathrm{~h}$ ist ein Auflösen des Embolus mit rechtsventrikulärer Entlastung und normaler Perfusion der PA zu dokumentieren. Die Katecholamine können noch am selben Tag, die Beatmung 2 Tage später beendet werden. Es tritt weder eine cerebrale Blutung als akute noch eine PVL als späte Komplikation auf. Die Entwicklung des Kindes verläuft erfreulicherweise bisher altersadäquat.

Schlussfolgerung: Neugeborene haben in der pädiatrischen Population das höchste Risiko für venöse Thrombembolien. Bei unserem FG ist eine Tage zuvor aufgetretene Phlebitis in Folge eines Einschwemmkatheters am Bein als Hauptursache anzunehmen

- PE werden insbesondere bei FG wahrscheinlich unterdiagnostiziert. Bei plötzlicher Verschlechterung der Oxygenierung, akut-RDS oder unklarer hämodynamischer Instabilität sollte an eine PE gedacht werden. Der V.a. eine PE sollte besonders dann bestehen, wenn in der Echokardiographie eine akute $\mathrm{RH}$-Belastung ohne die typischen dopplerechokardiographischen Befunde einer PH besteht

- eine Lyse stellt wahrscheinlich die einzig mögliche, lebensrettende Behandlung des von einer zentralen PE vital bedrohten ELBW-FG dar, auch wenn derzeit in der Literatur hierfür noch eine Kontraindikation besteht

\section{P114}

Frühgeborenes mit atypischem hämolytisch-urämischem Syndrom (aHUS)

\section{F. Ullmann' ', A. Müller', H. Reutter', B. Hoppe ${ }^{3}$, M. Feldkötter ${ }^{3}$}

'Universitätsklinik Zentrum f. Kinderheilkunde, Neonatologie und Pädiatrische Intensivmedizin, Bonn, Deutschland, ${ }^{2}$ Zentrum für Kinderheilkunde, Universität Bonn, Neonatologie und Pädiatrische Intensivmedizin, Bonn, Deutschland, ${ }^{3}$ Universitäts-Kinderklinik, Bonn, Deutschland

Das atypische hämolytisch-urämische Syndrom (aHUS) ist eine seltene Erkrankung, die durch eine unkontrollierte Aktivierung des Komplementsystems verursacht wird. Typisch ist eine Symptomtrias aus hämolytischer Anämie, Thrombozytopenie und akutem Nierenversagen. Vielen Fällen liegt eine genetische Mutation im klassischen oder alternativen Weg des Komplementsystems zugrunde. Der Krankheitsbeginn variiert von der Neonatalperiode bis in das Erwachsenenalter, häufig wird eine Infektion als Auslöser beobachtet. Wir berichten über ein Mädchen, das als drittes Kind einer Viertgravida mit 30+4 Schwangerschaftswochen an unserem Universitätsklinikum geboren wurde. Die Schwangerschaft wurde im letzten Trimenon durch eine intrauterine Wachstumstrestriktion und ein Oligohydramnion kompliziert. Unmittelbar postnatal zeigten sich eine ausgeprägte pulmonal hypertensive Krise mit Nierenfunktionseinschränkung sowie eine schwere transfusionspflichtige Thrombozytopenie mit multiplen Hämatomen und petechialen Einblutungen. Im Verlauf waren wiederholt Transfusionen von Thrombozyten- und Erythrozytenkonzentraten aufgrund einer persistierenden Thrombozytopenie und Anämie notwendig. In der 4. und 8. Lebenswoche kam es zu einem Anstieg der Retentionsparameter und einer LDH-Erhöhung bei persistierender Thrombozytopenie. In der 3. Lebenswoche ließ sich ein Thrombus an der Mitralklappe nachweisen, in der 7. Lebenswoche Darstellung eines Thrombus im intrahepatischen Segment der V. cava inferior sowie im Konfluenz der Lebervenen. Nach Ausschluss infektiöser Ursachen, einer Heparin-induzierte Thrombozytopenie sowie einer Auto- und Alloimmunthrombozytopenie wurden Untersuchungen auf das Vorliegen eines aHUS durchgeführt: Die gängigen Komplementfaktoren, Cobalamin und Methylmalonsäure waren normwertig, Autoantikörper gegen Faktor H nicht nachweisbar. Die Diagnose atypisches HUS gelang schließlich durch den Nachweis der lokalen Aktivierung des Komplementsystems auf menschlichen mikrovaskulären Endothelzellen (HMEC-1, Noris et al, Blood 2014). Im Alter von drei Monaten begannen wir eine Therapie mit dem humanisierten monoklonalen anti-C5-Antikörper „Eculizimab“, der die Aktivierung der terminalen Komplementkaskade selektiv inhibiert. Unter wöchentlicher Infusion konnte eine Regredienz der Symptome erreicht werden. 
Der dargestellte Fall zeigt, dass sich ein aHUS bereits in der Neonatalperiode manifestieren kann. Bei entsprechender Klinik sollte dies daher differentialdiagnostisch bedacht und abgeklärt werden. Dabei zeigen ca. 30 \% der Patienten keine Auffälligkeiten im Komplementsystem und benötigen, wie in diesem Fall, weitere Tests. Molekulargenetische Untersuchungen vervollständigen die Diagnostik.

\section{P115}

\section{Faktor XIII und Routineparameter der Gerinnung VLBW Frühgeborener}

B. Ackermann' ', H. Rolle' , N. Wolf', F. Pulzer' ' U. Thome', M. Knüpfer ${ }^{1}$ 'Universitätskinderklinik, Neonatologie, Leipzig, Deutschland

Hintergrund: Normwerte für Gerinnungsparameter in VLBW Frühgeborenen sind noch unzureichend definiert. Faktor XIII als wichtiger Faktor der Thrombusstabilisierung und Modulator der Fibrinolyse kann weder über übliche extrinsische noch intrinsische Parameter erfasst werden. Eine prophylaktische Gabe von Faktor XIII an Hochrisiko-Frühgeborene scheint das Risiko einer intraventrikulären Blutung zu senken. Bisher liegen keine Referenzwerte für Faktor XIII bei VLBW Frühgeborenen vor. Methoden: Wir führten eine prospektive Beobachtungsstudie an 100 VLBW Frühgeborenen $(<1500 \mathrm{~g} ;<32 \mathrm{SSW})$ durch. Ausschlusskriterien waren schwere angeborene Fehlbildungen bzw. fehlende Einwilligung der Eltern. Direkt postnatal wurden über Nabelvenen-, Nabelarterien- oder über periphere Venenverweilkatheter Faktor XIII und Routineparameter der Gerinnung (Quick, PTT, AT3, Fibrinogen, D-Dimere) sowie ein maschinelles Differentialblutbild vor der ersten Heparingabe bestimmt.

Ergebnisse: 100 Patienten wurden eingeschlossen und hatten ein mittleres Geburtsgewicht von $921 \pm 262 \mathrm{~g}$ bei einem mittleren Gestationsalter (GA) von $192 \pm 15 \mathrm{~d}(27+3 \mathrm{SSW} \pm 2 \mathrm{SSW})$. Fünf zunächst rekrutierte Patienten wurden ausgeschlossen, da sie die Einschlusskriterien nicht erfüllten (GG>1500 g, $n=2 ; \mathrm{GA}>32+0$ SSW, $n=3)$. Die Parameter werden als Mittelwert \pm SD angegeben, zusätzliche Angabe des Median bei relevanter Streuung der Messwerte: Faktor XIII 35,4 $\pm 11,7 \%$; PTT 66,2 $\pm 17,0$ s; Quick 60,3 $\pm 16,3 \%$; AT3 25,6 $\pm 8,1 \%$; Fibrinogen 1,86 $\pm 1,3$ g/l, Median $1,34 \mathrm{~g} / \mathrm{l}(0,28-5,93)$; D-Dimere 11,2 $\pm 15,9 \mathrm{mg} / \mathrm{l}$, Median 3,95 mg/l (0,1170,8); Thrombozyten $187 \pm 71 \mathrm{gpt} / \mathrm{l}, 185$ (22-458). D-Dimer- und Fibrinogen-Werte zeigten eine ausgeprägte Streuung, kein Patient mit einem Amnioninfektionssyndrom hatte ein Fibrinogen $<1 \mathrm{~g} / \mathrm{l}(2,2 \pm 1,3 \mathrm{~g} / \mathrm{l})$.

Für Faktor XIII zeigte sich eine signifikante Korrelation mit steigendem GA (-23,0\% + 0,304.GA[d]). Äquivalent hierzu gruppierte Mittelwerte für $<26$ SSW: $29,9 \pm 10,4 \%$; 26-28 SSW: $35,3 \pm 10,3 \%$; $28-30$ : 36,9 $\pm 10,5 \%$; 30-32 SSW $46 \pm 13,8 \%$.

Schlussfolgerung: Unsere Untersuchungen zeigen die großen Abweichungen der Gerinnungstests Frühgeborener von den üblichen Normen. Faktor XIII ist ein interessanter, bislang unzureichend untersuchter Parameter in der komplexen Entwicklung des pro- und antikoagulatorischen Systems Frühgeborener. Weitere Untersuchungen sind notwendig, um unreife-bedingte Normabweichungen von therapiebedürftigen Laborwerten zu unterscheiden.

\section{P116}

Reduktion reaktiver Sauerstoffspezies bei der Aufnahme von Hämoglobin in neonatalen Makrophagen: Die Rolle des CD163-Signalweges

S. Dreschers' ${ }^{1}$, C. Platen ${ }^{2}$, B. Dennecke ${ }^{3}$, K. Ohl' ${ }^{4}$, K. Tenbrock ${ }^{2}$, T. Orlikowsky ${ }^{5}$ ${ }^{1}$ Med. Einrichtungen der RWTH Aachen, Kinderklinik Neonatologie, Köln, Deutschland, ${ }^{2}$ Med. Einrichtungen der RWTH Aachen, Kinderklinik, Aachen, Deutschland, ${ }^{3}$ Universitätsklinik Aachen, IZKF, Aachen, Deutschland, ${ }^{4}$ Kinder- und Jugendmedizin, Grundlagenforschung AG PD Dr. Tenbrock, Aachen, Deutschland, ${ }^{5}$ Universitätsklinik für Kinder- und Jugendmedizin, Sektion für Neonatologie, Aachen, Deutschland

Hintergrund: Toll-like Rezeptoren (TLR) auf Makrophagen (MF) spielen in der Vermittlung der angeborenen Immunantwort eine zentrale Rolle. Nach Bindung bakterieller Komponenten kommt es unter Aktivierung von Signalmediatoren (TIRAP, Phosphatidyl-inositol-3-Kinase (PI3K/ AKT) zur Gen-Aktivierung, die u. a. zur Sekretion des anti-inflammatorischen Zytokins IL-10 führt. Über den Scavenger-Rezeptor CD163, der die Hämoglobin-Aufnahme vermittelt, wird der oben beschriebene Signalweg ebenfalls aktiviert. Neonatale MF sind entwicklungsbedingt hohen Konzentrationen freien Hämoglobins ( $\mathrm{HbF}$ ) ausgesetzt. Dies könnte bei intrazellulärem Abbau einen Stress durch reaktive Sauerstoff-Spezies hervorrufen und zum Zelltod führen.

Hypothese: Die CD163 Expression ist bei neonatalen MF (CBMF) im Vergleich zu adulten MF (PBMF) stark reduziert. Damit wird die Aufnahme von Hämoglobin und die Aktivierung von $\mathrm{Pi} 3 \mathrm{~K} / \mathrm{AKT}$ reduziert.

Methoden: Polarisierung von PBMF und CBMF mittels Zytokinen/ Wachstumsfaktoren für 5 Tage. FACS-basierte Charakterisierung (CD14, TLR4, CD163) basal, nach Aufnahme von Erythrozyten oder nach Infektion mit E. coli. Analyse der AKT-Phosphorylierung mittels Immunoblot, IL-10-Sekretion via ELISA. Intrazelluläre Bestimmung des NO-Gehalts. Vergleichende Transkriptanalyse der Hämoxygenase-1 zwischen PBMF und CBMF.

Ergebnisse: In unstimuliertem Zustand wurde CD163 bei PBMF deutlich höher exprimiert als bei CBMF. TLR4 und CD163 wurden nach Infektion mit E. coli hoch-reguliert ( $p<0,05$ vs. nicht-infiziert) und induzierten IL-10-Sekretion (PBMF $>$ CBMF; $p<0,05$ ). Im Gegensatz zur identischen Phagozytoseraten bei bakterieller Infektion fand sich bei Aufnahme von Hämoglobin eine deutlich reduzierte Kapazität von CBMF $(p<0,05$ vs. PBMF). Ebenfalls war nach Aufnahme von Hämoglobin die AKT bei PBMF durch Phosphorylierung stärker aktiviert und ihr intrazellulärer NO- Gehalt höher ( $p<0,05$ vs. CBMF). Transkripte der Häm-Oxygenase-1 waren bei CBMF ca. 9-fach herunterreguliert $(p<0,05)$.

Schlussfolgerung: Über die Aktivierung von TLR4 wird bei PBMF die CD163 Expression gesteuert. Bei CBMF unterbleibt dieser Prozess. Die geringere CD163 und Häm-Oxygenase-1 Expression bildet einen Schutzmechanismus, der bei hohem Hämoglobingehalt eine Übersteuerung des CD163 und PI3K/AKT Signalwegs vermeidet und die zytotoxische Produktion reaktiver Sauerstoff-Spezies vermindert.

Tab. P115

\begin{tabular}{|llllllll} 
& $\mathbf{n}$ & MW & Median & SD & Min & Max & Korrelation mit GA \\
\hline Faktor XIII [\%] & 100 & 35,4 & 35 & 11,7 & 11 & 85 & $0,429^{* *}$ \\
\hline Antithrombin III [\%] & 97 & 25,6 & 25,0 & 8,1 & 3 & 49 & 0,103 \\
\hline Quick [\%] & 96 & 60,3 & 61 & 16,3 & 17 & 101 & 0,28 \\
\hline aPTT [s] & 95 & 66,2 & 66,2 & 17,0 & 18,4 & 121,2 & $-0,118$ \\
\hline Thrombozyten[gpt/l] & 99 & 187,6 & 185 & 70,8 & 22 & 458 & $-0,14$ \\
\hline Fibrinogen [g/l] & 86 & 1,86 & 1,35 & 1,29 & 0,28 & 5,93 & $-0,72$ \\
\hline D-Dimere [mg/l] & 94 & 11,21 & 3,95 & 15,88 & 0,11 & 70,8 & $-0,168$
\end{tabular}




\section{P117}

\section{Vom Hau(p)tbefund zur (Verdachts-)diagnose: Fall eines 7 Tage alten Neugeborenen mit initial unspezifischem Exanthem}

E. Resch' ${ }^{1}$, S. Dettmers' ${ }^{1}$, T. Rothoeft ${ }^{1}$, U. Schauer ${ }^{2}$, T. Lücke', N. Teig ${ }^{4}$ 'Universitätskinderklinik Bochum, katholisches Klinikum Bochum, Neonatologie und pädiatrische Intensivmedizin, Bochum, Deutschland, ${ }^{2}$ Universitätskinderklinik Bochum, katholisches Klinikum Bochum, Allergologie, Immunologie und Pneumologie, Bochum, Deutschland, ${ }^{3}$ Universitätskinderklinik Bochum, katholisches Klinikum Bochum, Neuropädiatrie, Bochum, Deutschland, ${ }^{4}$ Universitätskinderklinik Bochum, katholisches Klinikum Bochum, Abteilung für Neonatologie und pädiatrische Intensivmedizin, Bochum, Deutschland

Hintergrund: Schuppende oder erythematöse Hautveränderungen, wie im folgendem Fall geschildert, sind in der Neonatalzeit häufig, meist jedoch transient und ohne Therapiebedarf, können jedoch auch Marker chronischer Erkrankungen sein.

Kasuistik: Wir berichten über ein männliches Neugeborenes der $37+1$ SSW. Geburtseinleitung bei Plazentainsuffizienz (APGAR 9/10/10, NApH 7,23, GG 2910 g, KL 50 cm, KU 33,5 cm); Mutter 2-Gravida, 1-Para; Eltern nicht konsanguin. In U1 und U2 fielen ein papulo-erythematöses Exanthem und eine periorale Hautschuppung auf, welche als Erythema neonatorum gewertet wurden. Bei gutem AZ Entlassung aus der Geburtsklinik. Im Verlauf der 1. Lebenswoche AZ-Verschlechterung und Trinkschwäche sowie Zunahme der Hautschuppung. Am 7. Lebenstag Vorstellung bei der Kinderärztin und Einweisung in die Klinik. Bei Aufnahme Patient in reduziertem AZ, wenig Reaktion auf die Untersuchungssituation, Körpertemperatur $33,4{ }^{\circ} \mathrm{C}$, ausgeprägte (nicht-bullöse) Exfoliation der Haut insbesondere an Gesicht, Thorax und Intertrigines sowie Hand- und Fußrückenödeme. Bei V.a. Staphylococcal scalded skin syndrome antibiotische Therapie. Bei Trinkschwäche Teilsondierung der Nahrung. Anhaltende Thermolabilität mit ausgeprägtem Bedarf externer Wärmezufuhr (Wärmebett-Temp. $38^{\circ} \mathrm{C}$ ). Im Verlauf Veränderung des Hautbildes mit progredienter Exfoliation und zunehmender Erythrodermie. Hand- und Fußrückenödeme nahmen zu und inguinal zeigten sich vergrößerte Lymphknoten. Im Labor auffallend waren eine Eosino-

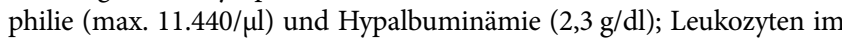
Verlauf von $6470 / \mu$ lauf $30.620 / \mathrm{ml}$ ansteigend, keine relevante CRP-Erhöhung (max. 12,4 mg/l). Auf Grund der Befundkonstellation wurde eine immunologische Diagnostik durchgeführt. Bei dem Neugeborenen waren keine B-Zellen, eine normale Zahl von NK-Zellen und ausschließlich aktivierte T-Zellen (memory Zellen) nachweisbar. Daraus ergab sich die Verdachtsdiagnose eines Omenn-Syndroms als Ausdruck eines schweren kombinierten Immundefekts mit einer Restfunktion autoreaktiver T-Zellen. Nach Befunderhalt an Lebenstag 14 Beginn einer Prophylaxe mit Cotrimoxazol, Beendigung der Ernährung mit Muttermilch und umgehende Verlegung des Patienten zur Stammzelltransplantation.

Schlussfolgerung: Erythematöse oder ichthyöse Hautveränderungen beim Neugeborenen können Ausdruck eines schweren Immundefektes sein. Da die Prognose dieser Immundefekte bei rechtzeitiger Diagnosestellung, d. h. vor Auftreten schwerer Infektionen, relativ günstig ist, sollte frühzeitig eine immunologische Basisdiagnostik durchgeführt werden.

\section{P118 \\ Granulozyten hemmen die T-Zell-Proliferation nach in vitro Infektion mit Escherichia coli}

B. Spring ', A. Leiber', N. Köstlin' ', J. Schwarz', T. Orlikowsky' ${ }^{2}$, C. Poets' ', C. Gille 'Eberhard-Karls-Universität Universitätsklinik für Kinderheilkunde und Jugendmedizin, Neonatologie, Tübingen, Deutschland, ${ }^{2}$ Universitätsklinik für Kinder- und Jugendmedizin, Sektion für Neonatologie, Aachen, Deutschland

Hintergrund: Prä- und postnatale bakterielle Infektionen sind Risikofaktoren für inflammatorische Erkrankungen in der Neonatalzeit wie der Bronchopulmonalen Dysplasie (BPD) oder der Perivenrikulären Leukomalazie (PVL). Pathogenetisch scheinen verlängerte Entzündungsreaktionen (,sustained inflammation“) wichtig zu sein. Unsere Vorergebnisse zeigen, dass myeloide Vorläuferzellen (myeloid derived suppressor cells, MDSC) im Nabelschnurblut (NSB) stark erhöht sind und hemmend auf Immuneffektorzellen wirken. Ob dies auch nach bakterieller Stimulation der Fall ist, ist unklar. Bisher wurde angenommen, dass reife Granulozyten keine suppressive Aktivität haben.

Fragestellung: Wie ist die suppressive Aktivität von MDSC und reifen Granulozyten aus NSB und dem peripheren Blut Erwachsener ohne und mit Infektion mit E. coli?

Methoden: Granulozyten (PB-Granulos) und periphere monokleäre Zellen gesunder Erwachsener (PBMC) sowie Granulozyten (CB-Granulos) und mononukleäre Zellen aus dem Nabelschnurblut (CBMC) wurden isoliert. MDSC wurden aus CBMC mittels magnetic activated cell sorting (MACS) weiter angereichert. PB-Granulos, CB-Granulos und MDSC wurden für eine Stunde mit Escherichia coli (E. coli zu Zellen: $50 \mathrm{zu}$ 1) infiziert. Die Infektion wurde mit Gentamycin gestoppt, und die gewaschenen Zellen zu CFSE-markierten PBMC (1:4) gegeben. Nach 4 Tagen Stimulation mit anti-CD3 (5 ug/ml) und IL-2 wurde die CD4- und CD8-T-Zell-Proliferation durchflusszytometrisch ermittelt. Als Kontrolle dienten stimulierte und unstimulierte PBMC ohne Zugabe weiterer Zellen.

Ergebnisse: Naive MDSC und MDSC nach Infektion mit E. coli hemmten die CD4- und CD8-T-Zell-Proliferation gleichermaßen effektiv. Naive PB-Granulos und CB-Granulos hemmten die T-Zell-Proliferation nicht. Nach Infektion mit E. coli hatten sowohl PB-Granulos als auch CB-Granulos einen hemmenden Effekt auf die CD4- und CD8-T-Zell-Proliferation. Die hemmende Potenz von CB-Gralunos war jedoch deutlich schwächer. Schlussfolgerung: Wir zeigen zum ersten mal, dass nicht nur myeloide Vorläuferzellen wie MDSC, sondern auch reife Granulozyten können im Rahmen eines Infektionsgeschehens immunsuppressive Funktionen haben. Die immunsuppressive Potenz von reifen Granulozyten scheint dabei im Nabelschnurblut vermindert zu sein. Möglicherweise spielt dies für verlängerte Entzündungsreaktionen in der Neonatalzeit eine Rolle.

\section{Postersitzung 12: Neurologie}

\section{P119}

\section{Neuroprotektive Effekte von 17ß-Estradiol und} Fetalzonensteroiden bei Hyperoxie-induzierter Schädigung unreifer Gliazellen

S. Hübner' ${ }^{1}$ D. Sunny ${ }^{1}$, M. Heckmann ${ }^{1}$

'Universitätsmedizin Greifswald, Kinderklinik, Greifswald, Deutschland

Hintergrund: Faktoren neurologischer Entwicklungsstörungen bei Frühgeborenen sind neben der Unreife selbst vor allem Hypoxie-Ischämie, Entzündungen und Hyperoxie. Frühgeburt geht mit drastischem Absinken von $17 \beta$-Estradiol (E2) und Progesteron einher. Jedoch werden Östrogenvorstufen, sogenannte Fetalzonensteroide (FZS) (hauptsächlich Dehydroepiandrosteron [DHEA]) in hoher Konzentration bis zum Termin weiter synthetisiert. Klinische Vorstudien zur Substitution von E2 und Progesteron zeigten keine eindeutigen Verbesserungen des neurologischen Outcomes.

Fragestellung: Haben FZS im Vergleich zu E2 eine neuroprotektive Wirkung im Modell Hyperoxie-geschädigter unreifer Gliazellen?

Methoden: Es wurden (1) die Rezeptoren bestimmt, die an der E2-vermittelten Protektion bei Hyperoxie-induziertem Zelltod beteiligt sind; (2) untersucht, ob DHEA und andere Metaboliten ebenfalls protektiv wirken, sowie (3) die daran beteiligten Rezeptoren und der Einfluss intrazellulärer Aromatasen. Schließlich wurden (4) Hormon-Einzelbehandlungen gegen die Kombination E2/FZS in Bezug auf Ausmaß und beteiligter Rezeptoren untersucht.

Ergebnisse: (1) Bei OLN-93 Zellen (unreife Oligodendrozyten [OL], Ratte), PDGFRa+ Primärzellen (unreife OL, Maus) und C6 Zellen (unreife Astrozyten, Ratte) vermitteln klassische Östrogen-Rezeptoren (ER) die Protektion durch E2. In C6 Zellen sind zusätzlich nicht-klassische ER be- 
teiligt. (2) Die Behandlung mit den FZS DHEA, Adiol und 16OH-DHEA induziert Dosis-abhängig Protektion bei allen Zellarten. (3) Der Effekt von DHEA wird dabei über klassische ER und den Androgen-Rezeptor (AR) vermittelt. Blockieren von Aromatasen (BA) verhindert die Umwandlung von FZS in östrogenere Moleküle. In C6 Zellen führt BA zur Reduktion der Protektion bei $16 \mathrm{OH}-\mathrm{DHEA}$. Im Gegensatz dazu, steigert BA bei OLN93 Zellen die Protektion bei allen FZS. (4) Die Kombination E2/FZS führt bei OLN-93 Zellen zu synergistischen Effekten mit allen FZS. Das steht im Kontrast zu C6 und PDGFR $a+$ Zellen, wo die Kombination E2/FZS keine Synergien aufweist. Bei C6 Zellen wird die Protektion bei der Kombination E2/DHEA über klassische ER und AR vermittelt.

Diskussion: E2, DHEA und Metaboliten wirken in allen Zellarten Dosis-abhängig protektiv. Abhängig vom Zelltyp vermitteln klassische ER, nicht-klassische ER oder AR den Effekt. Bei C6 und PDGFRa+ Zellen führt die Kombination E2/FZS nicht zu synergistischen Effekten, da die E2- und FZS-vermittelte Protektion hauptsächlich über die klassischen ER wirken, was vermutlich zu einer Sättigung am ER führt. Das steht im Kontrast zu den OLN-93 Zellen, wo die E2/FZS Kombination Synergien zeigt. Hier scheint der AR eine genauso ausgeprägte Wirkstärke zu besitzen wie klassische ER. Die BA-Experimente unterstützen diese Ergebnisse. Da FZS im $\mu \mathrm{M}$-Bereich im Frühgeborenen akkumulieren, könnte eine Sättigung durch Bindungskonkurrenz am ER bei E2-Substitution stattfinden und so den klinischen Effekt einer Substitution von E2 bei Frühgeborenen abschwächen.

\section{P120}

\section{Nachweis von Mykoplamsen pneumonia im Liquor} bei einem Neugeobrenen mit beidseitigem Mediainfarkt: Möglicher kausaler Zusammenhang?

\section{S. Kunzmann', R. Wößner', C. Willaschek², J. Wirbelauer', C. Speer}

'Universitäts-Kinderklinik Würzburg, Würzburg, Deutschland, ${ }^{2}$ Caritas Krankenhaus Bad Mergentheim, Kinderklinik, Bad Mergentheim, Deutschland

Hintergrund: Ischämische Infarkte treten in der Perinatalperoide häufiger als in jedem anderen Alter von Kindheit und Adoleszenz auf. Neben der Asphyxie sind diese die häufigste Ursache von neonatalen Krampfanfällen und eine der Hauptursache für das Auftreten einer Zerebralparese. Die Pathogenese der Infarkte ist letztlich nicht sicher geklärt, am ehesten werden thrombembolische Ereignisse vermutet.

Fallbeschreibung: Bei einem eutrophes Neugeborenes nach unauffälligem Schwangerschaftsverlauf und problemloser postnataler Adaption $(40+0$ SSW, GG 3150 g; Apgarwerte: 1 9/5 10/10 10; Nabelarterien-pH: 7,31; Blasensprung: zur Geburt, eilige Sectio am Termin bei fetaler Bradykardie, grünes Fruchtwasser sowie bekannte Insertio velamentosa.) traten am 1. Lebenstag tonisch-klonische zerebrale Anfälle auf, die nach Einleitung einer anti-epileptischen Therapie mit Phenobarbital sistierten. Das EEG zeigte keinen auffälligen Befund. In der zerebralen Sonographie, sowie im cNMR Nachweis ausgeprägter Ischämieareale im Bereich der Mediastromgebiete beidseits. Ab dem 6. Lebenstag milder zusätzlicher Sauerstoffbedarf bei unauffälligem Röntgen-Thorax. Die Familienanamnese ist bis auf ein Klippel-Trenaunay-Syndrom des Vaters unauffällig. Eine breite Abklärung zur Ursache der zerebralen Ischämie (u. a. hämatologische, metabolische, kardiologische und Vaskulopathie-Diagnostik) ergab keine auffälligen Befunde. In den infektiologischen Untersuchungen positive PCR-Reaktion auf Mykoplasma pneumoniae im Liquor (Leukozyten 17/ $\mu$, Erythrozyten

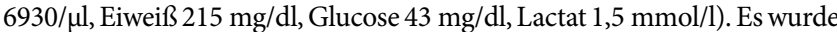
eine antimikrobielle Therapie mit Clarithromycin über 21 Tage bei weiterhin klinisch-neurologisch unauffälligem Kind durchgeführt.

Diskussion: Mykoplasmen-Infektionen können verschiedene Entzündungsreaktionen im ZNS (u.a. Enzephalitis, Meningitis, Myelitis) bei Kindern und Erwachsenen auslösen. Auch bei Früh- und Neugeborenen wurden selten Meningoenzephalitiden beschrieben, die häufig erst später diagnostiziert wurden (Glaser, Speer et al. Exp Rev Anti Infect Ther 2015). Einzelne Berichte bei Erwachsenen weisen bei zerebralen Ischämien daneben auf eine Beteiligung von Mykoplasmen bei Vaskulopathien hin. Eine solche Assoziation ist für das Neugeborenenalter, insbesondere als möglicher Auslöser von „strokes", bisher nicht beschrieben worden. Bei Neugeborenen mit zerebralem Infarkt sollte deshalb auch nach einer möglichen Mykoplasmen-Infektion gezielt gesucht werden. Sollte sich unsere Beobachtung bestätigen lassen, könnte dies evtl. mit zur Klärung der Genese von einigen zerebralen Infarkten beitragen mit auch neuen, ggf. auch präventiven, therapeutischen Implikationen

\section{P121}

Entwicklung eines Scoringssystems zur sonographischen Beurteilung der Entwicklung der Gyrierung bei Frühgeborenen $<\mathbf{3 2}+\mathbf{0} \mathrm{SSW}$

\section{E. Zanke', U. Felderhoff-Müser' ${ }^{2}$, A. Stein}

'UK Essen, Klinik für Kinderheilkunde 1, Essen, Deutschland, 2Universitätsklinikum Essen, Direktorin der Klinik für Kinderheilkunde I, Zentrum für Kinder- und Jugendmedizin, Essen, Deutschland, ${ }^{3}$ Klinik für Kinder und Jugendmedizin der Universität, Klinik für Kinderheilkunde I, Essen, Deutschland

Hintergrund: Die kortikale Entwicklung Frühgeborener wurde bisher in postmortalen anatomischen Studien, intrauterin 2D- und 3D-sonographisch sowie postnatal in MRT-Studien untersucht. Im Gegensatz zu höher auflösenden aber aufwendigeren MRT- Untersuchungen wäre die Sonographie geeignet, in regelmäßigen bettseitigen Kontrolluntersuchungen mit standardisierter und objektiver Beurteilung des Kortex Aussagen über die morphologische Hirnreifung Frühgeborener zu machen.

Ziel: Entwicklung eines Scoringsystems zur objektiven Beurteilung der Gyrierungsentwicklung basierend auf der sonographischen Vermessung kortikaler Strukturen. Als Grundlage dienen bereits bekannte Scoringsysteme aus MRT-Studien und aus ultraschallbasierten Untersuchungen mit jedoch geringen Fallzahlen.

Methode: Es wurden retrospektiv 371 serielle sonographische Aufnahmen aus Routineuntersuchungen von 96 Frühgeborenen ausgewertet, die zwischen 2009 und 2013 im Universitätsklinikum Essen unter einem Gestationsalter von 32 Schwangerschaftswochen geboren wurden. Zur Anwendung des Scores lagen 90 Untersuchungen vom 1.-3. Lebenstag in verwertbarer Qualität vor. Der Score wurde weiterhin zur longitudinalen Beurteilung der kortikalen Entwicklung bei Frühgeborenen ohne postnatale Hirnblutungen in den sonographischen Verlaufsuntersuchungen am 14. $(n=65)$ und 28. $(n=77)$ Lebenstag eingesetzt. Von 56 Frühgeborenen lagen verwertbare Untersuchungen zu allen 3 Zeitpunkten vor.

Ergebnisse: Aus einer Vielzahl erhobener Messwerte wurden aufgrund der einfachen und sicheren Identifikation und Vermessung im Koronarschnitt durch den 3. Ventrikel die Tiefe des Sulcus cinguli, die Länge der Inselrinde und die Weite der Öffnung der Fissura Sylvii in einen Score eingeschlossen. Die Messwerte jeder Struktur wurden in jeweils drei Kategorien mit den Punktwerten 0,1 und 2 eingeteilt und zu einem Scoresummenwert addiert. Es zeigt sich eine signifikante Korrelation zwischen Scoresummenwert in der Ultraschalluntersuchung am 1.-3. Lebenstag und Gestationsalter bei Geburt $(p<0,001)$. Auch bei der Anwendung des Scores in den sonographischen Verlaufsuntersuchungen am 14. und 28. Lebenstag ergab sich eine signifikante Korrelation mit dem Gestationsalter (jeweils $p<0,001$ ).

Schlussfolgerung: Der entwickelte Score stellt eine einfache und objektive Möglichkeit zur bettseitigen Beurteilung der kortikalen Entwicklung bei Frühgeborenen dar.

Ausblick: In einem nächsten Schritt ist eine prospektive Evaluation des Scores erforderlich. Danach wäre es möglich, den Score auch für Frühgeborene mit intrakraniellen Blutungen, periventrikulärer Leukomalazie oder posthämorrhagischem Hydrozephalus in der postnatalen Entwicklung anzuwenden und den Einfluss dieser Faktoren auf die kortikale Entwicklung zu untersuchen. 


\section{P122}

Prognose von Frühgeborenen mit schwerer intraventrikulärer Blutung ('III) ohne/mit periventrikulärer hämorrhagischer Infarzierung oder zystischer periventrikulärer Leukomalazie

M. Leber' ', P. Kutz' ' S. Supcun-Ritzler' ', L. Stüwe-Kunz' , C. Roll'

${ }^{1}$ Vestische Kinder- und Jugendklinik Datteln, Universität Witten/Herdecke, Neonatologie und pädiatrische Intensivmedizin, Datteln, Deutschland

Hintergrund: In der Neonatalzeit sonographisch darstellbare zerebrale Läsionen sind wesentliche Risikofaktoren für eine eingeschränkte kognitive und motorische Langzeitentwicklung Frühgeborener.

Fragestellung: Entwicklung Frühgeborener mit schwerer Hirnblutung oder periventrikulärer Leukomalazie im korrigierten Alter von 2 Jahren. Material und Methoden: Frühgeborene mit einem Gestationsalter $<30$ Wochen, Jahrgang 2006-2012, mit Hirnblutung Grad III (IVH III) ohne oder mit periventrikulärem hämorrhagischem Infarkt (PHI) sowie Frühgeborene mit zystischer periventrikulärer Leukomalazie (PVL), Ergebnisse der 2 Jahres-Untersuchungen (Bayley Scales of Infant Development II).

Ergebnisse: Im 7-Jahres-Zeitraum wurden 394 Frühgeborene mit einem Gestationsalter $<30$ Wochen behandelt. Von den 34 (8,6\%) während des stationären Aufenthaltes verstorbenen Kindern hatten 12 eine IHV III (davon 11 mit PHI). 22 Frühgeborene überlebten mit schweren zerebralen Läsionen: 4 mit IHV III, 14 mit IHV III + PHI, 4 mit PVL. Das Gestationsalter der Frühgeborenen mit IVH III/IVH III + PHI lag im Median bei 25 Wochen (Range 23-28), mit PVL bei 29 Wochen (Range 25-29). Alle wurden im korrigierten Alter von 2 Jahren untersucht. Bei IVH III lag der MDI bei 3/4 Kindern im Normbereich, bei 1 Kind bei 82, der PDI bei allen Kindern im Normbereich, 1 Kind hatte eine CP; bei IVH III + PHI lag der MDI bei 4/14 Kindern im Normbereich, bei 3 zwischen 84 und 70, bei 3 zwischen 69 und 50 und bei $4<50$, der PDI bei einem Kind im Normbereich, bei 5 zwischen 84 und 70, bei einem 69 und bei $7<50,12 / 14$ Kindern hatten eine CP. Bei allen 4 Kindern mit PVL lagen PDI und MDI $<50$ bzw. waren nicht testbar, alle hatten eine $\mathrm{CP}$.

Diskussion und Schlussfolgerung: Das Gestationsalter der Frühgeborenen mit PVL war deutlich höher als das der Frühgeborenen mit schweren Hirnblutungen. Während die kognitive und motorische Entwicklung im Alter von 2 Jahren bei allen 4 Kindern mit PVL ungünstig war, zeigten die Ergebnisse bei Frühgeborenen mit schweren Hirnblutungen eine große Spannbreite und waren besser als erwartet.

\section{P123}

\section{Die Effekte von Doxapram auf die Aktivität des Hirnstammes}

\section{S. Kruszynski', H. Koch'2, J. Brandes ${ }^{2}$, H. Lerche' ${ }^{2}$, C. Poets}

${ }^{1}$ Eberhard-Karls-Universität Universitätsklinik für Kinderheilkunde und Jugendmedizin, Neonatologie, Tübingen, Deutschland, ${ }^{2}$ Neurologische Universitätsklinik Tübingen, Epileptologie, Tübingen, Deutschland

Hintergrund: Zur Therapie der Apnoe-Bradykardie-Hypoxämie-Symptomatik (ABHS) Frühgeborener stehen Lagerungstechniken, nicht-invasive Beatmungsformen und Coffeincitrat als Atemanaleptikum zur Verfügung. Dennoch persistiert bei einem Teil der Kinder die Symptomatik, sodass an einigen Zentren eine Therapie mit Doxapram in Erwägung gezogen wird. Über dessen genauen Wirkungsmechanismus und Sicherheit liegen aber bisher kaum Daten vor. Wesentlicher Bestandteil des neuronalen Netzwerkes zur Generation und Regulation der Atemaktivität ist ein umschriebener Bereich der ventro-lateralen Medulla oblongata, der sogenannte Pre-Bötzinger-Komplex (PreBötC). Dieser ist essentiell für die normale inspiratorische Aktivität und spielt auch im Rahmen der Schnappatmung eine Rolle (Lieske et al 2000).

Fragestellung: Im Rahmen dieser Studie wurde erstmals der direkte Einfluss von Doxapram auf den isolierten Hirnstamm mit dem darin enthaltenen PreBötC und dem Hypoglossuskern (XII) untersucht. Beide stehen in enger anatomischer Beziehung zueinander. Unsere Hypothese war, dass Doxapram zu einer höheren Aktivität der Neurone im PreBötC führt und darüber eine Stimulation der motorischen Efferenzen des Hypoglossuskerns bewirkt.

Material und Methoden: Es wurden neonatale (postnatale Tage (P) 0-5 (PreBötC $n=13$, XII $n=12$ )) und juvenile (P 9-14 (PreBötC $n=16$, XII $n=7)$ ) Mäuse (CD1/NMRI) beider Geschlechter eingesetzt. Nach Präparation eines Hirnstammschnittes in künstlicher Zerebrospinalflüssigkeit erfolgte die Ableitung der Aktivitäten (Frequenz und Amplitude) des PreBötC und des Hypoglossuskerns mittels Mikroglaselektroden vor und nach $\mathrm{Zu}$ gabe von Doxapram sowie unter normo- und hypoxischen Bedingungen. Ergebnisse: Doxapram stimulierte sowohl den PreBötC als auch den Hypoglossuskern. Es kam altersunabhängig zu einer Zunahme der Frequenz (PreBötC 26,9\% (SD $\pm 30,2 \%, p<0,0001$ ), XII 33,8 \% (SD $\pm 18,4 \%$, $p<0,0001))$. Zudem kam es zu einem Anstieg der Amplitude im Hypoglossuskern $(40,2 \%(\mathrm{SD} \pm 24,6 \%, p=0,0002))$; der Anstieg im PreBötC $(7,5 \%)$ war dagegen nicht signifikant. Unter hypoxischen Bedingungen bewirkte Doxapram keine Änderungen der Aktivität. In der Erholungsphase nach einer Hypoxie zeigte die Amplitude des PreBötC nach Zugabe von Doxapram anhaltend niedrigere Werte $(p<0,0001)$ im Vergleich zur Erholungsphase ohne Doxapram. Die Aktivität des Hypoglossuskerns wurde in dieser Phase durch Doxapram nicht beeinflusst.

Diskussion: Doxapram wirkte im Mausmodell zentral stimulierend auf den PreBötC und den Hypoglossuskern, was zu der schon zuvor bei Frühgeborenen gezeigten Erniedrigung des $\mathrm{CO}_{2}$ unter Doxapram passt (Poets et al 1999). Diese Ergebnisse deuten auf Interaktionen zwischen respiratorischen Kerngebieten und könnten erste Schritte zum Verständnis der Wirkung von Doxapram auf die ABHS darstellen. Ob die im Hypoglossuskern gemessenen Effekte durch direkte Stimulation des Kerngebiets oder durch Veränderungen in der neuronalen Verbindung zwischen dem PreBötC und dem Hypoglossuskern entstehen, soll im Weiteren mittels intrazellulärer Ableitungen von einzelnen Hypoglossusneuronen untersucht werden.

\section{P124}

Eignet sich die Kombination aus frühem aEEG und der kranialen MRT am Entbindungstermin zur Vorhersage des neurologischen Outcomes bei sehr unreifen Frühgeborenen?

T. Storbeck', B. Hüning ${ }^{1}$, N. Bruns' ${ }^{1}$, F. Dransfeld ${ }^{1}$, J. Hobrecht' ${ }^{1}$ J. Karpienski', S. Sirin'², B. Schweiger ${ }^{2}$, C. Weiß3', U. Felderhoff-Müser' ${ }^{1}$ H. Müller ${ }^{1}$

'Universitätsklinikum Essen, Kinderheilkunde I, Essen, Deutschland, ${ }^{2}$ Universitätsklinikum Essen, Institut für diagnostische und interventionelle Radiologie und Neuroradiologie, Essen, Deutschland, ${ }^{3}$ Fakultät für Klinische Medizin Mannheim, Universität Heidelberg, Abteilung für Medizinische Statistik, Mannheim, Deutschland

Hintergrund: Sehr unreife Frühgeborene haben ein hohes Risiko für neurologische Spätfolgen. Deshalb ist es sehr wichtig, Hoch-Risiko-Kinder zu identifizieren, um geeignete Fördermaßnahmen zu initiieren.

Fragestellung: Wir haben untersucht, ob ein innerhalb der ersten 72 Lebensstunden abgeleitetes aEEG (amplitude integrated electroencephalography) und eine kraniale MRT (Magnetresonanztomografie) am errechneten Entbindungstermin (ET) das neurologische Outcome von sehr unreifen Frühgeborenen im korrigierten Alter von 2 Jahren voraussagen kann.

Patienten und Methoden: Bei 38 sehr unreifen Frühgeborenen, die im Zeitraum März 2011 bis Dezember 2012 geboren wurden, war sowohl ein aEEG in den ersten 72 Lebensstunden, eine kraniale MRT am ET (Interpretation erfolgte mittels modifiziertem Kidokoro-Score) sowie eine BSID II-Testung mit korrigiert 24 Monaten durchgeführt worden. Das mittlere Gestationsalter betrug $28+2$ (Bereich: $23+4-31+4$ ) Wochen, das mittlere Geburtsgewicht 1093 (Bereich: 450-2085) g. Um unabhängige Faktoren, die mit dem aEEG (Burdjalov Score (BS)), den MRT-Daten, den klinischen Parametern und dem neurologischen Outcome assoziiert sind, zu identifizieren, wurde eine multiple Regressionsanalyse verwendet.

Ergebnisse: Eine intraventrikuläre Blutung (IVH, $p=0,0060$ ) und die interhemisphärische Distanz (IHD, $p=0,0052$ ) waren unabhängige Prädiktoren für den Mental Developmental Index (MDI); der BS am Tag 1 $(p=0,0201)$ sowie die IHD $(p=0,0142)$ sind Prädiktoren für den Psycho- 
motor Development Index (PDI). Es zeigte sich ein Zusammenhang zwischen dem Volumen der Basalganglien/Thalami und der Biparietalweite (BPW, $p<0,0001)$, dem BS am Tag $3(p=0,0129)$ und dem Gesamt-BS am Tag 1-3 $(p=0,0028)$. Das Geburtsgewicht $(p=0,0225)$, der BS am Tag 1 $(p=0,0163)$, der transzerebelläre Durchmesser $(p<0,0001)$ und das Auftreten von Krämpfen $(p=0,0245)$ waren mit der Biparietalweite korreliert. Die interhemisphärische Distanz war mit dem Kopfumfang beim MRT assoziiert $(p=0,0139)$. Die Biparietalweite $(p<0,0001)$ und der BS am Tag 1 $(p=0,0199)$ korrelierten mit dem transzerebellären Durchmesser und das Auftreten einer IVH mit dem MRT Score $(p<0,0001)$.

Schlussfolgerung: Die Parameter eines in den ersten 72 Lebensstunden abgeleiteten aEEGs sind mit der veränderten Hirnreife beim MRT am ET assoziiert. Das aEEG und die kraniale MRT sind insbesondere in Kombination Methoden, die zur Abschätzung des neurologischen Outcomes beitragen können.

\section{P125}

\section{Hypothermie-Behandlung eines Frühgeborenes von 33 + 1 SSW nach schwerer perinataler Asphyxie- ein Fallbericht}

\section{F. Brevis' ${ }^{1}$ T. Rosenbaum ${ }^{2}$ \\ ${ }^{1}$ Klinikum Duisburg, Kinderintensivstation, Duisburg, Deutschland, \\ ${ }^{2}$ Kinderklinik, Lüdenscheid, Deutschland}

Hintergrund: Die Hypothermie-Behandlung von Neugeborenen und Frühgeborenen ${ }^{3} 36$ SSW nach perinataler Asphyxie gehört mittlerweile zum Standard neonatologischer Therapien. Aufgrund der unzureichenden wissenschaftlichen Datenlage wird in den offiziellen Leitlinien die Hypothermie-Behandlung bei jüngeren Frühgeborenen nicht empfohlen.

Fragestellung: Lassen sich Frühgeborene mit einem niedrigeren Gestationsalter behandeln?

Material und Methoden: Wir berichten über ein Frühgeborenes der 33+ 1 SSW, das per Notsectio bei vorzeitiger Plazentalösung in einer auswärtigen Geburtsklinik entbunden wurde. Die Erstversorgung vor Ort erfolgte durch die Kollegen der Anästhesie, die das Kind bei fehlender Spontanatmung und Bradykardie 25 Minuten lang kardiopulmonal reanimierten. Bei Ankunft unseres neonatologischen Teams war das Kind bereits $35 \mathrm{~min}$ alt, spontanatmend unter CPAP-Beatmung. Apgar 3/5/7, NsapH 6,75. Geburtsgewicht $2070 \mathrm{~g}$ (75. Perzentile).

Ergebnisse: Die erste Blutgasanalyse, noch in der auswärtigen Klinik, zeigte eine schwere Azidose ( $\mathrm{pH} 6,93, \mathrm{pCO}_{2} 50 \mathrm{mmHg}, \mathrm{pO}_{2} 72 \mathrm{mmHg}, \mathrm{BE}$ $-24 \mathrm{mmol} / \mathrm{l})$. Klinisch imponierten neben einem reduzierten Allgemeinzustand, eine deutliche muskuläre Hypotonie sowie respiratorische Insuffizienz. Die Eltern wurden noch vor Ort über die Option einer Hypothermie-Behandlung als individuellen Heilversuch aufgeklärt und es erfolgte eine Einverständniserklärung. Die Durchführung eines Sarnat-Scores oder Thompson-Scores ließ sich nach Intubation nicht mehr vollständig bewerkstelligen. Das unmittelbar nach Aufnahme auf unsere neonatologische Intensivstation begonnene aEEG wies eine schwere Hirnfunktionsstörung mit initialer Niedervoltage nach, die im Verlauf in ein Burst-Suppression-Muster überging. Bei wiederholt nachweisbaren epilepsietypischen Potentialen kam es am 3. Lebenstag zu kurzen Krampfanfällen, die eine kurzzeitige Behandlung mit Phenobarbital erforderlich machte. Die Hypothermie-Behandlung über 72 Stunden wurde klinisch wie auch laborchemisch gut vertragen. Im Alter von 7 Tagen konnten in einer zerebralen MRT puncted lessions im Marklager als Verdacht auf generalisierte hypoxische Läsionen nachgewiesen werden, eine Diffusionsstörung ließ sich nicht feststellen. Bei der Abschlussuntersuchung vor Entlassung fand sich eine zentrale Koordinationsstörung mit Tonusregulationsstörung im Rahmen der Frühgeburtlichkeit sowie eine deutliche muskuläre Hypotonie sowie Strecktendenzen der Beine. Diskussion: Bei engmaschigen neuropädiatrischen Kontrollen im Alter von 6 Wochen, 2, 4 und 6 Monaten konnten altersentsprechende EEG sowie bei neuropädiatrischen Untersuchungen altersentsprechende Befunde erhoben werden.

Schlussfolgerung: Nach ausführlicher Aufklärung und Einverständniserklärung lässt sich eine Hypothermie-Behandlung als individuellen Heilversuch durchführen. In unserem Fall konnte trotz schwerer hypo- xisch-ischämischer Enzephalopathie eine positive neurologische Entwicklung erzielt werden.

\section{Literatur}

1. Gunn AJ, Bennet L. Brain cooling for preterm infants. Clin. Perinatol. 2008; 35: 735-748

2. Hall NJ, Eaton S, Peters MJ et al. Mild controlled hypothermia in preterm neonates with advanced necrotizing enterocolitis. Pediatrics 2010; 125: e300-8

3. Austin T, Shanmugalingam S, Clarke P. To cool or not to cool? Hypothermia treatment outside trial criteria. Arch Dis Child Fetal Neonatal Ed 2012

4. Saliba E. Acta Paediatr. 2015, Feb; 104 (2): 114-115

\section{P126}

\section{Neonatale Krampfanfälle und Therapieansprechen} auf Phenobarbital: Monozentrische Erfahrung

S. Pfahl' ${ }^{1}$, D. Tibussek ${ }^{2}$, T. Höhn ${ }^{3}$, H. Sabir ${ }^{4}$

${ }^{1}$ Med. Einricht. d. Universität Kinderklinik, Pädiatrische Intensivstation/ Neonatologie, Düsseldorf, Deutschland, ${ }^{2}$ Med. Einricht. d. Universität Kinderklinik, Düsseldorf, Deutschland, ${ }^{3}$ Med. Einricht. d. Universität Kinderklinik, Allgemeine Pädiatrie, Düsseldorf, Deutschland, ${ }^{4}$ Med. Einricht. d. Universität Kinderklinik, Neonatologie und pädiatrische Intensivmedizin, Düsseldorf, Deutschland

Hintergrund: Neugeborenenkrampfanfälle sind der häufigste neurologische Notfall auf Neugeborenen-Intensivstationen. Lange ist Phenobarbital (PBT) Mittel der ersten Wahl zur Behandlung gewesen. Zuletzt ist ein deutlicher Trend Richtung Levetiracetam auch zur Primärtherapie erkennbar. Dies ist möglicherweise auf publizierte niedrige Response-Raten auf PBT und Sorge um neuronale Apoptose zu erklären. Wir untersuchten in unserem Zentrum die Therapie von Neugeborenenkrampfanfällen und Ansprechen auf Phenobarbital.

Methode: Eine retrospektive Studie von Neugeborenen, die mit klinisch und/oder elektrophysiologisch diagnostizierten Krampfanfällen auf unserer Neugeborenenintensivstation aufgenommen wurden, wurde durchgeführt. Akten der Patienten wurden von einem Neonatologen und einem Neuropädiater gesichtet, um Krampfanfälle zu bestätigen und demographische Daten, Tag und Art der Anfälle, Ätiologie sowie Art, Dosierung, Dauer der Therapie und Therapieansprechen zu dokumentieren.

Ergebnisse: Zwanzig Neugeborene mit Krampfanfällen innerhalb der ersten 72 Lebensstunden wurden identifiziert. Ein breites ätiologisches Spektrum wurde gefunden, bei 2 Neonaten fand sich keine Ätiologie. Mittel der ersten Wahl war Phenobarbital $(n=19)$ oder Levetiracetam $(n=1)$. Nach PBT zeigten 14 von 19 Neonaten Anfallsfreiheit (64\%). PBT Medikamentenspiegel lagen bei allen Patienten $<50 \mathrm{mg} / \mathrm{dl}$. Non-Responder waren Patienten mit schweren Grunderkrankungen (schwerste HIE $(n=1)$, schwerste Stoffwechselkrankheit $(n=3)$, epileptische Encephalopathie $(n=1)$, die auch auf weitere Add-on Therapie nicht ansprachen. Ein Patient erhielt nur Levetiracetam.

Schlussfolgerung: Das Ansprechen auf Phenobarbital ist generell gut. Non-responder waren Patienten mit schweren, zugrundeliegenden Erkrankungen, die auch auf Mittel der 2. oder 3.Wahl nicht ansprachen, dabei auch Levetiracetam. Bis zur besseren Studienlage, die auch Langzeiteffekte von Levetiracetam untersucht, sollte Phenobarbital Mittel der ersten Wahl zur Behandlung von Neugeborenen-Krampfanfällen bleiben. 
P127

Das neurologische Outcome im korrigierten Alter von 2 Jahren der Frühgeborenen mit einem frühen vorzeitigen Blasensprung (PPROM - preterm premature rupture of membranes) von mindestens 7 Tagen

H. Müller', T. Storbeck', N. Bruns' ${ }^{1}$, D. Katzer ${ }^{2}$, J. Hobrecht ${ }^{1}$, G. WössnerStegmann ${ }^{2}$, A. Köninger ${ }^{3}$, A. Müller'², U. Felderhoff-Müser', S. Bagci ${ }^{2}$

'Universitäts-Kinderklinik Essen, Klinik für Kinderheilkunde I, Essen, Deutschland, ${ }^{2}$ Zentrum für Kinderheilkunde, Universität Bonn, Neonatologie und Pädiatrische Intensivmedizin, Bonn, Deutschland, ${ }^{3}$ Klinik für Frauenheilkunde und Geburtshilfe, Universitätsklinikum Essen, Essen, Deutschland

Hintergrund: Ein früher vorzeitiger Blasensprung (PPROM) ist ein häufiger Grund für die Frühgeburtlichkeit. Es besteht zudem ein hohes Risiko für das Auftreten eines Amnioninfektionssyndroms (AIS), das zu Schäden des unreifen Gehirns führen kann.

Fragestellung: Es soll untersucht werden, ob sich eine Prolongation der Schwangerschaft bei PPROM ohne AIS negativ auf das neurologische Outcome im korrigierten Alter von 2 Jahren (BSIDII) auswirken kann.

Patienten und Methoden: Wir haben eine Fall-Kontroll-Studie mit Kindern der Universitätskinderkliniken Essen und Bonn durchgeführt. Dabei wurde auf ein identisches Geburtsgewicht und Gestationsalter geachtet, und jeweils ein Kind hatte einen vorzeitigen Blasensprung von mindestens 7 Tagen und kein Amnioninfektionssyndrom, ein Kind hatte ein AIS und ein Kind hatte weder einen vorzeitigen Blasensprung noch ein AIS Dies führte zu 3 Gruppen mit jeweils 20 Frühgeborenen (PPROM-, AIS-, Kontrollgruppe).

Ergebnisse: Das Geburtsgewicht und Gestationsalter bei Entbindung (Mittelwert \pm SEM) der Gruppen (PPROM: $1056 \pm 88 \mathrm{~g}, 27,8 \pm 0,5 \mathrm{SSW}$; AIS: $1038 \pm 72$ g, 27,8 $\pm 0,5$ SSW; Kontrolle: $1097 \pm 91$ g, 27,8 $\pm 0,5$ SSW; jeweils $p>0,05)$ war identisch. Die Blasensprung-Dauer in der PPROM-Gruppe betrug im Median 22,4 (10,9-39,3) Tage. Beim Vergleich bezüglich mütterliches Alter, vorzeitige Wehen, Präeklampsie oder HELLP-Syndrom, Plazentainsuffizienz, Plazentalösung, vaginale Blutung und pathologischer Doppler gab es keinen signifikanten Unterschied zwischen den Gruppen. Dagegen gab es signifikante Unterschiede beim maternalen CRP (VBS vs AIS: $p=0,031$ ), bei einem Oligohydramnion (VBS vs AIS und VBS vs Kontrolle jeweils $p<0,0001$ ) und bei einem pathologischen CTG (VBS vs AIS: $p=0,018$; AIS vs Kontrolle: $p=0,016$ ). Das korrigierte Alter zum Zeitpunkt des Bayley-Tests unterschied sich zwischen den Gruppen nicht (PPROM: $25 \pm 0,9$ Monate; AIS: $24 \pm 0,7$ Monate; Kontrolle: $24 \pm 0,7$ Monate; $p>0,05$ ). Es zeigte sich kein signifikanter Unterschied beim MDI- und PDI-Score zwischen den einzelnen Gruppen (Mittelwert \pm SEM): Der MDI-Score betrug bei der PPROM-Gruppe $101 \pm 3$, bei der AIS-Gruppe $98 \pm 3$ und bei der Kontroll-Gruppe $96 \pm 4$ (PPROM vs AIS: $p=0,316$; PPROM vs Kontrolle: $p=0,297$; AIS vs Kontrolle: $p=0,665)$. Bei der PPROM-Gruppe war ein PDI-Score von $96 \pm 2$, bei der AIS-Gruppe von $89 \pm 4$ und bei der Kontroll-Gruppe von $90 \pm 4$ zu beobachten (PPROM vs AIS: $p=0,151$; PPROM vs Kontrolle: $p=0,569$; AIS vs Kontrolle: $p=0,776$ ). Es wurde zudem eine multiple Regressionsanalyse durchgeführt, wobei sich nur der 1-Minuten-APGAR-Wert als Prädiktor für den MDI $(p=0,034)$ und kein Prädiktor für den PDI herausstellte, d.h. auch die Faktoren PPROM und AIS trugen nicht zur Abschätzung des MDI und PDI bei.

Schlussfolgerung: Das Vorliegen eines frühen vorzeitigen Blasensprungs ohne AIS scheint keinen Einfluss auf das neurologische Outcome im Alter von korrigiert 2 Jahren zu haben.
P128

Hirnatrophie eines Frühgeborenen durch präpartalen Vitamin B12 Mangel

V. Lieftüchter', C. Jansen', S. Herber-Jonat' ', K. Förster', M. Klemme', A. Flemmer

${ }^{1}$ Klinikum der Universität München, Marchioninistraße 15, 81377 München, Neonatologie der Kinderklinik am Perinatalzentrum Großhadern, München, Deutschland

Hintergrund: Ein Vitamin B 12 Mangel kann präpartal/intrauterin zu einer kindlichen Dystrophie und in der Folge einer kognitiven Entwicklunsgverzögerung führen. Vitamin B12 ist als Coenzym an der DNA Methylierung, der Hämobglobinbildung und dem Fett- und Eiweißmetabolismus beteiligt.

Kasuistik: Wir berichten von einem Frühgeborenen der 33 1/7 SSW (SGA bei einem $G G<3$. Perz.), welches per Notsectio bei schwerer Präeklampsie mit generalisiert tonisch-klonischem Krampfanfall der Mutter geboren wurde. Die 37-jährige Mutter (G3P2) ernährt sich seit vielen Jahren streng vegan und ist untergewichtig. Der Junge passte sich initial zögerlich an und benötigte im Rahmen eines milden ANS für wenige Tage eine nicht-invasive Atemunterstützung. Bereits im pränatalen Ultraschall zeigte sich eine Seitenventrikel-Erweiterung beidseits und verkürzte Röhrenknochen. In den postnatalen Schädelsonographien bestätigte sich der Befund. Zudem fiel das Frühgeborene mit auffälliger Facies (Dolichocephalus, prominente Sutura metopica, prominente breite Nasenwurzel und hypoplastische Nasenflügel, schmales Lippenrot und Mundspalte, kurzes Zungenbändchen, prominente Maxilla und Mikroretrognathie, tiefsitzenden Ohren), sowie einer häutigen Syndaktylie beidseits und einer Hypospadie auf. In der cMRT zeigte sich eine asymmetrische Volumenminderung des $\mathrm{Pa}$ renchyms und frontale Polymikrogyrie. Vor allem der rechte Seitenventrikel erschien erweitert. Aufgrund der mütterlichen Anamnese bestand der Verdacht eines schweren mütterlichen und kindlichen Vitamin B12 Mangels. Nach laborchemischer Bestätigung behandelten wir das Kind mit Vitamin B12 Gaben i.m.. In den laborchemischen Verlaufskontrollen zeigte sich eine rasche Normalisierung der Methylmalonsäure (Verlaufsmarker). In der Kontroll-cMRT Untersuchung zeigten sich eine zunehmende Gyrierung und Reifung des Gehirns bei weiterhin für das Alterskollektiv erweiterten inneren Liquorräumen sowie V.a. Polymikrogyrie an einzelnen Stellen. Laboruntersuchungen: Typischerweise geht ein Vitamin B12 Mangel mit einer Erhöhung von Methylmalonsäure im Plasma (3466 nmol/l (Norm 73-271nmol/l) und Homocystein $(22,1 \mu \mathrm{mol} / \mathrm{l}$ (Norm $<13,9 \mu \mathrm{mol} / \mathrm{l}$ ) einher. Die Serumspiegel von Vitamin B12 (hier: $453 \mathrm{pg} / \mathrm{ml}$ (Norm 250-900 pg/ml)) bleiben meist normal. Eine megaloblastäre Anämie fiel nicht auf. Das Karyogramm war unauffällig.

Schlussfolgerung: Bei Müttern, die sich über einen langen Zeitraum streng vegan ernähren, besteht das Risiko eines Vitamin B 12-Mangels für sie selbst und das ungeborene Kind. Bei entsprechender Anamnese sollte daher eine Bestimmung der Vit. B12 Metabolite und ggf. eine Vit. B12 Supplementation erfolgen und überwacht werden.

\section{P128a}

Endogenous potential of brain repair: Characterisation of neural progenitor cells in the cerebrospinal fluid of preterm infants

E. Bonilla', R. Spaull', K. Luyt ${ }^{2}$, J. Uney ${ }^{1}$, A. Heep ${ }^{2}$

'University of Bristol, School of Clinical Sciences, Stem Cell and Neurodegeneration Research Group, Bristol, United Kingdom, ${ }^{2}$ University of Bristol, School of Clinical Sciences, Neonatal Neurology Group, Bristol, United Kingdom

Background: Neural progenitor cells (NPCs) populate the periventricular subependymal germinal matrix playing a crucial role in the neuroplasticity of the developing brain. Preterm infants are highly vulnerable to perinatal brain injury. Evidence from post-mortem studies and animal models of neonatal hydrocephalus demonstrate disruption of the subventricular zone and migration of neural progenitor cells into the cerebrospinal fluid (CSF). 
Aims: To establish protocols to identify and characterize NPCs and related gene expression in CSF from preterm infants with post-haemorrhagic ventricular dilataion (PHVD).

Patients, Methods: CSF samples $(n=18)$ from 3 infants (27 weeks median gestational age at birth) undergoing external CSF drainage via Ommaya reservoir for clinical indications were studied. The study was approved by NRES committee (15/YH/0251). i) Immunocytochemistry for stem cell markers (SOX2, glial fibrillary acidic protein, nestin), ii) RNA extraction and digital/quantitative PCR (SOX2, PAX6, EMX-2, Prom1, APP), iii) immunocytochemical cell staining (SOX2) and iv) flourescence assisted cell sorting (FACS) using CD133 surface antigen.

Results: Cell count 3.5/ $\mathrm{\mu l}$ (mean) and $5.2 \mathrm{ml} \mathrm{CSF} / \mathrm{sample}$ (mean). Immunocytochemistry revealed the presence of SOX2+ cells; digital PCR presented gene expression for SOX2, PAX6, Prom1 and APP. Results of FACS analysis for $\mathrm{CD} 133$ were inconclusive.

Conclusions: Our study supports the presence of NPCs in the CSF of preterm infants following perinatal brain injury. The digital PCR technique was found to be ideal for measuring gene expression in these samples with low RNA concentration. Future work will aim to isolate and culture these cells and trial in disease models to support clinical applications for infants affected by brain injury.

\section{Postersitzung 13: Erstversorgung}

\section{P129}

Peripartale Asphyxie als Folge einer intrauterin beginnenden Hämophagozytische Lymphohistiozytose (HLH)

\section{R. John ${ }^{1}$, F. Schmidt-Wankel', G. Reichelt' ', C. Wiethoff' \\ 'Sana Klinikum Offenbach, Neonatologie und pädiatrische Intensiv, Offenbach, Deutschland}

Die HLH ist eine altersunabhängig auftretende, lebensbedrohliche Erkrankung, bei der es zu einer überschießenden Abwehr- und Inflammationreaktion mit Makrophagenaktivierung und Zytokinsturm kommt. Zu den klinischen Symptomen und diagnostischen Kriterien zählen: Fieber, progressive Zytopenie, Hypofribrinogenämie, Hämophagozytose, Ferritin $>500 \mu \mathrm{g} / \mathrm{L}$, Leberfunktionsstörungen, Koagulopathie und neurologische Symptome. Wir berichten über ein hypotrophes Neugeborenes der 38. SSW, Blutgruppe der Mutter O Rhesus negativ, das bei pathologischen CTG per Sectio entbunden wurde. Bei Erstversorgung: Zeichen eines Hydrops fetalis mit Ascites, Pleuraergüssen, generalisiertem und Hirnödem, GG 2146 g, APGAR 2/3/5, muskulär hyperton, pH 6,9; BZ 7 mg/dl; Laktat 124 mg/dl; BE 14,1 mmol/1; Hb 13,3 g/dl, Thrombozyten 95/nl; Leukozyten 11/nl; Stabkernige $2 \%$, Fibrinogen 0.8 g/l, LDH 2272 U/l. Blutgruppe des Kindes 0 Rhesus positiv. Entsprechend der AWMF-Leitlinie für peripartale Asphyxie wurde eine Hypothermiebehandlung eingeleitet. Zusätzlich erfolgte bei persistierender Laktatazidose, schwerster Glucoseutilisationsstörung und erhöhten Ammoniak eine „Metabolische Notfalltherapie“. Bei progressiver Leukozytopenie wurde mit dem Verdacht auf eine HLH die Diagnostik entsprechend erweitert und unmittelbar eine Notfall-Dexamethason-Stoßtherapie eingeleitet. Darunter kam es zu einem Anstieg der Leukozyten. Klinisch verschlechtere sich der Patient zunehmend und verstarb am 5. Lebenstag im Multiorganversagen. Eine Obduktion und weiterführende genetische Untersuchungen wurden abgelehnt. Die Leberfunktionsstörung mit Koagulopathie und die neurologische Symptomatik sind durch peripartale Asphyxie, ein Hydrophs fetalis durch mögliche Rhesusinkompatibilität erklärbar. Panzytopenie, Hypofibrininogenämie, Hyperferritinämie und die Erhöhung des IL-2 Rezeptors gehören zu den Kriterien der HLH. Das klinisch führende Symptom Fieber wurde durch die Hypothermiebehandlung kaschiert. Da weder infektiologische noch metabolische Ursachen nachweisbar waren, ist hier von einer primären (familiären) HLH auszugehen. Aufgrund der ungünstigen Prognose ist eine frühe Diagnose und Therapie unerlässlich. Bei ungewöhnlichen Befundkonstellationen im Rahmen einer Asphyxie sollten seltene Erkankungen wie eine HLH in Betracht gezogen werden.

\section{P130}

Brain activity cycling im amplituden-integrierten EEG was sehen wir in der Polysomnographie?

L. Schmidt' , A. Hausmann' ', Z. Rona', V. Giordano', T. Werther', S. Vanhatalo², A. Berger ${ }^{1}$, K. Klebermass-Schrehof ${ }^{1}$

${ }^{1}$ Universitätsklinik für Kinder- und Jugendheilkunde; Medizinische Universität Wien, Abteilung für Neonatologie, pädiatrische Intensivmedizin und Neuropädiatrie, Wien, Österreich, ${ }^{2}$ University of Helsinki, Department of Neurophysiology and Neurobiology, Helsinki, Finnland

Hintergrund: Brain activity cycling (BAC) ist bereits bei sehr unreifen Frühgeborenen im amplituden-integrierten EEG (aEEG) beschrieben und ist ein Hinweis auf eine intakte Entwicklung des Gehirns. Die gegenwärtige Einteilung des BAC in Quiet (QS) und Active State (AS) im aEEG ist nicht mit der herkömmlichen Einteilung im EEG bzw. Polysomnographie in Active und Quiet Sleep zu vergleichen. Dennoch zeigen die derzeitigen BAC Daten eine Korrelation zwischen der Integrität des zentralen Nervensystems und dem späteren entwicklungsneurologischen Outcome. Daher könnten sie vielversprechende prognostische Marker sein. Das Ziel dieser Studie ist die Analyse der Entstehung von Brain Activity Cycling bei Frühgeborenen, die vor der 28. Schwangerschaftswoche geboren sind in jeweils 10 Minuten Abschnitten Active und Quiet State im aEEG sowie in der Polysomnographie. Methoden und Patienten: Polysomnographien (konventionelle EEGs mit Aufzeichnung von Herzfrequenz, Atemfrequenz und Videoüberwachung) wurden alle zwei Wochen für einen Zeitraum von mindestens 3 Stunden bis zum errechneten Geburtstermin an Frühgeborenen kleiner als 28 Schwangerschaftswochen durchgeführt. Anhand der gleichzeitigen durchgeführten aEEGs (C3-C4) wurden jeweils 10 Minuten QS und AS Abschnitte ausgewählt. Die Interburst-Intervalle (IBI) und die Burst-Dauer (B) wurden im QS und AS visuell analysiert und zusätzlich wurden - unter Verwendung eines automatisierten NLEO- Algorithmus - SAT (= spontaneous activity transients) Prozente (SAT\%) berechnet.

Ergebnisse: Hundertneun Polysomnographien wurden bei 44 Frühgeborenen analysiert. Die mittleren IBIs sanken sowohl in QS und AS mit zunehmendem Gestationsalter (GA) (QS 7,38- 2 ( $\left.\mathrm{r}^{2} 0226\right)$, AS 5- $2\left(\mathrm{r}^{2} 0101\right)$ ) und die mittlere BD stiegen sowohl in QS und AS mit zunehmendem GA (QS 5,69- 22 ( $\left.\mathrm{r}^{2} 0149\right)$, AS 10,83- 26 ( $\left.\mathrm{r}^{2} 0035\right)$ ). Die anhand des automatisierten NLEO- Algorithmus analysierten SAT\% zeigten ebenfalls eine Zunahme in QS und AS mit steigendem GA.

Schlussfolgerung: BAC konnte in unserer Daten schon bei sehr unreifen Frühgeborenen nachgewiesen werden und es zeigte sich eine Korrelation von QS und AS mit steigendem GA. Neue Methoden, insbesondere automatisierte- Analysen, Definitionen und Referenzwerte für Frühgeborenen unter der 28. SSW werden die klinische Anwendbarkeit erweitern und vereinfachen.

\section{P131}

\section{Handy oder Handicap? - Extremfrühgeborene im Schulalter}

F. Seeger ${ }^{1}$, R. Dürr' ${ }^{1}$ U. Bernbeck', A. Aichler ${ }^{1}$, H. Bühler-Weidle', C. West ${ }^{1}$, R. Rauch

'Rems-Murr-Kliniken Winnenden, Pädiatrie, Winnenden, Deutschland

Hintergrund: Es gibt es wenige umfassende Studien zum Langzeit-Outcome von Extrem-Frühgeborenen. Ein Schwerpunkt lag bisher auf den Folgen der bronchopulmonalen Dysplasie. Einige Arbeiten sind weit vor der Ära der nicht-invasiven Beatmung entstanden. Pränatal stellen Eltern heutzutage Fragen nicht nur zum Risiko von Organschäden, sondern v. a. zur Teilhabe des Kindes am gesellschaftlichen Leben.

Fragestellung: Wir untersuchten, 1. welche somatischen Einschränkungen bei den in unserem Level1-Zentrum betreuten Extrem-Frühgeborenen vorliegen und 2. in wie weit ihre intellektuelle und emotionale Situation ihnen eine Teilhabe am Leben Gleichaltriger erlaubt.

Methoden: Es wurden Eltern aller überlebenden Frühgeborenen unserer Level 1-Neonatologie mit einem Geburtsgewicht $<1500 \mathrm{~g}$ aus den Geburtsjahren 2006-2008 zur Nachuntersuchung eingeladen, 24 nahmen teil. Sie wurden jeweils von spezialisierten Fachärzten kardial (Echo, EKG), pulmonal (Lungenfunktion, Spiroergometrie) und neuropädiatrisch un- 
tersucht. Zudem wurde eine psychologische Leistungstestung (K-ABC II) und eine emotionale Untersuchung vorgenommen.

Resultate: In der Neonatalperiode wiesen 12 (50\%) schwere Komplikationen (ANS III-IV ${ }^{\circ}$, BPD, Pneumothorax, IVH III-IV ${ }^{\circ}$, NEC, operativer PDA-Verschluss) auf. Von 8 Kindern mit auffälligen Bailey-Testungen hatten zum Studienzeitpunkt noch 2 eine schwere Cerebralparese. Alle anderen waren neurologisch unauffällig und nicht in ärztlicher Therapie. Die Regelschule bzw. -Kindergarten besuchten 20 von 24 Kindern (83\%), 17 waren Mitglied in einem Sportverein (71\%). Bei 2 Kindern lagen milde kardiologische Auffälligkeiten vor (Sinusbradykardie, bikuspide Aortenklappe bei Klinefelter-Syndrom). Obwohl 23/24 Kindern pp. Atemunterstützung hatten, lagen FEV1 (84\%) und Vitalkapzität (82\%) ebenso wie die Ergebnisse der Spiroergometrie im Normbereich. In der IQ-Testung waren 15 von 18 (83\%) untersuchten Kindern normal. Bei 5 Kindern ergaben sich leichtere emotionale Beeinträchtigungen.

Zusammenfassung: Insgesamt lag ein gutes somatisches und psychisches Langzeit-Outcome unserer Patienten vor. Im Vergleich zur Bailey-Testung mit 2 Jahren zeigte sich eine weitere neurologische Erholung bis zum Schulalter. Lediglich $25 \%$ der Kinder erhielten weitere Förderung. Schwachpunkt der Untersuchung ist ein Bias durch Verlust von Patientenkontakten. Größere Studien zum Langzeit-Outcome von Extremfrühgeborenen sind nötig, um in Pränatalgesprächen konkretere Aussagen treffen zu können.

\section{P132}

\section{Volumensubstitution in der neonatalen Erstversorgung: Balancierte Vollelektrolytlösung vs. $\mathrm{NaCl} 0,9 \%$}

\section{Heinecke ${ }^{1}$, J. Kersten ${ }^{2}$, S. Schmidtke ${ }^{3}$, D. Singer ${ }^{4}$}

${ }^{1}$ Altonaer Kinderkrankenhaus, Hamburg, Deutschland, ${ }^{2}$ Universitätsklinikum Eppendorf, Institut für Medizinische Biometrie und Epidemiologie, Hamburg, Deutschland, ${ }^{3}$ Asklepios Klinik Barmbek, Abteilung Neonatologie, Hamburg, Deutschland, ${ }^{4}$ Universitätsklinikum Eppendorf, Sektion Neonatologie und Pädiatrische Intensivmedizin, Hamburg, Deutschland

Hintergrund und Fragestellung: Die internationalen Reanimationsleitlinien empfehlen zur Volumensubstitution in der neonatalen Erstversorgung isotone kristalloide Infusionslösungen, machen über deren genaue Zusammensetzung aber keine Angaben. Generell hat sich bei den kristalloiden Lösungen ein Wechsel von der „physiologischen“ Kochsalzlösung zu sog. balancierten Vollelektrolytlösungen vollzogen, weil $\mathrm{NaCl} 0,9 \%$ besonders nach großvolumiger Zufuhr eine hyperchlorämische "Dilutionsazidose“ verursachen soll. Es wurde daher untersucht, ob sich nach Umstellung von $\mathrm{NaCl}$ 0,9\% auf eine balancierte Lösung (SterofundinR ISO, B. Braun Melsungen AG) Änderungen im Säure-Basen- und Elektrolythaushalt der im Kreißsaal erstversorgten Neu- und Frühgeborenen erkennen ließen.

Material und Methoden: Retrospektive Beobachtungsstudie an $114 \mathrm{Neu}$ und Frühgeborenen, von denen 65 (in dem halben Jahr vor der Umstellung) $\mathrm{NaCl}$ 0,9\% und 49 (in dem halben Jahr danach) SterofundinR ISO erhalten hatten. Die Gruppen entsprachen sich in Reifealter und Geburtsgewicht, der mittlere Nabelarterien-pH war in der Sterofundin-Gruppe niedriger als in der $\mathrm{NaCl}$-Gruppe $(7,1 \pm 0,1$ vs. $7,2 \pm 0,1 ; p=0.02)$ Das mittlere Infusionsvolumen betrug $7,3 \pm 3,4 \mathrm{ml} / \mathrm{kg}$ in der NaCl- und $7,6 \pm 3,2 \mathrm{ml} / \mathrm{kg}$ in der Sterofundin-Gruppe $(p=0,62)$. Aus der routinemäßig abgenommenen ersten und zweiten Blutgasanalyse im Kreißsaal und auf der neonatologischen Intensivstation (d. h. vor und nach erfolgter Volumensubstitution) wurden die direkt messbaren Parameter $\mathrm{pH}, \mathrm{pCO}_{2}$, Laktat und Chlorid ausgewertet.

Ergebnisse und Diskussion: In der Entwicklung der H+-Konzentration über die Zeit (Abklingrate einer milden perinatalen Azidose) zeigte sich zwischen der NaCl-Gruppe $(-0,196 \pm 0,04 \mathrm{nmol} / \mathrm{l} / \mathrm{min})$ und der Sterofundin-Gruppe $(-0,204 \pm 0,04 \mathrm{nmol} / \mathrm{l} / \mathrm{min})$ kein signifikanter Unterschied $(p=0,89)$. Das Gleiche galt für den Zeitverlauf von $\mathrm{pCO}_{2}$ (NaCl: $-0,13 \pm 0,30 \mathrm{mmHg} / \mathrm{min}$; Sterofundin: $-0,15 \pm 0,36 \mathrm{mmHg} / \mathrm{min}$; $p=0,62)$ und Laktat $(\mathrm{NaCl}:-0,024 \pm 0,004 \mathrm{mmol} / \mathrm{l} / \mathrm{min}$; Sterofundin: $-0,018 \pm 0,004 \mathrm{mmol} / \mathrm{l} / \mathrm{min} ; p=0,32$ ) als Parameter für die respiratorische und die metabolische Komponente des Säure-Basen-Status. Ebensowenig erwiesen sich die absoluten Chloridkonzentrationen in der zweiten
Blutentnahme (NaCl: $108 \pm 0,49 \mathrm{mmol} / \mathrm{l}$; Sterofundin: $107 \pm 0,44 \mathrm{mmol} / \mathrm{l}$; $p=0,09)$ als verschieden (Mean \pm SE). Die Studie ist durch das retrospektive Design, die begrenzte Fallzahl und die gewählten Parameter limitiert; außerdem bleibt offen, ob sich bei größeren Infusionsvolumina $(\geq 10 \mathrm{ml} / \mathrm{kg}$ ) deutlichere Unterschiede gezeigt hätten. Das Ergebnis reiht sich jedoch in die kontroverse Diskussion um die praktische Relevanz balancierter Vollelektrolytlösungen in der Volumenersatztherapie ein.

Schlussfolgerung: Für die Volumensubstitution in der neonatalen Erstversorgung scheinen $\mathrm{NaCl}$ 0,9\% und eine balancierte Vollelektrolytlösung hinsichtlich ihrer Effekte auf den Säure-Basen- und Elektrolythaushalt gleichwertig zu sein.

\section{P133}

\section{Stimulation bei reifen Neugeborenen während der Erstversorgung}

G. Pichler', N. Baik', M. Rock', K. Binder', L. Mileder', B. Schwaberger', J. Stadler', B. Urlesberger

'Univ. Klinik für Kinder- und Jugendheilkunde, Abt. für Neonatologie, Graz, Österreich

Hintergrund: In den ERC-Guidelines für Neugeborenen-Erstversorgung werden das Abtrocken und eine „kurze“ Stimulation empfohlen. Effekte einer Stimulation wurden bisher jedoch nicht untersucht.

Zielsetzung: Ziel dieser Studie war es, bei den reifen Neugeborenen zu untersuchen, welche Körperregion wie lange während der Erstversorgung stimuliert wurden und ob die Stimulation einen Effekt auf die Vitalparameter hatte.

Material und Methode: Es wurde eine Analyse von Videoaufzeichnungen, welche im Rahmen von prospektiven Beobachtungs-Studien an der Abteilung für Neonatologie/Medizinischen Universität Graz bei reifen Neugeborenen während der Erstversorgung gemacht wurden, durchgeführt. Die Videoaufzeichnungen wurden von zwei Untersuchern hinsichtlich Dauer und Körperregion von Stimulationen analysiert. Peripher-arterielle Sauerstoffsättigung $\left(\mathrm{SpO}_{2}\right)$ und Herzfrequenz $(\mathrm{HF})$ wurden kontinuierlich mittels Pulsoxymetrie gemessen. Um eventuelle Effekte der Stimulation auf die Vitalparameter zu untersuchen, wurden $\mathrm{SpO}_{2}$ und $\mathrm{HF}$ vor und nach der Stimulation verglichen.

Ergebnisse: Insgesamt wurden 52 reife Neugeborene in dieser Studie analysiert. Das mittlere Gestationsalter betrug 38,9 $\pm 0,9$ Wochen und das mittlere Geburtsgewicht $3301 \pm 414$ g. 28 von 52 reifen Neugeborenen wurden im Rahmen der Erstversorgung stimuliert. Es wurden im Median 2 (Range 1-13) Stimulationen pro Neugeborenes durchgeführt. Die Stimulation erfolgte bei 8 reifen Neugeborenen an der Brust, bei 5 an den Füßen und bei 15 an mehreren Körperregionen (Brust, Fuß). Im Vergleich vor und nach der Stimulation konnte kein signifikanter Unterschied sowohl von Herzfrequenz (vor: $158 \pm 43 / \mathrm{min}$ versus nach: $159 \pm 46 / \mathrm{min}$; $p>0,05$ ) als auch von $\mathrm{SpO}_{2}$ (vor: $69 \pm 17 \%$ versus nach: $69 \pm 19 \% ; p>0,05$ ) beobachtet werden.

Schlussfolgerung: Die vorliegende Beobachtungstudie beschreibt erstmalig Stimulationen während der Erstversorgung bei reifen Neugeborenen, wobei die Stimulationen keinen Effekt auf $\mathrm{HF}$ und $\mathrm{SpO}_{2}$ hatten.

\section{P134}

Status quo in pediatric and neonatal simulation in the European German-speaking countries: The DACHI survey

M. Wagner', E. Heimberg' ${ }^{2}$, L. Mileder ${ }^{3}$, A. Staffler', A. Berger' ${ }^{1}$, R. Loellgen ${ }^{5}$ ${ }^{1}$ Medical University of Vienna, Division of Neonatology, Pediatric Intensive Care and Neuropediatrics; Department of Pediatrics, Vienna, Österreich, 2University Children's Hospital Tuebingen, Department for Pediatric Cardiology, Pulmonology and Intensive Care Medicine, Tuebingen, Deutschland, ${ }^{3}$ Medical University of Graz, Division of Neonatology; Department of Pediatrics and Adolescent Medicine, Graz, Österreich, ${ }^{4}$ Central Teaching Hospital of Bolzano, Department of Neonatology, Bolzano, Italien, ${ }^{5}$ Inselspital Bern, Pediatric Emergency Department, Bern, Schweiz 
Background: Simulation has acquired wide acceptance as an important component of education in health care and as a patient safety tool. Successful recognition and resuscitation of critically ill or injured children requires proficient technical and non- technical skills1,2. While the simulated practice of these competencies has been well established in most Anglo-Saxon countries3, simulation training is still not established in many European countries.

Research question: The authors of this article and founding members of the Netzwerk Kindersimulation4 (Pediatric Simulation Network) aimed to survey all pediatric and neonatal health care institutions and departments in Germany (D), Austria (A), Switzerland (CH) and the German-speaking part of Italy, South Tyrol (I) (DACHI) on their current status of pediatric and neonatal simulation-based training.

Methodology: We composed a comprehensive online survey including 26 questions on the distribution and organization, target groups, resources, instructor and participant education as well as research activities among all pediatric institutions and departments in DACHI. The questionnaire was validated according to established models5 including a pre-test and distributed electronically by the online tool SurveyMonkey ${ }^{\circ}$. RESULTS After dispatching a total of 472 surveys ( $n=359$ in D, $n=41$ in A, $n=65$ in $\mathrm{CH}$ and $n=7$ in South Tyrol) we achieved a $25.8 \%$ response rate, with 62 responders from $\mathrm{D}$ ( $17.3 \%$ out of all $\mathrm{D}), 33$ from $\mathrm{A}$ ( $80.5 \%$ out of all $\mathrm{A}), 21$ from $\mathrm{CH}(32.3 \%$ out of all $\mathrm{CH})$ and 6 from I ( $85.7 \%$ out of all I), at the time of abstract submission. $67.2 \%$ of all surveyed institutions already perform simulation-based training, including algorithm training (89.4\%), simulation-based skills training (60.6\%), high fidelity simulation training $(53 \%)$ and other courses (10.6\%). Only $14.3 \%$ of all inquired institutions actively conduct research in the area of simulation, whereas $66.1 \%$ did express an interest in research regarding simulation. The most frequently stated impediments for establishing pediatric simulation-based training were lack of personnel (64.9\%) and financial (59.5\%) resources.

Discussion and conclusion: The significant response rate of our survey underlines the presence of a previously unknown, but strong interest in pediatric and neonatal simulation in the DACHI region. The strength of this questionnaire lies in the identification of a large number of already existing simulation-based training programs in the DACHI region. Our findings provide an initial position for the advocacy and expansion of simulation in pediatrics. The better knowledge of national and international resources, along with the recent foundation of the Netzwerk Kindersimulation4 will support a more effective collaboration in education, training, quality improvement, research and resource management among all simulation enthusiasts in this region aiming to ultimately increase patient safety of children and neonates.

\section{P135}

\section{Das Netzwerk Kindersimulation e.V. -}

\section{Konzept und praktische Umsetzung}

L. Mileder', M. Wagner' ${ }^{2}$, R. Löllgen ${ }^{3}$, A. Staffler', A. Paulun ${ }^{5}$, R. Klein ${ }^{6}$, S. Trepels-Kottek', E. Heimberg ${ }^{8}$

'Univ.-Klinik für Kinder- und Jugendheilkunde, Medizinische Universität Graz, Klinische Abteilung für Neonatologie, Graz, Österreich, ${ }^{2}$ Medizinische Universität Wien, Abteilung für Neonatologie, Pädiatrische Intensivmedizin und Neuropädiatrie, Univ.-Klinik für Kinder- und Jugendheilkunde, Wien, Österreich, ${ }^{3}$ Inselspital Bern, Notfallzentrum für Kinder und Jugendliche, Bern, Schweiz, ${ }^{4}$ Zentrales Lehrkrankenhaus Bozen, Abteilung für Neonatologie, Bozen, Italien, ${ }^{5}$ Asklepios Klinik Sankt Augustin, Neonatologie und Pädiatrische Intensivmedizin, Sankt Augustin, Deutschland, ${ }^{6}$ Managing Human Resources, München, Deutschland, ${ }^{7}$ Uniklinik RWTH Aachen, Kinderklinik Sektion Neonatologie und Kinderintensivmedizin, Aachen, Deutschland, ${ }^{8}$ Eberhard-Karls-Universität Universitätsklinik für Kinderheilkunde und Jugendmedizin, Interdisziplinäre Pädiatrische Intensivstation, Tübingen, Deutschland

Hintergrund: Simulationsbasierte Aus- und Weiterbildung führt zu signifikanten Verbesserungen von kognitiven, technischen und nichttechnischen Fertigkeiten und ist mit einem positiven Effekt auf Patientensicherheit und -outcome assoziiert. Der zunehmende Einzug von Simulation in die medizinische Ausbildung spiegelt sich nicht zuletzt in der Gründung internationaler Fachgesellschaften wider. Die Anwendung von medizinischer Simulation ist im deutschsprachigen Raum jedoch nach wie vor heterogen. Fragestellung: Bislang existieren keine umfassenden Empfehlungen bezüglich der Ausbildung und Zertifizierung von Instruktoren und im Speziellen keine Standards zur Durchführung, Dauer und Häufigkeit von simulationsbasiertem Training. Als eine mögliche Ursache dafür kann das bisherige Fehlen einer Fachgesellschaft angesehen werden, die sich dezidiert der simulationsbasierten pädiatrischen Aus- und Weiterbildung im deutschsprachigen Raum widmet.

Material und Methoden: Die Idee zur Gründung des Netzwerkes Kindersimulation basiert auf einer Kooperation von Personen, die im deutschsprachigen Raum in simulationsbasierter pädiatrischer Aus- und Weiterbildung aktiv tätig sind. Im Rahmen der 2. Kindernotfalltage 2015 in Garmisch-Partenkirchen fand ein erstes Treffen mit 47 Teilnehmern statt. Das zweite Netzwerktreffen fand, dank Unterstützung der Gesellschaft für Neonatologie und Pädiatrische Intensivmedizin (GNPI), im Zuge der 41. Jahrestagung in Stuttgart statt. Zu den herausragenden Ergebnissen dieses Treffens zählen die Entscheidung zur Gründung eines unabhängigen gemeinnützigen Vereins und die Präsentation von Homepage (https:// www.netzwerk-kindersimulation.org/) und Vereinslogo.

Ergebnisse: Das Netzwerk Kindersimulation wurde am 17. Oktober 2015 in München in Anwesenheit von 34 Personen aus Deutschland, Österreich, der Schweiz, Südtirol und Luxemburg gegründet. Als Vereinszweck wurde der „Ausbau und die Verbesserung der Aus- und Weiterbildung sowie Forschung in der präklinischen und klinischen Versorgung von Kindern und Jugendlichen durch simulationsbasierte Konzepte und dadurch die Optimierung der Patientensicherheit, in diesem Fall der pädiatrischen Patienten jeden Alters" definiert. Seit der Eintragung in das deutsche Vereinsregister am 25. November 2015 ist das Netzwerk Kindersimulation als „Netzwerk Kindersimulation e. V.“ gemeldet.

Diskussion: Es ist die primäre Aufgabe des Netzwerkes Kindersimulation e. V., den Paradigmenwechsel von ,see one, do one, teach one“ $\mathrm{zu}$,see one, simulate many, do one" zu unterstützen und zu fördern. Die Aufklärung von medizinischem Fachpersonal und politischen Verantwortungsträgern, Forschungstätigkeiten und Kooperationen mit Fachgesellschaften werden dazu beitragen, die Aus- und Weiterbildung in der präklinischen und klinischen Pädiatrie, Neonatologie, Kinderchirurgie, Kinderintensivund -notfallmedizin im gesamten deutschsprachigen Raum so effektiv und sicher wie möglich zu gestalten.

\section{P136}

Arterial Oxygen Saturation $\left(\mathrm{SpO}_{2}\right)$ and cerebral regional tissue oxygenation $\left(\mathrm{CrSO}_{2}\right.$ ) during automated control of inspired oxygen in a model of delivery room management in preterm lambs

M. Hütten', T. Goos' ${ }^{2}$ P. Andriessen ${ }^{3}$, D. Ophelders ${ }^{4}$, T. Mohns ${ }^{3}$, I. Reiss ${ }^{5}$, J. Dankelman ${ }^{6}$, B. Kramer

'und Maastricht University Medical Center, Neonatology, Maastricht, NL, Neonatologie, Klinik für Kinder- und Jugendmedizin, RWTH Aachen, Aachen, Deutschland, 'Department of Pediatrics, Division of Neonatology, Erasmus Medical Centre, Rotterdam, Department of Biomechanical Engineering, Delft University of Technology, Delft, NL, Rotterdam, Niederlande, ${ }^{3}$ Máxima Medical Centre, Department of Pediatrics, Veldhoven, Niederlande, ${ }^{4}$ Maastricht University Medical Center, Department of Pediatrics, Maastricht, Niederlande, ${ }^{5}$ Erasmus MC-Sophia Children's Hospital, Dept. of Pediatrics, Division of Neonatology, Rotterdam, Niederlande, ${ }^{6}$ Department of Biomechanical Engineering, Delft University of Technology, Delft, NL, Delft, Niederlande

Introduction: Hyperoxemia and hypoxemia may contribute to morbidity and mortality of preterm infants, e. g. by compromised neurodevelopmental outcome. Hypoxemia has been shown to influence cerebral oxygenation. Automated closed-loop control of the fraction of inspired oxygen $\left(\mathrm{FiO}_{2}\right)$ helps to keep preterm infants receiving ventilation within predefined limits of arterial oxygen saturation $\left(\mathrm{SpO}_{2}\right)$ during neonatal intensive 
care, but might already be beneficial in the delivery room. We hypothesized that automated $\mathrm{FiO}_{2}$ control in the delivery room might help to stabilize cerebral regional tissue oxygenation $\left(\mathrm{CrSO}_{2}\right)$ in a preterm lamb model of preterm respiratory distress.

Materials and methods: In a comparison trial of 22 preterm lambs receiving automated or manual $\mathrm{FiO}_{2}$ control directly after operative delivery, 12 animals received additional $\mathrm{CrSO}_{2}$ measurement by near-infrared spectroscopy (NIRS, InvosTM, Covidien). All animals received standardized resuscitation including endotracheal intubation and followed by volume-controlled ventilation for 3 hours. $\mathrm{SpO}_{2}$ measured by pulse oximetry was used as target variable for $\mathrm{FiO}_{2}$. Baseline $\mathrm{crSO}_{2}$ was defined as $\mathrm{crSO}_{2}$ after initial stabilization.

Results: Baseline $\mathrm{CrSO}_{2}$ did not differ between groups, but animals receiving automated $\mathrm{FiO}_{2}$ control reached baseline $\mathrm{crSO}_{2}$ earlier (median $412 \mathrm{sec}$, IQR [242-623 sec] vs. $833 \mathrm{sec}$ [783-883 sec]). During subsequent ventilation, animals receiving automated $\mathrm{FiO}_{2}$ control spent more time within the $\mathrm{SpO}_{2}$ target range, although missing statistical significance $(94.4 \%$ [85.8-97.6\%] vs. $84.4 \%$ [75.9-90.4\%], $p=0.082$ ). In contrast, deviation from average $\mathrm{CrSO}_{2}$ from a $+/-5 \%$ range showed a high variability in both groups (time outside range $13.9 \%$ [9.1-37.3\%] vs. 9.6\% [1.7-34.0\%], n.s.). We did not observe a correlation between average $\mathrm{SpO}_{2}$ and $\mathrm{crSO}_{2}$ or $\mathrm{FiO}_{2}$ and $\mathrm{CrSO}_{2}$.

Conclusions: Automated $\mathrm{FiO}_{2}$ is a promising tool for $\mathrm{SpO}_{2}$ control during delivery room management and postnatal ventilation in preterm infants. Although $\mathrm{crSO}_{2}$ measurement during transition was feasible, the high postnatal variability of $\mathrm{CrSO}_{2}$ we observed indicates that stabilizing $\mathrm{SpO}_{2}$ by automated $\mathrm{FiO}_{2}$ control might only have minor effects on cerebral oxygenation directly after birth.

\section{P137}

\section{Morbus Hämolyticus Neonatorum ohne Hyperbilirubinämie und Hydrops Fetalis}

S. Schwartz' ', A. Zimmermann'1, W. Müller' ${ }^{2}$ S. Paepke ${ }^{3}$, E. Rieger-Fackeldey ${ }^{1}$ ${ }^{1}$ Kinderklinik Schwabing, Klinikum Rechts der Isar der TU München, Neonatologie, München, Deutschland, ${ }^{2}$ Institut für Klinische Chemie, Klinikum Rechts der Isar der TU München, München, Deutschland, ${ }^{3}$ Frauenklinik und Poliklinik, Klinikum Rechts der Isar der TU München, München, Deutschland

Hintergrund: Der Morbus Hämolyticus Neonatorum wird durch transplazentare Übertragung von mütterlichen Antikörpern gegen Antigene der kindlichen Erythozytenmembran verursacht. Meist handelt es sich um Anti-D-AK, seltener gegen Antikörper gegen A- oder B-Antigene. Dadurch kommt es zur Hämolyse beim Fetus mit schwerer Hyperbilirubinämie bis Kernikterus sowie schwerer Anämie bis Hydrops fetalis.

Fall: Ein Neugeborenes aus $40+2$ SSW fällt postnatal durch reduzierten AZ, Blässe und muskuläre Hypotonie auf. In der laborchemischen Untersuchung $\mathrm{Hb}$ 10,5 g/dl, Hkt 34,3\%, IL 6636 pg/ml, LDH 243U/l Es erfolgten eine Transfusion mit $15 \mathrm{ml} / \mathrm{kg}$ EK der Blutgruppe A Rh negativ sowie die sofortige antibiotische Behandlung. Mutter: 1 G, 1 P, Blutgruppe laut Mutterpass 0 rh neg. Rhesusprophylaxe in der Schwangerschaft erhalten. Kein Hinweis auf Amnioninfektionssyndrom, GBS neg. Keine frühere Schwangerschaft bekannt, vor 2 Jahren nach Absetzen der Verhütung jedoch sehr unregelmäßige Menses. Diagnostik: Kind Blutgruppe A Rh positiv, stark erhöhter Anti-D-Titer, der nicht durch die Rhesusprophylaxe zu erklären war. Bei der Mutter wurde die seltene Blutgruppe Ax (Ax01/O02) molekulargenetisch festgestellt. Behandlung: Nach der ersten Transfusion mit A rh neg EK erneut rasch absinkender Hkt bis 19,7\% und $\mathrm{Hb}$ bis 6,4\%. 2. Transfusion mit EK 0 rh neg nach 7 Tagen, im weiteren Verlauf war nach 14 Tagen eine weitere Transfusion erforderlich. Das Kind wurde gesund nach Hause entlassen. Diskussion: Die Mutter hatte vermutlich 2 Jahre zuvor einen unerkannten Frühabort mit Rhesussensibilisierung. In der 2. Schwangerschaft kam es zur Übertragung von Anti-D-AK zu einem späten Zeitpunkt auf den Feten und trotz Rhesusprophylaxe zur kindlichen Hämolyse ohne Hyperbilirubinämie. Die initial transfundierten Erythrozyten der BG A wurden bei irregulären $\mathrm{AK}$ gegen $\mathrm{A}$ ebenfalls rasch hämolysiert.
Schlussfolgerung: Vermutlich kam es aufgrund einer späten AK-Übertragung in der Schwangerschaft nicht zu einem Hydrops. Warum bei ausgeprägter Hämolyse keine Hyperbilirubinämie auftrat, bleibt ungeklärt. Erschwerend kamen irreguläre AK gegen BG A hinzu bei seltener und erst später korrekt bestimmter Blutgruppe der Mutter.

\section{P138}

\section{Seltene Differentialdiagnose des akuten Abdomens}

S. Haufe' ${ }^{1}$, E. Robel-Tillig²

${ }^{1}$ St. Georg Leipzig, Neonatologie, Leipzig, Deutschland, ${ }^{2}$ Klinikum St. Georg gGmbH, Neonatologie, Leipzig, Deutschland

Hintergrund: Das klinische Bild des akuten Abdomens bei FG kann unterschiedliche Ursachen haben. Neben häufigen Diagnosen wie Perforationen oder NEC kann auch Gefäßläsionen durch NVK kausal verantwortlich sein.

\section{Fallbericht:}

- FG der 26.SSW, GG 900 g, klinisch unauffälliger Verlauf

- CPAP, Infusionstherapie über NVK

- Am 8. LT akute ausgeprägte abdominelle Distension, massives Labienund OS-Ödem re $>$ li,

- Tachykardie, pulmonal stabil

- Labor: CRP<1 mg/l, Leuko 24,5, SBH mäßige metabolische Azidose

- Sono - Abdomen: massiv freie Flüssigkeit

- Rö - Thorax: unveränderte Position des NVK auf Höhe des Zwerchfells

- Punktat der Aszites: leicht blutig tingierte weiße seröse Flüssigkeit

Differenzialdiagnosen:

- NEC

- Spontane intestinale Perforation

- inkarzerierte Leistenhernie

bei Sonographie- und Punktionsbefund und klinisch relativ stabiler Situation:

- !!!Dislokation des NVK trotz röntgenologisch korrekter Lage

Vorgehen:

- Entfernung des NVK

- Anlage eines peripher-zentralen Katheters

- Volumensubstitution nach intraabdominellen „Verlusten“

Schlussfolgerung: Dislozierte Gefäßkatheter können zur akuten Ergüssen in Körperhöhlen mit akuter klinischer Symptomatik führen

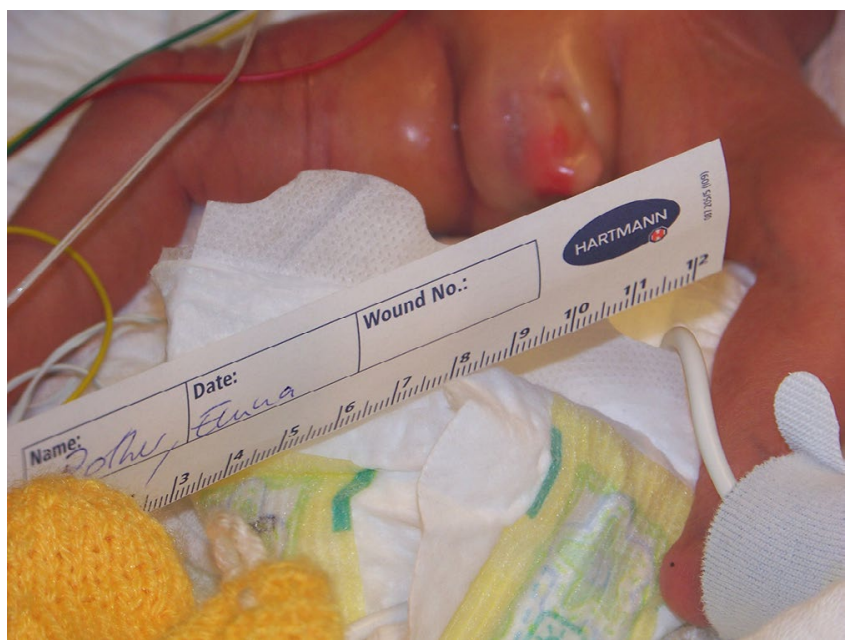


P139

\section{Infusionsbedingte schwere symptomatische Hyperglykämien -} eine Umfrage auf deutschen Kinderintensivstationen

\section{A. Große Lordemann', C. Dohna Schwake', U. Felderhoff-Müser \\ 'Universitätsklinikum Essen, Kinderklinik 1, Essen, Deutschland}

Hintergrund: Die intravenöse Gabe von Glukose-Elektrolytlösungen ist eine Standardanwendung in Kliniken, um eine unzureichende enterale Aufnahme von Glukose kurz- oder langfristig auszugleichen. Bei der infusionsbedingten schweren Hyperglykämie handelt es sich um eine akzidentell zu hohe Gabe von Glukose intravenös. Einzelfälle finden sich häufiger in der Laienpresse als in der wissenschaftlichen Literatur.

Fragestellung: Erfassung epidemiologischer und klinischer Daten, sowie Darstellung und kritische Bewertung verschiedener Therapieoptionen bei infusionsbedingter, schwerer Hyperglykämie. Material und Methoden: Retrospektive Erhebung in 105 deutschen Abteilungen für neonatologische und pädiatrische Intensivmedizin. Fälle von akzidenteller, schwerer Hyperglykämie wurden anonymisiert mittels eines Fragebogens gemeldet. Ergebnisse: Von den 105 kontaktierten Intensivstationen antworteten 50 (Rücklaufquote 47,6\%). Es wurden insgesamt 12 Fälle (Alter 2 Tage bis 15 Jahre) einer akzidentellen, schweren Hyperglykämie im Zeitraum 20012015 gemeldet. Die verabreichten i.v.-Lösungen beinhalteten Glukosekonzentrationen von $12,5 \%$ bis $70 \%$ und waren bei 10 Patienten in zu hoher Laufgeschwindigkeit verabreicht worden. Bei einem Patienten war eine falsche Zusammensetzung der Infusionslösung festgestellt worden, einmal blieb die Ursache unklar. Der maximal gemessene Blutzuckerwert betrug $2240 \mathrm{mg} /$ dl, der minimal gemeldete Wert $539 \mathrm{mg} / \mathrm{dl}$. Im Durchschnitt dauerte es 7,5 Stunden, bis der Blutzucker $<180 \mathrm{mg} / \mathrm{dl}$ war (2-23 Stunden). In den 12 Stunden nach Diagnosestellung trat bei 2 Patienten eine Hyponatriämie, bei einem Patienten eine Hypoglykämie und bei 5 Patienten eine metabolische Azidose auf. In 8 Fällen wurde Insulin verabreicht, was zu einer Senkung des Blutzuckerwertes $<180 \mathrm{mg} / \mathrm{dl}$ in durchschnittlich 6,5 Stunden führte (10 Stunden ohne Insulin). Zweimal war eine Hämodialyse und 6x eine Intubation notwendig. Bei 5 Patienten traten Krampfanfälle auf. In der bildgebenden Diagnostik wurde bei zwei Patienten ein Hirnödem, bei zwei Patienten intrazerebrale Blutungen und bei einem Patienten Hygrome gesehen. Zwei der Kinder verstarben. Bei einem Patienten entwickelte sich eine Zerebralparese mit psychomotorischer Retardierung. Ein Patient blieb niereninsuffizient. In 4 Fällen erfolgte eine kritische Aufarbeitung des Zwischenfalls innerhalb der Klinik. Diskussion oder Schlussfolgerung: Die infusionsbedingte schwere Hyperglykämie stellt eine potentiell lebensbedrohliche vermeidbare Komplikation einer parenteralen Ernährung dar. In nur vier Fällen wurde der Vorfall intern aufgearbeitet, und es wurden Konsequenzen für das weitere Handeln gezogen. Die 12 gemeldeten Patienten sind aus unserer Sicht nur als Spitze eines Eisbergs aus Behandlungskomplikationen zu sehen. Jede Klinik ist in der Verantwortung, Sicherheitssysteme (CIRS, 4-Augen-Prinzip u. a.) zur Vermeidung dieser Komplikationen zu etablieren und zu kontrollieren.

\section{Postersitzung 14: PICU}

\section{P140}

\section{Toxic-Schock-Syndrom durch Streptococcus pyogenes} bei einem dreijährigen Mädchen

L. Schulze' ${ }^{1}$ S. Buchholtz' ${ }^{1}$, A. Wroblewski², H. Graffstädt ${ }^{3}$, H. Giest ${ }^{3}$, B. Schmidt ${ }^{4}$ 'St. Joseph Krankenhaus Berlin Tempelhof, Neonatologie und Pädiatrische Intensivmedizin, Berlin, Deutschland, ${ }^{2}$ St. Joseph Krankenhaus Berlin Tempelhof, Pädiatrische Intensivmedizin, Berlin, Deutschland, ${ }^{3}$ St. Joseph Krankenhaus Berlin Tempelhof, Kinderchirurgie, Berlin, Deutschland, ${ }^{4} \mathrm{St}$. Joseph Krankenhaus, Berlin Tempelhof, Kinder-/Jugendmedizin, Berlin, Deutschland

Einleitung. Durch Streptokokken verursachte Schleimhautinfektionen gehören zu den häufigsten bakteriellen Erkrankungen im Kindesalter mit einem Altersgipfel zwischen 6 und 12 Lebensjahren. Das Streptokok-
ken-Toxic-Schock-Syndrom (STSS) zählt, wie auch der Scharlach, zu den toxinvermittelten Erkrankungen und erreicht als eigene Krankheitsentität eine Letalitätsrate von bis zu $80 \%$.

Fallbericht. Wir berichten über ein dreijähriges Mädchen, welches mit Fieber und Abgeschlagenheit vorstellig wird. Klinisch imponiert ein scarlatiformes Exanthem mit ausgeprägter Tonsillitis und Tachydyspnoe. Das Mädchen befindet sich in stark reduziertem Allgemeinzustand mit Vigilanzminderung bei noch stabilen Vitalparametern. Paraklinisch sehen wir stark erhöhte Entzündungswerte. Es kommt rasch zur kardiopulmonalen Dekompensation sowie Intubation und intensivmedizinischer Maximaltherapie mit ausgeprägtem Katecholaminbedarf zzgl. Hydrocortison. Es zeigt sich eine massive Zunahme des Exanthems mit Haarausfall und großflächiger Blasenbildung. Anhand des klinischen Bildes eines Scharlachs und eines positiven Streptokokkenschnelltests beginnen wir eine Therapie mit Penicillin und entsprechend dem fulminanten Verlauf mit Ampicillin, Gentamicin, Metronidazol und Cefotaxim. Die Sepsis Diagnostik erbringt den Nachweis eines Streptococcus pyogenes sowohl in der initialen Blutkultur als auch im Punktat des Pleuraempyems rechts. Rasch entsteht ein ausgeprägtes Kapillarleck mit massiven Ödemen und weiterer Befundzunahme unter der bei intravasalem Volumenmangel notwendigen Substitution von Volumen als Elektrolytlösung zzgl. Erythrozyten-, Thrombozytenkonzentraten, Fresh Frozen Plasma sowie später auch Albumin. Das Mädchen entwickelt ein abdominelles Kompartmentsyndrom (ACS), welches bei Anurie, zunehmendem Leber- und drohendem Beatmungsversagen durch Medianlaparotomie mit anschließender Versorgung des offenen Situs abdominalis entlastet wird. Hierunter stabilisiert sich die Situation mit Rückgang der Entzündungswerte. Am 12. stationären Tag gelingt der Bauchdeckenverschluss mit Anlage eines passageren Anus praeter. Postoperativ kommt es erneut zu Fieber, Exanthemausbruch und Anstieg der Entzündungswerte. Die Computertomographie des Thorax zeigt abgekapselte residuelle pleurale Flüssigkeitsformationen. Unter erweitertem Antibiotikaregime kommt es zur deutlichen Stabilisierung des Allgemeinzustandes mit Regredienz des Exanthems. Das Mädchen wird nach 25 Tagen erfolgreich extubiert. Nach Ausschwemmen der Ödeme und unter prokinetischer Therapie kann eine regelrechte Magendarmpassage erreicht werden, so dass die Rückverlagerung des Anus praeter nach 16 Tagen gelingt. Nach 35 Tagen wird das Mädchen in stabilem Allgemeinzustand in eine Rehabilitationsklinik entlassen. Schlussfolgerung. Das STSS ist eine seltene, aber aufgrund seiner Toxin-vermittelten Inflammation schwerwiegende Erkrankung mit fulminantem klinischem Verlauf und einer hohen Letalität. Unser Fall zeigt die Erkrankung im vollen Ausmaß, einhergehend mit einem ausgeprägten Kapillarleck, massiven Ödemen und einem sich rasch entwickelnden ACS. Die Medianlaparotomie mit Versorgung des offenen Situs abdominalis im stationären Setting stellt hierbei neben der raschen Diagnosestellung und damit einhergehender adäquater intensivmedizinischer Maximaltherapie die lebensrettende Maßnahme für das Mädchen dar.

\section{P141}

Die Confusion Assessment Method für die pädiatrische Intensivstation (pCAM-ICU) - Validität und Reliabilität der deutschsprachigen Übersetzung

A. Lütz' , D. Gensel', J. Müller' ', H. Krude'2, C. Spies'

${ }^{1} K$ linik für Anästhesiologie mit Schwerpunkt operative Intensivmedizin Campus Charité Mitte und Campus Virchow-Klinikum, Berlin, Deutschland, ${ }^{2}$ Charité Campus Virchow, Inst. f. Experimentelle päd. Endokrinologie, Berlin, Deutschland

Zielsetzung: Die häufigste psychiatrische Komplikation bei pädiatrischen und erwachsenen Intensivpatienten ist das Delir. Die Inzidenz unter intensivpflichtigen Kindern wird mit $13 \%$ bis $28 \%$ angegeben (1). Bisher konnte bereits eine signifikant längere Krankenhausbehandlung festgestellt werden (2). Ein routinemäßiges Delirscreening auf pädiatrischen Intensivstationen wird empfohlen (3). Hierfür steht seit 2012 die Confusion Assessment Method für die Pädiatrische Intensivstation (pCAM-ICU) zur Verfügung (4). Ziel dieser Arbeit ist es die Validität und Reliabilität für die deutsche Version der pCAM-ICU zu überprüfen. 
Methodik: Die prospektive, observierende Kohortenstudie wurde von der Ethikkommission der Charité Universitätsmedizin Berlin (Nr. EA2/073/11) genehmigt. Eingeschlossen wurden Kinder im Alter von 5 bis 17 Jahren. Patienten mit einer intensivstationären Behandlungsdauer von weniger als 24 Stunden und nicht deutschsprechende Patienten wurden ausgeschlossen. Nach einem vorgegebenen Ablaufschema wurden die Delirmessungen täglich durchgeführt. Die Delirdiagnostik mittels der Kriterien des diagnostischen und statistischen Manuals psychischer Störungen (DSM-IV, Referenzstandard) wurde von einem geschulten Intensivmediziner erhoben.

Ergebnisse: Eingeschlossen und analysiert wurden die Daten von $64 \mathrm{~Pa}-$ tienten. Es ergab sich eine Delirinzidenz von 23,4\% $(n=15)$ diagnostiziert durch die DSM-IV-Kriterien. Die Messungen mit der pCAM-ICU zeigten eine Inzidenz von 17,2\% ( $n=11)$. Für die erste Messung mit der pCAMICU verglichen mit dem Referenzstandard ergab sich eine Sensitivität von $77 \%$ (95\% KI, 46-95\%) und eine Spezifität von 98\% (90-100\%). Der positive prädiktive Wert lag bei 90,9\% (59-100\%) und der negative prädiktive Wert bei 94,3\% (84-99\%). Außerdem konnte eine Interrater-Reliabilität mit k=0,91 (95\%KI, 0,69-0,95) gezeigt werden.

Schlussfolgerung: Die pCAM-ICU stellt ein valides und reliables Messinstrument für das Delirmonitoring unter intensivpflichtigen Kindern im deutschsprachigen Raum dar. Die Notwendigkeit für die routinemäßige Implemtierung eines Delirscreenings wird durch die ermittelte Delirinzidenz von $23 \%$ verdeutlicht.

\section{Literatur}

1. Daoud A, et al., Crit Care 18(5):489 (2014)

2. Smeets IA, et al., Eur Child Adolesd Psychatry 19, 389-93 (2010)

3. Baron R, et al., Ger Med Sci 13:Doc19 (2015)

4. De Grahl C, Luetz A, et al., Ger Med Sci 10:Doc07 (2012)

\section{P142}

\section{Venoarterielle ECMO als Bridging-Verfahren bei kardiogenem Schock bei einer 15 Jahre alten Patientin mit AML bei V.a. Linezolid-induzierte-Mitochondriopathie}

\section{S. Kunzmann', R. Muellenbach ${ }^{2}$, M. Kredel', P. Nordbeck ${ }^{3}$, C. Bühler', A. Buck ${ }^{5}$, H. Hänscheid ${ }^{5}$, S. Hackenberg ${ }^{6}$, M. Wölfl', M. Eyrich', J. Wirbelauer ${ }^{1}$ P. Schlegel}

${ }^{1}$ Klinik für Kinderheilkunde, Würzburg, Deutschland, ${ }^{2}$ Klinik für Anästhesiologie, Würzburg, Deutschland, ${ }^{3}$ Klinik für Innere Medizin I, Würzburg, Deutschland, ${ }^{4}$ Klinik für Chirurgie I, Würzburg, Deutschland, ${ }^{5}$ Klinik für Nuklearmedizin, Würzburg, Deutschland, ${ }^{6}$ Klinik für HNOErkrankungen des Universitäts-Klinikums, Würzburg, Deutschland

Einleitung: Die Indikationsstellung zur ECMO-Therapie ist bei onkologischen Kindern mit unklarer Prognose oftmals aufgrund der erheblichen medizinischen (u. a. Blutungs-, Infektions-Risiko) und ethischen Probleme bei fehlender Evidence schwierig.

Fallbeschreibung: Bei einer 15 Jahre alten Patienten mit einer AML (M4, ZNS+; AML-BFM Protokoll) kam es in der prolongierten Panzytopenie-Phase nach dem zweiten Induktions-Chemotherapie-Block (L 200/ $\mu \mathrm{l}, \mathrm{Hb} 8 \mathrm{~g} / \mathrm{dl}, \mathrm{T} 21.000 / \mu \mathrm{l})$ unter einer breiten antimikrobiellen Therapie (u. a. Linezolid) zu einem kardiogenen Schock unklarer Ursache. Trotz Intensivierung der Therapie (Intubation mit Katecholamin-, Milrinon-, Vasopressin- und Hydrocortison-Therapie) trat ein progredientes linkskardiales Pumpversagen auf (EF 15\%, ZVS 49\%, Laktat 19 mmol/l, pH 6,9, BE - $22 \mathrm{mmol} / \mathrm{l})$. Nach Aufklärung der Eltern Anlage einer venoarteriellen extrakorporalen Membranoxygenierung (va-ECMO) durch das ECMO-Team der Klinik für Anästhesiologie (Blutfluss 3,5 L/min). Bei ANV AKIN III wurde zusätzlich mit einer venovenösen Citrat-Hämodiafiltration (CVVHDF) begonnen. Trotz va-ECMO mit einem Blutfluss von 3,5 L/ min kam es zu einem weiteren Anstieg des Laktats auf $22 \mathrm{mmol} / \mathrm{L}$, so dass eine weitere arterielle ECMO-Kanüle (vaa-ECMO) mittels Dacron-Prothese zur Erhöhung des ECMO-Blutfluss auf 5-6 L/min angelegt wurde. Bei hochgradig eingeschränkter linksventrikulärer Funktion ohne antegraden Auswurf über die Aortenklappe wurde eine weitere ECMO-Kanüle per Atrioseptostomie zum Venting des Blutes linksatrial platziert (vava-ECMO). Darunter konnte eine suffiziente Entlastung des linken
Herzens sowie Blutflüsse $>6 \mathrm{~L} / \mathrm{min}$ erreicht werden. Trotz Hochdosis-Katecholamintherapie mit Vasopressin und Noradrenalin konnte für ca. 1216 Std. nur ein MAD von 30-45 mmHg erzielt werden. Die zerebrale Oxymetrie war jedoch trotz dieser MAP Werte unter ECMO-Blutflüssen $>6 \mathrm{~L} /$ min konstant zwischen $70-80 \%$. Nach ca. 48 Std. kam es unter inotroper Therapie mit Enoximon zu einer Verbesserung der kardialen Pumpfunktion, so dass die ECMO-Blutflüsse reduziert werden konnten. Nach 7 Tagen konnte die ECMO entfernt werden. Aufgrund einer Critical Illness Polyneuropathie und Myopathie (CIP/CIM) gestaltete sich die Entwöhnung von der Beatmung schwierig, die Patientin wurde tracheotomiert und konnte danach sukzessive von der Beatmung entwöhnt werden.

Im weiteren Verlauf deutliche Verbesserung des Gesamtzustandes, sodass trotz einer fortbestehender Herzinsuffizienz eine allogene Fremdspendertransplantation nach reduzierter Konditionierung mit einem 188Re-antiCD66 AK zur Myelosuppression durchgeführt werden konnte. 10 Monate nach ECMO-Therapie und 5 Monate nach SZT befindet sich die Patientin in molekularer Remission in stabilem Allgemeinzustand.

Diskussion: Dieser Fall zeigt, dass die Indikation zur ECMO-Therapie in individuellen Fällen auch bei schwer erkrankten onkologischen Patienten bei initial unklarem Krankheitsbild mit unsicherer Prognose unter Einbeziehung aller Subdisziplinen einer Klinik der Maximalversorgung in Erwägung gezogen werden sollte. Als mögliche Ursachen für den kardiogenen Schock kommen differentialdiagnostisch eine septische Kardiomyopathie sowie eine Linezolid-induzierte akute Mitochondriopathie in Frage. Bei der Gabe von Linezolid sollte stets auch an diese sehr seltene, aber beschriebene Nebenwirkung gedacht werden.

\section{P143}

Fulminante Pneumokkoken-Meningoenzephalitis durch seltenen non-Vakzine Serotyp 23A

L. Schröder', G. Hischebeth', M. van der Linden ${ }^{3}$, M. Born ${ }^{4}$, H. Reutter', T. Dresbach', A. Müller?

'Universitätsklinikum Bonn, Pädiatrische Intensivmedizin und Neonatologie, Bonn, Deutschland, ${ }^{2}$ Universitätsklinikum Bonn, Institut für Mikrobiologie, Immunologie und Parasitologie, Bonn, Deutschland, ${ }^{3}$ Universitätsklinikum Der RWTH Aachen, Institut für Medizinische Mikrobiologie, Aachen, Deutschland, ${ }^{4}$ Universitätsklinikum Bonn, Radiologie, FE Kinderradiologie, Bonn, Deutschland

Einleitung: Streptococcus pneumoniae ist einer der führenden Pathogene von bakteriellen Meningitiden bei Kindern weltweit. Die Zunahme von non-Vakzine Serotypen führt zu einer zunehmenden klinischen Problematik.

Fallbericht: Wir berichten über eine 12 Monate alte Patientin mit Down-Syndrom, AV-Kanal, Herzinsuffizienz und Tracheotomie mit Heim-Beatmung, welche sich auf unserer PICU vorstellte mit prolon-
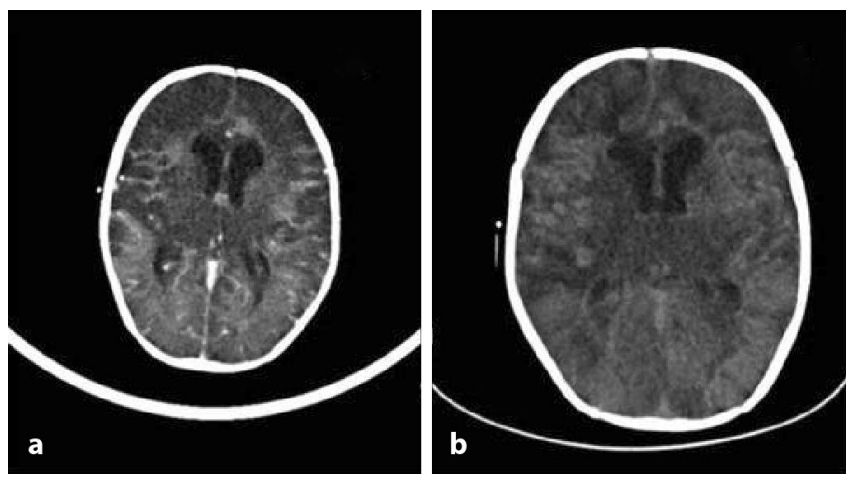

Abb. P143.1 $\Delta$ Initiales CCT am zweiten (a) und neunten Tag (b) nach Aufnahme. Zu beachten sind die ausgedehnten hypo- und hyperintensen Parenchym-Veränderungen beidseits. In Abb. P143.1 b zeigt sich die Progression des Befundes 

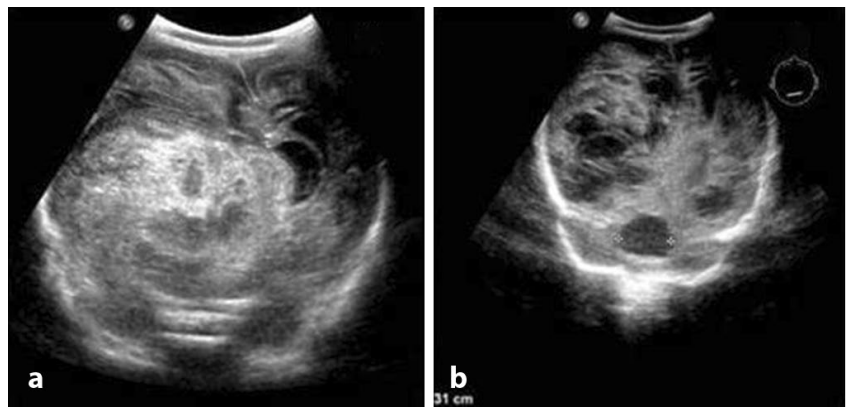

Abb. P143.2 $\triangle$ Sonographie Hirn am Tag 18 (a) und Tag 29 (b) nach Aufnahme. Die periventrikulären Strukturen sind nicht mehr abgrenzbar und das Parenchym zeigt sich bds. inhomogen

giertem Fieber und reduziertem Allgemeinzustand. Es fiel eine reduzierte Spontanmotorik und ein für sie ungewöhnliches Verhalten auf. In der klinischen Untersuchung zeigte sich v. a. eine blasse/marmorierte Haut und eine verlängerte Rekapillarisierungszeit. Ein eindeutiger Meningismus fehlte. Laborchemisch zeigte sich eine Leukozytose und deutlicher CRP-Anstieg (192 mg/l). Die Liquordiagnostik ergab eine Pleozytose (1161 L./ul), erhöhtes Laktat und Protein. In der Liquor- und Blutkultur bestätigte sich S. pneumoniae (Serotyp 23A). Die antibiotische Therapie wurde auf Penicillin-G umgestellt.

Im initialen CCT zeigte sich bereits eine massive Zerebritis, eine Ventrikulitis und beginnende Hirn-Infarzierungen (siehe - Abb. P143.1 a/b). Klinisch zeigte die Patientin Zeichen eines schweren Hirnödems (siehe - Abb. P143.1 a/b). Ein Verlaufs-CCT wurde bei sekundärem CRP-Anstieg und anhaltendem Fieber durchgeführt (siehe - Abb. P143.1 b). Nach zwei Wochen zeigte sich sonographisch eine massive parenchymatöse Einblutung der rechten Hemisphäre (siehe 1 Abb. P143.2 a/b). Die Patientin entwickelte im Verlauf einen schweren Panhypopituaitarismus. Nach sechs Wochen konnte die Patientin mit kontrollierter Heimbeatmung und $24 \mathrm{~h}$-Pflegedienst entlassen werden.

Diskussion: War der Serotyp 23A in der Prä-Vakzine Ära noch ein selten anzutreffender Serotyp, nimmt die Prävalenz in den letzten Jahren deutlich zu. Gen-shifts entlang der Pneumokokken-Stämme führen zu neuen Ungleichgewichten in der Pneumokokken-Population. Kinder mit Down-Syndrom leiden häufig unter einer Immunschwäche auf Grund einer T-Zell-Reifungsstörung, B-Lymphozyten Reduktion und IgG-Subklassen Mangel. S. pneumoniae führt bei diesen Kindern häufig zu rekurrierenden Infekten und ist somit eine stetige Gefahrenquelle. Der ungewöhnlich schwere Verlauf des Mädchens könnte durch eine adverse Immunreaktion und Immunschwäche zurück zu führen sein.

Die Schwere der pathologischen intrazerebralen Veränderungen ist für eine Pneumokokken-Meningitis sehr ungewöhnlich. Es gibt jedoch Fallberichte über ähnliche Verläufe bei Kindern. Genaue Inzidenzen über zerebrovaskuläre Komplikationen bei Kindern im Rahmen von Pneumokokken-Meningitiden fehlen. Es ist anzunehmen, dass die Diagnostik und Therapie im vorliegenden Fall verzögert wurden. Die syndromale Erkrankung und Vorerkrankungen der Patientin könnten zu einer Verschleierung der Symptomatik geführt haben. Zusammenfassung: Durch Pneumokokken-Impfstoffe nicht abgedeckte Serotypen stellen eine stete Bedrohung im klinischen Alltag dar und verdeutlicht den Nutzen einer Einführung von Pneumokokken-Impfstoffen mit einer breiteren Serotypen-Abdeckung.

\section{P144}

Metalloproteasen steuern den Phagozytose-induzierten Zelltod (PICD) von Monozyten

\section{S. Dreschers', M. Haas', C. Platen', C. Gille ${ }^{2}$, T. Orlikowsky ${ }^{3}$}

${ }^{1}$ Med. Einrichtungen der RWTH Aachen, Kinderklinik, Aachen, Deutschland, ${ }^{2}$ Eberhard-Karls-Universität Universitätsklinik für Kinderheilkunde und Jugendmedizin, Neonatologie, Tübingen, Deutschland, ${ }^{3}$ Universitätsklinik für Kinder- und Jugendmedizin, Sektion für Neonatologie, Aachen, Deutschland

Hintergrund: Die Regulation des intrazellulären Signallings durch „Abschneiden" (Cleaving) von Liganden und/oder Rezeptoren an der Plasmamembran kann durch Enzyme (Metalloproteasen) geschehen. Dieser Vorgang kann die Liganden/Rezeptoren auf der Zelloberfläche kritisch beeinflussen und dadurch die Transmission eines Signals regulieren. Mehrere Arbeiten zeigten die Funktion der Todesrezeptoren (TNF Superfamilie: CD95/CD95L und TNF-a/TNFR1) im PICD nach bakteriellen Infektionen von Monozyten Erwachsener (PBMO). In neonatalen Monozyten (CBMO) ist die PICD-Induktion signifikant reduziert, was durch niedrige Expression und Sekretion von CD95L bzw. Internalisierung von TNF/ TNFR1-Komplexen zustande kommt.

Hypothese: Eine reduzierte Expression/Funktion von Metalloproteasen auf der Zelloberfläche von CBMO ist für das reduzierte pro-apoptotische Signalling (durch TNF-a/TNFR1 und CD95/CD95L vermittelt) verantwortlich. Die Expression der Metalloproteasen ADAM17 und MMP-9, welche TNF-a, TNFR1, CD95L und weitere Moleküle von der Zelloberfläche abschneiden, regulieren die Induktion von PICD in Monozyten.

Methoden: Die Expression von Metalloprotease-9 (MMP-9), ADAM17 und TNF-a wurden mittels intra- und extrazellulärem FACS, TNF-a- und CD95-Sekretion via ELISA untersucht. Die Aktivität von Metalloproteasen wurde mit einem Inhibitor (GM6001) blockiert. Die Auswirkungen auf den PICD wurden nach Infektion von Monozyten mit E. coli mittels hypodiploiden Zellkernen sowie TUNEL-Assay nachgewiesen.

Ergebnisse: Nicht-infizierte PBMO und CBMO wiesen vergleichbare Enzymdichten von ADAM17 und MMP-9 auf. Infektion mit E.coli führte zur Hoch-Regulation von MMP-9 (6-fach) und ADAM17 (2-fach) auf der Plasmamembran von PBMO ( $p<0,01$ vs. nicht-infiziert). Die mittlere ADAM17 Expression auf CBMO stieg nach Infektion ebenfalls an, jedoch im Gegensatz zu PBMO wurde MMP-9 auf CBMO nicht hoch-reguliert. Während sich in infizierten CBMO und PBMO intrazellulär vergleichbare Konzentrationen von TNF-a und CD95L nachweisen ließen, sezernierten PBMO ungefähr das 2,5-fache an TNF-a und CD95L im Vergleich zu CBMO $(p<0,05)$. Die Vor-Inkubation mit dem Metalloprotease-Inhibitor GM6001 erhöhte die Dichte von TNF-a und CD95L an der Plasmamembran und verringerte die Konzentration der entsprechend löslichen Moleküle. Die Inhibition von MMP-9 nach Infektion reduzierte den PICD von PBMO signifikant, wobei TNFR1 und CD95L auf der Oberfläche nachweisbar blieb.

Schlussfolgerung: Die pro-apoptotischen Mechanismen, die bei Monozyten zum PICD führen, sind bei CBMO aufgrund der Metalloprotease-abhängigen Depletion von CD95L und TNF-a reduziert.

\section{P145}

Untersuchungen zur Rolle des Toll-like Rezeptors 2 (TLR2) in der Candida albicans Infektion beim Neugeborenen

S. Dreschers', P. Saupp², M. Hornef ${ }^{3}$, J. Morschhäuser', T. Orlikowsky ${ }^{5}$

${ }^{1}$ Med. Einrichtungen der RWTH Aachen, Kinderklinik, Aachen, Deutschland, 2Universitätsklinik Düsseldorf, Kinderklinik, Köln, Deutschland, ${ }^{3}$ Universitätsklinik Aachen, Mikrobiologie, Aachen, Deutschland, ${ }^{4}$ Universität Würzburg, Mikrobiologie, Würzburg, Deutschland, ${ }^{5}$ Universitätsklinik für Kinder- und Jugendmedizin, Sektion für Neonatologie, Aachen, Deutschland

Hintergrund: Phagozytose von Bakterien führt zum Phagozytose-induzierten Zelltod (PICD) von Monozyten. Der PICD von neonatalen Monozyten (CBMO) ist gegenüber Monozyten von Erwachsenen (PBMO) 
reduziert. Invasive Candida-Infektionen sind beim extremen Frühgeborenen (ELBW) häufig und weisen hohe Defektheilungsraten auf. Toll-like Rezeptoren (TLR) sind zelluläre Strukturen der angeborenen Immunität und dienen der Abwehr von Mikroben.

Hypothese: Bei Monozyten wird die Expression von TLR2 und das TLR2-vermitteltes Signalling durch C. albicans moduliert, was zu funktionellen Konsequenzen (TNF-Produktion, PICD) führt. Der TLR2-Signalweg ist in die funktionellen Unterschiede von CBMO und PBMO bei C. albicans Infektionen involviert.

Methoden: Phänotypisierung (TLR2, TLR4, CD14), intrazelluläre Charakterisierung (P38-Phosphorylierung, TNF-a Sekretion, Caspase-8, -3) von Monozyten aus Nabelschnurblut (CBMO) oder vom Erwachsenen (PBMO). Infektion von GFP-exprimierenden C. albicans und Analyse von Phagozytose, PICD (FACS, TUNEL). Nachweis der TLR2-Beteiligung mittels anti-TLR2-blockierenden Antikörpers (anti-TLR2 Ab), Nachweis der TLR2-Spezifität mit TLR-Agonisten (LPS, Pam3Cys, FSL-1, Zymosan). Ergebnisse: CBMO und PBMO phagozytierten C. albicans vergleichbar. Nach Infektion kam es bei PBMO mit niedriger basaler TLR2-Expression zur Hoch-Regulation des Rezeptors mit konsekutiver MAP Kinase P38-Phosphorylierung und Sekretion von TNF-a ( $p<0,005$ vs. CBMO) Die Apoptose-induzierenden intrazellulären Konzentrationen von Caspase- 8 and -3 waren deutlich höher in PBMO ( $p<0,05$ vs. CBMO), was zur signifikanten Erhöhung des PICD bei PBMO führte. Die beschriebenen Phänomene waren durch anti-TLR2 Ab signifikant, bis zu $80 \%$ blockierbar.

Schlussfolgerung: In der C.albicans Infektion ist das TLR2-vermittelte $\mathrm{Si}$ gnalling kritisch für Monozytenfunktionen. CBMO weisen ein reduziertes TLR2-Signalling auf, was für die schweren Verläufe der invasiven Candida-Infektion beim Frühgeborenen mitverantwortlich sein kann.

\section{P146 \\ Erfolgreiche ECMO Therapie mit Restitution ad integrum nach kindlichem Beinaheertrinken mit ausgeprägter Hypothermie}

\section{H. Topf', D. Salleck' ${ }^{2}$ R. Cesnejvar ${ }^{3}$, T. Völkl \\ 'Universitäts-Kinderklinik, Neonatologie und pädiatrische Intensivmedizin, Erlangen, Deutschland, ${ }^{2}$ Klinikum Nürnberg Süd, Nephrologie/ Hypertensiologie, Nürnberg, Deutschland, ${ }^{3}$ Kinderherzchirurgische Abteilung, Erlangen, Deutschland}

Vorgeschichte: Wir berichten über einen 6 Jahre alten Patienten, der am Abend des vierten Advent beim Spielen nahe des elterlichen Gartens in ein trübes Flussgewässer gefallen und beinahe ertrunken ist. Erst ca. 10 min nach dem Unfall konnte der Patienten von ins Wasser gesprungenen Anwesenden geborgen werden. An Land wurde unmittelbar durch bereits hinzugezogene Hilfskräfte mit der cardiopulmonalen Reanimation begonnen und durch den im Verlauf eintreffenden Notarzt fortgeführt werden. Bei Ankunft des Notarztes wies der Junge bereits eine Unterkühlung von $24^{\circ} \mathrm{C}$ auf. Nach repetitiven Gaben von Adrenalin und erfolgreicher Intubation konnte der Patient mit leicht arrhythmischem Eigenrhythmus in die Klinik verlegt werden. In Absprache mit dem Rettungsdienst erfolgte die Übernahme in unser Zentrum über den Schockraum.

Verlauf: Bei Ankunft in unsere Klinik lag bei dem $20 \mathrm{~kg}$ schweren Patienten ein unregelmäßiger Sinusrhythmus mit ausreichendem systemischen Blutdruck bei augeprägter Hyopthermie vom $27^{\circ} \mathrm{C}$ vor. Noch im Schockraum erfolgte die Anlage eines arteriellen Katheters, eines zentarlen Coolguard Katheters und Blasenkatheters bevor das CT mit unauffälligen Werten für ZNS und Halswirbelsäule erfolgte. Die BGA bei Aufnahme zeigte einen $\mathrm{pH}$ von 6,8 (temp. korrigiert 6,9), einen base excess von -21 , ein Laktat von $13,8 \mathrm{mmol} / 1, \mathrm{pO}_{2} 96,6$ und $\mathrm{pCO}_{2} 47$ bei $100 \% \mathrm{iO}_{2}$. Nach Übernahme auf die pädiatrische Intensivstation erfolgte das cerebrale monitoring mittels kontinuierlicher NIRS Messung und intermittierendem EEG und transkranieller Dopplersonographie. Neuroprotektiv erfolgte eine Sedierung mittels Fentanyl und Midazolam. Eine antibiotische Therapie mit Cefuroxim und Metronidazol wurde eingeleitet. In den Folgestunden gestaltete sich die Beatmung zunehmend schwierig mit abnehmender Sättigung bis $76 \%$ und $\mathrm{pO}_{2}$ Werten um 34, so dass die Anla- ge einer veno-venösen ECMO erfolgte. Hierunter zeigte sich ein stabiler Patient, mit gleichmäßiger Erwärmung von ca. $0,4^{\circ} \mathrm{C} / \mathrm{h}$ und sich normalisierenden Laborparametern. Nach 60 Stunden konnte bei stabilen Beatmungs- und Kreislaufverhältnissen die ECMO Therapie beendet werden, hierbei waren vorübergehend kurzfristig Katecholamine erforderlich. Nach weiteren 24 Stunden konnte die Beatmung beendet werden, eine überbrückende sedierende Therapie mit Clonidin wurde durchgeführt. Der Kostaufbau gestaltete sich problemlos, bei gutem Allgemeinzustand erfolgte die Verlegung auf die neuropädiatrische Nachsorgestation. Ein am Tag acht nach Ereignis durchgeführtes MRT ergab einen unauffälligen Befund, die Verlaufsuntersuchung im EEG ergaben keine Pathologie. Die neurolgische Feinuntersuchung vor Entlassung war ebenfalls altersgerecht, sodass der Patient an Tag 10 nach dem Ereignis ohne den Bedarf eine Rehabilitationsmaßnahme nach Hause entlassen werden konnte. Die restlichen Ferientage verbrachte der Patient im Kreis der Familie bevor er regulär den Schulbesuch seiner Grundschule am ersten Schultag nach den Ferien antreten konnte.

Zusammenfassung: Die ECMO-therapie ist ein elegantes Verfahren zur Wiedererwärmung und Überbrückung einer Ateminsuffizienz nach Ertrinkungsunfällen. Das gute neurologische outcome des Patienten ist aber größtenteils auf die sehr gute präklinische Versorgung zurückzuführen, die einen wichtigen Teil der Versorgungskette darstellt. Aus diesem Grund sollte darüber nachgedacht werden einen größeren Wert auf regelmäßige Erste Hilfe Kurse für alle Mitbürger zu legen.

\section{P147}

\section{Erstmanifestation eines Hyperglykämisches Hyperosmolares Syndrom (HHS) bei einem Neugeborenen}

\section{Berwald' ', I. Beger', M. Engler', C. Wiethoff'}

'Sana Klinkum Offenbach, Neonatologie und pädiatrische Intensiv, Offenbach, Deutschland

Das HHS ist eine sehr seltene, akut lebensbedrohliche Erkrankung, die im Kindesalter mit einer hohen Letalität $(>35 \%)$ und einer hohen Morbidität ( $>50 \%)$ einhergeht. Der bis dato jüngste beschriebene Patient war 18 Monate alt. Das HHS ist charakterisiert durch extrem hohe Blutzuckerspiegel (BZ) mit Hyperosmolalität ohne wesentliche Ketoazidose. Auf Grund des extrem hohen BZ wird oft ein Diabetes mellitus (DM) angenommen. Der DM kann sich in zwei unterschiedlichen Formen, entweder als diabetische Ketoazidose (DKA) oder als HHS primärmanifestieren. Wohingegen bei Erwachsenen das HHS mit zunehmendem Alter häufiger auftritt, ist die Erstmanifestation durch ein HHS im Kindesalter eine extreme Rarität. Die im Gegensatz zur DKA deutlich höhere Mortalität beruht bei Erwachsenen auf Komorbiditäten, im Kindesalter scheinen andere Faktoren beteiligt zu sein. Wir berichten über ein 24 Tage altes Neugeborenes (NG), das im hypovolämen Schock mit ausgeprägter Exsikose ( $>25 \%$ des KG), Zentralisation und Sauerstoffbedarf (6 1/min) eingewiesen wurde. Bei Einweisung: Tachy-dyspnoe (AF $>70 / \mathrm{min}$ ), Tachykardie (HF $>200 / \mathrm{min}$ ), Blutdruck 72/57 mmHg, stuporös, Körperkerntemperatur $38,6^{\circ} \mathrm{C}$. Laborchemisch zeigte sich ein $\mathrm{pH}$ von $6,9, \mathrm{HCO} 3-6,4 \mathrm{mmol} / \mathrm{l}$, $\mathrm{pCO}_{2} 29,3 \mathrm{mmHg}$, Natrium $175 \mathrm{mmol} / \mathrm{l}$, Kalium 7,2 mmol/l, BZ $1194 \mathrm{mg} /$ dl, Osmolalität 418 mosm/l, Laktat 35,3 mg/dl, Kreatinin 1,6 mg/dl, Ammoniak $296 \mu \mathrm{g} / \mathrm{dl}$, Ketone im Urin schwach positiv, spez. Gewicht $>1025$. Keine Infektionszeichen. Bis auf einen DM der Großmutter und Trinkschwäche sowie entfärbte, dünne Stühle am Vortag unauffällige Anamnese. Bei Aufnahme erhielt das NG eine isotone Kochsalzinfusion über Knochennadel. Nach Anlage einer NIV-Beatmung und Kathetern (ZVK, periphere Arterie) erfolgte initial die Therapie entsprechend den Leitlinien für DKA. Darunter kam es zur Senkung des Blutzuckers ( $100-150 \mathrm{mg} /$ dl/h) aber zu einem Anstieg des Natriums. Nur durch exzessive Steigerung der Volumenzufuhr war das Natrium (Maximal $183 \mathrm{mmol} / \mathrm{l}$ ) zu senken. Nach 3 Tagen bestand Normoglykämie und Stabilisierung, so dass die Beatmung und die Insulinzufuhr beendet werden konnten. Im Weiterem normalisierten sich die Elektrolyte und Retentionswerte. Transient bestand ein erhöhter Glucosebedarf $(18.5 \mathrm{~g} / \mathrm{kgKG} / \mathrm{die})$. In der initialen Blutkultur konnte eine Klebsiella pneumonia nachgewiesen werden. Eine 
genetische Diagnostik bzg. des DM wurde veranlasst. Im weiteren Verlauf war das NG unauffällig. Bei der HHS entsteht neben der schweren intravasalen auch eine extravasale Dehydratation, die beide zu einer extremen Laktatazidose führen können. Eine forcierte Rehydrierung des intra- und extravaskulären Raumes und ein Elektrolytausgleich sind daher substantiell um Komplikationen wie Thrombose, Rhabdomyolyse, maligne Hyperthermie, und Hirnödem zu vermeiden.

\section{P148 \\ Unilateraler hämorrhagischer Thalamusinfarkt und IVH bei einem zuvor gesunden Säugling}

\section{S. Pfahl', D. Klee', S. Sarikaya' ', E. Mayatepek', D. Tibussek' \\ ${ }^{1}$ Med. Einricht. d. Universität Kinderklinik, Düsseldorf, Deutschland}

Einseitige hämorrhagische Infarkte im Thalamus begleitet von einer intraventrikulären Blutung (IVH) sind im Säuglingsalter sehr selten. Bei Neugeborenen hingegen ist dieser Befund bekannt und gilt als nahezu pathognomonisch für einen zerebrale Sinusvenenthrombose (CSVT). Wir berichten hier über einen zweieinhalb Monate alten Säugling mit einseitigem hämorrhagischen Thalamusinfarkt und IVH. Differenzialdiagnosen und therapeutische Ansätze werden diskutiert. Ein zweieinhalb Monate alter, zuvor gesunder Säugling wird mit Fieber, schrillem Schreien und Irritabilität seit 2 Tagen in unserer Notfallambulanz vorgestellt. Keine anamnestischen Hinweise auf Krampfanfälle. Die klinische Untersuchung erbrachte keinen weiteren wegweisenden Befund. Zum Ausschluss einer ZNS-Infektion erfolgte eine Lumbalpunktion, die xanthochromen Liquor mit Liquorpleozytose zeigte. Die erweiterte Diagnostik zum Ausschluss einer schweren bakteriellen Infektion, inklusive CRP, Blutbild, Urinstatus, Blut- und Liquorkultur, blieb ohne pathologischen Befunde. Im Schädelultraschall zeigte sich eine Raumforderung im Bereich des Thalamus. Eine folgende zerebrale Kernspintomographie ergab eine unilaterale thalamische Blutung mit intraventrikulärer Blutung. Mehrfache sonographische Verlaufskontrolluntersuchungen zeigten die sich in Auflösung befindliche Blutung ohne Hinweis auf Hydrozepahlus. Der Patient zeigte eine rasche klinische Besserung. Im poststationären Verlauf zeigten sich keine neurologischen Auffälligkeiten und eine altersentsprechende Entwicklung. Die Befundkonstellation aus unilateralem hämorrhagischen Thalamusinfarkt mit IVH kommt auch jenseits der Neugeborenen-Periode vor und sollte auch hier immer an eine CSVT denken lassen. Wir stellen die Hypothese einer spontan rekanalisierten CVST als wahrscheinlichste Diagnose bei unserem Patienten auf. In vergleichbaren Fällen sollte frühzeitig an eine CVST gedacht werden und entsprechende Bildgebung erfolgen.

\section{P149 \\ Leukozytendepletion durch Austauschtransfusion bei maligner Pertussis}

D. Stavropoulou', J. Krause', R. Hentschel', M. Schmitt', B. Keyser', H. Fuchs' ${ }^{1}$ Institut/Klinik: Universitätsklinikum Freiburg, Zentrum für Kinder- und Jugendmedizin, Neonatologie/Intensivmedizin, Freiburg, Deutschland, ${ }^{2}$ Oberschwabenklinik, Krankenhaus St. Elisabeth, Klinik für Kinder- und Jugendmedizin, Ravensburg, Deutschland

Hintergrund: Infektionen durch Bordetella pertussis haben bei Säuglingen $<3$ Monate eine erhebliche Mortalität aufgrund von Komplikationen wie respiratorischem Versagen, pulmonaler Hypertonie, Enzephalitis und kardialem Versagen. Eine assoziierte Hyperleukozytose $>100.000 / \mu \mathrm{l}$ bedingt eine fast $100 \%$ ige Mortalität bei konventioneller Therapie aufgrund von Hyperviskositätssyndrom und pulmonalarterieller Thrombose. Retrospektive Kohorten und Fallberichte legen eine Verbesserung des Outcomes durch Leukozytendepletion nahe.

Fallbericht: Wir berichten über einen 73 Tage alten Jungen der bei maligner Pertussis-Infektion mit respiratorischem Versagen und Hyperleukozytose durch Austauschtransfusion behandelt wurde. Bei respiratorischen Symptomen, Dyspnoe, Sauerstoffbedarf, zunehmenden stakkatoartigen Hustenanfällen und Leukozytose wurde früh an eine Pertussis-Infektion gedacht und entsprechend eine Makrolid-Therapie eingeleitet. Die Bestätigung der Befunde erfolgte mittels PCR-Nachweis. Bei rasch progredienter respiratorischer Symptomatik mit zunehmender Tachypnoe und Sauerstoffbedarf trotz Therapie mit HFNC wurde bei weiter ansteigenden Leukozytenzahlen bis $>140.000 / \mu$ l eine Austauschtransion zur Leukozytendepletion durchgeführt. Eine pulmonale Hypertonie lag bis dahin aber noch nicht vor. Durch Austausch des 1,5fachen Blutvolumens sind die Leukozyten auf 34.000/ $\mu \mathrm{l}$ abgefallen. Mit Abfall der Leukzytenzahlen kam es zu einer dramatischen und eindrücklichen Besserung der respiratorischen Symptomatik und des Gesamtzustandes des Säuglings. Nach nur 6 Tagen konnte das Kind von der Intensivstation entlassen werden.

Diskussion und Schlussfolgerung: Bei typischer respiratorischer Symptomatik und Hyperleukozytose muss bei kleinen Säuglingen auch an eine Pertussis-Infektion gedacht werden. Gemäß aktueller Expertenmeinung sollte bei rasch progredientem respiratorischem oder kardialem Versagen im Rahmen der Pertussis-Erkrankung ab Leukozyten $>50000 / \mu \mathrm{l}$, in jedem Fall aber ab Leukozyten $>100.000 / \mu$, eine Leukozytendepletion vorgenommen werden. Unsere Erfahrung bestätigt die beschriebene erhebliche Verbesserung der respiratorischen Symptomatik durch diese Therapiemaßnahme.

\section{P150}

Management und Verlauf einer schweren PVL-positiven c-MRSA Pleutopneumonie bei einem einjährigen Kind

\section{A. Wendt ${ }^{1}$, H. Sallmon ${ }^{2}$, A. Gratopp ${ }^{1}$, B. Niggemann}

'Charité Universitätsmedizin Berlin, Campus Virchow Klinikum, Klinik für pädiatrische Pneumologie und Immunologie, interdiszipl. Kinderintensivstation, Berlin, Deutschland, ${ }^{2}$ Charité Universitätsmedizin Berlin, Campus Virchow Klinikum, Klinik für Neonatologie, Berlin, Deutschland

Einleitung: cMRSA Infektionen nehmen weltweit zu, erstmalige Beschreibung in Deutschland 2002. Bis 95\% der Infektionen sind mit dem Panton-Valentine Leukozidin (PVL, Pathogenitätsfaktor) positivem cMRSA. Nekrotisierende Pneumonien und Fasziitiden sind assoziiert, eine nosokomiale Übertragung ist möglich. cMRSA Nachweis in Wunden erfordert die Bestimmung des nasalen Trägertums mit entsprechender Sanierung. Infektketten in Krankenhäusern wurden beschrieben, die strikte Einhaltung von Hygienevorschriften ist unerlässlich.

Fall: Aufnahme eines einjährigen Flüchtlingskindes aus einer Flüchtlingsunterkunft mit Verdacht auf eine Pneumonie und Meningitis mit Tachydyspnoe und Meningismus nach anamnestisch 10tägigem Fieber. Der Großvater hat eine nachgewiesene thorakale Weichteilinfektion zum Aufnahmezeitpunkt des Kindes. Liquordiagnostisch Ausschluß einer Meningitis, radiologisch Nachweis einer ausgeprägten Pleuropneumonie mit Spannungspneumothorax rechts. Anlage zweier Thoraxdrainagen (apikal und basal) und anschließende Beatmungspflichtigkeit für 7 Tage bei klinisch manifestem ARDS mit schwerer Oxygenierungsstörung. Es erfolgte eine kalkulierte antibiotische Therapie mit Cefotaxim, Gentamycin und Clarithromycin, Umstellung auf Vancomycin Monotherapie intravenös für zwei Wochen bei Nachweis von MRSA im Pleurasekret. Entfernung der apikalen Thoraxdranage nach 3 Tagen, Wechsel der basalen nach 2 Wochen auf eine Pigtaildrainage bei frustranen Entwöhnungsversuchen mit rezidivierenden Spannungspneumothoraces. Entwicklung einer Sepsis ohne Erregernachweis 5 Tage nach Beendigung der Vancomycintherapie mit Verdacht auf eine pneumogene cMRSA Sepsis. Daher wurde eine kalkulierte Antibiotische Therapie mit Clindamycin und Rifampicin initiiert, bei anhaltendem Fieber Eskalation um Vancomycin und Meropenem mit subsequenter Entfieberung. Es erfolgte eine cMRSA Eradikation mit Octenisan ${ }^{\oplus}$ Waschungen und Octenisan ${ }^{\oplus}$ Nasengel (Octenidine $\mathrm{HCl}$ ). Die Erregeranalyse ergab einen community acquired MRSA (cMRSA, PVL-Bildner, Vancomycin sensibel). Der gleichzeitige Nachweis des Erregers beim Großvater spricht für eine intrafamiliäre Erregerübertragung auf das Kind. Risikofaktoren, wie prädisponierende Grunderkrankungen, vorherige antibiotische Therapien oder Krankenhausaufenthalte fehlten, ein zugrundeliegender Immundefekt konnte beim Kind ausgeschlossen werden. Die 
Hygieneabstriche (MRSA/MRGN) nach einmonatigem stationären Aufenthalt waren negativ aus Rachen, Nase, und Rektum. Eine Meldung an das zuständige Gesundheitsamt zur Durchführung von MRSA-Screening der gesamten Familie und eine Empfehlung der getrennten Unterbringung des Großvaters vom Kind sind erfolgt.

Diskussion: 1) cMRSA als Ursache schwerer Pleuropleumonien muss bei Hautinfektionen von Familienmitgliedern bedacht, und in die Kalkulation der antibiotischen Therapie mit einbezogen werden 2) eine MRSA Eradikation zur Vermeidung von Übertragung und Infektion vor allem kleiner Kinder ist dringend notwendig 3) Besondere Krankheitsschwere durch PVL-assoziierte nekrotisierende Weichteilinfekte bzw. nekrotisierende Pneumonien

\section{P151}

Lungenfunktion in VLBW-Frühgeborenen mit persistierendem Ductus arteriosus nach pharmakologischer und chirurgischer Therapie

H. Sallmon', P. Barikbin', S. Wilitzki', J. Photiadis', C. Bührer', P. Koehne', G. Schmalisch'

'Universitätsklinikum Charité, Neonatologie, Berlin, Deutschland, ${ }^{2}$ Deutsches Herzzentrum Berlin, Kinderherzchirurgie, Berlin, Deutschland

Hintergrund: Die Indikationen und die optimale Strategie zur Behandlung eines persistierenden Ductus arteriosus (PDA) bei sehr unreifen Frühgeborenen werden kontrovers diskutiert. Insbesondere die Nebenwirkungen einer pharmakologischen oder chirurgischen Therapie sowie die Effekte auf das Langzeitoutcome sind weitgehend unklar.

Fragestellung: Ziel dieser Studie war die Untersuchung der Lungenfunktion bei Frühgeborenen mit PDA nach erfolgreicher pharmakologischer Therapie im Vergleich zu Kindern, die einer sekundären Ligatur bedurften (nach erfolglosem pharmakologischen Therapieversuch).

Methoden: 114 VLBW-Frühgeborene (Geburtsgewicht $<1500 \mathrm{~g}$ ), einschließlich 94 ELBW-Frühgeborene (Geburtsgewicht $<1000 \mathrm{~g}$ ), die wegen eines hämodynamisch signifikanten PDA behandelt wurden, erhielten im Alter von durchschnittlich 48 Wochen post menstruationem eine Lungenfunktionsuntersuchung. Alle Kinder wurden initial pharmakologisch therapiert, wobei 40 (35\%) eine sekundäre Ligatur benötigten. Die Lungenfunktionsuntersuchungen umfassten die Bestimmung von Atemvolumina, Atemmechanik (Okklusionstestung) und FRCVmax (rapid thoracoabdominal compression technique) sowie Ganzkörperplethysmographie, SF6-Auswaschtestung, die Messung des fraktionierten exhalierten Stickstoffmonoxids (FeNO) und arterialisierte kapilläre Blutgasuntersuchungen.

Ergebnisse: Am Tag der Lungenfunktionsuntersuchung waren beide Gruppen hinsichtlich Gewicht und korrigiertem Alter vergleichbar. Die Kinder mit sekundärer Ductusligatur waren allerdings unreifer bei Geburt $(p<0,001)$, zeigten eine verminderte respirtorische Compliance $(p<0,001)$, niedrigere VmaxFRC $(p=0,006)$, eine erhöhte Resistence (Raw; $p<0,001)$ und hatten schlechtere Blutgasanalysewerte $(p<0,001)$. Mittels multivariater Analyse konnte die Ducutusligatur als unabhängiger Risikofaktor für eine erhöhte Resistance und eine verminderte Compliance identifiziert werden.

Diskussion und Schlussfolgerungen: Im Alter von 48 Wochen post menstruationem zeigen VLBW-Frühgeborene nach sekundärer Ductusligatur eine eingeschränkte Lungenfunktion im Vergeich zu Kindern nach erfolgreicher pharmakologischer Ductustherapie.

\section{Postersitzung 15: Ernährung Frühgeborene}

\section{P152}

\section{Postnatal Growth Velocity Calculation:} Accuracy of Different Methods

A. Ali', N. Rochow', R. Iskander', G. Fusch' ', C. Fusch ${ }^{1}$

${ }^{1}$ McMaster University, Division of Neonatology, Department of Pediatrics, Hamilton, Kanada

Introduction: Postnatal growth in premature infants is an indirect measure of health status, nutritional adequacy, and long-term health outcome. Growth is usually assessed as an increase in weight over time. However, an absolute weight gain has different clinical implications depending on the weight of the infant. Hence, growth velocity (GV) that is normalized for body weight expressed as $\mathrm{g} / \mathrm{kg} /$ day is an important parameter of growth assessment, for clinical management and research. Currently, there is no agreement for the calculation of GV. This study aims to assess the accuracy of different GV calculations.

Objective: to compare different methods of growth velocity calculation in real infant dataset.

Methods: Real weight data of 94 infants ( $<29$ weeks gestational age) was used with six different methods of GV calculation: 1) 2-point linear 2) 2-point exponential 3) daily average method 4) linear regression 5) exponential regression 6) generalized reduced algorithm. The first two are 2-point methods whereas the first and last weights are used for GV calculation. The third is a modification of a 2-point method where daily GV is calculated using two points, and averaged over a period. The next three are regression methods where all available weight data are incorporated into the calculation. We used the mean growth velocity of all methods as a reference, and correlated it with GV estimated from the individual methods. Results: The mean variation between the six methods was $3.7 \pm 2.2 \mathrm{~g} / \mathrm{kg} /$ day. GV calculated with the generalized reduced algorithm method has the most agreement with the line of identity. The regression methods have better agreement with line of identity compared to the 2-point methods (Fig. A). Based on the R2, the best methods are as follows. First, the generalized reduced algorithm (0.94), exponential regression (0.93), linear regression (0.92), 2-point exponential (0.90), 2-point linear (0.89) and lastly, the daily average method (0.87).

Conclusion: The GV estimates vary depending on the method of calculation. 2-point methods overestimate low growth rates and underestimate high growth rates. This has significant implications for clinical trials as it could mask a potential effect. Since clinical trials are often powered to detect a difference of $2-3 \mathrm{~g} / \mathrm{kg} /$ day, such a difference in growth rate may be clinically significant and meaningful. Incorporating all available information appears to be better than using only 2 points. GV calculation needs to be standardized to allow for comparison across nutritional studies.

\section{P153}

Body Composition Assessment in Preterm Infants: Comparison of Air Displacement Plethysmography and Dual-Energy X-Ray Absorptiometry

\section{A. Ali', G. Fusch', N. Rochow', S. Atkinson ${ }^{2}$, C. Fusch ${ }^{1}$}

${ }^{1}$ McMaster University, Division of Neonatology, Department of Pediatrics, Hamilton, Ontario, Kanada, ${ }^{2}$ McMaster University, Department of Pediatrics, Hamilton, Kanada

Introduction: Fat and lean mass accretion in preterm infants determines the risk for chronic diseases. Assessing nutrition is the best way to curtail inappropriate growth of these body compartments. Infant body composition is an emerging field promising a more clinically relevant assessment of adequacy of nutrition compared to the current practice of weight gain evaluation. Hence, there is a need to establish the validity of body composition techniques. Air displacement plethysmography (ADP) has been independently validated against established reference methods. However, 
there is little to no literature comparing ADP with DXA, particularly in the preterm population.

Objectives: To compare body composition estimates from DXA with ADP Methods: 72 concurrent DXA (Hologic QDR4500) and ADP (PEAPOD, COSMED) measurements were compared from 72 preterm infants (born $<30$ weeks of gestation). Measurements were performed at three points: $<40$ weeks of corrected gestational age, term and 3 months corrected age ( $n=21,33$, and 18 respectively). Infants were measured with DXA while wearing a disposable diaper, swaddled in a cotton blanket, and laying supine on the scanning bed. ADP measurements were performed with the infant nude, wearing only a wig cap. In addition, total mass measurements from DXA and ADP were compared against a third method, an electronic scale (Smart Scale Model 65).

Results: DXA and ADP were significantly correlated for total body mass $\left(R^{2}=0.997\right)$, absolute fat mass $\left(R^{2}=0.910\right)$, absolute fat-free mass $\left(R^{2}=0.961\right)$ and \%fat mass $\left(R^{2}=0.696\right)$. However, the Bland-Altman analysis revealed significant bias $(p \leq 0.001)$ in all these estimates. Both the DXA and ADP total mass against the independent electronic scale showed a high correlation $\left(R^{2}=0.995\right.$ and $R^{2}=0.999$ respectively). However, only the DXA total mass showed a significant bias from the electronic scale $(p<0.001)$ in the Bland-Altman analysis.

Conclusions: Body composition estimates by DXA and ADP were highly correlated, but significantly biased in preterm infants. DXA mass deviates systematically from both the independent scale and the ADP scale, and $\%$ fat mass is underestimated compared to ADP. Literature suggests that this discrepancy is not attributable to the mass of the diaper or blanket. Further studies are needed to identify the basis of the large inter-method biases.

\section{P154}

Laktobezoare als seltene Ursache eines Ileus beim Frühgeborenen

K. Ruf', F. Seitz' ${ }^{2}$ J. Wirbelauer', S. Kunzmann'

'Universitäts-Kinderklinik Würzburg, Würzburg, Deutschland, ${ }^{2}$ Chirurgische Universitätsklinik I, Abteilung für Kinderchirurgie, Würzburg, Deutschland

Hintergrund: Laktobezoare aus Bestandteilen unverdauter Milch sind in Einzelfallberichten als seltene Ursache gastrointestinaler Probleme Frühund Neugeborener bis hin zu Magen- und Darmperforationen beschrieben worden. Als Risikofaktoren wird neben der Organunreife oft die Ernährung mit Formula-Nahrung genannt. In seltenen Fällen können jedoch auch Muttermilchzusätze die Bildung von Laktobezoaren begünstigen.

Fallbericht: Der Nahrungsaufbau erfolgte bei dem eutrophen weiblichen Zwillingsfrühgeborenen $28+3$ SSW (GG 940 g) problemlos. Vollständige enterale Ernährung mit Muttermilch ab dem 7. Lebenstag, Anreicherung mit Frauenmilchsupplement (FMS Aptamil?) ab dem 10. Lebenstag. Wegen eines akuten Abdomens erfolgte am 15. Lebenstag eine explorative Laparotomie, in der sich eine langstreckig-ödematöse Dünndarmwand sowie trüber Aszites letztlich unklarer Genese zeigte. Intraoperativ keine Malrotation, kein Hinweis auf NEC, keine Perforation. Nach doppelläufiger Ileostoma-Anlage Abgang von Stuhl über das Stoma ab dem 2. Postoperativem Tag, erneut problemloser Kostaufbau mit Muttermilch ab dem 3. postoperativen Tag. Ab dem 14. postoperativen Tag erfolgte bei einer Nahrungsmenge von $170 \mathrm{ml} / \mathrm{kgKG}$ die Anreicherung mit FMS. Innerhalb von 3 Tagen entwickelte das Frühgeborene einen Subileus, das Stoma förderte wenig flüssige Stühle, während zunehmend Resistenzen - am ehesten Skyballa entsprechnend - zu palpieren waren. Es bestanden keine systemischen Entzündungszeichen, kein Hinweis auf eine Hohlorganperforation, jedoch deutlich distendierte Dünndarmschlingen. Auch eine Magen-Darm-Passage unter Röntgen-Durchleuchtung blieb ohne wegweisenden Befund. Bei Ileussymptomatik erfolgte so am 24 Tage mach der Laparotomie eine Re-Laparotomie, in der zahlreiche Laktobezoare geborgen wurden, die eine vollständige Dünndarmobstruktion bewirkt hatten Eine Perforation bestand nicht. Postoperativ gestaltete sich der Nahrungsaufbau mit Muttermilch erneut problemlos. Der in gleicher Weise ernährte Zwillingsbruder blieb stets klinisch unbeeinträchtigt.
Diskussion: In einzelnen Fallserien wird von Laktobezoaren berichtet, die zur Ileussymptomatik führten. Als Risikofaktoren wird neben der Frühgeburtlichkeit und einem niedrigen Geburtsgewicht vor allem die Ernährung mit Formula-Nahrung genannt. Sehr selten wird eine mögliche Assoziation von Laktobezoaren bei Supplementierung der Muttermilch mit Energie und Mineralstoffen spekuliert. Insbesondere intestinale Vorerkrankungen wie beispielsweise eine nekrotisierende Enterokolitis scheinen prädisponierend zu sein für die Bildung von Laktobezoaren. In unserem Fall konnte keine klare Kausalität für die Entstehung der Laktobezoare hergestellt werden; neben der Frühgeburtlichkeit kann als Risiko erhöhend das Ileostoma und die hohe Osmolarität der angereicherten Muttermilch die Entwicklung der Laktobezoare begünstigt haben. Unbekannt ist bislang, ob eine weitere intestinale Störung - beispielswiese eine zystische Fibrose - als Risikofaktor angesprochen werden muss. Möglicherweise stehen Milchnahrungen mit einer höheren Osmolarität und die Entwicklung von Laktobezoaren in einem engeren Zusammenhang, so dass insbesondere bei kleinen Frühgeborenen sowie bei Frühgeborenen mit vorerkrankten Gastrointestinaltrakt die Gabe von Nahrungszusätzen nur bei strenger Indikation und unter guter Beobachtung erfolgen sollte.

\section{P155}

\section{Is there a difference in body composition in infants after necrotizing enterocolitis (NEC) surgery in comparison to infants without NEC surgery?}

\section{Binder', S. Uthaya' ', N. Longford' ', C. Gale', N. Modi'}

'Imperial College London, Faculty of Medicine, Department of Neonatal Medicine, London, United Kingdom

Introduction: Necrotizing entercolitis (NEC) is the most common intra-abdominal emergency in preterm infants and surgical management is required in up to $42 \%$. Preterm infants who have surgery for necrotising enterocolitis (NEC) are at high risk of growth failure. A cardinal goal of subsequent neonatal care is to achieve sufficient nutritional intake to support adequate growth and development after NEC surgery. There is a paucity of published data describing the body composition of infants after NEC surgery. The aim of this preliminary study is to evaluate the body composition in infants after NEC surgery in comparison to infant without NEC surgery.

Methods: In this study, we included a subset of preterm infants who underwent NEC surgery, born between 2010-2013 and recruited into the NEON study (REC 09/H0707/88). The control group consisted of preterm infants without clinically diagnosed NEC. Body composition was measured by MRI in both groups at term, as previously described. Adipose tissue volume (ATV) was quantified directly and non-adipose tissue mass (non-ATM) was calculated as follows: ATV was converted into adipose tissue mass $(\mathrm{ATM}=\mathrm{ATVx} 0.9)$ and subtracted from weight $(\mathrm{kg})$ (non-ATM = weight-ATM). Linear regression was used to compare ATV and non-ATM between the surgery group and control groups, after adjusting for body size (length) and sex. The primary objective of this study was to investigate differences in body composition (ATV and non-ATM) between the two groups.

Results: Six preterm infants ( 5 male and 1 female) after NEC surgery (mean birth weight: $797 \pm 69$ grams) and 23 preterm infants (16 male and 7 female) without NEC surgery (birth weight: $902 \pm 170$ grams) were implemented in the study. Mean gestational age was $25+3 \pm 2.4$ weeks in the NEC surgery group and $26+3 \pm 1.2$ weeks in the control group. After adjustment for length and sex mean total ATV was significantly lower in the NEC surgery group in comparison with control infants: 0.55 litre $(95 \% \mathrm{CI} 0.38$ to 0.72$)$ in the NEC surgery group and 0.84 litre $(95 \% \mathrm{CI}$ 0.75 to 0.93 ) in the control group; $p=0.003$. Mean non-ATM was also lower in the NEC surgery group but this difference did not reach statistical significance: $2.90 \mathrm{~kg}$ (95\%CI 2.33 to 3.47 ) in the NEC surgery group and $3.33 \mathrm{~kg}$ (95\%CI 3.04 to 3.62 ) in the control group; $p=0162$. The average hospital stay (NEC surgery group: $140 \pm 36$ days vs. control group: $112 \pm 46$ days, $p=0,031$ ) and the duration time of parenteral nutrition (NEC surgery 
group: $67 \pm 49$ days vs. control group $17 \pm 7$ days, $p=0,01$ ) were significantly prolonged in the NEC surgery group.

Conclusion: Following surgical NEC, preterm infants have altered body composition compared to unaffected infants, with lower adiposity predominating. Future work should explore the long-term impact of this pattern of altered body composition, and the potential role of nutritional management in ameliorating it, in this group of infants.

\section{P156}

\section{Profiling Fatty Acid Concentrations in Hypertriglyceridemic} Plasma from Preterm Infants

\section{A. Choi', G. Fusch', N. Rochow', H. Abed ', C. Fusch'}

${ }^{1}$ McMaster University, Division of Neonatology, Department of Pediatrics, Hamilton, Ontario, Kanada

Background: Parenteral lipid emulsions are used as a primary source of energy and essential fatty acids during the critical development period for preterm infants. However, infants receiving total parenteral lipid emulsions are more likely to have higher circulating plasma triglyceride levels (TG) than those fed with breast milk. Since preterm infants are at risk for developing hypertriglyceridemia (i. e. TG $\geq 1.5 \mathrm{mmol} / \mathrm{L}$ ), profiling fatty acid concentrations in hypertriglyceridemic plasma (hyper TG) would provide a better understanding of the nature of hypertriglyceridemia whether single fatty acid or accumulation of certain fatty acids causes elevated TG level.

Aim: To determine how the fatty acid profile of preterm infants with hyper TG differs from those with normal TGs.

Methods: Fatty acids in total lipids in hyper TG $(n=88)$ and normal TG plasma samples $(n=116)$ from preterm infants were measured using GCMS and analyzed using relative and absolute quantification. This study was approved by the Hamilton Integrated Research Ethics Board (HIREB) of McMaster University.

Preliminary results: Absolute concentrations of majority of fatty acids are elevated in hypertriglyceridemic plasma (average TGs: $2.1 \pm 0.7 \mathrm{mmol} / \mathrm{L}$ ) compared to fatty acids in plasma with normal TG level (average TGs: $0.8 \pm 0.3 \mathrm{mmol} / \mathrm{L}$ ). However, relative quantification does not show the same trend of fatty acid elevations (• Fig. P156).

Conclusion: Relative quantification does not seem to accurately represent all fatty acids present in the plasma since individual fatty acids rise in proportion to the total fatty acids. Hypertriglyceridemia in preterm infants may be dependent on nutrient intake, altered absorption or altered metabolism of fat. Future comparisons of fatty acid profiles in nutrition sources to resulting plasma profiles may reveal differences in fatty acid uptakes, which might influence the use of specific fatty acids in lipid emulsions.

\section{P157}

\section{Postnatal weight loss of healthy preterm infants and crossing of percentiles on growth charts during postnatal adaptation}

N. Rochow' , E. Landau-Crangle', P. Raja', G. Fusch', J. Poesch'², M. Heckmann ${ }^{3}$, S. Seigal ${ }^{4}$, D. Campbell, C. Fusch ${ }^{7}$

${ }^{1}$ McMaster University, Division of Neonatology, Department of Pediatrics, Hamilton, Ontario, Kanada, ${ }^{2}$ Universitätsklinikum Heidelberg, Klinik für Kinder- und Jugendmedizin, Heidelberg, Deutschland, ${ }^{3}$ Ernst-Moritz-Arndt Universität, Kinderklinik, Greifswald, Deutschland, ${ }^{4}$ St. Joseph's Healthcare, Hamilton, Kanada, ${ }^{5}$ St. Michael's Hospital, Toronto, Kanada

Background: During postnatal adaptation, preterm infants undergo preterm contraction of extracellular water spaces (PreCES) with postnatal weight loss (PWL) and adjustment to a new percentile. We hypothesized that infants with lower birth weights will cross more percentiles during PreCES due to lower fat mass and higher total body water.

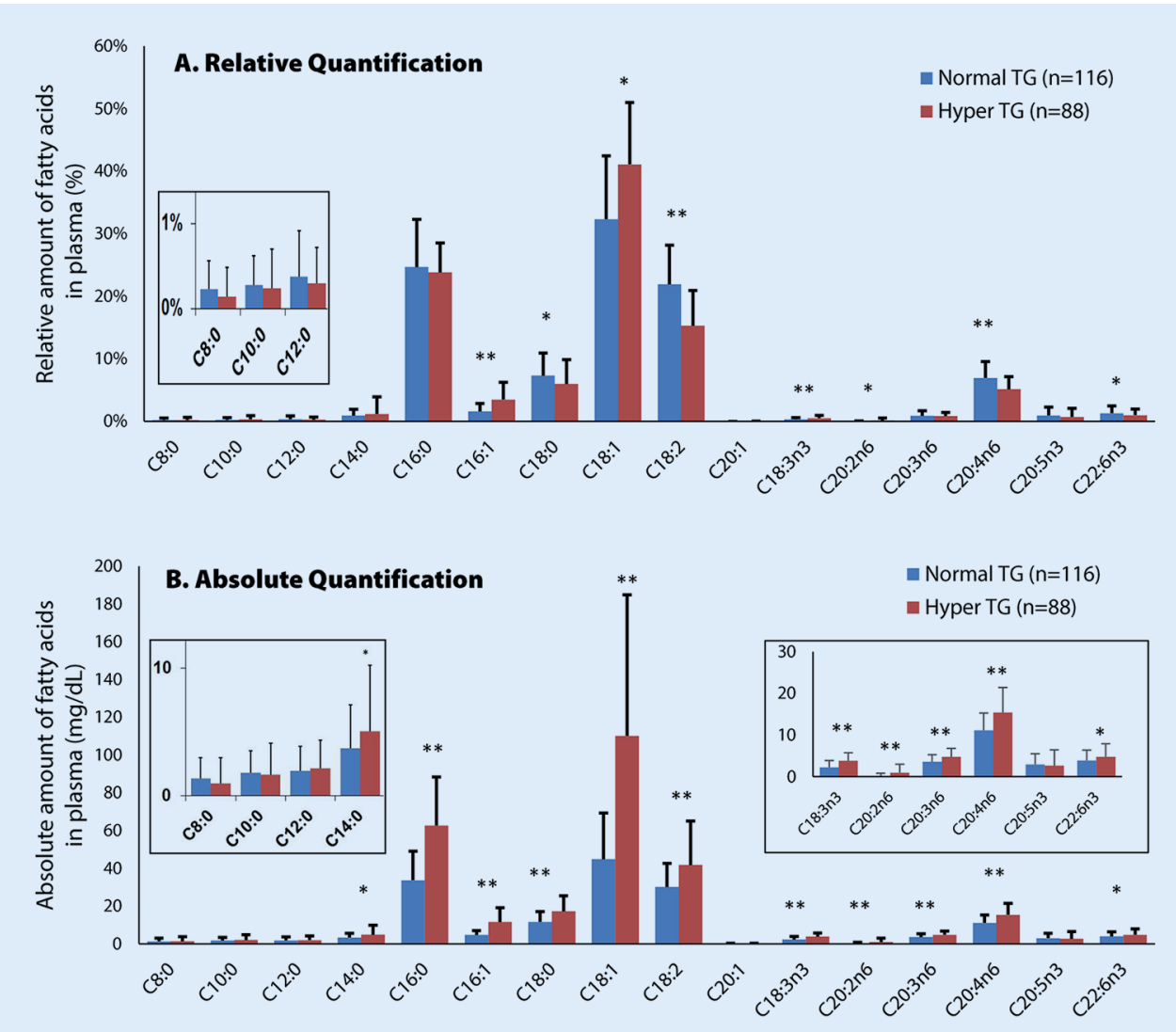



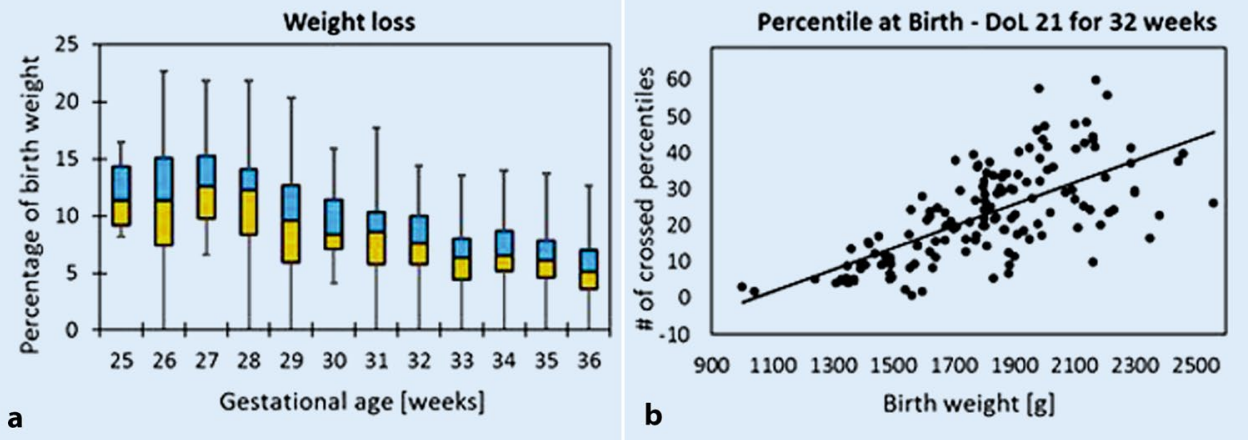

Fig. P157a \& b 4

Objective: To analyze PWL and change in percentiles during PreCES. Methods: International, multi-center study at five NICUs. Daily weights until day of life(DoL) 21 for infants (A): 30-36 weeks GA and (B): 24-29 weeks GA, admitted from 2008-2012 with undisturbed postnatal adaptation were analyzed. Exclusion criteria: (A) and (B): maternal diabetes/substance use, nosocomial sepsis (positive blood culture until DoL 21); (A): nCPAP >3 days, not on full enteral feeds by DoL 10, (B): mechanical ventilation on $\mathrm{DoL}>3, \mathrm{FiO}_{2} \geq 0.3$ within first $21 \mathrm{DoL}, \mathrm{NEC}>$ stage $2, \mathrm{IVH}>2$, PVL.

Results: 1188 healthy infants included. PWL was higher in infants born at earlier GAs. Maximum weight loss was for (A): $7 \%$ and (B): $11 \%$ by DoL 5 . While relative PWL was similar, infants born at higher birth weight percentiles crossed more percentiles compared to those born at lower percentiles. - Fig. P157a shows weight loss for GA. 1 Fig.P157b shows data for infants born at 32 weeks GA.

Conclusions: This study provides a robust estimate for physiological PWL in preterm infants after undisturbed PreCES. Crossing more percentiles during PWL for infants with higher birth weights could be explained by skewed percentile distributions of reference growth charts, which do not take into account PreCES.

\section{P158}

\section{Predicting Preterm Infants individual Growth Trajectories using} an Innovative Growth Trajectory Calculator Tool

E. Landau-Crangle', N. Rochow' ', T. Fenton', K. Liu', G. Fusch', M. Marrin', C. Fusch ${ }^{1}$

${ }^{1}$ McMaster University, Division of Neonatology, Department of Pediatrics, Hamilton, Ontario, Kanada, ${ }^{2}$ Alberta Children's Hospital Research Institute, Calgary, Kanada, ${ }^{3}$ McMaster University, Mathematics and Statistics, Hamilton, Kanada

Background/Objectives: Current growth charts provide no target for how a preterm infant should grow and ignore the physiological postnatal adaptation and weight loss in preterm and term infants between birth and day of life (DoL) 14-21. This includes a one-time, irreversible loss of extracellular water. Subsequently, preterm infants downshift to a "new“ growth trajectory earlier than term infants. The period of stable growth, from the end of postnatal adaptation by DoL 21 until 42 0/7 weeks postmenstrual age (PMA) should be similar in preterm infants to healthy fetuses remaining in utero until term, however evidence is scarce. The objective is to compare two approaches to generate individual growth trajectories for the period of stable growth and evaluate the difference to the term WHO growth standards (WHOGS) target weight corresponding to the birth weight percentile at $420 / 7$ weeks PMA.

Methods: Two approaches were tested for infants born at 24-34 weeks PMA and for birth weights at 7 major percentiles. Postnatal-Percentile Approach: growth following the percentile achieved at DoL 21 until term; Growth-Velocity Approach (figure-red line, marked „T“): increasing weight using day-specific Fenton median growth velocities until term. Results: Difference between predicted and target weights at 42 0/7 weeks with Postnatal-Percentile Approach: up to 930 g; Growth-Velocity Ap- proach: accurate and precise match with term WHOGS target weights at $420 / 7$ weeks when optimized by a single factor of 1.0017 .

Conclusion: This study showed that individual growth trajectories for preterm infants could be predicted by applying the optimized Fenton day-specific median growth velocity for the period of stable growth. These predicted trajectories accurately and precisely matched with the WHOGS target weight at 42 0/7 weeks PMA. A growth trajectory calculator tool, which can be used at bedside by clinicians to predict individual growth trajectories for preterm infants, can be developed from these results.

\section{P159}

Einfluss einer höheren Eiweißzufuhr auf Serum-

Wachstumsfaktoren und ihre Bindungsproteine bei unreifen Frühgeborenen Ergebnisse einer prospektiven, randomisiert kontrollierten Interventionsstudie

C. Bleeker', M. Mathes', J. Bürkle', K. Weber' ${ }^{2}$, C. Wiechers', C. Poets', G. Binder' ${ }^{2}$ A. Franz' ${ }^{1}$, C. Maas ${ }^{1}$

${ }^{1}$ Eberhard-Karls-Universität Universitätsklinik für Kinderheilkunde und Jugendmedizin, Neonatologie, Tübingen, Deutschland, ${ }^{2}$ Eberhard-KarlsUniversität Universitätsklinik für Kinderheilkunde und Jugendmedizin, Pädiatrische Endokrinologie, Tübingen, Deutschland

Hintergrund und Fragestellung: Die Sekretion von Wachstumsfaktoren wie z. B. Insulin-like growth factor-I (IGF-I) wird auch durch Ernährungsfaktoren wie z. B. die Proteinzufuhr beeinflusst und kann sich durch unterschiedliches Nahrungsangebot rasch ändern. Ziel dieser Untersuchung war zu prüfen, ob eine höhere enterale Eiweißzufuhr zu einer bedeutsamen Veränderung der Serumspiegel verschiedener Wachstumsfakto-

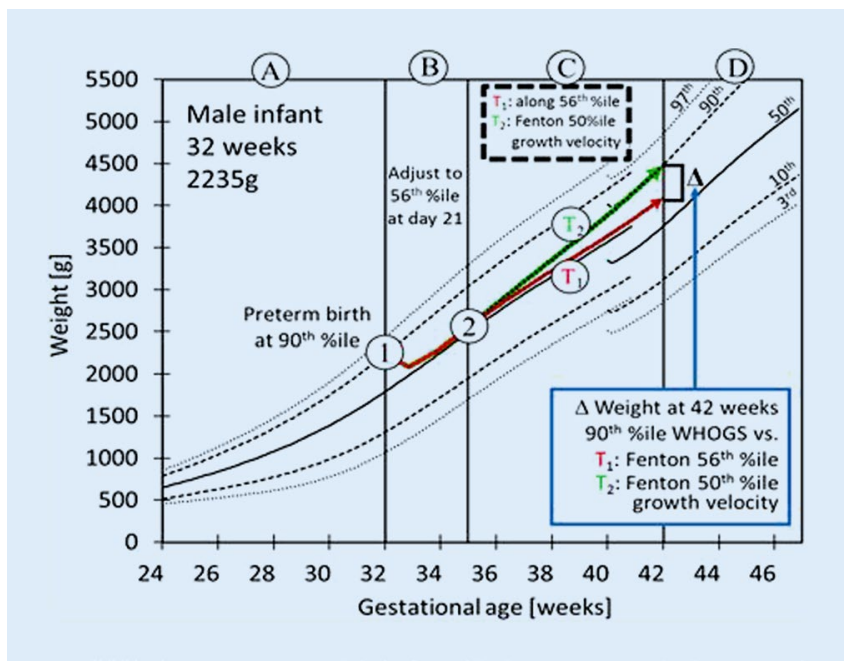

Abb.P158 4 
ren und ihrer Bindungsproteine bei überwiegend muttermilchernährten Frühgeborenen (FG) im Verlauf des postnatalen stationären Aufenthalts führt.

Patienten und Methoden: Prospektive, monozentrische, randomisiert kontrollierte Interventionsstudie. Eingeschlossen wurden FG mit einem Gestationsalter (GA) bei Geburt $<32$ SSW und einem Geburtsgewicht $(\mathrm{GG})<1500$ g. Primär wurde die Hypothese geprüft, ob eine an den Empfehlungen der ESPGHAN orientierte Eiweißzufuhr von ca. $4,5 \mathrm{~g} / \mathrm{kg} / \mathrm{d}$ im Vergleich zu einer Standardernährung mit ca. 3,5-4,0 g/kg/d Eiweiß zu einer Verbesserung des Wachstums führt, was nicht zutraf. A priori geplant war außerdem die hier berichtete Analyse der Serumspiegel von IGF-I, IGF-II, IGFBP-3, IGFBP-1 und Wachstumshormon im Verlauf des postnatalen stationären Aufenthalts aus bis zu drei Blutproben jedes Probanden (Bestimmung mittels in-house Radioimmunassays).

Ergebnisse: Untersucht wurden 139 Blutproben von 60 Frühgeborenen (30 in der Standardeiweiß- und 30 in der Hocheiweiß-Gruppe, medianes GA bei Geburt 30,0 vs. 29,7 Wochen, medianes GG 1215 vs. 1193 g). Trotz einer im Mittel um 0,57 g/kg/d unterschiedlichen Proteinzufuhr (3,7 in der Standardeiweiß- vs. $4,3 \mathrm{~g} / \mathrm{kg} / \mathrm{d}$ in der Hocheiweißgruppe) unterschieden sich die Spiegel von IGF-I, IGF-II, IGFBP-3, IGFBP-1 und Wachstumshormon nicht signifikant zwischen den Gruppen an den drei Beobachtungszeitpunkten (Median im Alter von 15, 35 und 54 Tagen). Der IGF-I-Spiegel lag im Median (p25/p75) an Beobachtungszeitpunkt 1 bei 37 (23/48) in der Standardeiweiß- vs. 32 (23/42) in der Hocheiweißgruppe, an Beobachtungszeitpunkt 2 bei 43 (34/55) vs. 43 (35/47) und an Beobachtungs-

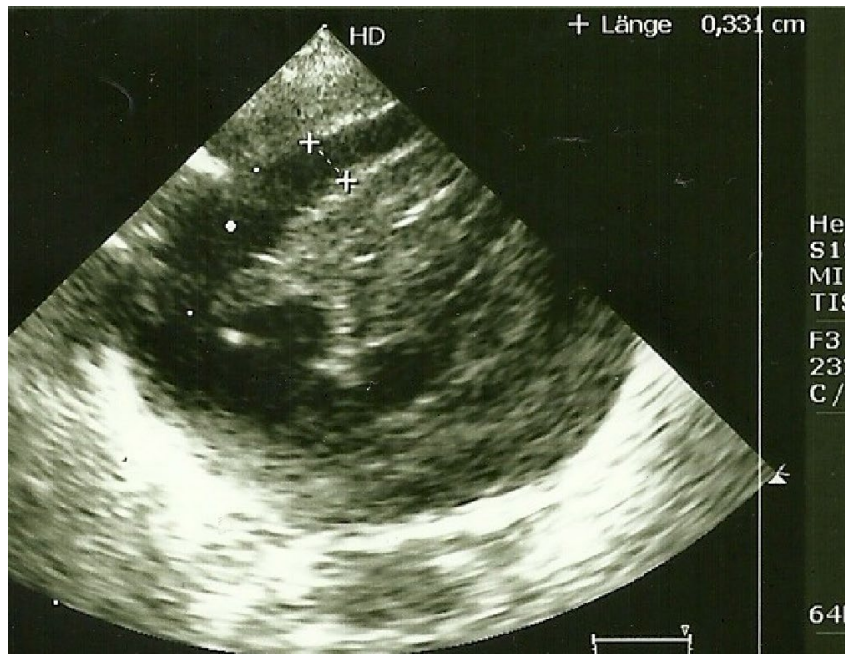

Abb. P160.1 $\Delta$ Perikarderguss, 9. Lebenstag

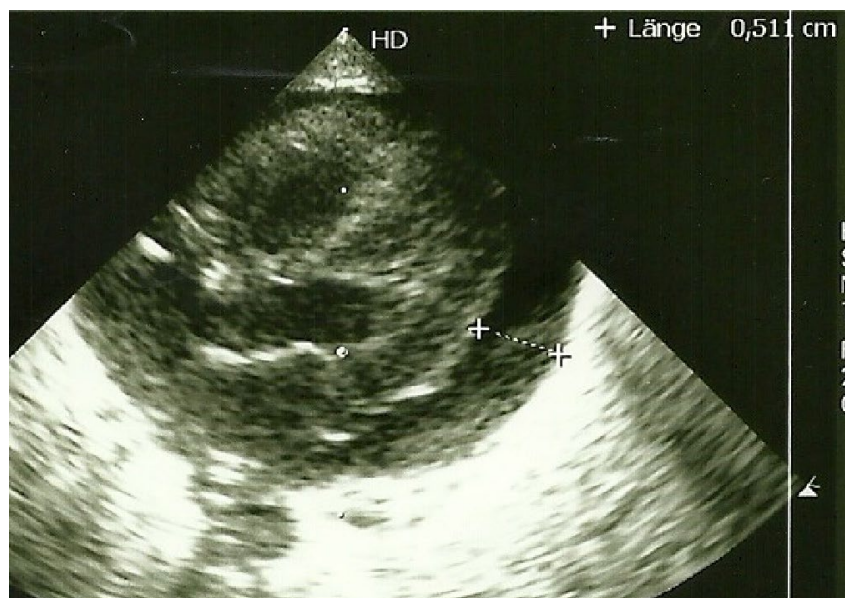

Abb. P160.2 $\Delta$ Perikarderguss, 9. Lebenstag zeitpunkt 3 bei 44 (43/51) vs. 46 (41/57) ng/ml. Auch die Veränderung des IGF-I-Spiegels im Verlauf unterschied sich zwischen den Gruppen nicht. Der Zusammenhang zwischen postmenstruellem Alter bei Probenentnahme und IGF-I-Spiegel war deutlich stärker $\left(\mathrm{r}^{2}=0,31, p<0,0001\right)$ als der zwischen postnatalem Alter und IGF-I-Spiegel $\left(r^{2}=0,13 p<0,0001\right)$. Schlussfolgerung: Die hier untersuchte Erhöhung der Eiweißzufuhr führte nicht zu einer signifikanten Veränderung der gemessenen Wachstumsfaktorenspiegel und ihrer Bindungsproteine. Der IGF-I-Spiegel wurde am stärksten durch das postmenstruelle Alter bei Probenentnahme bestimmt.

\section{P160}

Infusoperikard - eine seltene Komplikation bei ELBWFrühgeborenen mit zentralem Einschwemmkatheter

A. Paulun', V. Umlauf', C. Fremerey', B. Wiebe'

'Asklepios Klinik Sankt Augustin GmbH, Neonatologie und Pädiatrische Intensivmedizin, Sankt Augustin, Deutschland

Fallbericht: Zwillings-Frühgeborenes, $25+4$ SSW, GG 640 g, Sectio aus mütterlicher Indikation. Komplikationslose Anlage eines zentralen Einschwemmkatheters am 2. Lebenstag. Im Rahmen einer Routineechokardiographie am 9. Lebenstag Feststellung eines zirkulären Perikardergusses mit rascher Progredienz und maximalem Durchmesser von $6 \mathrm{~mm}$. Echoardiographisch Darstellung der Katheterspitze im rechten Vorhof. Nach sofortiger Beendigung der Infusion und Entfernen des Katheters

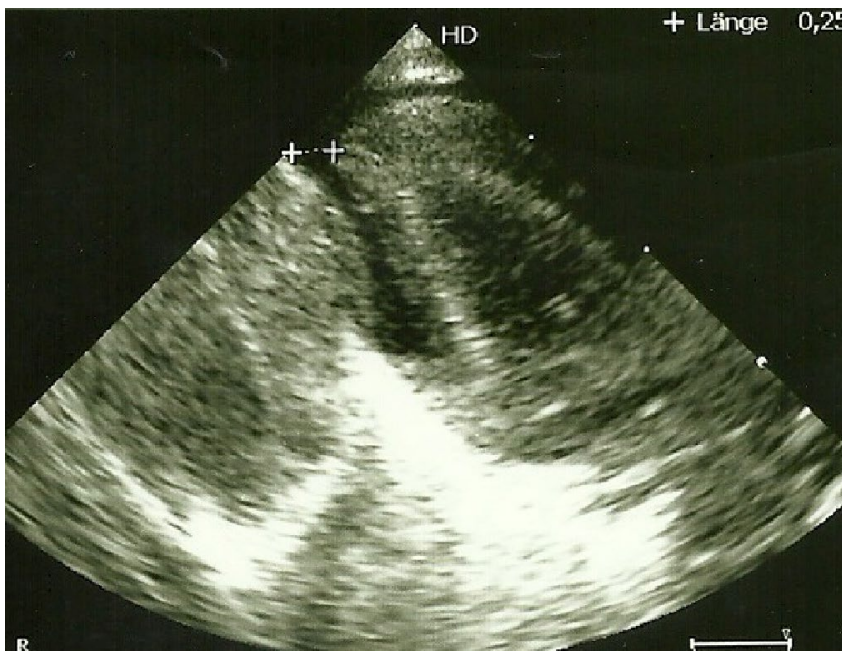

Abb. P160.3 $\Delta$ Perikarderguss, 9. Lebenstag

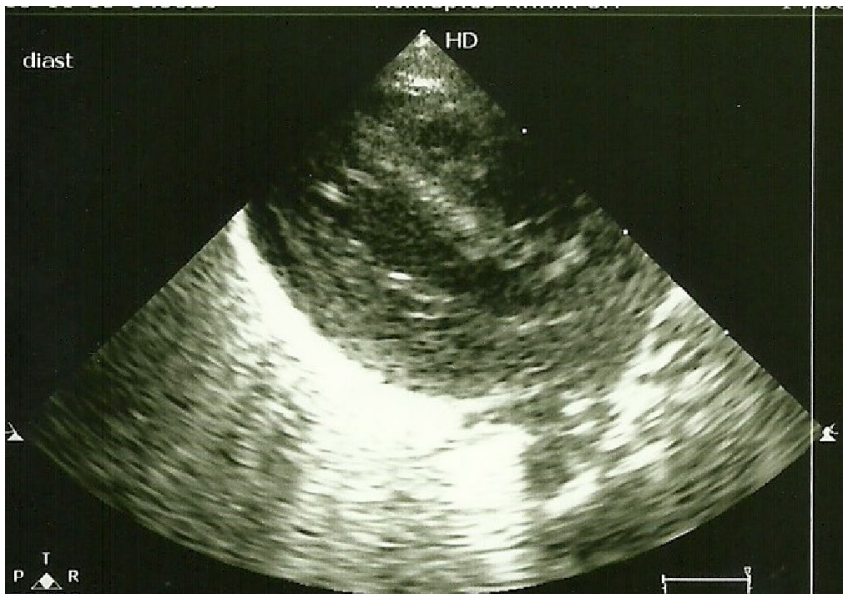

Abb. P160.4 \ Perikarderguss im Verlauf, 11. Lebenstag (Diastole) 


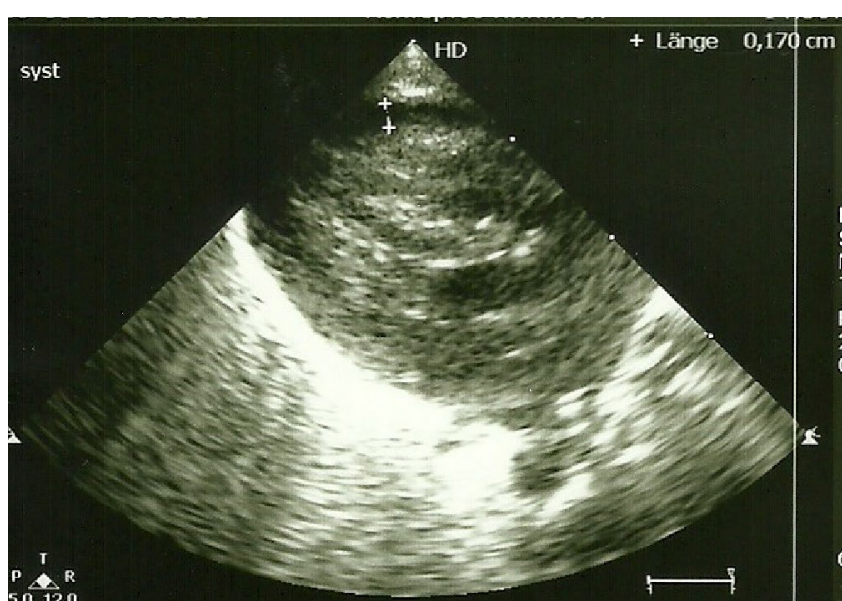

Abb. P160.5 $\Delta$ Perikarderguss im Verlauf, 11. Lebenstag (Systole)

Erguss rasch rückläufig. Vollständige Resorption binnen 12 Tagen. Zu keinem Zeitpunk kardiorespiratorische Instabilität des Frühgeborenen.

Definition Infusoperikard: Perikarderguss mit der Maximalvariante der Perikardtamponade als Komplikation eines zentralvenösen Katheters. Die Punktatzusammensetzung entspricht der Infusionslösung. Nach Entfernen des Katheters rasch rückläufiger Befund.

Inzidenz bei Frühgeborenen: Angaben variieren zwischen 0,18 und 1,4\% aller zentralen Einschwemmkatheter mit einer mittleren Mortalität von $34 \%$. Pathogenese: Ätiologie unklar. Diskutiert werden direkte Perforationen des Myokards, Bildung myokardialer Thromben oder Nekrosen, langsame Erosionen durch mechanische Reize oder Anhaftung der Katheterspitze an das Myokard mit Diffusion der Infusionslösung. Auftreten meist 2 bis 3 Tage nach Katheteranlage mit einer Varianz von 0,2 bis 37 Tagen. Zeitintervall von Beginn des Perikardergusses bis zum Auftreten klinischer Symptome unbekannt.

Lage der Katheterspitze im rechten Vorhof vs. Lage in der Vena cava superior: In unserem Fall Darstellung der Katheterspitze im rechten Vorhof zum Zeitpunkt der Diagnosestellung. Ein vermuteter Zusammenhang zwischen Lage der Katheterspitze im rechten Vorhof und erhöhter Inzidenz eines Infusoperikards konnte in einer retrospektiven Studie aus Großbritannien nicht bestätigt werden. Die tatsächliche Lage der Katheterspitze zum Zeitpunkt der Entstehung des Perikardergusses bleibt häufig unklar, da insbesondere bei kleinen Frühgeborenen eine geringe Bewegung von Kopf oder Armen ausreicht, um den Katheter um bis zu 2 cm zu verschieben.

Klinik: Wird der Perikarderguss nicht, wie in unserem Fall, zufällig in einer routinemäßig durchgeführten Echokardiographie diagnostiziert, kann es im Verlauf zu einer plötzlichen kardiorespiratorischen Verschlechterung bis hin zum plötzlichen Herzstillstand kommen.

Therapie und Prävention: Bislang gibt es keine eindeutigen Empfehlungen zur Prävention dieser seltenen, möglicherweise aber fatalen Komplikation. Bei plötzlich einsetzender kardiorespiratorischer Verschlechterung und einliegendem zentralen Einschwemmkatheter ohne andere Ursache muss echokardiographisch ein Perikarderguss ausgeschlossen werden. Bei konkretem Verdacht ist die Infusion zu beenden und der Katheter zu entfernen. Bei kardialer Beeinträchtigung sollte eine Perikardpunktion erwogen werden. Durch dieses Vorgehen kann eine deutliche Senkung der Mortalität von $75 \%$ auf $8 \%$ erreicht werden.

Das Wichtigste ist, daran zu denken!

\section{P161}

Fokale intestinale Perforation bei einem Frühgeborenen mit Anomalie des Mesenteriums

S. Krauter', D. Schopen ', T. Bielefeld ${ }^{2}$, A. Trotter ${ }^{3}$

${ }^{1}$ Hegau Bodensee Klinikum Singen, Neonatologie, Singen, Deutschland, ${ }^{2} \mathrm{Hegau}-K$ linikum GmbH, Kinderklinik, Singen, Deutschland, ${ }^{3} \mathrm{Hegau}-$ Bodensee-Klinikum Singen,Klinik für Kinder und Jugendliche, Neonatologie, Singen, Deutschland

Hintergrund: Die nekrotisierende Enterokolitis (NEC) sowie die fokale intestinale Perforation (FIP) sind für sehr kleine Frühgeborene typische erworbene gastrointestinale Erkrankungen (1). Pathogenetisch wird das Zusammentreffen von intestinaler Ischämie, Kolonisation mit potentiell pathogenen Bakterien sowie intraluminalen Faktoren bei immunologischer Unreife beschrieben.

Bei der FIP findet sich eine intestinale Perforation in Abwesenheit von Zeichen einer Entzündung oder Nekrose wie bei einer NEC. Die häufigste Lokalisation der FIP ist das terminale Ileum (2).

Das Gestationsalter und das Geburtsgewicht bei Kindern mit FIP sind signifikant geringer als bei Kindern, die an einer NEC erkranken. Die Inzidenz der FIP bei Kindern $<1500$ g liegt zwischen $1 \%$ und $2 \%$ (2), bei Kindern $<1000$ g steigt sie auf bis zu $9 \%$ an (3), wobei ihre Prognose meist besser als die einer NEC mit Perforation ist.

Kasuistik: Männliches Extrem-Frühgeborenes (ELBW), 595 g, sekundäre Sectio bei vorzeitigem Blasensprung und Muttermundseröffnung. RDS-Prophylaxe insg. vier Gaben.Vollständiger oraler Nahrungsaufbau am 10. LT komplikationslos erreicht. Bei zunehmend pulmonaler Verschlechterung 7-tägige Hydrocortisontherapie mit 3-4 mg/kg/d (8.-14. LT). Seit dem 14. LT progrediente Rötung am rechten Unterbauch mit verhärtetem und druckdolentem Abdomen, makroskopisch sichtbar distendierte Darmschlingen, kaum Peristaltik. Nahrungskarenz, i. v.antibiotische Therapie. Sonographischer Nachweis distendierter Darmschlingen im re Unterbauch mit Pendelperistaltik sowie Subileuszeichen. Radiologisch keine freie Luft oder Pneumatosis intestinalis.

Verlauf: Primäre Laparotomie am 19. LT bei fortbestehender Ileussymptomatik. Intraoperativ Darstellung einer ca. $1 \mathrm{~cm}$ grossen Perforation am terminalen Ileum wenige Zentimeter vor der Bauhin'schen Klappe ohne Hinweis auf nekrotische oder entzündlich veränderte weitere Dünndarmanteile. Auffallende Mesenteriallücke im Bereich des distalen Ileums über eine Strecke von ca. $5 \mathrm{~cm}$. Deutlich hypotrophes distales Ileum post perforationem. Anlage eines doppelläufigen Stomas. Seit dem 3. postoperativen Tag vorsichtiger Nahrungsaufbau. Dreitägige Nahrungskarenz bei Stuhlpropf am 13. postoperativen Tag, im Verlauf dann unkomplizierter Nahrungsaufbau. Schlussfolgerung/Fazit: Bei fokaler intestinaler Perforation muss auch an eine Mesenteriallücke als mögliche Anomalie des Mesenteriums gedacht werden, welche ursächlich für das Entstehen der FIP aufgrund der dort bestehenden Mangelversorgung („letzte Wiese“) sein kann. Bei unserem Fall war möglicherweise die Kombination von Hydrocortisongabe und bestehender Mesenterialanomalie ausschlaggebend für die Entstehung der FIP.

\section{Literatur}

1. Necrotizing enterocolitis and focal intestinal perforation in neonatal intensive care units in the state of Baden-Württenberg, Germany. Böhler et al. Pediatr Rep, 2014, Feb 17, vol 6 (1), 5194

2. Spontaneous gastrointestinal perforation in very-low-birth-weight infants - a rare complication in a neonatal intensive care unit. Resch B. et al. Pediatr Surg Int., 1998, Mar 13 (2-3):165-167

3. Discharge outcomes of extremely low birth weight infants with spontaneous intestinal perforations. Attridge JT et al. J Perinatol., 2006, Jan 26 (1): 49-54 


\section{P162}

\section{Ein wachstumsverzögertes Zwillingsfrühgeborenes mit Nephrocalcinose und Hypercalcämie - frühe genetische Diagnosesicherung}

\section{E. Altunlu' , N. Sailer' , B. Zirn'², A. Longin ${ }^{3}$, K. Niethammer', C. von} Schnakenburg ${ }^{4}$

${ }^{1}$ Klinikum Esslingen $\mathrm{GmbH}$, Klinik für Kinder und Jugendliche, Esslingen, Deutschland, ${ }^{2}$ genetikum ${ }^{\oplus}$, Stuttgart, Deutschland, ${ }^{3}$ Klinikum Esslingen $\mathrm{GmbH}$, Klinik für Radiologie, Esslingen, Deutschland, ${ }^{4}$ Klinik für Kinder und Jugendliche Klinikum Esslingen GmbH, Esslingen, Deutschland

Fallbericht: Bei einer Zwillingsschwangerschaft wurde wegen Wachstumsstillstand bei normaler Fruchtwassermenge und pathologischem Dopplerfluss eines Feten in der $29+4$. SSW die Sectio nach vorheriger Lungenreifetherapie durchgeführt. Geminus I war ein Junge, GG 1390 g, mit unproblematischem postpartalen Verlauf, der im Alter von 40 Minuten bei Stöhnen und $\mathrm{FiO}_{2}$ bis 0.35 am CPAP eine endotracheale Surfactantgabe bei Spontanatmung erhielt und anschließend $14 \mathrm{~d}$ mit CPAP bei $\mathrm{FiO}_{2} 0.21$ behandelt wurde. Es zeigten sich im Verlauf eine milde periventrikuläre Leukomalazie (ab 10.-14. LT) sowie eine milde Nephrocalcinose IIb bei Entlassung. Geminus II, ein Mädchen, wog bei Geburt 890 g, erhielt ebenfalls Surfactant über eine endotracheal platzierte Magensonde, wurde aber im Alter von 8 Stunden wegen zunehmenden Apnoen und steigendem $\mathrm{FiO}_{2}$ intubiert und für 4 Tage beatmet; anschließend CPAP-Atemhilfe über 15 Tage. Wegen einer Glukoseverwertungsstörung erfolgte bis zum 14. LT eine Insulinsubstitution. Als somatische Auffälligkeiten zeigten sich im Verlauf ein auffälliger Gesichtsschädel (nach lateral abfallende Lidachse, etwas tiefsitzende Ohren, antevertierte Nares, langes Philtrum, schmales Oberlippenrot) eine valvuläre Pulmonalstenose und eine Gedeihstörung (gestörtes Längen- und Kopfwachstum).

Diagnose: Letztlich führte eine unter Vitamin D und normaler Calciumsubstitution aufgetretene Hypercalcämie (bis 3,6 mmol/l) bei einer sich entwickelnden Nephrocalcinose in Verbindung mit o.g. Auffälligkeiten zur molekulargenetisch bestätigen Diagnose eine Williams-Beuren-Syndroms mit einer interstitiellen Deletion im langen Arm eines Chromosoms 7. Diskussion: Anhand des Falles wird die seltene Differentialdiagnose eines Williams-Beuren-Syndroms vorgestellt, welches ungewöhnlich früh im Reifealter von 35+6 SSW mit der Präsentation von fortbestehender Gedeihstörung, Trinkschwäche, valvulärer Pulmonalstenose und diagnoseweisender Hypercalcämie diagnostiziert wurde. Diffentialdiagnostische und therapeutische Konsequenzen werden bei der Fallpräsentation diskutiert. Beide Zwillinge konnten im Reifealter von 40+3 SSW mit sozialmedizinischer Nachsorge und häuslicher Krankenpflege wegen Notwendigkeit der Teilsondierung bei Geminus II nach Hause entlassen werden.

\section{P163}

\section{Late-onset Volvulus ohne Malrotation bei extremer Frühgebürtlichkeit}

\section{A. Tocut ${ }^{1}$, G. Selzer ${ }^{2}$}

'Evangelisches Krankenhaus Hamm, Neonatologie und pädiatrische Intensivmedizin, Hamm, Deutschland, ${ }^{2}$ Evangelisches Krankenhaus Hamm gGmbh, Ltd. Arzt Klinik für Neonatologie und pädiatrische Intensivmedizin, Hamm, Deutschland

Hintergrund: Die Entität des Volvulus ohne Malrotation ist distinkt vom typischen Bild des Volvulus ohne Malrotation beim extrem Neugeborenen, hinbezüglich Risikofaktoren, Zeitspanne des Auftrettens,Diagnoseu und Prognose, benötigt jedoch eine schnelle Diagnosestellung und Therapie um einer hohen Morbidität und Mortalität vorzubeugen.

Material und Methoden: retrospektive Fallstudie (2015) im Vergleich zu Ergebnissen von case-control Studien (2007-2013) und klinischen Fallstudien.

Ergebnisse: Ein extrem Frühgeborenes der 24+0 SSW, mit einem Geburtsgewicht von $650 \mathrm{~g}$ erlitt eine akute Verschlechterung des Allgemeinzustandes und mit erneuter Notwendigkeit der maschinellen Beatmung in der 6 . Lebenswoche (40. Lebenstag). Als Risikofaktoren konnte die rektale Mani- pulationen oder Bauchmassage ausgeschlossen werden (letzte Darmspülung 10 Tage vor Beginn der Symptomatik, keine erhöhte Frequenz von Bauchmassage bei regelmäßigen Stuhlgang). Langzeit Beatmung (HFO bis zur 4. Lebenswoche mit einem medianem CDP von 11,3 $\pm 2,1$ und NCPAP mit einem PEEP von 5,8 $\pm 1,3$ bis zur Reintubation) lagen jedoch vor. Es fanden sich keine klinischen oder paraklinischen Unterschiede zwischen Volvulus und nekrotisierender Enterocolitis. Hier fanden sich massiv erhöhte Entzündungsparameter und radiologisch luftdistendierte Darmschlingen, die mit dem Bild einer NEC übereinstimmten. Der Patient wurde 40 Stunden nach Eintritt der Symptomatik laparotomiert. Intraoperativ zeigte sich ein $180^{\circ}$ Volvulus der Darmschlingen im Oberbauch ante perforationem. Nach Retorquierug keine Erholung, sodass $20 \mathrm{~cm}$ Ileum entfernt werden. Postoperativ stabiler Verlauf, die Patientin hat überlebt.

Diskussion: Volvulus ohne Malrotation ist eine seltene Entität die leicht mit einer nekrotisieerende Enterkolitis verwechselt werden könnte und mit einer hohen Mortalität ausgeht. Typisch ist das späte(> 30 Lebenstagen) und plötzliche Auftreten der Symptomatik. Vor allem bei Langzeitbeatmung und respiratorische Unterstützung mit hohem endexpiratorischem Druck bei extremen Frühgeborenen sollte der Volvulus differnetialdiagnostisch in Betracht gezogen werden. Da die Risikofaktoren Teil der Behandlung bei den meisten Frühgeborenen sind, kann man nicht über eine Prävention sondern eher über eine frühe Diagnosestellung sprechen.

\section{P164}

\section{Rascher enteraler Nahrungsaufbau bei Extremfrühgeborenen:} erhöhte Rate abdomineller Komplikationen?

F. Belling-Dierks', E. Frieauff', K. Glaser', V. Rücker' ${ }^{2}$ C. Speer ${ }^{1}$

'Universitäts-Kinderklinik Würzburg, Würzburg, Deutschland, ${ }^{2}$ Institut für Klinische Epidemiologie und Biometrie der Universität Würzburg, Würzburg, Deutschland

Hintergrund: Der enterale Nahrungsaufbau bei sehr unreifen Frühgeborenen wird kontrovers diskutiert. Insbesondere der optimale Anfangszeitpunkt und die Steigerungsrate der enteralen Nahrungszufuhr sind Gegenstand der Diskussion.

Ergebnisse: aktueller Studien sprechen für Vorteile eines rascheren enteralen Nahrungsaufbaus bei Frühgeborenen mit einem Geburtsgewicht unter $1500 \mathrm{~g}$ (VLBW-Frühgeborene).

Fragestellung: In der vorliegenden Studie wurde die Auswirkung einer rascheren Steigerung der enteralen Nahrungsmenge auf die abdominelle Komplikationsrate (Nekrotisierende Enterokolitis und intestinale Perforation) untersucht.

Material und Methoden: Alle zwischen 2008 und 2013 im Perinatalzentrum der Universitätsklinik Würzburg geborenen Frühgeborenen $(=24+0$ SSW $)$ mit einem Geburtsgewicht $<1500 \mathrm{~g}$ wurden in diese retrospektive Kohortenstudie eingeschlossen. Im Zeitraum 2008-2010 sah das Ernährungsregime eine tägliche Steigerung der Nahrungsmenge um $10 \mathrm{ml} / \mathrm{kg} / \mathrm{Tag}$ vor. Ab 2011 erfolgte ein rascherer enteraler Nahrungsaufbau mir einer Steigerungsrate von $20 \mathrm{ml} / \mathrm{kg} / \mathrm{Tag}$. Wir verglichen die Rate abdomineller Komplikationen und das Gesamt-Outcome der zwischen 2008 und 2010 geborenen Frühgeborenen nach langsamen Nahrungsaufbau mit der Vergleichskohorte der von 2011 bis 2013 geborenen Frühgeborener nach rascherem Nahrungsaufbau. Mittels multivariabler linearer Regressionsanalysen wurde auf mögliche Confounder adjustiert und mittels logistischer Regression der Einfluss des Nahrungsaufbaus auf das Outcome untersucht.

Ergebnisse: Insgesamt wurden 301 VLBW Frühgeborene in die Studie eingeschlossen. Beide Gruppen waren vergleichbar bezüglich ihrer demographischen und perinatalen Charakteristika. Eine univariate logistische Regressionsanalyse zeigte keine signifikanten Unterschiede in der Inzidenz abdomineller Komplikationen zwischen beiden Gruppen $(p=0,4)$. Ebenso waren Mortalität und die Rate an Late onset-Septitiden in beiden Gruppen nahezu identisch. Im Gegensatz dazu waren die Dauer von Krankenhausaufenthalt (KA) und teilparenteraler Ernährung (PEN) in der rascheren Nahrungsaufbaugruppe signifikant reduziert (KA: $-8,35$ Tage, $p=0,012$; PEN: $-7,13$ Tage, $p<0,001)$. Höhergradige intraventrikuläre Hämorrha- 
gien, periventrikuläre Leukomalazie und ein persistierender Ductus arteriosus Botalli traten in beiden Gruppen in vergleichbarer Häufigkeit auf. Schlussfolgerung: Rascherer enteraler Nahrungsaufbau führt zu keiner Erhöhung der abdominellen Komplikationsrate bei sehr unreifen Frühgeborenen und konnte zu einer signifikanten Verringerung der Dauer von Krankenhausaufenthalt und teilparenteraler Ernährung dieser Patienten führen.

\section{Postersitzung 01: Pflege}

\section{Pfl.P01}

\section{Baden durch die Eltern - sind frühgeborene Kinder belastet?}

F. Fischer', J. Bergander', A. Butter' , M. Sommerfeld' ' J. Reichert ${ }^{2}$, M. Rüdiger ${ }^{1}$ 'Univ. Klinikum Carl Gustav Carus, Klinik und Poliklinik für Kinder- und Jugendmedizin, Neonatologie, Pädiatrische Intensivmedizin, Dresden, Deutschland, ${ }^{2}$ Universitätsklinikum Carl Gustav Carus Kinderklinik, Neonatologie/Pädiatrische Intensivmedizin, Dresden, Deutschland

Neben der medizinischen Versorgung ist eine individualisierte, beziehungsorientierte Pflege für die Entwicklung frühgeborener Kinder von entscheidender Bedeutung. Hierbei steht die Unterstützung der Herausbildung einer sicheren Eltern-Kind-Bindung im Mittelpunkt. Deshalb sollen die Eltern möglichst frühzeitig zur selbstständigen Pflege und Versorgung ihres Kindes befähigt werden. Das hierzu erforderliche Wissen wird ihnen durch Pflegekräfte vermittelt. Für das Erwerben praktischer Fertigkeiten hat sich eine standardisierte Anleitung durch speziell geschultes Pflegepersonal bewährt. Im Mittelpunkt dieser Anleitung steht beim Baden des Kindes durch seine Eltern die Unterstützung und Förderung der Eltern-Kind-Interaktion. Nach drei Anleitungen haben die Eltern in der Regel die erforderliche Sicherheit beim Baden ihres Kindes erworben. Eine dieser Anleitungen dauert etwa 75 Minuten und damit wesentlich länger, als wenn das Kind durch eine Pflegekraft gebadet wird.

Fragestellung: Zeigen frühgeborene Kinder nach dem Baden durch ihre angeleiteten Eltern, die gezielt auf eine adäquate sensorische Stimulation des Kindes und die Eltern-Kind-Interaktion beim Baden achten, besondere Belastungsreaktionen?

Methode: Ausgewertet werden die im Rahmen des kardiorespiratorischen Routinemonitorings erfassten Vitalparameter Atem- und Herzfrequenz sowie die Sauerstoffsättigung. Verglichen werden die Vitalwerte über einen jeweils 10-minütigen Beobachtungszeitraum vor und nach dem Baden des Kindes durch seine Eltern für alle drei Anleitungen; signifikante Unterschiede werden in ihrer Bedeutung als Stressindikatoren analysiert. In die Studie werden alle Kinder mit einem Geburtsgewicht von 1000 bis $1250 \mathrm{~g}$ eingeschlossen, die stationär im Perinatalzentrum Dresden aufgenommen wurden; es wird eine Teilnehmerzahl von 20 angestrebt. Raumtemperatur, Temperatur am Versorgungsplatz und Körpertemperatur des Kindes sowie in die Beobachtungszeit fallende medizinische und pflegerische Maßnahmen werden zur Kontrolle erfasst.

Ergebnisse: Die Studie ist noch nicht beendet; Voruntersuchungen haben bereits gezeigt, dass die im Routinemonitoring aufgezeichneten kardiorespiratorischen Parameter im vorher-nachher-Vergleich geeignet sind, das Stresslevel des Kindes relativ zuverlässig und objektiv zu indizieren.

Diskussion: Die Ergebnisse der Studie werden es ermöglichen, die für ein frühgeborenes Kind durch das Baden durch seine Eltern entstehenden Belastungen zu objektivieren. Dies ist insofern von Bedeutung, weil das Baden eine Situation darstellt, in der eine sensorische Stimulation des Kindes durch seine Eltern und eine gezielte Förderung der Eltern-Kind-Interaktion erfolgen kann. Allerdings können förderliche Wirkungen auf die Entwicklung des Kindes nur in Abhängigkeit von der Einhaltung seiner Belastungsgrenzen erwartet werden.

\section{Pfl.P02 \\ Intensiviertes Bonden/Känguruhen zur Förderung des Stillens und der ausschließlichen Muttermilchernährung}

\section{Braches' ${ }^{1}$ J. Adler ${ }^{2}$}

'Städtisches Klinikum Solingen, Neonatologisch/pädriatrische Intensivstation, Solingen, Deutschland, ${ }^{2}$ Städtisches Klinikum Solingen, Solingen, Deutschland

Hintergrund: Als dritte, von der WHO und UNICEF Initiative Babyfreundlich zertifizierte Kinderklinik in Deutschland ist eines unserer Ziele, die von uns betreuten Frühgeborenen und kranken Neugeborenen ausschließlich gestillt bzw. muttermilchernährt zu entlassen. In der Vergangenheit haben wir aber immer wieder erlebt, dass viele Mütter über die mehrwöchigen Krankenhausaufenthalte Probleme hatten, ihre Laktation in ausreichendem Maße zu etablieren und aufrecht zu erhalten.

Fragestellung: Mit welchen Maßnahmen können wir in der Praxis eine ausreichende Milchbildung und -erhaltung von Müttern frühgeborener oder kranker Neugeborener günstig beeinflussen.

Material und Methoden: Wir erläutern verschiedene Möglichkeiten, wie wir vorhandene Methoden intensiviert, Ressourcen ermittelt und genutzt haben. $\mathrm{Zu}$ erörtern sind unsere klinischen Beobachtungen in Bezug auf Effektivität und wir benennen Hindernisse sowie Grenzen, die wir erfahren haben.

Ergebnis: Als Ergebnis stellen wir vor, in welcher Form die Mutter-Kind-Paare von der neuen Vorgehensweise profitiert haben und welche Veränderung wir in der Still-Statistik erheben konnten.

Diskussion/Schlussfolgerung: Es ist mittlerweile gut belegt, dass ausschließliche Ernährung mit Muttermilch gerade zu früh geborenen und kranken Neugeborenen in besonderem Maße zu Gute kommt. Die Kunst ist es, den Müttern und Kindern eine Betreuung zukommen zu lassen, welche für die Etablierung und Erhaltung einer für lange Zeit ausreichenden Laktation besonders günstig ist. Daraus ziehen wir unsere Schlussfolgerung, die auch andere Kinderkliniken ermutigen soll, intensivere Stillförderung zu betreiben.

\section{Pfl.P03}

Transition to Home - Ein APN geleitetes interprofessionelles Betreuungsmodell in der Übergangsversorgung von Familien mit einem frühgeborenen Kind

T. Kohler' ', L. Stoffel', M. Gruber', K. Hirter' ${ }^{2}$, N. Schütz Hämmerli³, E. Cignacco ${ }^{4}$

'Universitätsklinik für Kinderheilkunde, Inselspital Bern, Abteilung

Neonatologie, Bern, Schweiz, ${ }^{2}$ Direktion Pflege Inselspital und

Spitalnetz Bern AG, Forschung und Entwicklung, Bern, Schweiz, ${ }^{3}$ Berner Fachhochschule Fachbereich Gesundheit Disziplin Geburtshilfe Forschung und Inselspital Bern Universitätsklinik für Kinderheilkunde, Bern, Schweiz, ${ }^{4}$ Berner Fachhochschule Fachbereich Gesundheit, Disziplin Geburtshilfe Forschung, Bern, Schweiz

Hintergrund: In der Schweiz kommen 7.2\% der Kinder zu früh zur Welt. Der Übergang vom Spital nach Hause ist für betroffene Familien besonders anspruchsvoll. Die physischen und emotionalen Belastungen, mangelnde Selbstwirksamkeit und -kompetenz der Eltern, sowie somatische Komplikationen der frühgeborenen Kinder führen zu verlängerten Hospitalisationen und Rehospitalisationen von bis zu 35\%. Professionelle Interventionen zur Stärkung der elterlichen Selbstwirksamkeit und -kompetenz, und weiterführende, koordinierte interprofessionelle Unterstützung nach Spitalaustritt sind im Schweizerischen Gesundheitssystem nicht etabliert. Die Eltern erleben dort eine Versorgungslücke.

Ziel: Um diese Versorgungslücke zu schliessen ist ein Advanced Practice Nurse (APN) geleitetes, interprofessionelles und nachhaltiges Modell zur Optimierung der Übergangsversorgung von Familien mit einem frühgeborenen Kind entwickelt und in der Praxis umgesetzt. In einer Pilotstudie ist die Durchführbarkeit der Interventionen evaluiert. Basierend darauf wird eine Interventionsstudie zur Überprüfung der Wirksamkeit und Kosteneffektivität des Modells durchgeführt. 
Methoden: Für die Entwicklung und Implementierung der APN-Rolle orientieren wir uns am 9-Schritte Modell nach PEPPA Participatory, Evidence-informed, Patient-centered, Process for APN role.

Ergebnisse und Schlussfolgerungen: Die Rolle und das Modell der APN sind entwickelt und werden in einem nächsten Schritt in die Praxis implementiert. Das familienzentrierte Modell beinhaltet bestehende spitalinterne und -externe Angebote. Rollen- und Modelldefinition: Die APN Neonatologie ist eine spezialisierte Pflegefachperson mit akademischem Abschluss. Sie übernimmt die Fallführung und ist als Drehscheibe zwischen den interprofessionellen Diensten tätig. Sie führt Bedarfserhebungen und Austrittsplanungen durch, berät, schult und begleitet Familien mit ihrem frühgeborenen Kind in der Übergangsphase vom Spital nach Hause. Die APN leitet ein Team von drei erfahrenen Neonatologie-Pflegefachpersonen, welche in familienzentrierter Pflege ausgebildet sind. Die Teammitglieder begleiten die Familien von Geburt an und während des gesamtem Klinikaufenthalts. Nach der Entlassung führt das APN-Team während sechs Monaten regelmässig Hausbesuche und Telefonberatungen durch. Resultate der Pilotstudie werden Ende 2018 erwartet.

\section{Pfl.P07 \\ ECPR (Angang unter Reanimation an die ECMO) bei kinderkardiologischen Patienten - Empfehlung der neuen ERC Leitlinie}

S. Dannemann', A. Ksellmann ${ }^{2}$

'Universitäts-Kinderklinik, Station 5, Bonn, Deutschland, ${ }^{2}$ Asklepios Kinderklinik St. Augustin, Abteilung Kinderherz-Intensivmedizin, Bonn, Deutschland

Zielsetzung: In der letzten Novellierung der ERC (European Resuscitation Council) Guideline 2015 wird zum ersten Mal die kardiale ECMO als Rescueverfahren bei Kreislaufstillstand von kardiologisch vorerkrankten Kindern empfohlen. Eine Vielzahl von Zentren verspricht sich durch diese Neuerung eine deutliche Verbesserung der Überlebensrate und des Outcome von herzkranken pädiatrischen Patienten. Im Kinderherzzentrum Sankt Augustin ist dieses Verfahren seit einigen Jahren bereits etabliert. „Ist eine Umsetzung der ECPR gemäß der neuen Empfehlungen des ERC möglich und wie groß ist der Benefit für den Patienten?"

Methode: Retrospektive Sichtung der ECMO Patientendaten der Jahre 2006-2013.

Ergebnis: Zwischen 2006-2013 wurden 71 Kinder mit einer ECPR behandelt. Das Körpergewicht lag zwischen 2,3 kg und $83 \mathrm{~kg}$, die Laufzeit der ECMO-Therapie lag zwischen $30 \mathrm{~h}$ bis $270 \mathrm{~h}$. Die Überlebensrate lag bei $46 \%($ ECPR Neo $=58 \%$, Päd. $=42 \%), 35 \%$ aller Patienten konnten nach ECMO-Therapie nach Hause entlassen werden.

Diskussion: Durch einen frühzeitig geplanten ECMO Angang unter Reanimation bei Patienten mit Herz-Kreislaufversagen und vorbestehendem Herzfehler kann die Überlebensrate deutlich gesteigert werden kann. Allerdings bedarf es hierzu eines klar strukturierten Konzeptes, eines interprofessionell geschulten Teams aus Pflege, Ärzten und Kardiotechnikern und örtlichen Strukturen, die eine ECMO Implantation in kurzer Zeit ermöglichen.

\section{Pflege Vorträge}

Invasive und Non-Invasive Beatmung

\section{Pfl.V01}

Praktische Erfahrungen mit der nicht-invasiven Beatmung auf einer pädiatrischen Intensivstation

A. Aurin' ${ }^{1}$ A. Radke'

'UKE Hamburg, Kinderintensiv, Hamburg, Deutschland

Die NIV - Beatmung hat sich in den letzten Jahren in der Pädiatrie als Therapieoption der akuten respiratorischen Insuffizienz (ARI)zunehmend etabliert und stellt aber nach wie vor, sowohl für das Pflegepersonal als auch für die Ärzte eine Herausforderung dar. Ein besonderes Augenmerk liegt in der Vielseitigkeit der unterschiedlichen Masken, der Anpassung, Fixierung und Beatmungseinstellung.

Indikationen, Kontraindikationen und Abbruchkriterien dürfen nicht außer acht gelassen werden. Um ein positives Ergebnis für die Patienten unter NIV - Beatmung zu erzielen, ist ein großer pflegerischer Aufwand in der Betreuung und Begleitung dieser Patienten notwendig. Dabei spielt die Zusammenarbeit aller beteiligten Berufsgruppen eine große Rolle. Ziel ist es, eine invasive Beatmung zu vermeiden, dennoch den richtigen Zeitpunkt zur Intubation zu stellen, um somit das Outcome der Patienten zu optimieren.

\section{Ethik - eine gemeinsame Sprache finden}

\section{Pfl.V02}

Was macht der Elefant auf der Intensivstation?

\section{Die Sprache der Pflege}

\section{Bergers' ${ }^{1}$ S. Spahl}

'Uniklinikum Hamburg Eppendorf, Kinderintensivstation, Hamburg, Deutschland, ${ }^{2}$ Uniklinikum Hamburg Eppendorf, Zentrum für Geburtshilfe, Kinder- und Jugendmedizin, Hamburg, Deutschland

„Wenn die Sprache nicht stimmt, so ist das, was gesagt wird, nicht das was gemeint ist.

Ist das, was gesagt wird, nicht das, was gemeint ist, so kommen die Werke nicht zustande." Legende von Konfuzius.

Theoretische Grundlagen der Sprache werden im Vortrag als Basiswissen kurz erläutert. Ausführlich eingegangen wird auf Redewendungen, Begrifflichkeiten, Verniedlichungen und mögliche Fehlinterpretationen, die durch die ,eigene Sprache“ Pflegender resultieren kann. Die Beispiele wurden auf insgesamt 10 Intensivstationen gesammelt und zusammengefasst. Dargestellt wird auch, welche Konsequenzen daraus gezogen werden müssen um auch professionell in der Pflegesprache dar zustehen.

\section{Pfl.V03}

Die „Harl.e.kin-Nachsorge“ - ein bayernweites Nachsorgemodell für früh- und risikogeborene Kinder und ihre Familien

K. Schwaier', S. Wolf', S. Schmidt-Goerke ${ }^{1}$

'Städtisches Klinikum München GmbH, Klinikum Schwabing, München, Deutschland, ${ }^{2}$ Lebenshilfe München Kinder und Jugend GmbH, München, Deutschland

Ziel der Harl.e.kin-Frühchen-Nachsorge ist die Betreuung von Familien mit Frühgeborenen oder Neugeborenen mit erheblichen Entwicklungsrisiken im Übergang von der Klinik nach Hause, solange Bedarf besteht. Die Geburt eines Früh- oder Risikoneugeborenen ist mit erheblichen Ängsten, Traumen und Problemen für die Familie und insbesondere für die Mütter verbunden. In den Kliniken gibt es in der Regel neben der medizinischen Betreuung, ein kompetentes Team aus Psychologen, Seelsorgern, Thera- 


\section{Abstracts}

peuten, die sich der Nöte dieser Familien annehmen. Nach der Entlassung reißt diese Betreuung ab. Hier greift die Harl.e.kin-Nachsorge ein. Die weitere Entwicklung eines Kindes verläuft umso günstiger, je besser die psychosoziale Situation des Kindes ist, in der es heranwächst. Diese zu optimieren, ist das Ziel der Harlekin-Frühchen-Nachsorge. Das Nachsorgeteam besteht aus Kinderkrankenschwestern der Klinik und aus Mitarbeiterinnen mit entsprechender Zusatzausbildung der regional zuständigen Frühförderstellen. Organisiert wird das Team von Sozialpädagogen, die bei Bedarf beratend tätig sind. Das Nachsorgeteam lernt die Familie auf Station kennen und betreut sie dann nach Entlassung des Kindes weiter- ganz nach individueller Situation - mit Hausbesuchen und telefonischer Beratung. Ziel ist die sinnvolle Überleitung in die Selbständigkeit oder, so erforderlich, in andere professionelle Betreuung. Das Harlekin-Nachsorge-System wird subsidiär finanziell unterstützt durch das Bayerische Staatsministerium für Arbeit und Soziales, Familie und Integration und fachlich begleitet über die Arbeitsstelle Frühförderung Bayern. In den letzten Jahren wurden über 4000 Familien in ganz Bayern im Rahmen der Harl.e.kin-Nachsorge betreut. 


\section{Autorenverzeichnis}

\begin{tabular}{|c|c|c|c|c|c|}
\hline A & & Berger, Angelika & FV01, FV22, FV25, FV28, & Bucher, Hans Ulrich & P052 \\
\hline Abdul-Khaliq, Hashim & FV57 & & FV36, FV51, FV60, P018, & Buchholtz, Stefan & P140 \\
\hline Abed, Haider & P156 & & P028, P059, P130, P134 & Buck, A.K. & P142 \\
\hline Ackermann, Benjamin & P053, P055, P115 & Berger, Hans & P089 & Bühler, C & P142 \\
\hline Adams, Ortwin & P097 & Berger, Stefanie & FV36 & Bühler-Weidle, Heike & P131 \\
\hline Adler, Jutta & Pfl.P02 & Bergers, Marlies & Pfl.V02 & Bührer, Christoph & P032, FV20, FV21, FV30, \\
\hline Ahmad, Hassan Mohamn & nad P046 & Bernbeck, Ulrich & P131 & & FV35, FV65, P011,P024, \\
\hline Aichler, Andrea & P131 & Bernhard, Wolfgang & FV04 & & P032, P043, P151 \\
\hline Alba-Alejandre, Irene & P102 & Berns, Monika & P032 & Burgmann, Johanna & FV52 \\
\hline Ali, Anaam & P152, P153 & Berwald, Mario & P147 & Burkhardt, Tilo & FV08 \\
\hline Allendorf, Antje & FV18 & Beyer, Wolfgang & P088 & Burkhardt, Wolfram & FV29 \\
\hline Altunlu, Engin & P162 & Bialas, Johanna & P075, P108 & Bürkle, Julia & FV04, P159 \\
\hline Altuntas, Özlem & P050 & Bielefeld, Thilo & P161 & Büssing, Arndt & $\mathrm{FV} 40$ \\
\hline Alvarez Tapia, Natalie & P103 & Biermayr, Marlene & P045 & Butter, Alin & Pfl.P01 \\
\hline Alzen, Gerhard & FV34 & Biesalski, Hans Konrad & FV33 & Buxmann, Horst & FV34, FV50, P023, P095, \\
\hline Andreas, Pomschar & P006 & Bigi, Sandra & P092 & & P105 \\
\hline Andriessen, Peter & P136 & Bigl, Arndt & P053 & & \\
\hline Apostolidou, Sofia & P044, P063 & Bigl, Nicole & P041 & & \\
\hline Arand, Jörg & FV07 & Binder, Christoph & FV01, P155 & C & \\
\hline Armbrust, Sven & P088 & Binder, Gerhard & P159 & Calder, Nuala DM & FV26 \\
\hline Arndt, Annett & P013 & Binder, Kathrin & FV43, P133 & Campbell, Douglas & P157 \\
\hline Arnold, Katrin & FV03, FV05, FV74 & Binder-Heschl, Corinna & FV09, FV11, FV63 & Carbon, Roman & P073 \\
\hline Artmann, Stefanie & P034 & Biskupski, Elisabeth & $\mathrm{FV} 29$ & Carnibella, Richard P. & FV19 \\
\hline Asgari, Siamak & P074 & Bittkowski, Nina & P036 & Cesnejvar, Robert & P146, P073 \\
\hline Asgeirsson, Alicia & FV69 & Blank, Douglas & FV09 & Cheung, Po-Yin & FV37 \\
\hline Assing, Blanca & FV41 & Blankenstein, Oliver & P064 & Choi, Arum & P156 \\
\hline Atkinson, Stephanie & P153 & Bläser, Annett & P055 & Christoph, Jürgen & P025 \\
\hline Aurin, Andrea & Pfl.V01 & Bleeker, Christine & FV04, P159 & Cignaccom, Eva Luzia & Pfl.P03 \\
\hline Austermann, Judith & FV52 & Blohm, Martin & P037 & Cordon, Ben & FV26 \\
\hline Avellina, Vanessa & FV02 & Boeckelmann, Moritz & P107 & Crossley, Kelly & FV09 \\
\hline Avenarius, Stefan & FV34 & Böhler, Thomas & FV07 & Czaba-Hnizdo, Christine & $\mathrm{FV} 28$ \\
\hline Avian, Alexander & FV37, P107, P111 & $\begin{array}{l}\text { Böhne, Carolin } \\
\text { Bohnhorst, Bettina } \\
\text { Bolz, Hanno Jörn }\end{array}$ & $\begin{array}{l}\text { P009 } \\
\text { FV34, FV38, P009 } \\
\text { P075 }\end{array}$ & Czernik, Christoph & FV30, FV35, P011 \\
\hline B & & Bonilla, Esteban & P128a & D & \\
\hline Backendorf, Alexander & P108 & Born, Mark & P143 & Dähnert, Ingo & P041 \\
\hline Bagci, Soyhan & FV61, P050, P051, P066, & Bösche, Christina & FV14 & Daifalla, Khaled & P080 \\
\hline & P068, P093, P127 & Bösing, Thomas & P090 & Dallèves, Fanny & P092 \\
\hline Bahlmann, Hagen & FV17 & Böttger, Ralf & FV34 & Dame, Christof & P052 \\
\hline Baik, Nariae & FV11, FV37, FV43, FV59, & & kham FV46, P064 & Dangl, Mercedes & FV01 \\
\hline & FV63, P133 & Braches, Claudia & Pfl.P02 & Dankelman, Jenny & P136 \\
\hline Bakry, Ahmed & P015 & Brait, Daniela & FV23 & Danne, Anne & P023 \\
\hline Bangen, Ursula & P048 & Brandes, Janine & P123 & Dawczynski, Kristin & P027 \\
\hline Barczyk-Kahlert, Katarzyr & na FV52 & Brandner, Johannes & P014, P113 & Degenhardt, Petra & FV21 \\
\hline Barikbin, Payman & FV21, P151 & Brandner, Ju. & P113 & Deinert, Isabell & P030 \\
\hline Bartels, Maren & P065 & Brasch, Frank & P069 & DeKoninck, Paul & FV19 \\
\hline Barthel, Michael & P090 & Braun, Wolfgang & P010 & DeMaio, Noemi & FV56 \\
\hline Bartmann, Peter & FV61, FV72, P050 & Brenner, Sebastian & FV46 & Dennecke, Bernd & P116 \\
\hline Bartz, Hans-Jürgen & P044 & Brentrup, A & P091 & Dettmers, Susanne & P117 \\
\hline Bates, Sarah & FV06 & Bressetti, Ilia & P012 & Dewitz, Ruth & FV18 \\
\hline Baumann, Nicole & FV72 & Breuer, Johannes & P039 & Dietrich, Olaf & P006 \\
\hline Baumgartner, Sigrid & FV60 & Brevis, Francisco & P125 & Dinger, Jürgen & P109, P110 \\
\hline Becker, Ingrid & P058 & Brickmann, Christian & FV32 & Dingley, John & FV24 \\
\hline Becker-Peth, Michael & FV31 & Brolund, Allan & P063 & Dittrich, Sven & P073 \\
\hline Beckmann, Matthias W. & P073, P080 & Brückner, Mag. Victoria & P018 & Dobermann, Helke & P027 \\
\hline Beger, Isabell & P147 & Bruckner-Tuderman, L. & P085 & Doberschütz, Nora & FV18 \\
\hline Belling-Dierks, Franziska & P164 & Bruder, Ingo & FV07 & Doherty, Dorota & $\mathrm{FV} 22$ \\
\hline Bendix, Ivo & FV23 & Brüning , Tanja & P108 & Dohna Schwake, Christia & $n \quad P 139$ \\
\hline Berg, Christoph & P050, P051 & Brunner, Barbara & P045 & Dransfeld, Frauke & P124 \\
\hline Bergander, Jörg & P020, Pfl.P01 & Bruns, Nora & P067, P124, P127 & Dresbach, Till & P066, P100, P143 \\
\hline & & Bubl, Benedikt Tobias & P092 & Dreschers, Stephan & P116 \\
\hline
\end{tabular}




$\begin{array}{ll}\text { Dreschers, Stephan } & \text { P144, } \\ \text { Dressel, Alexander } & \text { P096 } \\ \text { Dreyhaupt, Jens } & \text { FV34 } \\ \text { Druschke, Diana } & \text { FV03, } \\ \text { Duangdala, Phouvieng } & \text { FV46 } \\ \text { Dür, Mona } & \text { P018 } \\ \text { Dürr, Renate } & \text { P131 } \\ \text { Dutta, Sourabh } & \text { P015 } \\ \text { Dworschak, Gabriel } & \text { P071 } \\ & \\ \text { E } & \\ \text { Edel, Birgit } & \text { P027 } \\ \text { Eger, Beate } & \text { FV71 } \\ \text { Ehlen, Michael } & \text { P103 } \\ \text { Ehren, Ramus } & \text { P075 } \\ \text { Ehrhardt, Harald } & \text { P006 } \\ \text { Eickelberg, Oliver } & \text { P006 } \\ \text { Eifinger, Frank } & \text { FV48 } \\ \text { el Helou, Salhab } & \text { P015 } \\ \text { Elias, Sandra } & \text { P047 } \\ \text { Enders, Martin } & \text { P095 } \\ \text { Engler, Matthias } & \text { P147 } \\ \text { Eppich, Walter } & \text { FV47 } \\ \text { Ertl-Wagner, Birgit } & \text { P006 } \\ \text { Essers, Jochen } & \text { FV12 } \\ \text { Eyrich, Matthias } & \text { P142 }\end{array}$

\section{F}

\begin{tabular}{ll} 
Faas, Dirk & FV34 \\
Fandrey, Joachim & FV23 \\
Farhang, Mariam & P068 \\
Faschingbauer, Florian & P073, P080 \\
Fassl, Selina & FV52 \\
Fauchère, Jean-Claude & P052 \\
Faust, Kirstin & P065 \\
Fehrholz, Markus & P001, P008 \\
Felderhoff-Müser, Ursula & FV23, FV41, P047, P051, \\
& P067, P075, P108, P121, \\
Feldkötter, Markus & P124, P127, P139 076, P114 \\
Fenton, Tanis & P158 \\
Fischer, Franziska & Pf.P01 \\
Fischer, Georg & FV60 \\
Fischer, Hendrik & FV30, P011 \\
Fleischer, Ingo & P086 \\
Fleischer, Ulrike & P042 \\
Flemmer, Andreas W. & FV19, FV56, P006, P021, \\
& P128 \\
Förster, Kai Martin & P006, P021, P128 \\
Förster-Waldl, Elisabeth & P028, P059 \\
Fouras, Andreas & FV19 \\
Fracassi, Sara & P075 \\
Franck, Peter & P026 \\
Franz, Axel & FV04, FV62, P159 \\
Franzel, Julia & P049, P097 \\
Freidl, Thomas & P107 \\
Freisinger, Peter & P040, P060, P085 \\
Fremerey, Christian & P103, P160 \\
Freund, Daniel & P029 \\
Frieauff, Eric & P078, P164 \\
Friepörtner, Mattias & P091, P104 \\
Friesenhagen, Judith & FV52 \\
Fritsche, Kathrin & P035 \\
Fruth, Anja & P079 \\
& \\
\hline
\end{tabular}

\begin{tabular}{|c|c|c|c|}
\hline Fuchs, Hans & $\begin{array}{l}\text { FV32, FV34, P026, P081, } \\
\text { P082 P106, P149 }\end{array}$ & Harps, Egmont & P094 \\
\hline \multirow[t]{2}{*}{ Fusch, Christoph } & P015, P054, P152, P153, & Härtel, Christoph & $\begin{array}{l}\text { FV14, FV53, FV55, P065, } \\
\text { P070 }\end{array}$ \\
\hline & P156, P157, P158 & Härtel, Katharina & P098 \\
\hline \multirow[t]{4}{*}{ Fusch, Gerhard } & P015, P054, P152, P153, & Hartwich, Jana & P037 \\
\hline & P156, P157, P158 & Hattinger-Jürgenssen, Ern & na $\mathrm{P} 113$ \\
\hline & & Haufe, Susann & P138 \\
\hline & & Häusler, Martin & FV70, FV75 \\
\hline G & & Hausmann, Alexandra & P130 \\
\hline Gajdos, Marek & P010 & Heckmann, Matthias & FV17, FV34, P096, P112, \\
\hline Gale, Christopher & P155 & & P119, P157 \\
\hline Garten, Lars & P043 & Heep, Axel & FV06, FV26, P128a \\
\hline Gaspar, Harald & P084 & Heimann, Konrad & P083 \\
\hline Gastmeier, Petra & P024 & Heimberg, Ellen & FV44, FV47, P134, P135 \\
\hline Gatzweiler, Eva & P076 & Heindel, Katrin & FV58 \\
\hline \multicolumn{2}{|c|}{ Gebauer, Corinna Mirjam FV34, P055 } & Heinecke, Lucie Susanna & P132 \\
\hline Geffers, Christine & P024 & Heinrich, Luise & FV03, FV05, FV74 \\
\hline Geier, Norbert & P086, P089 & Heinzel, Oliver & FV44 \\
\hline Gensel, Dennis & P141 & Hennig, Judith & P020 \\
\hline \multicolumn{2}{|c|}{ Genzel-Boroviczény, Orsolya FV34, P034, P102 } & Hensel, Kai O. & P057 \\
\hline Gesser, Maren & P108 & Hentschel, Roland & FV07, FV16, FV34, P016, \\
\hline \multicolumn{2}{|c|}{ Giannikopoulou, Dimitra P091 } & & P017, P026, P061, P081, \\
\hline Giest, Henning & P140 & & P149 \\
\hline Gilberg, Eberhard & P088 & Herberg, Ulrike & P039 \\
\hline Gille, Christian & FV53, FV54, P118, P144 & Herber-Jonat, Susanne & FV56, P006, P021, P128 \\
\hline Giordano, Vito & $\mathrm{FV} 25, \mathrm{FV} 28, \mathrm{P} 130$ & Herndl, Elisabeth & P028, P059 \\
\hline Glaser, Kirsten & P001, P008, P164 & Hero, Barbara & P004 \\
\hline Glöckler, Martin & P073 & Herting, Egbert & FV14, FV55, P065, P070 \\
\hline Goeral, Katharina & $\mathrm{FV} 25, \mathrm{FV} 28$ & Hess, Johannes & P080 \\
\hline Goos, Tom & P136 & Hilberath, Johannes & P014 \\
\hline Göpel, Wolfgang & FV14, P065, P070 & Hilgendorff, Anne & P006 \\
\hline Göral, Katharina & FV51 & Hilger, Alina & FV15 \\
\hline Goretzki, Sarah & P075 & Hinner, Patricia & FV71, P019 \\
\hline Gortner, Ludwig & FV33, P033 & Hirter, Kathrin & Pfl.P03 \\
\hline Gottschalk, Ingo & P072 & Hischebeth, Gunnar T.R. & P143 \\
\hline Gräber, Stefan & FV57 & Hoberg, Kathrin & FV70, FV75 \\
\hline Graffstädt, Heiko & P140 & Hobrecht, Julia & $\mathrm{P} 124, \mathrm{P} 127$ \\
\hline Gratopp, Alexander & P150 & Hoehn, Thomas & P097 \\
\hline Gratzki, Nils & P073 & Hoenes, Marco & FV12 \\
\hline Grgic-Mustafic, Renata & FV59 & Hofbeck, Michael & FV44, FV47 \\
\hline Grinstein, Lev & P099 & Hohmann, Melanie & P069 \\
\hline Große Lordemann, Anja & P139 & Höhn, Thomas & FV46, FV73, P049, P064, \\
\hline Gruber, Michaela & Pfl.P03 & & P126 \\
\hline Gründler, Kerstin & FV62 & Hooper, Stuart & FV09, FV19 \\
\hline Grünzinger, Laura & FV57 & Hoppe, Bernd & P039, P072, P076, P114 \\
\hline Gudowius, Peter & P063 & Hornef, Mathias & P145 \\
\hline Guthmann, Florian & FV69, P025, P101 & Horsch, Sandra & P094 \\
\hline & & Hoyer, Heike & P027 \\
\hline & & Hoyer, Peter F & P075 \\
\hline $\mathrm{H}$ & & Hübener, Christoph & P006 \\
\hline Haas, Martin & & Huber, Sara & FV02 \\
\hline Habbig, Sandra & P072 & Hübner, Stephanie & P119 \\
\hline Haberl, Hannes & P056 & Huckenbeck, Wolfgang & P064 \\
\hline Hackenberg, Stephan & P142, P003 & Humberg, Alexander & P070 \\
\hline Hafner, Erich & FV51 & Hummler, Helmut & FV12, FV34, P010, P046, \\
\hline Haftel, Lior & P074 & & P056, P084 \\
\hline Hagemann Gysling, Kersti & tin $\mathrm{P} 092$ & Hüning, Britta & FV41, P108, P124 \\
\hline Hahn, Sigrid & P032 & Hürter, Hanna & FV50 \\
\hline Haiden, Nadja & FV01 & Hüseman, Dieter & FV65 \\
\hline Hajiyeva, Khadija & FV61 & Hütten, Matthias Christian & in FV33, P087, P136 \\
\hline Hallschmid, Manfred & FV02 & & \\
\hline Hamelmann, Eckard & P090 & & \\
\hline Hanke, Kathrin & FV14, P070 & I & \\
\hline Hänscheid, H & P142 & Ifflaender, Sascha & FV29, FV71 \\
\hline Hansen, Gesine & FV34 & Innocenti, Patricia & FV56 \\
\hline Harnisch, Sabine & FV16 & Iskander, Renata & P152 \\
\hline & & Ives, Kevin & P005 \\
\hline
\end{tabular}




\begin{tabular}{|c|c|c|c|c|c|}
\hline \multicolumn{2}{|l|}{ J } & Koss, Sarah & P029, P030 & Luksch, Hella & P099 \\
\hline Jani, Jacques & FV19 & Kostelka, Martin & P041 & Lüsebrink, Natalia & P105 \\
\hline Jansen, Christine & P128 & Köstenberger, Martin & FV59 & Lütz, Alawi & P141 \\
\hline Jenetzky, Ekkehart & FV15 & Köstlin, Natascha & FV53, FV54, P118 & Luyt, Karen & P128a \\
\hline Jenke, Andreas & P057 & Kovarova, Marketa & FV04 & Lwabi, Peter & P041 \\
\hline Jiang, Pingping & P057 & Kramer, Boris W. & FV22, FV33, P136 & & \\
\hline Jobe, Alan H. & $\mathrm{FV} 22$ & Krause, Jens Christian & P149 & & \\
\hline \multirow[t]{3}{*}{ John, Ruby } & P129 & Krauter, Sarah & P161 & $M$ & \\
\hline & & Kredel, M & P142 & Maas, Christoph & FV04, P159 \\
\hline & & Kreissl, Alexandra & FV01 & Maes, Elke & $\mathrm{FV} 24, \mathrm{FV} 27$ \\
\hline \multicolumn{2}{|l|}{ K } & Kremens, Bernhard & P075 & Maier, Rolf Felix & P098 \\
\hline Kaar, Katharina & P014 & Krempl, Christine & P008 & Mair, Eva-Maria & P056 \\
\hline Kalache, Karim & $\mathrm{FV} 21$ & Kreuzahler, Theresa & P008 & Marrin, Michael & P158 \\
\hline Kallapur, Suhas & $\mathrm{FV} 22$ & Kribs, Angela & FV31, FV45, P004, P022, & Marsch, Florian & FV15 \\
\hline \multirow{2}{*}{$\begin{array}{l}\text { Karnberger, Agnes } \\
\text { Karpienski, Julia }\end{array}$} & FV11 & & P048, P058, P072 & Martitz, Janine & P052 \\
\hline & P124 & Kriebel, Thomas & P035, P038 & Mathes, Michaela & FV04, P159 \\
\hline Kasper, David & FV51 & Krude, Heiko & P141 & Mattner, Barbara & P018 \\
\hline Kasprian, G. & FV25 & Kruszynski, Sandra & P123 & Maurer, Ellen & P033 \\
\hline Kasser, Severin & FV39 & Ksellmann, Anne & Pfl.P07 & Maurer, Kathrin & P045 \\
\hline \multirow{2}{*}{$\begin{array}{l}\text { Kathemann, Simone } \\
\text { Katzer, David }\end{array}$} & P075 & Kugler, Katrin & P018 & Maurer-Fellbaum, Ute & FV64, FV66 \\
\hline & FV61, P050, P051, P127 & Kühn, Thomas & FV46 & Mayatepek, Ertan & P097, P148 \\
\hline Kause, Franziska & FV15 & Kühne, Benjamin & FV45 & Mayer, Benjamin & FV12, P046 \\
\hline Kehl, Sven & P073 & Kujawa, Madelene & P021 & Meese, Eckart & P033 \\
\hline Keller, Titus & P022, P048, P058 & Kummer, Sebastian & P049 & Mehler, Katrin & P048, P058, P072 \\
\hline Kelzon, Svetlana & P103 & Kuntz, Martin & P026 & Meier, Petra & P053 \\
\hline Kemp, Matthew & FV22 & Kunzmann, Steffen & P001, P003, P008, P078, & Melichar, Volker & P073 \\
\hline Kerl, Kornelius & P091 & & P120, P142, P154 & Mendler, Marc Robin & FV12, P010, P046 \\
\hline Kerr, Lionel & FV19 & Küster, Helmut & P036 & Menz, Heike & P109 \\
\hline Kerry, Matthew & FV44 & Kutz, Patrizia & P122 & Menzel, Felix & P002 \\
\hline Kersten, Jan Felix & P132 & Kwan, Celia & P054 & Messner, Hubert & FV56 \\
\hline Kerwat, Martina & P098 & & & Metze, Boris & FV35 \\
\hline Keyser, Brigitte & P149 & & & Meyer, Thomas & P078 \\
\hline Kiechl-Kohlendorfer, Urs & ula P045 & $\mathrm{L}$ & & Michna, Dariusz & P108 \\
\hline Kieslich, Matthias & P105 & Landau-Crangle, Erin & P157, P158 & Mildenberger, Eva & P079 \\
\hline Kipfmueller, Florian & FV58 & Lang, Esther & FV44 & Mileder, Lukas & FV43, P133 \\
\hline Kirchgäßner, Christoph & FV45 & Lange, Anja & FV17, P096 & Mileder, Lukas Peter & FV11, FV59, FV63, P134, \\
\hline Kirchner, Alexander & P043 & Langer, Juliane & P052 & & P135 \\
\hline Kitchen, Marcus J. & FV19 & Langhammer, Kristina & FV31 & Minke, Ann-Katrin & FV35 \\
\hline Klarer, Noemi & FV39 & Längler, Alfred & FV40 & Minnich, Bernd & P014 \\
\hline Klebermaß-Schrehof, Ka & trin FV25, FV28, P130 & Lapaire, Olav & FV39 & Mitschke, Thomas & P035 \\
\hline Klee, Dirk & P148 & Leber, Melanie & P122 & Miura, Yuichiro & $\mathrm{FV} 22$ \\
\hline Kleemann, Beate & P062 & Lee, Katie & FV19 & Möbius, Marius Alexande & er $\mathrm{P} 029, \mathrm{P} 030$ \\
\hline Klein, Robert & P135 & Leiber, Anja & P118 & Modi, Neena & P155 \\
\hline Klein, Ruth & $\mathrm{P} 022$ & Leidinger, Petra & P033 & Mögel, Michael & FV13 \\
\hline Kleinlein, Barbara & FV34 & Leistner, Christiane & P103 & Mohns, Thilo & P136 \\
\hline Klemme, Mathias & $\mathrm{FV} 56, \mathrm{P} 021, \mathrm{P} 128$ & Lerche, Holger & P123 & Mohrmann, Matthias & FV07 \\
\hline Kling, Pamela J. & P052 & Lieftüchter, Victoria & $\mathrm{P} 021, \mathrm{P} 128$ & Möllmann, Cornelia & P016, P017, P081 \\
\hline Klotz, Daniel & FV32, P016, P017 & Lindner, Wolfgang & P084 & Molnar, Zoltan & P005 \\
\hline Knies, Ralf & P039 & Lindtner, Claudia & FV36 & Monz, Dominik & FV33, P033 \\
\hline Knöppel, Carmen & P098 & Linnemann, Knud & P112 & Morfeld, Christine & P101 \\
\hline Knüpfer, Matthias & P053, P055, P115 & Lipp, Patrick & P096 & Morra, Deanna & P015 \\
\hline Koch, Arite & FV13, FV29 & Lippert, Burkard & P086 & Morschhäuser, Joachim & P145 \\
\hline Koch, Christian & P053 & Liu, Kai & P158 & Moser, Katja & P065 \\
\hline Koch, Henner & P123 & Liu, Xun & FV24 & Mottaghy, Felix M. & P083 \\
\hline Koehne, Petra & P151 & Loellgen, Ruth Mari & P134 & Moxham, Alison & FV09 \\
\hline Kohler, Therese & Pfl.P03 & Lohmeier, Klaus & FV73 & Muche, Rainer & FV34 \\
\hline Köhler, Siegmund & P098 & Löllgen, Ruth & P135 & Mücke, Sonja & FV65 \\
\hline Koller, Brigitte & P052 & Longford, Nicholas & P155 & Muehler, Eberhard & P039 \\
\hline Kollertz, P. & P090 & Longin, Andreas & P162 & Muellenbach, Ralf & P142 \\
\hline Köninger, Angela & P067, P127 & Louwen, Frank & FV50 & Müller, Andreas & FV15, FV58, FV61, P050, \\
\hline Konstantelos, Dimitrios & P109 & Lücke, Thomas & P117 & & P051, P066, P068, P071, \\
\hline Kopf, Nina & FV64, FV66 & Lüdeke, Clara & FV50 & & P076, P093, P100, P114, \\
\hline Körber, Friederike & FV48 & Ludwig, Michael & FV15 & & P127, P143 \\
\hline Körner, H.T. & P069 & Ludwikowski, Barbara & FV69 & Müller, Dominik & FV20 \\
\hline Korsch, Eckhard & P075 & Luger, Beate & FV02 & Müller, Fabian & P074 \\
\hline Koslowski, Andrea & FV08 & Luibl, Sebastian & P077 & Müller, Hanna & P051, P067, P075, P124, \\
\hline & & Lukacs, Zoltan & P064 & & P127 \\
\hline
\end{tabular}




$\begin{array}{ll}\text { Müller, Judith } & \text { P141 } \\ \text { Müller, Stefan } & \text { P039 } \\ \text { Müller, Tessa } & \text { FV63 } \\ \text { Müller, Werner } & \text { P137 }\end{array}$

$\mathrm{N}$

$\begin{array}{ll}\text { N., N. } & \text { Pfl.P05, Pfl.P06 } \\ \text { Nagel, Manuela } & \text { FV65 } \\ \text { Nährlich, Lutz } & \text { P006 } \\ \text { Näther, Nicole } & \text { P109 } \\ \text { Nelle, Mathias } & \text { P092 } \\ \text { Neubauer, Rajko } & \text { P042 } \\ \text { Neubauer, Vera } & \text { P045 } \\ \text { Neuberger, Patrick } & \text { FV10 } \\ \text { Neunhoeffer, Felix } & \text { FV44 } \\ \text { NeoVita Study Group } & \text { P007 } \\ \text { Newnham, John P. } & \text { FV22 } \\ \text { Nicolai, Thomas } & \text { P086 } \\ \text { Niethammer, Klaus } & \text { P162 } \\ \text { Niggemann, Bodo } & \text { P150 } \\ \text { Nikiforou, Maria } & \text { FV33 } \\ \text { Nitsch-Felsecker, Patrizia P004 } \\ \text { Nitzsche, Katharina } & \text { FV13, P030, P110 } \\ \text { Nohr, Donatus } & \text { FV33 } \\ \text { Nordbeck, P } & \text { P142 } \\ \text { Notheis, Gundula } & \text { P102 } \\ \text { Nufer, Janaina } & \text { FV10 } \\ \text { Nusche, Andrea } & \text { P085 } \\ \text { Nußbaum, Claudia } & \text { P034 }\end{array}$

\section{0}

$\begin{array}{ll}\text { Oak, Prajakta } & \text { P006 } \\ \text { Oberthuer, André } & \text { FV45, P048, P058 } \\ \text { Oberweis, Sheila } & \text { P066 } \\ \text { Obrecht, Denise } & \text { P037 } \\ \text { Odd, David } & \text { FV06, FV26 } \\ \text { Ohl, Kim } & \text { P116 } \\ \text { Olischar, Monika } & \text { FV25, FV28 } \\ \text { Omran, Heymut } & \text { P091 } \\ \text { Ophelders, Daan } & \text { FV33, P136 } \\ \text { Orlikowsky, Thorsten W. } & \text { FV53, FV70, FV75, P083, } \\ & \text { P087, P116, P118, P144, } \\ & \text { P145 } \\ \text { Ortkras, Theresa } & \text { FV52 } \\ \text { Osredkar, Damjan } & \text { FV27 } \\ \text { Ostermeir, Anna-Lena } & \text { FV54 } \\ \text { Ott, Hagen } & \text { FV69 } \\ \text { Otten, Jörg-Elard } & \text { FV16 }\end{array}$

P

$\begin{array}{ll}\text { Paepke, Stefan } & \text { P137 } \\ \text { Pagel, Julia } & \text { FV53 } \\ \text { Palasz, Joanna } & \text { FV23 } \\ \text { Panagiotou, Panagiota } & \text { P090 } \\ \text { Panou, Evelina } & \text { P068 } \\ \text { Pansy, Jasmin } & \text { FV59, FV63, FV66, FV67, } \\ & \text { P111 } \\ \text { Papastergiou, Charikleia } & \text { P091, P104 } \\ \text { Paul, Pia } & \text { FV14, P070 } \\ \text { Paul, Thomas } & \text { P036 } \\ \text { Paulun, Annika } & \text { P135, P160 } \\ \text { Payne, Matthew } & \text { FV22 } \\ \text { Perez, Anna } & \text { FV49 }\end{array}$

\begin{tabular}{|c|c|c|c|}
\hline Peter, Corinna & FV38, P009, P069 & \multicolumn{2}{|c|}{ Rittenschober-Böhm, Judith FV22, FV51 } \\
\hline Peter, Ruef & FV07 & Robel-Tillig, Eva & FV68, P042, P138 \\
\hline Peters, Jochen & FV34 & Rochow, Niels & P054, P152, P153, P156 \\
\hline Petrasch, Maurice & P086 & & P157, P158 \\
\hline Petrasch, Renate & P086 & Rock, Marie & FV43, P133 \\
\hline Pfahl, Sebastian & FV73, P126, P148 & Rödel, Jürgen & P027 \\
\hline Pfeifer, Jochen & FV57 & Rodgers, Jennifer & $\mathrm{FV} 22$ \\
\hline Pfeifer, Yvonne & P027 & Rodgers, Karyn & FV09 \\
\hline Pfister, Marc & FV39 & \multicolumn{2}{|c|}{ Rodriguez Cabrera, Luis Alberto $\mathrm{P} 030$} \\
\hline Photiadis, Joachim & P151 & \multicolumn{2}{|c|}{ Roehr, Charles Christoph FV19, FV21, P005, P012 } \\
\hline \multirow[t]{2}{*}{ Pichler, Gerhard } & FV11, FV37, FV43, FV59, & Rohde, Oliver & FV34 \\
\hline & FV63, P133 & Röher, Katharina & P044 \\
\hline Pichler-Stachl, Elisabeth & FV37, FV64 & Roll, Claudia & P108, P122 \\
\hline Piening, Brar & P024 & Rolle, Hanna & P115 \\
\hline \multicolumn{2}{|c|}{ Pietruschka, Sascha Alexander P088 } & Rolle, Udo & $\mathrm{FV} 18$ \\
\hline Pilz-Klement, Ines & P094 & Römer, Dirk & P061 \\
\hline Pimpel, Birgit & FV51 & Römer, Susanne & $\mathrm{FV} 20$ \\
\hline Pirr, Sabine & FV38, FV52, P009 & Römer, Tristan & P087 \\
\hline Pitterle, Lisa & FV60 & Rompel, Oliver & P073 \\
\hline Platen, Christopher & P116, P144 & Rona, Zsofia & P130 \\
\hline Pletz, Mathias & P027 & Ronovsky, Marianne & FV36 \\
\hline Poeschl, Johannes & P157 & Röschinger, Wulf & P049 \\
\hline \multirow[t]{2}{*}{ Poets, Christian F. } & FV02, FV04, FV53, FV54, & Rosenbaum, Thorsten & P125 \\
\hline & FV62, P118, P123, P159 & Rösen-Wolff, Angela & P099 \\
\hline Pogodda, Sven & P088 & Rossi, Rainer & FV34 \\
\hline Pogorzelski, David & P015 & Roth, Bernhard & FV31, P004, P058 \\
\hline Pohler, Jutta & P035, P038 & Roth, Johannes & FV52 \\
\hline Polglase, Graeme & FV09 & Rothe, Karin & $\mathrm{FV} 20$ \\
\hline Pollak, Daniela & FV36 & Rothoeft, Tobias & P117 \\
\hline Poralla, Silvia & P093, P100 & Rücker, Viktoria & P164 \\
\hline Postberg, Jan & P057 & Rüdiger, Mario & FV03, FV05, FV13, FV29, \\
\hline \multicolumn{2}{|c|}{ Prieto Betancourt, Nicole P039 } & & FV71, FV74, P019, P020 \\
\hline Proquitté, Hans & P027 & & P029, P030, P109, P110 \\
\hline \multirow[t]{3}{*}{ Pulzer, Ferdinand } & P053, P055, P115 & & Pfl.P01 \\
\hline & & Ruef, Peter & P086, P089 \\
\hline & & Ruf, Katharina & P003, P154 \\
\hline $\mathbf{R}$ & & Ruhnau, Johanna & P096 \\
\hline Rabenau, Holger & FV50 & Ruschewski, Wolfgang & P036 \\
\hline Radke, Andrea & Pfl.V01 & Russell, Geoff & FV06 \\
\hline Ragazzi, Monica & P092 & & \\
\hline Raith, Wolfgang & $\begin{array}{l}\text { FV64, FV66, FV67, P107, } \\
\text { P111 }\end{array}$ & S & \\
\hline Raja, Preeya & P157 & Sabir, Hemmen & FV24, FV27, FV46, FV73, \\
\hline Rajendran, Gauthamen & P005 & & P049, P097, P126 \\
\hline Rajwich, Lea & P034 & Sadeghi, Kambis & P028, P059 \\
\hline Rascher, Wolfgang & FV34, P080 & Sailer, Nives & P162 \\
\hline Rasenack, Regina & FV16 & Salleck, Daniel & P146 \\
\hline Rath, Matthias & P088 & Sallmon, Hannes & $\mathrm{P} 052, \mathrm{P} 150, \mathrm{P} 151$ \\
\hline Rauch, Ralf & P131 & Salzer-Muhar, Ulrike & FV60 \\
\hline Rauthe, Stefan & P078 & Sander, Franziska & P063 \\
\hline Rechenauer, Tobias & P080 & Sandkötter, Julia & P091 \\
\hline Reichelt, Gabriele & P129 & Sangild, Per T. & P057 \\
\hline Reichert, Felix & P024 & Sarikaya, Sevgi & P148 \\
\hline \multirow[t]{2}{*}{ Reichert, Jörg } & FV03, FV05, FV71, FV74, & Sass, Steffen & P006 \\
\hline & P019, P020, P110, Pfl.P01 & Sauer, Harald & FV57 \\
\hline Reiss, Irwin & P136 & Saupp, Peter & P145 \\
\hline Rellensmann, Georg & P091 & Scaal, Martin & FV48 \\
\hline Repa, Andreas & FV01 & Schara, Ulrike & P108 \\
\hline Resch, Elisabeth & P117 & Schauer, Uwe & P117 \\
\hline \multirow[t]{2}{*}{ Reutter, Heiko } & FV15, P050, P068, P071, & Scheibe, Madlen & FV03, FV05, FV74 \\
\hline & P076, P114, P143 & Schenk, Wilfried & FV34 \\
\hline Richter, Jost Wigand & P101 & Scheuchenegger, Anna & FV67, P111 \\
\hline \multicolumn{2}{|c|}{ Richter, Manuela Friederike P101 } & Scheuern, Andrea & P084 \\
\hline Richter-Werkle, Renate & FV73 & Schiza, Barbara & P095 \\
\hline Rickenbacher, Hanna & FV39 & Schlatter, Sara-Maria & FV16 \\
\hline Riebe, Katharina & P040, P060 & Schlegel, P.G. & P142 \\
\hline Rieger-Fackeldey, Esther & P137 & Schleicher, Erwin & FV04 \\
\hline
\end{tabular}




\begin{tabular}{|c|c|c|c|c|c|}
\hline Schlößer, Rolf & FV18, FV34, FV50, P023, & Siedentopf, Jan-Peter & FV65 & U & \\
\hline & P095, P105 & Silwedel, Christine & P078 & Udin ten Cate, Floris & P048 \\
\hline Schmalisch, Gerd & FV21, FV30, P011, P032, & Singer, Dominique & FV49, P037, P044, P132 & Ullmann, Fabian & P114 \\
\hline & P151 & Singer, Katharina & P102 & Ullrich, Tim & FV30, P011 \\
\hline Schmeh, Isabella & P079 & Sirin, Selma & P124 & Umlauf, Volker & P160 \\
\hline Schmid, Manuel & FV12, FV34, FV46 & Sizonenko, Stéphane V. & FV23 & Uney, James & P128a \\
\hline Schmidt, Beatrix & P002, P140 & Sliutz, Gerhard & FV51 & Urban, Helen & P110 \\
\hline Schmidt, Johanna & FV32 & Söder, Stephan & P080 & Urlesberger, Berndt & FV11, FV25, FV37, FV43, \\
\hline Schmidt, Lisa & FV25, FV28, P130 & Sommerfeld, Marion & P019, Pfl.P01 & & FV59, FV63, FV64, FV66, \\
\hline Schmidt, Simone & FV46 & Spahl, Sonja & Pfl.V02 & & FV67, P107, P111, P133 \\
\hline Schmidt-Goerke, Silvia & Pfl.P04 & Spanaus, Katharina & FV08 & Urlesberger, Pia & FV37 \\
\hline Schmidtke, Susanne & P132 & Spaull, Robert & P128a & Uthaya, Sabita & P155 \\
\hline Schmidt-Wankel, Franzisk & ка P129 & Speer, Christian P. & P001, P008, P120, P164 & v. Osten, Heimke & P112 \\
\hline Schmitt, Jochen & FV03, FV05, FV74, P110 & Sperl, W. & P113 & & \\
\hline Schmitt, Mariangela & P149 & Spies, Claudia & P141 & & \\
\hline Schmölzer, Georg & FV37 & Spittler, Andreas & P028, P059 & V & \\
\hline Schmutz, Jan & FV44, FV47 & Spring, Bärbel & FV53, FV54, P118 & Valter Markus & \\
\hline Schneider, Hans Christop & h P040, P085, P060 & Stadler, Jasmin & FV43, P133 & van de Looii. Yohan & FV23 \\
\hline Schneider, Heike & P036 & Staffler, Alex & FV56, P134, P135 & van den Anker, John & FV39 \\
\hline Schneider, Hendryk & P082, P106 & Stähling, Ann-Christin & P067 & $\begin{array}{l}\text { van den Anker, Jonn } \\
\text { van den Bruck. Rhea }\end{array}$ & $\begin{array}{l}\text { FV39 } \\
\text { P057 }\end{array}$ \\
\hline Schneider, Mara & P079 & Stangl, Oliver & P077 & $\begin{array}{l}\text { van den Bruck, Khea } \\
\text { van der Linden Mark }\end{array}$ & $\begin{array}{l}\text { P05/ } \\
\text { P143 }\end{array}$ \\
\hline Schoberer, Anne & FV70, FV75 & Stavropoulou, Dimitra & FV16, P061, P149 & van der Linden, Mark & $\begin{array}{l}\text { P143 } \\
\text { FV49 }\end{array}$ \\
\hline Schoberer, Mark & FV70, P083, P087 & Stein, Anja & P047, P121 & $\begin{array}{l}\text { van der Meer, Frauke } \\
\text { Vanhatalo, Sampsa }\end{array}$ & $\begin{array}{l}\text { V449 } \\
\text { P130 }\end{array}$ \\
\hline Schomburg, Lutz & P052 & Steinau, Gerd & P087 & Vásquez-Jienez, Jaime & P083 \\
\hline Schön, Gerhard & P044 & Stenzel, Martin & P081 & Verena, Rybicki & P057 \\
\hline Schoner, Katharina & P090, P098 & Stephan, Victoria & FV03, FV05, FV74 & Vettorazzi, Eik & FV49 \\
\hline Schopen, Daniela & P161 & Stihsen, Birgit & FV51 & Viemann, Dorothee & $\begin{array}{l}F V 49 \\
\text { FV52 }\end{array}$ \\
\hline Schrading, Simone & P087 & Stoffel, Liliane & Pfl.P03 & $\begin{array}{l}\text { Viemann, Dorothee } \\
\text { Vierzig. Anne }\end{array}$ & FV48, P004, P072 \\
\hline Schrapel, Christian & FV14 & Stöger, Julian & FV67, P111 & $\begin{array}{l}\text { Vierzig, Anne } \\
\text { Vochem, Matthias }\end{array}$ & FV10 \\
\hline Schreiber, Mandy & FV68 & Storbeck, Tobias & P067, P124, P127 & $\begin{array}{l}\text { Vochem, I Iatthias } \\
\text { Vogelgesang Antie }\end{array}$ & P096 \\
\hline Schreiner, Christine & P093 & Storz, Lena & FV02 & Vogelgesang, Antje & P096 \\
\hline Schröder, Lukas & FV58 & Ströbele, Stephanie & P084 & Vogelmann, Margit & FV53 \\
\hline Schröder, Lukas & P143 & Strube, Juliane & P081 & Vogl, Thomas & $F \sqrt{F 2}$ \\
\hline Schubert; Sören & FV56 & Stüwe-Kunz, Laura & P122 & Völkl, Thomas M.K. & P080, P146 \\
\hline Schulte-Kemna, Eva & P089 & Sülz, Sandra & FV31 & von der Wense, Axel & P044, P063 \\
\hline Schulte-Kemna, Felix & P089 & Sunny, Donna & P119 & von Köckritz-Blickwede, & Maren FV52 \\
\hline Schulz, Stefan & FV51 & Supcun-Ritzler, Sirma & P122 & von Schnakenburg, Chri & tian P162 \\
\hline Schulze, Andreas & P006, P021 & Szekessy, David & $\begin{array}{l}\text { P122 } \\
\text { P043 }\end{array}$ & & \\
\hline Schulze, Lizzy & P002, P140 & & & & \\
\hline Schumacher, Johannes & FV15 & & & W & \\
\hline Schumann Stefan & FV/32 & & & Wagner, Marlene & P033 \\
\hline & FV3L & $\mathrm{T}$ & & Wagner, Michael & FV15 P134 P125 \\
\hline Schünemann, Marion & P084 & Taylan, Christina & P072 & wagner, Ivicnael & $\mathrm{FV} 25, \mathrm{PI} 34, \mathrm{P} 135$ \\
\hline Schupp, Wiebke & FV16 & te Pas, Arjan & FV09, FV19 & Wahl, Holger & FV33 \\
\hline Schütz Hämmerli, Natasc & ha Pfl.P03 & Teig, Norbert & FV34, P117 & Waitz, Markus & P010 \\
\hline Schwab, Frank & P024 & Tenbrock Klaus & P116 & Wald, Martin & P014, P113 \\
\hline Schwaberger, Bernhard & FV11, FV43, FV59, FV63, & $\begin{array}{l}\text { lenbrock, Klaus } \\
\text { Tesch, Falko }\end{array}$ & P116 & Waldhör, Thomas & FV51 \\
\hline & P133 & $\begin{array}{l}\text { Tesch, Falko } \\
\text { Thanhäuser Margarita }\end{array}$ & FV05 & Wallace, Megan & FV19 \\
\hline Schwaier, Karin & Pfl.P04 & Thanhäuser, Margarita & FV01 & Wannenmacher, Bardo & P041 \\
\hline Schwalm, Hanno & P091, P104 & Theis, Fabian & P006 & Waßermann, Undine & FV40 \\
\hline Schwartz Sabine & P137 & Thiel, Michael & FV40, FV42 & Weber, Claudia & P046 \\
\hline Schwarz Christonh & FV62 & Thiele, Holger & FV15 & Weber, Karin & P159 \\
\hline Schuarz lulian. & $\begin{array}{ll}\text { FVbL } \\
\text { P118 }\end{array}$ & Thio Lluch, Marta & FV19 & Weber, Lutz & P072 \\
\hline Schwarz, Jullan & P118 & Thome, Ulrich & FV34, P053, P055, P115 & Weber, Romy & FV02 \\
\hline Schweiger, Bernd & P124 & Thoresen, Marianne & FV24, FV27 & Weidenbach, Michael & P041 \\
\hline Schwindt, Eva & FV28 & Tibussek, Daniel & P126, P148 & Weishaupt, Eva & FV41 \\
\hline Seeger, Fabienne & P131 & Timme, Katharina & FV34 & Weiß, Christel & P124 \\
\hline Segerer, Hugo & FV34 & Tocut, Alexandru & P163 & Weisser Christof & $\begin{array}{l}P 124 \\
P 014\end{array}$ \\
\hline Seidel, Karoline & P009 & Topf, Hans-Georg & FV34, P073, P146 & Welser, Cnirstor & P014 \\
\hline Seidenberg, Jürgen & FV34 & Torresani, Toni & P064 & Weißhaar, Guido & FV45 \\
\hline Seidenspinner, Silvia & P008 & Trawöger, Rudolf & P045 & Wellmann, Sven & FV08, FV39 \\
\hline Seigal, Sandra & P157 & Trepels-Kottek Sonia & FV70 FV75 P083 P087 & Wendt, Anke & P150 \\
\hline Seipolt, Barbara & FV71 & 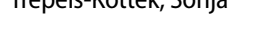 & $\begin{array}{l}\text { FV/0, FV/5, PU83, PU8/ } \\
\text { P135 }\end{array}$ & Weninger, Manfred & FV28 \\
\hline Seitz, Florian & P154 & Tröger, Birte & $\begin{array}{l}\text { PIS5 } \\
\text { FV55 }\end{array}$ & Werlein, Anna & P098 \\
\hline Selzer, Georg & P091, P104, P163 & Trntter Andreas & $\begin{array}{l}\text { FVbb } \\
\text { P161 }\end{array}$ & Wermuth, Marieke & P069 \\
\hline Senqpiel, Tobias & FV38 & & P161 & Werther, Tobias & P130 \\
\hline Serdar, Meray & FV23 & Tschernig, Thomas & FV33 & Wessely, Isabelle & P059 \\
\hline Shen, René Liang & P057 & Tutdibi, Erol & FV33, P033 & West, Christiane & P131 \\
\hline Shunova, Anna & FV04 & & & Weyers, W. & P089 \\
\hline
\end{tabular}




\section{Autorenverzeichnis}

Whybra-Trümpler, Catharina P079

Wiebe, Beatrix P160

Wiechers, Cornelia FV02, P159

Wiedemann, Holger P063

Wieg, Christian FV14, P065, P077

Wiegand, Gesa $\quad$ FV62

Wiethoff, Christiane Maria P129, P147

Wilbaux, Melanie FV39

Wilitzki, Silke FV21, P151

Willaschek, C P120

Willruth, Arne $\quad$ P050, P051

Winkler, Stefan P099

Wirbelauer, Johannes $\quad$ P120, P142, P154

Wirth, Stefan P057

Wisgrill, Lukas P028, P059

Witt, Armin FV51

Wittekindt, Boris P023

Wolf, Nadine $\quad$ P055, P115

Wolf, Sabine $\quad$ Pfl.P04

Wölfl, M P142

Wolke, Dieter $\quad$ FV72

Wood, Tommy $\quad$ FV27

Wosnik, Annette FV44

Wößner, Rupert P120

Wössner-Stegmann, Gaby P127

Wroblewski, Andreas P002, P140

Wurster, Torsten $\quad$ FV56

Y

Yacoub, Lisa P041

Z

Zahra, Valerie $\quad$ FV09

Zanke, Elsa $\quad$ P121

Zemlin, Michael P098

Zernickel, Maria $\quad$ FV34

Zhang, Rong FV15

Ziehenberger, Evelyn FV67, P111

Zimmermann, Andrea FV34, P137

Zimmermann, Roland FV08

Zimmermann, Ulrich P110

Zirn, Birgit P162

Zivanovic, Sanja $\quad$ P012

Zmyj, Norbert FV41

Zöllner, Christian P044

Zöllner, Nicola P109

Zur, Bernd P050 